\title{
Zum Ermüdungsverhalten von Stumpfnahtverbindungen bei sehr hohen Lastwechselzahlen
}

\author{
Von der Fakultät für Bauingenieurwesen und Geodäsie \\ der Gottfried Wilhelm Leibniz Universität Hannover \\ zur Erlangung des Grades
}

Doktor der Ingenieurwissenschaften

- Dr.-Ing. -

genehmigte Dissertation

von

Dipl.-Ing. Stefanie Steppeler

geboren am 19. März 1983 in Gütersloh 
Referent:

Korreferent:

Tag der Promotion:
Prof. Dr.-Ing. Peter Schaumann

Prof. Dr.-Ing. Michael Vormwald 


\section{Vorwort}

Die vorliegende Arbeit entstand während meiner Tätigkeit als wissenschaftliche Mitarbeiterin am Institut für Stahlbau der Gottfried Wilhelm Leibniz Universität Hannover. Mein besonderer Dank gilt meinem Doktorvater, Herrn Professor Dr.-Ing. Peter Schaumann, der mich bei der Anfertigung dieser Arbeit betreut und die Erstberichterstattung übernommen hat. Die fachlichen und fachübergreifenden Gespräche waren für mich von äußerst großem Wert. Sein Engagement und sein großes Interesse an meiner Arbeit haben mir wichtige Impulse gegeben und die Durchführung der Arbeit in einem hervorragenden Umfeld ermöglicht.

Für die Übernahme des Korreferates danke ich Herrn Professor Dr.-Ing. Michael Vormwald ganz herzlich. Sein entgegengebrachtes Interesse an meiner Arbeit und seine Diskussionsbereitschaft haben wichtige Anregungen gegeben. Ebenfalls sei Herrn Professor Dr.-Ing. Ludger Lohaus für das Mitwirken in der Promotionskommission sowie Herrn Professor Dr.-Ing. Martin Achmus für die Übernahme des Vorsitzes herzlich gedankt.

Die Grundlage der vorliegenden Arbeit bilden die Untersuchungen im Rahmen des NTH-Top-Down-Projektverbundes "Strategien und Methoden des Life-CycleEngineerings für Ingenieurbauwerke und Gebäude“ (Teilprojekt 3 „Integrierende Modellierungs-, Monitoring- und Bewertungsmethoden für mechanische Degradation und Ermüdung"), das von der Niedersächsisch Technischen Hochschule (NTH) gefördert wurde. Für die finanzielle Unterstützung bedanke ich mich herzlich.

Einen großen Dank möchte ich all meinen Kolleginnen und Kollegen am Institut für Stahlbau für die sehr gute Zusammenarbeit aussprechen. Das äußerst angenehme Arbeitsklima sowie die stete Diskussionsbereitschaft bei fachlichen Fragestellungen haben mir sehr geholfen. Insbesondere bedanke ich mich bei Mareike Collmann und Malte Gottschalk für die vielen gewinnbringenden Gespräche und ihre beständige Unterstützung. Bei Beiden möchte ich mich auch ganz herzlich für die kritische und sorgfältige Durchsicht dieser Arbeit bedanken.

Für die außerordentlich gewissenhafte Vorbereitung und tatkräftige Unterstützung bei der Durchführung der experimentellen Untersuchungen danke ich den Herren KarlHeinz Hentschel und Christian Fricke für ihren Einsatz. Desweiteren gilt Herrn Dr.-Ing. Attila Alt mein besonderer Dank für die kontinuierliche Diskussionsbereitschaft und Unterstützung im Bezug auf die Prüfvorrichtung. 
Weiterhin möchte ich mich bei den wissenschaftlichen Hilfskräften am Institut für Stahlbau für die tatkräftige Unterstützung bei den experimentellen Untersuchungen sowie den umfangreichen Auswertungen bedanken. Zudem gilt mein Dank den Studentinnen und Studenten, deren Abschlussarbeiten für mich eine große Unterstützung waren. An dieser Stelle sei besonders den Herren Matthias Bode und Robert Lee Gates sowie Frau Laura Marie Wagner gedankt.

Der abschließende Dank gilt Gerko sowie meiner Familie und meinen Freunden, die mir stets Rückhalt gegeben haben und durch ihre große Geduld und Unterstützung wesentlich zum Gelingen dieser Arbeit beigetragen haben.

Stefanie Steppeler 


\section{Kurzfassung}

Hochdynamisch beanspruchte Bauteile und Konstruktionen sind während ihrer Lebensdauer vielfach mehr als $10^{9}$ Lastwechseln ausgesetzt. Daher kommt dem Ermüdungsverhalten von Konstruktionselementen wie Schweißverbindungen im Hinblick auf eine sichere Betriebszeit und eine wirtschaftliche Bemessung eine besondere Bedeutung zu. In gültigen Regelwerken basiert die Bemessung von Stahlkonstruktionen gegen Ermüdung auf experimentell ermittelten Wöhlerlinien. Ab dem Abknickpunkt wird üblicherweise von einer Dauerfestigkeit ausgegangen. Allerdings ist der Verlauf der Ermüdungsfestigkeitskurven in diesem Bereich nicht abschließend geklärt. Die Existenz einer Dauerfestigkeit wird prinzipiell in Frage gestellt. Ermüdungsversuche an Schweißverbindungen mit mehr als $10^{7}$ Lastwechseln sind bisher aufgrund eingeschränkter Möglichkeiten in der Versuchsführung nur begrenzt verfügbar.

In dieser Arbeit wird das Ermüdungsverhalten einer axial beanspruchten Stumpfnahtverbindung aus Stahl bei konstanter Spannungsschwingbreite bis zu sehr hohen Lastwechselzahlen untersucht. Hierbei stehen die Weiterentwicklung der Prüftechnik, um Ermüdungsversuche in einem akzeptablen Zeitraum zu realisieren, sowie die Ausweitung der experimentellen Untersuchungen, die einen Beitrag zur Klärung der Fragestellung des Vorliegens einer Dauerfestigkeit leisten, im Vordergrund.

In einer hochfrequenten Prüfvorrichtung werden Ermüdungsversuche bis $5 \cdot 10^{8}$ Lastwechsel in einem akzeptablen Zeitraum realisiert. Neben der Untersuchung zeitlich veränderlicher Messgrößen während des Versuchs werden die optimierte Vorspannvorrichtung sowie die um eine frequenzabhängige Sollwertvorgabe erweiterte Regelung der Prüfvorrichtung vorgestellt. Zudem wird ein Kriterium zur Auswertung der Anrissschwingspielzahl definiert und die Größe dieses Anrisses untersucht.

Im Zeitfestigkeits- und Übergangsbereich werden Schwingversuche durchgeführt, um den Einfluss von Prüffrequenz und Mittelspannung auf die Ermüdungsfestigkeit zu untersuchen. Dabei zeigt sich kein signifikanter Einfluss der Prüffrequenz, wobei die Ergebnisse keine einheitliche Aussage zulassen. Der Einfluss einer erhöhten Mittelspannung ist im Zeitfestigkeitsbereich in Form einer flacheren Wöhlerlinie zu erkennen. Der Vergleich der Ergebnisse mit der normierten Auswertung von Versuchen aus der Literatur wird vorgenommen. Die Einstufung des Konstruktionsdetails in die entsprechende Kerbfallklasse gemäß Eurocode 3 wird annähernd bestätigt.

Die Ergebnisse dieser Arbeit zeigen, dass bei der untersuchten Schweißverbindung von einer Dauerfestigkeit auszugehen ist, da weder späte Brüche noch eine Änderung des Schädigungsmechanismus festgestellt wurden. Zudem zeigen Durchläufer in der erneuten Prüfung keine eindeutigen Hinweise auf eine Vorschädigung. 


\section{Abstract}

Components and structures under significant dynamic loading must often endure more than $10^{9}$ load cycles during their design life. In consequence, the fatigue behaviour of constructional elements such as welded joints becomes relevant ensuring secure service life and cost-effective design. In the regulations governing steel structures, the fatigue strength curves are established from fatigue tests. Below a certain cyclic stress, a fatigue limit has usually been assumed. However, the behaviour of the fatigue strength curve in this area is a topic of ongoing research. The existence of a fatigue limit is put into question in principal. In the case of welded joints, the experimental data beyond $10^{7}$ load cycles has not been extensively available until now, mainly due to the limitations of testing procedures.

This work examines the fatigue behaviour of an axially loaded butt weld under constant amplitude loading up to very high cycles. The focus lies on technical advances of fatigue testing, which allow for the realisation of experiments within reasonable time frames, as well as on making a contribution to clarify the question of the existence of a fatigue limit for welded steel joints by way of experimental results.

An acceptable period of testing time needed to reach $5 \cdot 10^{8}$ load cycles is realised by employing a high frequency testing device. Besides the investigation of time dependent data gathered during the test, the optimized preload device and the controller, which was extended by a frequency dependent setpoint setting, are presented.

High and very high cycle fatigue tests are conducted in order to investigate the influence of test frequency and mean stress on the fatigue strength. No significant influence of test frequency is observed, but the results do not allow for a consistent conclusion. The influence of increased mean stress is evident in the high cycle regime, resulting in a shallower fatigue strength curve. A comparison of the test results with normalised fatigue tests taken from literature is given. The classification of the constructional detail into the detail category according to Eurocode 3 is approximately confirmed.

The results of this work illustrate that the investigated welded joint shows a fatigue limit, as neither late cracks, nor a change in damage mechanism is detected. Furthermore, the repeated tests of specimens without failure indicate no precise evidence of pre-damage. 


\section{Schlagwörter}

Schweißverbindungen, Stahl, Ermüdungsversuche, sehr hohe Lastwechselzahlen, Wöhlerlinie, Prüffrequenz, Spannungsverhältnis

\section{Keywords}

Welded joints, Steel, Fatigue tests, Very high cycle fatigue (VHCF), SN-curve, Test frequency, Stress ratio 


\section{Inhaltsverzeichnis}

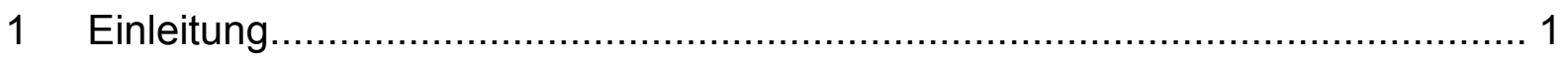

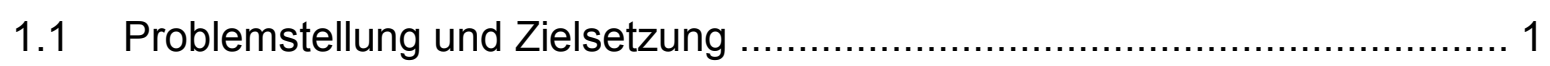

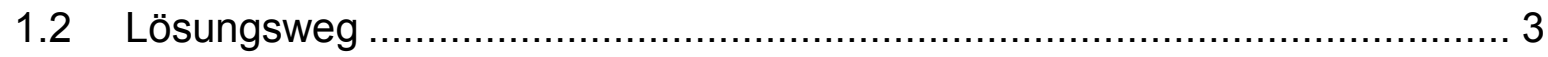

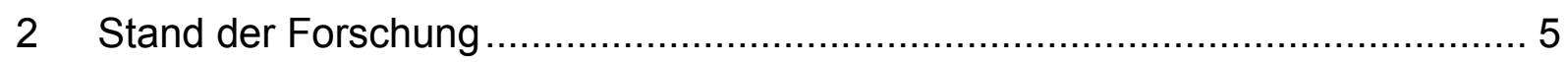

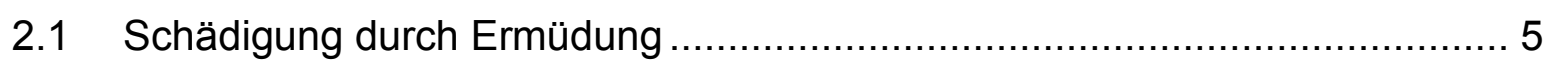

2.2 Ermüdungsfestigkeitskurven metallischer Werkstoffe ............................... 6

2.3 Einflussgrößen auf die Ermüdungsfestigkeit ............................................ 9

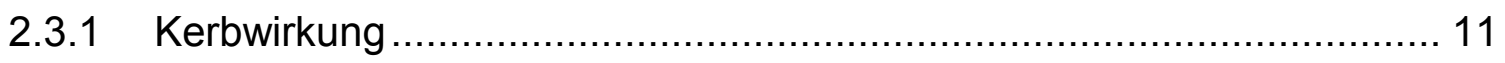

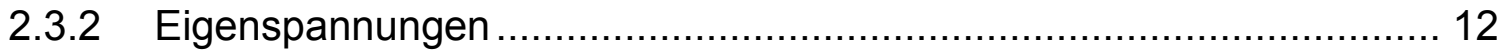

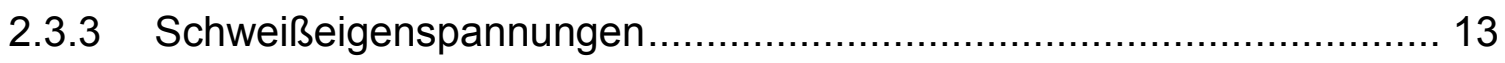

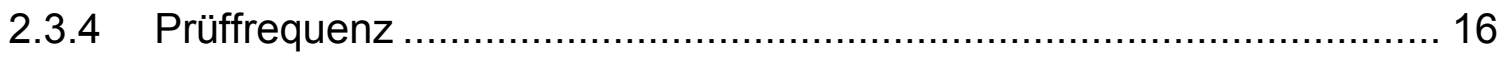

2.4 Ermüdungsversuche an Schweißverbindungen aus Stahl ...................... 18

2.5 Normative Regelungen und Empfehlungen ......................................... 25

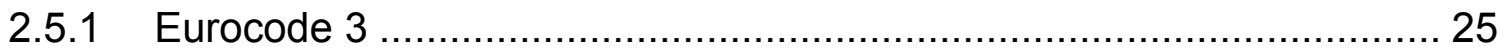

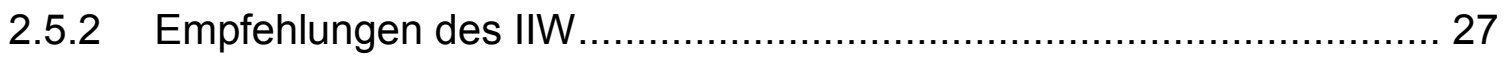

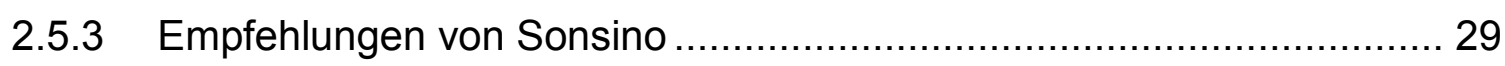

2.6 Prüftechniken im Bereich sehr hoher Lastwechselzahlen ........................ 30

2.7 Fazit im Hinblick auf die Untersuchungen der vorliegenden Arbeit ............. 31

3 Herstellung der Probekörper und Charakterisierung des Ausgangszustandes . 33

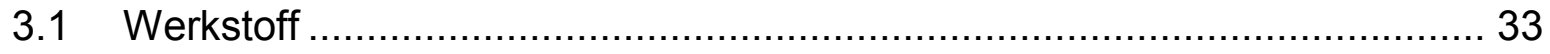

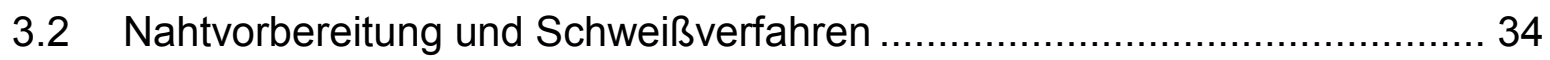

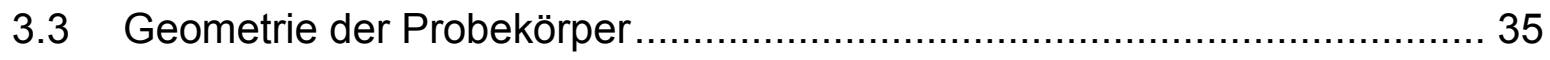

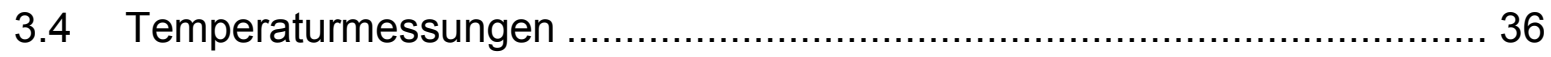

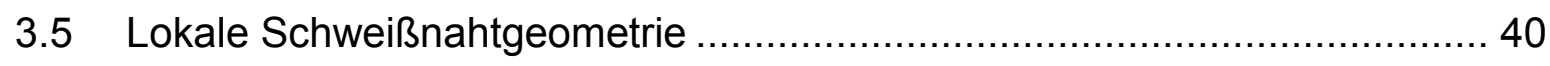

3.5.1 Aufnahme der lokalen Schweißnahtgeometrie …................................ 41

3.5.2 Auswertung der lokalen Nahtgeometrieparameter ............................. 42

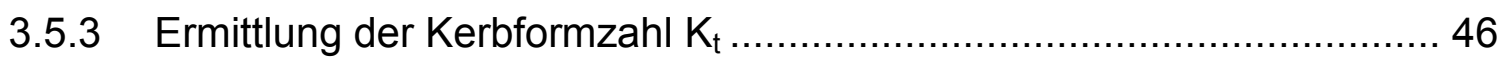




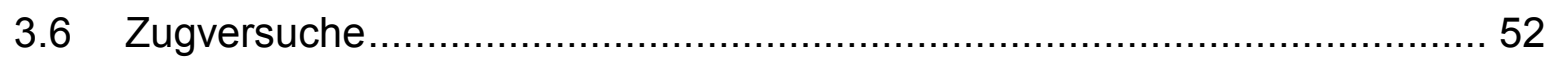

3.7 Metallographische Untersuchung ………......................................... 53

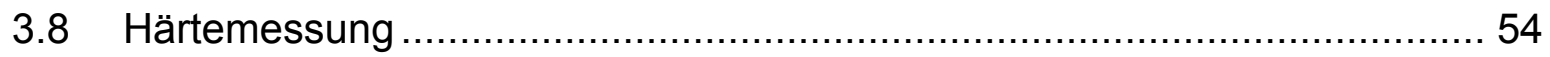

3.9 Zusammenfassung und Erkenntnisse ………................................. 56

4 Weiterentwicklung der Prüftechnik ............................................................ 59

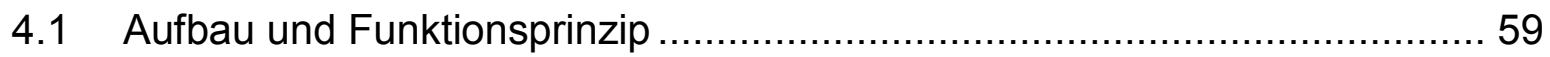

4.2 Versuchsablauf und Weiterentwicklungen ............................................. 61

4.2.1 Vorspannvorgang des Probekörpers ................................................ 61

4.2.2 Festlegung von Prüfkraftamplitude und Abbruchkriterien .................... 62

4.2.3 Start des Versuchs und Aufzeichnung der Messdaten ........................ 63

4.2.4 Weiterentwicklungen der Prüfvorrichtung ......................................... 63

4.3 Zeitlich veränderliche Messgrößen..................................................... 64

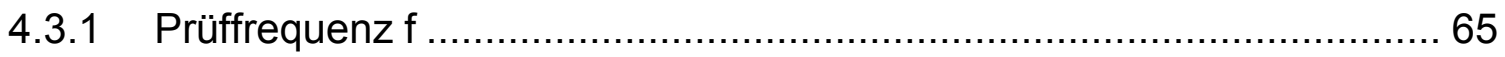

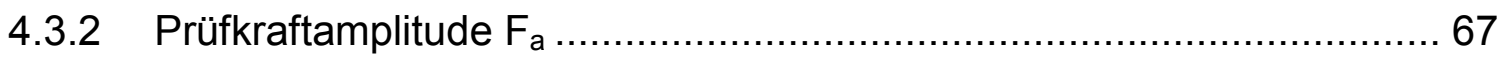

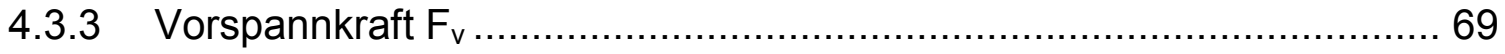

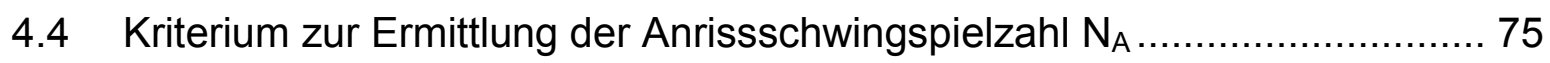

4.5 Untersuchung zur Größe des vorliegenden Anrisses .............................. 76

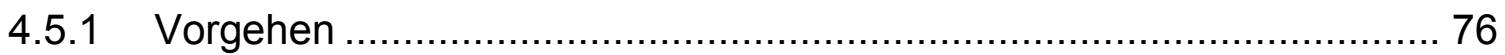

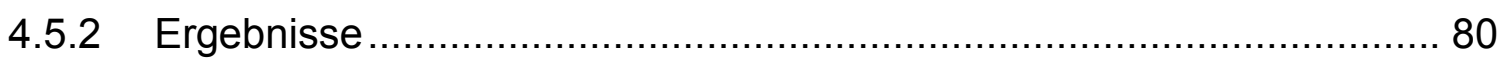

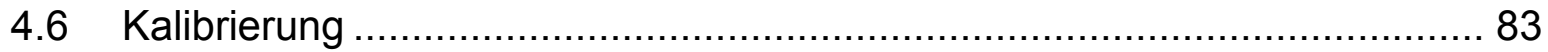

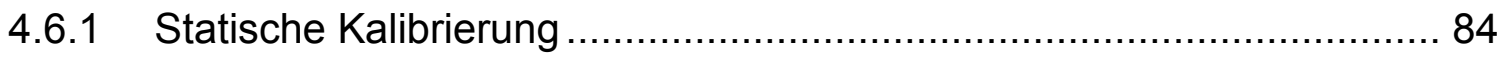

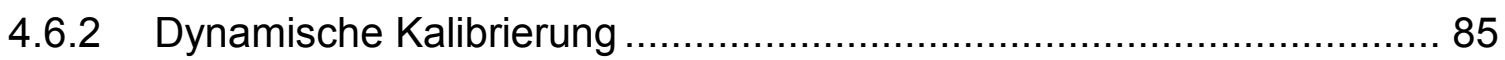

4.7 Zusammenfassung und Erkenntnisse ………................................... 91

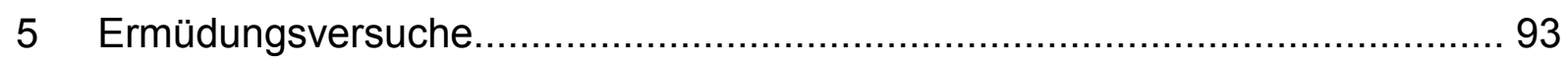

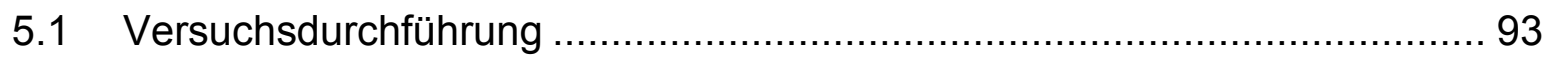

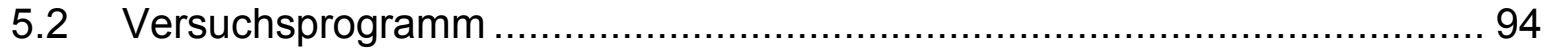

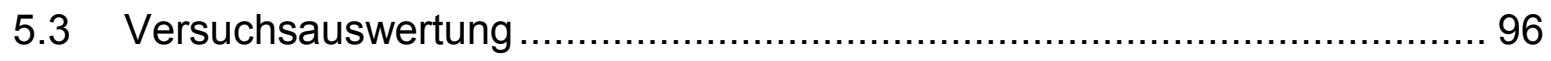

5.3.1 Untersuchung des Zeitfestigkeitsbereichs ....................................... 96

5.3.2 Untersuchung des Übergangsbereichs .......................................... 97 
5.3.3 Erneute Prüfung von Durchläufern.................................................... 100

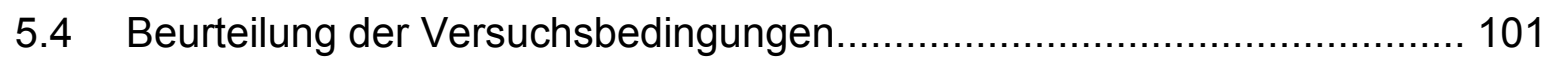

5.4.1 Soll- und Istwerte von Kraftamplitude und Vorspannkraft ................... 101

5.4.2 Vorspannkraftabfall zu Versuchsbeginn.......................................... 102

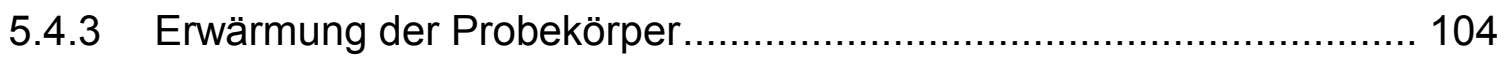

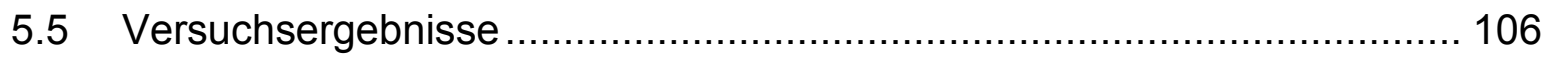

5.5.1 Allgemeine Auswertung und Darstellung der Versuchsergebnisse..... 107

5.5.2 Einfluss der Prüffrequenz............................................................... 111

5.5.3 Einfluss der Mittelspannung ...................................................... 117

5.5.4 Bewertung der hochgesetzten Durchläufer..................................... 119

5.5.5 Beurteilung der Bruchflächen...................................................... 122

5.5.6 Verhalten im Bereich sehr hoher Lastwechselzahlen ....................... 126

5.5.7 Vergleich der Versuchsergebnisse mit Sonsino et al ......................... 127

5.5.8 Vergleich der Versuchsergebnisse mit Eurocode 3 .......................... 129

5.6 Zusammenfassung und Erkenntnisse .............................................. 131

6 Zusammenfassung und Ausblick............................................................ 135

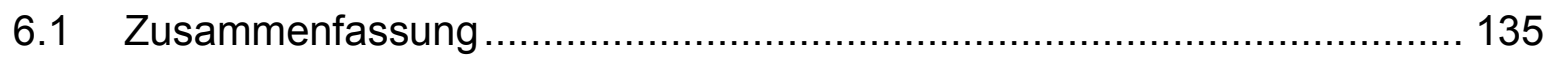

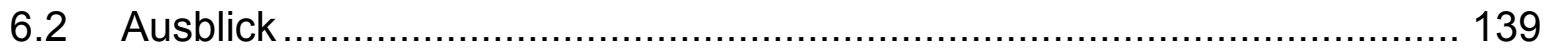

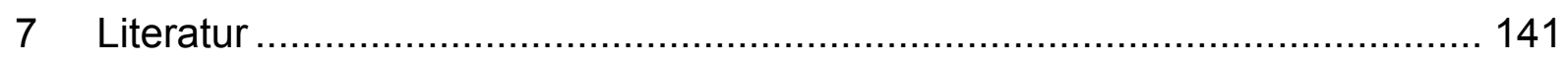

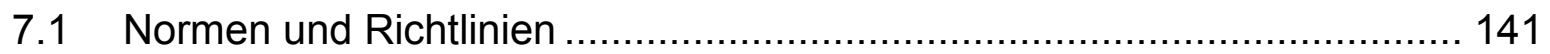

7.2 Fachbücher, Monographien, Aufsätze und Berichte............................... 143

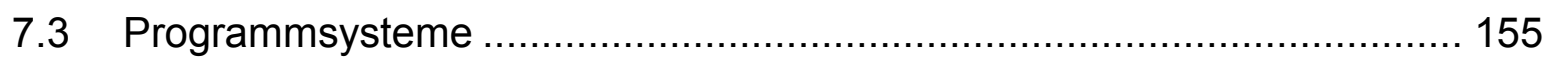

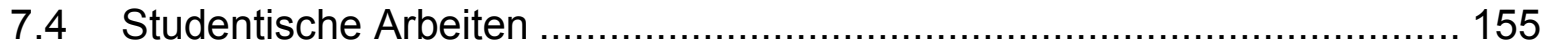

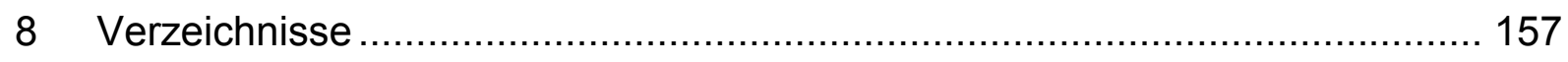

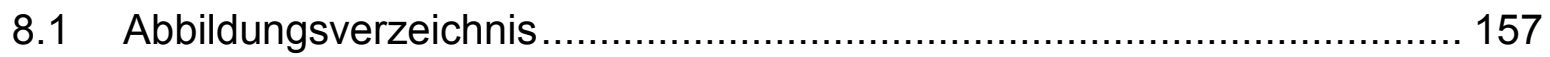

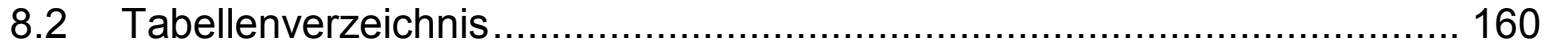

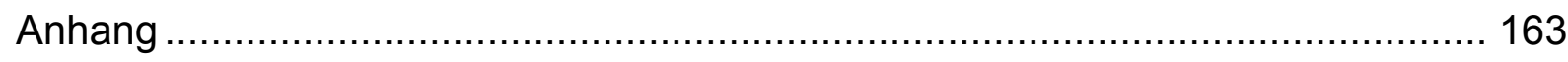




\section{Bezeichnungen}

\section{Lateinische Buchstaben}

\begin{tabular}{|c|c|c|}
\hline A & {$[\%]$} & Bruchdehnung \\
\hline$A_{N}$ & {$\left[\mathrm{~mm}^{2}\right]$} & Nennprüfquerschnittsfläche \\
\hline $\mathrm{b}$ & {$[\mathrm{kN} / \mathrm{V}]$} & Achsenabschnitt einer Geraden \\
\hline b & {$[\mathrm{mm}]$} & Spalt nach DIN EN ISO 9692-1 \\
\hline b & {$[\mathrm{mm}]$} & Maß der Breiten in DIN EN ISO 5817 \\
\hline$b_{N}$ & {$[\mathrm{~mm}]$} & Breite am Nahtübergang \\
\hline$b_{W}$ & {$[\mathrm{~mm}]$} & Breite am Wurzelübergang \\
\hline C & {$[\mathrm{mm}]$} & Absatz nach DIN EN ISO 9692-1 \\
\hline E & {$\left[\mathrm{N} / \mathrm{mm}^{2}\right]$} & Elastizitätsmodul \\
\hline f & {$[\mathrm{Hz}]$} & Prüffrequenz \\
\hline $\mathrm{F}$ & {$[\mathrm{kN}]$} & Kraft \\
\hline $\mathrm{F}_{\mathrm{a}}$ & {$[\mathrm{kN}]$} & Kraftamplitude \\
\hline$F_{m}$ & {$[\mathrm{kN}]$} & Mittellast \\
\hline$F_{v}$ & {$[\mathrm{kN}]$} & Vorspannkraft \\
\hline$f_{y}$ & {$\left[\mathrm{~N} / \mathrm{mm}^{2}\right]$} & Streckgrenze bzw. Fließgrenze \\
\hline h & {$[\mathrm{mm}]$} & Maß der Höhe in DIN EN ISO 5817 \\
\hline$h_{N}$ & {$[\mathrm{~mm}]$} & Höhe am Nahtübergang \\
\hline$h_{W}$ & {$[\mathrm{~mm}]$} & Höhe am Wurzelübergang \\
\hline $\mathrm{k}$ & {$[-]$} & Neigungsexponent der Wöhlerlinie \\
\hline$k^{*}$ & {$[-]$} & Neigungsexponent der Wöhlerlinie nach dem Abknickpunkt \\
\hline kf & {$[\mathrm{kN} / \mathrm{V}]$} & Übertragungsfaktor \\
\hline kfd & {$[\mathrm{kN} / \mathrm{V}]$} & dynamischer Kalibrierfaktor \\
\hline $\mathrm{kfd}_{\text {Bezug }}$ & {$[\mathrm{kN} / \mathrm{V}]$} & Korrekturfaktor der frequenzabhängigen Sollwertkorrektur \\
\hline $\mathrm{kfd}_{\text {linear }}$ & {$[\mathrm{kN} / \mathrm{V}]$} & Korrekturfaktor der frequenzabhängigen Sollwertkorrektur \\
\hline kfs & {$[\mathrm{kN} / \mathrm{V}]$} & statischer Kalibrierfaktor \\
\hline $\mathrm{K}_{\mathrm{t}}$ & {$[-]$} & Spannungskonzentrationsfaktor (Kerbformzahl) \\
\hline $\mathrm{m}$ & {$[\mathrm{kN} /(\mathrm{Hz} \cdot \mathrm{V})]$} & Steigung einer Geraden \\
\hline $\mathrm{n}$ & {$[-]$} & Anzahl der Versuche \\
\hline $\mathrm{N}$ & {$[-]$} & Schwingspielzahl \\
\hline $\mathrm{N}_{\mathrm{A}}$ & {$[-]$} & Anrissschwingspielzahl \\
\hline$N_{B}$ & {$[-]$} & Bruchschwingspielzahl \\
\hline $\mathrm{N}_{\mathrm{G}}$ & {$[-]$} & Grenzschwingspielzahl \\
\hline $\mathrm{N}_{\mathrm{k}}$ & {$[-]$} & Knickschwingspielzahl \\
\hline $\mathrm{p}_{\%}$ & {$[\%]$} & prozentuale Abweichung \\
\hline $\mathrm{p}_{\mathrm{k}}$ & {$[\% / \mathrm{Hz}]$} & Korrekturfaktor der frequenzabhängigen Sollwertkorrektur \\
\hline
\end{tabular}




\begin{tabular}{|c|c|c|}
\hline$P_{u ̈}$ & [\%] & Überlebenswahrscheinlichkeit \\
\hline r & {$[\mathrm{mm}]$} & Kerbradius \\
\hline $\mathrm{R}$ & {$[-]$} & Spannungsverhältnis \\
\hline$r^{2}$ & {$[-]$} & Determinationskoeffizient \\
\hline $\mathrm{R}_{\text {Abfall }}$ & {$[-]$} & Spannungsverhältnis nach Vorspannkraftabfall \\
\hline $\mathrm{R}_{\mathrm{eH}}$ & {$\left[\mathrm{N} / \mathrm{mm}^{2}\right]$} & Streckgrenze \\
\hline $\mathrm{R}_{\mathrm{m}}$ & {$\left[\mathrm{N} / \mathrm{mm}^{2}\right]$} & Zugfestigkeit \\
\hline$r_{N}$ & {$[\mathrm{~mm}]$} & Kerbradius am Nahtübergang \\
\hline $\mathrm{R}_{\text {Start }}$ & {$[-]$} & Spannungsverhältnis bei Versuchsbeginn \\
\hline $\mathrm{R}_{\mathrm{t}}$ & {$[\mu \mathrm{m}]$} & Rauhtiefe \\
\hline$r_{W}$ & [mm] & Kerbradius am Wurzelübergang \\
\hline $\mathrm{t}$ & {$[\mathrm{mm}]$} & Blechdicke \\
\hline $\mathrm{T}$ & {$\left[{ }^{\circ} \mathrm{C}\right]$} & Temperatur \\
\hline$t_{8 / 5}$ & [s] & Abkühlzeit von $800^{\circ} \mathrm{C}$ auf $500^{\circ} \mathrm{C}$ \\
\hline$t_{A}$ & [mm] & Anrisstiefe \\
\hline $\mathrm{T}_{\text {Anstieg }}$ & {$\left[{ }^{\circ} \mathrm{C}\right]$} & Temperatur auf näherungsweise konstantem Plateau \\
\hline $\mathrm{T}_{\max }$ & {$\left[{ }^{\circ} \mathrm{C}\right]$} & Spitzentemperatur \\
\hline$t_{\min }$ & {$[\mathrm{mm}]$} & minimale Anrisstiefe \\
\hline $\mathrm{T}_{\mathrm{N}}$ & {$[-]$} & Streuspanne über die Schwingspielzahl \\
\hline $\mathrm{T}_{\text {Start }}$ & {$\left[{ }^{\circ} \mathrm{C}\right]$} & Temperatur bei Versuchsbeginn \\
\hline$t_{v}$ & {$[\mathrm{~mm}]$} & Kantenversatz \\
\hline $\mathrm{T}_{\sigma}$ & {$[-]$} & Streuspanne über die Spannungsschwingbreite \\
\hline$U_{p}$ & [V] & Probendehnung \\
\hline$U_{R}$ & [V] & Rahmendehnung \\
\hline$W_{t}$ & {$[\mu \mathrm{m}]$} & Welligkeitstiefe \\
\hline
\end{tabular}

\section{Griechische Buchstaben}

$\begin{array}{lll}\alpha & {\left[{ }^{\circ}\right]} & \text { Öffnungswinkel in DIN EN ISO 9692-1 } \\ \alpha & {\left[{ }^{\circ}\right]} & \text { Nahtübergangswinkel in DIN EN ISO } 5817 \\ \Delta \mathrm{F} & {[\mathrm{kN}]} & \text { Kraftdifferenz } \\ \Delta \mathrm{N} & {[-]} & \text { Schwingspielzahldifferenz } \\ \Delta R & {[-]} & \text { Spannungsverhältnisdifferenz } \\ \Delta T & {[\mathrm{~K}]} & \text { Temperaturdifferenz } \\ \Delta t_{\mathrm{M}} & {[\mathrm{s}]} & \text { Dauer des Markierungsblocks } \\ \Delta \mathrm{t}_{\mathrm{V}} & {[\mathrm{s}]} & \text { Dauer des Versuchsblocks } \\ \Delta \sigma & {\left[\mathrm{N} / \mathrm{mm}^{2}\right]} & \text { Spannungsschwingbreite } \\ \Delta \sigma_{\mathrm{C}} & {\left[\mathrm{N} / \mathrm{mm}^{2}\right]} & \text { Charakteristische Ermüdungsfestigkeit bei } \mathrm{N}=2 \cdot 10^{6}\end{array}$




$\begin{array}{lll}\Delta \sigma_{D} & {\left[\mathrm{~N} / \mathrm{mm}^{2}\right]} & \text { Spannungsschwingbreite der Dauerfestigkeit } \\ \Delta \sigma_{\mathrm{M}} & {\left[\mathrm{N} / \mathrm{mm}^{2}\right]} & \text { Spannungsschwingbreite des Markierungsblocks } \\ \Delta \sigma_{V} & {\left[\mathrm{~N} / \mathrm{mm}^{2}\right]} & \text { Spannungsschwingbreite des Versuchsblocks } \\ \Theta & {\left[^{\circ}\right]} & \text { Anstiegswinkel } \\ \Theta_{\mathrm{N}} & {\left[^{\circ}\right]} & \text { Anstiegswinkel am Nahtübergang } \\ \Theta_{\mathrm{W}} & {\left[^{\circ}\right]} & \text { Anstiegswinkel am Wurzelübergang } \\ \rho_{V} & {\left[^{\circ}\right]} & \text { Winkelverzug } \\ \sigma_{\mathrm{a}} & {\left[\mathrm{N} / \mathrm{mm}^{2}\right]} & \text { Spannungsamplitude } \\ \sigma_{E} & {\left[\mathrm{~N} / \mathrm{mm}^{2}\right]} & \text { Eigenspannung } \\ \sigma_{\mathrm{m}} & {\left[\mathrm{N} / \mathrm{mm}^{2}\right]} & \text { Mittelspannung } \\ \sigma_{\max } & {\left[\mathrm{N} / \mathrm{mm}^{2}\right]} & \text { Maximale Kerbspannung } \\ \sigma_{\mathrm{N}} & {\left[\mathrm{N} / \mathrm{mm}^{2}\right]} & \text { Nennspannung } \\ \sigma_{\mathrm{o}} & {\left[\mathrm{N} / \mathrm{mm}^{2}\right]} & \text { Oberspannung } \\ \sigma_{\mathrm{u}} & {\left[\mathrm{N} / \mathrm{mm}^{2}\right]} & \text { Unterspannung }\end{array}$

\section{Abkürzungen}

EC3 Eurocode 3 Teil 1-9

FEM Finite-Elemente-Methode

FGA Fine Granular Area

GBF Granular-Bright Facet

GW Grundwerkstoff

HCF High Cycle Fatigue

IIW International Institute of Welding

LCF Low Cycle Fatigue

MAG Metall-Aktivgas

ODA Optical Dark Area

SG Schweißgut

TE Thermoelement

VHCF Very High Cycle Fatigue

WEZ Wärmeeinflusszone

ZFP Zerstörungsfreie Prüftechnik 


\section{Einleitung}

\subsection{Problemstellung und Zielsetzung}

In der Verbindungstechnik des Stahlbaus wird zwischen lösbaren und unlösbaren Verbindungen unterschieden. Die klassische lösbare Verbindung stellt die Schraubverbindung dar, wohingegen das Schweißen als Standardfügeverfahren zur Herstellung unlösbarer Verbindungen anzusehen ist. Schweißverbindungen in Stahlkonstruktionen sind ein wesentliches Konstruktionselement. Auf der einen Seite ist das Schweißen ein schnelles, effizientes und kostengünstiges Fügeverfahren. Auf der anderen Seite gehören Schweißverbindungen aufgrund der geometrischen und strukturellen Kerbwirkung zu den ermüdungskritischen Details einer Konstruktion. Im Hinblick auf die wirtschaftliche Bemessung und die sichere Betriebszeit einer Struktur kommt dem Ermüdungsverhalten von Konstruktionsdetails wie Schweißverbindungen eine besondere Bedeutung zu.

Im Bezug auf die Ermüdungsfestigkeit werden Stahlkonstruktionen nach gültigen Regelwerken wie dem Eurocode 3 (EC3) [DIN EN 1993-1-9, 2010] oder den Empfehlungen des International Institute of Welding (IIW) [IIW, 2009] ausgelegt. Die klassische Beschreibung der Ermüdungsfestigkeit eines Werkstoffes oder Bauteils erfolgt über Ermüdungsfestigkeitskurven (Wöhlerlinien). Ermüdungsfestigkeitskurven lassen sich in Abhängigkeit der Schwingspielzahl N grundsätzlich in drei Bereiche einteilen:

- Low Cycle Fatigue (LCF):

$\mathrm{N}<10^{5}$

- High Cycle Fatigue (HCF):

$10^{5} \leq \mathrm{N} \leq 10^{7}$

- Very High Cycle Fatigue (VHCF) $\quad \mathrm{N}>10^{7}$

Traditionell wird bei konstanter Spannungsschwingbreite von einem Abknickpunkt der Ermüdungsfestigkeitskurve im Übergangsbereich zur Dauerfestigkeit ausgegangen, ab dem die Ermüdungsfestigkeitskurve einen horizontalen Verlauf aufweist. Spannungsschwingbreiten unterhalb der Dauerfestigkeit werden somit als nicht schädigungswirksam deklariert. Experimentelle Untersuchungen haben allerdings gezeigt, dass die Ermüdungsfestigkeit nach dem Abknickpunkt weiterhin abfallen kann. Die Existenz einer Dauerfestigkeit wird somit prinzipiell in Frage gestellt [Sonsino, 2005a].

Die grundlegende Relevanz des Ermüdungsverhalten von Werkstoffen bis in den Bereich sehr hoher Lastwechselzahlen basiert auf der stetig steigenden Anzahl hochdynamisch beanspruchter Bauteile und Konstruktionen wie beispielsweise Maschinenteile und Eisenbahnräder sowie Brücken und Komponenten von Windener- 
gieanlagen. Diese sind häufig zyklischen Beanspruchungen von bis $10^{9}$ Lastwechselzahlen und darüber hinaus ausgesetzt. Das allgemeine wissenschaftlichtechnische Interesse wird durch einen von der DFG finanzierten Paketantrag [DFG-Paketantrag] und das zuletzt initiierte Schwerpunktprogramm der DFG „Life $\infty$ “ [DFG-SPP1466] widergespiegelt. Außerdem wurde in weiteren Forschungsvorhaben das Ermüdungsverhalten unterschiedlicher Bauteile sowie verschiedener Konstruktionswerkstoffe bis in den Bereich hoher und sehr hoher Lastwechselzahlen untersucht. Zur Thematik des Ermüdungsverhaltens bis in den Bereich sehr hoher Lastwechselzahlen haben bereits fünf internationale Tagungen in Paris [Bathias, 1999], Wien [Stanzl-Tschegg, 2001], Kyoto [Sakai, 2004], Ann Arbor [Allison, 2007] und Berlin [Berger, 2011] stattgefunden.

Der Überblick der normierten Auswertung von Ermüdungsversuchen an Schweißverbindungen aus Stahl in Abb. 1-1 zeigt, dass bisher lediglich vereinzelt experimentelle Ergebnisse für Schweißverbindungen im Bereich sehr hoher Lastwechselzahlen vorliegen [Sonsino, 2005b]. Zur weiteren Absicherung der Ermüdungsfestigkeitskurven nach $10^{8}$ Lastwechseln sind die Versuche in diesem Bereich auszuweiten. Bisher wurden im Bereich sehr hoher Lastwechselzahlen zudem vornehmlich Untersuchungen an glatten Werkstoffproben und bei konstanten Spannungsschwingbreiten durchgeführt, was für Bauteile als weniger interessant einzustufen ist [Bacher-Höchst, 2008].

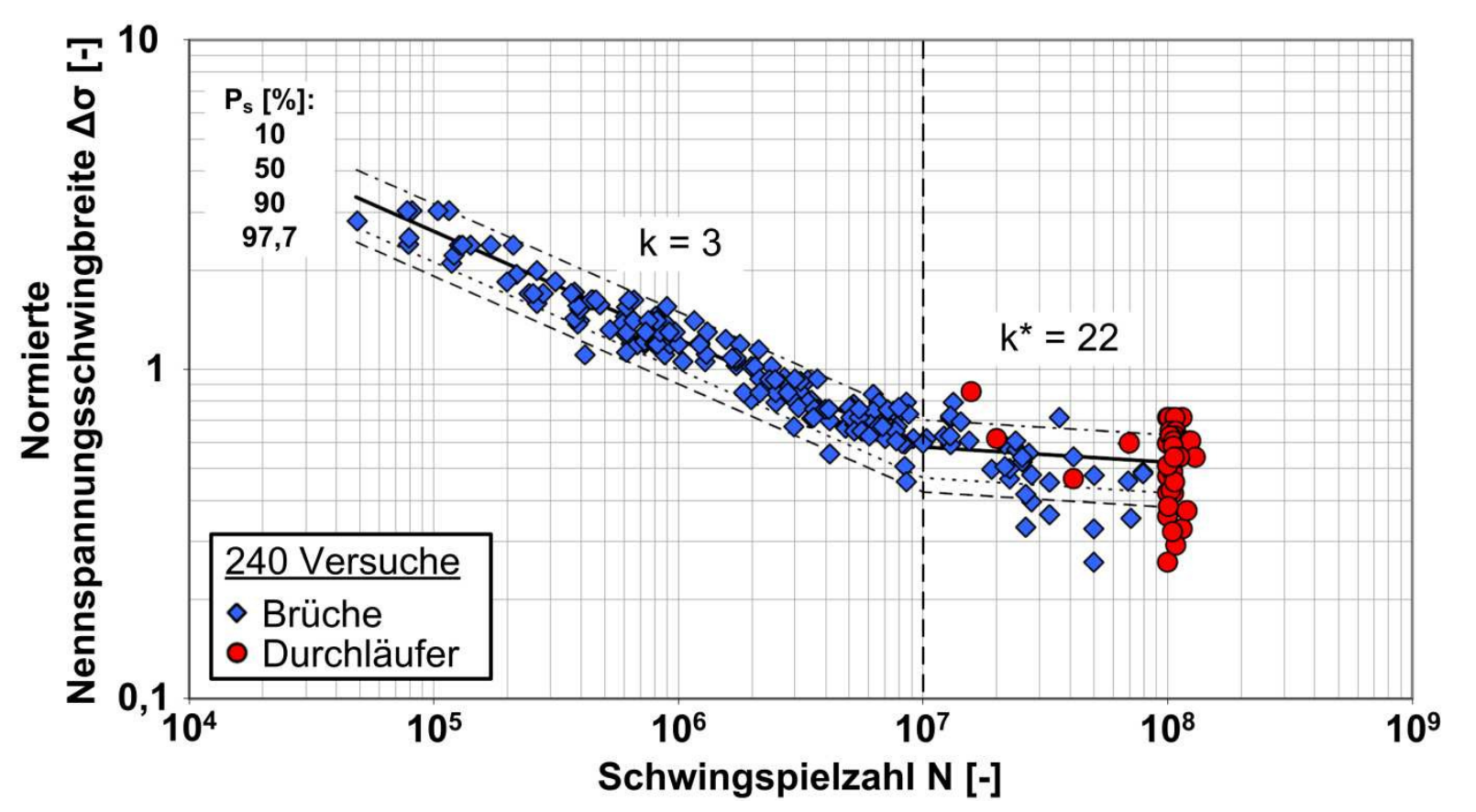

Abb. 1-1: Normierte Auswertung von Ermüdungsversuchen an Schweißverbindungen aus Stahl [Sonsino, 2005b] 
Die Empfehlungen des IIW wurden bereits überarbeitet [Hobbacher, 2009]. Für Schweißverbindungen aus Stahl wird im Bereich sehr hoher Lastwechselzahlen ein kontinuierlicher Abfall der Ermüdungsfestigkeitskurven um 10 \% pro Dekade bei konstanter Spannungsschwingbreite ab einem Abknickpunkt von 107 Lastwechseln angenommen, was einer Neigung von 22 entspricht [IIW, 2009]. Wohingegen im Eurocode 3 [DIN EN 1993-1-9, 2010] bei konstanter Spannungsschwingbreite weiterhin von einem Abknickpunkt der Ermüdungsfestigkeitskurve zur Dauerfestigkeit bei $5 \cdot 10^{6}$ Lastwechseln ausgegangen wird.

Die Problematik in der Versuchsdurchführung im Bereich sehr hoher Lastwechselzahlen besteht in der Realisierung eines angemessenen Prüfzeitraums. Hierzu sind Prüfmethoden mit hohen Frequenzen erforderlich. Die Ultraschallprüftechnik mit Frequenzen von bis zu $20 \mathrm{kHz}$ stellt prinzipiell ein geeignetes Prüfverfahren dar, jedoch ist im Hinblick auf die Prüfung von Schweißverbindungen die Herstellung relativ kleiner und ungestörter Probekörper als äußerst problematisch anzusehen [Sonsino, 2005b]. Aufgrund der erforderlichen speziellen Probekörpergeometrie ist die Prüfung von Bauteilen oder bauteilähnlichen Proben bisher nicht möglich [Pyttel, 2011].

Die experimentellen Untersuchungen an Schweißverbindungen sind bis in den Bereich sehr hoher Lastwechselzahlen auszuweiten, um die bisherigen Annahmen weiter absichern zu können. Es besteht somit Forschungsbedarf im Bereich der Weiterentwicklung von Prüftechniken, die eine Prüfung bauteilähnlicher Probekörper, zu denen Schweißverbindungen zählen, bis in den Bereich sehr hoher Lastwechselzahlen in einem akzeptablen Zeitumfang ermöglichen.

Vor diesem Hintergrund liegt die Zielsetzung der vorliegenden Arbeit zum Einen in der Weiterentwicklung der Prüftechnik, um Ermüdungsversuche an Schweißverbindungen im Bereich sehr hoher Lastwechselzahlen in einem akzeptablen Prüfzeitraum zu realisieren. Zum Anderen ist das Ziel der vorliegenden Arbeit die Ausweitung der experimentellen Untersuchungen an Schweißverbindungen bei konstanter Spannungsschwingbreite bis in den Bereich sehr hoher Schwingspielzahlen, um somit einen Beitrag zur Klärung der Fragestellung des Vorliegens einer Dauerfestigkeit von Schweißverbindungen aus Baustahl zu leisten.

\subsection{Lösungsweg}

Um die geschilderte Problemstellung zu behandeln und die formulierten Fragestellungen der Zielsetzung in der vorliegenden Arbeit zu beantworten, wird zunächst der aktuelle Stand der Forschung in Kapitel 2 beschrieben. Hierbei werden die im Kontext dieser Arbeit stehenden wesentlichen Einflüsse auf die Ermüdungsfestigkeit von 
Schweißverbindungen erörtert sowie ein Überblick von durchgeführten Ermüdungsversuchen an Schweißverbindungen aus Stahl, die in den Bereich sehr hoher Lastwechsel reichen, dargestellt. Außerdem wird eine Übersicht zu Prüftechniken zum Einsatz im Bereich sehr hoher Lastwechselzahlen gegeben.

In Kapitel 3 erfolgen die Beschreibung der Herstellung der Probekörper sowie die Charakterisierung des Ausgangszustandes. Diese umfasst Temperaturmessungen während des Schweißvorgangs, die Aufnahme der lokalen Nahtgeometrie und Zugversuche sowie metallographische Untersuchungen und Härtemessungen.

Die Weiterentwicklung der Prüftechnik wird in Kapitel 4 dargestellt. Dazu werden der Aufbau und das Funktionsprinzip sowie der Versuchsablauf und die Weiterentwicklungen der für die Ermüdungsversuche eingesetzten Prüfvorrichtung erläutert. Desweiteren wird auf die zeitlich veränderlichen Messgrößen während des Versuchs sowie das gewählte Kriterium zur Ermittlung der Anrissschwingspielzahl eingegangen. Außerdem werden Untersuchungen zur Markierung der Rissfront auf der Bruchfläche im Bezug auf die Ermittlung der Anrissgröße beim Erreichen dieses Kriteriums in der vorgestellten Prüfvorrichtung ausgewertet. Desweiteren werden die Kalibrierung der Prüfvorrichtung und die Umsetzung der frequenzabhängigen Sollwertkorrektur behandelt.

In Kapitel 5 erfolgt die Beschreibung und Auswertung der durchgeführten experimentellen Untersuchungen. Es handelt sich hierbei um Ermüdungsversuche an axial beanspruchten im Schweißzustand belassenen Stumpfnahtverbindungen der in Kapitel 3 beschriebenen und charakterisierten Probekörper mit konstanter Spannungsschwingbreite. In drei Versuchsreihen werden der Einfluss von Prüffrequenz und Zugeigenspannungen, die näherungsweise als Mittelspannungen berücksichtigt werden, sowie die Fragestellung des Auftretens später Brüche bei Schweißverbindungen untersucht. Hierzu wurden Ermüdungsversuche im Zeitfestigkeits- und Übergangsbereich bei unterschiedlichen Prüffrequenzen und Spannungsverhältnissen bis in den Bereich sehr hoher Lastwechselzahlen durchgeführt. Außerdem erfolgt der Vergleich der Versuchsergebnisse der vorliegenden Arbeit mit der normierten Auswertung von Ermüdungsversuchen aus der Literatur und mit Eurocode 3.

Den Abschluss der vorliegenden Arbeit bildet Kapitel 6 mit der Zusammenfassung der erlangten Erkenntnisse und dem Ausblick auf weiterführende Untersuchungen. 


\section{Stand der Forschung}

\subsection{Schädigung durch Ermüdung}

Metallische Strukturen können bei zyklischer Beanspruchung deutlich unterhalb der statischen Festigkeit versagen. Diese Schädigung wird als Materialermüdung bezeichnet und umfasst komplexe Vorgänge im Material, hervorgerufen durch zyklische Beanspruchungen. Die Initiierung eines Ermüdungsrisses bei Metallen wird durch Versetzungsbewegungen hervorgerufen. Charakteristisch ist die Bildung von Gleitbändern in parallel zur maximalen Schubspannung orientierten Gleitebenen mit Gleitstufen an der Werkstoffoberfläche in Form von Extrusionen und Intrusionen [Macherauch, 1976] [Schijve, 2009]. An diesen Stellen, aber auch an Korngrenzen oder nicht-metallischen Einschlüssen, können Mikrorisse auftreten. Im Allgemeinen wachsen Ermüdungsrisse anfangs in Richtung der Gleitbänder und setzen sich daraufhin rechtwinklig zur Richtung der maximalen Hauptnormalspannung fort [Seeger, 1996] [Sander, 2008].

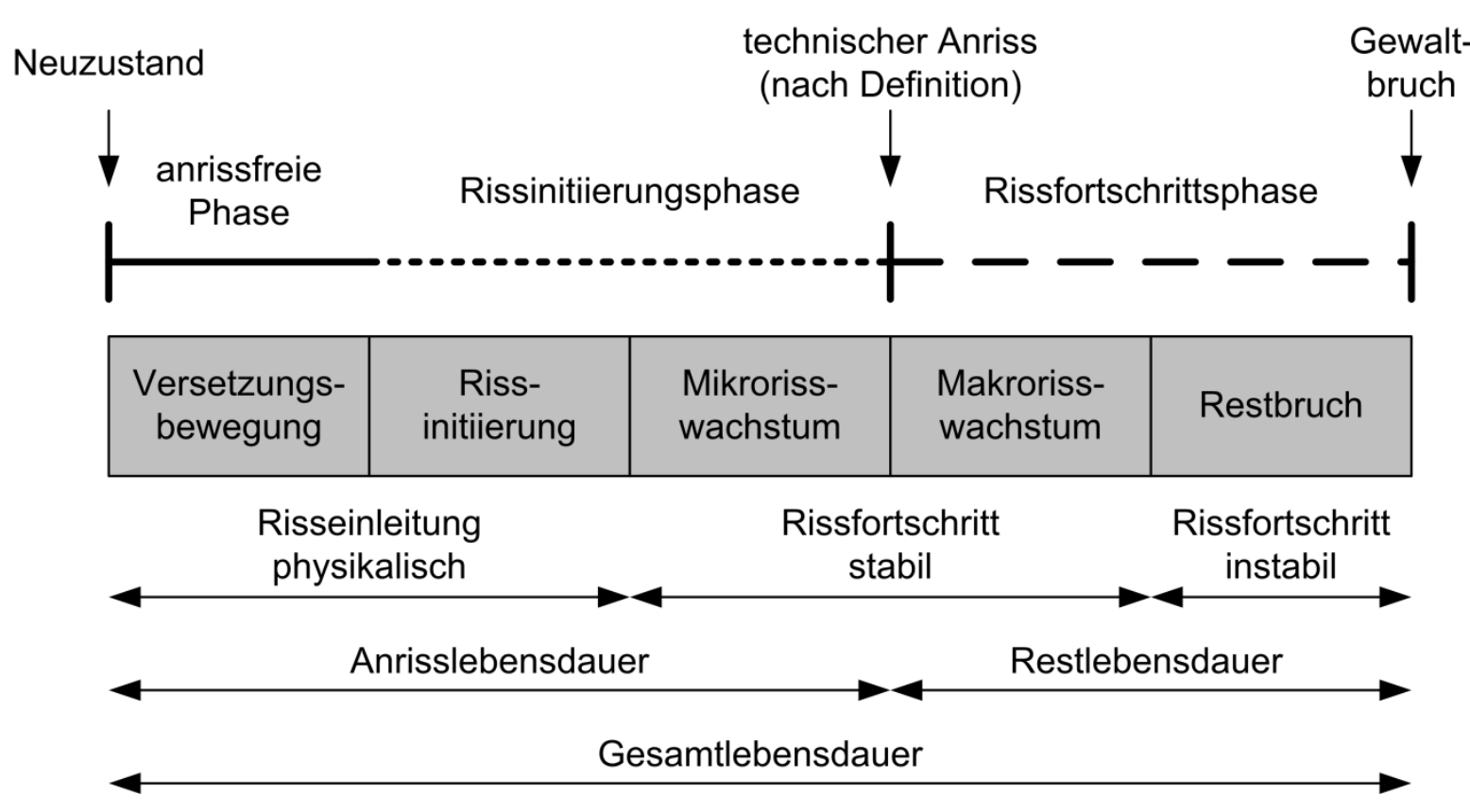

Abb. 2-1: Phasen des Ermüdungsprozesses [Gudehus, 1999] [Radaj, 2007]

Der Ablauf der Ermüdungsschädigung metallischer Strukturen unter zyklischer Beanspruchung ist in Abb. 2-1 schematisch dargestellt. Die Gesamtlebensdauer einer Struktur vom anrissfreien Neuzustand bis zum Gewaltbruch kann in die Phase der Anrisslebensdauer (Rissinitiierungsphase) und der Restlebensdauer (Rissfortschrittsphase) unterteilt werden. Nach der physikalischen Risseinleitung durch Versetzungsbewegungen der Gleitbänder bis zur Risskeimbildung folgen die Phasen 
des stabilen und instabilen Risswachstums. Mikrorisse wachsen zu Makrorissen zusammen. Die Rissfront schreitet immer weiter voran, bis der Restbruch eintritt.

Ein charakteristischer Punkt zwischen der Rissinitiierungsphase und der Rissfortschrittsphase ist der technische Anriss. Der technische Anriss wird von Gudehus und Zenner [Gudehus, 1999] als ein mit üblichen zerstörungsfreien Prüftechniken erkennbarer Riss definiert. Zu den üblichen zerstörungsfreien Prüfungen zur Detektion von Oberflächenrissen gehören beispielsweise das Farbeindringverfahren, die Durchstrahlprüfung und die Ultraschallprüfung. Die Größenordnung des technischen Anrisses wird mit einer Oberflächenlänge von ca. $1-2 \mathrm{~mm}$ und einer Tiefe von ca. 0,5 mm angegeben [Seeger, 1996] [Radaj, 2000] [Radaj, 2007].

\subsection{Ermüdungsfestigkeitskurven metallischer Werkstoffe}

Die klassische Beschreibung der Ermüdungsfestigkeit eines Werkstoffes oder Bauteils erfolgt über experimentell ermittelte Wöhlerlinien (Ermüdungsfestigkeitskurven). Im Wöhler-Versuch wird eine Probe einer konstanten zyklischen Beanspruchung unterworfen und bis zum Eintritt des Versagens geprüft. Als Versagen der Probe gilt vorwiegend der Bruch. Es ist ebenfalls üblich, einen Anriss bestimmter Größe als Versagen zu definieren. Um die Ermüdungsfestigkeitskurve abzuleiten, werden Proben auf unterschiedlichen Beanspruchungsniveaus bis zum Eintritt des definierten Versagens geprüft.

Die Darstellung der Ermüdungsfestigkeitskurve erfolgt üblicherweise in doppeltlogarithmischer Auftragung der Beanspruchungsgröße über der ertragbaren Schwingspielzahl. In Abhängigkeit der ertragbaren Schwingspielzahl kann die Ermüdungsfestigkeitskurve in unterschiedliche Bereiche (siehe Abb. 2-2) mit charakteristischen Verläufen unterteilt werden: Kurzzeitfestigkeit, Zeitfestigkeit und Dauerfestigkeit.

Der Bereich mit ertragbaren Schwingspielzahlen von kleiner als $10^{5}$ wird als Kurzzeitfestigkeit bzw. Low Cycle Fatigue (LCF) bezeichnet. Der Bereich von $10^{5}$ bis $10^{7}$ Schwingspielzahlen umfasst die Zeitfestigkeit bzw. High Cycle Fatigue (HCF). Die Dauerfestigkeit beschreibt im traditionellen Sinn einen Grenzwert, ab dem eine Beanspruchungsgröße beliebig oft ohne Eintritt eines Versagens ertragen werden kann. In experimentellen Untersuchungen hat sich allerdings gezeigt, dass es zu einem weiteren Abfall der Ermüdungsfestigkeit auch unterhalb der Dauerfestigkeit kommen kann [Sonsino, 2005a]. Für diesen Bereich mit ertragbaren Schwingspielzahlen von größer als $10^{7}$ hat sich der Begriff Very High Cycle Fatigue (VHCF) etabliert [Pyttel, 2011]. 


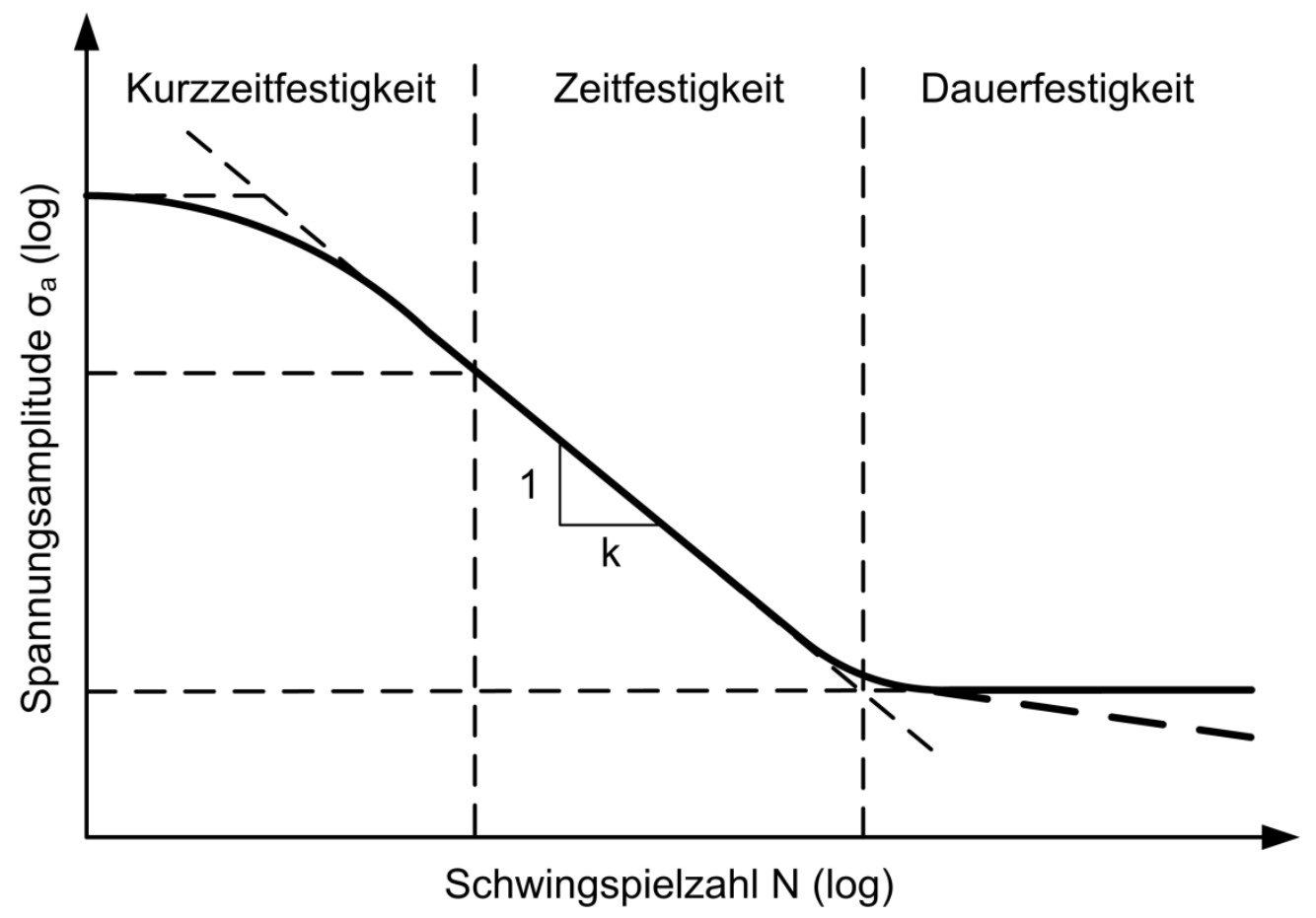

Abb. 2-2: Schematischer Verlauf der Wöhlerlinie [Haibach, 2006]

In Abb. 2-2 ist schematisch der klassische Verlauf der Wöhlerlinie dargestellt. Im Zeitfestigkeitsbereich erfolgt die Beschreibung der Ermüdungsfestigkeitskurve in doppeltlogarithmischer Darstellung über eine Gerade mit einem Neigungsexponenten k. Diese Gerade mündet im Kurzzeitfestigkeitsbereich horizontal in die Zugfestigkeit ein. Im Übergangsbereich zur Dauerfestigkeit wird idealisiert von einem Abknickpunkt der Ermüdungsfestigkeitskurve in einen horizontalen Verlauf oder einem weiteren Abfall der Ermüdungsfestigkeit ausgegangen. Bei Metallen mit kubischflächenzentriertem Gitter wie beispielsweise austenitischen Stählen oder Aluminiumund Kupferlegierungen kommt es zu einer weiteren Abnahme der Ermüdungsfestigkeit nach dem Abknickpunkt. Im Allgemeinen zeigen Metalle mit kubischraumzentriertem Gitter wie beispielsweise ferritische Stähle und Titanlegierungen, begründet durch die Behinderung von Versetzungen durch Kohlenstoff- oder Stickstoffatome auf Zwischengitterplätzen, ein horizontales Auslaufen der Ermüdungsfestigkeitskurve [Bannantine, 1990] [Haibach, 2006] [Radaj, 2007] [Sonsino, 2007].

Liegen korrosive Einflüsse oder erhöhte Temperaturen vor oder treten regelmäßig Überlasten auf, kann es auch bei Metallen mit kubisch-raumzentriertem Gitter wie beispielsweise Baustahl oder Titanlegierungen zu einem weiteren Abfall der Ermüdungsfestigkeit kommen [Bannantine, 1990] [Radaj, 2007]. Außerdem zeigen aktuelle Untersuchungen im VHCF-Bereich, dass nicht nur Metalle mit kubischflächenzentriertem Gitter einen Abfall der Ermüdungsfestigkeit bei sehr hohen Schwingspielzahlen aufweisen können, sondern auch einige Metalle mit kubisch- 
raumzentriertem Gitter, ohne dass korrosiver Einflüsse oder erhöhte Temperaturen sowie regelmäßige Überlasten vorhanden sind [Bathias, 2001] [Sonsino, 2007] [Pyttel, 2011].

Aus zahlreichen Versuchsergebnissen bis in den Bereich sehr hoher Schwingspielzahlen haben Pyttel et al. [Pyttel, 2011] vier schematische Verläufe von Wöhlerlinien bis in den VHCF-Bereich abgeleitet: Die klassische Wöhlerlinie mit einem horizontalen Auslaufen der Ermüdungsfestigkeitskurve (siehe Abb. 2-3, A) ist charakteristisch für niedrigfeste (unlegierte und niedriglegierte) Stähle [Mayer, 2007] [Sakai, 2007] und stark gekerbte Proben [Akiniwa, 2006] [Schwerdt, 2009]. Der Anriss geht hierbei von der Oberfläche aus. Ein weiterer Abfall der Ermüdungsfestigkeit ab dem Abknickpunkt (siehe Abb. 2-3, B) ist charakteristisch für Metalle mit kubischflächenzentriertem Gitter [Pyttel, 2007] und bei Werkstoffen mit kubischraumzentriertem Gitter unter korrosiven Medien oder erhöhter Temperatur [Bannantine, 1990] [Radaj, 2007]. Der Anrissort kann sich hierbei von der Oberfläche ins Werkstoffinnere verlagern. Das Ausbilden eines Plateaus mit anschließendem weiterem Abfalls der Ermüdungsfestigkeitskurve (siehe Abb. 2-3, C) oder einem kontinuierlichen Abfall der Ermüdungsfestigkeitskurve ohne Abknickpunkt (siehe Abb. 2-3, D) ist charakteristisch für hochlegierte Kugellagerstähle und für auf hohe oder höchste Festigkeiten vergütete niedriglegierte Stähle [Shiozawa, 2001] [Sakai, 2007] [Sonsino, 2007]. Der Anrissort verlagert sich dabei im Bereich hoher Schwingspielzahlen von der Oberfläche ins Werkstoffinnere.
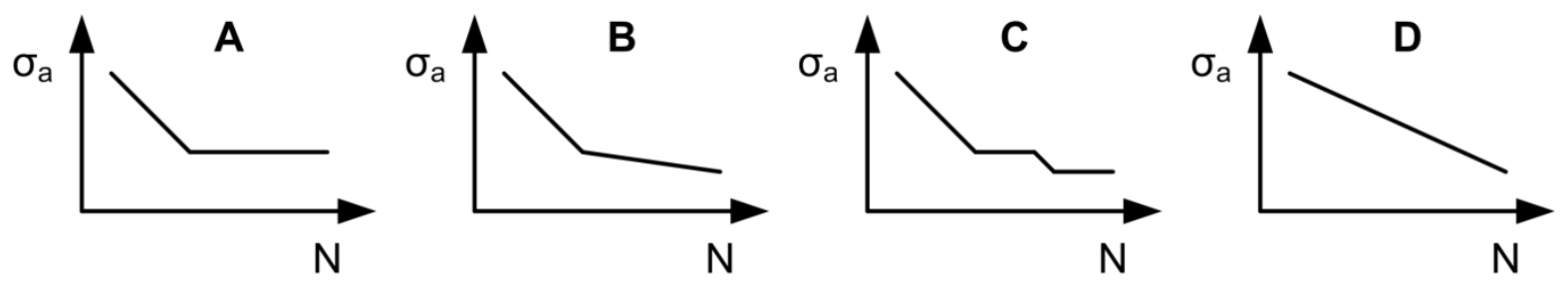

Abb. 2-3: Schematische Verläufe von Wöhlerlinien bis in den VHCF-Bereich in doppeltlogarithmischer Darstellung [Pyttel, 2011]

In Abhängigkeit der definierten Bereiche der ertragbaren Schwingspielzahlen (LCF, HCF und VHCF) unterscheidet Bathias et al. [Bathias, 2001] allgemein drei unterschiedliche Risseinleitungsarten glatter Proben (siehe Abb. 2-4). Im LCF-Bereich kann es zu einer Risseinleitung an mehreren Stellen der Oberfläche kommen, wohingegen im HCF- und VHCF-Bereich vorwiegend eine Stelle der Risseinleitung vorliegt. Kennzeichnend für den HCF-Bereich ist die Risseinleitung an der Oberfläche. Im Gegensatz dazu können im VHCF-Bereich Risse im Inneren des Bauteils eingeleitet werden. 

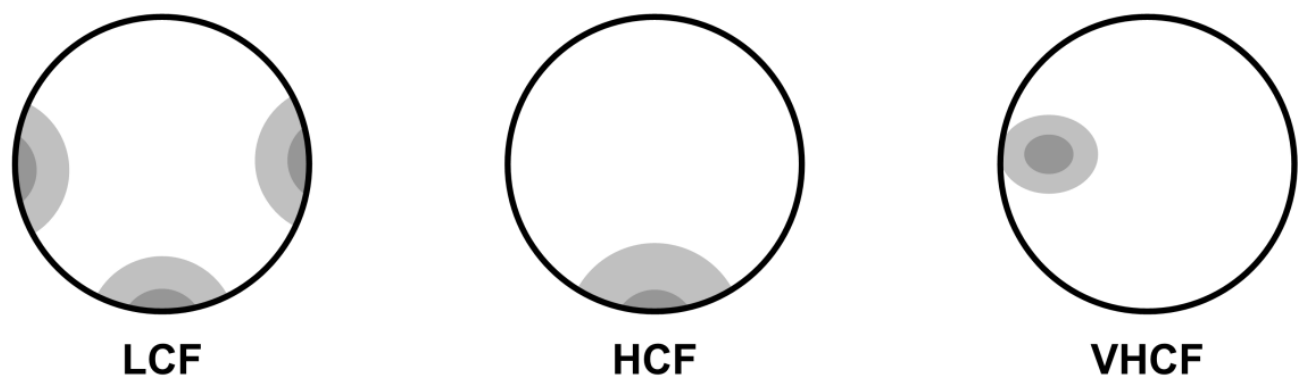

Abb. 2-4: Risseinleitungsarten glatter Proben im LCF-, HCF- und VHCF-Bereich

[Bathias, 2001]

Ursächlich für die Risseinleitung im Inneren des Bauteils sind vielfach nichtmetallische Einschlüsse, aber auch Defekte wie Poren oder die Mikrostruktur des Werkstoffes selbst [Bathias, 2001] [Sander, 2008]. Bei einem nicht-metallischen Einschluss als Rissausgangsort konnte das typische Erscheinungsbild eines optisch dunklen Bereichs festgestellt werden, der als fish-eye [Nishijima, 1999] oder Optical Dark Area (ODA) [Murakami, 2002] bezeichnet wird. Der charakteristische Bereich der Bruchfläche um einen nicht-metallischen Einschluss wird von Sakai [Sakai, 2007] als Fine Granular Area (FGA) sowie von Shiozawa et al. [Shiozawa, 2001] als Granular-Bright Facet (GBF) bezeichnet. Unterschiedliche Erklärungen zu vorherrschenden Schädigungsmechanismen sind beispielsweise bei Murakami et al. [Murakami, 2002] oder bei Sakai [Sakai, 2007] zu finden.

Ein weiterer Abfall der Ermüdungsfestigkeitskurve kann auch bei Schweißverbindungen im Bereich sehr hoher Lastwechselzahlen beobachtet werden [Sonsino, 2005a] [Sonsino, 2005b]. Dies wird durch hohe Zugeigenspannungen sowie die Belastungsart Torsion begründet [Seeger, 1992] [Ritter, 1994] [Sonsino, 1994]. Ein Überblick zu Ermüdungsversuchen an Schweißverbindungen aus Stahl bis in den Bereich sehr hoher Lastwechselzahlen ist Kapitel 2.4 zu entnehmen.

\subsection{Einflussgrößen auf die Ermüdungsfestigkeit}

Allgemein ist die Ermüdungsfestigkeit von Werkstoffen bzw. Strukturen abhängig von Werkstoffart und -zustand, Oberflächengüte, Proben- oder Bauteilgröße, Belastungsart, Temperatur, Korrosion, Mittel- und Eigenspannung sowie Spannungskonzentrationen [Gudehus, 1999] [Haibach, 2006] [Radaj, 2007]. In Abb. 2-5 ist ein Überblick der Einflussgrößen auf die Ermüdungsfestigkeit gegeben. 


\begin{tabular}{|c|c|c|}
\hline Werkstoff & Werkstück & Oberfläche \\
\hline $\begin{array}{l}\text { Art } \\
\text { Legierung } \\
\text { Korngefüge } \\
\text { Gefüge }\end{array}$ & $\begin{array}{l}\text { Kerbwirkung } \\
\text { Größeneinfluss }\end{array}$ & $\begin{array}{c}\text { Rauheit } \\
\text { Härte } \\
\text { Eigenspannungen }\end{array}$ \\
\hline Umgebung & Beanspruchungsart & Beanspruchungsablauf \\
\hline $\begin{array}{l}\text { Temperatur } \\
\text { Korrosion }\end{array}$ & $\begin{array}{l}\text { Mittelspannung } \\
\text { Mehrachsigkeit }\end{array}$ & $\begin{array}{l}\text { Amplitudenspektrum } \\
\text { Amplitudenfolge } \\
\text { Mittelwertfolge } \\
\text { Ruhephasen }\end{array}$ \\
\hline
\end{tabular}

Abb. 2-5: Einflussgrößen auf die Ermüdungsfestigkeit [Radaj, 2000]

Bedingt durch den Fügeprozess weisen Schweißverbindungen im Vergleich zum Grundwerkstoff charakteristische Eigenschaften auf, welche die Ermüdungsfestigkeit von Schweißverbindungen beeinflussen können. Zu diesen zählen Werkstoffinhomogenitäten, Schweißfehler und -imperfektionen, Schweißeigenspannungen und -verzug sowie die geometrischen Schweißnahtparameter [Gurney, 1979] [Maddox, 1991] [Radaj, 2000].

Durch den Einsatz von Zusatzwerkstoff findet eine Vermischung mit dem Grundwerkstoff statt. Dies kann zu Mikroporen und Einschlüssen im Bereich von Naht- und Wurzelübergang führen. In Abhängigkeit der Temperatureinwirkung während des Schweißens treten in der Wärmeeinflusszone unterschiedliche Mikrostrukturen (Korngröße und Härte) auf [Radaj, 2000]. Schweißeigenspannungen resultieren aus der konzentrierten Wärmezufuhr während des Schweißprozesses und können im Nahtbereich in Höhe der Streckgrenze vorliegen [Gurney, 1979] [Maddox, 1991]. Die lokale Kerbwirkung im Schweißnahtbereich beeinflusst die Ermüdungsfestigkeit einer Struktur deutlich [Heeschen, 1986]. Die geometrischen Schweißnahtparameter wie Kerbradius, Anstiegswinkel oder Breite und Höhe der Naht- und Wurzelüberhöhung streuen in hohem Maße und schlagen sich in der Höhe der resultierenden Spannungskonzentrationen nieder. Zu typischen Schweißfehlern und -imperfektionen zählen Risse, Poren, Lunker, Einbrandkerben, Bindefehler oder Fugenversatz [Radaj, 2000]. Bei äußerer Beanspruchung führt Schweißverzug zu Zusatzspannungen.

Aus zahlreichen Untersuchungen geht hervor, dass im Bezug auf die Ermüdungsfestigkeit zu den für Schweißverbindungen maßgeblichen Einflussfaktoren die aus der Nahtgeometrie resultierende lokale Kerbwirkung sowie der aus dem Schweißprozess resultierende Eigenspannungs- und Gefügezustand zählen [Gurney, 1979] [Heeschen, 1986] [Maddox, 1991] [Sonsino, 1994] [Nitschke-Pagel, 1995] [Radaj, 2000]. 
Im Folgenden werden die für Schweißverbindungen maßgeblichen sowie in unmittelbarem Zusammenhang mit der Zielsetzung der vorliegenden Arbeit stehenden Einflussgrößen näher behandelt. Hierzu zählen die Kerbwirkung, die Eigenspannungen und die Prüffrequenz.

\subsubsection{Kerbwirkung}

Bei der Kerbwirkung handelt es sich um lokale Spannungserhöhungen, die an Kerben auftreten. Die Ermüdungsfestigkeit einer Struktur kann durch Kerben deutlich verringert werden. Allgemein wird zwischen Form-, Werkstoff- und Belastungskerben unterschieden, die einzeln und in überlagerter Form auftreten können [Radaj, 2007]. Bei Schweißverbindungen treten im Bereich der Schweißnaht Formkerben hinsichtlich der lokalen Nahtgeometrie und Werkstoffkerben hinsichtlich der durch die Wärmewirkung des Schweißens beeinflussten Werkstoffbereiche auf, wobei der Einfluss der lokalen Nahtgeometrie als maßgeblich angesehen wird [Nitschke-Pagel, 1995] [Lachmann, 2003] [Nitschke-Pagel, 2006].

Aus diesem Grund kommt der Verbesserung der Nahtgeometrie im Bezug auf die Steigerung der Ermüdungsfestigkeit eine besondere Bedeutung zu. Eine Vielzahl an Untersuchungen widmet sich demnach unter anderem Maßnahmen wie dem blechebenen Abarbeiten der Naht und dem WIG-Aufschmelzen sowie den Hämmer- und Strahlverfahren im Hinblick auf die Erzielung einer verbesserten Nahtgeometrie und letztendlich einer Steigerung der Ermüdungsfestigkeit [Heeschen, 1986] [Heeschen, 1990] [Nitschke-Pagel, 2006] [Dürr, 2007] [Weich, 2008]. Beim Einsatz höherfester Stähle sind Maßnahmen zur Verbesserung der Nahtgeometrie ebenfalls bedeutend, da von einer Zunahme der Kerbempfindlichkeit mit steigender Festigkeit des Werkstoffes ausgegangen werden kann [Signes, 1967] [Gurney, 1979].

Bei Schweißverbindungen treten die maximalen Kerbspannungen an den Übergängen von Naht- und Wurzelüberhöhung auf. Bei vorhandenen Schweißnahtenden können die maximalen Spannungserhöhungen dort auftreten [Kaffenberger, 2012]. Die lokale Nahtgeometrie im Bereich der Schweißnaht wird vielfach durch definierte geometrische Parameter wie Kerbradius und Anstiegswinkel sowie Breite und Höhe der Naht- und Wurzelüberhöhung beschrieben und ist abhängig von der Nahtvorbereitung sowie vom Schweißverfahren und den Schweißparametern. Zahlreiche Arbeiten zum Einfluss der Nahtgeometrieparameter auf die Ermüdungsfestigkeit von Schweißverbindungen, welche die lokale Nahtgeometrie an Grundwerkstoffproben mit nachgebildetem Nahtprofil sowie an realen Schweißnähten untersucht haben, wurden von Heeschen [Heeschen, 1986] zusammengetragen. Der vorherrschende Einfluss der Nahtgeometrie auf die Ermüdungsfestigkeit wird durch diese Arbeiten belegt, wobei Kerbradius und Anstiegswinkel häufig als maßgebliche Einflussfakto- 
ren angesehen werden. Sowohl ein großer Anstiegswinkel als auch ein kleiner Kerbradius führen zu einer Minderung der Ermüdungsfestigkeit.

Die Kerbformzahl $\mathrm{K}_{\mathrm{t}}$ stellt ein Maß zur Beschreibung der geometrischen Kerbwirkung dar und beschreibt das Verhältnis von maximaler Kerbspannung $\sigma_{\max } z$ zur Nennspannung $\sigma_{N}$ unter Voraussetzung linearelastischen Werkstoffverhaltens, wobei sich die Nennspannung hierbei im Allgemeinen auf den ungestörten Querschnitt bezieht. Allerdings bewirkt die Kerbwirkung aufgrund von Effekten der Mikro- und Makrostützwirkung keine Abminderung der Ermüdungsfestigkeit in der Höhe des Anstiegs der Kerbformzahl. Der Effekt der elastisch-plastischen Makrostützwirkung im Zeitfestigkeitsbereich beruht auf dem Kerbspannungsabbau durch lokales Fließen im Kerbgrund. Der Effekt der elastischen Mikrostützwirkung besagt, dass die Dauerfestigkeit nicht von der maximalen Kerbspannung, sondern von der über ein kleines Werkstoffelement im Kerbgrund gemittelten Kerbspannung bestimmt wird [Radaj, 2007].

Zur Ermittlung der Kerbformzahl liegen analytische Näherungsformeln für Schweißverbindungen beispielsweise von Rainer [Rainer, 1979] oder Anthes et al. [Anthes, 1993] vor. Diese basieren auf gezielten Parameterstudien an idealisierten Nahtgeometrien und ermöglichen die Berechnung der Kerbformzahl in Abhängigkeit von den Nahtgeometrieparametern. Ein Überblick analytischer Formulierungen von Kerbformzahlen für unterschiedliche Schweißverbindungen kann bei Radaj und Sonsino [Radaj, 2000] oder bei Radaj et al. [Radaj, 2006] entnommen werden. Desweiteren kann die Ermittlung der Kerbformzahl durch numerische Berechnungen erfolgen. Hinweise und Empfehlungen zur numerischen Ermittlung von Kerbspannungen sind bei Fricke [Fricke, 2010] angegeben.

\subsubsection{Eigenspannungen}

Eigenspannungen stellen innere Beanspruchungen in Bauteilen dar, die nicht auf die Einwirkung äußerer Beanspruchungen zurückzuführen sind. Innerhalb des Bauteils herrscht Gleichgewicht. Bei der Einwirkung von Lastspannungen kommt es zu einer Überlagerung mit den Eigenspannungen. Die Entstehungsursachen von Eigenspannungen sind vielschichtig, wobei Bauteile in der Regel durch Fertigungsprozesse wie beispielsweise Walzen, Gießen, Schweißen oder Härten mit Eigenspannungen behaftet werden.

In Abhängigkeit vom Wirkungsbereich wird zwischen Eigenspannungen I. Art, II. Art und III. Art unterschieden [Macherauch, 1973], wobei es sich bei einem Eigenspannungszustand immer um eine Überlagerung dieser Eigenspannungsarten handelt. Die Dimension des Wirkungsbereichs erstreckt sich hierbei über mehrere Atomabstände (III. Art), die Korngröße (II. Art) und makroskopische Bereiche mehrerer Körner (I. Art). Makroskopische Eigenspannungen I. Art, zu denen Schweißeigenspan- 
nungen zählen, sind vornehmlich von praktischer Bedeutung [Dilthey, 2005] [Radaj, 2007]. Begründet wird dies mit der zentralen Relevanz makroskopischer Eigenspannungen im Hinblick auf das Festigkeitsverhalten metallischer Werkstoffe. Jedoch basiert dieser Sachverhalt vor allem auf der komplizierten Ermittlung mikroskopischer Eigenspannungen [Nitschke-Pagel, 1995].

Der Einfluss von Eigenspannungen auf die Ermüdungsfestigkeit ist mit dem Einfluss von Mittelspannungen vergleichbar. Durch zyklische Beanspruchung kann es allerdings zu einer Veränderung der Eigenspannungen in Form eines Abbaus durch Plastizierungen kommen. Im Allgemeinen wirken sich vorliegende Druckeigenspannungen positiv sowie Zugeigenspannungen negativ auf die Ermüdungsfestigkeit aus [Haibach, 2006] [Radaj, 2007].

\subsubsection{Schweißeigenspannungen}

Der resultierende Schweißeigenspannungszustand setzt sich aus einer Überlagerung von Schrumpf- und Umwandlungseigenspannungen zusammen, wie in zahlreichen Arbeiten [Rappe, 1972] [Wohlfahrt, 1983] [Schimmöller, 1992] [NitschkePagel, 1995] beschrieben und im Folgenden kurz dargestellt. Im lokal begrenzten Nahtbereich des konzentrierten Wärmeeintrags beim Schweißen kommt es zu unterschiedlichen Temperaturdehnungen des Werkstoffs, woraus beim Abkühlen ein Eigenspannungszustand im Bauteil resultiert. Bei Schweißeigenspannungen wird zwischen in Nahtrichtung wirkenden Längseigenspannungen und senkrecht zur Naht wirkenden Quereigenspannungen unterschieden (siehe Abb. 2-6).

Temperaturbedingte Ausdehnungen im Nahtbereich werden durch kältere Umgebungsbereiche behindert, was Wärmespannungen zur Folge hat. Mit steigender Temperatur sinkt die Streckgrenze metallischer Werkstoffe, sodass es im Schweißbereich zum Teil zu einer Überschreitung der Streckgrenze kommt. In diesem Bereich treten plastische Stauchungen auf. Nach dem Abkühlvorgang kommt es zu Schrumpfungen, die durch die Stützwirkung kälterer Umgebungsbereiche behindert werden. Daraus resultieren in Längsrichtung Zugeigenspannungen im Nahtbereich sowie Druckeigenspannungen in den Umgebungsbereichen. Die in Längsrichtung wirkenden Zugeigenspannungen und die Dehnungsbehinderung in Querrichtung führen zu Zugeigenspannungen in Nahtmitte sowie Druckeigenspannungen an den Nahtenden.

Neben den aus Schrumpfungsbehinderung resultierenden Eigenspannungen können Gefügeumwandlungen ebenfalls Eigenspannungen verursachen. Eine Gefügeumwandlung ist mit einer Volumenzunahme, die wiederum durch kältere Umgebungsbereiche behindert wird, verbunden, sodass es zu Druckeigenspannungen im Nahtbereich und zu Zugeigenspannungen in den Umgebungsbereichen kommen kann. 

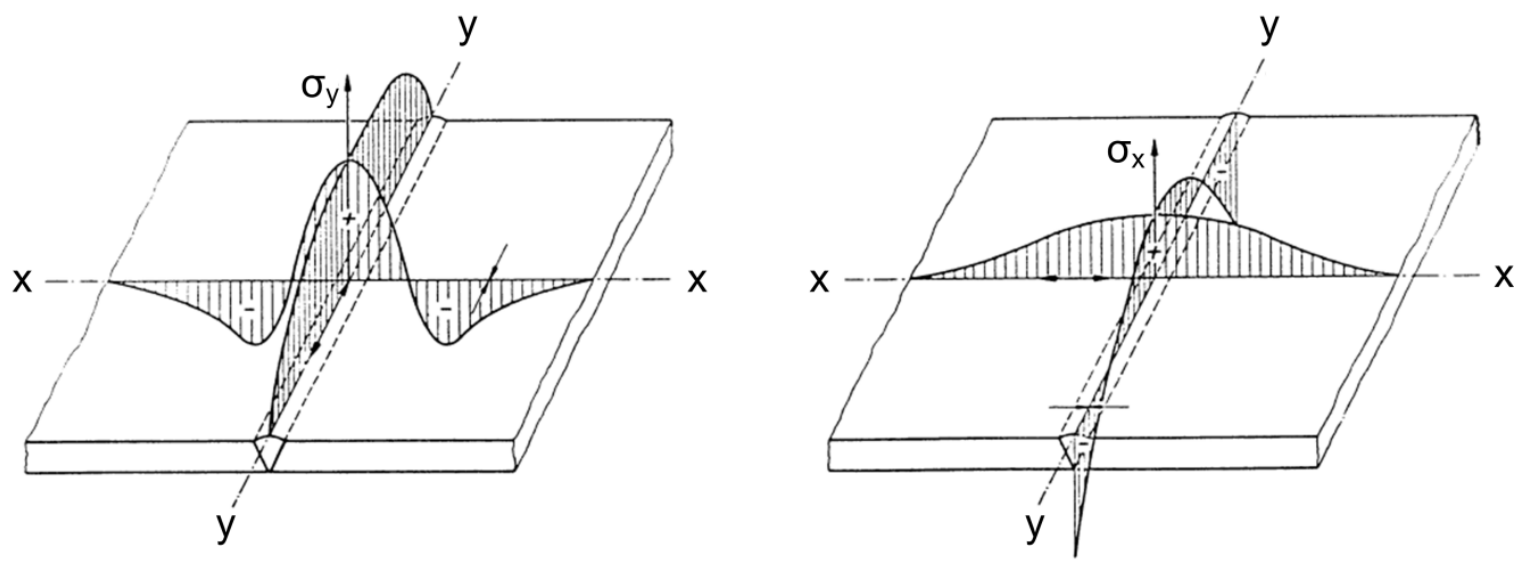

Abb. 2-6: Eigenspannungen einer Stumpfnahtverbindung in Längsrichtung (links) und in Querrichtung (rechts) zur Naht [Rappe, 1972]

Resultierende Eigenspannungsverteilungen aus der Überlagerung von Schrumpfund Umwandlungseigenspannungen werden maßgeblich von der Abkühlgeschwindigkeit und den Umwandlungseigenschaften des Werkstoffes beeinflusst. Die Festigkeitseigenschaften von Werkstoffen sowie die Herstellungsbedingungen von Bauteilen bestimmen vornehmlich die Höhe der Eigenspannungen [Nitschke-Pagel, 1995]. Die Ausprägung von Eigenspannungen in unterschiedlicher Höhe oder auch unterschiedlichen Vorzeichens sind abhängig von Werkstoff und Geometrie, Anordnung und Ausführung der Schweißnähte sowie von Nahtform und Schweißbedingungen [Wohlfahrt, 1983] [Krebs, 2012].

Bei Schweißverbindungen in Bauteilen mit ausreichender Steifigkeit wird im ungünstigsten Fall davon ausgegangen, dass Zugeigenspannungen $\sigma_{E}$ in Höhe der Streckgrenze $f_{y}$ des Grundwerkstoffes vorliegen [Gurney, 1979] [Maddox, 1982] [Maddox, 1991]. In der Modellvorstellung von Gurney [Gurney, 1979] wirkt eine konstante Oberspannung aus der Überlagerung von Last- und Eigenspannungen in Höhe der Streckgrenze. Es findet ein Abbau der Eigenspannungen durch Plastizierung in Höhe der die Streckgrenze überschreitenden Zuglastspannung statt. Es stellen sich demnach dieselben resultierenden Spannungsverläufe bei unterschiedlichen Spannungsverhältnissen $\mathrm{R}$ ein, wie Abb. 2-7 verdeutlicht.

Folglich ist die Ermüdungsfestigkeit im vorliegenden Fall unabhängig von der Mittelspannung [Gurney, 1972] [Harrison, 1981], was auch in gültigen Regelwerken wie dem Eurocode 3 [DIN EN 1993-1-9, 2010] verankert ist. Experimentelle Untersuchungen an Längssteifen von Gurney und Maddox [Gurney, 1972] und großformatigen Biegeträgern von Fischer et al. [Fischer, 1974] [Fischer, 1997] bestätigten diese Annahme. Eine Bestätigung durch Eigenspannungsmessungen liegt jedoch nicht vor [Krebs, 2012]. 


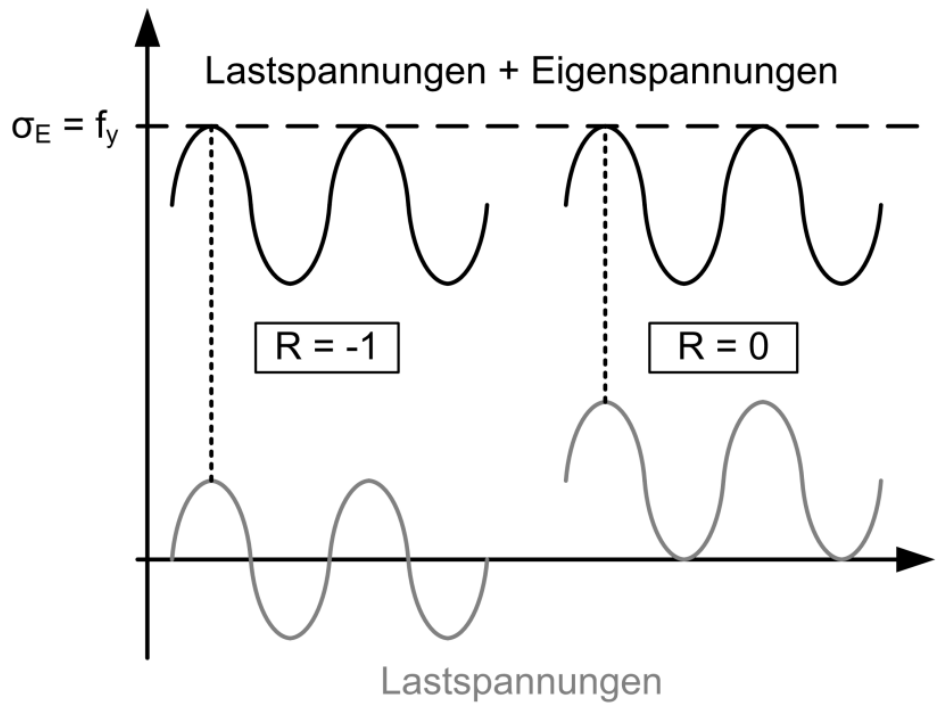

Abb. 2-7: Überlagerung von Last- und Eigenspannungen [Gurney, 1979]

Bei kleinen Probekörpern ist nicht von Zugeigenspannungen in Höhe der Streckgrenze auszugehen [Gurney, 1977]. Es sind geringe bis mäßige Zugeigenspannungen zu erwarten, sodass ein Mittelspannungseinfluss gemäß Abb. 2-8 vorliegt [Haibach, 2006]. Um dennoch Aussagen über den Eigenspannungseinfluss aus der Prüfung von kleinen Probekörpern zu gewinnen, empfiehlt sich einen Prüfung bei hohen Zugmittelspannungen in Form von Spannungsverhältnissen von $R>0,5$ [Gurney, 1972] [IIW, 2009]. Im elastischen Bereich können Zugeigenspannungen näherungsweise als Mittelspannungen betrachtet werden [Sonsino, 1994].

Der Einfluss von Schweißeigenspannungen auf die Ermüdungsfestigkeit von Schweißverbindungen wurde vorwiegend durch qualitative Vergleiche aus Untersuchungen von im Schweißzustand belassenen und spannungsarm geglühten Probekörpern untersucht [Otha, 1986] [Sonsino, 1987] [Nakamura, 1988], wobei Messungen von Eigenspannungen nur vereinzelt durchgeführt wurden. Diese Untersuchungen liefern zum Teil gegensätzliche Ergebnisse im Hinblick auf einen ermüdungsfestigkeitssteigernden Einfluss des Spannungsarmglühens [Nitschke-Pagel, 1995] [Krebs, 2012]. Außerdem liegen Untersuchungen mit definiert eingeprägten Eigenspannungszuständen vor [Gurney, 1977] [Maddox, 1982]. Quantitative Bewertungen durch systematische Messung von Eigenspannungen liegen nur begrenzt vor [Nitschke-Pagel, 1995] [Lachmann, 2003]. 


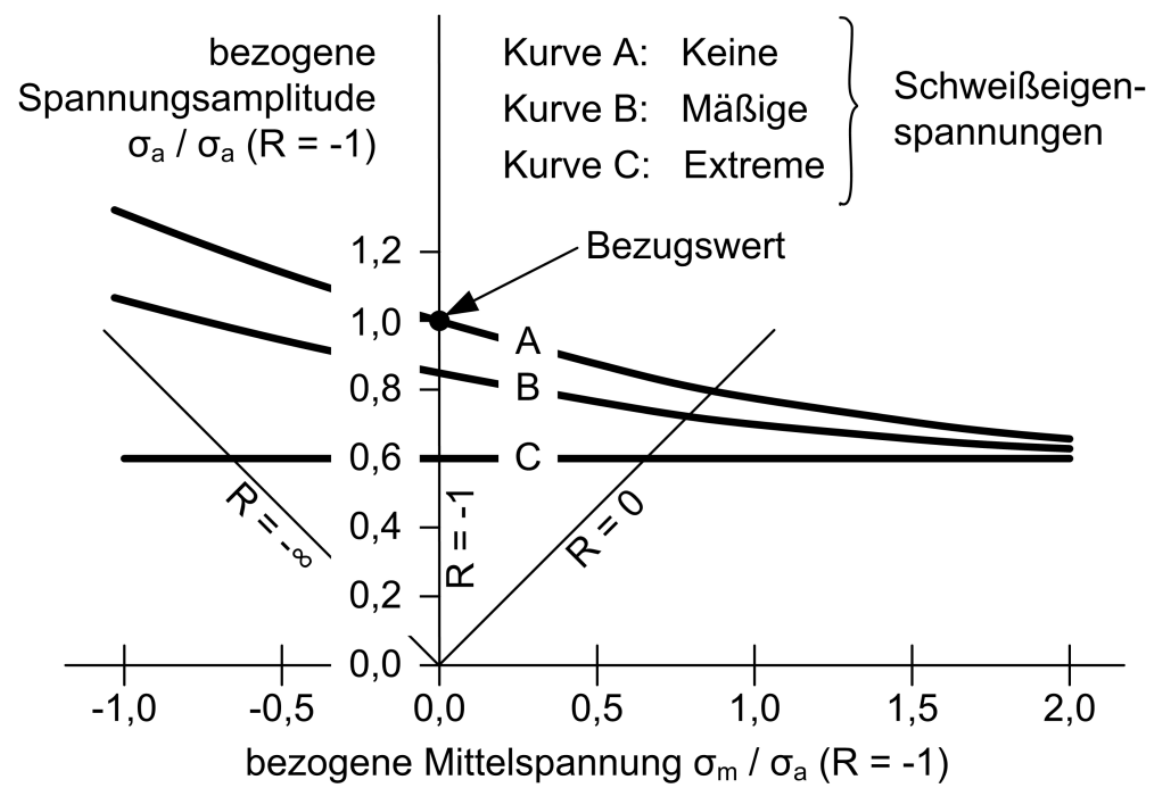

Abb. 2-8: Einfluss der Höhe der schweißbedingten Zugeigenspannungen auf die Mittelspannungsabhängigkeit [Haibach, 2006]

Aus den aufgezeigten Untersuchungen geht hervor, dass neben der geometrischen Kerbwirkung die durch den Schweißprozess bedingte resultierende Eigenspannungsverteilung als maßgebliche Einflussgröße auf die Ermüdungsfestigkeit von Schweißverbindungen angesehen wird. Die Ermüdungsfestigkeit von Schweißverbindungen im eigenspannungsarmen Zustand kann in gleichem Maße durch eine geringe Zunahme der Zugeigenspannung oder der Zugmittelspannung deutlich reduziert werden [Nitschke-Pagel, 1995]. Mit steigender Festigkeit des Werkstoffes nimmt der Einfluss der Eigenspannungen zu. Es besteht eine gegenseitige Abhängigkeit von Eigen- und Mittelspannungen bei gleichzeitiger Wirkung, die durch die Höhe der Streckgrenze begrenzt ist. Der Einfluss der Mittelspannung wirkt sich demnach in Abhängigkeit der Höhe der Zugeigenspannungen aus. Der Einfluss von Eigenspannungen auf die Ermüdungsfestigkeit ist als nicht vollständig geklärt anzusehen. Vergleichende Auswertungen von Versuchsergebnissen aus der Literatur wie von Krebs et al. [Krebs, 2012] belegen dies durch festgestellte Widersprüche der Untersuchungen.

\subsubsection{Prüffrequenz}

Untersuchungen zum Einfluss der Prüffrequenz auf die Ermüdungsfestigkeit an unterschiedlichen Materialen reichen vom klassischen Prüffrequenzbereich $<100 \mathrm{~Hz}$ bis in den Bereich deutlich erhöhter Prüffrequenzen von bis zu $20 \mathrm{kHz}$, wobei sich die Erkenntnisse zum Teil unterscheiden [Stephenson, 1958] [Mayer, 1999]. Bei der Beurteilung des Einflusses der Prüffrequenz auf die Ermüdungsfestigkeit sind materialabhängige sowie äußere Effekte zu unterscheiden [Holper, 2004] 
[Tsutsumi, 2009]. Zu den dem Material innewohnenden Effekten zählt die durch die Erhöhung der Prüffrequenz ansteigende Dehngeschwindigkeit. Zu den äußeren Effekten zählen Einflüsse wie hohe Temperaturen, Korrosion oder die Erwärmung des Probekörpers infolge innerer Reibung.

Eine Erhöhung der Dehngeschwindigkeit kann sich auf die Plastizität an der Rissspitze und somit auf die Rissfortschrittsgeschwindigkeit auswirken [Holper, 2004]. Bei Materialien, deren mechanische Eigenschaften eine Dehngeschwindigkeitsabhängigkeit aufweisen, kann es bei hohen Dehngeschwindigkeiten zu einer Erhöhung von Streckgrenze und Zugfestigkeit kommen, was eine verringerte Ausbreitung der plastischen Zone an der Rissspitze zur Folge haben kann. Aufgrund der extrem kurzen Rissöffnungszeiten kann die plastische Zone an der Rissspitze ebenfalls deutlich kleiner sein als bei Versuchen mit herkömmlichen Prüffrequenzen. Eine geringere Ausbreitung der plastischen Zone an der Rissspitze kann die Rissfortschrittsgeschwindigkeit reduzieren und die Lebensdauer dadurch verlängern [Tsutsumi, 2009].

Die Dehngeschwindigkeitsabhängigkeit des Materials kann auf den Kristallaufbau zurückgeführt werden [Laird, 1982]. Bei geringen Dehngeschwindigkeiten verhalten sich kubisch-raumzentrierte und kubisch-flächenzentrierte Metalle ähnlich. Der Einfluss auf die Ermüdungsfestigkeit ist in der Regel gering. Im Falle hoher Dehngeschwindigkeiten kann für Materialien mit kubisch-raumzentriertem Gitter eine Beeinflussung der Ermüdungsfestigkeit beobachtet werden. Generell zeigt sich, dass ein dominanter Einfluss besonders bei hohen plastischen Dehnungen im Bereich der Kurzzeitfestigkeit vorliegen kann [Mayer, 1999]. Im Bereich sehr hoher Lastwechselzahlen liegen in der Regel sehr geringe plastische Dehnungsamplituden vor, sodass sich der Einfluss der Prüffrequenz auch bei kubisch-raumzentrierten Materialien in diesem Fall als gering erweisen kann [Müller-Bollenhagen, 2011].

Grundsätzlich wird der Einfluss der Prüffrequenz auf die Ergebnisse von Ermüdungsversuchen bei Stahl von Radaj und Vormwald [Radaj, 2007] für Frequenzen bis $1000 \mathrm{~Hz}$ als gering sowie von Haibach [Haibach, 2006] als untergeordnet eingestuft. Frequenzen von 0,1 bis $100 \mathrm{~Hz}$ werden von Gudehus und Zenner [Gudehus, 1999] mit einem sehr geringen Einfluss auf die Ermüdungsfestigkeit eingeordnet. Diese Angaben gelten für den Fall, dass Korrosion, hohe Temperaturen oder Beanspruchungen im Bereich der Fließgrenze ausgeschlossen werden können [Bannantine, 1990] [Radaj, 2007] [Schijve, 2009]. Desweiteren ist eine erhöhte Erwärmung der Probe durch innere Reibung auszuschließen [Koller, 1997]. 
Vorausgesetzt es herrschen Umgebungseinflüsse wie Korrosion oder hohe Temperaturen vor oder es besteht eine Dehngeschwindigkeitsabhängigkeit des Materials, so kann bei Ermüdungsversuchen mit der Ultraschallprüftechnik bei Prüffrequenzen von bis zu $20 \mathrm{kHz}$ von einem Einfluss der Prüffrequenz auf die Ergebnisse ausgegangen werden [Pyttel, 2011] [Stanzl-Tschegg, 2014].

Bei der Beurteilung von Prüffrequenzeinflüssen ist es wichtig, Einflüsse aus innerer Reibung und der daraus resultierenden Probekörpererwärmung auszuschließen. Da im Gegensatz zur herkömmlicher Prüftechnik in der Ultraschallprüftechnik von einer deutlichen Erwärmung des Probekörpers infolge innerer Reibung auszugehen ist, ist dieser Erwärmung durch Kühlung und/oder dem Betrieb in Sequenzen aus Puls und Pause entgegenzuwirken [Tsutsumi, 2009] [Stanzl-Tschegg, 2014].

Um die Aussagekraft und Zuverlässigkeit von mit der Ultraschallprüftechnik erzielten Versuchsergebnissen sicherzustellen, sind nach Pyttel et al. [Pyttel, 2011] Untersuchungen zum Einfluss der Prüffrequenz unerlässlich, wobei zu beachten ist, dass das gleiche Probekörpervolumen verwendet wird, um einen zusätzlichen Größeneinfluss zu vermeiden. In Sonsino [Sonsino, 2007] wird darauf hingewiesen, dass ein stetiger Übergang von Versuchsergebnissen bei geringen Prüffrequenzen zu Versuchsergebnissen, die mit der Ultraschallprüftechnik erzielt wurden, nicht immer gegeben ist. Die Lastüberwachung im Bereich kleiner als $10^{6}$ Lastwechsel wird von Sonsino [Sonsino, 2007] aufgrund der sehr kurzen Kontrollzeiten als ungenau eingestuft. Zudem wird angemerkt, dass die Probekörpererwärmung im Inneren trotz Kühlung nicht zu vernachlässigen ist.

In den normativen Regelungen zum Dauerschwingversuch in der Werkstoffprüfung [DIN 50100, 1978] ist keine Begrenzung der maximalen Prüffrequenz enthalten. Im Gegensatz dazu ist in der Norm zum Schwingfestigkeitsversuch bei Axialbelastung von Verbindungselementen mit Gewinde [DIN 969,1997] eine maximale Prüffrequenz von $250 \mathrm{~Hz}$ festgelegt sowie eine Begrenzung der maximalen Prüffrequenz auf einen Wert, bei dem sich die Probe auf nicht mehr als $50{ }^{\circ} \mathrm{C}$ erwärmt.

\subsection{Ermüdungsversuche an Schweißverbindungen aus Stahl}

Für Schweißverbindungen aus Baustahl ist der Zeitfestigkeitsbereich gut mit Ermüdungsversuchen abgedeckt. Es liegt ein umfangreicher, in mehreren Teilen veröffentlichter Wöhlerlinienkatalog von Olivier und Ritter [Olivier, 1979-1985] vor. Dieser enthält eine einheitliche statistische Auswertung von Versuchsdaten für unterschiedliche Nahtformen wie Stumpfstoß, Kreuzstoß und Längs- sowie Quersteife aus einer Vielzahl an Literaturstellen. Die Beurteilung der Versuchsergebnisse aus dem Wöhlerlinienkatalog von Olivier und Ritter bezüglich des Abknickpunktes der Ermüdungs- 
festigkeitskurven vom Zeit- in den Dauerfestigkeitsbereich ist allerdings als problematisch anzusehen. Zum Einen ist der Umfang der einzelnen Versuchsreihen im Übergangsbereich begrenzt und zum Anderen liegen hauptsächlich Versuchsdaten mit Grenzschwingspielzahlen deutlich unterhalb von $10^{7}$ Lastwechseln vor. Häufig wurden Versuche bereits bei $2 \cdot 10^{6}$ Lastwechseln ohne Bruch beendet.

Die Auswertung von Ritter [Ritter, 1994] im Hinblick auf den Abknickpunkt der Ermüdungsfestigkeitskurven vom Zeit- in den Dauerfestigkeitsbereich für unterschiedliche Nahtformen wie Stumpfstoß, Kreuzstoß und Längssteife auf Basis des Wöhlerlinienkatalogs [Olivier, 1979-1985] und Versuchsdaten aus weiteren Veröffentlichungen ergab, dass Versuchsreihen mit und ohne eindeutig erkennbaren Abknickpunkt vorliegen. Das Verhalten der entsprechenden Versuchsreihen ohne eindeutig erkennbaren Abknickpunkt wurde auf den Eigenspannungszustand und den Ausgangsort des Bruches von der Schweißnahtwurzel zurückgeführt, wobei sich Abknickpunkte bei wesentlich höheren Schwingspielzahlen auch im Bereich von mehr als $10^{7}$ Lastwechseln andeuten. Allerdings sind eindeutige Aussagen bezüglich eines späteren Abknickpunktes aufgrund der zu geringen Anzahl von Versuchsdaten im Bereich sehr hoher Lastwechselzahlen nicht möglich. Das Verhalten eines nicht eindeutig erkennbaren Abknickpunktes zeigte sich besonders ausgeprägt bei Längssteifen und schubbeanspruchten Kehlnähten, konnte aber auch bei weiteren Details wie Stumpfstößen festgestellt werden. Das unterschiedliche Verhalten der Ermüdungsfestigkeitskurven konnte für das Detail Längssteife auf den Eigenspannungszustand zurückgeführt werden [Ritter, 1984] [Sonsino, 1987].

Die Ermüdungsversuche von Otha et al. [Otha, 1986] an axial beanspruchten Stumpfnähten aus Stahl SPV50 $\left(f_{y}=579 \mathrm{~N} / \mathrm{mm}^{2}\right)$ mit einer Blechdicke von $20 \mathrm{~mm}$, die bis zu einer Grenzschwingspielzahl von $10^{7}$ Lastwechseln geprüft wurden, weisen Brüche im Bereich von $2 \cdot 10^{6}$ bis $10^{7}$ Lastwechseln auf (siehe Abb. 2-9). Im Hinblick auf die Berücksichtigung von hohen Zugeigenspannungen wurden unterschiedliche konstante Spannungsverhältnisse $R$ untersucht. Es zeigte sich eine abnehmende Ermüdungsfestigkeit bei steigendem Spannungsverhältnis. Die vorgestellte Methode zur Erzielung konservativer Versuchsdaten sieht die Versuchsführung bei variablem Spannungsverhältnis vor, wobei die Oberspannung konstant in Höhe der Streckgrenze des Grundmaterials liegt. Es zeigten sich geringere Ermüdungsfestigkeiten in den Ergebnissen der vorgestellten Methode als bei der Versuchsführung mit konstantem Spannungsverhältnis. Desweiteren wurden von Otha et al. [Otha, 1997] Ermüdungsversuche an groß- und kleinformatigen Stumpfnähten des Stahls SM490B durchgeführt. Der Vergleich der Ergebnisse der großformatigen Proben mit hohen Eigenspannungen im Bereich der Streckgrenze, die bei einem Spannungsverhältnis von $\mathrm{R}=0$ geprüft wurden, mit den Ergebnissen der kleinformatigen Proben 
ohne Eigenspannungen, die mit konstanter Oberspannung in Höhe der Streckgrenze des Grundmaterials geprüft wurden, liefert eine sehr gute Übereinstimmung. Es ist ein kontinuierlicher Abfall der Ermüdungsfestigkeit bis $10^{7}$ Lastwechseln zu erkennen, wobei einzelne Versuche bis $5 \cdot 10^{7}$ Lastwechseln durchgeführt wurden.

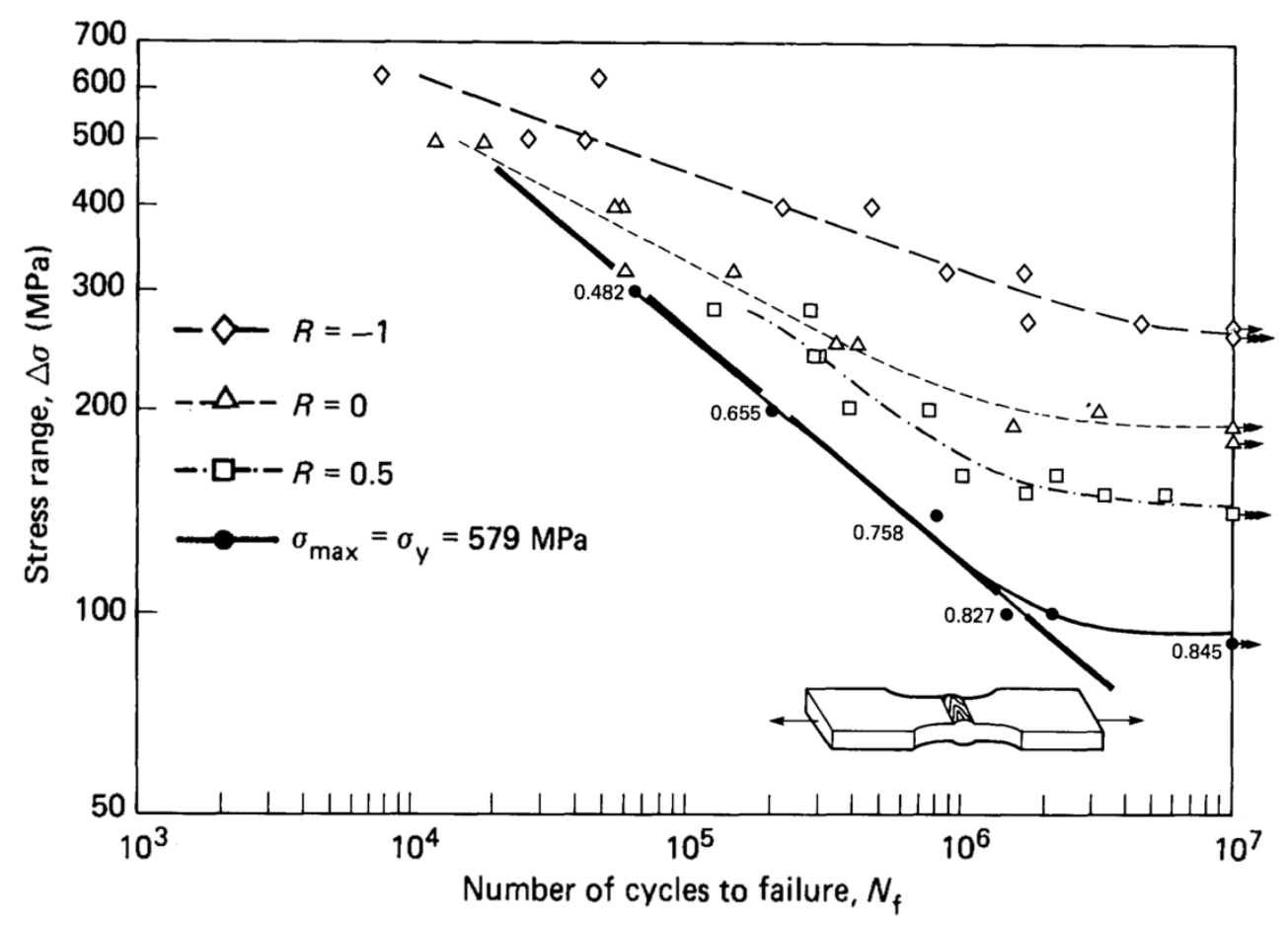

Abb. 2-9: Ermüdungsversuche an einer Stumpfnahtverbindung aus Otha et al. [Otha, 1986]

In Nakamura et al. [Nakamura, 1988] wurden ebenfalls Versuche an axial beanspruchten Stumpfnähten aus Stahl SM50B $\left(f_{y}=431 \mathrm{~N} / \mathrm{mm}^{2}\right)$ durchgeführt. Die Ergebnisse von Versuchen an schmalen Proben mit geringen Eigenspannungen unter Anwendung der von Otha et al. [Otha, 1986] beschriebenen Methode und an breiten Proben mit hohen Eigenspannungen bei konstantem Spannungsverhältnis zeigen eine gute Übereinstimmung. Einzelne Versuche wurden bis zu einer Grenzschwingspielzahl von $5 \cdot 10^{7}$ Lastwechseln durchgeführt, wobei Brüche bis $10^{7}$ festgestellt wurden.

In Zhao et al. [Zhao, 2012] wurden mit der Ultraschallprüftechnik Ermüdungsversuche bei einer Prüffrequenz von $20 \mathrm{kHz}$ an axial beanspruchten Stumpfnähten aus kohlenstoffarmem Stahl $\left(f_{y}=400 \mathrm{~N} / \mathrm{mm}^{2}\right)$ mit abgeschliffenen Oberflächen und an Grundwerkstoffproben durchgeführt. Die Versuche reichen bis zu Grenzschwingspielzahlen von 3.10 $10^{9}$ Lastwechseln. Abb. 2-10 zeigt, dass beide Ermüdungsfestigkeitskurven mit steigender Lastwechselzahl abfallen. Es treten auch nach $10^{7}$ Lastwechseln Brüche auf. Die Lebensdauer der abgeschliffenen Schweißproben ist geringer als die der Grundwerkstoffproben. Im Zeitfestigkeitsbereich werden die Riss- 
ausgangsorte an der Oberfläche an sehr kleinen Riefen lokalisiert. Ein Abknickpunkt ist zwischen $10^{7}$ und $10^{8}$ Lastwechseln erkennbar. Nach dem Abknickpunkt verlaufen beide Ermüdungsfestigkeitskurven flacher und es kommt zu einer Verlagerung der Rissausgangsorte ins Innere. Ermüdungsrisse werden an inneren Defekten wie Poren oder Einschlüssen initiiert. Da bei den abgeschliffenen Schweißproben viele größere innere Defekte vorliegen, fällt die Ermüdungsfestigkeitskurve dieser Proben stärker ab als die des Grundwerkstoffes.

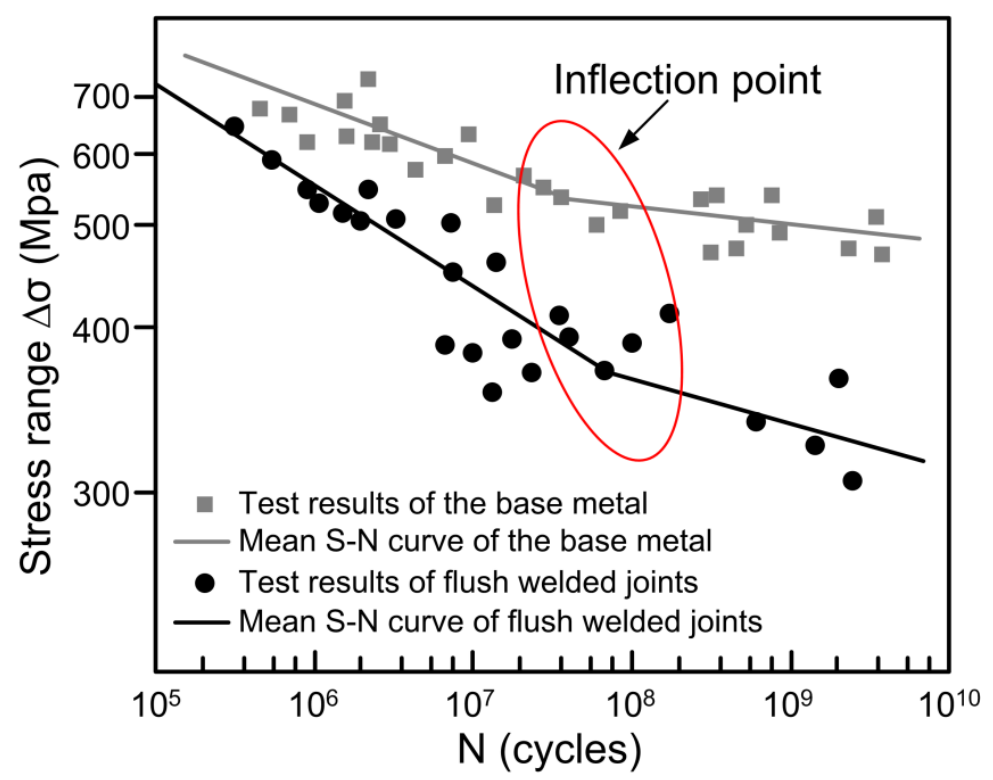

Abb. 2-10: Ermüdungsversuche an abgeschliffenen Schweißproben und Grundwerkstoffproben aus Zhao et al. [Zhao, 2012]

In Ermüdungsversuchen unter Axialbeanspruchung an großformatigen Rohren mit Umfangsnähten und an kleinformatigen Proben, die als Streifen aus Rohren mit Umfangsnähten oder stumpf gestoßenen Blechen geschnitten wurden, untersuchten Maddox und Razmjoo [Maddox, 1998] unterschiedliche Schweißverfahren sowie einseitig und zweiseitig geschweißte Nähte. Die Proben aus Baustahl $\left(f_{y}=443 \mathrm{~N} / \mathrm{mm}^{2}\right)$ wurden zum Teil bis zu einer Grenzschwingspielzahl von $10^{7}$ Lastwechseln bei konstanter Mittelspannung und somit variablem Spannungsverhältnis geprüft. Es zeigten sich Brüche bis in den Bereich der Grenzschwingspielzahl. Die Ergebnisse der kleinformatigen Proben lieferten höhere Ermüdungsfestigkeiten als die der großformatigen Proben, was auf den Einfluss von Eigenspannungen zurückgeführt wurde. Zur Erzielung einer besseren Übereinstimmung der Ergebnisse wurde die Prüfung von kleinformatigen Proben bei hohen Spannungsverhältnissen empfohlen. Desweiteren liegen Ermüdungsversuche an Rohren im Originalmaßstab mit Umlaufnähten von Buitrago et al. [Buitrago, 2003] unter Umlaufbiegung bis zu einer Grenzschwingspielzahl von $10^{8}$ Lastwechseln vor. Es traten Ermüdungsbrüche im Bereich von $10^{7}$ bis 
$10^{8}$ auf, die allerdings nicht darauf hinweisen, dass ein Wechsel des Neigungsexponenten der Wöhlerlinie bzw. ein Abknickpunkt vorliegt.

Geschweißte T- und Y-Stöße aus Baustahl St 52-3 mit unterschiedlichen Nahtformen wurden von Olivier [Olivier, 2000] basierend auf Versuchsdaten aus Olivier et al. [Olivier, 1989] [Olivier, 1994], die bis in den Grenzschwingspielzahlbereich von $10^{7}$ Lastwechseln und in Einzelfällen auch darüber hinaus reichen, unter Biegung untersucht. Es zeigte sich die Tendenz, dass Abknickpunkte der Ermüdungsfestigkeitskurven vom Zeit- in den Dauerfestigkeitsbereich bei deutlich mehr als $2 \cdot 10^{6}$ Lastwechseln liegen können. Es konnten Brüche bis in den Bereich von $10^{7}$ Lastwechseln beobachtet werden. Desweiteren wurde eine Abhängigkeit der Lage des Abknickpunktes von der Kerbwirkung unter Biegung festgestellt. Bei geringer Kerbwirkung ist ein Abknickpunkt zwischen $2 \cdot 10^{6}$ bis $5 \cdot 10^{6}$ Lastwechseln zu erkennen, wohingegen bei starker Kerbwirkung höhere Schwingspielzahlen festzustellen sind. Allerdings ließ eine zu geringe Anzahl von Versuchsdaten im Bereich von $10^{8}$ Lastwechseln keine eindeutige Ermittlung von Abknickpunkten zu.

Untersuchungen an durch Torsion schubbeanspruchten Kehlnähten aus niedrigfestem Baustahl bis zu Grenzschwingspielzahlen von $10^{7}$ Lastwechseln von Seeger und Olivier [Seeger, 1987] und aus höherfestem Baustahl bis zu Grenzschwingspielzahlen von vereinzelt $10^{8}$ Lastwechseln von Seeger und Olivier [Seeger, 1992] weisen ein unterschiedliches Verhalten bezüglich des Abknickpunktes vom Zeit- in den Dauerfestigkeitsbereich auf. Für niedrigfesten Baustahl ist die Tendenz eines Abknickpunktes bei $5 \cdot 10^{6}$ Lastwechseln zu beobachten. Im Gegensatz dazu ist für den hochfesten Baustahl eine Fortführung der Zeitfestigkeitsgeraden bis in den Bereich von $10^{8}$ Lastwechseln zu erkennen. Im Schweißzustand belassene und spannungsarm geglühte Proben verhalten sich ähnlich, sodass Eigenspannungseinflüsse als Ursache ausgeschlossen wurden.

Flachstäbe mit Quersteifen aus Baustahl St 37-2 unter Biegewechselbeanspruchung wurde von Haibach [Haibach, 1971] in einem umfangreichen Versuchsprogramm mit insgesamt 160 Versuchen im Bereich von $10^{6}$ bis $10^{7}$ untersucht (siehe Abb. 2-11). Die Dauerfestigkeit wurde bei $10^{7}$ Lastwechseln ermittelt, die im Verhältnis um 1:1,2 niedriger ist, als die üblicherweise bei $2 \cdot 10^{6}$ Lastwechseln ermittelte Dauerfestigkeit. Ein weiterer Abfall der Dauerfestigkeit oberhalb von $10^{7}$ Lastwechseln wird mit etwa $5 \%$ erwartet. 


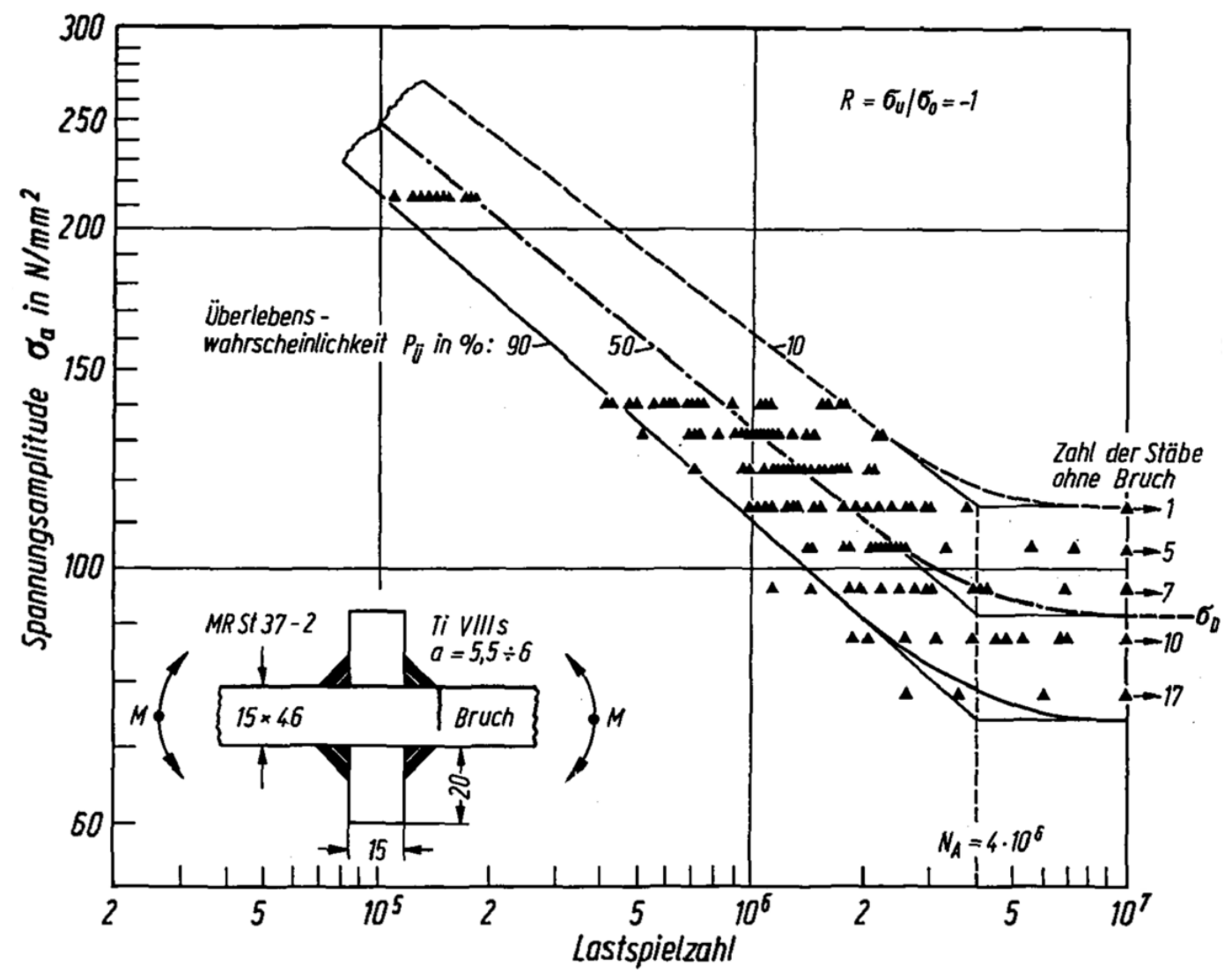

Abb. 2-11: Ermüdungsversuche an Flachstäben mit Quersteifen aus Haibach [Haibach, 1971]

In [Pöting, 2002] wurden Ermüdungsversuche an einem Kreuzstoß aus Baustahl S235JRG2 unter axialer Beanspruchung bis zu einer Grenzschwingspielzahl von $10^{7}$ Lastwechseln durchgeführt, um eine Erweiterung der Ermüdungsdatenbasis für Schweißverbindung im HCF-Bereich zu erzielen und somit einen Beitrag zur Verbesserung der Lebensdauervorhersage für Schweißverbindungen zu leisten. Es traten Brüche bis in den Bereich von 2.10 $10^{6}$ Lastwechseln auf. Für die untersuchte Schweißverbindung wurde ein Abknickpunkt zur Dauerfestigkeit zwischen $10^{6}$ und $10^{7}$ Lastwechseln ermittelt.

In Maddox [Maddox, 1982] wurde der Einfluss von Zugeigenspannungen auf die Ermüdungsfestigkeit von Proben mit aufgeschweißten Längssteifen untersucht. Hierbei wurden Ermüdungsversuche an im Schweißzustand belassenen und spannungsarm geglühten Proben mit aufgeschweißten Längssteifen aus Stählen unterschiedlicher Zugfestigkeiten bei unterschiedlichen Spannungsverhältnissen durchgeführt. Einige Versuche wurden im Bereich geringer Spannungsschwingbreiten bis zu einer Grenzschwingspielzahl von $10^{7}$ Lastwechseln geprüft, wobei auch Brüche bis in diesen Bereich aufgetreten sind. Es zeigte sich, dass die im Schweißzustand belassenen Proben mit hohen Zugeigenspannungen keine starke Abhängigkeit vom Spannungs- 
verhältnis aufweisen, wobei die Ermüdungsfestigkeiten der im Schweißzustand belassenen Proben nicht signifikant von den spannungsarm geglühten Proben abweichen.

In Kulka et al. [Kulka, 1987] und Sonsino et al. [Sonsino, 1987] werden ebenfalls Ergebnisse von Ermüdungsversuchen an im Schweißzustand belassenen und spannungsarm geglühten Proben aus Baustahl St 52-3 mit aufgeschweißten Längssteifen verglichen. Die Versuche wurden bis zu einer Grenzschwingspielzahl von $10^{8}$ durchgeführt. Bei den im Schweißzustand belassenen Proben konnte ein deutlicher Abfall der Ermüdungsfestigkeit bis $10^{7}$ Lastwechseln festgestellt werden (siehe Abb. 2-12). Dieser Effekt wird auf den Eigenspannungszustand aus dem Schweißprozess zurückgeführt. Desweiteren wurden spannungsarm geglühte Proben bei einer Mittelspannung in Höhe der gemessenen Eigenspannungen geprüft. Die Ergebnisse lagen hierbei im Streuband der Ergebnisse der im Schweißzustand belassenen Proben. Es konnte gezeigt werden, dass sich ein Ansatz von Zugeigenspannungen als Mittelspannungen im elastischen Bereich als gute Näherung erweist [Sonsino, 1994].

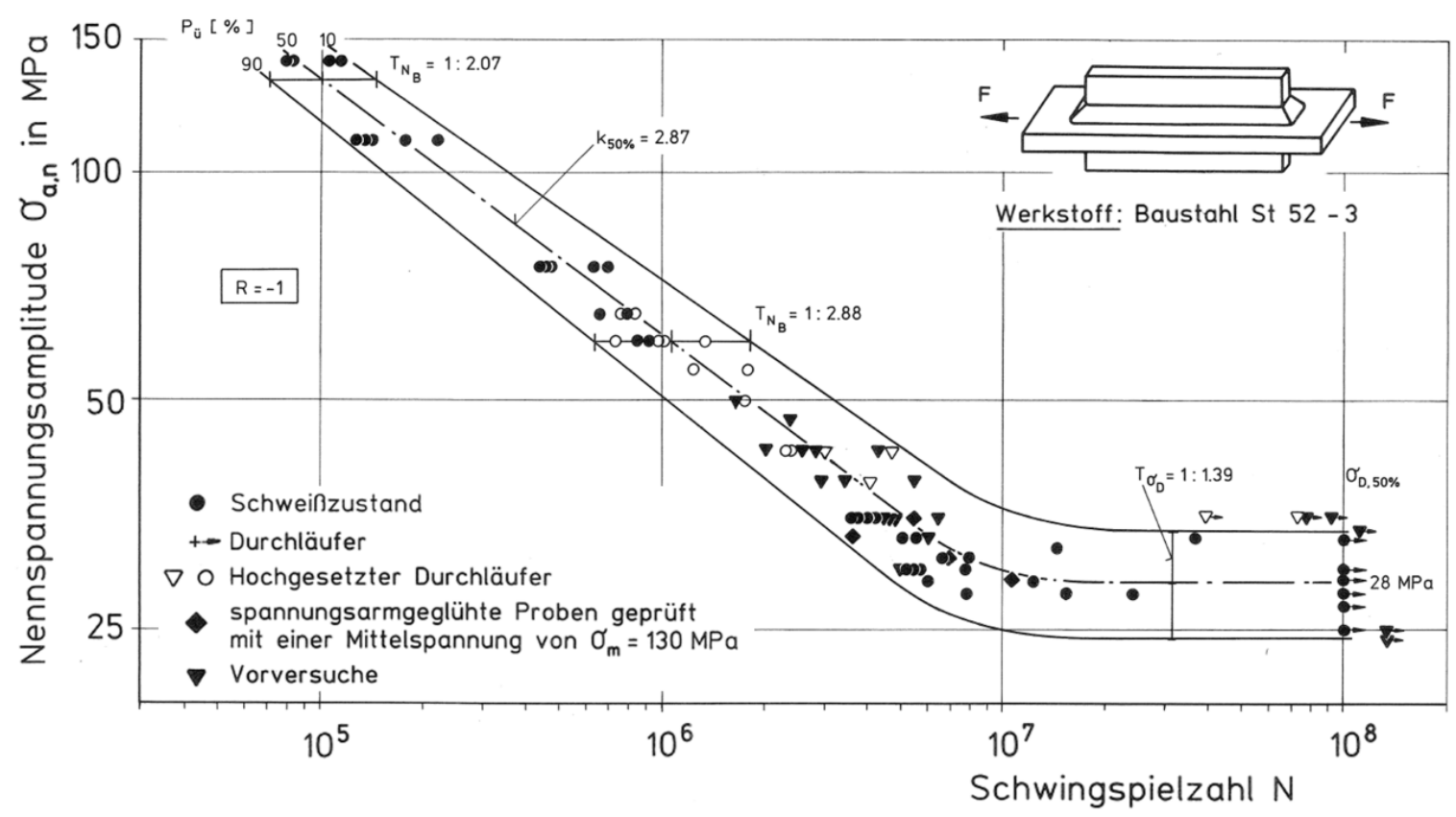

Abb. 2-12: Ermüdungsversuche an Längssteifen aus Kulka et al. [Kulka, 1987]

Die Versuchsdaten aus der Literatur belegen, dass Abknickpunkte von Ermüdungsfestigkeitskurven vom Zeit- in den Dauerfestigkeitsbereich bei Schweißverbindungen mit unterschiedlichen Nahtformen aus Stahl im Bereich von $10^{6}$ bis $10^{8}$ Lastwechseln liegen können, wenn keine erhöhten Temperaturen oder korrosive Umgebungsbedingungen vorliegen. Der beobachtete weitere Abfall der Ermüdungsfestigkeit wird auf Zugeigenspannungen, resultierend aus dem Schweißprozess, aber auch die Belastungsart zurückgeführt. Der Umfang vorhandener Versuchsergebnisse ab einer 
Schwingspielzahl von $10^{7}$ Lastwechseln ist als begrenzt einzustufen und Versuchsergebnisse an Schweißverbindungen mit mehr als $10^{8}$ Lastwechseln liegen kaum vor. Die Prüfung bauteilähnlicher Proben ist mit der Ultraschallprüftechnik bisher nicht möglich, sodass lediglich abgeschliffene Schweißproben untersucht wurden. In den meisten Fällen liegen keine systematischen Untersuchungen des Übergangsbereichs vor. Es sind nur vereinzelte Ergebnisse im Bereich sehr hoher Lastwechselzahlen vorhanden, die lediglich eine Tendenz für das Auftreten später Brüche darstellen. Es lassen sich keine systematischen Aussagen ableiten.

\subsection{Normative Regelungen und Empfehlungen}

Im Bauwesen werden Stahlkonstruktionen nach gültigen Regelwerken wie dem Eurocode 3 [DIN EN 1993-1-9, 2010] oder den Empfehlungen des IIW [IIW, 2009] ausgelegt. Im Allgemeinen erfolgt die Bemessung gegen Ermüdung nach dem Nennspannungskonzept, was durch die einfache Anwendbarkeit begründet ist. Das in den gültigen Regelwerken und Empfehlungen verankerte Nennspannungskonzept basiert auf experimentell ermittelten Ermüdungsfestigkeitskurven, wobei der Bereich der sehr hohen Lastwechselzahlen in den meisten Fällen experimentell nicht ausreichend abgedeckt ist. Es erfolgt die Einstufung von Konstruktionsdetails in Kerbfallklassen. Die Ermüdungsfestigkeitskurven sind über die charakteristische Ermüdungsfestigkeit einer Kerbfallklasse bei $2 \cdot 10^{6}$ Lastwechseln definiert. Außerdem existieren Empfehlungen von Sonsino [Sonsino, 2005a] [Sonsino, 2007], die Angaben über den Verlauf vom Ermüdungsfestigkeitskurven unterschiedlicher Werkstoffe und Werkstoffzustände im Bereich sehr hoher Lastwechselzahlen enthalten.

\subsubsection{Eurocode 3}

Für die Bemessung von Kerbdetails für Längsspannungsschwingbreiten sind im Eurocode 3 [DIN EN 1993-1-9, 2010] Ermüdungsfestigkeitskurven mit einem Neigungsexponenten $k$ von 3,0 und einem Abknickpunkt $N_{k}$ bei $5 \cdot 10^{6}$ Lastwechseln angegeben (siehe Abb. 2-13). Ab dem Abknickpunkt weist die Ermüdungsfestigkeitskurve für konstante Längsspannungsschwingbreiten einen horizontalen Verlauf auf. Für variable Längsspannungsschwingbreiten gelten die erweiterten Ermüdungsfestigkeitskurven, für die im Bereich $5 \cdot 10^{6}<\mathrm{N}<10^{8}$ eine Änderung des Neigungsexponenten auf $k=5$ vorsehen ist. Ab dem Abknickpunkt $N_{k}$ bei $10^{8}$ Lastwechseln weist die Ermüdungsfestigkeitskurve ebenfalls einen horizontalen Verlauf auf. 


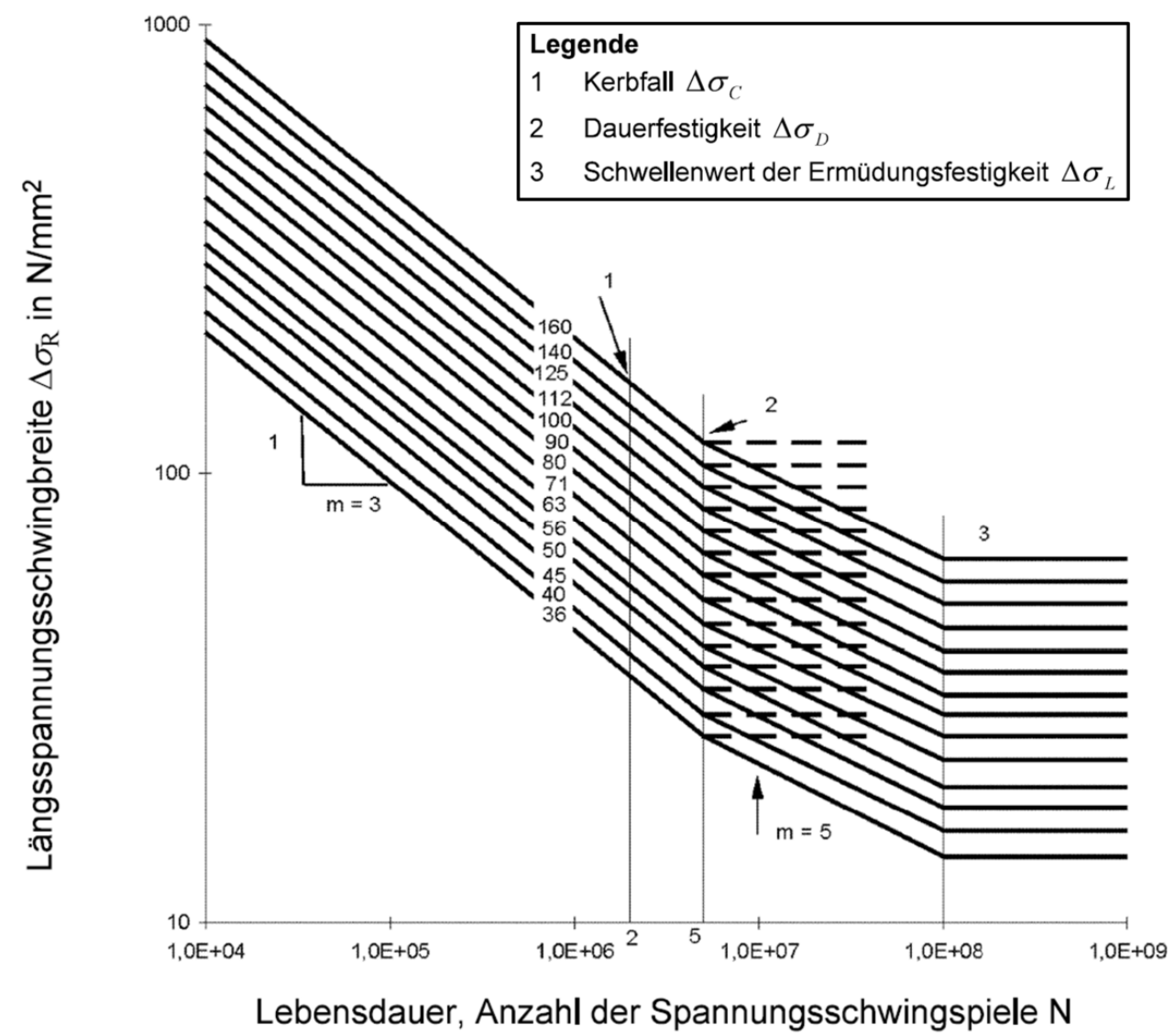

Abb. 2-13: Ermüdungsfestigkeitskurve für Längsspannungsschwingbreiten aus Eurocode 3 [DIN EN 1993-1-9, 2010]

Die im Eurocode 3 angegebenen Ermüdungsfestigkeitskurven sind statistisch als $95 \%$-Quantil für Überleben mit etwa $75 \%$ Vertrauenswahrscheinlichkeit ermittelt worden. Die Wöhlerlinien sind für ein Spannungsverhältnis von $\mathrm{R}=0$ angegeben. Beim Vorliegen hoher Zugeigenspannungen besteht gemäß Eurocode 3 keine Abhängigkeit der Ermüdungsfestigkeit von der Mittelspannung. Für nichtgeschweißte oder spannungsarm geglühte Strukturen kann unter Druckbeanspruchung der Mittelspannungseinfluss auf die Ermüdungsfestigkeit berücksichtigt werden (vergleiche Abb. 2-14, links). Hierbei darf der im Druckbereich liegende Teil der Spannungsschwingbreite von $R=0$ bis $R= \pm \infty$ auf $60 \%$ reduziert werden. Wenn die Spannungsschwingbreite vollständig im Druckbereich liegt, wird ein konstanter Wert angesetzt. Dies entspricht einer maximalen Erhöhung der zulässigen Spannungsschwingbreite von $67 \%$, wenn die Spannungsschwingbreite vollständig im Druckbereich liegt. Der Größeneinfluss wird bei Durchmessern größer als $30 \mathrm{~mm}$ und bei Blechdicken größer als 25 mm über Abminderungsfaktoren berücksichtigt. 


\section{Kurve $A$ geringe Eigenspannungen Kurve $B$ mäßige Eigenspannungen \\ Kurve $\mathrm{C}$ hohe Eigenspannungen}
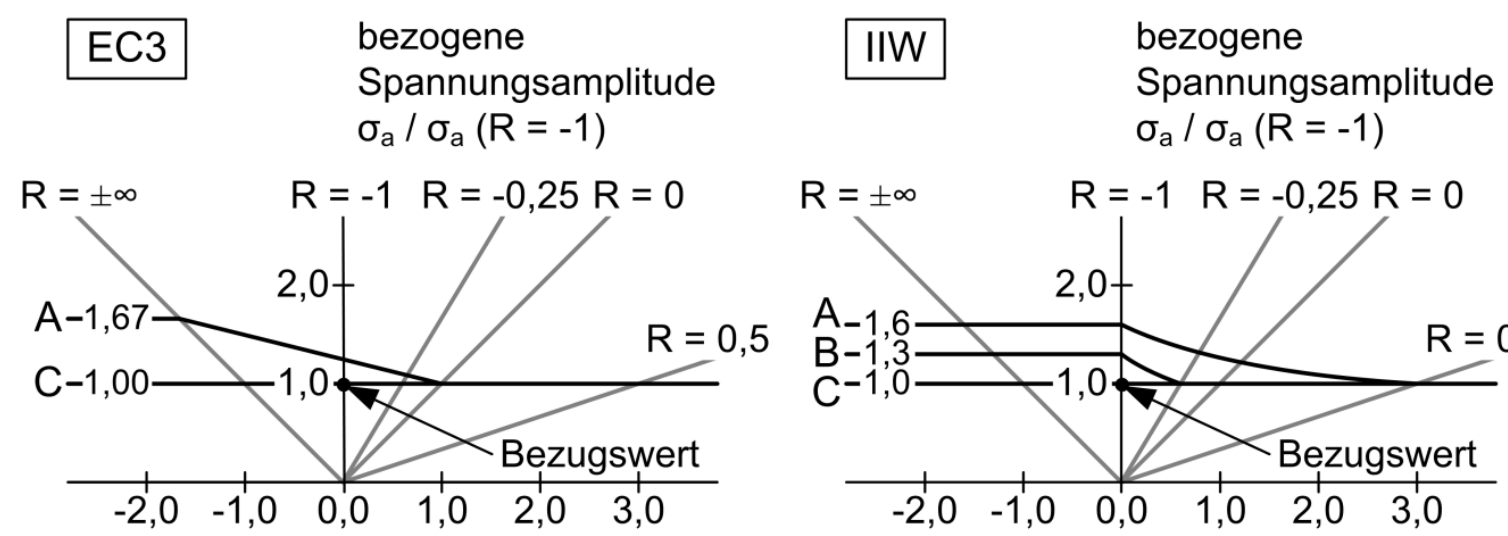

bezogene Mittelspannung $\sigma_{\mathrm{m}} / \sigma_{\mathrm{a}}(\mathrm{R}=-1)$

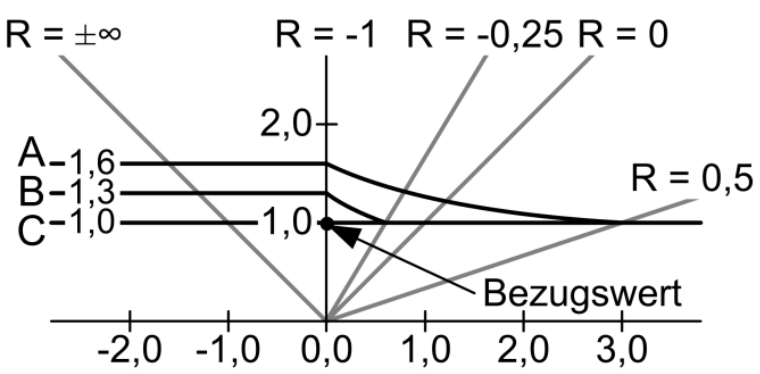

bezogene Mittelspannung $\sigma_{m} / \sigma_{a}(R=-1)$

Abb. 2-14: Einfluss schweißbedingter Zugeigenspannungen auf die Mittelspannungsabhängigkeit nach Eurocode 3 (links) und nach den Empfehlungen des IIW (rechts)

[Haibach, 2006]

\subsubsection{Empfehlungen des IIW}

Die Empfehlungen des IIW [IIW, 2009] sehen für die Bemessung von Kerbdetails bei konstanten Längsspannungsschwingbreiten ebenfalls Ermüdungsfestigkeitskurven mit einem Neigungsexponenten $k$ von 3,0 vor. Der Abknickpunkt $N_{k}$ liegt allerdings erst bei $10^{7}$ Lastwechseln. Desweiteren wird zwischen Standardanwendungen und Anwendungen im Bereich sehr hoher Lastwechselzahlen unterschieden. Für Standardanwendungen weist die Ermüdungsfestigkeitskurve für konstante Längsspannungsschwingbreiten ab dem Abknickpunkt analog zum Eurocode 3 einen horizontalen Verlauf auf. Für Anwendungen im Bereich sehr hoher Lastwechselzahlen weist die Ermüdungsfestigkeitskurve für konstante Längsspannungsschwingbreiten $a b$ dem Abknickpunkt einen Neigungsexponenten $k^{*}$ von 22 auf (siehe Abb. 2-15). Dies entspricht einer weiteren Abnahme der Ermüdungsfestigkeit um $10 \%$ pro Dekade. Desweiteren wird darauf hingewiesen, dass Anwender in diesem Fall die aktuell relevante Fachliteratur hinzuziehen sollten. 


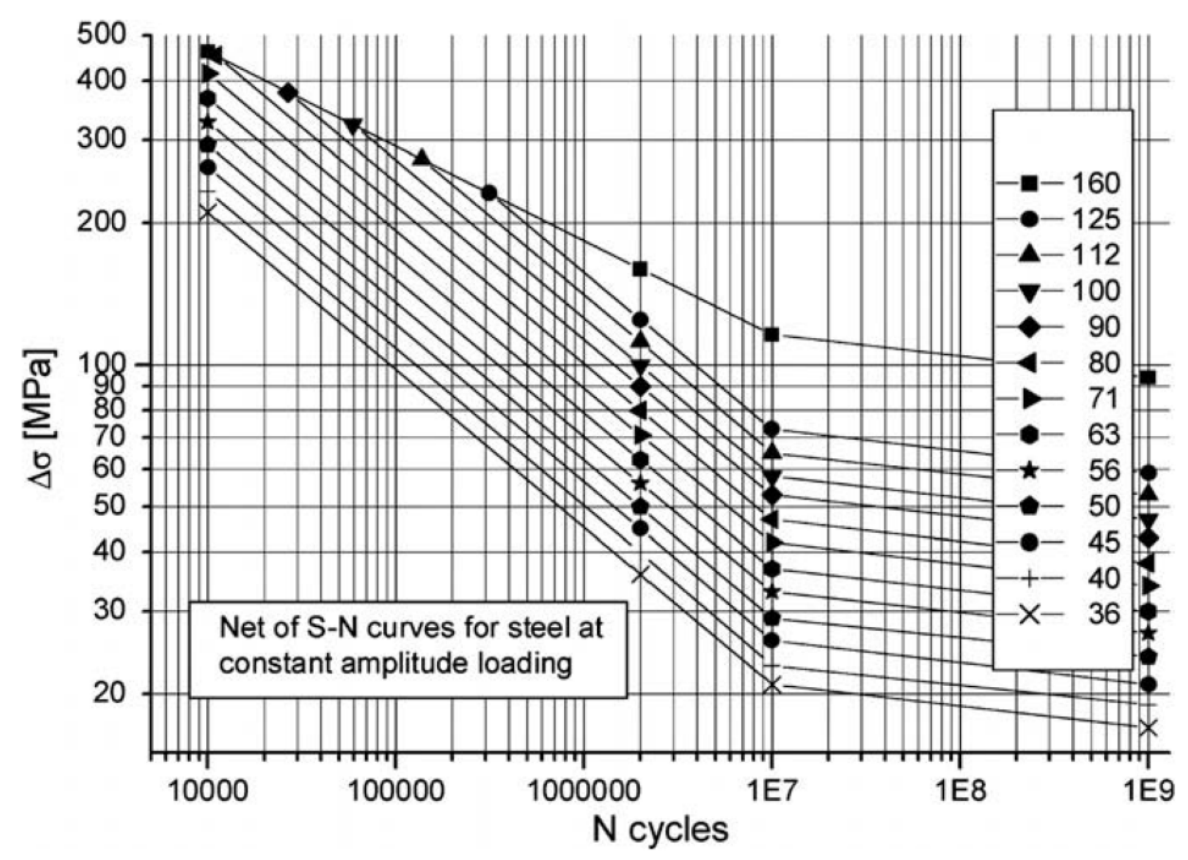

Abb. 2-15: Ermüdungsfestigkeitskurven für konstante Längsspannungsschwingbreiten bei Anwendungen im VHCF-Bereich aus den Empfehlungen des IIW [IIW, 2009]

Die in den Empfehlungen des IIW [IIW, 2009] angegebenen Ermüdungsfestigkeitskurven sind statistisch für eine Überlebenswahrscheinlichkeit von $95 \%$ mit einer Vertrauenswahrscheinlichkeit von $75 \%$ ermittelt worden. Die Wöhlerlinien sind für ein Spannungsverhältnis von $\mathrm{R}=0,5$ angegeben. Analog zum Eurocode 3 besteht beim Vorliegen hoher Zugeigenspannungen keine Abhängigkeit der Ermüdungsfestigkeit von der Mittelspannung. Über einen Erhöhungsfaktor kann der Mittelspannungseinfluss auf die Ermüdungsfestigkeit für nichtgeschweißte oder spannungsarmgeglühte sowie für einfache kleinformatige dünnwandige Strukturen mit kurzen Schweißnähten berücksichtigt werden (vergleiche Abb. 2-14, rechts). In ähnlicher Größenordnung wie im Eurocode 3 liegt der konstante Wert bei geringen Zugeigenspannungen. Allerdings wird dieser bereits ab einem Spannungsverhältnis von $R=-1$ als konstant angesetzt. Zudem wird bereits bei mäßigen Zugeigenspannungen eine Mittelspannungsabhängigkeit in geringerem Maße berücksichtigt. Es kann beim Vorliegen geringer bzw. mäßiger Zugeigenspannungen eine maximale Erhöhung der Kerbfallklasse von 60 \% bzw. 30 \% angesetzt werden, wenn die Spannungsschwingbreite im Wechsellastbereich oder darüber hinaus im Druckbereich liegt. Der Größeneinfluss bei Blechdicken größer als $25 \mathrm{~mm}$ wird ebenfalls über einen Reduktionsfaktor berücksichtigt. 


\subsubsection{Empfehlungen von Sonsino}

In Sonsino [Sonsino, 2005a] [Sonsino, 2007] werden Empfehlungen zu Abknickpunkten, Neigungsexponenten nach dem Abknickpunkt und Streumaßen für unterschiedliche Werkstoffe und Werkstoffzustände angegeben. Ein Auszug ist in Tab. 2-1 gegeben. Für spannungsarm geglühte Schweißverbindungen wird der Abknickpunkt $N_{k}$ bei $10^{6}$ Lastwechseln definiert. Ab dem Abknickpunkt wird der Neigungsexponent $k^{*}$ zu 45 angenommen, was einem Abfall von $5 \%$ pro Dekade entspricht. Für Schweißverbindungen mit hohen Zugeigenspannungen wird der Abknickpunkt $N_{k}$ bei $10^{7}$ Lastwechseln angesetzt. Ab dem Abknickpunkt wird der Neigungsexponent $k^{*} z u 22$ zugrundegelegt, was einem Abfall von $10 \%$ pro Dekade entspricht. Dieser Fall wurde, wie zuvor in Kapitel 2.5.2 beschrieben, in die Empfehlungen des IIW aufgenommen. Als Streumaß $1: T_{\sigma}$ wird 1,50 angegeben.

Tab. 2-1: Empfohlene Abknickpunkte, Neigungen nach dem Abknickpunkt und Streumaße, Auszug aus [Sonsino, 2005a] [Sonsino, 2007]

\begin{tabular}{|l|l|c|c|c|}
\hline Werkstoff & Abknickpunkt $\mathbf{N}_{\mathbf{k}}$ & $\mathbf{k}^{*}$ & $\begin{array}{c}\text { Abfall pro } \\
\text { Dekade in \% }\end{array}$ & $\mathbf{1 : \mathbf { T } _ { \boldsymbol { \sigma } }}$ \\
\hline Stahl, nicht geschweißt & $\begin{array}{l}5 \cdot 10^{5}, \text { hochfest } \\
2 \cdot 10^{6}, \text { Baustähle }\end{array}$ & 45 & 5 & 1,20 \\
\hline Stahl, geschweißt & $\begin{array}{l}1 \cdot 10^{6}, \text { spannungsarm geglüht } \\
1 \cdot 10^{7}, \text { hohe Zugeigenspannungen }\end{array}$ & $\begin{array}{c}45 \\
22\end{array}$ & $\begin{array}{c}5 \\
10\end{array}$ & 1,50 \\
\hline Stahlguss & $5 \cdot 10^{5}$, hochfest & 45 & 5 & 1,40 \\
& $2 \cdot 10^{6}$, mittelfest & & & \\
\hline
\end{tabular}

Diese Empfehlungen basieren auf Erfahrungen und Auswertungen von Versuchsdaten ([Nakamura, 1988], [Sonsino, 1994], [Maddox, 1998], [Buitrago, 2003]), die bis in den Bereich sehr hoher Lastwechselzahlen reichen. Die Auswertung in Sonsino et al. [Sonsino, 2005b] erfolgte für insgesamt 240 Versuchsergebnisse verschiedener Schweißverbindungen bei Prüffrequenzen bis $125 \mathrm{~Hz}$ und Grenzschwingspielzahlen von bis zu $10^{8}$ Lastwechseln (vergleiche Abb. 1-1). Es wird deutlich, dass bisher nur wenige experimentelle Ergebnisse für Schweißverbindungen aus Stahl im Bereich sehr hoher Lastwechselzahlen vorliegen. Das Auftreten von Durchläufern wird als Hinweis auf eine Veränderung des Schädigungsmechanismus gedeutet. Zur weiteren Absicherung der Ermüdungsfestigkeitskurven nach $10^{8}$ Lastwechseln wird eine Ausweitung der experimentellen Untersuchungen mit Grenzschwingspielzahlen von größer als $10^{8}$ Lastwechseln empfohlen. 


\subsection{Prüftechniken im Bereich sehr hoher Lastwechselzahlen}

Die Problematik bei der Versuchsführung im Bereich sehr hoher Lastwechselzahlen besteht in der Realisierung eines angemessenen Prüfzeitraums. Hierzu sind Prüftechniken mit hohen Prüffrequenzen erforderlich. Bei herkömmlicher Prüftechnik mit Prüffrequenzen bis $100 \mathrm{~Hz}$ kann nur über die zeitgleiche Prüfung einer Vielzahl von Probekörpern zu einem wirtschaftlichen Ergebnis gelangt werden, was eine zielorientierte Versuchsplanung voraussetzt [Schwerdt, 2009].

Schwingversuche an Schweißverbindungen im Zeitfestigkeitsbereich werden vorwiegend mit servohydraulischen Prüfmaschinen und Resonanzprüfmaschinen bei Prüffrequenzen im Bereich von kleiner als $100 \mathrm{~Hz}$ durchgeführt. In Pyttel et al. [Pyttel, 2011] und Stanzl-Tschegg [Stanzl-Tschegg, 2014] sind maximale Prüffrequenzen unterschiedlicher Prüfmaschinen angegeben und in Tab. 2-2 zusammengefasst, wobei anzumerken ist, dass es immer auf die Anwendung und Ausstattung der jeweiligen Prüfmaschine ankommt. Servohydraulische Prüfmaschinen werden üblicherweise in einem Prüffrequenzbereich von 20 bis $50 \mathrm{~Hz}$ eingesetzt, wobei auch servohydraulische Prüfmaschinen existieren, die maximale Prüffrequenzen von $400 \mathrm{~Hz}$ (oder auch $1000 \mathrm{~Hz}$ ) realisieren können. Resonanzprüfmaschinen arbeiten mit Prüffrequenzen von 50 bis $250 \mathrm{~Hz}$, was für Untersuchungen im Bereich von größer als $10^{8}$ Lastwechseln als unwirtschaftlich anzusehen ist.

Tab. 2-2: Prüfmaschinen mit maximaler Prüffrequenz und entsprechender Versuchsdauer bis zu $10^{9}$ Lastwechseln [Pyttel, 2011] [Stanzl-Tschegg, 2014]

\begin{tabular}{|l|l|l|}
\hline Prüfmaschine & Max. Prüffrequenz & Versuchsdauer bis $\mathbf{N}=\mathbf{1 0}^{\mathbf{9}}$ \\
\hline Resonanzprüfmaschine & $250 \mathrm{~Hz}$ & 46 Tage \\
\hline Servohydraulische Prüfmaschine & $400 \mathrm{~Hz}(1000 \mathrm{~Hz})$ & 29 Tage (12 Tage) \\
\hline Ultraschallprüfmaschine & $20 \mathrm{kHz}$ & $\begin{array}{l}\text { 0,6 bis 6 Tage (in Abhängigkeit } \\
\text { der Pausenzeiten) }\end{array}$ \\
\hline
\end{tabular}

Die Ultraschallprüftechnik hingegen, mit Prüffrequenzen von bis zu $20 \mathrm{kHz}$, hat sich in diesem Bereich etabliert und wird verstärkt eingesetzt. Bei Ultraschallprüfmaschinen handelt es sich allerdings nicht um Universalprüfmaschinen. Probekörper mit einer speziell ausgelegten, symmetrischen Geometrie, die auch als HourglassProben bezeichnet werden, und einem relativ geringen Werkstoffvolumen können dehnungskontrolliert geprüft werden. Um eine übermäßige Probekörpererwärmung zu vermeiden, wird die Ultraschallprüfmaschine neben der Möglichkeit der Kühlung in Sequenzen aus Puls (25-100 ms) und Pause (25-1000 ms) betrieben [Schwerdt, 2009] [Stanzl-Tschegg, 2014]. Prinzipiell stellt die Ultraschallprüftechnik 
zur Erschließung des Bereichs sehr hoher Lastwechselzahlen ein geeignetes Prüfverfahren dar. Im Hinblick auf die Prüfung von Schweißverbindungen ist die Herstellung und Prüfung relativ kleiner und ungestörter Probekörper jedoch als äußerst problematisch anzusehen [Sonsino, 2005b]. Aufgrund der speziell erforderlichen Probekörpergeometrie ist die Prüfung von Bauteilen oder bauteilähnlichen Probekörpern sowie die Prüfung bei variablen Beanspruchungsamplituden bisher nicht möglich [Schwerdt, 2009] [Pyttel, 2011].

Erste Untersuchungen mit der Ultraschallprüftechnik zu Schweißverbindungen aus Aluminium wurden von Cremer et al. [Cremer, 2010] [Cremer, 2011] durchgeführt. Mit der Ultraschallprüftechnik werden hier zum Einen Flachproben aus Grund- und Zusatzwerkstoff und zum Anderen Hourglass-Proben der charakteristischen Bereiche der Schweißnaht (Grundwerkstoff, Wärmeinflusszone und Schmelzbad) geprüft. Die Hourglass-Probekörper werden aus den jeweiligen Bereichen verschweißter Bleche gefertigt. Bisher wurden hingegen keine Ermüdungsversuche mit der Ultraschallprüftechnik an geschweißten Probekörpern ohne bearbeitete Oberflächen durchgeführt. Allerdings ist eine bestimmte Kalibrierungsmethode zur Umsetzbarkeit von Ermüdungsversuchen mit der Ultraschallprüftechnik an realen Schweißverbindungen mit Nahtüberhöhung in der Entwicklung [Cremer, 2013]. Weitere Untersuchungen mit der Ultraschallprüftechnik an Schweißverbindungen aus Stahl sind bei Zhao et al. [Zhao, 2012] zu finden, wobei auch hier die Oberflächen der Schweißproben bearbeitet und mit Ergebnissen an Grundwerkstoffproben verglichen wurden.

\subsection{Fazit im Hinblick auf die Untersuchungen der vorliegenden Arbeit}

Im Bezug auf die Ermüdungsfestigkeit von Schweißverbindungen sind die aus der Nahtgeometrie resultierende lokale Kerbwirkung sowie der aus dem Schweißprozess resultierende Eigenspannungszustand als maßgebliche Einflussfaktoren anzusehen. Da die geometrischen Schweißnahtparameter in hohem Maße streuen und sich in der Höhe der hieraus resultierenden Spannungskonzentrationen niederschlagen, ist besonderes Augenmerk auf die Herstellung der Probekörper zu legen, um die Reproduzierbarkeit des Schweißprozesses zu gewährleisten und die Streuung zu minimieren. Die im Nahtbereich von realen Schweißverbindungen im ungünstigsten Fall auftretenden Zugeigenspannungen in Höhe der Streckgrenze liegen bei kleinen Probekörpern in der Regel nicht vor. Um dennoch Aussagen über den Eigenspannungseinfluss bei der Prüfung von kleinen Probekörpern zu gewinnen, empfiehlt sich die Prüfung bei hohen Zugmittelspannungen, da Zugeigenspannungen im elastischen Bereich näherungsweise als Mittelspannungen aufgefasst werden können. Um die Aussagekraft und Zuverlässigkeit von Versuchsergebnissen, die bei erhöhten Prüf- 
frequenzen erzielt wurden, sicherzustellen, sind Untersuchungen zum Einfluss der Prüffrequenz erforderlich.

Für Schweißverbindungen aus Stahl ist der Umfang an vorhandenen Ermüdungsversuchen ab einer Schwingspielzahl von $10^{7}$ Lastwechseln als begrenzt einzustufen. Versuchsergebnisse mit mehr als $10^{8}$ Lastwechseln liegen nur vereinzelt vor. Dies ist auf die begrenzten Möglichkeiten bei der Versuchsführung zurückzuführen. Es sind Prüftechniken mit hohen Prüffrequenzen erforderlich, um Ermüdungsversuche in einem angemessenen Prüfzeitraum zu realisieren. Die Prüfung bauteilähnlicher Proben wie Schweißverbindungen ist mit der Ultraschallprüftechnik bisher nicht möglich. Um die experimentellen Untersuchungen von Schweißverbindungen im Bereich sehr hoher Lastwechselzahlen dennoch ausweiten zu können, bedarf es der Weiterentwicklung anderer Prüftechniken. Es existieren Empfehlungen für den Verlauf der Ermüdungsfestigkeitskurven von Schweißverbindungen im Bereich sehr hoher Lastwechselzahlen, die bereits Eingang die Empfehlungen des IIW gefunden haben. AIlerdings können festgestellte Brüche bei hohen Schwingspielzahlen in bisherigen Untersuchungen aufgrund des geringen Probenumfangs lediglich als Tendenz eines weiteren Abfalls der Ermüdungsfestigkeitskurve angesehen werden und lassen keine systematischen Aussagen bezüglich eines Abknickpunktes und des weiteren Verlaufs zu. Der beobachtete weitere Abfall der Ermüdungsfestigkeit von Schweißverbindungen im Bereich sehr hoher Lastwechselzahlen wird auf aus dem Schweißprozess resultierenden Zugeigenspannungen aber auch die Belastungsart zurückgeführt. 


\section{Herstellung der Probekörper und Charakterisierung des Aus- gangszustandes}

Zur Ausweitung der experimentellen Untersuchungen an Schweißverbindungen bei konstanter Spannungsschwingbreite bis in den Bereich sehr hoher Schwingspielzahlen werden Ermüdungsversuche an bauteilähnlichen Schweißverbindungen durchgeführt. Das Standarddetail der Stumpfnahtverbindung sowie der häufig eingesetzte Baustahl S355J2 $+\mathrm{N}$ wurden gewählt. Die für die Ermüdungsversuche eingesetzte Prüfvorrichtung (siehe Kapitel 4) weist zur Probekörperaufnahme einen Durchlass von $6 \mathrm{~mm}$ auf. Die Blechdicke wurde auf $4 \mathrm{~mm}$ festgelegt, sodass Probekörper mit Naht- und Wurzelüberhöhung problemlos eingebaut werden können.

Im Folgenden wird die Herstellung der Probekörper beschrieben. Hierzu werden Angaben zum untersuchten Werkstoff, zur vorgenommenen Nahtvorbereitung und dem eingesetzten Schweißverfahren sowie der Geometrie der bauteilähnlichen Probekörper gemacht. Zur Bewertung der Reproduzierbarkeit des Schweißprozesses und zur Prüfung der Qualität der Probekörper wird der Ausgangszustand charakterisiert. Herstellungsbegleitend wurden Temperaturmessungen während der Schweiß- und Abkühlphase durchgeführt. Die lokale Nahtgeometrie wurde untersucht. Außerdem wurden Zugversuche, metallographische Untersuchungen und Härtemessungen durchgeführt. Die Ergebnisse werden im Folgenden dargestellt.

\section{$3.1 \quad$ Werkstoff}

Die Stahlsorte S355J2 +N gemäß DIN EN 10025-1 [DIN EN 10025-1, 2005] wurde für die Untersuchungen gewählt. Das Material der Blechdicke $4 \mathrm{~mm}$ stammt aus einer Stahlcharge. Die chemische Zusammensetzung des Werkstoffes ist in Tab. 3-1 angegeben. Die werkstoffmechanischen Kennwerte des Grundwerkstoffs wurden im Zugversuch nach DIN EN ISO 6892-1 [DIN EN ISO 6892-1, 2009] ermittelt. Die Zugversuche am Grundwerkstoff ergaben einen Mittelwert der Streckgrenze von $503,4 \mathrm{~N} / \mathrm{mm}^{2}$ und einen Mittelwert der Zugfestigkeit von 590,0 N/mm². Das Versuchsprotokoll ist Anhang A.1 zu entnehmen.

Tab. 3-1: Chemische Zusammensetzung des Werkstoffes $\$ 355 \mathrm{~J} 2+\mathrm{N}(\mathrm{t}=4 \mathrm{~mm})$

\begin{tabular}{|}
\begin{tabular}{|c|c|c|c|c|c|c|c|c|c|}
\hline Element & $\mathbf{C}$ & $\mathbf{M n}$ & $\mathbf{S i}$ & $\mathbf{P}$ & $\mathbf{S}$ & $\mathbf{C r}$ & $\mathbf{N i}$ & $\mathbf{C u}$ & $\mathbf{M o}$ \\
\hline$[\%]$ & 0,161 & 1,384 & 0,004 & 0,011 & 0,002 & 0,017 & 0,079 & 0,106 & 0,004 \\
\hline Element & $\mathbf{A l}$ & $\mathbf{B}$ & $\mathbf{C o}$ & $\mathbf{S b}$ & $\mathbf{S n}$ & $\mathbf{T i}$ & $\mathbf{V}$ & $\mathbf{M g}$ & $\mathbf{Z r}$ \\
\hline$[\%]$ & 0,033 & 0,0003 & 0,001 & 0,080 & 0,014 & 0,028 & 0,025 & 0,011 & 0,000 \\
\hline
\end{tabular}
\end{tabular}




\subsection{Nahtvorbereitung und Schweißverfahren}

Bleche der Abmessungen $150 \times 150 \times 4 \mathrm{~mm}$ wurden einlagig mit einer Stumpfnaht in Wannenlage gefügt. Die Nahtvorbereitung der Bleche erfolgte in Anlehnung an die Empfehlungen in DIN EN ISO 9692-1 [DIN EN ISO 9692-1, 2004], wie in Abb. 3-1 dargestellt. Die Bleche wurden mit einem Flankenwinkel von $22,5^{\circ}$ vorbereitet. Im Vorfeld der Schweißung wurden die Bleche bei einem Spalt von $0,7 \mathrm{~mm}$ mit zwei Heftnähten im Randbereich versehen. Die gehefteten Bleche wurden kurz geflammt, damit Rückstände auf den Blechen im Bereich der Schweißnaht verbrennen und nicht als Einschlüsse oder Fremdstoffe in der Schweißnaht verbleiben. Daraufhin wurden die Proben im Schweißstand ausgerichtet und mit einem Öffnungswinkel von $45^{\circ}$ verschweißt.
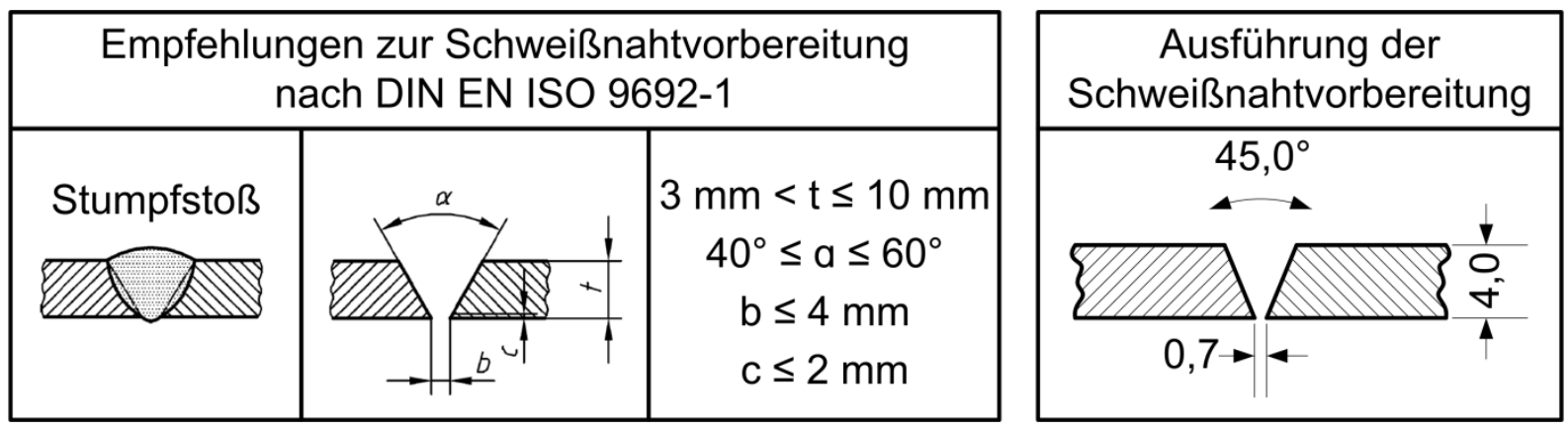

Abb. 3-1: Schweißnahtvorbereitung in Anlehnung an DIN EN ISO 9692-1 [DIN EN ISO 9692-1, 2004]

Das Fügen der Bleche erfolgte durch das Metall-Aktivgasschweißen (MAGSchweißen) mit Impulstechnik, wobei der Brenner mechanisiert über einen Traktor geführt wurde. Die Schweißparameter wurden während der Schweißung konstant gehalten und sind in Tab. 3-2 zusammengefasst.

Tab. 3-2: Überblick der Schweißparameter

\begin{tabular}{|l|c|}
\hline \multicolumn{2}{|l|}{ Schweißparameter } \\
\hline Stromstärke & $76,0 \mathrm{~A}$ \\
\hline Spannung & $19,5 \mathrm{~V}$ \\
\hline Schweißgeschwindigkeit & $376,5 \mathrm{~mm} / \mathrm{min}$ \\
\hline Drahtvorschub & $3,8 \mathrm{~m} / \mathrm{min}$ \\
\hline Drahtelektrode (G 42 3 M G3Si1) & $1,0^{\mathrm{mm}}$ \\
\hline Schutzgas (M21 ArC 18) & $14,0^{\circ} / \mathrm{min}$ \\
\hline Brennerwinkel & $15,0^{\circ}$ \\
\hline Düsenabstand & $15,0^{\mathrm{mm}}$ \\
\hline
\end{tabular}


Während des Schweißprozesses wird der Brenner von einem Traktor in einer definierten Geschwindigkeit über die Probe geführt. Durch diese Mechanisierung des Schweißprozesses wurde die Reproduzierbarkeit der Probekörper verbessert, um die Streuung zu minimieren. Der Aufbau des Schweißstandes, bestehend aus der Lagerung der Proben, der Führung des Brenners über den Traktor und dem Schweißgerät der Firma Cloos (Quineo Puls 450), ist in Abb. 3-2 dargestellt. Die Konstruktion zur Lagerung der Probe und die Schienen zur Führung des motorbetriebenen Traktors sind fest auf dem Schweißtisch montiert. Die gehefteten Bleche werden auf einer Konstruktion aus U-Profilen ausgerichtet und während des Schweißens an den Rändern lose gelagert.

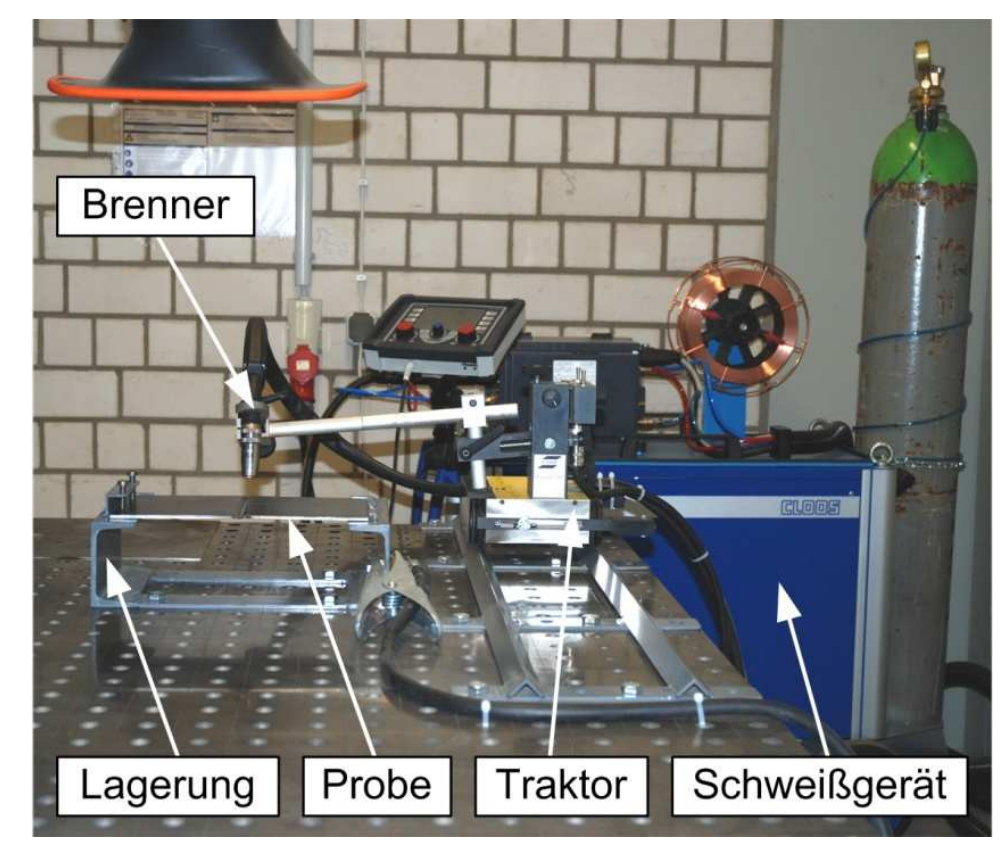

Abb. 3-2: Aufbau des Schweißstands

\subsection{Geometrie der Probekörper}

Die Probekörper wurden aus den verschweißten Blechen gefräst. Zuvor wurden Schlackereste durch Bürsten entfernt. Die Geometrie der Probekörper ist auf die Randbedingungen der Prüfvorrichtung abgestimmt und in Abb. 3-3 dargestellt. Die Schweißnaht befindet sich in Probekörpermitte. In diesem Bereich ist der Prüfquerschnitt tailliert und weist eine Breite von $25 \mathrm{~mm}$ auf, woraus sich eine Nennprüfquerschnittsfläche $A_{N}$ von $100 \mathrm{~mm}^{2}$ ergibt. Zur Vermeidung eines Rissausgangs vom Rand der Probekörper und zur Reduktion des Kanteneinflusses wurden die Ränder der Probekörper nach dem Zuschnitt poliert. Außerdem wurden die Probekörper zur Befestigung in der Prüfvorrichtung mit zwei Durchgangsbohrungen in den Endbereichen versehen. 


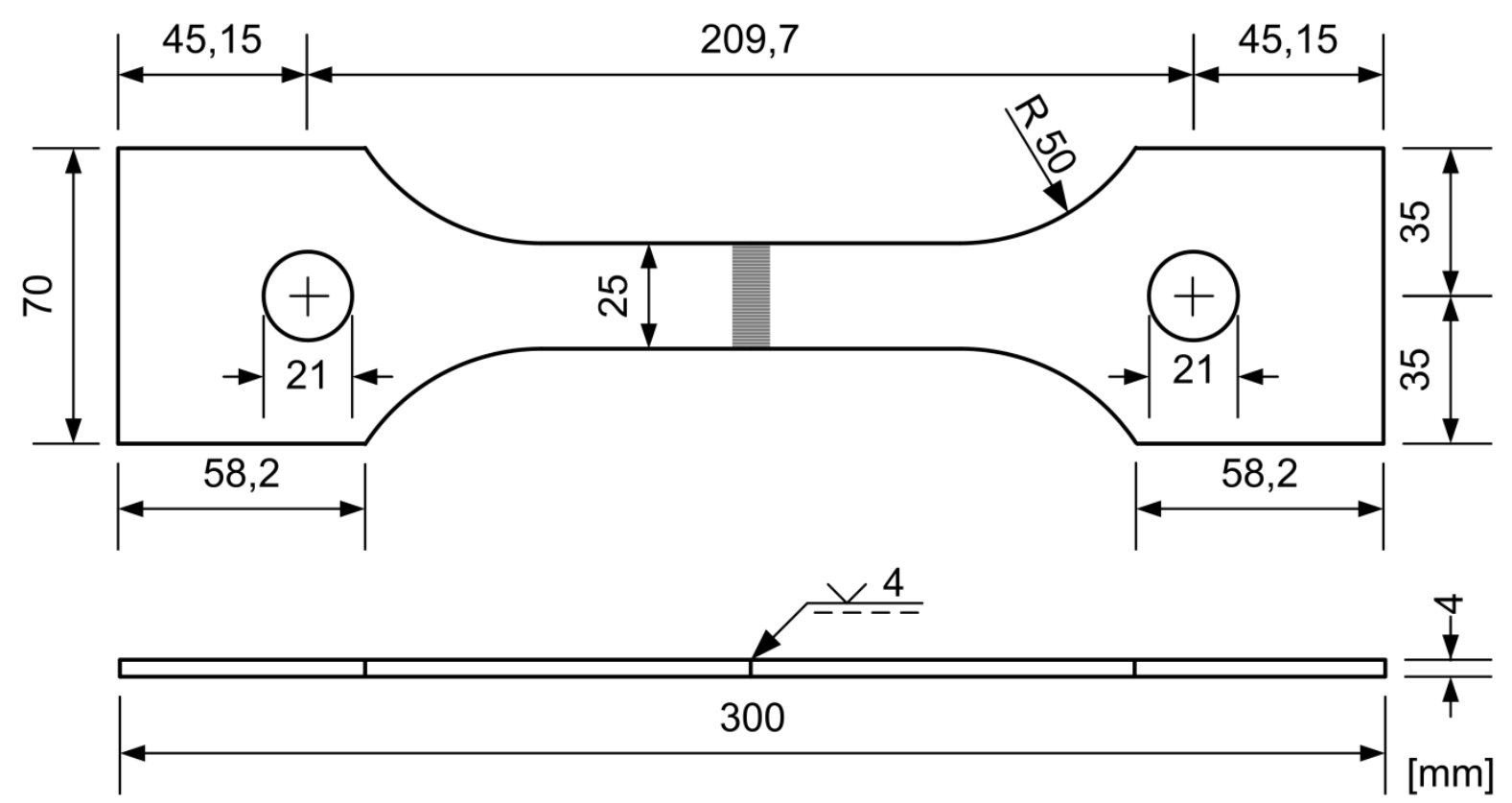

Abb. 3-3: Geometrie der Probekörper

\subsection{Temperaturmessungen}

Der Temperatur-Zeit-Verlauf ausgewählter Schweißproben während der Schweißund Abkühlphase wurde punktuell mit Thermoelementen (Typ K) gemessen. Hierzu wurden jeweils sechs Thermoelemente paarweise (1-2 / 3-4 / 5-6) auf den Schweißproben positioniert. Die Position der aufgeschweißten Sensoren ist im Querschnitt in Abb. 3-4 und in der Draufsicht in Abb. 3-5 dargestellt. Es erfolgte eine Anordnung an zwei Positionen:

- Position 1 (Thermoelemente 1, 3 und 5)

$4 \mathrm{~mm}$ Abstand von der Fase/ $6 \mathrm{~mm}$ Abstand von der Schmelzbadmitte

- Position 2 (Thermoelemente 2, 4 und 6)

$9 \mathrm{~mm}$ Abstand von der Fase/ $11 \mathrm{~mm}$ Abstand von der Schmelzbadmitte

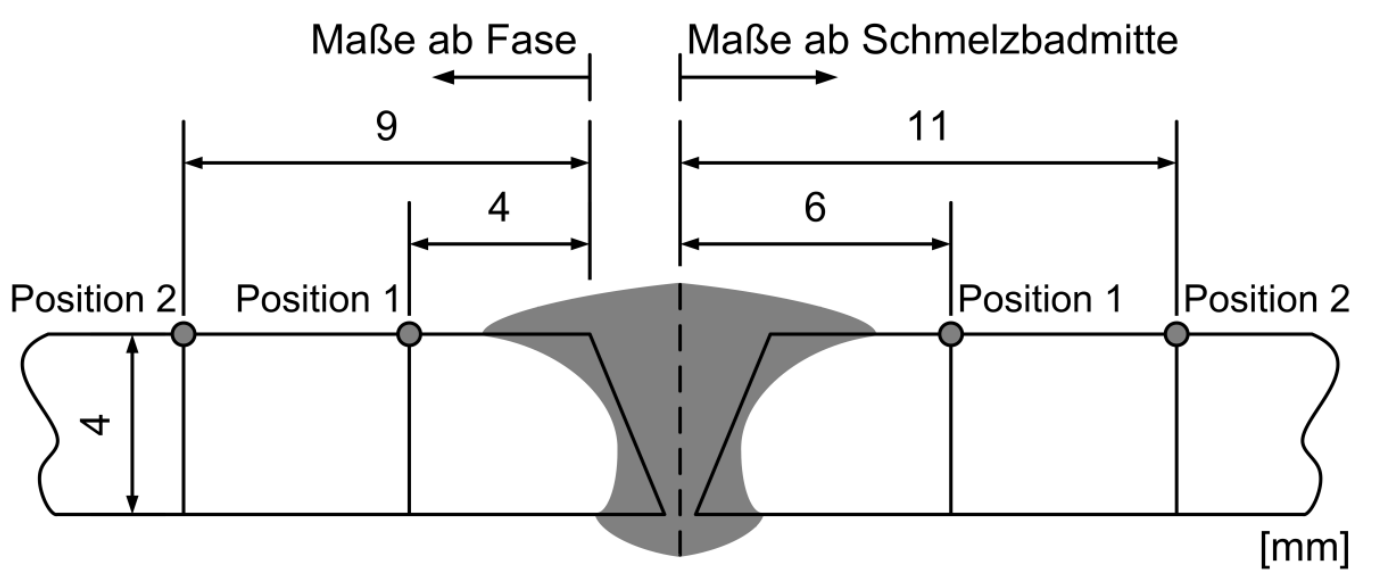

Abb. 3-4: Position der Thermoelemente auf der Schweißprobe im Querschnitt 
Die Thermoelemente wurden parallel zur Schweißnaht in Blechmitte im Abstand von $20 \mathrm{~mm}$ positioniert. Diese gewählte Anordnung der Thermoelemente erlaubt die Messung nebeneinander- und gegenüberliegender Temperatur-Zeit-Verläufe unmittelbar in der Nähe der Schweißnaht.

Überblick Schweißprobe
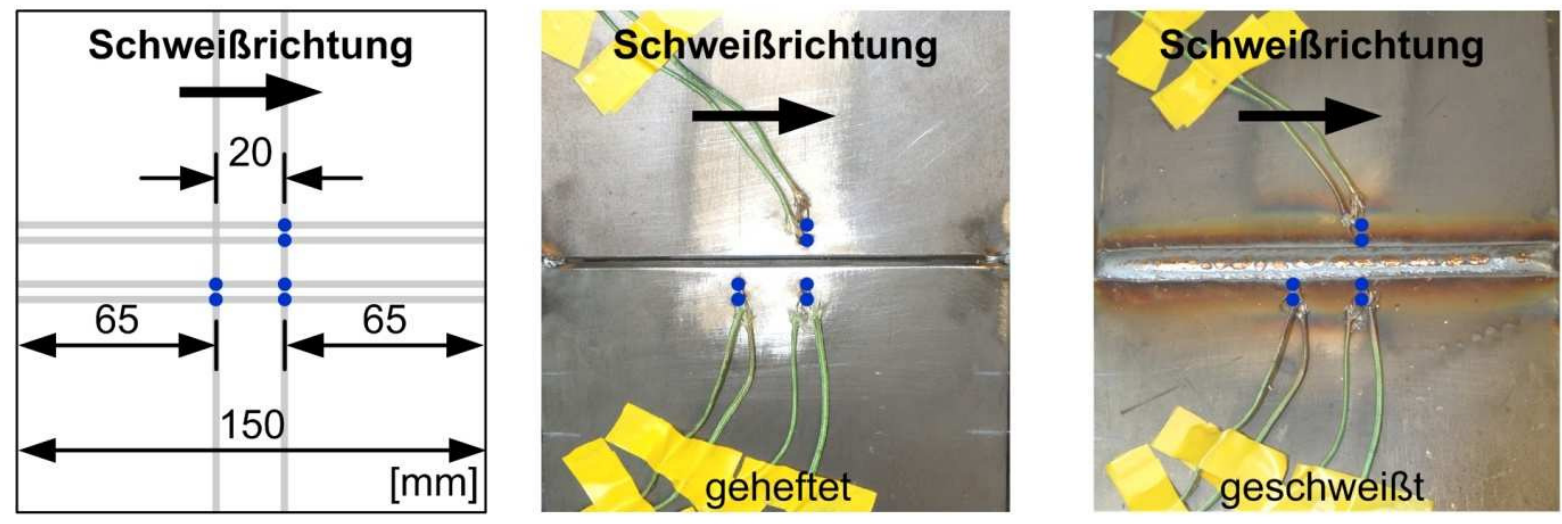

Detail in Blechmitte
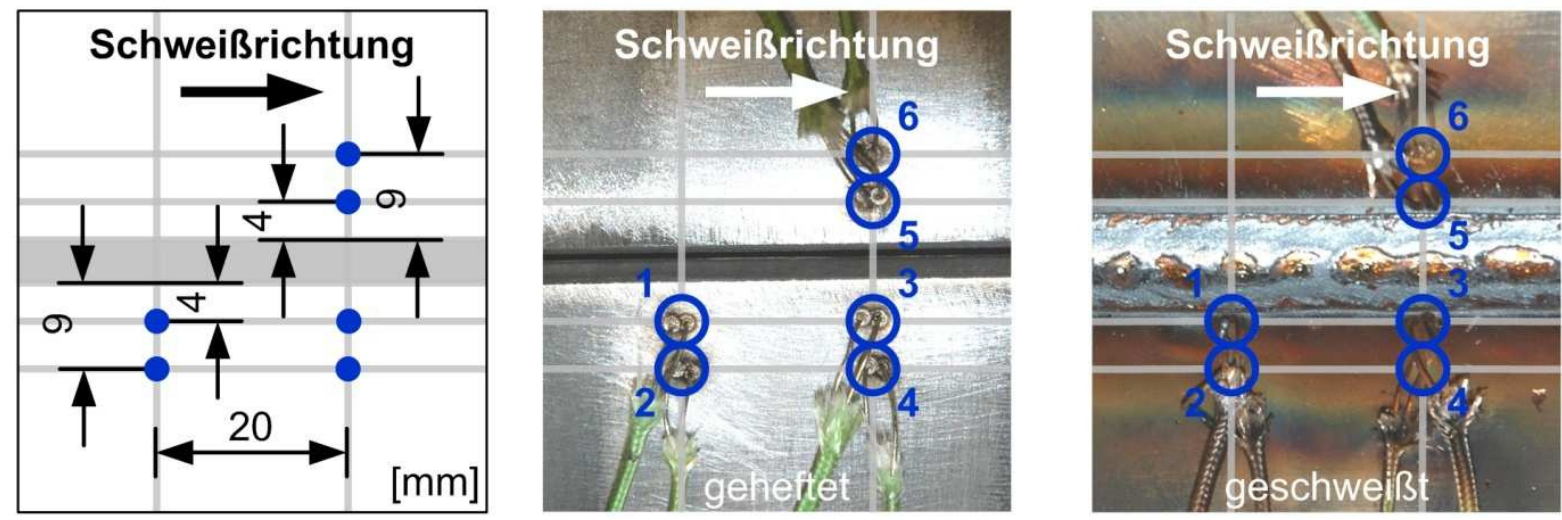

Abb. 3-5: Position der Thermoelemente auf der Schweißprobe in der Draufsicht

Es wurden insgesamt sechs Messungen durchgeführt. In Tab. 3-3 sind die erreichten Spitzentemperaturen $T_{\max }$ der jeweiligen Thermoelemente bei den einzelnen Messungen abgebildet. Bei Messung 1 fielen Thermoelement 1 und 2 sowie bei Messung 4 Thermoelement 3 aus. Es zeigt sich erwartungsgemäß, dass die Thermoelemente in Position 1 höhere Spitzentemperaturen als die in Position 2 erreichen.

Die erreichten Spitzentemperaturen $T_{\max }$ aller Thermoelemente der einzelnen Messungen in Position 1 (Thermoelemente 1, 3 und 5) haben einen Mittelwert von $899,5^{\circ} \mathrm{C}$ und eine Standardabweichung von $87,7^{\circ} \mathrm{C}$. Dies entspricht $9,7 \%$ bezogen auf den Mittelwert. Die erreichten Spitzentemperaturen $\mathrm{T}_{\max }$ aller Thermoelemente der einzelnen Messungen in Position 2 (Thermoelemente 2, 4 und 6) haben einen Mittelwert von $515,4^{\circ} \mathrm{C}$ und eine Standardabweichung von $19,6^{\circ} \mathrm{C}$. Dies entspricht 3,8 \% bezogen auf den Mittelwert. 
Es zeigt sich deutlich, dass die Streuung der erreichten Spitzentemperatur in Position 1 mit 9,7\% wesentlich höher ist als in Position 2 mit 3,8\%. Dies ist auf den sehr hohen Temperaturgradienten in unmittelbarer Nähe des Schmelzbades bzw. der Schmelzgrenze zurückzuführen. Durch den Schweißprozess wird sehr lokal begrenzt und äußerst konzentriert Wärme in das Blech eingebracht. Die Temperaturen verändern sich innerhalb kleinster Abstände vom Schmelzbad erheblich. Somit machen sich geringste Abweichungen in der Position der Thermoelemente in den Spitzentemperaturen deutlich bemerkbar. Eine Vielzahl an Einflussgrößen, welche die Lage der Thermoelemente beeinflussen, kann hierfür ursächlich sein. Es sind hierbei unter anderem zu nennen: Nahtvorbereitung, Heftung der Bleche, Positionierung der Thermoelemente mittels Aufschweißung, Kontaktierung der Thermoelemente, Ausrichtung des Bleches auf der Lagerungskonstruktion, Führung des Brenners durch den Traktor, Zündung des Lichtbogens, etc.

Tab. 3-3: Spitzentemperaturen $T_{\max }$

\begin{tabular}{|c|c|c|c|c|c|c|}
\hline $\begin{array}{c}\text { Messung } \\
{[-]}\end{array}$ & $\begin{array}{c}\mathbf{T}_{\max \_} \\
{\left[{ }^{\circ} \mathbf{C}\right]}\end{array}$ & $\begin{array}{c}\mathbf{T}_{\max \_}{ }^{\circ} \\
{\left[{ }^{\circ} \mathbf{C}\right]}\end{array}$ & $\begin{array}{c}\mathbf{T}_{\max \_3} \\
{\left[{ }^{\circ} \mathbf{C}\right]}\end{array}$ & $\begin{array}{c}\mathbf{T}_{\max \_4} \\
{\left[{ }^{\circ} \mathbf{C}\right]}\end{array}$ & $\begin{array}{c}\mathbf{T}_{\max \_5} \\
{\left[{ }^{\circ} \mathbf{C}\right]}\end{array}$ & $\begin{array}{c}\mathbf{T}_{\max \_6} \\
{\left[{ }^{\circ} \mathbf{C}\right]}\end{array}$ \\
\hline 1 & - & - & 810,6 & 511,1 & 847,5 & 514,9 \\
\hline 2 & 934,6 & 520,4 & 747,3 & 477,4 & 769,3 & 494,3 \\
\hline 3 & 902,9 & 523,6 & 1009,0 & 506,8 & 839,4 & 500,2 \\
\hline 4 & 1047,0 & 528,6 & - & 550,6 & 1011,0 & 493,1 \\
\hline 5 & 885,4 & 511,2 & 928,9 & 512,5 & 895,7 & 527,1 \\
\hline 6 & 980,7 & 543,9 & 837,1 & 543,6 & 945,1 & 502,2 \\
\hline
\end{tabular}

Die Temperatur-Zeit-Verläufe der einzelnen Thermoelemente während der Schweißund Abkühlphase sind exemplarisch für Messung 5 in Abb. 3-6 dargestellt. Die Temperatur-Zeit-Verläufe aller Messungen können Anhang $B$ entnommen werden. Bei $10,0 \mathrm{~s}$ beginnt die Schweißung. Die Schweißphase dauert 23,9 s. Daran schließt die Abkühlphase an. Die zeitliche Verschiebung des Thermoelementpaares 1-2 zu den Thermoelementpaaren 3-4 und 5-6 ist deutlich erkennbar. Die gegenüberliegenden Thermoelementpaare 3-4 und 5-6 beschreiben einen nahezu identischen Temperatur-Zeit-Verlauf und unterscheiden sich lediglich durch die Spitzentemperaturen $T_{\max }$ um $33,2^{\circ} \mathrm{C}$ sowie $14,6{ }^{\circ} \mathrm{C}$. Die nahezu identische Aufheiz- und Abkühlgeschwindigkeit zeigt sich deutlich. Die nebeneinander liegenden Thermoelementpaare 1-2 und 3-4 beschreiben ebenfalls einen nahezu identischen, aber zeitlich versetzten, Temperatur-Zeit-Verlauf und unterscheiden sich lediglich durch die Spitzentemperaturen $T_{\max }$ um $43,5^{\circ} \mathrm{C}$ sowie $1,3^{\circ} \mathrm{C}$. Dieser Sachverhalt lässt auf eine symmetrische, homogen verlaufende Schweißung schließen. 


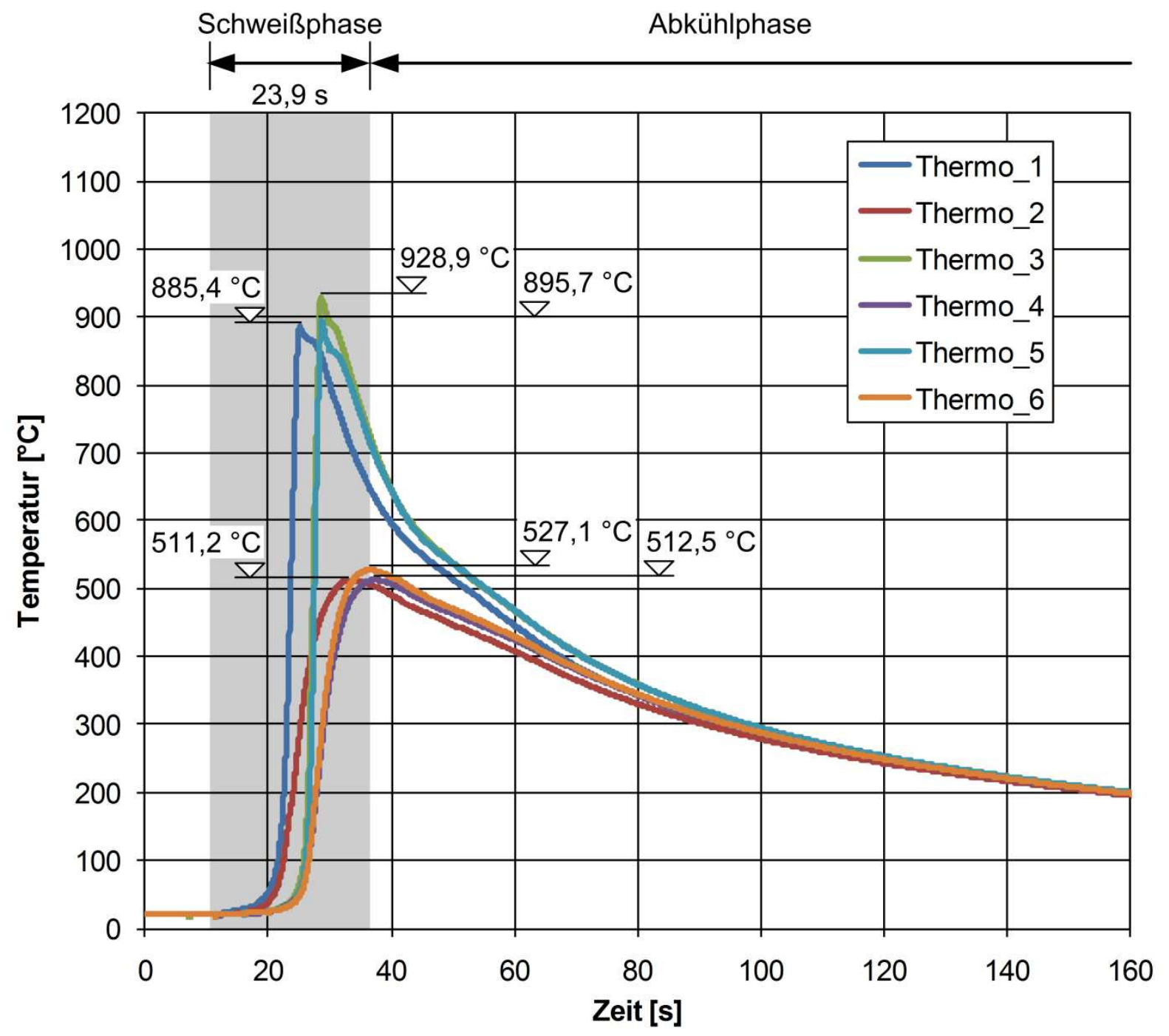

Abb. 3-6: Temperatur-Zeit-Verläufe der einzelnen Thermoelemente während der Schweiß- und Abkühlphase in Messung 5

Der Temperatur-Zeit-Verlauf während der Schweiß- und Abkühlphase hat einen ausschlaggebenden Einfluss auf die mechanischen Eigenschaften der Schweißverbindung [SEW 088, 1993] [Schulze, 2010]. Zur Beurteilung dieses Verhaltens wird in der Regel die Abkühlzeit $t_{8 / 5}$ als Betrachtungsgröße herangezogen. Hierbei handelt es sich um die Zeit, in der die Temperatur der Schweißnaht von $800{ }^{\circ} \mathrm{C}$ auf $500{ }^{\circ} \mathrm{C}$ abgekühlt ist. Während dieser Zeit finden die wichtigen Umwandlungsprozesse des Stahls statt [Schulze, 2010]. Diese Abkühlzeiten wurden für die Thermoelemente 1, 3 und 5 aller Messungen ausgewertet und in Tab. 3-4 zusammengetragen. Bei Messung 1 fiel Thermoelement 1 und bei Messung 4 fiel Thermoelement 3 aus. Die Thermoelemente 3 und 5 bei Messung 2 haben keine $800{ }^{\circ} \mathrm{C}$ erreicht. Der Mittelwert der Abkühlzeit $t_{8 / 5}$ aller Thermoelemente 1, 3 und 5 beträgt 22,4 s. Die Standardabweichung ergibt sich zu 1,5 s. Dies entspricht 6,7 \% bezogen auf den Mittelwert. 
Tab. 3-4: Abkühlzeit $t_{8 / 5}$

\begin{tabular}{|c|c|c|c|}
\hline $\begin{array}{c}\text { Messung } \\
{[-]}\end{array}$ & $\begin{array}{c}\mathbf{t}_{\mathbf{8 / 5} \mathbf{1}} \\
{[\mathbf{s}]}\end{array}$ & $\begin{array}{c}\mathbf{t}_{\mathbf{8 / 5} \mathbf{3}} \\
{[\mathbf{s}]}\end{array}$ & $\begin{array}{c}\mathbf{t}_{\mathbf{8} / \mathbf{5}} \mathbf{5} \\
{[\mathbf{s}]}\end{array}$ \\
\hline 1 & - & 24,6 & 23,1 \\
\hline 2 & 23,1 & - & - \\
\hline 3 & 22,1 & 21,8 & 22,3 \\
\hline 4 & 20,5 & - & 18,8 \\
\hline 5 & 22,0 & 21,3 & 21,9 \\
\hline 6 & 23,4 & 24,6 & 23,5 \\
\hline
\end{tabular}

Zur Beurteilung der gemessenen Abkühlzeiten $t_{8 / 5}$ sind nach SEW 088 [SEW 088, 1993] sowie bei Degenkolbe et al. [Degenkolbe, 1985] Richtwerte der Abkühlzeit $t_{8 / 5}$ von 10 bis $25 \mathrm{~s}$ für die dort behandelten Stähle angegeben. Die gemessenen Werte liegen knapp unterhalb des oberen Richtwertes und sind demnach eingehalten. Allerdings ist $z u$ beachten, dass die Messung der Abkühlzeit $t_{8 / 5}$ üblicherweise durch das Eintauchen eines Thermoelementes in das flüssige Schmelzbad und dessen Aufzeichnung des Temperatur-Zeit-Verlaufs erfolgt [Degenkolbe, 1985]. Im vorliegenden Fall wurden die Thermoelemente lediglich möglichst nah am Schmelzbad in der Wärmeeinflusszone auf dem Blech positioniert, sodass die nach SEW 088 angegebenen Richtwerte lediglich zur Einstufung der Größenordnung der gemessenen Werte herangezogen werden können.

\subsection{Lokale Schweißnahtgeometrie}

Da die lokale Schweißnahtgeometrie als bedeutende Einflussgröße auf die Ermüdungsfestigkeit von Schweißverbindungen anzusehen ist, wird diese im Hinblick auf die Abschätzung der Kerbwirkung an den Naht- und Wurzelübergängen ausgewertet. Bei der lokalen Schweißnahtgeometrie handelt es sich um in größeren Bereichen streuende Parameter [Radaj, 2000], die mit unterschiedlichsten Methoden ermittelt werden können.

Die Erfassung der lokalen Schweißnahtgeometrie kann anhand von mikroskopischen oder fotographischen Aufnahmen metallographischer Schliffe [Radaj, 1970] [Zeine El Dine, 2007] oder Abdrücken [Ruge, 1980] [Kuhlmann, 2006] sowie über induktive Wegaufnehmer und Tastschnittgeräte [Heeschen, 1986] oder Laserdistanzmessungen [Lachmann, 2003] [Weich, 2008] [Schaumann, 2013a] sowie 3DScannern [Kaffenberger, 2012] erfolgen. Die Auswertung der ermittelten lokalen Schweißnahtgeometrie erfolgte hierbei visuell oder zeichnerisch beispielsweise mit Schablonen sowie rechnerisch oder automatisiert mit entwickelten Algorithmen. 


\subsubsection{Aufnahme der lokalen Schweißnahtgeometrie}

Die lokale Schweißnahtgeometrie wurde mit einer Laserdistanzmessung berührungslos erfasst. Die Abstandsmessung basiert auf dem Prinzip der Triangulation. Ein Laserstrahl trifft auf das Messobjekt auf und wird auf der Oberfläche diffus reflektiert. Die Position des Laserpunktes wird durch den Empfänger des Sensors detektiert und der Abstand zum Messobjekt wird berechnet. Die gemessenen Daten stehen in digitaler Form zur Auswertung bereit.

Das Profil der Nahtgeometrie wurde beidseitig mit einer Lasermessung erfasst. Zwei Punktlaser wurden an einer Gabelkonstruktion (siehe Abb. 3-7, links) befestigt. Die Probe wurde mit konstanter Geschwindigkeit durch die Mitte des Versuchsaufbaus aus Schaumann et al. [Schaumann, 2013a] bewegt. Die Punktlaser wurden mit konstanter Geschwindigkeit von 1,82 mm/s über eine Strecke von $50 \mathrm{~mm}$ geführt, wobei sich der Schweißnahtbereich in der Mitte befindet (siehe Abb. 3-7, rechts). Die Messdaten wurden mit einer Frequenz von $100 \mathrm{~Hz}$ aufgezeichnet. Hieraus ergeben sich ca. 55 Messpunkte pro mm (dies entspricht einer Schrittweite von ca. $20 \mu \mathrm{m}$ ). Auf einer Probe wurden jeweils drei Profile gemessen, wobei sich ein Profil in Probenmitte und zwei weitere jeweils rechts und links im Abstand von $5 \mathrm{~mm}$ zur Probenmitte befinden.
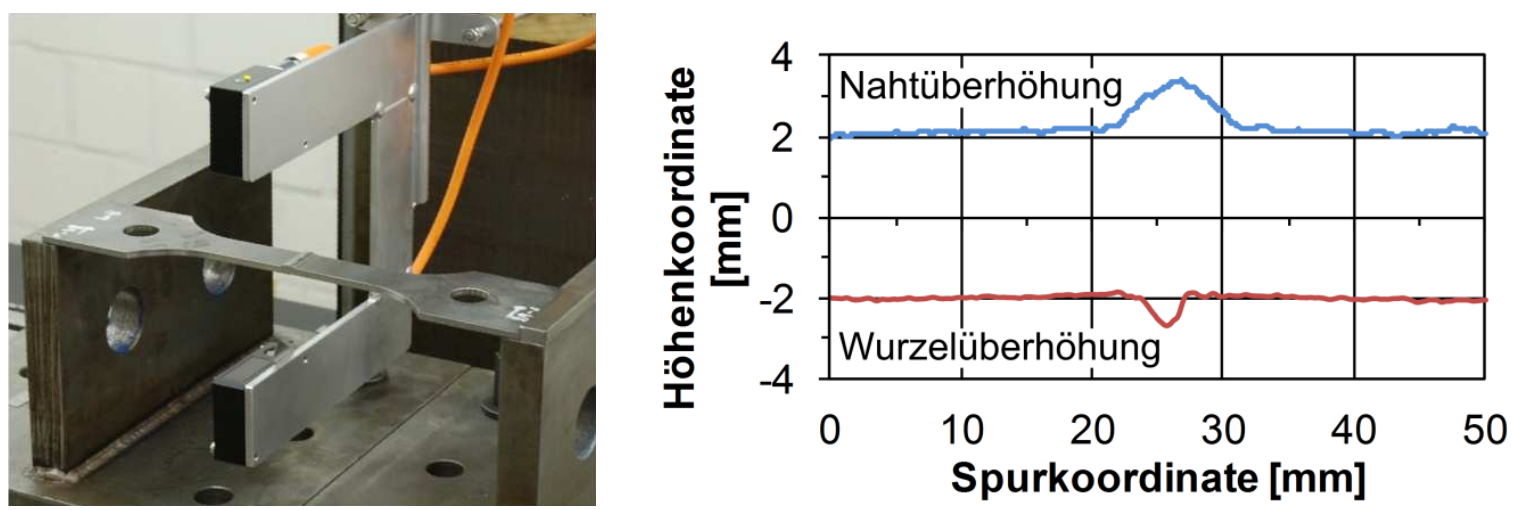

Abb. 3-7: Aufbau der Lasermessung (links) und Beispiel eines Messschriebs (rechts)

Die Punktlaser der Firma Welotec (Typ OWLF 4007 FA S1) weisen nach Angaben des Herstellers eine Auflösung von 4 bis $20 \mu \mathrm{m}$ auf. Die Auflösung eines Lasers entspricht der kleinsten erfassbaren Änderung der Messgröße. Im Bezug auf die Genauigkeit der Messung kommen weitere Einflüsse wie der Linearitätsfehler und die Umgebungsbedingungen hinzu. Der Linearitätsfehler wird vom Hersteller mit einem Bereich von 12 bis $60 \mu \mathrm{m}$ angegeben. Die angegebenen Bereiche von Auflösung und Linearitätsfehler der Laser ergeben sich aus dem gewählten Messaufbau und liefern die minimale sowie maximale Genauigkeit von 16 bis $80 \mu \mathrm{m}$. Die Genauigkeit des Profilverlaufs im Bezug auf die theoretisch ideale Oberfläche wird zudem von weiteren Einflüssen wie beispielsweise der Bewegung des Messobjektes (Vibrationen) 
beeinflusst. Außerdem wird die Rauheit und Welligkeit der Oberfläche in der Messung erfasst. Die Rauheit einer Oberfläche ist als Abweichung von der idealen Oberfläche definiert, die durch den Werkstoff selbst sowie durch Bearbeitungsmechanismen existiert. Im vorliegenden Fall wurde die Rauheit der Proben im Tastschnittverfahren ermittelt. Es ergab sich im Mittel eine Rauhtiefe $R_{t}$ von $12,4 \mu m$ sowie eine Welligkeitstiefe $W_{t}$ von 5,1 $\mu \mathrm{m}$. Die Ergebnisse sind im Anhang $C$ aufgeführt.

In Untersuchungen von Radaj [Radaj, 1970] sind an den Nahtübergängen von Schweißverbindungen Kerbradien $r$ im Bereich von $0,01 \mathrm{~mm}$ bis $3,0 \mathrm{~mm}$ ermittelt worden, wobei Kerbradien $r$ in der Größenordnung von 0,01 mm Bindefehlern zugeordnet wurden. Zudem konnte kein Einfluss der Blechdicke auf den Kerbradius festgestellt werden. Bei Stumpfnähten, die mit Lichtbogenhandschweißen gefügt wurden, konnten größere Kerbradien $r$ im Bereich von $1 \mathrm{~mm}$ bis $3 \mathrm{~mm}$ festgestellt werden als bei Stumpfnähten, die mit Metallschutzgasschweißen gefügt wurden. Hier ergaben sich Kerbradien $r$ im Bereich von 0,1 mm. Von Lachmann [Lachmann, 2003] wurden aus Laserdistanzmessungen an MAG geschweißten DV-Nähten (S355J2G3, $\mathrm{t}=10 \mathrm{~mm}$ ) mittlere Mikrokerbradien $\mathrm{r}$ der Decklage von 0,67 mm und der Gegenlage von 0,76 mm ermittelt. Von Weich [Weich, 2008] wurde ebenfalls aus Laserdistanzmessungen an MAG geschweißten DV-Nähten (S355J2, $t=16 \mathrm{~mm}$ ) ein mittlerer Kerbradius $r$ von 1,33 mm ermittelt. Für diesen Erwartungsbereich der Größenordnung des Kerbradius $r$ wird die Genauigkeit des Messaufbaus als ausreichend eingestuft.

\subsubsection{Auswertung der lokalen Nahtgeometrieparameter}

Das Profil der lokalen Nahtgeometrie wurde an insgesamt 20 Proben mit jeweils 3 Spuren beidseitig ermittelt. Es wurden somit insgesamt 60 Oberflächenprofile aufgenommen und ausgewertet. Die lokalen Nahtgeometrieparameter an den Übergängen der Naht- und Wurzelüberhöhung sind in Abb. 3-8 definiert.

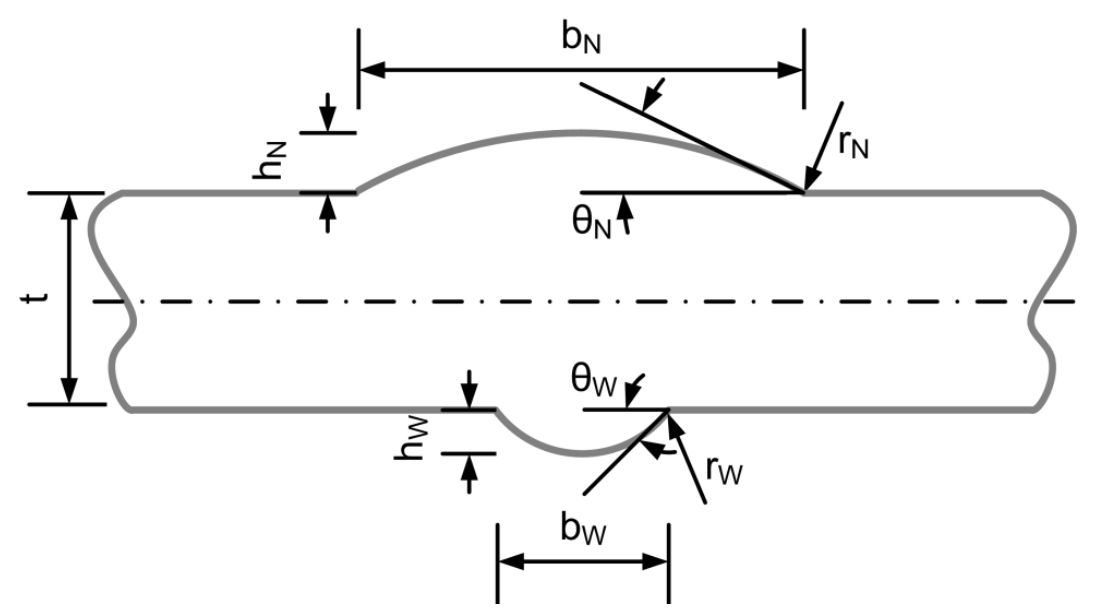

Abb. 3-8: Definition der lokalen Nahtgeometrieparameter an den Übergängen 
Es werden die folgenden lokalen Nahtgeometrieparameter für die Naht- und Wurzelübergänge ausgewertet:

- Breite an Naht- und Wurzelübergang ( $b_{N}$ und $\left.b_{W}\right)$

- Höhe an Naht- und Wurzelübergang $\left(h_{N}\right.$ und $\left.h_{W}\right)$

- Kerbradius der Übergange (links und rechts) an Naht- und Wurzelüberhöhung $\left(r_{N}\right.$ und $\left.r_{W}\right)$

- Anstiegswinkel der Übergange (links und rechts) an Naht- und Wurzelüberhöhung $\left(\theta_{\mathrm{N}}\right.$ und $\left.\theta_{\mathrm{W}}\right)$

Die Auswertung der lokalen Nahtgeometrieparameter der im Schweißzustand belassenen Probekörper erfolgte automatisiert auf Grundlage des von Zeine El Dine [Zeine El Dine, 2007] beschriebenen Algorithmus (siehe Anhang D). Dieser Algorithmus setzt voraus, dass die Übergänge mit Kreisbögen angenähert werden können. In Abb. 3-9 ist die Auswertung der lokalen Nahtgeometrie an einem exemplarischen Übergang dargestellt.

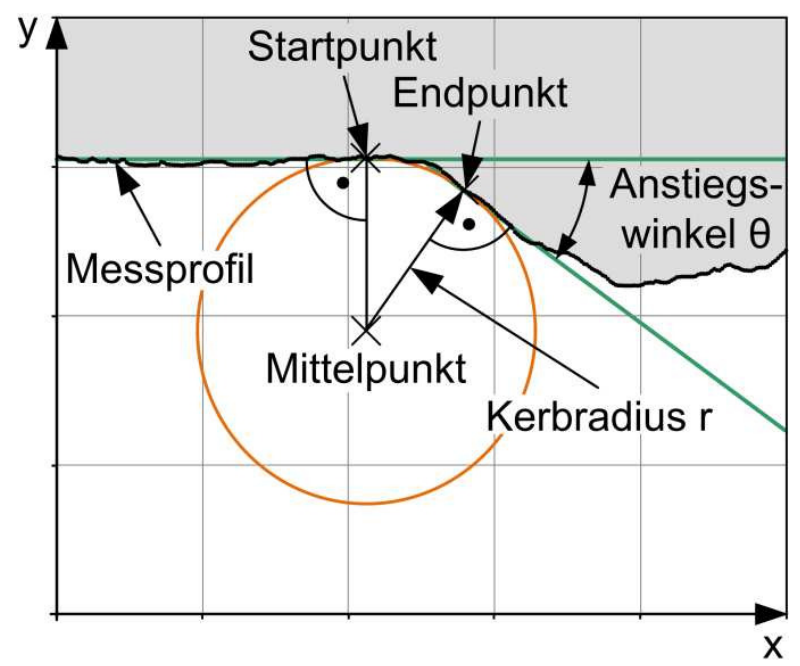

Abb. 3-9: Darstellung der Auswertung der lokalen Nahtgeometrie an einem exemplarischen Übergang

Für mögliche Startpunkte werden sämtliche Kreisbögen mit unterschiedlichen Kerbradien $r$ ermittelt, die anhand des Quotienten aus der Summe der Abstände der Punkte des gemessenen Profils zum idealisierten Kreisbogen und der quadrierten Anzahl der entlang dem idealisierten Kreisbogen liegenden Punkte des gemessenen Profils bewertet werden. Anhand des Kreisbogens mit dem geringsten Quotienten wird der Kerbradius $r$ mit der besten Annäherung festgelegt. Der Anstiegswinkel $\theta$ wird als Steigung der Tangente im letzten Profilpunkt entlang dem Kreisbogen ermittelt. Naht- und Wurzelbreite $\left(b_{N}\right.$ und $\left.b_{W}\right)$ sowie Naht- und Wurzelhöhe $\left(h_{N}\right.$ und $\left.h_{W}\right)$ 
werden über die Koordinaten der Startpunkte der ermittelten Kreisbögen berechnet. Außerdem werden Kantenversatz tv und Winkelverzug $\rho_{v}$ ausgewertet. Der Winkelverzug wird als Differenz der Neigungen der, über lineare Regressionsrechnung im Bereich außerhalb der unmittelbaren Schweißnaht ermittelten Mittellinien beider Bleche, berechnet. Zur Ermittlung des Kantenversatzes wird anhand der Koordinaten der Startpunkte aller vier Kreisbögen der Nahtübergänge die Schweißnahtmitte bestimmt. Der Kantenversatz wird über die Höhendifferenz der Schnittpunkte der Mittellinien in Nahtmitte berechnet. Weitere Details zum Ablauf können Zeine El Dine [Zeine El Dine, 2007] entnommen werden. Der Algorithmus wurde in Excel programmiert und an die vorliegende Situation angepasst. Es erfolgte die automatisierte Auswertung der einzelnen Profilschriebe für die Naht- und Wurzelübergänge.

Die Ergebnisse der lokalen Nahtgeometrieparameter aller ausgewerteten Oberflächenprofile sind in Tab. 3-5 zusammengefasst. Die Einzelergebnisse aller Messungen sind in Anhang E.1 in tabellarischer Form angegeben. Die Auswertung von Kerbradius und Anstiegswinkel erfolgte jeweils für die Übergänge an Naht- und Wurzelüberhöhung auf der rechten und linken Seite, wobei die Ergebnisse in Tab. 3-5 jeweils zusammengefasst für die Naht- und Wurzelüberhöhung angegeben sind.

Tab. 3-5: Ergebnisübersicht der Auswertung der lokalen Nahtgeometrieparameter

\begin{tabular}{|c|c|c|c|c|c|c|c|}
\hline & & & $\begin{array}{l}\text { Mittel- } \\
\text { wert }\end{array}$ & $\begin{array}{c}\text { Standard- } \\
\text { abweichung }\end{array}$ & $\begin{array}{l}\text { Maxi- } \\
\text { mum }\end{array}$ & $\begin{array}{l}\text { Mini- } \\
\text { mum }\end{array}$ & $\begin{array}{l}\text { Variation- } \\
\text { koeffizient }\end{array}$ \\
\hline \multirow{2}{*}{ Breite } & $\mathbf{b}_{\mathrm{N}}$ & {$[\mathrm{mm}]$} & 10,31 & 0,89 & 12,04 & 8,23 & $8,7 \%$ \\
\hline & $b_{w}$ & {$[\mathrm{~mm}]$} & 4,20 & 0,57 & 5,57 & 2,73 & $13,7 \%$ \\
\hline \multirow{2}{*}{ Höhe } & $\mathbf{h}_{\mathrm{N}}$ & {$[\mathrm{mm}]$} & 1,15 & 0,22 & 1,72 & 0,70 & $19,4 \%$ \\
\hline & $h_{w}$ & [mm] & 0,89 & 0,25 & 1,44 & 0,40 & $28,1 \%$ \\
\hline \multirow{2}{*}{ Kerbradius } & $r_{N}$ & [mm] & 2,67 & 1,13 & 4,96 & 0,53 & $42,4 \%$ \\
\hline & $r_{W}$ & {$[\mathrm{~mm}]$} & 1,19 & 0,56 & 3,10 & 0,29 & $47,2 \%$ \\
\hline \multirow{2}{*}{$\begin{array}{l}\text { Anstiegs- } \\
\text { winkel }\end{array}$} & $\Theta_{\mathrm{N}}$ & {$\left[{ }^{\circ}\right]$} & 28,24 & 8,73 & 69,97 & 14,04 & $30,9 \%$ \\
\hline & $\Theta_{w}$ & {$\left[{ }^{\circ}\right]$} & 35,33 & 10,26 & 69,35 & 15,57 & $29,0 \%$ \\
\hline Winkelverzug & $\rho_{v}$ & {$\left[^{\circ}\right]$} & 0,68 & 0,26 & 1,32 & 0,21 & $38,1 \%$ \\
\hline $\begin{array}{l}\text { Kanten- } \\
\text { versatz }\end{array}$ & $t_{v}$ & {$[\mathrm{~mm}]$} & 0,11 & 0,09 & 0,37 & 0,00 & $83,2 \%$ \\
\hline
\end{tabular}

Die Streuungen, ausgedrückt als Variationskoeffizienten, der Breite und Höhe von Naht- und Wurzelüberhöhung fallen am geringsten aus, wohingegen Kerbradius und Kantenversatz die höchsten Streuungen aufweisen. Hierbei zeigt sich mit Ausnahme des Anstiegswinkels, dass die Streuung am Nahtübergang geringer ist als am Wur- 
zelübergang. Die ermittelten Streuungen von Kerbradius und Anstiegswinkel liegen für die beiden Übergänge an der Naht- und Wurzelüberhöhung jeweils dicht beieinander. Als bedeutende Einflussparameter auf die Ermüdungsfestigkeit werden Kerbradius und Anstiegswinkel angesehen (vergleiche Kapitel 2.3.1). Der Mittelwert für den Kerbradius am Nahtübergang $r_{N}$ beträgt 2,67 mm und ist deutlich höher als der Mittelwert für den Kerbradius am Wurzelübergang $r_{w}$ mit 1,19 mm. Der Mittelwert des Anstiegswinkels am Nahtübergang $\theta_{N}$ beträgt $28,24^{\circ}$ und ist niedriger als der Mittelwert des Anstiegswinkels am Wurzelübergang $\theta_{w}$ mit $35,33^{\circ}$.

Im Hinblick auf die Beurteilung der Kerbwirkung deutet sich an, dass die Kerbwirkung am Wurzelübergang größer als am Nahtübergang ist, da an den Wurzelübergängen im Mittel geringere Kerbradien und höhere Anstiegswinkel vorliegen. Als Anrissort wird somit vorwiegend der Wurzelübergang erwartet. Bei Betrachtung der Extremwerte zeigt sich jedoch, dass sich die Minima und Maxima von Kerbradius und Anstiegswinkel an den jeweiligen Übergängen von Naht- und Wurzelüberhöhung nur geringfügig unterscheiden. Im Einzelfall könnte somit von einer Verlagerung des Anrissortes vom Wurzelübergang zum Nahtübergang ausgegangen werden. Die Auswertung der lokalen Nahtgeometrie in Tab. 3-5 bestätigt, dass es sich trotz mechanisierter Fertigung um stark streuende Größen handelt. Bei Betrachtung der Einzelergebnisse der drei Profilspuren auf den jeweiligen Proben zeigt sich, dass besonders bei Kerbradius und Anstiegswinkel große Streuungen auf einer Probe vorliegen können.

In DIN EN ISO 5817 [DIN EN ISO 5817, 2006] sind typische Unregelmäßigkeiten von Schmelzschweißverbindungen definiert, die in einer normalen Fertigung erwartet werden können. Durch die Festlegung von Bewertungsgruppen sind die einzuhaltenden Toleranzen für Unregelmäßigkeiten angegeben. Die Angaben beziehen sich auf keine spezielle Anwendung und sind als Grundbezugsdaten anzusehen. In Abb. 3-10 sind die Grenzwerte für Oberflächenunregelmäßigkeiten für Bewertungsgruppe B nach DIN EN ISO 5817 [DIN EN ISO 5817, 2006] für den vorliegenden Fall dargestellt. Die Überprüfung der ermittelten lokalen Nahtgeometrieparameter zeigt, dass die Anforderungen an die Oberflächenunregelmäßigkeiten von Bewertungsgruppe B in Anlehnung an DIN EN ISO 5817 [DIN EN ISO 5817, 2006] für Naht- und Wurzelüberhöhung sowie für den Kantenversatz von allen Probekörpern eingehalten sind. Lediglich beim Übergangswinkel unterschreiten die ermittelten Werte, besonders an der Wurzelüberhöhung, zum Teil diese Anforderungen. Im Mittel beträgt der Übergangswinkel an der Nahtüberhöhung $152,7^{\circ}$, wohingegen sich der Mittelwert des Übergangswinkels an der Wurzelüberhöhung zu $144,7^{\circ}$ ergibt. 


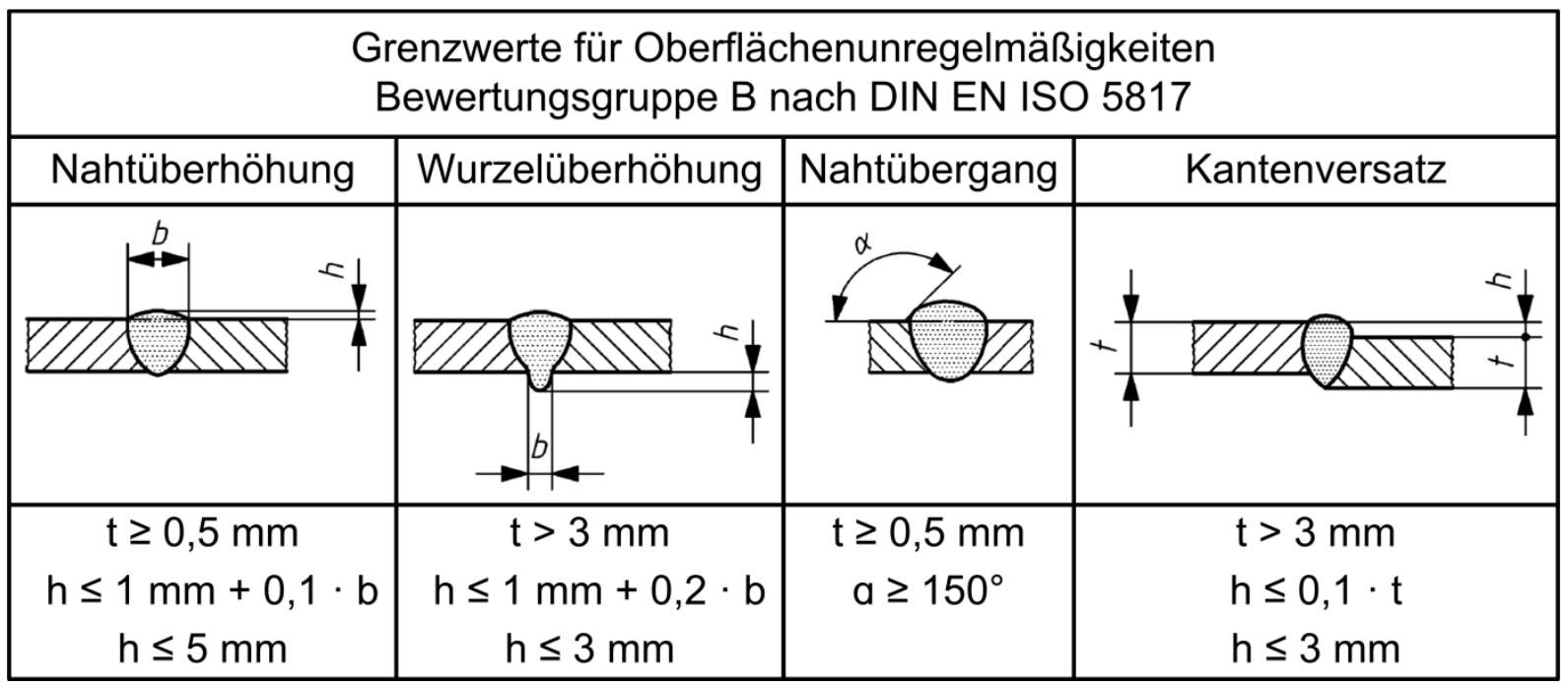

Abb. 3-10: Grenzwerte für Oberflächenunregelmäßigkeiten für Bewertungsgruppe B nach DIN EN ISO 5817 [DIN EN ISO 5817, 2006]

\subsubsection{Ermittlung der Kerbformzahl $\mathrm{K}_{\mathrm{t}}$}

Zur Einordnung der Höhe der Kerbwirkung der Übergänge an der Naht- und Wurzelüberhöhung sowie zur Abschätzung des erwarteten Anrissortes werden die Kerbformzahlen $\mathrm{K}_{\mathrm{t}}$ als Maß für die Kerbwirkung ermittelt. Hierzu werden analytische Näherungsformeln aus der Literatur angewendet und numerische Berechnungen mit der Finite-Elemente-Methode (FEM) durchgeführt. Die analytisch und numerisch ermittelten Ergebnisse werden im Folgenden dargestellt und verglichen.

\section{Analytische Ermittlung}

Von Anthes et al. [Anthes, 1993] wurden analytische Näherungsformeln zu Ermittlung der Kerbformzahl $\mathrm{K}_{\mathrm{t}}$ entwickelt. Diese wurden aus einer numerisch ermittelten Datenbasis, die durch Variation der lokalen Nahtgeometrieparameter an einem geometrisch idealisierten Modell erzeugt wurde, abgeleitet. Die Näherungsformel für doppeltsymmetrische Stumpfstöße ist vom Verhältnis der Blechdicke t zum Kerbradius $r$ sowie vom Anstiegswinkel $\theta$ abhängig. Von Rainer [Rainer, 1979] wurden ebenfalls analytische Näherungsformeln zu Ermittlung der Kerbformzahl $K_{t}$ vorgestellt, die auf numerischen Berechnungen an einem geometrisch idealisierten Modell basieren. Als Ersatzsystem für doppeltsymmetrische Stumpfstöße wird hier der abgesetzte Flachstab verwendet. In die Näherungsformel geht das Verhältnis der Überhöhung h zum Kerbradius $r$ sowie das Verhältnis der Blechdicke $t$ zum Kerbradius $r$ ein. Außerdem kann über Abminderungsfaktoren die Kerbformzahl für den unsymmetrischen einseitig geschulterten Ersatzstab ermittelt werden, der als Ersatzsystem für eine V-Naht ohne Wurzel angesetzt wird. Allerdings sind die Anwendungsgrenzen im vorliegenden Fall nicht erfüllt, sodass die Näherungsformel für doppeltsymmetrische Stumpfstöße verwendet wird. 
Obwohl beide Näherungsformeln für doppeltsymmetrische Stumpfstöße hergeleitet wurden, werden sie im vorliegenden Fall des nicht doppeltsymmetrischen Stumpfstoßes zur Abschätzung der Kerbformzahl herangezogen. Für die ermittelten lokalen Nahtgeometrieparameter an den Naht- und Wurzelübergängen wurden die Kerbformzahlen mit diesen beiden Näherungsformeln ermittelt. Die Ergebnisse sind in Tab. 3-6 zusammengefasst. Die Einzelergebnisse können Anhang E.2 entnommen werden.

Tab. 3-6: Zusammenfassung der ermittelten Kerbformzahlen $K_{t}$ nach Anthes et al. [Anthes, 1993] und nach Rainer [Rainer, 1979]

\begin{tabular}{|c|c|c|c|c|}
\hline & $\begin{array}{c}\mathbf{K}_{\mathbf{t}} \mathbf{N}_{-} \text {Anthes } \\
{[-]}\end{array}$ & $\begin{array}{c}\mathrm{K}_{\mathrm{t} \_\mathrm{W}} \text { _Anthes } \\
{[-]}\end{array}$ & $\begin{array}{c}\mathrm{K}_{\mathrm{t} \_N \_R a i n e r} \\
{[-]}\end{array}$ & $\begin{array}{c}\mathbf{K}_{\mathbf{t}} \mathbf{w}_{\text {_Rainer }} \\
{[-]}\end{array}$ \\
\hline Mittelwert & 1,38 & 1,57 & 1,37 & 1,69 \\
\hline Standardabweichung & 0,12 & 0,16 & 0,21 & 0,25 \\
\hline Maximum & 1,87 & 2,16 & 2,16 & 2,36 \\
\hline Minimum & 1,22 & 1,27 & 1,14 & 1,24 \\
\hline Variationskoeffizient & $8,5 \%$ & $10,0 \%$ & $15,3 \%$ & $14,6 \%$ \\
\hline
\end{tabular}

Für den Nahtübergang ergibt sich nach der Näherungsformel von Anthes et al. im Mittel eine geringfügig höhere Kerbformzahl $K_{\mathrm{t} \_}{ }_{\text {_Anthes }}$ von 1,38 als nach der Näherungsformel von Rainer mit einer Kerbformzahl $K_{t \_}{ }_{-}$Rainer von 1,37. Bei Betrachtung der Standardabweichung sowie des Variationskoeffizienten und der Extremalwerte zeigt sich, dass die Ergebnisse nach der Näherungsformel von Rainer eine größere Streuung aufweisen als die Ergebnisse nach der Näherungsformel von Anthes et al.. Am Wurzelübergang ergibt sich nach der Näherungsformel von Anthes et al. im Mittel eine etwas geringere Kerbformzahl $K_{t} W_{\_}$Anthes von 1,57 als nach der Näherungsformel von Rainer mit einer Kerbformzahl $\mathrm{K}_{\mathrm{t}} \mathrm{W}_{-}$Rainer von 1,69. Auch für diesen Fall ist die Streuung der Kerbformzahlen nach der Näherungsformel von Rainer größer als nach der Näherungsformel von Anthes et al..

In der Regel weisen die Ergebnisse der beiden Näherungsformeln an denselben Übergängen die maximalen Kerbformzahlen auf. Im Hinblick auf die Beurteilung der möglichen Anrissorte kann abgeleitet werden, dass der Anriss aufgrund der auftretenden maximalen Kerbformzahlen vorwiegend am Wurzelübergang erwartet wird. Vereinzelt tritt die maximale Kerbformzahl eines Profilschriebs am Nahtübergang auf, wie beispielsweise bei Profilschrieb M-2 in Tab. 3-7. Werden allerdings alle drei Profilschriebe einer Probe betrachtet, so zeigt sich in den meisten Fällen bei einem der anderen beiden Profilschriebe einer Probe (hier M-1 und M-3) eine höhere Kerbform- 
zahl am Wurzelübergang. Im Einzelfall kann es somit zu einer Verlagerung des Anrissortes vom Wurzel- zum Nahtübergang kommen. Die aufgenommenen Profilschriebe zeigen deutlich, dass sich die lokale Nahtgeometrie und damit auch die ermittelten Kerbformzahlen in jedem Schnitt ändern können.

Tab. 3-7: Vergleich der Kerbformzahlen $K_{t}$ der einzelnen Profilschriebe von Probe $M$

\begin{tabular}{|c|c|c|c|c|c|c|c|c|}
\hline & \multicolumn{2}{|c|}{$\begin{array}{c}\mathrm{K}_{\mathrm{t} \_\mathrm{N}} \text { Anthes } \\
{[-]}\end{array}$} & \multicolumn{2}{|c|}{$\begin{array}{c}\mathrm{K}_{\mathrm{t} \_} \mathrm{w}_{-} \text {Anthes } \\
{[-]}\end{array}$} & \multicolumn{2}{|c|}{$\begin{array}{c}\mathrm{K}_{\mathrm{t} \_\mathrm{N}} \text { Rainer } \\
{[-]}\end{array}$} & \multicolumn{2}{|c|}{$\begin{array}{c}\mathbf{K}_{\mathrm{t} \_w \_R a i n e r} \\
{[-]}\end{array}$} \\
\hline & links & rechts & links & rechts & links & rechts & links & rechts \\
\hline$M-1$ & 1,30 & 1,22 & 1,59 & 1,66 & 1,21 & 1,15 & 1,78 & 1,82 \\
\hline M-2 & 1,29 & 1,55 & 1,54 & 1,48 & 1,26 & 1,80 & 1,60 & 1,54 \\
\hline$M-3$ & 1,34 & 1,28 & 1,44 & 1,51 & 1,29 & 1,18 & 1,49 & 1,63 \\
\hline
\end{tabular}

\section{Numerische Ermittlung}

Die numerische Ermittlung der Kerbformzahl $\mathrm{K}_{\mathrm{t}}$ erfolgte mit der Methode der Finiten Elemente, wobei das Programmsystem ANSYS ${ }^{\circledR}$ (Version 13.0) [ANSYS, 2010] verwendet wurde. Die Untersuchungen wurden am ebenen System unter Verwendung der 8-Knoten-Elemente PLANE183 (ebener Dehnungszustand) und Zugrundelegung linearelastischen Materialverhaltens durchgeführt. Die Geometrie des Modells der Schweißnaht wurde idealisiert über Geraden und Kreisbögen aus den aufgenommenen Profilspuren angenähert, sodass die Berechnung unter Verwendung der ermittelten lokalen Nahtgeometrieparameter erfolgen konnte. Das numerische Modell ist in Abb. 3-11 (oben) dargestellt. Kantenversatz und Winkelverzug wurden hierbei nicht berücksichtigt. Die Bereiche außerhalb der Schweißnaht werden als Geraden und die Übergänge an der Naht- und Wurzelüberhöhung als anschließende Kreisbögen, die dem jeweiligen Kerbradius entsprechen, modelliert. Beim Erreichen des Anstiegswinkels schließt ein Gegenbogen an, der über die Naht- bzw. Wurzelmitte mit maximaler Überhöhung bis zum Erreichen des gegenüberliegenden Anstiegswinkels fortgeführt wird.

Bei Betrachtung der Extremalwerte der erfassten lokalen Nahtgeometrieparameter zeigt sich, dass die Kerbradien sowohl am Nahtübergang als auch am Wurzelübergang im Bereich von 0,29 mm bis 4,96 mm variieren können. Vor diesem Hintergrund erwies sich eine flexible Vernetzungsstrategie als sinnvoll, bei der im ersten Schritt eine einheitliche Elementkantenlänge für alle Elemente vorgegeben wird und im zweiten Schritt eine Verfeinerung des Netzes im Bereich des jeweiligen Kerbradius vorgenommen wird. In einer Konvergenzstudie wurde der Grad der Diskretisierung ausgehend vom minimalen Kerbradius ermittelt. Im Bereich der Kerbradien wird 
für alle Berechnungen eine einheitliche Elementkantenlänge von ungefähr $0,025 \mathrm{~mm}$ gewählt. Als Beanspruchung wurde eine Nennzugspannung $\sigma_{N}$ von $1 \mathrm{~N} / \mathrm{mm}^{2}$ aufgebracht, sodass bei der Auswertung der 1 . Hauptspannung $\sigma_{1}$ die Kerbformzahlen $\mathrm{K}_{\mathrm{t}}$ direkt abgelesen werden können. In Abb. 3-11 (unten) ist die Verteilung der 1. Hauptspannung $\sigma_{1}$ für eine exemplarische Berechnung dargestellt. Die Auswertung der Kerbformzahlen erfolgt für alle vier Übergänge.

\section{Numerisches Modell}

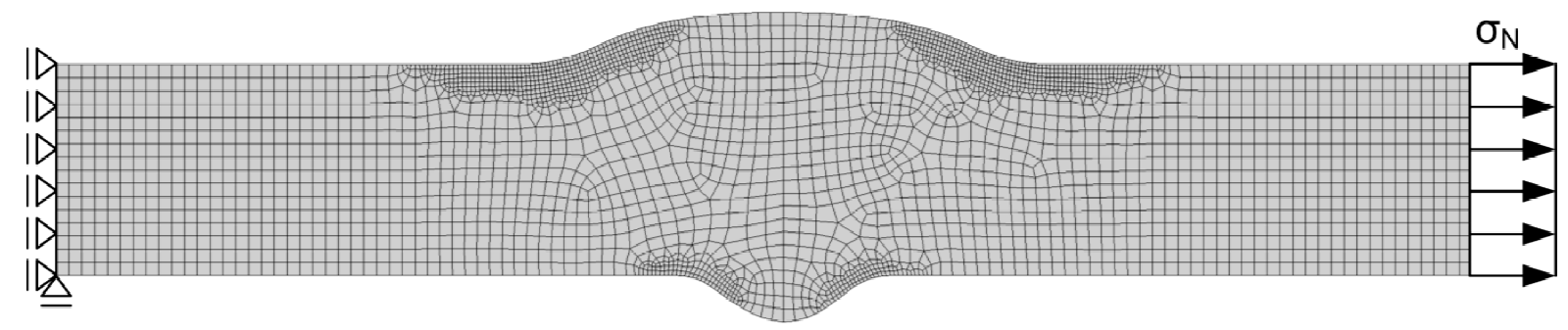

Verteilung der 1. Hauptspannung $\sigma_{1}$

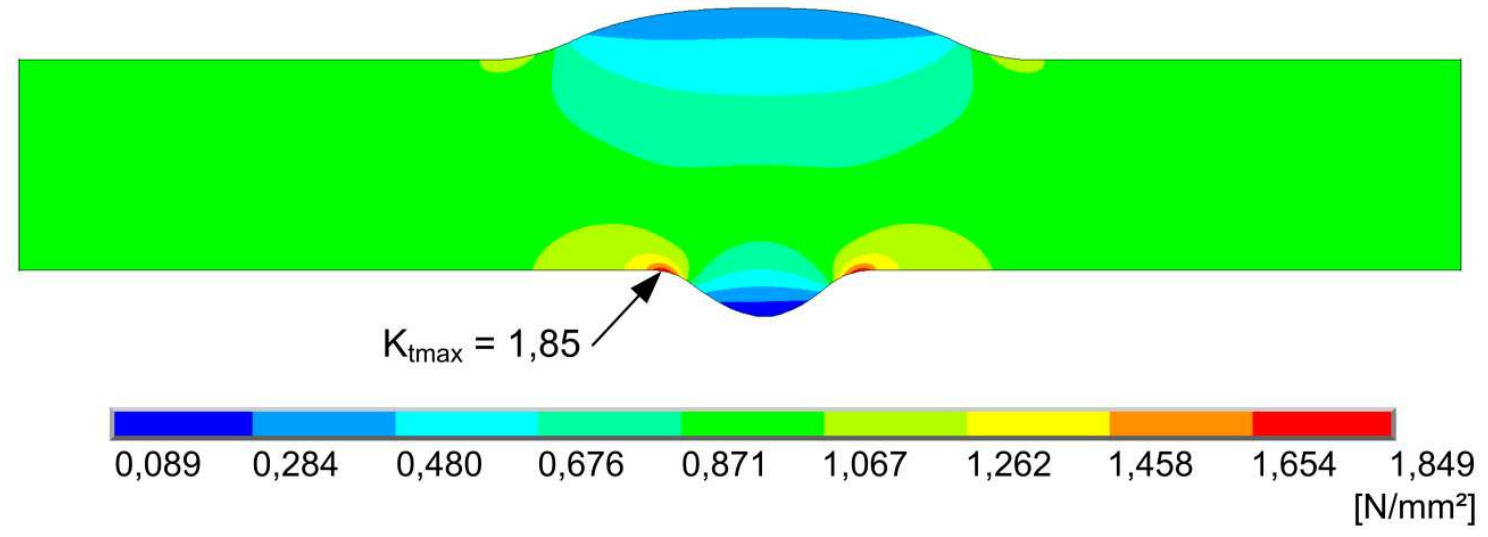

Abb. 3-11: Darstellung des numerischen Modells (oben) und der Verteilung der 1. Hauptspannung $\sigma_{1}$ (unten)

Für alle gemessenen Profilspuren wurde anhand der idealisierten Geometrie im numerischen Modell eine Auswertung der Kerbformzahlen für alle Übergänge vorgenommen. Die Ergebnisse der Berechnungen für die Übergänge an Naht- und Wurzelüberhöhung wurden jeweils zusammengefasst ausgewertet und sind in Tab. 3-8 dargestellt. Die Einzelergebnisse können Anhang E.2 entnommen werden. Am Nahtübergang ergibt sich im Mittel eine geringere Kerbformzahl $K_{\mathrm{t}_{\_} \mathrm{N}_{\text {FEM }}}$ Von 1,30 als am Wurzelübergang mit einem Wert von 1,92. Die Standardabweichung und die Extremalwerte am Wurzelübergang sind größer als am Nahtübergang, wobei die Variationskoeffizienten nahezu identisch sind. Im Hinblick auf die Beurteilung der möglichen Anrissorte zeigt sich bei der numerischen Ermittlung der Kerbformzahlen, dass die Maximalwerte ausschließlich am Wurzelübergang auftreten und somit dort der Anriss vermutet wird. Allerdings kann eine Verlagerung des Anrissortes in den 
Bereich des Nahtübergangs im Einzelfall nicht ausgeschlossen werden, da es sich bei den untersuchten Oberflächenprofilen lediglich um eine Stichprobe handelt.

Tab. 3-8: Zusammenfassung der numerisch ermittelten Kerbformzahlen $\mathrm{K}_{\mathrm{t}_{-} \mathrm{FEM}}$

\begin{tabular}{|c|c|c|}
\hline & $\begin{array}{c}\mathbf{K}_{\mathrm{t} \_ \text {N_FEM }} \\
{[-]}\end{array}$ & $\begin{array}{c}\mathbf{K}_{\mathrm{t} \_ \text {_FEM }} \\
{[-]}\end{array}$ \\
\hline Mittelwert & 1,30 & 1,92 \\
\hline Standardabweichung & 0,13 & 0,20 \\
\hline Maximum & 1,83 & 2,57 \\
\hline Minimum & 1,15 & 1,57 \\
\hline Variationskoeffizient & $10,3 \%$ & $10,6 \%$ \\
\hline
\end{tabular}

\section{Vergleich der Ergebnisse}

Zur Einordnung der Höhe der Kerbwirkung der Übergänge und zur Abschätzung des erwarteten Anrissortes wurden als Maß für die Kerbwirkung die Kerbformzahlen analytisch mit den Näherungsformeln von Anthes et al. und von Rainer sowie numerisch mit der Methode der Finiten Elemente ermittelt. Die Ergebnisse werden im Folgenden abschließend verglichen. In Tab. 3-9 sind die Mittelwerte der analytisch und numerisch berechneten Kerbformzahlen am Nahtübergang gegenübergestellt. Es ist zu erkennen, dass der Mittelwert der numerisch ermittelten Kerbformzahl $\mathrm{K}_{\text {t_N_FEM }}$ mit 1,30 geringfügig unterhalb der Mittelwerte nach den beiden Näherungsformeln ermittelten Kerbformzahlen $\mathrm{K}_{\mathrm{t} \_ \text {N_Anthes }}$ von 1,38 und $\mathrm{K}_{\mathrm{t} \_ \text {N_Rainer }}$ von 1,37 liegt. Die Standardabweichung und der Variationskoeffizient der numerisch ermittelten Kerbformzahlen liegen in der Größenordnung der Ergebnisse nach der Näherungsformel von Anthes et al. und sind demnach geringer als die Ergebnisse nach der Näherungsformel von Rainer.

Tab. 3-9: Vergleich der Ergebnisse der analytisch und numerisch ermittelten Kerbformzahlen $\mathrm{K}_{\mathrm{t} \_\mathrm{N}}$ am Nahtübergang

\begin{tabular}{|c|c|c|c|}
\hline & $\begin{array}{c}\mathbf{K}_{\mathbf{t} \_ \text {N_Anthes }} \\
{[-]}\end{array}$ & $\begin{array}{c}\mathbf{K}_{\mathbf{t} \_ \text {N_Rainer }} \\
{[-]}\end{array}$ & $\begin{array}{c}\mathbf{K}_{\mathbf{t} \_ \text {N_FEM }} \\
{[-]}\end{array}$ \\
\hline Mittelwert & 1,38 & 1,37 & 1,30 \\
\hline Standardabweichung & 0,12 & 0,21 & 0,13 \\
\hline Maximum & 1,87 & 2,16 & 1,83 \\
\hline Minimum & 1,22 & 1,14 & 1,15 \\
\hline Variationskoeffizient & $8,5 \%$ & $15,3 \%$ & $10,3 \%$ \\
\hline
\end{tabular}


In Tab. 3-10 werden die Mittelwerte der analytisch und numerisch berechneten Kerbformzahlen am Wurzelübergang verglichen. Es zeigt sich, dass im Mittel die numerisch ermittelte Kerbformzahl $\mathrm{K}_{\mathrm{t} \_ \text {W_FEM }}$ von 1,92 oberhalb der Mittelwerte nach den beiden Näherungsformeln ermittelten Kerbformzahlen $\mathrm{K}_{\mathrm{t} \_ \text {w_Anthes }}$ von 1,57 und $\mathrm{K}_{\mathrm{t} \_\mathrm{W} \_ \text {Rainer }}$ von 1,69 liegt. Die Standardabweichung und der Variationskoeffizient der numerisch ermittelten Kerbformzahlen liegen in der Größenordnung der Ergebnisse nach der Näherungsformel von Anthes et al. und sind demnach geringer als die Ergebnisse nach der Näherungsformel von Rainer.

Tab. 3-10: Vergleich der Ergebnisse der analytisch und numerisch ermittelten Kerbformzahlen $K_{t \_} w$ am Wurzelübergang

\begin{tabular}{|c|c|c|c|}
\hline & $\begin{array}{c}\mathbf{K}_{\mathbf{t} \_\mathbf{W}} \text { Anthes } \\
{[-]}\end{array}$ & $\begin{array}{c}\mathrm{K}_{\mathrm{t}} \mathrm{W}_{\mathbf{L}} \text { Rainer } \\
{[-]}\end{array}$ & $\begin{array}{c}\mathrm{K}_{\mathrm{t} \_\mathrm{W}_{-} \mathrm{FEM}} \\
{[-]}\end{array}$ \\
\hline Mittelwert & 1,57 & 1,69 & 1,92 \\
\hline Standardabweichung & 0,16 & 0,25 & 0,20 \\
\hline Maximum & 2,16 & 2,36 & 2,57 \\
\hline Minimum & 1,27 & 1,24 & 1,57 \\
\hline Variationskoeffizient & $10,0 \%$ & $14,6 \%$ & $10,6 \%$ \\
\hline
\end{tabular}

Zur Einordnung der beiden Näherungsformeln im Hinblick auf die Abschätzung der Kerbformzahlen für den vorliegenden Fall wurden die prozentualen Abweichungen $\mathrm{p}_{\%}$ der Ergebnisse nach den beiden Näherungsformeln von der numerisch ermittelten Kerbformzahl $\mathrm{K}_{\mathrm{t} \_ \text {FEM }}$ für alle Einzelberechnungen ausgewertet. Die Mittelwerte der prozentualen Abweichungen sind für die Naht- und Wurzelübergänge in Tab. 3-11 gegenübergestellt. Die prozentualen Abweichungen am Nahtübergang sind für beide Näherungsformeln deutlich geringer als am Wurzelübergang. Desweiteren sind die nach der Näherungsformel von Rainer ermittelten prozentualen Abwei-

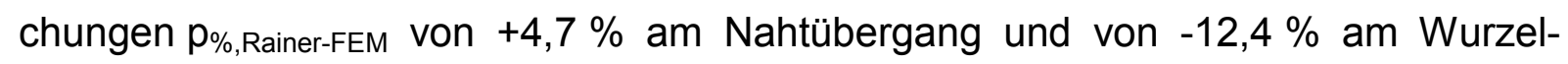
übergang geringer als die nach der Näherungsformel von Anthes et al. ermittelten

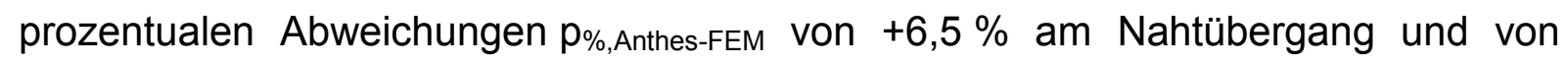
$-18,3 \%$ am Wurzelübergang.

Tab. 3-11: Vergleich der mittleren prozentualen Abweichungen $\mathbf{p}_{\%}$

\begin{tabular}{|c|c|c|}
\hline & $\begin{array}{c}\mathbf{p}_{\% \_ \text {Anthes-FEM }} \\
{[\%]}\end{array}$ & $\begin{array}{c}\mathbf{p}_{\% \_ \text {Rainer-FEM }} \\
{[\%]}\end{array}$ \\
\hline Nahtübergang & $+6,5$ & $+4,7$ \\
\hline Wurzelübergang & $-18,3$ & $-12,4$ \\
\hline
\end{tabular}


Aus dem Vergleich der numerisch ermittelten Ergebnisse mit den Ergebnissen der beiden Näherungsformeln für doppeltsymmetrische Stumpfnähte geht hervor, dass die Kerbformzahl am Nahtübergang durch beide Näherungsformeln gut abgeschätzt werden kann. Im Bereich des Wurzelübergangs zeigt sich allerdings, dass die numerisch ermittelten Ergebnisse im Vergleich zu den Ergebnissen der beiden Näherungsformeln für doppeltsymmetrische Stumpfnähte im Mittel eine höhere Kerbformzahl aufweisen. Die Ergebnisse nach der Näherungsformel von Rainer weisen im Mittel eine geringere prozentuale Abweichung zu den numerisch ermittelten Ergebnissen auf als die Ergebnisse nach der Näherungsformel von Anthes et al.. Aus diesem Grund ist zur Abschätzung der Kerbwirkung an den Naht- und Wurzelübergängen im vorliegenden Fall die Näherungsformel von Rainer zu empfehlen.

Die Näherungsformeln von Anthes et al. und von Rainer sind für doppeltsymmetrische Stumpfstöße ermittelt worden. Bei der numerischen Ermittlung der Kerbformzahlen wurde berücksichtigt, dass es sich im vorliegenden Fall jedoch um eine nicht doppeltsymmetrische Stumpfnaht handelt. Dies machte sich in den Ergebnissen in der Form bemerkbar, dass die maximalen Kerbformzahlen der Einzelberechnungen ausschließlich am Wurzelübergang auftraten. Im Hinblick auf die Abschätzung des Anrissortes wird abschließend erwartet, dass der Anriss vorwiegend am Wurzelübergang auftritt. Im Einzelfall kann jedoch nicht ausgeschlossen werden, dass es zu einer Verlagerung des Anrissortes vom Wurzel- zum Nahtübergang kommen kann, da es sich bei den untersuchten Oberflächenprofilen lediglich um eine Stichprobe handelt.

\subsection{Zugversuche}

Zur Überprüfung der Tragfähigkeit der Schweißverbindung wurden drei Zugversuche am Grundwerkstoff und sechs Zugversuche an Schweißverbindungen gemäß DIN EN ISO 6892-1 [DIN EN ISO 6892-1, 2009] an Flachzugproben durchgeführt. Die Zugversuche an den Schweißverbindungen ergaben einen Mittelwert der Streckgrenze von 499,7 N/mm² und einen Mittelwert der Zugfestigkeit von 583,2 N/mm². Im Vergleich dazu ergaben die Zugversuche am Grundwerkstoff einen Mittelwert der Streckgrenze von $503,4 \mathrm{~N} / \mathrm{mm}^{2}$ und einen Mittelwert der Zugfestigkeit von $590,0 \mathrm{~N} / \mathrm{mm}^{2}$. Die Versuchsprotokolle sind Anhang A.1 und A.2 zu entnehmen.

Sowohl die Zugfestigkeit als auch die Streckgrenze des Grundwerkstoffs und der Schweißverbindungen sind nahezu identisch. Alle Zugversuche an Schweißverbindungen haben im Grundwerkstoff versagt. Aus den Zugversuchen geht somit hervor, dass die Schweißverbindung der Anforderung einer mindestens gleichwertigen Festigkeit im Vergleich zum Grundwerkstoff gerecht wird. 


\subsection{Metallographische Untersuchung}

Die mit dem Schweißprozess verbundenen Eigenschafts- und Gefügeänderungen von Wärmeeinflusszone und Schmelzbad sind vornehmlich abhängig von Verfahrensparametern, welche die Abkühlgeschwindigkeit beeinflussen, und der chemischen Zusammensetzung des Grund- und Zusatzwerkstoffes [Schulze, 2010]. Abb. 3-12 zeigt zunächst die Schweißnaht im Makroschliff. Die Bereiche Grundwerkstoff, Wärmeeinflusszone und Schmelzbad sind klar zu erkennen. Das Gefüge wurde an einzelnen Stellen im Mikroschliff (Aufnahmen 1 bis 4) bei 200-facher Vergrößerung aufgenommen.

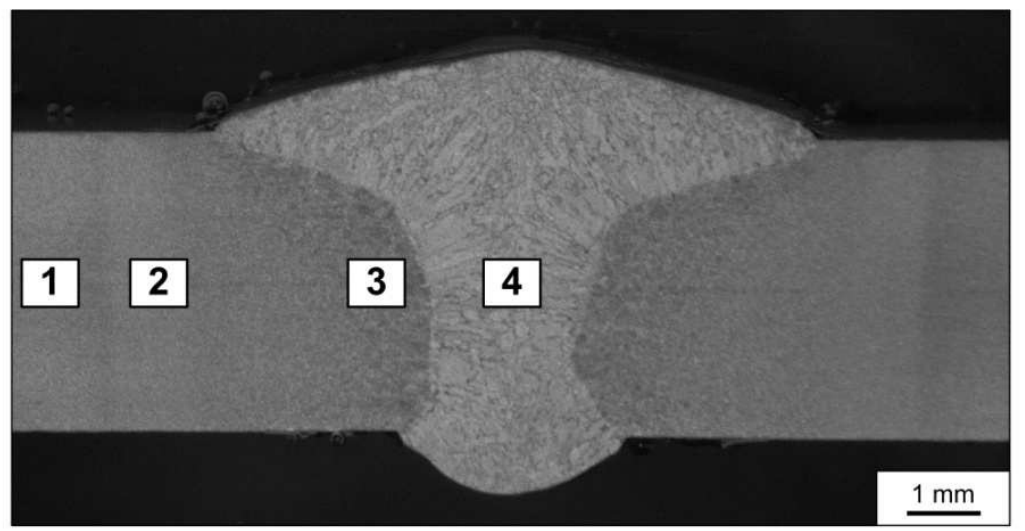

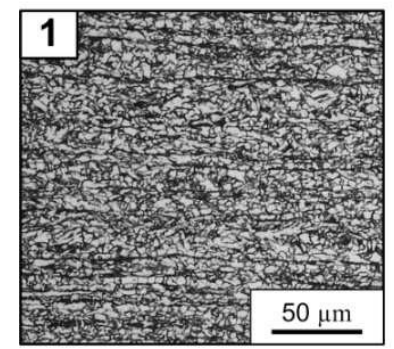

Grundwerkstoff

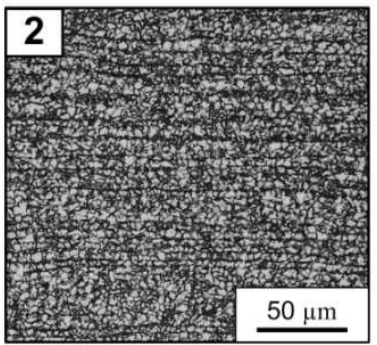

Feinkornzone

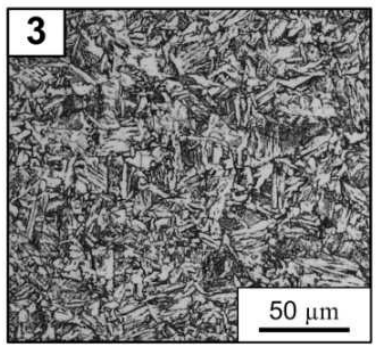

Grobkornzone

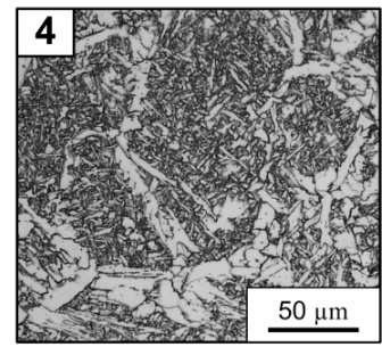

Schmelzgut

Abb. 3-12: Metallographische Untersuchung

Aufnahme 1 zeigt das Gefüge des Grundwerkstoffs. Im Grundwerkstoff S355J2 +N liegt ferritisch-perlitisches Gefüge vor, das eine manganlegierungstypische Walzzeiligkeit aufweist [Schulze, 2010]. Aufnahme 4 zeigt das Gefüge des Schmelzbades. Im Bereich des Schmelzbades ist das charakteristische Gussgefüge mit länglichen, schmalen, senkrecht zur Schweißnahtkante in Richtung Nahtmitte gerichteten Kristallen zu erkennen [Lohrmann, 1995] [Schulze, 2010]. Aufnahme 2 und 3 zeigen das Gefüge der Wärmeeinflusszone, das vornehmlich durch die Wärmeeinbringung und die damit verbundenen charakteristischen Temperatur-Zeit-Verläufe bestimmt wird [Schulze, 2010]. In der Nähe der Schmelzgrenze liegt ein Bereich mit hohem Kornwachstum vor, der ein sehr grobes Korn aufweist. Dieser Bereich wird als Grobkornzone bezeichnet. Im Vergleich dazu erfolgt eine Kornverfeinerung im Übergangsbe- 
reich zum Grundwerkstoff, wo ein deutlich feineres Korn vorliegt. Dieser Bereich wird als Feinkornzone bezeichnet.

\subsection{Härtemessung}

Um die Härte von Schweißgut, Wärmeeinflusszone und Grundwerkstoff beurteilen zu können, erfolgt die Härteprüfung nach Vickers für Lichtbogenschweißverbindungen gemäß DIN EN ISO 9015-1 [DIN EN ISO 9015-1, 2011] und DIN EN ISO 9015-2 [DIN EN ISO 9015-2, 2011]. Zulässige Höchstwerte der Härte in der Wärmeeinflusszone sind in DIN EN ISO 15614-1 [DIN EN ISO 15614-1, 2008] angegeben. Für den vorliegenden Baustahl ergibt sich ein zulässiger Höchstwert der Härte von 380 HV10. Die Höchstwerte der Härte treten in der Grobkornzone der Wärmeeinflusszone nahe der Schmelzlinie auf, wobei die Härte allgemein durch die Abkühlgeschwindigkeit und die chemische Zusammensetzung des Stahls sowie eine eventuelle Wärmebehandlung beeinflusst wird.

Zwei Härtereihen im Abstand von ca. 0,5 mm unterhalb der Oberfläche an Naht- und Wurzelüberhöhung sowie eine Härtereihe in Blechmitte wurden durch Schweißgut (SG), Wärmeeinflusszone (WEZ) und Grundwerkstoff (GW) gelegt. In Abb. 3-13 (oben) ist die Lage der Härtereihen im Makroschliff dargestellt. Der Abstand der Eindrücke beträgt 0,3 mm. Die Prüfkraft betrug 9,807 N. In Abb. 3-13 (unten) sind exemplarisch die Ergebnisse einer Härtemessung HV1 der einzelnen Härtereihen über den Abstand von der Nahtmitte aufgetragen. Die einzelnen Härtereihen an Naht- und Wurzelüberhöhung sowie in Blechmitte zeigen entsprechend ihrer Lage einen ähnlichen Verlauf. Die Härtewerte im Grundwerkstoff liegen im Mittel bei $188 \mathrm{HV} 1$ und nehmen in der Wärmeeinflusszone bis zur Schmelzlinie um bis zu $44 \mathrm{HV} 1$ zu. Im Bereich der Grobkornzone der Wärmeeinflusszone in der Nähe der Schmelzlinie kommt es zu Aufhärtungen, wobei die einzelnen Härtereihen Maximalwerte der Härte von im Mittel 232 HV1 aufweisen. Das Schweißgut weist im Mittel eine Härte von 206 HV1 auf. Die ermittelten maximalen Härtewerte liegen deutlich unterhalb der angegebenen zulässigen Höchstwerte der Härte von 380 HV10 in DIN EN ISO 15614-1 [DIN EN ISO 15614-1, 2008]. 


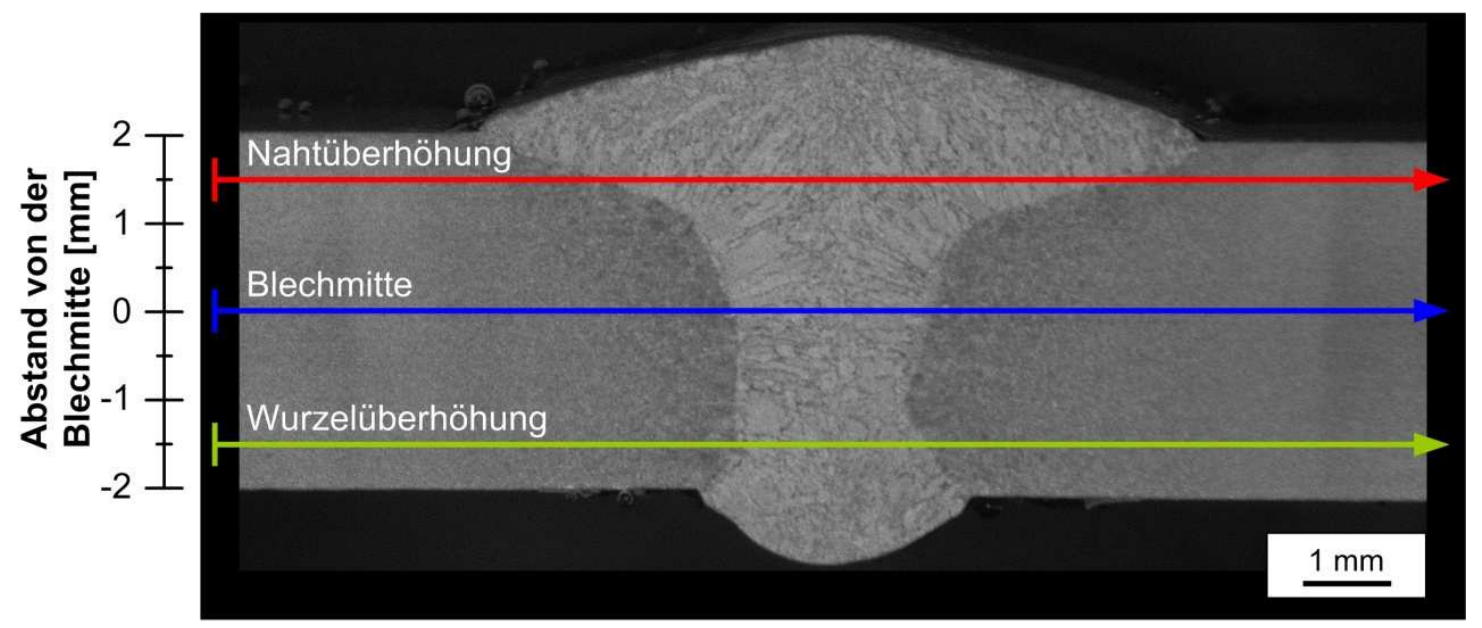

Abstand von der Nahtmitte [mm]

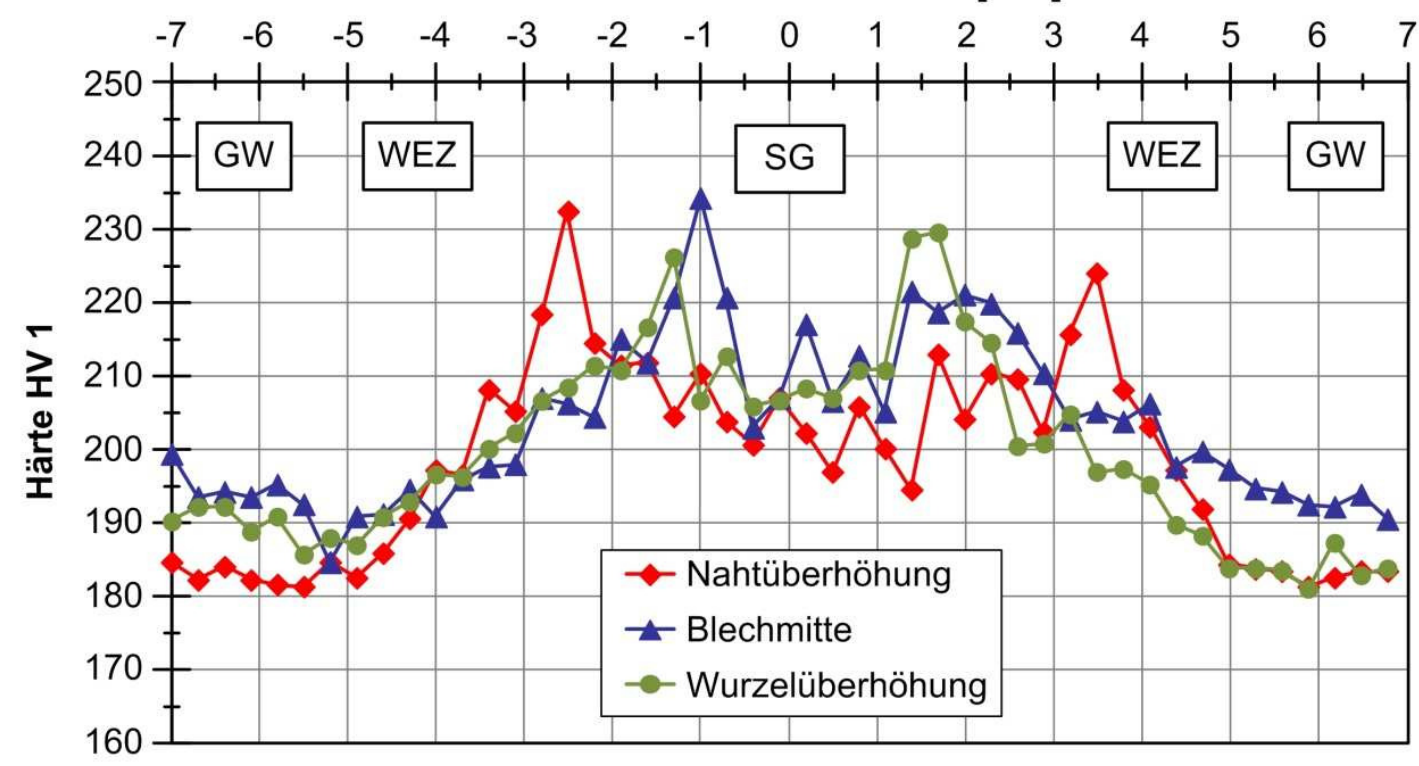

Abb. 3-13: Lage der Härtereihen im Makroschliff (oben) und Härtemessung HV1 der einzelnen Härtereihen (unten)

In Hanus et al. [Hanus, 1996] werden zwei empirische Formel von Düren und von Yurioka zur Ermittlung der Härte in Abhängigkeit von der chemischen Zusammensetzung und der Abkühlzeit $t_{8 / 5}$ aufgeführt. Die Härte in der Wärmeeinflusszone wurde auf Grundlage der chemischen Zusammensetzung des vorliegenden Baustahls (siehe Kapitel 3.1) und der mittleren ermittelten Abkühlzeit $t_{8 / 5}$ von 22,4 s (siehe Kapitel 3.4) ermittelt. Es ergibt sich nach Düren eine Härte in der Wärmeeinflusszone von 228 HV10 und nach Yurioka von 241 HV10. Die gemessene maximale Härte in Wärmeeinflusszone beträgt im Mittel $232 \mathrm{HV} 1$ und liegt demnach genau im Erwartungsbereich der analytischen Formeln von Düren und Yurioka. 


\subsection{Zusammenfassung und Erkenntnisse}

Zunächst wurde die Herstellung der Probekörper unter Angabe des untersuchten Werkstoffs sowie der vorgenommenen Nahtvorbereitung und des eingesetzten Schweißverfahrens beschrieben. Die Geometrie der bauteilähnlichen Probekörper wurde vorgestellt. Die Bewertung der Reproduzierbarkeit des Schweißprozesses und die Überprüfung der Qualität der Probekörper erfolgten durch die Charakterisierung des Ausgangszustandes.

In den herstellungsbegleitend durchgeführten Temperaturmessungen während der Schweiß- und Abkühlphase der Probekörper zeigte sich die Reproduzierbarkeit des Schweißprozesses. Da der Temperatur-Zeit-Verlauf der Schweiß- und Abkühlphase einen ausschlaggebenden Einfluss auf die mechanischen Eigenschaften der Schweißverbindung haben kann, wurden zur Beurteilung die Abkühlzeiten $t_{8 / 5}$ ausgewertet. Es wurde eine mittlere Abkühlzeit $t_{8 / 5}$ von $22,4 \mathrm{~s}$ ermittelt, die im oberen Bereich angegebener Richtwerte von 10 bis $25 \mathrm{~s}$ liegt.

Die Untersuchung der lokalen Schweißnahtgeometrie belegte, dass es sich trotz mechanisierter Fertigung bei den lokalen Nahtgeometrieparametern um in hohem Maße streuende Größen handelt. Die Streuungen von Breite und Höhe der Naht- und Wurzelüberhöhung sind am geringsten, wohingegen die Streuungen von Kerbradius und Kantenversatz am höchsten sind. Am Wurzelübergang zeigten sich im Mittel kleinere Kerbradien sowie größere Anstiegswinkel als am Nahtübergang, wobei sich die Extremalwerte der Naht- und Wurzelübergänge nur geringfügig unterscheiden.

Als Maß zur Beurteilung der Kerbwirkung wurden die Kerbformzahlen für die einzelnen Übergänge analytisch anhand von Näherungsformeln sowie numerisch mit Hilfe der Methode der Finiten Elemente ermittelt und verglichen. Hierbei stellte sich heraus, dass die Ergebnisse der beiden Näherungsformeln, die für doppeltsymmetrische Stumpfstöße hergeleitet wurden, nur geringfügig voneinander abweichen. Die Maximalwerte der Kerbformzahlen traten vorwiegend am Wurzelübergang auf, wobei es vereinzelt zu einer Verlagerung der maximalen Kerbformzahlen vom Wurzel- zum Nahtübergang kam. Im Gegensatz dazu ergaben sich die maximalen Kerbformzahlen bei der numerischen Ermittlung ausschließlich am Wurzelübergang. Am Nahtübergang wurde im Mittel eine Kerbformzahl $K_{\mathrm{t} \_ \text {N_FEM }}$ von 1,30 ermittelt. Dieser Wert liegt im Bereich der im Mittel mit den Näherungsformeln berechneten Ergebnisse von 1,38 und 1,37. Am Wurzelübergang wurde in der numerischen Berechnung im Mittel eine Kerbformzahl $\mathrm{K}_{\mathrm{t} \_\mathrm{W}}$ FEM von 1,92 bestimmt. Dieser Wert liegt im Vergleich zu den im Mittel mit den Näherungsformeln berechneten Ergebnissen von 1,57 und 1,69 deutlich höher. Zur Abschätzung der Kerbwirkung am Naht- und Wurzelübergang lieferten die Ergebnisse der Näherungsformel für doppeltsymmetrische Stumpfstöße 
von Rainer im Mittel geringere Abweichungen von den numerischen Ergebnissen. Allerdings sind die nach der Näherungsformel von Rainer ermittelten Werte am Wurzelübergang geringer als die numerisch ermittelten Werte. Im Hinblick auf die Abschätzung des Anrissortes in den Ermüdungsversuchen wird vorwiegend erwartet, dass der Anriss am Wurzelübergang auftritt.

Die Zugversuche am Grundwerkstoff und an der Schweißverbindung belegten, dass die Schweißverbindung der Anforderung mindestens gleichwertiger Festigkeitseigenschaften im Vergleich zum Grundwerkstoff gerecht wird. Die metallographische Untersuchung der Schweißnahtbereiche Grundwerkstoff, Wärmeeinflusszone und Schmelzbad zeigte keine Auffälligkeiten. Die maximalen Härtewerte im Bereich der Grobkornzone der Wärmeeinflusszone in der Nähe der Schmelzlinie liegen bei $232 \mathrm{HV} 1$ und sind somit deutlich geringer als der zulässige Höchstwert der Härte von 380 HV10. Der im Mittel gemessene maximale Härtewert stimmte sehr gut mit den analytisch berechneten Härtewerten überein.

Im Hinblick auf die Ermüdungsversuche sind die Probekörper als repräsentativ zu beurteilen. 


\section{Weiterentwicklung der Prüftechnik}

Um Ermüdungsversuche im Bereich sehr hoher Lastwechselzahlen in einem angemessenen Zeitraum realisieren zu können, sind Prüfmaschinen mit hohen Prüffrequenzen erforderlich. Zu diesem Zweck wurde basierend auf dem Prinzip gemäß Deutscher Patentschrift [Deutsches Patent 10204258.6, 2005] eine Prüfvorrichtung für Schweißverbindungen mit einer Prüffrequenz von bis zu $400 \mathrm{~Hz}$ entwickelt. Ein Resonanzkörper wird elektromagnetisch über Wechselstrommagnete in seiner Biegeeigenform harmonisch angeregt. Diese Prüfvorrichtung wurde bereits für Versuchsreihen zur Ermittlung der Zeitfestigkeit von Schweißverbindungen eingesetzt [Schaumann, 2008] [Keindorf, 2009] [Keindorf, 2010]. Im Rahmen der vorliegenden Arbeit wurden die Vorspannvorrichtung sowie die Regelung für Versuche im Langzeitbereich weiterentwickelt.

Die Prüfvorrichtung ist keine Universalprüfmaschine, sondern speziell für einen möglichst standardisierten Probekörper geformt und dimensioniert. Die vorliegende Ausführung kann bei einer Prüffrequenz von bis zu $400 \mathrm{~Hz}$ stumpf gestoßene Schweißverbindungen mit einer Blechdicke von bis zu $5 \mathrm{~mm}$ axial mit einer maximalen Prüfkraftamplitude von $50 \mathrm{kN}$ und einer maximalen Vorspannkraft von $60 \mathrm{kN}$ belasten. Ein Versuch mit beispielsweise $10^{9}$ Lastwechseln dauert 30 Tage. Ähnliche Prüfvorrichtungen basierend auf demselben Prüfprinzip kamen bei Versuchen zur Ermittlung der Dauerfestigkeit von Schraube-Mutter-Verbindungen zum Einsatz [Alt, 2005] [Alt, 2007].

Im Folgenden werden Aufbau und Funktionsprinzip der verwendeten Prüfvorrichtung erläutert und die Weiterentwicklungen vorgestellt. Desweiteren werden die zeitlich veränderlichen Messgrößen betrachtet, das Kriterium für die Ermittlung der Anrissschwingspielzahl definiert und die Untersuchung zur Größe des vorliegenden Anrisses vorgestellt. Außerdem wird die statische sowie dynamische Kalibrierung der Prüfvorrichtung erläutert.

\subsection{Aufbau und Funktionsprinzip}

Die Prüfvorrichtung besteht aus einem Regelkreis, der sich aus Resonanzkörper, Wechselstrommagneten, Dehnungsmessstreifen, Messverstärker, Regelungseinheit und Leistungsverstärker zusammensetzt. Der Resonanzkörper ist in Abb. 4-1 dargestellt und hat die Form eines geschlossenen rechteckigen Rahmens mit verlängerten Längsseiten (Hebelarmen). Die Lagerung des Resonanzkörpers erfolgt reaktionskräftefrei in den Rahmenecken über Passstifte auf einer Grundplatte. Die Wechselstrommagnete sind an den Enden der Hebelarme ebenfalls auf der Grundplatte befestigt. 


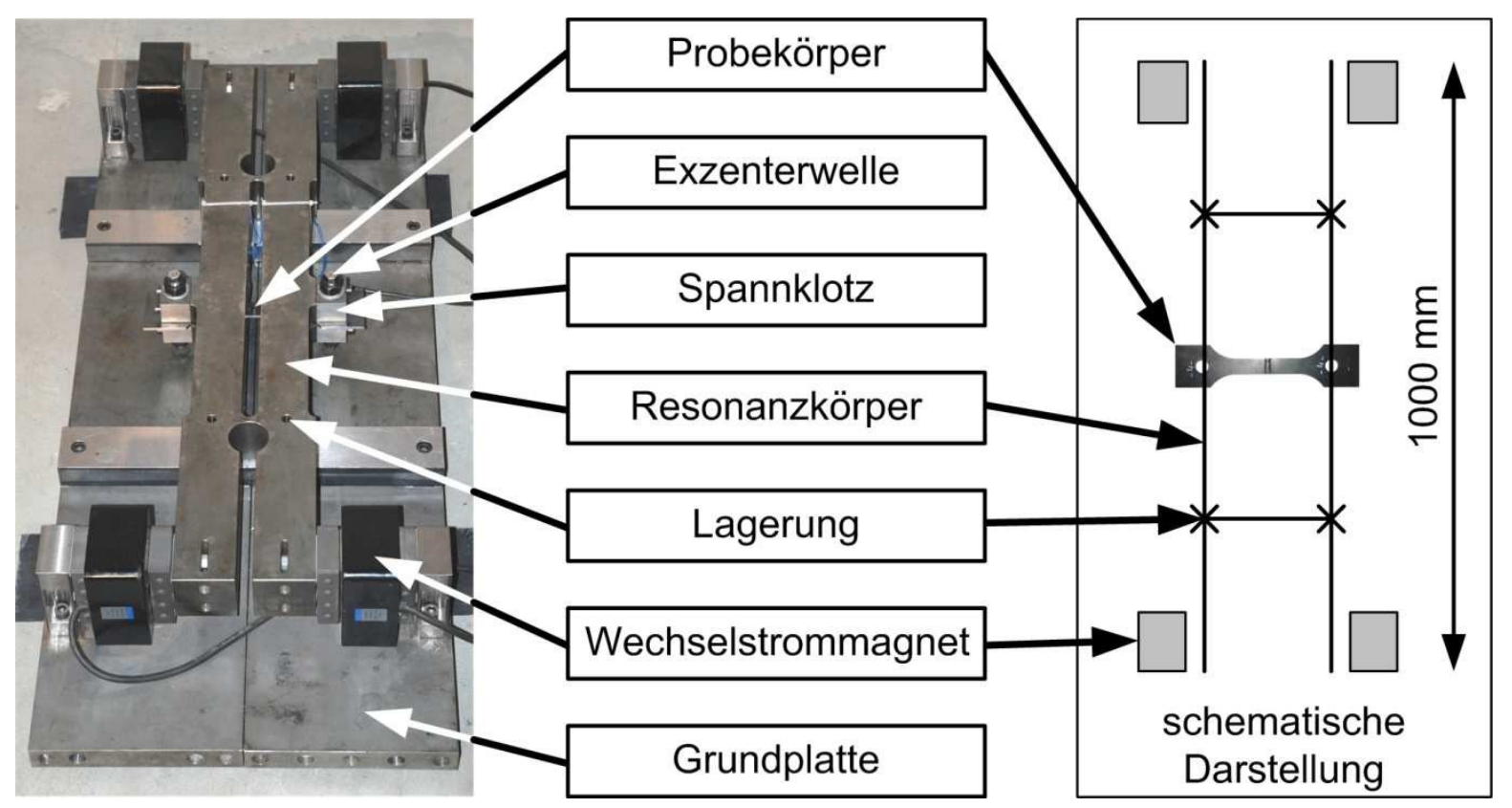

Abb. 4-1: Aufbau der Prüfvorrichtung

Das Funktionsprinzip der Prüfvorrichtung wird in Abb. 4-2 schematisch verdeutlicht. Zur Vereinfachung ist der Biegeanteil des Rahmens infolge Vorspannung nicht mit abgebildet. Die Prüfvorrichtung wird elektromagnetisch über die Wechselstrommagnete in der Biegeeigenform des Resonanzkörpers harmonisch angeregt. Die Rahmenecken stellen hierbei die Knotenpunkte der Biegeeigenschwingung des Resonanzkörpers dar. Durch die Anordnung des Probekörpers in der Mitte der Prüfvorrichtung wird dieser rein axial beansprucht.
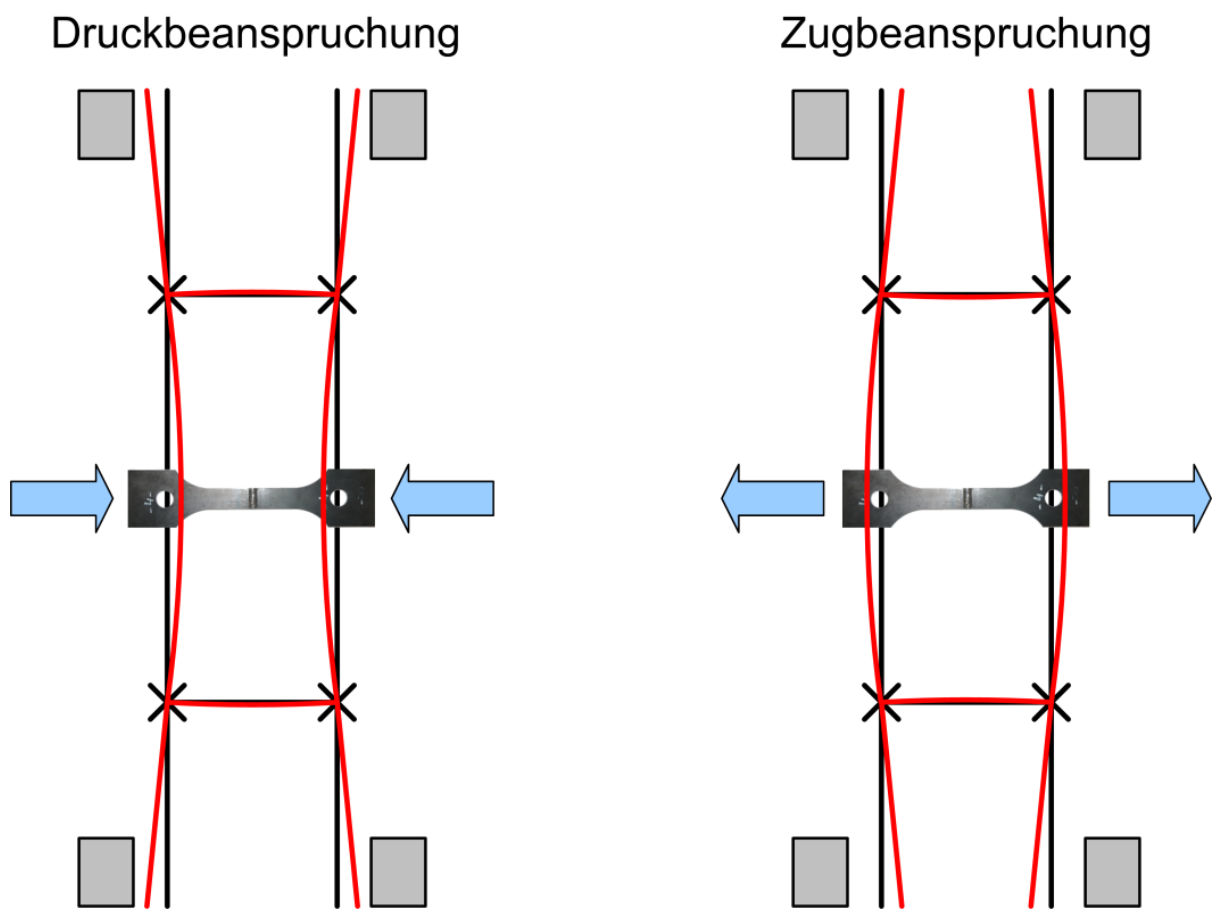

Abb. 4-2: Funktionsprinzip der Prüfvorrichtung 
In Abhängigkeit von der Höhe der Vorspannkraft und der Prüfkraftamplitude ergibt sich das Spannungsverhältnis. Zunächst wird der Probekörper statisch mit Exzenterwellen über Spannklötze gegen den Resonanzkörper verspannt. Im Betrieb wird die aufgebrachte Vorspannkraft mit der Prüfkraftamplitude überlagert und kann prinzipiell nicht nachgeregelt werden. Auf den Außen- und Innenseiten des Resonanzkörpers sind Dehnungsmessstreifen appliziert. Über den Messverstärker erhält die Regelungseinheit ein elektrisches Signal der Dehnung des Resonanzkörpers. Die elektromagnetische Anregung des Resonanzkörpers erfolgt über Rückkopplung dieses Signals der Eigenschwingung, das proportional zur Beanspruchung des Probekörpers ist.

Die Kalibrierung dieses Signals erfolgt über mit Dehnungsmessstreifen versehene Kalibrierproben (siehe Kapitel 4.6). Bei der Prüfvorrichtung wird das gleichbleibende Verhältnis zwischen Beanspruchung von Probekörper und Rahmen genutzt. Die Prüfvorrichtung wird über die Rahmendehnung geregelt, wobei von der Rahmendehnung auf die Prüfkraft im Probekörper geschlossen wird. Um dies zu realisieren, ist die statische und dynamische Kalibrierung der Prüfvorrichtung erforderlich. Prinzipiell ist für unterschiedliche Arten von Probekörpern jeweils eine Kalibrierung des Prüfrahmens erforderlich.

Die Regelungseinheit gleicht das gemessene Signal der Rahmendehnung mit dem Sollwert ab und steuert über den Leistungsverstärker die Wechselstrommagnete an. Die Aufzeichnung und Auswertung der Messdaten erfolgt mit einem Computer über eine Messkarte mit der Software LabVIEW 8.5 [LabVIEW, 2007]. Weitere Details zur Prüfvorrichtung und Einzelheiten zur Regelung können der Deutschen Patentschrift [Deutsches Patent 10204258.6, 2005], Alt [Alt, 2005] und Schaumann et al. [Schaumann, 2008] entnommen werden.

\subsection{Versuchsablauf und Weiterentwicklungen}

\subsubsection{Vorspannvorgang des Probekörpers}

In Abb. 4-3 ist die Vorspannvorrichtung im Detail dargestellt. Der Probekörper wird über zwei symmetrisch angeordnete Exzenterwellen gegen den Prüfrahmen vorgespannt. Die Exzenterwellen sind jeweils drehbar in zwei Spannklötzen gelagert. Die Spannklötze liegen an den Außenseiten des mittig mit einem Schlitz versehenen Prüfrahmens an. Die Spannklötze geben den zylindrischen Exzenter der Exzenterwellen frei, auf dem der beidseitig mit einer Bohrung versehene Probekörper steckt. Beim Verdrehen der Exzenterwellen werden die Spannklötze über die Exzenterwelle gegen den Rahmen gedrückt, während der Probekörper über den Exzenter durch Zug belastet wird. 
Nach Abschluss des Vorspannvorgangs werden Spannklötze, Probekörper und Exzenterwelle in axialer Richtung der Exzenterwelle mit Hilfe einer Mutter M20 je Exzenterwellenende verspannt, um die Rückdrehung der Exzenterwelle unter den dynamischen Einflüssen im Versuch zu verhindern. Die Spannklötze sind auf der dem Probekörper zugewandten Seite mit einer Rändelung versehen, sodass beim Verspannen ein zusätzlicher Formschluss zur Übertragung der Prüfkräfte entsteht. Die Spannklötze werden zuletzt über jeweils zwei Schrauben M8 am Prüfrahmen fixiert, um gegen Verlust bei der Vormontage zu sichern und eine Seitwärtsbewegung während des Versuchs zu verhindern.

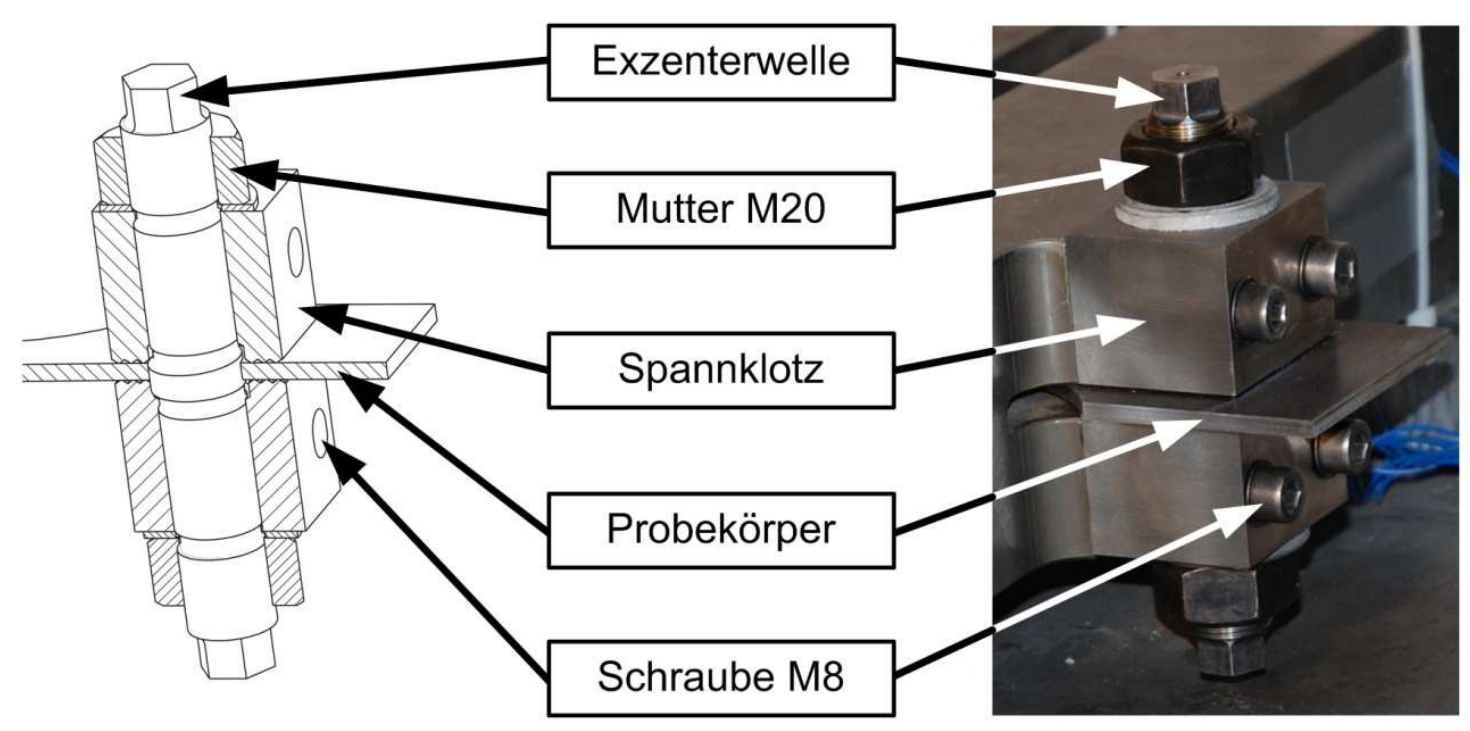

Abb. 4-3: Vorspannvorrichtung

Die Vorspannkraft kann im Betrieb prinzipiell nicht nachgeregelt werden, sodass durch einen ersten manuell gesteuerten Lastzyklus Setzungserscheinungen vorweggenommen werden. Die Vorspannkraftverluste werden damit erheblich reduziert. Beim Vorspannvorgang wird die Kraft zunächst auf Höhe der Oberlast gesteigert und anschließend vollständig wieder aufgehoben. Daraufhin wird die endgültige Vorspannkraft eingestellt.

\subsubsection{Festlegung von Prüfkraftamplitude und Abbruchkriterien}

Der Sollwert der Prüfkraftamplitude $F_{a}$ wird an der Regelungseinheit eingestellt. Desweiteren sind die Abbruchkriterien des Versuchs zu definieren. Als Abbruchkriterien sind ein Vorspannkraftabfall auf ein festgelegtes Niveau oder das Erreichen einer festgelegten Grenzschwingspielzahl vorgesehen. Fällt die Vorspannkraft des Probekörpers deutlich ab, so ist von einem Riss im Probekörper auszugehen und der Versuch wird beendet. Als Abbruchkriterium für die Vorspannkraft wird ein Abfall auf ein festgelegtes Kraftniveau $F_{v, A b b r u c h}$ festgelegt. Bei einem Spannungsverhältnis von $R=0,1$ wird beispielsweise das Kraftniveau für den Abbruch auf das der Prüfkraft- 
amplitude festgelegt $\left(F_{v, A b b r u c h}=F_{a}\right)$, sodass die Probe keine Druckbeanspruchungen erhält. Wenn bei konstant bleibender Vorspannkraft die Grenzschwingspielzahl erreicht wird, ist der Versuch ebenfalls beendet.

\subsubsection{Start des Versuchs und Aufzeichnung der Messdaten}

Zum Starten des Versuchs wird der Resonanzkörper durch einen Stoß mit einem Gummihammer im Bereich der Hebelarme in seiner ersten Biegeeigenschwingungsform ausgelenkt. Der sich selbst überlassene Resonanzkörper beginnt mit Resonanzfrequenz gedämpft in seiner ersten Biegeeigenschwingungsform auszuschwingen. Die Regelungseinheit erkennt diese Eigenschwingung und koppelt sie, wie oben beschrieben, zum weiteren Resonanzbetrieb über Elektromagnete zurück.

Während der Versuchslaufzeit werden die zeitlich veränderlichen Messgrößen des Prüfrahmens über die Schwingspielzahl $N$ aufgezeichnet und in einer Textdatei gespeichert. Zu den zeitlich veränderlichen Messgrößen des Prüfrahmens zählen Prüfkraftamplitude $F_{a}$, Vorspannkraft $F_{v}$, und Prüffrequenz f. Bei den Kalibrierproben werden zusätzlich die zeitlich veränderlichen Messgrößen der Probe (Vorspannkraft $F_{v}$ und Prüfkraftamplitude $F_{a}$ ) aufgezeichnet. Diese Messgrößen stehen zur Auswertung der Versuche zur Verfügung. Für ausgewählte Proben werden zusätzlich Temperaturmessungen über die Versuchslaufzeit durchgeführt.

\subsubsection{Weiterentwicklungen der Prüfvorrichtung}

Bislang wurden die Probekörper über eine separate Vorspannvorrichtung bestehend aus Vorspannblock, Schubblech und Stellschraube auf das gewünschte Vorspannkraftniveau gebracht [Schaumann, 2008]. Beim Erreichen des festgelegten Vorspannkraftniveaus wurde der Probekörper mit Hilfe von Spannklötzen fixiert. Im Anschluss wurde die Vorspannvorrichtung entfernt. Das Lösen der Vorspannvorrichtung führte zu einer Umlagerung des Kraftflusses und einem damit verbundenen Verlust an Vorspannkraft. Durch das Vorspannen mit Exzenterwellen entfällt die separate Vorspannvorrichtung. Die Vorspannkraft kann direkt eingestellt werden und der durch die Umlagerung des Kraftflusses hervorgerufene Verlust entfällt. Durch das Fixieren der Spannklötze nach dem Vorspannen wird durch die Rändelung der Oberflächen der Spannklötze ein zusätzlicher Formschluss herbeigeführt. Dieser wird erst durch Änderungen der Kraft beispielsweise durch Setzungen oder die Überlagerung mit der dynamischen Beanspruchung aktiviert und am Kraftfluss beteiligt.

Bisher wurde eine gleichbleibende Proportionalität zwischen der Beanspruchung von Prüfrahmen und Probekörper angenommen. Eine Kalibrierung des Prüfrahmens erfolgte somit für den jeweiligen Probekörper. In den Langzeitversuchen hat sich allerdings herausgestellt, dass sich die Steifigkeit von Probekörper und Einspannung er- 
heblich verändern kann. Mit der Änderung der Steifigkeit geht eine Änderung der Proportionalität der Beanspruchungen einher. Der zu Beginn des Versuchs gültige Kalibrierfaktor verliert seine Richtigkeit. Die Regelung wurde um eine frequenzabhängige Sollwertvorgabe erweitert, um den Einfluss des Steifigkeitsverlustes auf den dynamischen Kalibrierfaktor zu kompensieren und im relevanten Frequenzbereich eine näherungsweise konstante Beanspruchung im Probekörper zu erzielen. Eine ausführliche Erläuterung der Funktionsweise ist Kapitel 4.6.2 zu entnehmen.

\subsection{Zeitlich veränderliche Messgrößen}

Während der Versuchslaufzeit werden die zeitlich veränderlichen Messgrößen der Prüfvorrichtung über die Schwingspielzahl N aufgezeichnet. Zu diesen zählen Prüfkraftamplitude $F_{a}$, Vorspannkraft $F_{v}$ und Prüffrequenz $f$. Wie in Kapitel 4.2 beschrieben, werden die Probekörper mit einer Vorspannkraft statisch vorgespannt und mit einer konstanten Prüfkraftamplitude dynamisch beansprucht. Die Rahmendehnung stellt die Regelgröße dar, um die Prüfkraftamplitude während des Versuchs konstant zu halten. Die Versuchsführung erfolgt mit einem konstanten Spannungsverhältnis R. Einen Überblick der zeitlich veränderlichen Messgrößen Prüfkraftamplitude $F_{a}$, Vorspannkraft $F_{v}$ und Prüffrequenz $f$ eines exemplarischen Versuchs mit Probenversagen aufgetragen über die Schwingspielzahl N gibt Abb. 4-4.

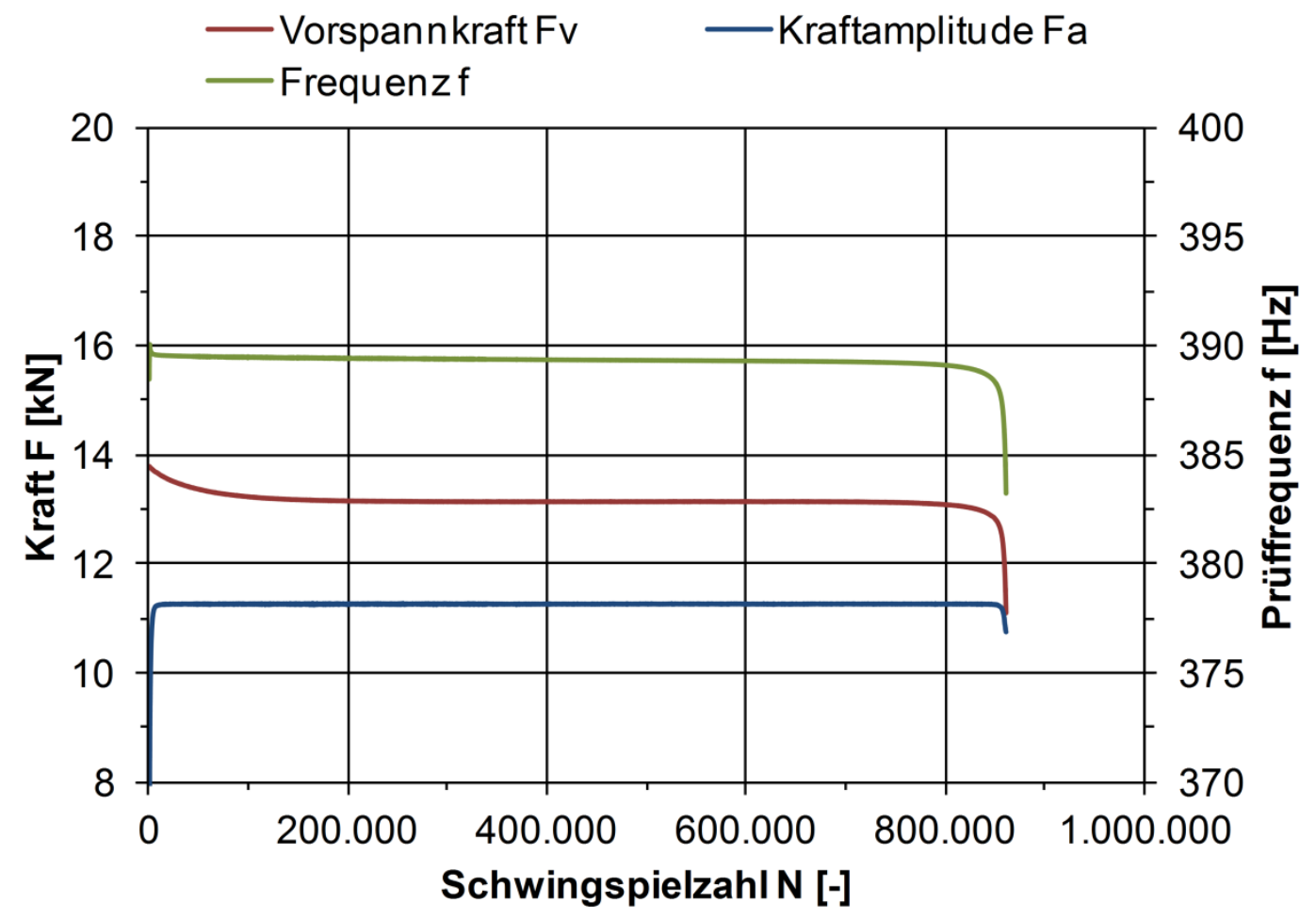

Abb. 4-4: Verlauf der zeitlich veränderlichen Messgrößen über die Schwingspielzahl N bei Probenversagen 
Die zeitlich veränderlichen Messgrößen eines exemplarischen Versuchs bis zum Erreichen der Grenzschwingspielzahl sind in Abb. 4-5 über die Schwingspielzahl N aufgetragen. Die charakteristischen Verläufe der einzelnen Messgrößen über die Versuchslaufzeit bei Probenversagen sowie beim Erreichen der Grenzschwingspielzahl $N_{G}$ werden im Folgenden näher beschrieben und erläutert.

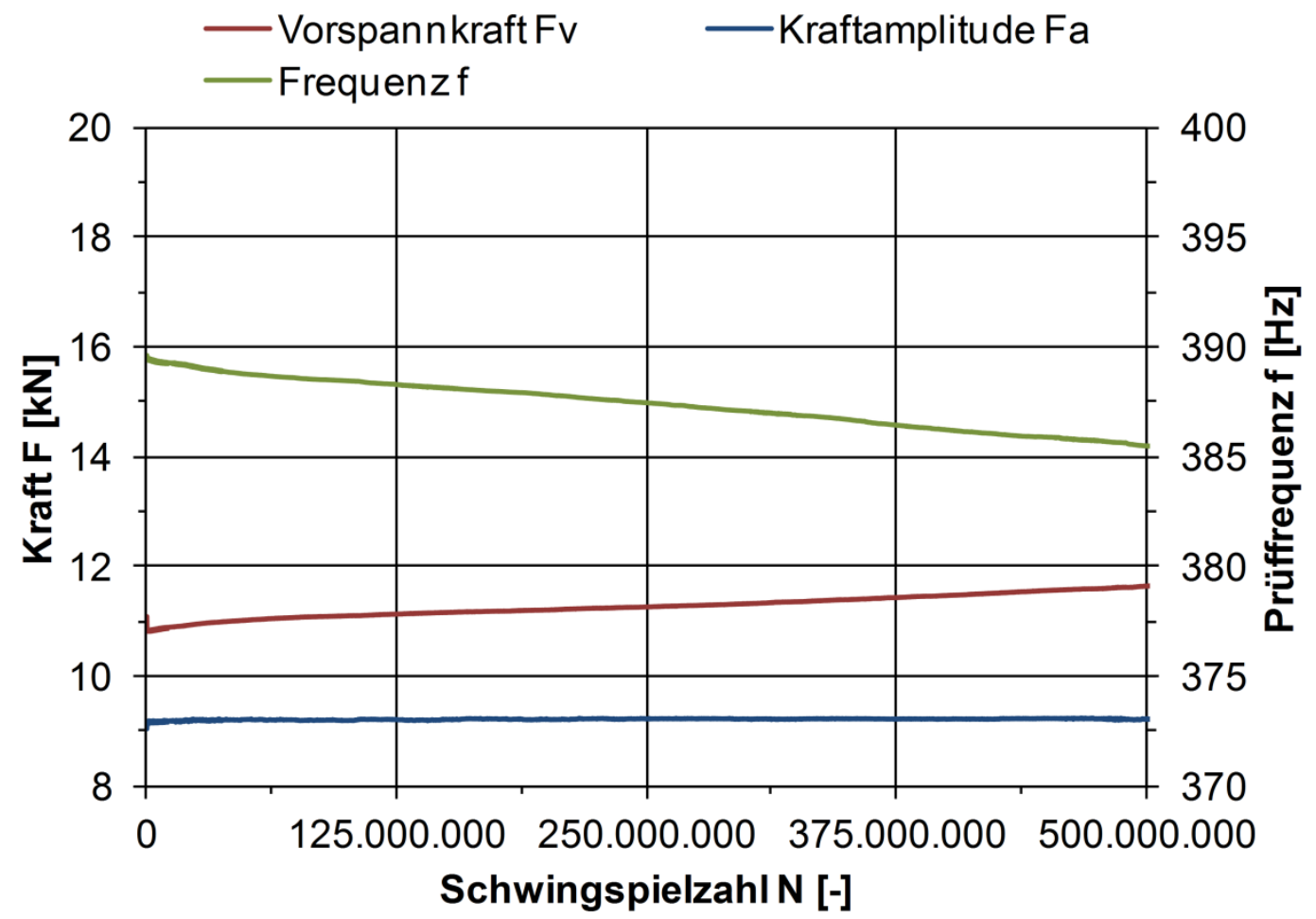

Abb. 4-5: Verlauf der zeitlich veränderlichen Messgrößen über die Schwingspielzahl N beim Erreichen der Grenzschwingspielzahl $N_{G}$

\subsubsection{Prüffrequenz $f$}

Die Prüffrequenz $f$ des Systems ist abhängig von der Steifigkeit des Systems. Diese variiert in geringem Maße, resultierend aus Streuungen der Probekörpersteifigkeit und der Einspannbedingungen, und verändert sich während der Versuchslaufzeit. Die Prüfvorrichtung verhält sich analog zu einem Ein-Massen-Schwinger mit einer Feder, die sich aus hintereinandergeschalteten Einzelfedern zusammensetzt. Die Steifigkeit der Einzelfedern wird durch den Probekörper, die Einspannung und den Prüfrahmen abgebildet. Der Kehrwert der Gesamtfedersteifigkeit des Systems ergibt sich aus der Summe der Einzelnachgiebigkeiten. Wenn sich die Steifigkeit einer Einzelfeder ändert, dann ändert sich auch die Gesamtfedersteifigkeit des Systems. Dies hat somit eine Änderung der Prüffrequenz zur Folge. Ein Abfall der Prüffrequenz bedeutet demnach einen Steifigkeitsverlust des Systems. Ursächlich für den Steifigkeitsverlust des Systems kann ein Steifigkeitsverlust von Prüfrahmen, Probe oder Einspannung sowie Kombinationen hieraus sein. 
Ein Steifigkeitsverlust des Prüfrahmens wäre auf eine Schädigung beispielsweise in Form eines Risses zurückzuführen. Dieser Steifigkeitsverlust würde sich von Versuch zu Versuch fortsetzen. Die Prüffrequenz würde mit stetig wachsendem Steifigkeitsverlust im Verlauf der gesamten Versuchsführung und letztendlich zum bis Versagen des Prüfrahmens immer geringer werden. Das Fortschreiten der Schädigung wäre hierbei abhängig von der Höhe der Beanspruchung.

Für das Ereignis Probenversagen ist in Abb. 4-6 der charakteristische Verlauf der Prüffrequenz $f$ über die Schwingspielzahl $N$ aufgetragen. Nach dem Vorgang des Hochfahrens und mit Erreichen des Sollwertes der Prüfkraftamplitude verläuft die Prüffrequenz nahezu konstant. Es ist lediglich ein leichter kontinuierlicher Abfall erkennbar. Da die Vorspannkraft in diesem Bereich konstant bleibt (vergleiche Abb. 4-4), wird dieser kontinuierliche leichte Abfall der Prüffrequenz auf einen Steifigkeitsverlust der Einspannung zurückgeführt.

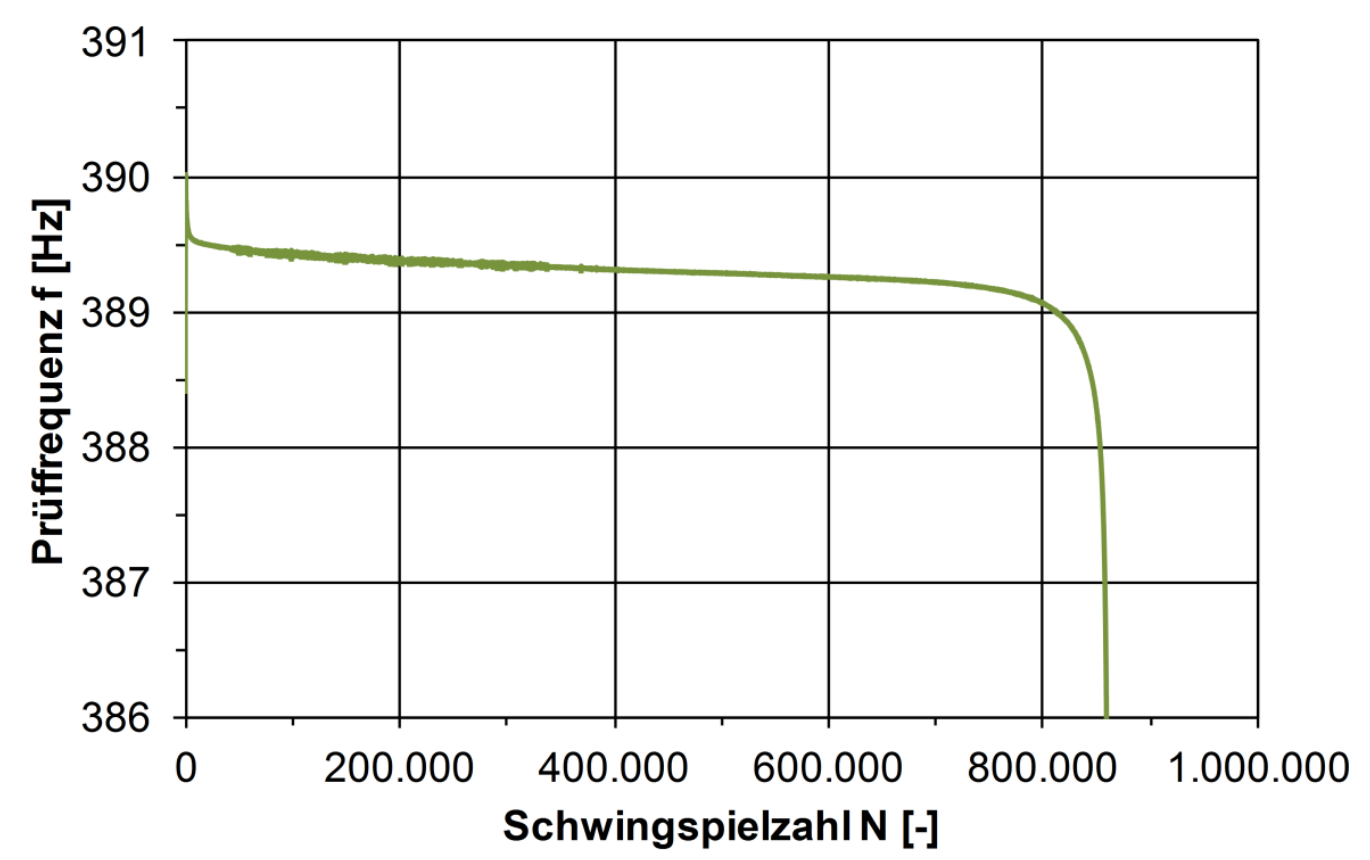

Abb. 4-6: Verlauf der Prüffrequenz $f$ über die Schwingspielzahl $N$ bei Probenversagen

Durch die Einprägung der Oberflächen der Spannklötze in die Probekörper im Zuge des Verspannungsvorgangs und die anschließende dynamische Beanspruchung der Probe kommt es zu Setzungserscheinungen. Die Oberflächen der Spannklötze mit Rändelung ebnen sich im Laufe des Versuchs in die Probe ein, sodass es zu einem Steifigkeitsverlust der Einspannung kommt. Dieser Steifigkeitsverlust setzt sich im Langzeitbereich kontinuierlich fort. Zu Versuchsende fällt die Prüffrequenz deutlich ab. Dieser Abfall geht mit einem deutlichen Abfall der Vorspannkraft einher, wie in Abb. 4-4 zu erkennen. Nach Versuchsende zeigt sich ein signifikanter Riss des Probekörpers. Dieser Prüffrequenzabfall ist auf den Steifigkeitsverlust des Probekörpers 
in Form einer Schädigung (Anriss und Rissfortschritt) zurückzuführen. Die Ausprägung dieses Abfalls ist abhängig von der Höhe der Beanspruchung.

Der charakteristische Verlauf der Prüffrequenz $f$ über die Schwingspielzahl $N$ bis zum Erreichen der Grenzschwingspielzahl $\mathrm{N}_{\mathrm{G}}$ in Abb. 4-7 zeigt einen kontinuierlichen $\mathrm{Ab}-$ fall der Prüffrequenz über die gesamte Versuchslaufzeit um mehrere Hertz. Der Probekörper wurde bis zu einer Grenzschwingspielzahl $N_{G}$ von 500.000.000 geprüft. Die Vorspannkraft nimmt in diesem Fall nicht ab. Nach Versuchsende konnte keine Schädigung des Probekörpers nachgewiesen werden. Dieser Frequenzabfall wird auf einen Steifigkeitsverlust der Einspannung zurückgeführt. Beispielsweise sind dies Setzungseffekte, bei denen sich die Rändelung der Spannklötze langsam in die Probekörper einebnet.

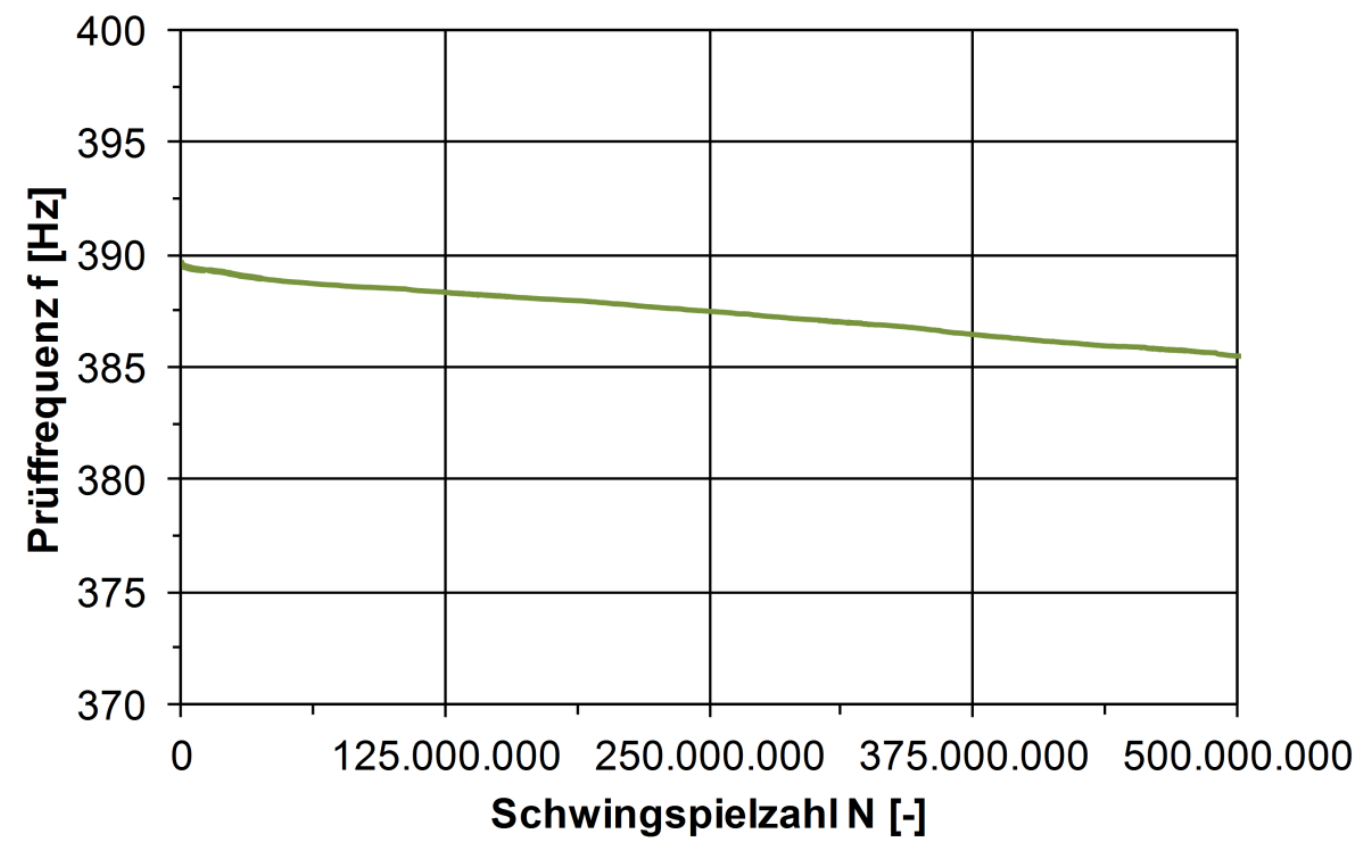

\section{Abb. 4-7: Verlauf der Prüffrequenz $f$ über die Schwingspielzahl $N$ bis zum Erreichen der Grenzschwingspielzahl $\mathbf{N}_{\mathrm{G}}$}

Die Prüffrequenz kann in Abhängigkeit der Steifigkeit des Systems von Versuch zu Versuch durch Streuungen der Probekörpersteifigkeit sowie der Einspannbedingungen variieren und sich während der Versuchslaufzeit durch Setzungserscheinungen sowie Schädigung verändern.

\subsubsection{Prüfkraftamplitude $F_{a}$}

Die Regelgröße stellt die Rahmendehnung dar. Bei Versuchsbeginn wird die Prüfkraftamplitude $F_{a}$ bis zum Erreichen des Sollwertes hochgefahren. Daraufhin wird die Prüfkraftamplitude bis zum Eintritt eines Abbruchkriteriums konstant gehalten. 
In Abb. 4-8 ist das Hochfahrverhalten von jeweils zwei Versuchen auf zwei unterschiedlichen Beanspruchungsniveaus der Prüfkraftamplitude $F_{a}$ über die Schwingspielzahl $\mathrm{N}$ dargestellt. Tab. 4-1 erläutert die im Folgenden verwendeten Bezeichnungen der Versuche.

Tab. 4-1: Bezeichnungen der Versuche

\begin{tabular}{|c|c|c|c|}
\hline Versuch & $\begin{array}{c}\text { Beanspruchungs- } \\
\text { niveau }\end{array}$ & $\begin{array}{c}\mathbf{F}_{\mathbf{a}} \\
{[\mathbf{k N}]}\end{array}$ & $\begin{array}{c}\mathbf{F}_{\mathbf{v}} \\
{[\mathbf{k N}]}\end{array}$ \\
\hline H1-1 & H1 & 14,0 & 17,1 \\
\hline H1-2 & H1 & 14,0 & 17,1 \\
\hline H2-1 & H2 & 11,2 & 13,7 \\
\hline H2-2 & H2 & 11,2 & 13,7 \\
\hline
\end{tabular}

Zu Beginn beschreiben alle Kurven in Abb. 4-8 einen nahezu identischen, linearen Verlauf bis ein Abknicken zum jeweiligen Prüfkraftniveau erfolgt. Innerhalb der ersten 5.000 Schwingspiele sind beide Prüfkraftniveaus erreicht. Erwartungsgemäß liegt eine Abhängigkeit der Anzahl der Lastwechsel bis zum Erreichen des Sollwertes der Prüfkraftamplitude von der Höhe des Prüfkraftniveaus vor. Das niedrigere Prüfkraftniveau wird früher erreicht als das höhere Prüfkraftniveau. Die Schwingspiele bis zum Erreichen des Sollwertes sind bei der Ermittlung der Anrissschwingspielzahl $\mathrm{N}_{\mathrm{A}}$ zu subtrahieren.

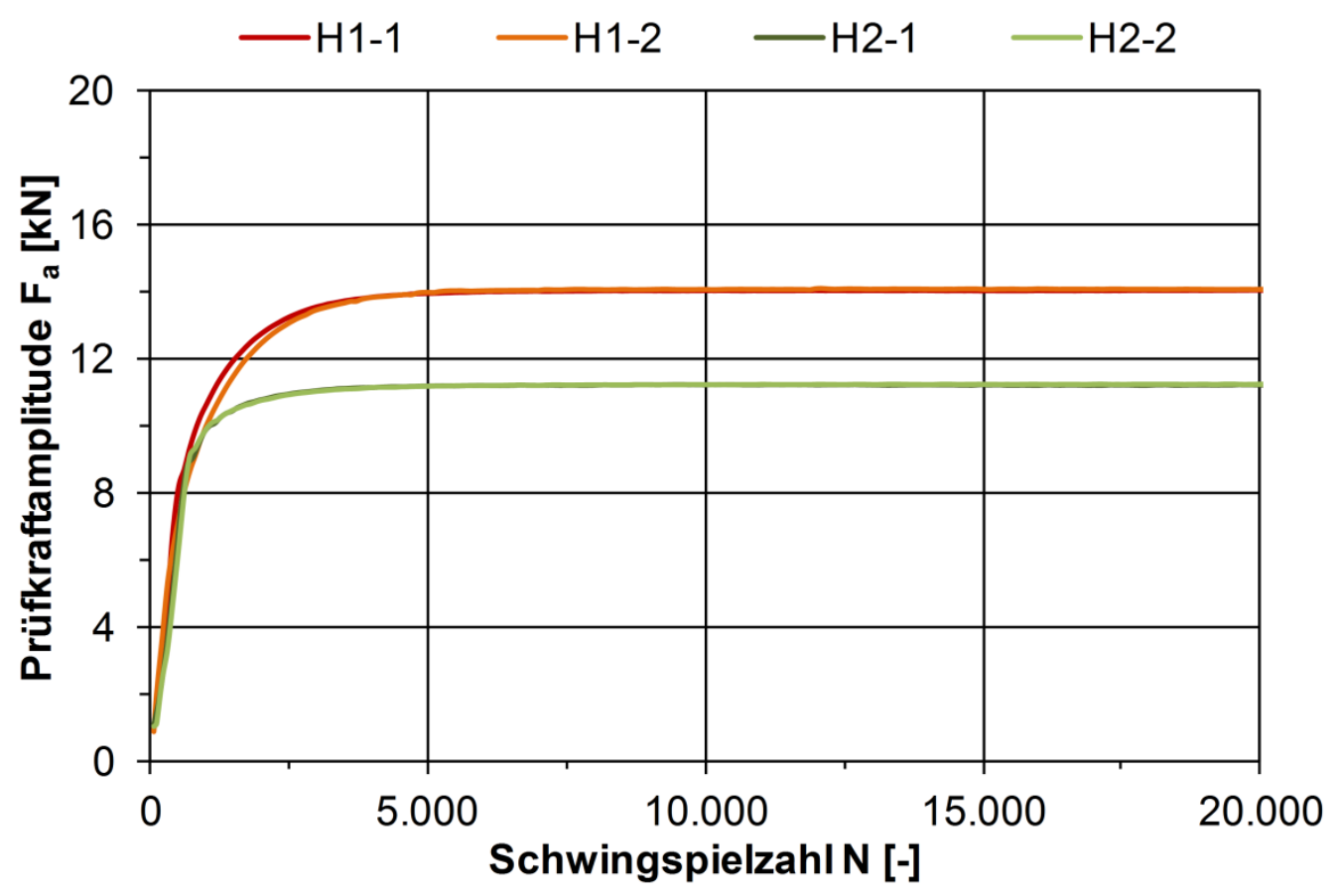

Abb. 4-8: Hochfahrverhalten der Prüfkraftamplitude $F_{a}$ auf den Beanspruchungsniveaus $\mathrm{H} 1$ und $\mathrm{H} 2$ 


\subsubsection{Vorspannkraft $F_{\mathrm{v}}$}

In Abb. 4-9 ist der charakteristische Verlauf der Vorspannkraft $F_{v}$ bei Probenversagen über die Schwingspielzahl $\mathrm{N}$ dargestellt. Die Vorspannkraft nimmt nach dem Vorspannvorgang (siehe Kapitel 4.2.1) zu Beginn des Versuchs erkennbar ab. Innerhalb der ersten 200.000 Lastwechsel ist ein Abfall der Vorspannkraft auf ein konstantes Niveau zu verzeichnen. Daraufhin bleibt die Vorspannkraft konstant. Da die Vorspannkraft nicht nachgeregelt werden kann, fällt das zu Versuchsbeginn vorliegende Spannungsverhältnis während des Versuchs geringfügig ab. Daher wird bei einem etwas geringeren Spannungsverhältnis geprüft als zu Versuchsbeginn eingestellt. Der Abfall der Vorspannkraft und damit des Spannungsverhältnisses sowie die Ausprägung des konstanten Niveaus sind abhängig von der Höhe des Beanspruchungsniveaus. Am Ende des Versuchs nimmt die Vorspannkraft nach Eintritt eines Anrisses aufgrund der fortschreitenden Schädigung der Probe bis zum Erreichen des Abbruchkriteriums stark ab.

Der beschriebene Abfall der Vorspannkraft zu Versuchsbeginn ist auf die Erwärmung des Probekörpers infolge innerer Reibung zurückzuführen. Durch die Erwärmung dehnt sich der Probekörper aus und die Vorspannkraft nimmt ab. Punktuelle Temperaturmessungen an ausgewählten Probekörpern während der Versuchslaufzeit bestätigen diesen Zusammenhang. Die Aufzeichnung der Temperaturen erfolgt mit Thermoelementen (TE), die im Abstand von $15 \mathrm{~mm}$ und $30 \mathrm{~mm}$ von der Schweißnahtmitte seitlich des Probekörpers in Blechmitte appliziert sind (siehe Abb. 4-9).

Die Temperaturdifferenz $\Delta T$ gegenüber der Temperatur bei Versuchsbeginn der Thermoelemente TE 1 und TE 2 sowie die Vorspannkraft $F_{v}$ sind in Abb. 4-9 über der Schwingspielzahl $\mathrm{N}$ aufgetragen. Der Verlauf der Temperaturdifferenz der beiden Thermoelemente ist annähernd parallel. Zu Versuchsbeginn steigt die Temperaturdifferenz linear an, flacht ab und nähert sich einem nahezu konstanten Niveau an. Es ist im vorliegenden Fall eine Temperaturerhöhung im Mittel von ca. 6,0 K zu verzeichnen. Bei Versuchsende steigt die Temperatur mit Versagen des Probekörpers erneut deutlich an. Beide Verläufe weisen einen Temperaturpeak mit maximalen Temperaturdifferenzen $\Delta T$ von $8,8 \mathrm{~K}$ und $7,2 \mathrm{~K}$ auf. Das näher zur Schweißnaht applizierte Thermoelement TE 1 weist erwartungsgemäß die höhere Maximaltemperatur auf. Nach Versuchsende kühlt der Probekörper beständig ab.

Der Zusammenhang zwischen der Erwärmung des Probekörpers und dem Abfall der Vorspannkraft ist deutlich zu erkennen. Der Abfall der Vorspannkraft ist abgeschlossen, wenn die Erwärmung der Probe ein annähernd konstantes Temperaturniveau erreicht hat. 


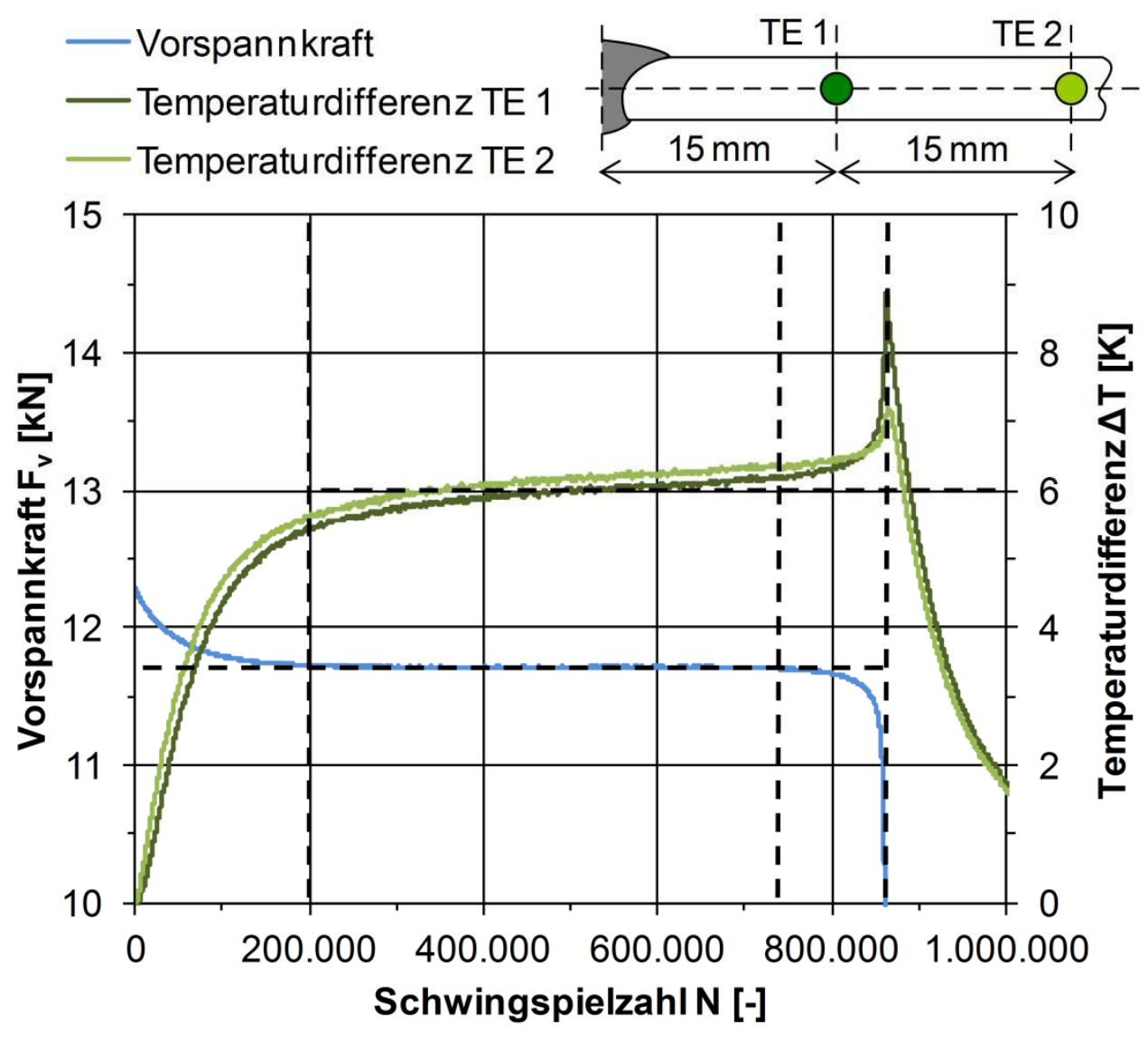

Abb. 4-9: Verlauf von Vorspannkraft $F_{\mathrm{v}}$ und Temperaturdifferenz $\Delta T$ gegenüber der Temperatur bei Versuchsbeginn während der Versuchslaufzeit

Die Ausprägung des konstanten Bereichs der Vorspannkraft $F_{v}$ ist von der erreichten Schwingspielzahl N abhängig wie Abb. 4-10 für zwei Versuche des Beanspruchungsniveaus $\mathrm{H} 2$ verdeutlicht. Versagt eine Probe bei geringer Schwingspielzahl, so ist die Erwärmung der Probe noch nicht vollständig abgeschlossen. Es stellt sich somit auch kein konstanter Bereich des Vorspannkraftverlaufs ein. Auf dem betrachteten Prüfkraftniveau ist dieser Vorgang nach 200.000 Schwingspielen weitgehend abgeschlossen und die Vorspannkraft hat einen konstanten Wert erreicht, auf dem sie bis zum Eintritt des Versagens nahezu verbleibt. Durch den deutlich früheren Eintritt des Versagens bei Versuch $\mathrm{H} 2-2$ sind auch die erreichten maximalen Temperaturdifferenzen geringer als bei Versuch $\mathrm{H} 2-1$. 

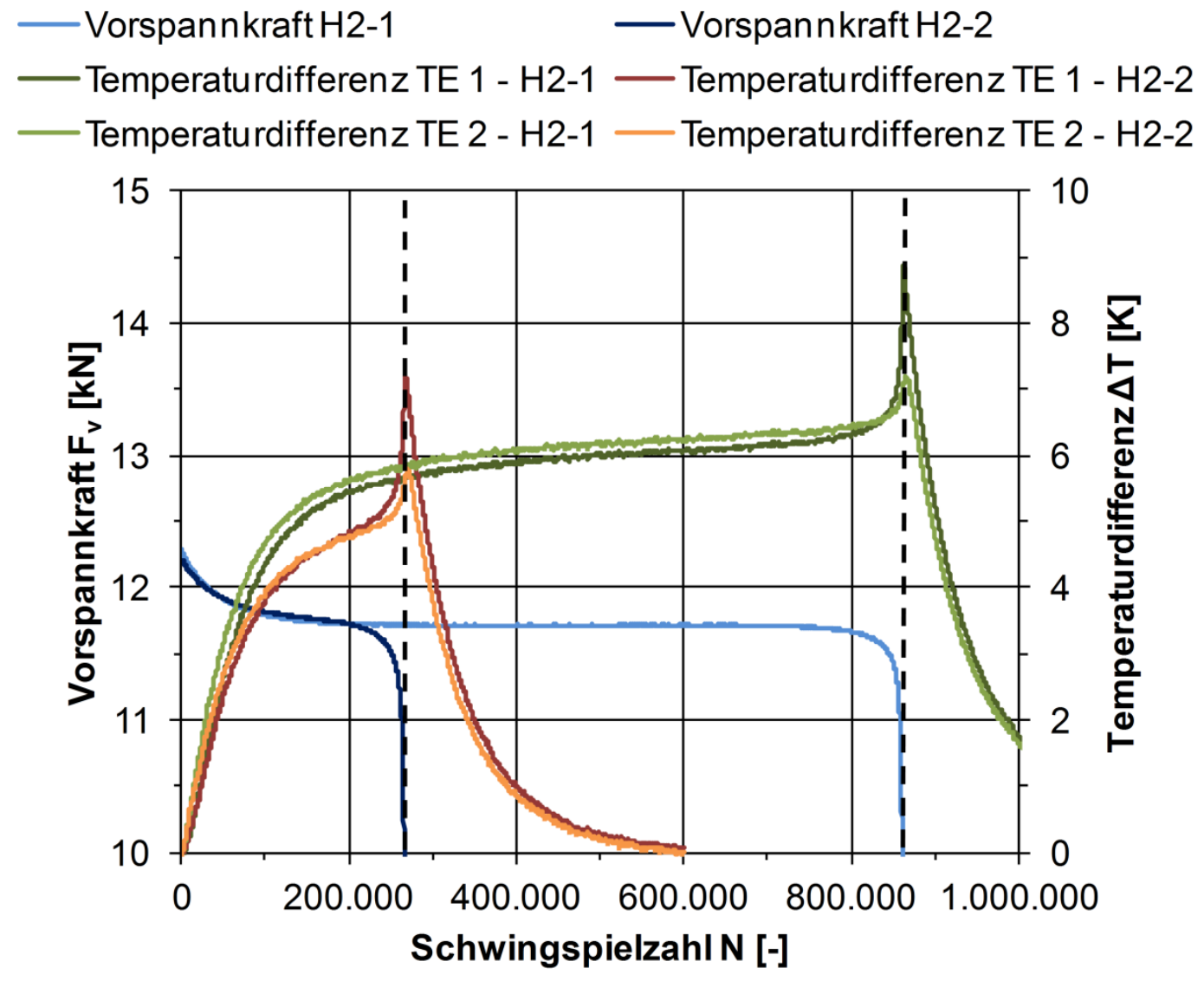

Abb. 4-10: Abhängigkeit des Verlaufes der Vorspannkraft $F_{v}$ von der erreichten Schwingspielzahl N für die Versuche von Beanspruchungsniveau H2

Die Erwärmung der Probekörper ist abhängig von der Höhe des Beanspruchungsniveaus. Mit steigender Amplitude nimmt die Erwärmung der Probekörper zu. Dies ist für die Versuche der Beanspruchungsniveaus $\mathrm{H} 1$ und $\mathrm{H} 2$ in Abb. 4-11 verdeutlicht. Es liegen geringe Abweichungen der Temperaturverläufe auf den Prüfkraftniveaus vor, die auf Abweichungen bei der Applikation der Thermoelemente sowie auf Raumtemperaturunterschiede zurückgeführt werden können. Der Einfluss des Zeitpunktes des Probenversagens wird deutlich. Die maximalen Temperaturdifferenzen weichen entsprechend voneinander ab.

Dieser Temperatureinfluss kann insgesamt zu einem steileren Verlauf der Ermüdungsfestigkeitskurven sowie einer Erhöhung der Streuung der Ergebnisse führen. Durch den Abfall des Spannungsverhältnisses ergeben sich günstigere Versuchsbedingungen, was zu höheren erreichten Schwingspielzahlen führen kann. Dies bedeutet insgesamt eine Verschiebung der Ermüdungsfestigkeitskurven hin zu höheren Schwingspielzahlen und einer Verbreiterung des Streubandes. 
—TE 1 - H2-1 —TE 1 - H2-2 —TE 1 - H1-1 - TE 1 - H1-2

$\longrightarrow$ TE 2 - H2-1 —TE 2 - H2-2 - TE 2 - H1-1 - TE 2 - H1-2

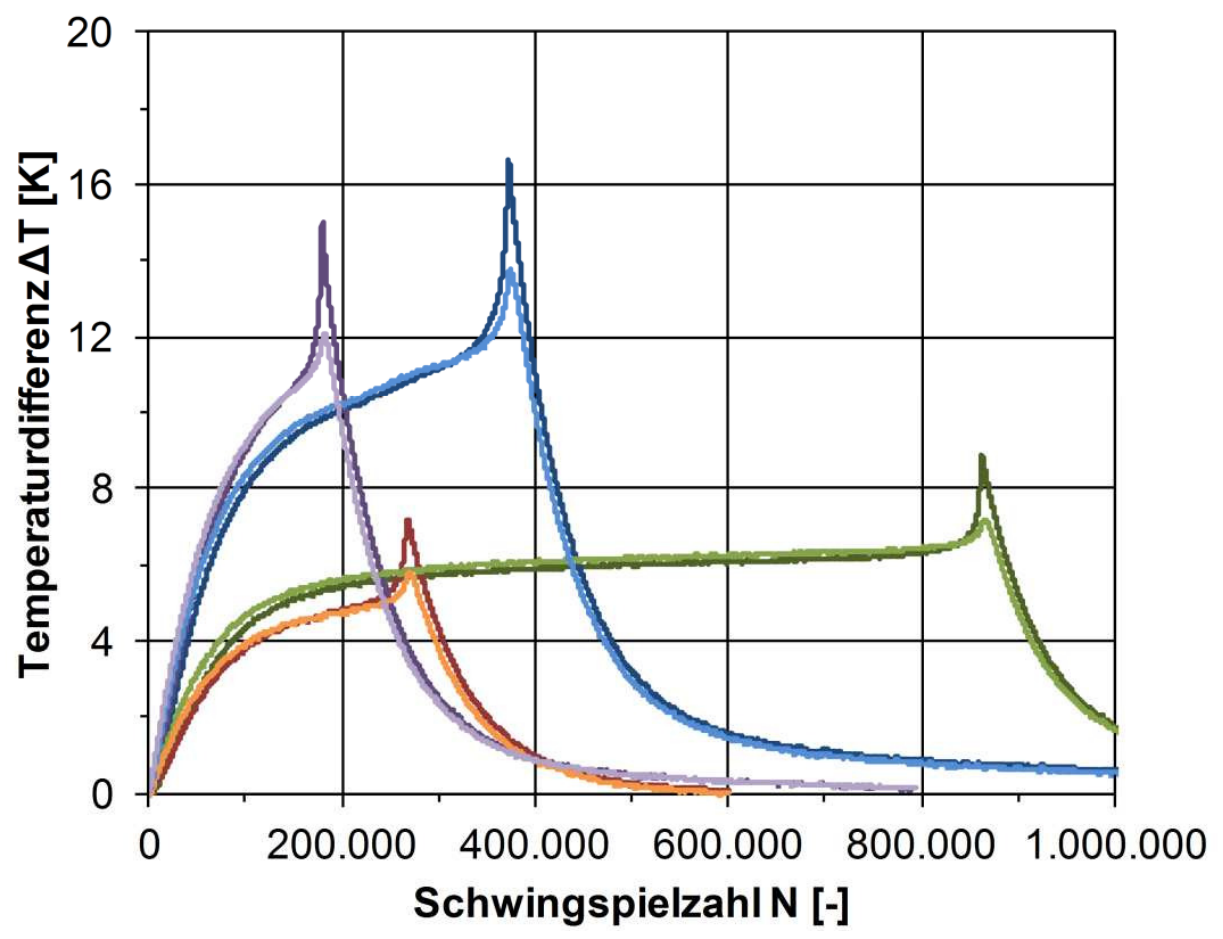

Abb. 4-11: Temperaturdifferenz $\Delta T$ der Versuche auf den Beanspruchungsniveaus $\mathrm{H} 1$ und $\mathrm{H} 2$

In Abb. 4-12 ist der Verlauf der Vorspannkraft $F_{v}$ über die Schwingspielzahl $N$ bis zum Erreichen der Grenzschwingspielzahl $\mathrm{N}_{\mathrm{G}}$ von 500.000.000 Lastwechseln dargestellt. Nach dem beschriebenen temperaturbedingten Vorspannkraftabfall zu Versuchsbeginn steigt die Vorspannkraft vereinzelt über die Versuchslaufzeit an. Es kommt zu Versuchsbeginn demnach zu einem geringfügigen Abfall und im Laufe des Versuchs zu einem geringfügigen Anstieg des ursprünglich vorgegebenen Spannungsverhältnisses. 


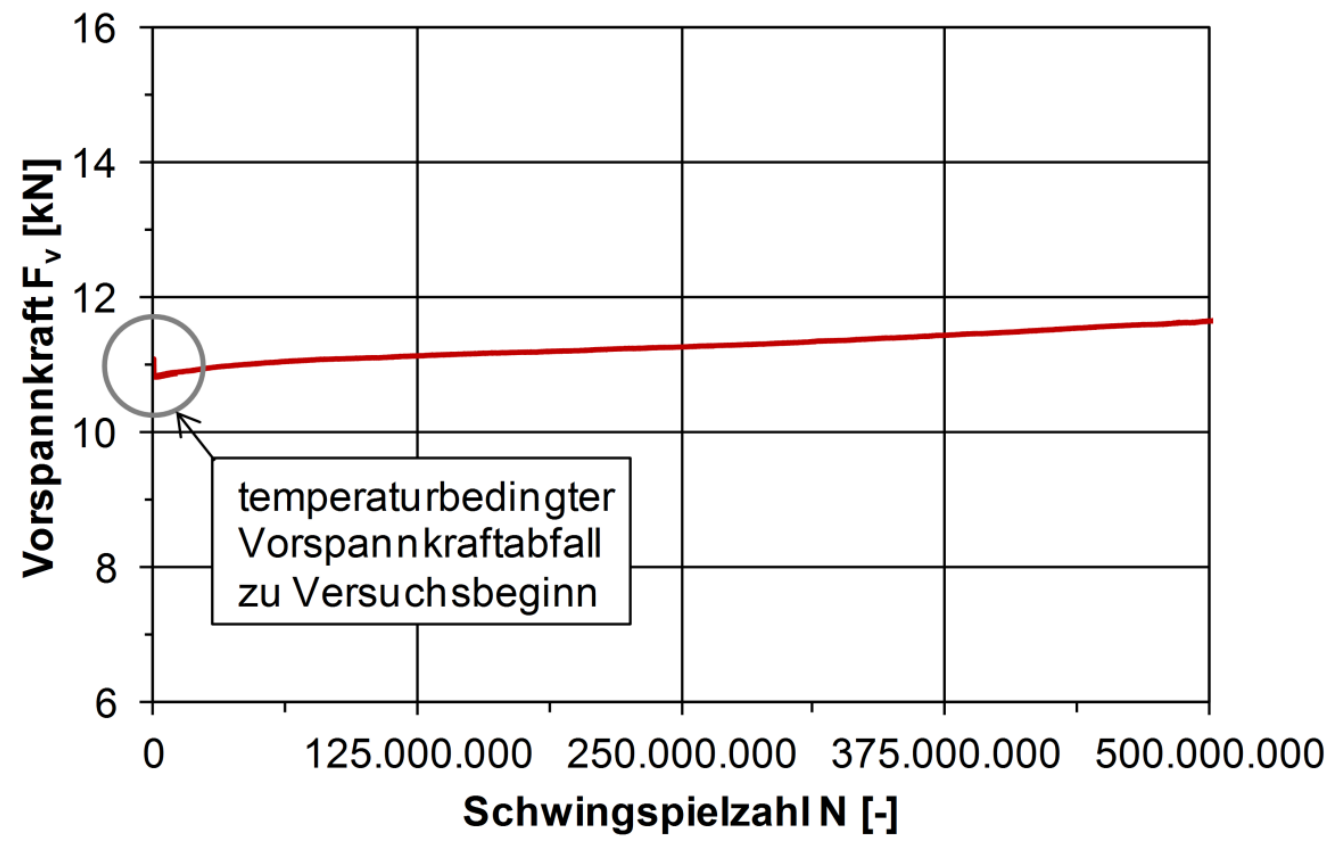

Abb. 4-12: Verlauf der Vorspannkraft $F_{\mathrm{v}}$ über die Schwingspielzahl $N$ bis zum Erreichen der Grenzschwingspielzahl

Der Verlauf von Vorspannkraft $\mathrm{F}_{\mathrm{v}}$ und Temperaturdifferenz $\Delta \mathrm{T}$ über die Versuchslaufzeit in Abb. 4-13 bei Versuchsbeginn (links) und bei Versuchsende (rechts) dargestellt. Nach Versuchsende bleibt die Vorspannkraft erhalten und fält nicht ab. Sie steigt um den zu Versuchsbeginn abgefallenen Wert an, was auf die Erwärmung des Probekörpers zurückzuführen ist. Da der Anstieg der Vorspannkraft sowohl auf dem Rahmen als auch auf der Probe gemessen wurde und nach Beendigung des Versuchs vorhanden ist, scheiden regelungstechnische Begründungen wie ein Anstieg der Regelspannung, der eine steigende Antriebsleistung und damit steigende Magnetkräfte zur Folge hätte, als Erklärungsmodell für den Vorspannkraftanstieg aus.

Eine andere Erklärung des dauerhaften Anstiegs der Vorspannkraft kann die durch Reibkorrosion verursachte Bildung einer Reaktionsschicht auf den Kontaktflächen der Spannklötze und des Rahmens sein. Im Bereich der Kontaktflächen der Spannklötze zum Prüfrahmen kommt es im Laufe der Versuchsführung zu Abnutzungserscheinungen, wie exemplarisch in Abb. 4-14 für den Zustand der Spannklötze nach einem Langzeitversuch dargestellt ist. 
- Temperaturdifferenz

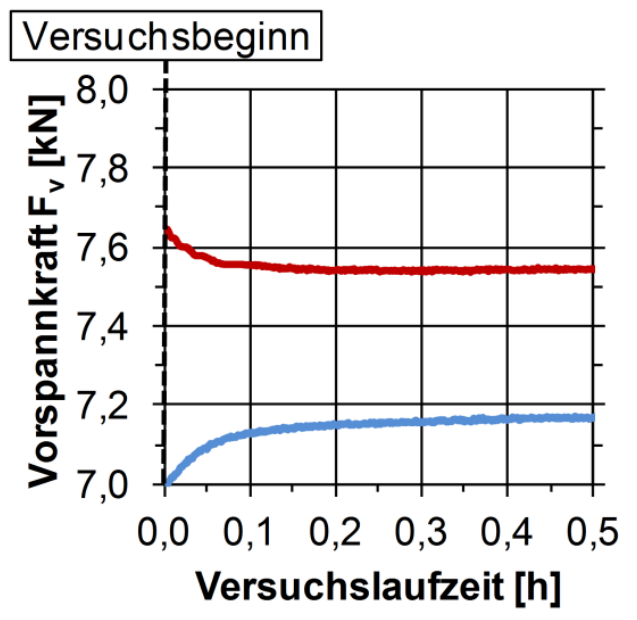

- Vorspannkraft

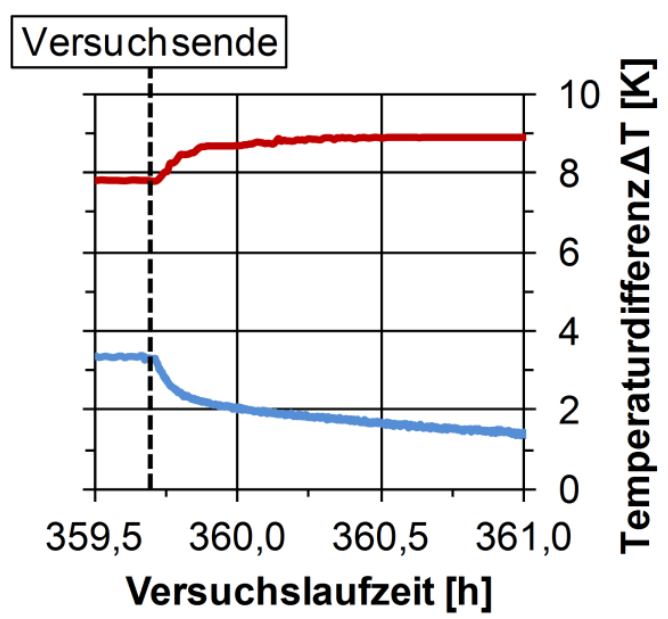

Abb. 4-13: Verlauf von Vorspannkraft $F_{\mathrm{v}}$ und Temperaturdifferenz $\Delta T$ über die Versuchslaufzeit bei Versuchsbeginn (links) und bei Versuchsende (rechts)

Bei mechanischer Beanspruchung von Werkstoffen kann es zu Korrosion kommen [Tostmann, 2001]. Liegt eine zyklische Bewegung von Werkstoffen gegeneinander vor, kann auch in geschmierten Kontaktflächen Reibkorrosion auftreten. Bei Reibkorrosion handelt es sich um eine Überlagerung von Verschleiß und Korrosion. Der Verschleiß wird durch Relativbewegungen von Werkstoffoberflächen hervorgerufen. Zersetzter Schmierstoff oxidiert in Verbindung mit dem Metallabrieb (Tribooxidation). Es kann zur Bildung einer festen Verbackung der Reaktionsprodukte durch Polymerisation kommen. Dieser Effekt konnte durch den Wechsel des Schmiermittels sowie die aufwendige Reinigung der Reibflächen minimiert werden.
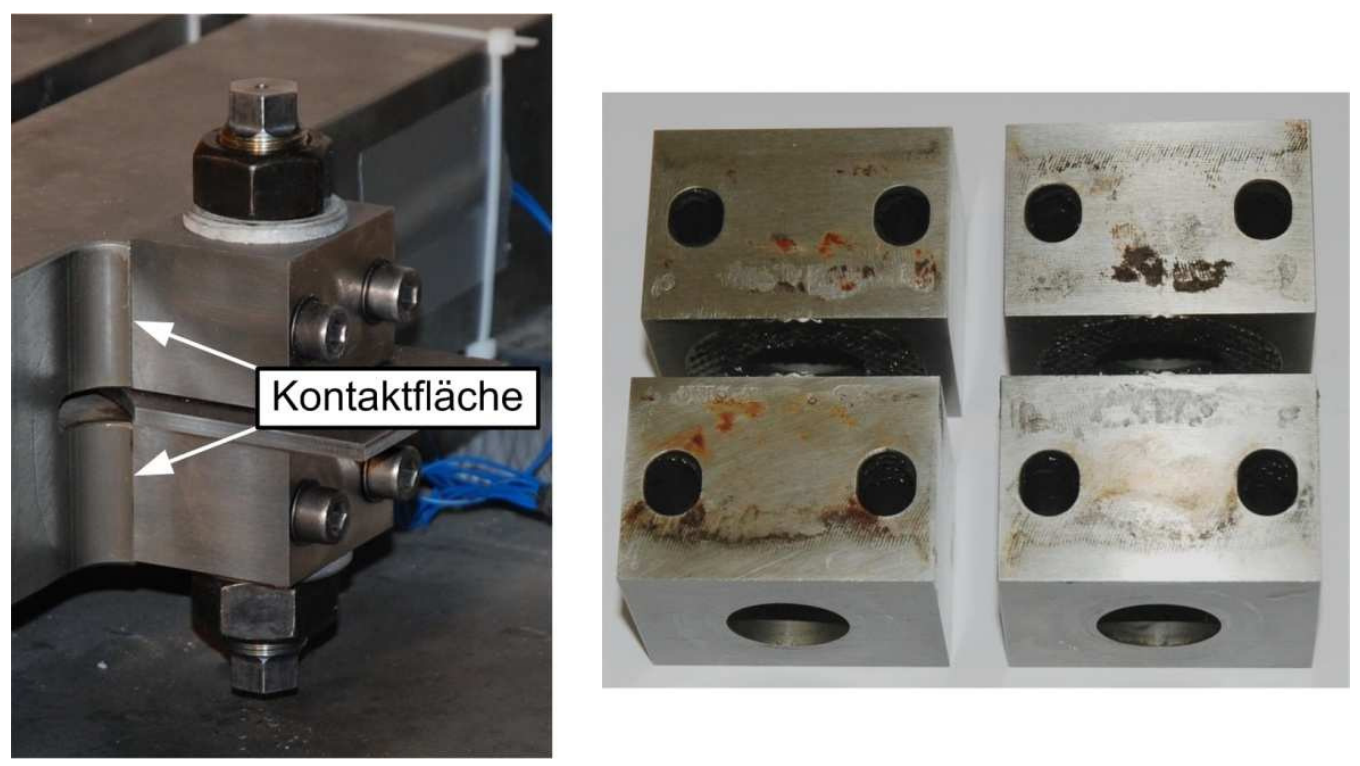

Abb. 4-14: Abnutzungserscheinungen auf den Kontaktflächen der Spannklötze zum Prüfrahmen nach Langzeitversuch 


\subsection{Kriterium zur Ermittlung der Anrissschwingspielzahl $\mathbf{N}_{\mathrm{A}}$}

Die Abbruchkriterien für den Versuch sind für den Fall ohne Probenversagen sowie für den Fall des Probenversagens definiert (vergleiche Kapitel 4.2.2). Im ersten Fall stellt das Abbruchkriterium das Erreichen einer festgelegten Grenzschwingspielzahl dar. Im zweiten Fall ist das Abbruchkriterium für den Abfall der Vorspannkraft auf ein definiertes Kraftniveau festgelegt. Hierbei weist die Probe bereits einen signifikanten Riss auf. Die ermittelte Abbruchschwingspielzahl stellt somit weder eine Anriss- noch eine Bruchschwingspielzahl dar. Die aufgestellte Proportionalitätsbeziehung zwischen Prüfkraft und zugehöriger Probendehnung verliert bei Eintritt eines Anrisses und dem damit verbundenen Risswachstum ihre Gültigkeit. Die Schwingspiele ab dem Anriss können exakter Weise nicht mehr gezählt werden, da die Prüfkraftamplitude nicht mehr korrekt aufgebracht werden kann. Die Anrissschwingspielzahl $\mathrm{N}_{\mathrm{A}}$ ist auszuwerten und es ist ein Kriterium für die Ermittlung der Anrissschwingspielzahl aus den zeitlich veränderlichen Messgrößen abzuleiten. Der Zeitpunkt des Anrisses wird indirekt ermittelt, wobei zunächst keine Information über die Anrissgröße vorliegt. Versuche zur Markierung der Rissfront auf der Bruchfläche in Kapitel 4.5 liefern eine qualitative Aussage zur vorliegenden Größe des Anrisses.

Zur Ableitung eines Kriteriums für den Anriss kann die Prüffrequenz sowie die Vorspannkraft herangezogen werden. Beide Größen fallen zu Versuchsende bei Probenversagen exponentiell ab. Da die Prüffrequenz nahezu konstant bleibt und die Vorspannkraft auf Grund unterschiedlicher Beanspruchungsniveaus variieren kann sowie einem anfänglichen Vorspannkraftabfall aus der Erwärmung unterworfen ist, wird die Prüffrequenz als Auswertungsgröße gewählt.

Der exponentielle Abfall der Prüffrequenz zu Versuchsende wird durch einen Steifigkeitsverlust der Probe hervorgerufen. Es ist von einem Anriss in der Probe auszugehen, der die beanspruchte Querschnittsfläche verringert und damit die Steifigkeit der Probe herabsetzt. Ebenso wird durch den wachsenden Anriss die Vorspannkraft abgebaut.

Der charakteristische Verlauf der Prüffrequenz über den Versuchsverlauf wurde in Kapitel 4.3.1 beschrieben. Nachdem die Prüfkraftamplitude erreicht wird, verläuft die Prüffrequenz nahezu konstant. Es zeigt sich lediglich ein leichter kontinuierlicher Abfall, bis es bei Eintritt des Anrisses zu einem deutlichen Abfall der Prüffrequenz kommt. Um die Anrissschwingspielzahl über den Verlauf der Prüffrequenz zu ermitteln, wird der Bereich des leichten kontinuierlichen Abfalls durch eine Regressionsgerade angenähert. Als Kriterium für die Ermittlung der Anrissschwingspielzahl wird die Abweichung der Prüffrequenz von der Regressionsgeraden eingeführt. Weicht der aktuelle Wert der Prüffrequenz um mehr als 0,01 \% vom zugehörigen Wert der Reg- 
ressionsgeraden ab, dann wird von einem Anriss in der Probe ausgegangen. Die Anrissschwingspielzahl wird an dieser Stelle abgelesen. Das Vorgehen wird in Abb. 4-15 veranschaulicht. Die prozentuale Abweichung wurde zu 0,01\% festgelegt, sodass übliche leichte Schwankungen im Frequenzverlauf nicht fälschlicherweise als Kriterium für den Anriss gewertet werden.

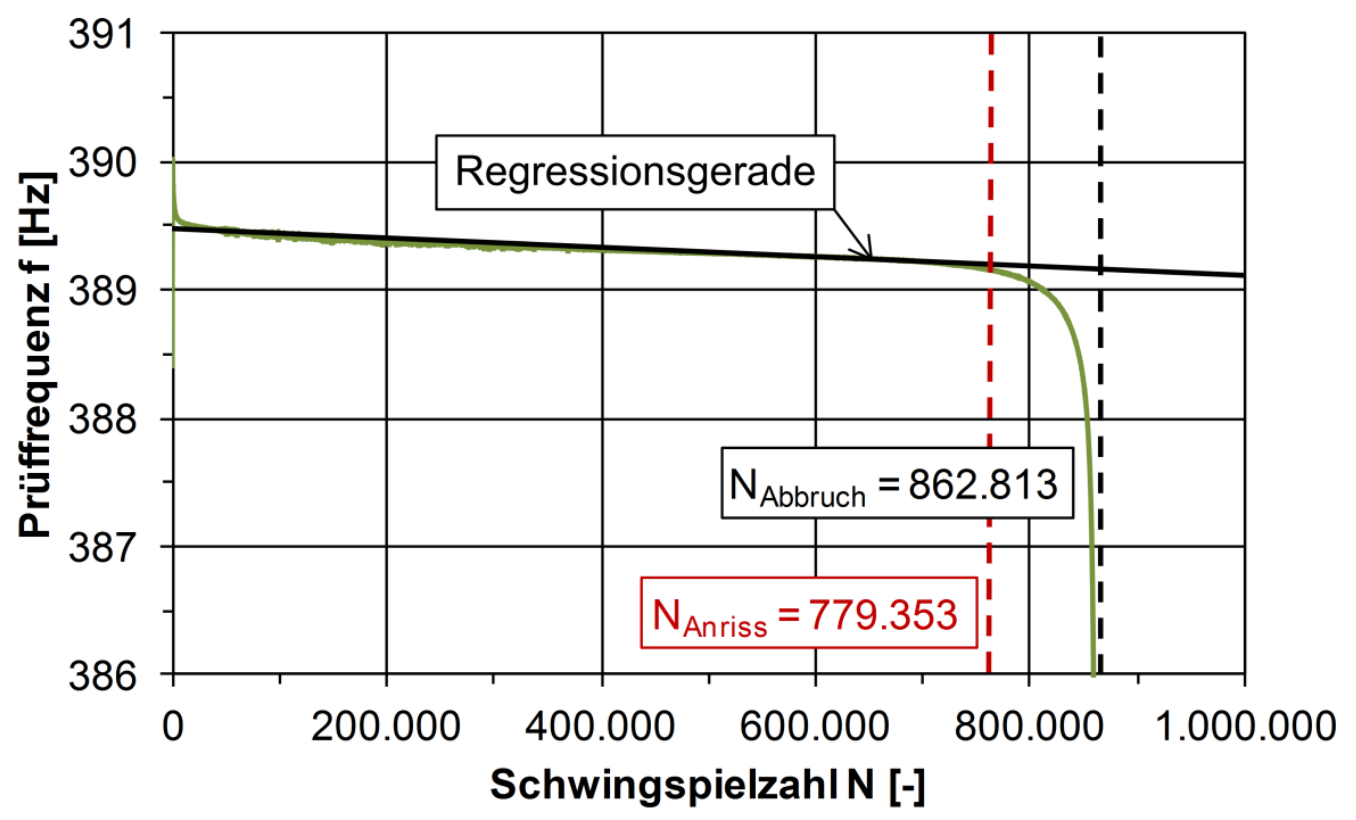

Abb. 4-15: Ermittlung der Anrissschwingspielzahl $\mathbf{N}_{A}$

\subsection{Untersuchung zur Größe des vorliegenden Anrisses}

Um Aufschluss über die Größe des vorliegenden Anrisses beim Erreichen des Kriteriums zur Ermittlung der Anrissschwingspielzahl in der vorgestellten Prüfvorrichtung zu gewinnen, werden Versuche zur Markierung der Rissfront auf der Bruchfläche anhand zufällig ausgewählter Proben durchgeführt. Das Ziel dieser Untersuchungen ist die qualitative Ermittlung der Anrissgröße beim Erreichen des in Kapitel 4.4 definierten Anrisskriteriums in der vorgestellten Prüfvorrichtung.

\subsubsection{Vorgehen}

Das Verfahren der Blocklastfolgen, das zur Untersuchung des Rissfortschrittsverhaltens eingesetzt wird, wird angewendet [Haydrych, 2001] [Dürr, 2007] [Weich, 2008]. Die Beanspruchung einer Probe mit konstanten Blöcken unterschiedlicher Beanspruchungsamplitude führt auf der Bruchfläche zu Rastlinien. Diese Rastlinien erscheinen farblich in unterschiedlichen Graustufen und formieren sich bogenartig auf der Bruchfläche. Diese Erscheinung wird durch Oxidationsvorgänge und unterschiedliche Rauheiten der Bruchfläche durch unterschiedliche Beanspruchungsamplituden hervorgerufen. Die Proben werden dabei mit konstanten Blöcken unterschiedlicher Beanspruchungsamplitude im Wechsel geprüft, wie Abb. 4-16 exemplarisch zeigt. 


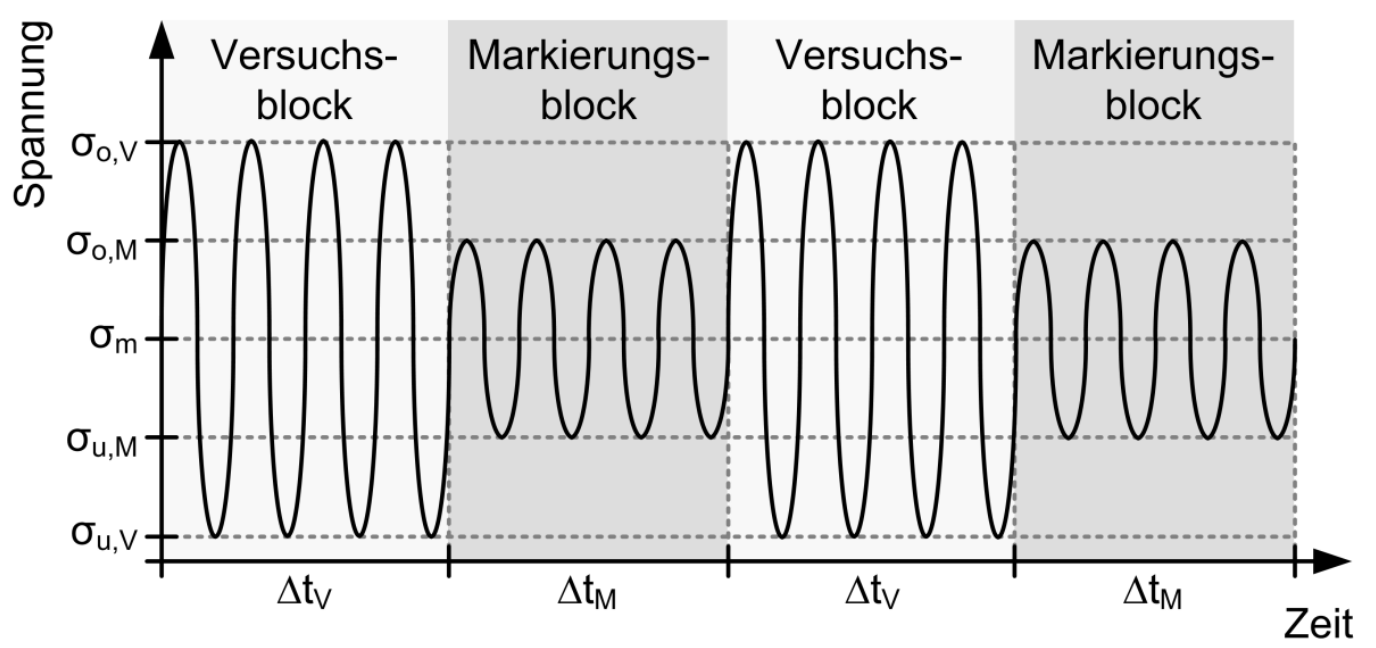

Abb. 4-16: Schematische Versuchsführung beim Verfahren der Blocklastfolgen

Hierbei wird zwischen dem Versuchs- und Markierungsblock unterschieden. Die Beanspruchungsschwingbreite des Markierungsblocks $\Delta \sigma_{M}$ wurde dabei halb so groß wie die des tatsächlichen Versuchsblocks $\Delta \sigma_{V}$ gewählt und führt zu einer dunkleren Färbung auf der Bruchfläche, wie in Abb. 4-17 zu erkennen ist.

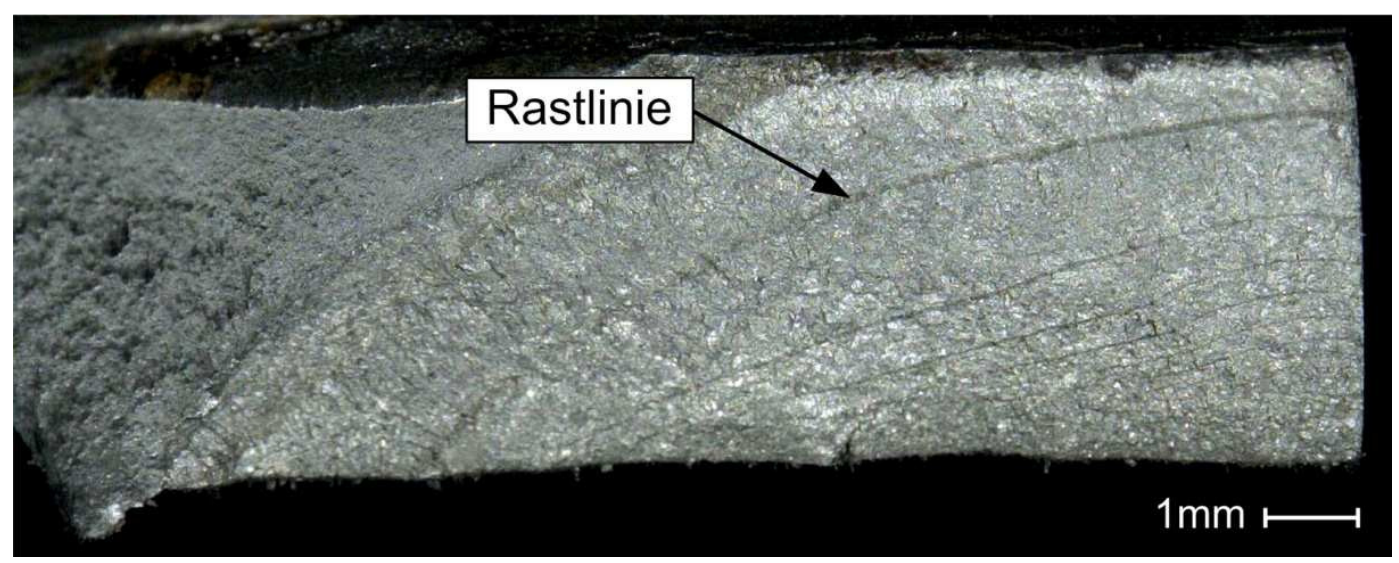

Abb. 4-17: Bruchfläche aus Versuchs- und Markierungsblock mit Rastlinien

Die aufgestellte Proportionalitätsbeziehung zwischen Prüfkraft und zugehöriger Probendehnung verliert bei Eintritt eines Anrisses und dem damit verbundenen Rissfortschritt seine Gültigkeit. Demnach kann die Prüfkraftamplitude nach dem Anriss nicht mehr exakt aufgebracht werden. Eine Untersuchung des Rissfortschrittsverhaltens ist hinfällig. Allerdings kann beim Erreichen des definierten Abbruchkriteriums für den Anriss eine qualitative Ermittlung der Größe des Anrisses vorgenommen werden.

In Abb. 4-18 sind die zeitlich veränderlichen Messgrößen (Kraftamplitude $F_{a}$, Vorspannkraft $F_{v}$ und Prüffrequenzf) während des Versuchs zur Markierung der Rissfront über die Schwingspielzahl N dargestellt. Es sind die einzelnen Versuchsund Markierungsblöcke mit ihren Auswirkungen auf die zeitlich veränderlichen Messgrößen zu erkennen. Die Dauer der Versuchs- und Markierungsblöcke betrug 
jeweils 30 Sekunden, was ungefähr 12.000 Lastwechseln entspricht. Hierin ist das Hoch- und Runterfahren bereits enthalten. Bei zwei Versuchen betrug die Dauer der Versuchsblöcke 40 Sekunden.

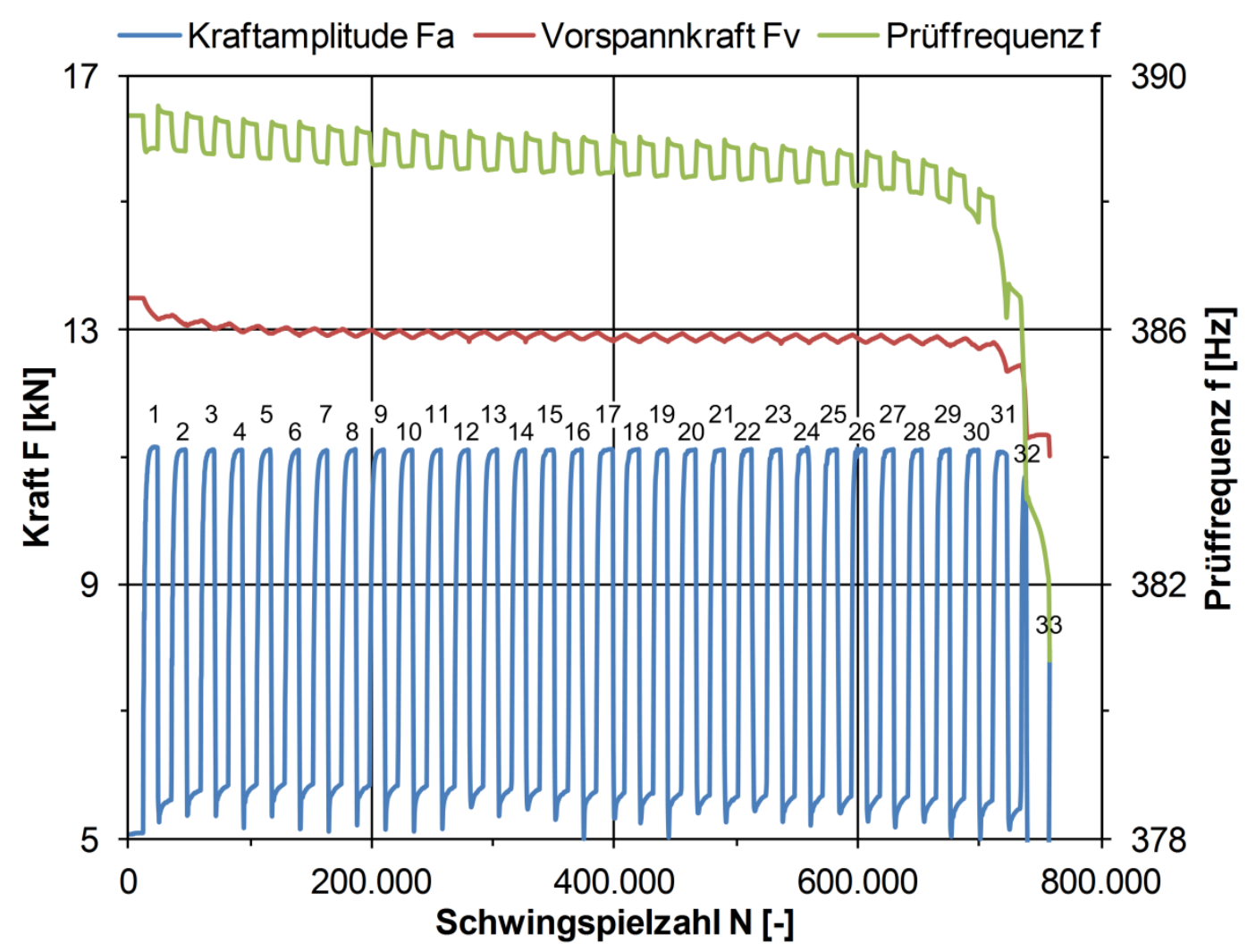

Abb. 4-18: Zeitlich veränderliche Messgrößen während des Versuchs R-1-1 zur Markierung der Rissfront auf der Bruchfläche

Bei Vernachlässigung der Markierungsblöcke zeigt Abb. 4-19 die Aneinanderreihung der Versuchsblöcke, wobei die Bereiche des Hoch- und Runterfahrens weiterhin enthalten sind. Um den kontinuierlichen Verlauf der zeitlich veränderlichen Messgrößen ohne den Einfluss des Hoch- und Runterfahrens zu erhalten, werden der jeweils letzte Wert der zeitlich veränderlichen Messgrößen der einzelnen Versuchsblöcke für die Auswertung des Kriteriums für den Anriss herangezogen. 


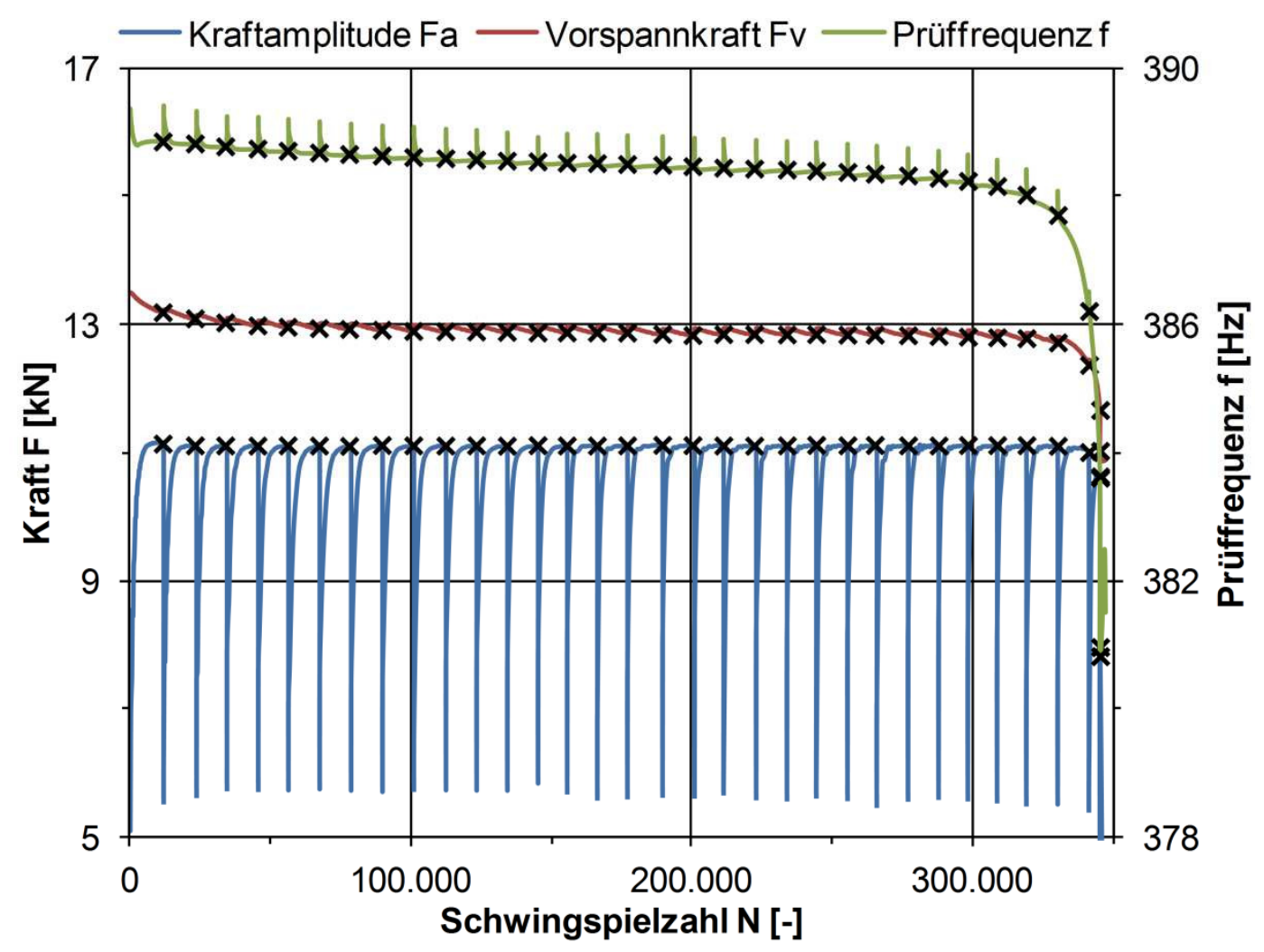

Abb. 4-19: Aufbereitung der zeitlich veränderlichen Messgrößen von Versuch R-1-1 zur Auswertung des Kriteriums für den Anriss

Die Ermittlung der Anrissschwingspielzahl $\mathrm{N}_{\mathrm{A}}$ erfolgt gemäß des in Kapitel 4.4 definierten Kriteriums und wird in Abb. 4-20 verdeutlicht. Eine Regressionsgerade nähert den leicht linear abfallenden Bereich des Verlaufs der Prüffrequenz an. Ein Anriss wird angenommen, wenn der aktuelle Wert der Prüffrequenz um mehr als 0,01\% vom zugehörigen Wert der Regressionsgeraden abweicht. Auf diese Weise kann der Versuchsblock, bei dem das Kriterium erreicht wird, ermittelt und auf der Bruchfläche durch Rückwärtszählen mit Hilfe der Rastlinien identifiziert werden. Die Vermessung und Dokumentation der Rastlinien erfolgte mit dem Lichtmikroskop (Keyence VHX1000D) bei 20-facher Vergrößerung. 


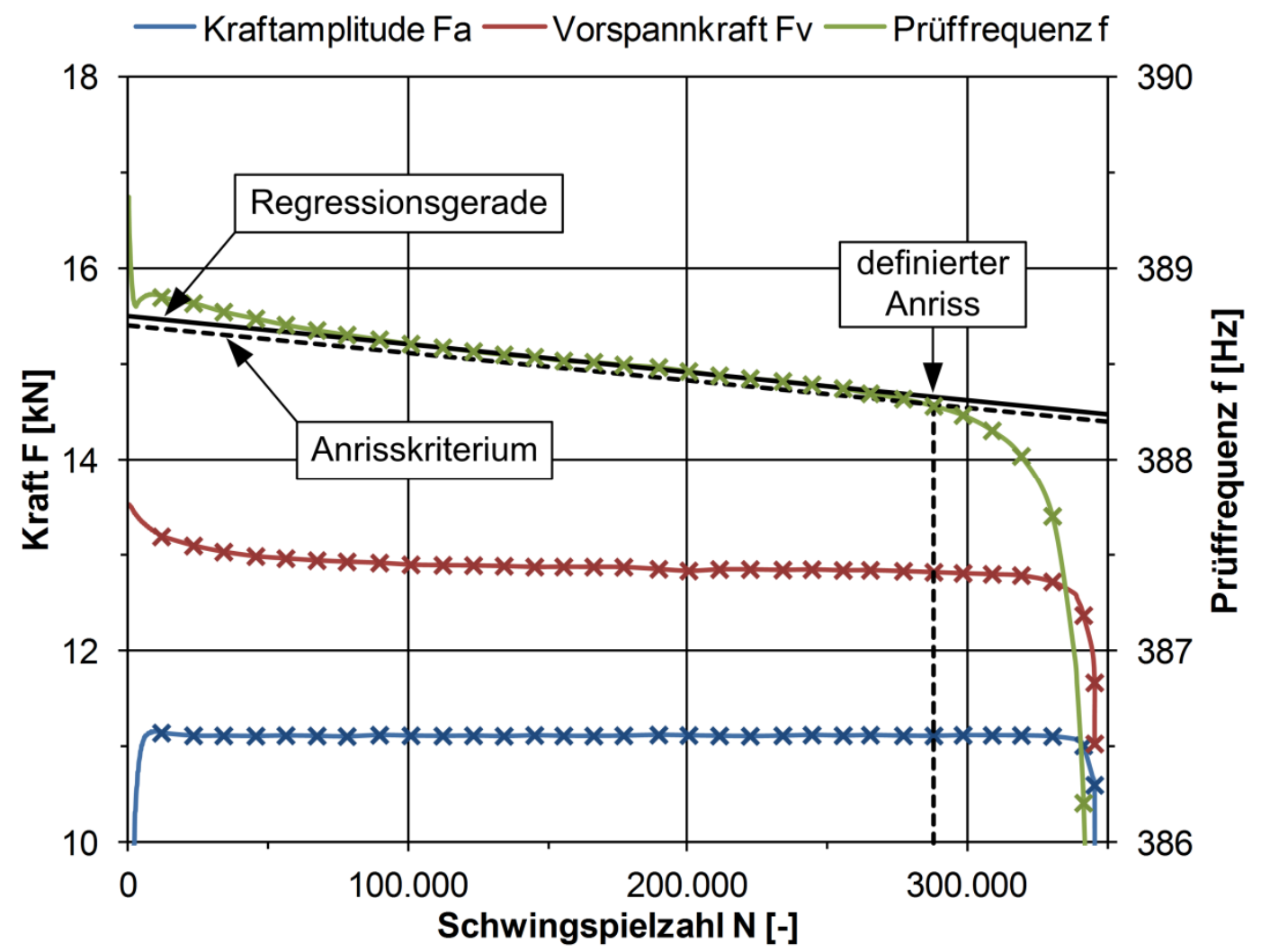

Abb. 4-20: Ermittlung der Anrissschwingspielzahl $\mathrm{N}_{\mathrm{A}}$ für Versuch R-1-1

Insgesamt wurden zur Markierung der Rissfront auf der Bruchfläche sechs Versuche im Zeitfestigkeitsbereich durchgeführt. Die Versuchsparameter sind in Tab. 4-2 zusammengefasst.

Tab. 4-2: Übersicht der Versuche zur Markierung der Rissfront auf der Bruchfläche

\begin{tabular}{|c|c|c|c|c|c|}
\hline $\begin{array}{c}\text { Versuchs- } \\
\text { reihe }\end{array}$ & $\begin{array}{c}\text { Spannungs- } \\
\text { verhältnis }\end{array}$ & $\begin{array}{c}\text { Prüf- } \\
\text { frequenz f }\end{array}$ & $\begin{array}{c}\mathbf{F}_{\mathbf{a}} \\
{[\mathbf{k N}]}\end{array}$ & $\begin{array}{c}\mathbf{F}_{\mathbf{v}} \\
{[\mathbf{k N}]}\end{array}$ & $\begin{array}{c}\text { Proben- } \\
\text { anzahl }\end{array}$ \\
\hline $\mathrm{R}-1$ & $\mathrm{R}=0,1$ & ca. $390 \mathrm{~Hz}$ & 11,1 & 13,6 & 3 \\
\hline $\mathrm{R}-2$ & $\mathrm{R}=0,5$ & ca. $390 \mathrm{~Hz}$ & 9,4 & 28,2 & 3 \\
\hline
\end{tabular}

\subsubsection{Ergebnisse}

Ein Überblick der Ergebnisse der Versuche zur Markierung der Rissfront auf der Bruchfläche ist in Tab. 4-3 gegeben. Es sind die Versuchsparameter sowie die Anzahl der geprüften Versuchsblöcke der einzelnen Versuche angegeben. Die Auswertung der aufbereiteten Verläufe liefert den Versuchsblock bzw. die Anrissschwingspielzahl beim Erreichen des Anrisskriteriums. Hieraus ergibt sich die Nummer der Rastlinie, welche die Ausbreitung des Anrisses beim Erreichen des Anrisskriteriums liefert, für das Rückwärtszählen. Die Verläufe der zeitlich veränderlichen Messgrößen 
während der einzelnen Versuche sowie die aufbereiteten Verläufe zur Auswertung des Kriteriums für den Anriss sind in Anhang $\mathrm{F}$ dargestellt.

Tab. 4-3: Überblick der Ergebnisse der Versuche zur Markierung der Rissfront

\begin{tabular}{|c|c|c|c|c|c|c|}
\hline $\begin{array}{c}\text { Ver- } \\
\text { such }\end{array}$ & $\begin{array}{c}\boldsymbol{\Delta \sigma} \\
{\left[\mathbf{N} / \mathbf{m m}^{2}\right]}\end{array}$ & $\begin{array}{c}\mathbf{R} \\
{[-]}\end{array}$ & $\begin{array}{c}\text { Versuchs- } \\
\text { blockanzahl }\end{array}$ & $\begin{array}{c}\text { Versuchsblock-Nr. } \\
\text { bei Anrisskriterium }\end{array}$ & $\begin{array}{c}\mathbf{N}_{\mathbf{A}} \\
{[-]}\end{array}$ & $\begin{array}{c}\text { Rastlinien- } \\
\text { Nr. }\end{array}$ \\
\hline R-1-1 & 221,9 & 0,1 & 33 & 27 & 288.165 & 7 \\
\hline R-1-2 & 221,9 & 0,1 & 39 & 33 & 370.644 & 7 \\
\hline R-1-3 & 221,9 & 0,1 & 25 & 21 & 314.252 & 5 \\
\hline R-2-1 & 187,5 & 0,5 & 52 & 44 & 482.599 & 7 \\
\hline R-2-2 & 187,5 & 0,5 & 65 & 55 & 603.662 & 11 \\
\hline R-2-3 & 187,5 & 0,5 & 29 & 20 & 242.868 & 9 \\
\hline
\end{tabular}

* Versuchsblockdauer 40 Sekunden (statt 30 Sekunden)

Die Anzahl der Versuchsblöcke bei der Prüfung mit einer Versuchsblockdauer von 40 Sekunden ist erwartungsgemäß geringer als bei der Prüfung mit einer Versuchsblockdauer von 30 Sekunden. Bei den Versuchen der Versuchsreihe R-2 sind die erreichten Versuchsblockanzahlen höher als bei Versuchsreihe R-1. Die Nummern der Rastlinien beim Erreichen des Anrisskriteriums liegen zwischen 5 und 11.

Die Rastlinien auf den Bruchflächen wurden mit Hilfe dem Lichtmikroskops ermittelt. Hierbei wurden jeweils beide Bruchflächen der jeweiligen Probe vermessen. Es wurden Tiefe und Breite der erkennbaren Rastlinien vermessen. Als Tiefe ist die maximale Ausdehnung der Rastlinie in Probendicke und als Breite die maximale Ausdehnung der Rastlinie in Probenbreite definiert (siehe Abb. 4-21). Die Übersichtsaufnahmen der Bruchflächen sowie die entsprechenden Ausschnitte mit Kennzeichnung der vermessenen Rastlinien sind in Anhang $F$ dargestellt. Ebenso sind die ermittelten Tiefen und Breiten der einzelnen Rastlinien (sofern vermessbar) tabellarisch aufgeführt.

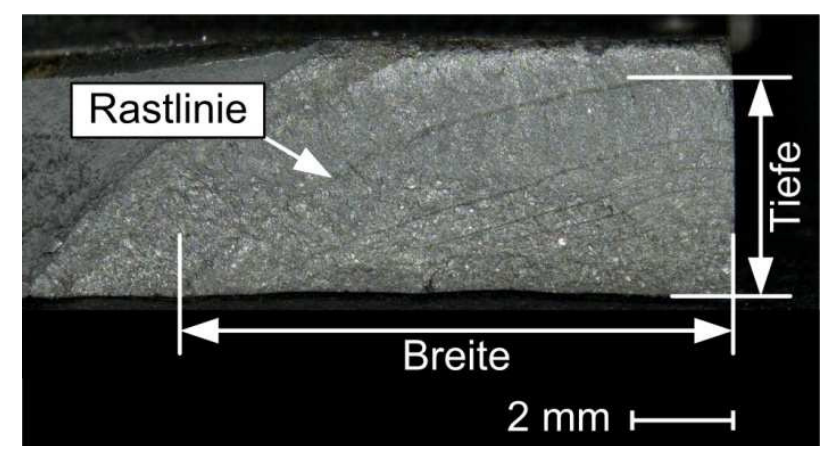

Abb. 4-21: Definition der Tiefe und Breite bei der Vermessung der Rastlinien 
Die Ergebnisse der Vermessung der Rastlinien sind in Tab. 4-4 zusammengefasst. Lediglich bei Versuch R-1-1 war die Anrisstiefe $t_{A}$ sichtbar und demnach auswertbar. In diesem Fall wurde eine Anrisstiefe $t_{A}$ von $1,5 \mathrm{~mm}$ gemessen. Aus diesem Grund wird für die Versuchsreihen die Rastlinien-Nr. beim Erreichen des Anrisskriteriums und die auf der Bruchfläche maximal sichtbare Rastlinien-Nr. sowie die zugehörige minimal messbare Risstiefe $t_{\text {min }}$ angegeben. Da die Rissbreiten lediglich in zwei Fällen und dort nur begrenzt vermessbar waren, sind diese hier nicht aufgeführt.

Tab. 4-4: Ergebnisse der Vermessung der Rastlinien

\begin{tabular}{|c|c|c|c|}
\hline $\begin{array}{c}\text { Ver- } \\
\text { such }\end{array}$ & $\begin{array}{c}\text { Rastlinien-Nr. } \\
\text { bei Anrisskriterium }\end{array}$ & $\begin{array}{c}\text { maximal sichtbare } \\
\text { Rastlinien-Nr. }\end{array}$ & $\begin{array}{c}\text { minimale Risstiefe } \mathbf{t}_{\text {min }} \\
\text { [mm] }\end{array}$ \\
\hline R-1-1 & 7 & 7 & $1,5^{* *}$ \\
\hline R-1-2 & 7 & 4 & 1,2 \\
\hline R-1-3* & 5 & 3 & 1,2 \\
\hline R-2-1 & 7 & 4 & 1,2 \\
\hline R-2-2 & 11 & 8 & 2,1 \\
\hline R-2-3* & 9 & 5 & 1,3 \\
\hline
\end{tabular}

*Versuchsblockdauer 40 Sekunden (statt 30 Sekunden) ** entspricht der Anrisstiefe $t_{A}$

Auf den Bruchflächen der einzelnen Proben sind zum Teil nur wenige Rastlinien sichtbar. Die Anzahl der erkennbaren Rastlinien liegt zwischen 3 und 8 Rastlinien. Eine Vermessung der Breite der Rastlinien war lediglich bei den Versuchen R-1-1 und R-2-2 bei den ersten Rastlinien (kurz vor dem Versagen) möglich. Beide Versuche weisen einen viertelelliptischen Eckanriss auf. Es zeigte sich in diesen beiden Fällen deutlich, dass die Anzahl der sichtbaren Rastlinien im Vergleich zu den anderen Versuchen deutlich höher ist und die Rastlinien auf den Bruchflächen deutlicher zu erkennen sind.

Im Mittel über alle 6 Versuche konnte eine minimale erkennbare Risstiefe $t_{\text {min }}$ von $1,4 \mathrm{~mm}$ gemessen werden. Allerdings ist hierbei das Anrisskriterium mit Ausnahme von Versuch R-1-1 bereits erreicht, sodass von Risstiefen kleiner diesem Wert beim Erreichen des Anrisskriteriums auszugehen ist. Es zeigt sich eine Abhängigkeit der minimal erkennbaren Risstiefe $t_{\min }$ von der Rissform. Im Fall der viertelelliptischen Eckanrisse der Versuche R-1-1 und R-2-2 wurden größere minimal erkennbare Risstiefen $t_{\min }$ von $1,5 \mathrm{~mm}$ und $2,1 \mathrm{~mm}$ gemessen als bei den übrigen Versuchen. Die übrigen Versuche weisen im Mittel eine minimal erkennbare Risstiefe $t_{\text {min }}$ von 1,2 mm auf, wobei sich die Rissfronten im Vergleich zu den viertelelliptischen Eckanrissen über einen Großteil der Probenbreite erstrecken. Allerdings sind die minimal erkenn- 
baren Risstiefen aufgrund der verschiedenen vorliegenden Rissformen und der unterschiedlichen Anzahl sichtbarer Rastlinien nur bedingt vergleichbar.

Die Versuche zur Markierung der Rissfront auf der Bruchfläche zeigen, dass bei viertelelliptischen Eckanrissen Anrisstiefen $t_{A}$ im Bereich von 1,5 mm erwartet werden. In den übrigen Fällen ist von Anrisstiefen $t_{A}$ kleiner als $1,2 \mathrm{~mm}$ auszugehen.

\subsection{Kalibrierung}

Zwischen Prüfkraft und zugehöriger Probendehnung besteht unter der Voraussetzung linearelastischen Werkstoffverhaltens eine Proportionalitätsbeziehung. Diese kann durch Einbau der Kalibrierproben in eine Universalprüfmaschine und Aufzeichnung von Prüfkraft und zugehörigem Spannungssignal der Probendehnung abgeleitet werden. Bei Kalibrierproben handelt es sich um mit Dehnungsmessstreifen versehene Probekörper (siehe Abb. 4-22). Im Nennspannungsquerschnitt auf Vorder- und Rückseite jeder Kalibrierprobe ist im Abstand von $25 \mathrm{~mm}$ zur Schweißnahtmitte jeweils ein Dehnungsmessstreifen $\left(0^{\circ} / 90^{\circ} \mathrm{T}\right.$-Rosette) appliziert. Die Dehnungsmessstreifen sind zu einer Vollbrücke verschaltet.

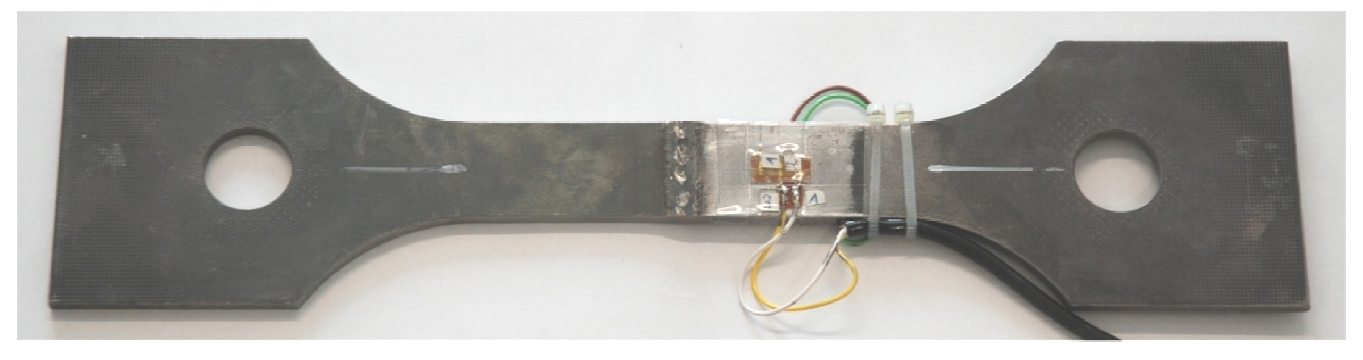

Abb. 4-22: Kalibrierprobe

Der Quotient aus Prüfkraft $F$ und zugehörigem Spannungssignal der Probendehnung $U_{P}$ liefert den Übertragungsfaktor kf der jeweiligen Kalibrierprobe, wie Gleichung (4-1) zeigt. Dieser wird für alle Kalibrierproben aus drei Durchläufen ermittelt, um auf die Prüfkraft des Probekörpers zu schließen. Die Ergebnisse sind in Anhang $\mathrm{G}$ dargestellt.

$$
k f[k N / V]=\frac{F[k N]}{U_{P}[V]}
$$

Zwischen der Prüfkraft des Probekörpers und dem zugehörigen Spannungssignal der Rahmendehnung besteht eine Proportionalitätsbeziehung. Durch Einbau der Kalibrierproben in die Prüfvorrichtung und Aufzeichnung der Spannungssignale der Proben- und Rahmendehnung in der Prüfvorrichtung wird diese abgeleitet. Die Prüfkraft des Probekörpers F wird hierbei aus dem Produkt von Übertragungsfaktor kf und Spannungssignal der Probendehnung $U_{P}$ ermittelt. Der Quotient aus Prüfkraft 
des Probekörpers und Spannungssignal der Rahmendehnung liefert die statischen und dynamischen Kalibrierfaktoren.

\subsubsection{Statische Kalibrierung}

Zur Ermittlung des statischen Kalibrierfaktors kfs wurden alle Kalibrierproben in die Prüfvorrichtung eingebaut und in drei Durchläufen auf sechs unterschiedliche Niveaus vorgespannt. Der Quotient aus Prüfkraft und Spannungssignal der Rahmendehnung liefert den statischen Kalibrierfaktor, wie die Gleichungen (4-2) und (4-3) zeigen.

$$
\begin{aligned}
& F_{m}=U_{m, P}[V] \cdot k f\left[\frac{k N}{V}\right]=k f s\left[\frac{k N}{V}\right] \cdot U_{m, R}[V] \\
& k f s\left[\frac{k N}{V}\right]=\frac{U_{m, P}[V] \cdot k f\left[\frac{k N}{V}\right]}{U_{m, R}[V]}
\end{aligned}
$$

In Abb. 4-23 ist der Zusammenhang dargestellt. Der statische Kalibrierfaktor wurde in einer Regressionsanalyse mit der Methode der kleinsten Fehlerquadrate zu 4,552 ermittelt.

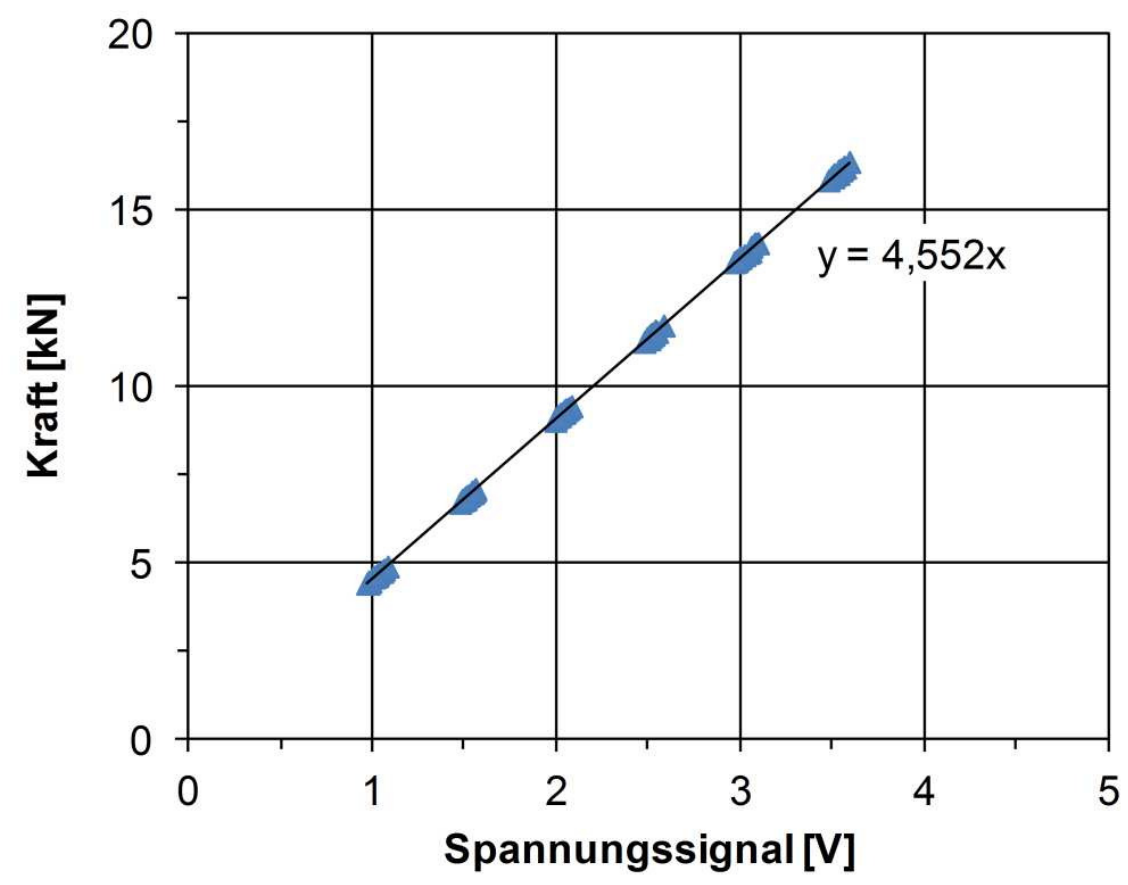

Abb. 4-23: Ermittlung des statischen Kalibrierfaktor kfs

Die Streuung der Messwerte um die ermittelte Funktion kann durch den Standardschätzfehler beschrieben werden. Dieser ist definiert als die Streuung der Regressionsresiduen und berechnet sich aus der Quadratwurzel der Residualvarianz. Der Standardschätzfehler, der die Streuung der Regressionsresiduen darstellt und somit Auskunft über die Streuung der Messwerte um die durch die Regression ermittelte 
Funktion gibt, wurde zu 0,028 ermittelt. Der Determinationskoeffizient $r^{2}$ als Kenngröße für den relativen Anteil der Varianz der abhängigen Größe, der durch die unabhängige Größe erklärt werden kann, ist als Quotient aus Regressionsvarianz und Gesamtvarianz definiert und ergibt sich zu 0,999.

\subsubsection{Dynamische Kalibrierung}

Analog zum statischen Kalibrierfaktor kfs ergibt sich der dynamische Kalibrierfaktor kfd. Das Spannungssignal der Rahmendehnung stellt die Regelgröße dar, die einer entsprechenden Probendehnung bzw. Prüfkraft des Probekörpers entspricht. Der Quotient aus Prüfkraftamplitude des Probekörpers und Spannungssignal der Rahmendehnung ergibt den dynamischen Kalibrierfaktor. Die Prüfkraft des Probekörpers ergibt sich hierbei ebenfalls aus dem Produkt von Spannungssignal der Probendehnung und Übertragungsfaktor kf.

\section{Frequenzabhängigkeit des dynamischen Kalibrierfaktors}

Der dynamische Kalibrierfaktor kfd bildet das Verhältnis von Probendehnung zu Rahmendehnung bei gleicher Prüfkraftkraftamplitude ab. Wenn sich die Steifigkeit des Systems (und damit die Prüffrequenz) verändert, dann ändert sich bei gleicher Prüfkraftamplitude das Verhältnis der Dehnungen, was einer Änderung und damit einer Frequenzabhängigkeit des dynamischen Kalibrierfaktors entspricht, wie die Gleichungen (4-4) und (4-5) zeigen.

$$
\begin{aligned}
& F_{a}(f)[k N]=U_{a, P}(f)[V] \cdot k f\left[\frac{k N}{V}\right]=k f d(f)\left[\frac{k N}{V}\right] \cdot U_{a, R}[V] \\
& k f d(f)\left[\frac{k N}{V}\right]=\frac{U_{a, P}(f)[V] \cdot k f\left[\frac{k N}{V}\right]}{U_{a, R}[V]}=\frac{F_{a}(f)[k N]}{U_{a, R}[V]}
\end{aligned}
$$

Bei Regelung einer konstanten Rahmendehnung nehmen Probendehnung bzw. Prüfkraftamplitude mit abfallender Prüffrequenz ab. In Abb. 4-24 ist dieser Zusammenhang exemplarisch für eine Probe mit einer erreichten Grenzschwingspielzahl $\mathrm{N}_{\mathrm{G}}$ von 500.000.000 Lastwechseln dargestellt. Während der Versuchslaufzeit von 15 Tagen ist die Prüffrequenz um mehrere Hertz abgefallen. Die Prüfkraftamplitude ist ebenfalls abgefallen. Bei Auftragung der Prüfkraftamplitude der Probe über der Prüffrequenz zeigt sich der abgebildete Zusammenhang, der die Frequenzabhängigkeit des dynamischen Kalibrierfaktors verdeutlicht.

Im Bereich kurzer Versuchslaufzeiten findet ein Abfall der Prüffrequenz um mehrere Hertz, verbunden mit einer deutlichen Reduktion der Vorspannkraft, innerhalb geringer Lastwechselzahlen statt. Es liegt ein signifikanter Riss in der Probe vor. Im Langzeitbetrieb zeigt sich ein kontinuierlicher Abfall der Prüffrequenz, wobei die Vorspannkraft nicht abfällt. Nach Erreichen der Grenzschwingspielzahl konnte nachweis- 
lich keine Schädigung des Probekörpers festgestellt werden, sodass von einem Steifigkeitsverlust der Einspannung ausgegangen wird.

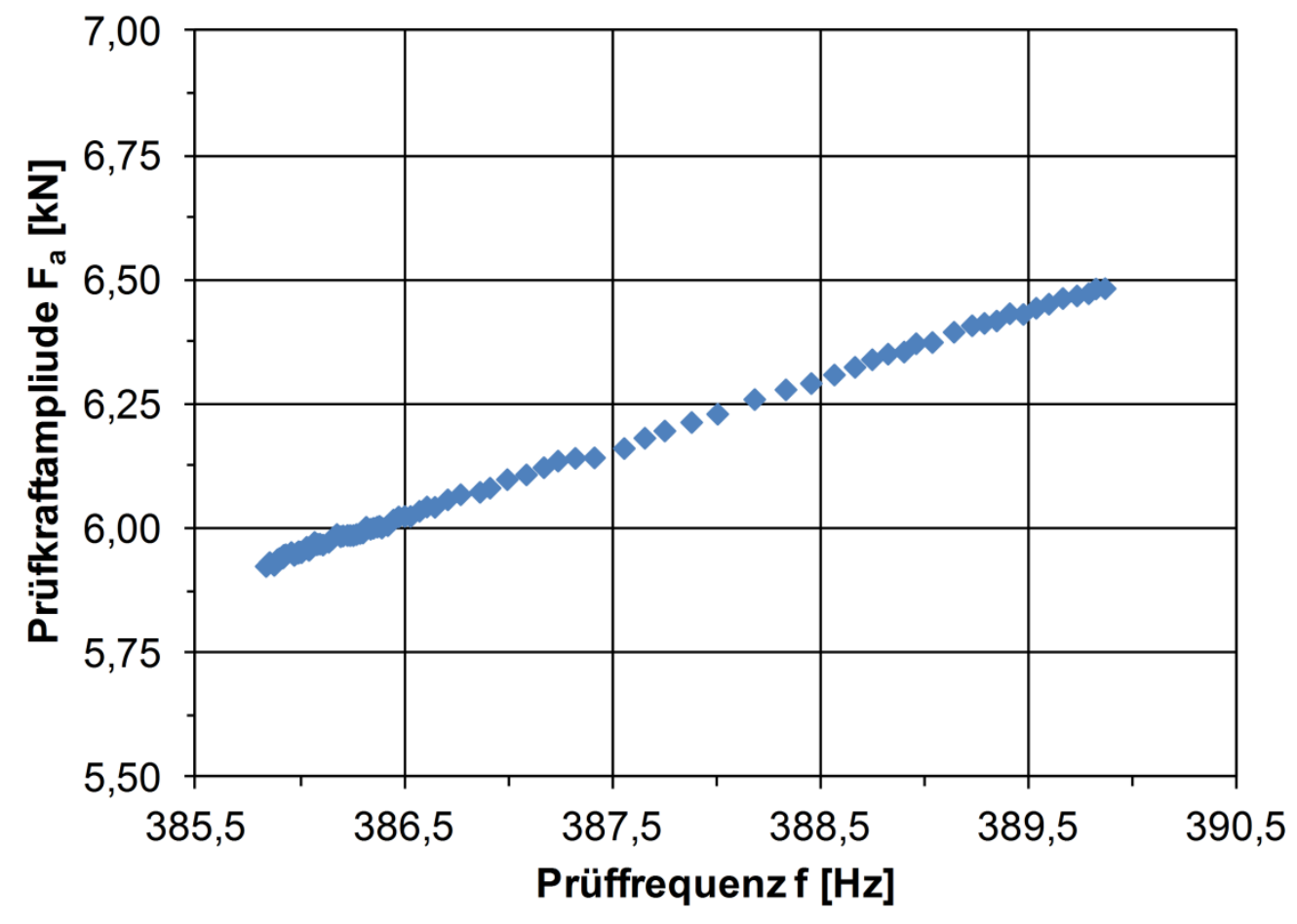

Abb. 4-24: Frequenzabhängigkeit des dynamischen Kalibrierfaktors kfd

Diese Frequenzabhängigkeit des dynamischen Kalibrierfaktors wirkt sich vornehmlich bei der Untersuchung des Übergangsbereichs mit entsprechend langen Versuchszeiten aus. Liegt im Zeitfestigkeitsbereich mit kurzen Versuchslaufzeiten eine geringe Streuung der Prüffrequenzen vor, kann in guter Näherung ein konstanter dynamischer Kalibrierfaktor angesetzt werden. Zur Vermeidung eines Abfalls der Prüfkraftamplitude im Langzeitbetrieb sind geeignete Strategien in der Versuchsführung erforderlich.

\section{Frequenzabhängige Sollwertvorgabe}

Aufgrund der Frequenzabhängigkeit des dynamischen Kalibrierfaktors kfd wurde eine frequenzabhängige Sollwertvorgabe in Zusammenarbeit mit dem Entwickler der Prüfvorrichtung [Deutsches Patent 10204258.6, 2005] etabliert. Bei konstanter Regelung der Rahmendehnung nimmt bei fallender Prüffrequenz die Prüfkraftamplitude bzw. Dehnungsamplitude der Probe ab. Um eine konstante Probendehnung zu realisieren, wird die Rahmendehnung in Abhängigkeit der Prüffrequenz geregelt. Abb. 4-25 zeigt schematisch das Verhalten der Probendehnung bei konstanter (links) und frequenzabhängiger (rechts) Regelung der Rahmendehnung. 


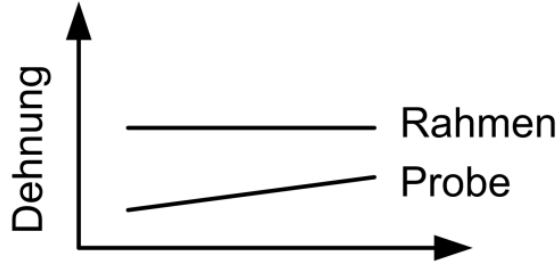

Frequenz

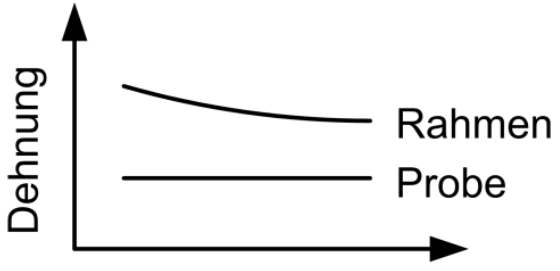

Frequenz

Abb. 4-25: Regelung Rahmendehnung: konstant (links) und frequenzabhängig (rechts)

Ziel ist es, eine konstante Probendehnung bzw. Prüfkraftamplitude durch Regelung einer frequenzabhängigen Rahmendehnung zu erhalten. Um eine konstante Probendehnung bzw. Prüfkraftamplitude für alle Prüffrequenzen zu erzielen, ist der Sollwert der Rahmendehnung in Abhängigkeit der Prüffrequenz mit dem Kehrwert der Funktion der festgestellten Frequenzabhängigkeit zu regeln, wie Gleichung (4-6) zeigt.

$$
F_{a}[k N]=U_{a, P}[V] \cdot k f\left[\frac{k N}{V}\right]=k f d(f)\left[\frac{k N}{V}\right] \cdot U_{a, R}(f)[V]=\text { konst. }
$$

Zur Umsetzung der frequenzabhängigen Sollwertvorgabe der Rahmendehnung steht eine lineare Sollwertvorgabe der Rahmendehnung in Abhängigkeit der Prüffrequenz zur Verfügung, wie Gleichung (4-7) zeigt. Diese lineare Sollwertvorgabe der Rahmendehnung stellt eine Näherungslösung dar, die für den relevanten Frequenzbereich von $385 \mathrm{~Hz}$ bis $391 \mathrm{~Hz}$ eine annähernd konstante Prüfkraftamplitude realisiert.

$$
U_{a, R}(f)[V]=\frac{F_{a}[k N]}{k f d_{\text {Bezug }}\left[\frac{k N}{V}\right]} \cdot\left(1+(388 \mathrm{~Hz}-f[H z]) \cdot \frac{p_{k}\left[\frac{\%}{H z}\right]}{100 \%}\right)
$$

Es werden der Korrekturfaktor $p_{k}$, der die prozentuale Änderung des Sollwertes der Rahmendehnung in Abhängigkeit der Prüffrequenz beschreibt, und der Korrekturfaktor $\mathrm{kdf}_{\text {Bezug, }}$, der den Bezugswert zum dynamischen Kalibrierfaktor darstellt, eingeführt. Durch Einsetzen von Gleichung (4-7) in Gleichung (4-6) ergibt sich die Näherungslösung in Gleichung (4-8). Die Prüfkraftamplitude wird im relevanten Frequenzbereich von $385 \mathrm{~Hz}$ bis $391 \mathrm{~Hz}$ annähernd konstant gehalten.

$$
\begin{aligned}
F_{a}[k N] & =U_{a, P}[V] \cdot k f\left[\frac{k N}{V}\right] \\
& \approx k f d(f)\left[\frac{k N}{V}\right] \cdot \frac{F_{a}[k N]}{k f d_{\text {Bezug }}\left[\frac{k N}{V}\right]} \cdot\left(1+(388 H z-f[H z]) \cdot \frac{p_{k}\left[\frac{\%}{H z}\right]}{100 \%}\right)
\end{aligned}
$$

Zur Ermittlung der Korrekturfaktoren $p_{k}$ und kfd $d_{\text {Bezug }}$ wird Gleichung (4-8) nach kfd(f) umgeformt, wie Gleichung (4-9) zeigt. Um eine optimale Näherungslösung mit minimalem Fehler zu erzielen, erfolgt die Ermittlung der Korrekturfaktoren der vorliegen- 
den Funktion der Sollwertkorrektur durch Annäherung an die Messwerte für die dynamischen Kalibrierfaktoren bei unterschiedlichen Prüffrequenzen.

$$
k f d(f)\left[\frac{k N}{V}\right]=\frac{U_{a, P}[V] \cdot k f\left[\frac{k N}{V}\right]}{U_{a, R}(f)[V]} \approx \frac{k f d_{\text {Bezug }}\left[\frac{k N}{V}\right]}{\left(1+(388 \mathrm{~Hz}-f[\mathrm{~Hz}]) \cdot \frac{p_{k}\left[\frac{\%}{H z}\right]}{100 \%}\right)}
$$

Die Datengrundlage der dynamischen Kalibrierfaktoren bei unterschiedlichen Prüffrequenzen wurde in mehreren Durchläufen für verschiedene Kalibrierproben in unterschiedlichen Verspannungssituationen geschaffen. Die ermittelten dynamischen Kalibrierfaktoren kfd sowie die Näherungslösung sind in Abhängigkeit der Prüffrequenz in Abb. 4-26 dargestellt. Die Ermittlung der Korrekturfaktoren durch eine Regressionsanalyse mit der Methode der kleinsten Fehlerquadrate liefert $\mathrm{kfd}_{\text {Bezug }}=4,041 \mathrm{kN} / \mathrm{V}$ und $\mathrm{p}_{\mathrm{k}}=2,203 \% / \mathrm{Hz}$. Der Standardschätzfehler wurde zu 0,0135 ermittelt. Der Determinationskoeffizient $r^{2}$ ergibt sich zu 0,993.

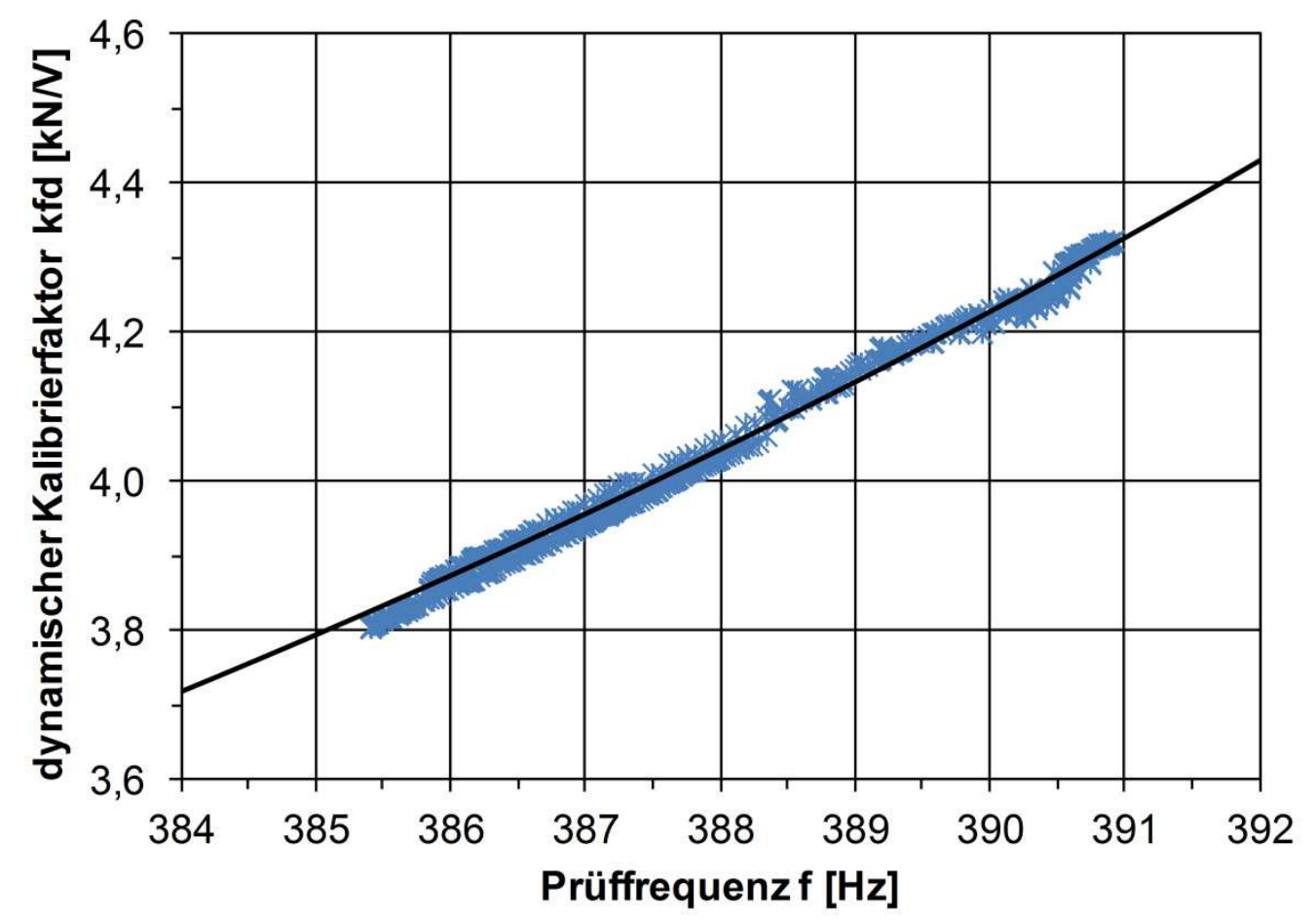

Abb. 4-26: Datengrundlage zur Ermittlung der Korrekturfaktoren $p_{k}$ und $\mathbf{k f d}_{\mathrm{Bezug}}$ der frequenzabhängigen Sollwertvorgabe

Der Standardschätzfehler von 0,0135 ließe sich auf 0,0128 verringern, wenn die Sollwertvorgabe in der Lage wäre, den Zusammenhang der Frequenzabhängigkeit des dynamischen Kalibrierfaktors nach Gleichung (4-10) mit kfd $\mathrm{d}_{\text {linear }}$ abzubilden. Die Parameter der Geradengleichung (Steigung $m$ und Achsenabschnitt $b$ ) ergeben sich durch eine Regressionsanalyse mit der Methode der kleinsten Fehlerquadrate zu 
$m=0,0903 \mathrm{kN} /(\mathrm{Hz} \cdot \mathrm{V})$ und $\mathrm{b}=-30,9910 \mathrm{kN} / \mathrm{V}$. Der Standardschätzfehler könnte somit auf 0,0128 verringert werden. Der Determinationskoeffizient $r^{2}$ ergibt sich dabei zu 0,994 .

$$
k f d_{\text {linear }}(f)\left[\frac{k N}{V}\right]=m\left[\frac{k N}{H z \cdot V}\right] \cdot f[H z]+b\left[\frac{k N}{V}\right]
$$

Allerdings steht zur Umsetzung der frequenzabhängigen Sollwertvorgabe der Rahmendehnung lediglich die zuvor beschriebene lineare Sollwertvorgabe der Rahmendehnung in Abhängigkeit der Prüffrequenz zur Verfügung. Abb. 4-27 zeigt den Verlauf der Prüfkraftamplitude eines Versuchslaufs beim Erreichen der Grenzschwingspielzahl $N_{G}$ von 500.000 .000 über die Prüffrequenz $\mathrm{f}$. Die Prüfkraftamplitude des Rahmens und der Probe im Vergleich zum Sollwert der Prüfkraftamplitude sind abgebildet. Es zeigt sich eine gute Übereinstimmung der Prüfkraftamplitude von Rahmen und Probe. Die Prüfkraftamplitude verhält sich bei einer Prüffrequenzänderung um mehrere Hertz nahezu konstant über die Versuchslaufzeit. Die Funktionsweise der frequenzabhängigen Sollwertvorgabe im relevanten Frequenzbereich wird hierdurch bestätigt.

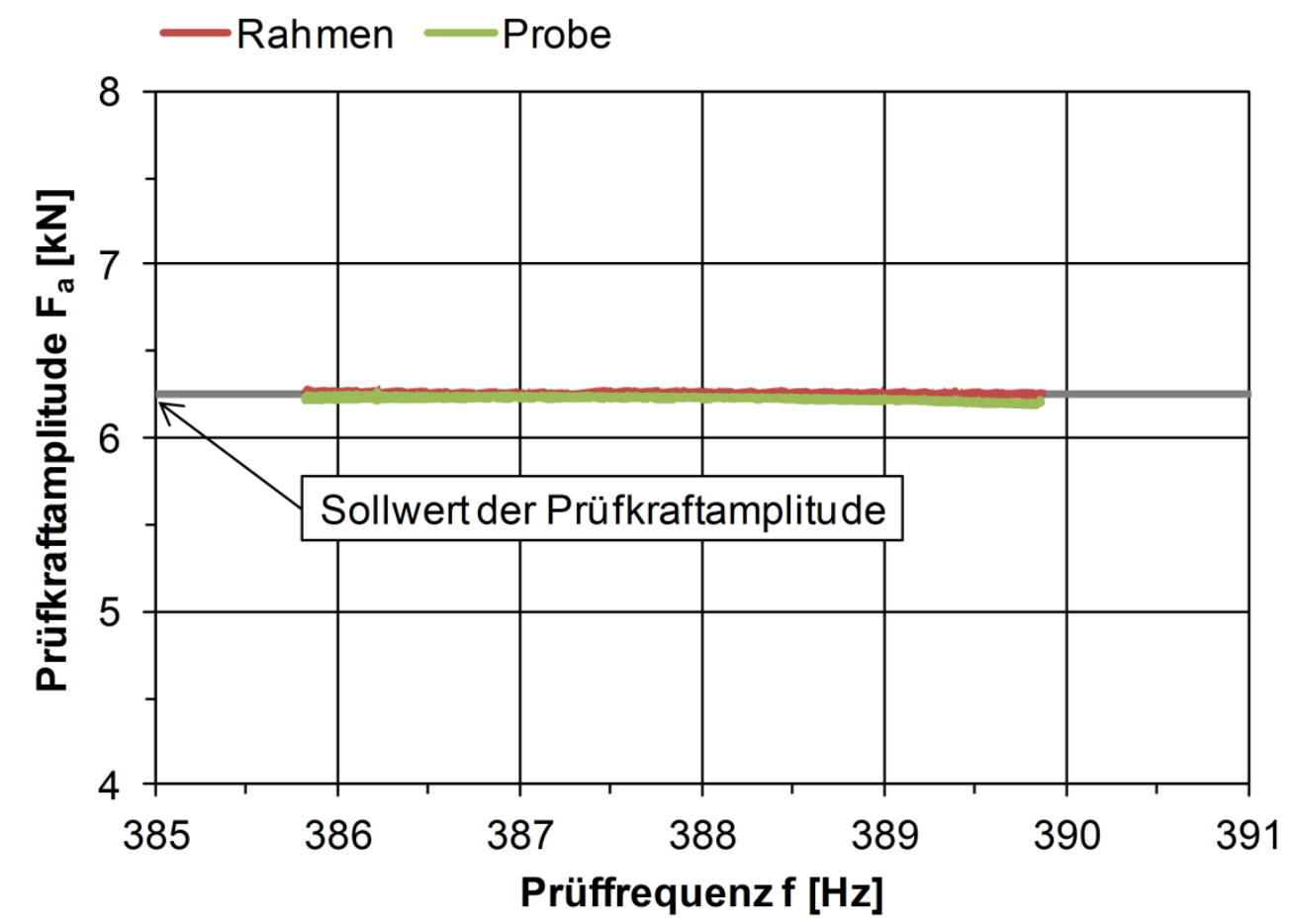

\section{Abb. 4-27: Verlauf der Prüfkraftamplitude $F_{\mathrm{a}}$ von Rahmen und Probe über die Prüffre- quenz $f$ beim Erreichen der Grenzschwingspielzahl $N_{G}$}

Die charakteristischen Verläufe der zeitlich veränderlichen Messgrößen von Probe und Rahmen sind im Vergleich für das Ereignis eines Probenversagens in Abb. 4-28 exemplarisch für eine Probe über die Schwingspielzahl $N$ aufgetragen. Die Prüffre- 
quenz verhält sich bis zum Eintritt des Anrisses konstant. Der durch den Anriss der Probe verursachte Steifigkeitsverlust führt zu einem starken Abfall der Prüffrequenz. Der Anriss ist gemäß der Definition in Kapitel 4.4 festgelegt. Bei Eintritt eines Anrisses und dem damit verbundenen Risswachstum verliert die aufgestellte Proportionalitätsbeziehung zwischen Prüfkraft und zugehöriger Probendehnung seine Gültigkeit. Demnach besitzen die Verläufe der zeitlich veränderlichen Messgrößen nach Eintritt eines Anrisses keine exakte Aussagekraft mehr.

Die Prüfkraftamplitude von Probe und Rahmen zeigt einen konstanten Verlauf bis zum Eintritt des Anrisses. Die Vorspannkraft von Probe und Rahmen weist den charakteristischen, temperaturbedingten Abfall der Vorspannkraft zu Versuchsbeginn auf und bleibt bis zum Eintritt des Anrisses konstant. Die Verläufe von Prüfkraftamplitude und Vorspannkraft von Probe und Rahmen weichen im Gültigkeitsbereich der Proportionalitätsbeziehung nur geringfügig voneinander ab.

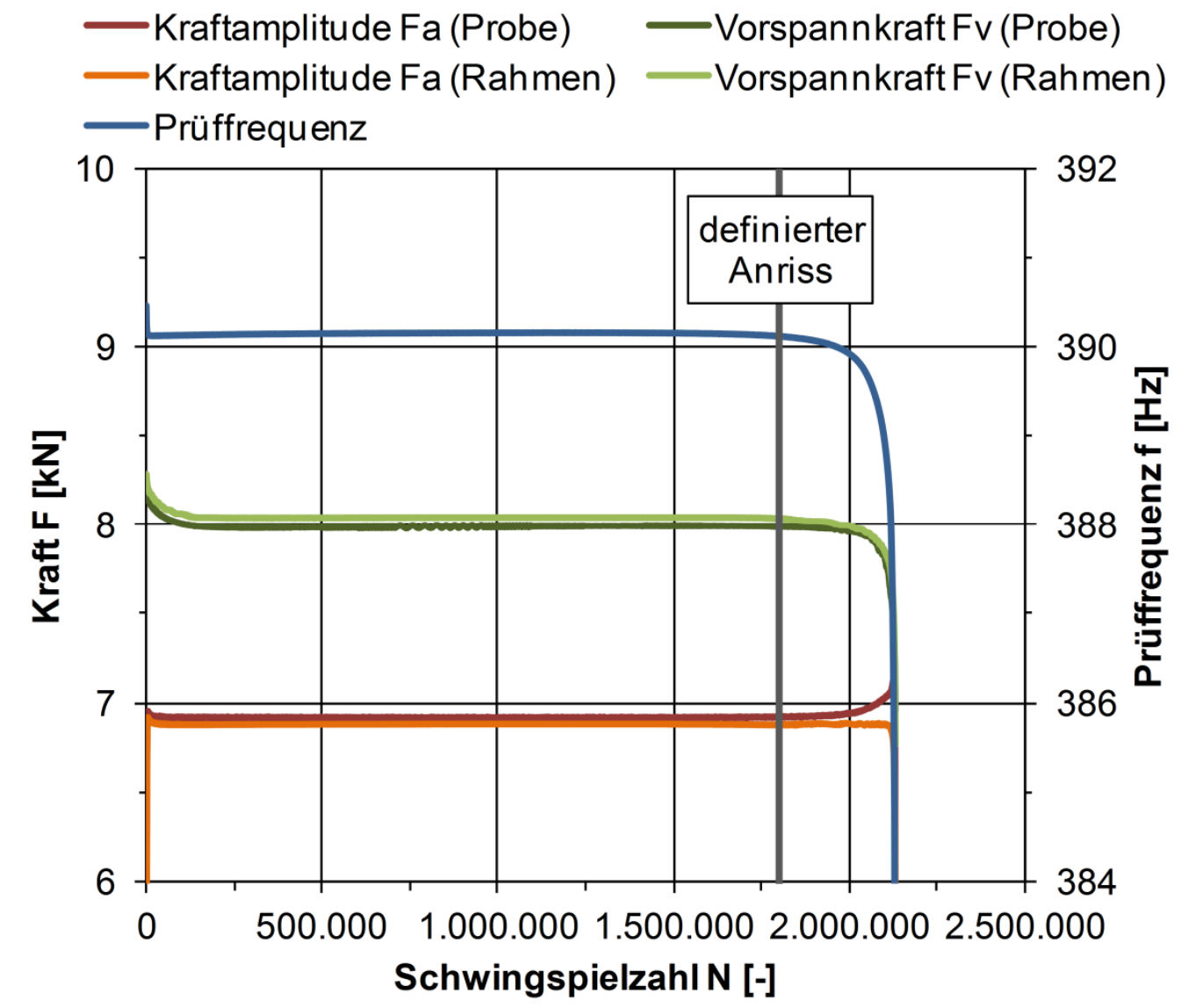

Abb. 4-28: Vergleich zeitlich veränderlicher Messgrößen von Probe und Rahmen über die Schwingspielzahl N bei Probenversagen 


\subsection{Zusammenfassung und Erkenntnisse}

Der Aufbau und das Funktionsprinzip der verwendeten Prüfvorrichtung mit einer Prüffrequenz von bis zu $400 \mathrm{~Hz}$, welche die Prüfung von Schweißverbindungen bis in den Bereich sehr hoher Lastwechselzahlen in einem akzeptablen Zeitraum ermöglicht, wurde erläutert. Desweiteren wurden die Weiterentwicklungen der Vorspannvorrichtung sowie der Regelung für Versuche im Langzeitbereich vorgestellt. Die separate Vorspannvorrichtung entfiel durch das Vorspannen mit Exzenterwellen und die Vorspannkraft kann nun direkt eingestellt werden. Der durch die Umlagerung des Kraftflusses hervorgerufene Verlust an Vorspannkraft entfällt somit. In den Langzeitversuchen stellte sich heraus, dass sich die Steifigkeit von Probekörper und Einspannung über den Prüfzeitraum auch ohne das Eintreten eines Versagens erheblich verändern kann. Mit der Änderung der Steifigkeit geht eine Änderung der Proportionalität der Beanspruchungen von Prüfrahmen und Probekörper einher, was einer Frequenzabhängigkeit des dynamischen Kalibrierfaktors entspricht. Die Regelung wurde um eine frequenzabhängige Sollwertvorgabe erweitert, um den Einfluss des Steifigkeitsverlustes auf den dynamischen Kalibrierfaktor zu kompensieren und im relevanten Frequenzbereich eine näherungsweise konstante Beanspruchung im Probekörper zu erzielen.

Die Untersuchung der zeitlich veränderlichen Messgrößen gab Aufschluss über das Verhalten der Prüfvorrichtung und die Aussagekraft der erzielten Versuchsergebnisse. Da die Vorspannkraft der Prüfvorrichtung im Laufe des Versuchs prinzipiell nicht nachgeregelt werden kann, führt der temperaturbedingte Abfall der Vorspannkraft zu Versuchsbeginn zu einem Abfall des Spannungsverhältnisses, was zu höheren erreichten Schwingspielzahlen führen kann. Da die Erwärmung der Probekörper mit steigender Höhe der Beanspruchungsamplitude zunimmt, kann dies insgesamt zu einem steileren Verlauf der Ermüdungsfestigkeitskurven und einer höheren Streuung der Ergebnisse im Zeitfestigkeitsbereich führen. Ein leichter Anstieg der Vorspannkraft im Langzeitbetrieb wurde auf Abnutzungserscheinungen, verursacht durch Reibkorrosion, im Bereich der Spannklötze zurückgeführt. Durch den Wechsel des Schmiermittels sowie die aufwendige Reinigung der Reibflächen konnte dieser Effekt minimiert werden.

Da die aufgestellte Proportionalitätsbeziehung zwischen Prüfkraft und zugehöriger Probendehnung bei Eintritt eines Anrisses seine Gültigkeit verliert, kann die Prüfkraftamplitude zu Versuchsende nicht mehr exakt aufgebracht werden. Die Definition des Kriteriums zur Ermittlung der Anrissschwingspielzahl gewährleistet eine einheitliche Auswertung der Versuchsergebnisse. Als Kriterium für die Ermittlung der Anrissschwingspielzahl wurde eine prozentuale Abweichung des aktuellen Wertes der 
Prüffrequenz von einer im leicht linear abfallenden Bereich eingeführten Regressionsgeraden von $0,01 \%$ definiert.

Die Versuche zur Markierung der Rissfront auf der Bruchfläche gaben Aufschluss über die Größe des vorliegenden Anrisses beim Erreichen des definierten Anrisskriteriums. Es konnte lediglich in einem Versuch die Anrisstiefe $t_{A}$ mit $1,5 \mathrm{~mm}$ gemessen werden. In den übrigen Versuchen waren deutlich weniger Rastlinien auf den Bruchflächen erkennbar, sodass es nur möglich war, die minimal erkennbaren Risstiefen zu ermitteln und damit die Größe des vorliegenden Anrisses beim Erreichen des Anrisskriteriums einzugrenzen. Die Breite der Rastlinien konnte nur in wenigen Fällen ermittelt werden. Es zeigte sich ein Unterschied der minimal erkennbaren Risstiefen in Abhängigkeit von der Rissform. Bei viertelelliptischen Eckanrissen kann von Anrisstiefen $t_{A}$ im Bereich von 1,5 $\mathrm{mm}$ ausgegangen werden, wohingegen in den übrigen Fällen Anrisstiefen $t_{A}$ kleiner als $1,2 \mathrm{~mm}$ vorliegen. 


\section{Ermüdungsversuche}

Um Aufschluss über das Ermüdungsverhalten von Schweißverbindungen im Bereich sehr hoher Lastwechselzahlen zu gewinnen und die Prüftechnik für Ermüdungsversuche mit hohen Prüffrequenzen weiterzuentwickeln, wurden Ermüdungsversuche an bauteilähnlichen Probekörpern durchgeführt.

In diesem Kapitel werden die einzelnen Versuchsreihen der durchgeführten experimentellen Untersuchungen beschrieben. Außerdem wird auf die Versuchsbedingungen der Versuchsreihen, die in der verwendeten Prüfvorrichtung geprüft wurden, eingegangen. Anhand der Ergebnisse aus den Schwingversuchen wird der Einfluss von Prüffrequenz und Mittelspannung auf die Ermüdungsfestigkeit sowie die Fragestellung des Auftretens später Brüche bei Schweißverbindungen untersucht. Es erfolgt der Vergleich der Versuchsergebnisse der vorliegenden Arbeit mit der normierten Auswertung von Ermüdungsversuchen aus Sonsino et al. [Sonsino, 2005b] und mit Eurocode 3 [DIN EN 1993-1-9, 2010].

\subsection{Versuchsdurchführung}

Die Ermüdungsversuche wurden bei Raumtemperatur unter rein axialer Belastung mit konstanter Beanspruchungsamplitude sowie bei konstanten Spannungsverhältnissen durchgeführt. Zur Ermittlung von Ermüdungsfestigkeitskurven wurden bauteilähnliche Probekörper auf unterschiedlichen Beanspruchungsniveaus geprüft. Es wurden Versuche im Zeitfestigkeits- und Übergangsbereich bei unterschiedlichen Prüffrequenzen und Spannungsverhältnissen bis in den Bereich sehr hoher Lastwechselzahlen durchgeführt, wobei der Schwerpunkt der Untersuchungen auf dem Übergangsbereich liegt.

Es kamen zwei unterschiedliche Prüfmaschinen zum Einsatz. Die Versuche bei einer Prüffrequenz $f$ von $20 \mathrm{~Hz}$ wurden kraftgeregelt in einer servohydraulischen Prüfmaschine mit einem sinusförmigen Beanspruchungs-Zeit-Verlauf durchgeführt. Die Versuche bei einer Prüffrequenz $f$ im Bereich von $390 \mathrm{~Hz}$ wurden rahmendehnungsgeregelt in der in Kapitel 4 vorgestellten hochfrequenten Prüfvorrichtung mit einem sinusförmigen Beanspruchungs-Zeit-Verlauf durchgeführt. Als Abbruchkriterium der Versuche wurde der vollständige Bruch bzw. der Anriss des Probekörpers oder das Erreichen einer in Abhängigkeit von der verwendeten Prüfmaschine festgelegten Grenzschwingspielzahl definiert. 
Bei Prüfung in der servohydraulischen Prüfmaschine wurden der Bruch des Probekörpers sowie das Erreichen einer Grenzschwingspielzahl $N_{G}$ von $5 \cdot 10^{6}$ Lastwechseln als Abbruchkriterium festgelegt. Im Gegensatz dazu wurde bei der Prüfung in der hochfrequenten Prüfvorrichtung der Anriss des Probekörpers gemäß der Definition in Kapitel 4.4 sowie das Erreichen einer Grenzschwingspielzahl $N_{G}$ von $5 \cdot 10^{8}$ Lastwechseln definiert. Die Proben mit Anriss wurden im Anschluss im statischen Zugversuch getrennt. Eine Übersicht der Prüfmaschinen mit Prüffrequenzen, festgelegter Grenzschwingspielzahl und zugehöriger Versuchsdauer gibt Tab. 5-1.

Tab. 5-1: Übersicht der Prüfmaschinen

\begin{tabular}{|c|c|c|c|}
\hline Prüfmaschine & $\begin{array}{c}\text { Prüffre- } \\
\text { quenz } \mathbf{f}\end{array}$ & $\begin{array}{c}\text { Grenzschwing- } \\
\text { spielzahl N }\end{array}$ & $\begin{array}{c}\text { Dauer bis zum Erreichen der } \\
\text { Grenzschwingspielzahl } \mathbf{N}_{\mathbf{G}}\end{array}$ \\
\hline $\begin{array}{c}\text { servohydraulische } \\
\text { Prüfmaschine }\end{array}$ & $20 \mathrm{~Hz}$ & 5.000 .000 & ca. 3 Tage \\
\hline $\begin{array}{c}\text { hochfrequente } \\
\text { Prüfvorrichtung }\end{array}$ & ca. $390 \mathrm{~Hz}$ & 500.000 .000 & ca. 15 Tage \\
\hline
\end{tabular}

Um Rückschlüsse auf eine möglicherweise vorliegende Schädigung der Probekörper aus der Beanspruchung bis zur festgelegten Grenzschwingspielzahl ziehen zu können, wurden die Durchläufer aus den Versuchen im Übergangsbereich auf den Beanspruchungsniveaus im Zeitfestigkeitsbereich bis zum Versagen der Probekörper geprüft.

\subsection{Versuchsprogramm}

Das Versuchsprogramm umfasst Ermüdungsversuche an insgesamt 89 Probekörpern. Einschließlich der hochgesetzten Durchläufer wurden insgesamt 111 Ermüdungsversuche an im Schweißzustand belassenen Stumpfnahtverbindungen der Stahlgüte S355J2 +N mit einer Blechdicke t von 4 mm (siehe Kapitel 3) durchgeführt. Anhand von drei Versuchsreihen wird der Einfluss von Prüffrequenz und Mittelspannung auf die Ermüdungsfestigkeit sowie die Fragestellung des Auftretens später Brüche bei Schweißverbindungen untersucht. Einen Überblick über das Versuchsprogramm mit den einzelnen Versuchsreihen gibt Tab. 5-2. Zur besseren Übersicht wurde jeweils eine Unterteilung in die Untersuchungsbereiche Zeitfestigkeitsbereich (A), Übergangsbereich (B) und hochgesetzte Durchläufer (C) vorgenommen. 
Tab. 5-2: Übersicht über das Versuchsprogramm

\begin{tabular}{|c|c|c|c|c|}
\hline $\begin{array}{c}\text { Versuchs- } \\
\text { reihe }\end{array}$ & $\begin{array}{c}\text { Spannungs- } \\
\text { verhältnis }\end{array}$ & $\begin{array}{c}\text { Prüffre- } \\
\text { quenz f }\end{array}$ & Untersuchungsbereich & $\begin{array}{c}\text { Proben- } \\
\text { anzahl }\end{array}$ \\
\hline $1-A$ & $\mathrm{R}=0,1$ & $20 \mathrm{~Hz}$ & Zeitfestigkeitsbereich & 18 \\
\hline $1-\mathrm{B}$ & $\mathrm{R}=0,1$ & $20 \mathrm{~Hz}$ & Übergangsbereich & 17 \\
\hline $1-\mathrm{C}$ & $\mathrm{R}=0,1$ & $20 \mathrm{~Hz}$ & hochgesetzte Durchläufer & $(8)$ \\
\hline $2-\mathrm{A}$ & $\mathrm{R}=0,1$ & $390 \mathrm{~Hz}$ & Zeitfestigkeitsbereich & 12 \\
\hline $2-\mathrm{B}$ & $\mathrm{R}=0,1$ & $390 \mathrm{~Hz}$ & Übergangsbereich & 16 \\
\hline $2-\mathrm{C}$ & $\mathrm{R}=0,1$ & $390 \mathrm{~Hz}$ & hochgesetzte Durchläufer & $(7)$ \\
\hline $3-\mathrm{A}$ & $\mathrm{R}=0,5$ & $390 \mathrm{~Hz}$ & Zeitfestigkeitsbereich & 10 \\
\hline $3-\mathrm{B}$ & $\mathrm{R}=0,5$ & $390 \mathrm{~Hz}$ & Übergangsbereich & 16 \\
\hline $3-\mathrm{C}$ & $\mathrm{R}=0,5$ & $390 \mathrm{~Hz}$ & hochgesetzte Durchläufer & $(7)$ \\
\hline
\end{tabular}

Das Ziel von Versuchsreihe 1 war es, eine Referenz-Wöhlerlinie für den Probekörper in einer herkömmlichen Prüfmaschine bei üblichen Prüffrequenzen zu ermitteln, um die Ergebnisse der hochfrequenten Prüfvorrichtung einzuordnen und bewerten zu können. Diese Versuchsreihe wurde mit einer Prüffrequenz $f$ von $20 \mathrm{~Hz}$ bei einem konstanten Spannungsverhältnis $R$ von 0,1 ermittelt. Sie umfasst insgesamt 35 Probekörper. Im Zeitfestigkeitsbereich (1-A) wurden auf drei Beanspruchungsniveaus jeweils 6 Probekörper geprüft. Im Übergangsbereich (1-B) wurden auf verschiedenen Laststufen 17 Probekörper geprüft. Die erzielten Durchläufer (8 Probekörper) wurden auf die Beanspruchungsniveaus im Zeitfestigkeitsbereich hochgesetzt (1-C).

Im Hinblick auf den Vergleich mit Versuchsreihe 1 soll durch Versuchsreihe 2 Aufschluss über den Einfluss der Prüffrequenz gewonnen werden. Hierzu wurde eine Wöhlerlinie in der hochfrequenten Prüfvorrichtung mit einer Prüffrequenz $f$ im Bereich von $390 \mathrm{~Hz}$ ebenfalls bei einem konstanten Spannungsverhältnis R von 0,1 ermittelt. Dabei wurde der Bereich der sehr hohen Schwingspielzahlen mit einbezogen. Diese Versuchsreihe umfasst insgesamt 28 Probekörper. Im Zeitfestigkeitsbereich (2-A) wurden auf zwei Beanspruchungsniveaus jeweils 6 Probekörper geprüft. Da die Prüfvorrichtung für den Langzeitbereich ausgelegt ist, war die Prüfung auf höheren Beanspruchungsniveaus aufgrund des Leistungsverstärkers nicht möglich. Im Übergangsbereich (2-B) wurden auf verschiedenen Laststufen 16 Probekörper geprüft. Die erzielten Durchläufer (7 Probekörper) wurden auf die Beanspruchungsniveaus im Zeitfestigkeitsbereich hochgesetzt (2-C). 
Um Aufklärung über den Einfluss von aus dem Schweißprozess resultierenden Zugeigenspannungen auf die Versuchsergebnisse zu gewinnen, wurde eine Wöhlerlinie in der hochfrequenten Prüfvorrichtung mit hoher Mittelspannung ermittelt. Die aus dem Schweißprozess resultierenden Zugeigenspannungen sind bei kleinen Probekörpern als gering einzustufen. Um dennoch Aussagen aus Versuchen mit kleinen Probekörpern treffen zu können, wurden näherungsweise Mittelspannungen angesetzt und die Prüfung bei einem erhöhten Spannungsverhältnis $R$ von 0,5 durchgeführt. Hierbei wurde der Bereich der sehr hohen Schwingspielzahlen mit einbezogen. Diese Versuchsreihe umfasst insgesamt 26 Probekörper. Im Zeitfestigkeitsbereich (3-A) wurden auf zwei Beanspruchungsniveaus jeweils 5 Probekörper geprüft. Die Prüfung auf höheren Beanspruchungsniveaus war wiederum aufgrund der hohen erforderlichen Vorspannkräfte mit der Exzenterwelle nicht möglich. Auf verschiedenen Laststufen wurden im Übergangsbereich (3-B) 16 Probekörper geprüft. Die erzielten Durchläufer (7 Probekörper) wurden auf die Beanspruchungsniveaus im Zeitfestigkeitsbereich hochgesetzt (3-C).

\subsection{Versuchsauswertung}

Ermüdungsversuche sind starken Streuungen unterworfen und können als zufallsbedingt aufgefasst werden. Die Anzahl der Proben ist jedoch aus wirtschaftlichen Gründen (Zeit und Kosten) begrenzt. Um dennoch Aussagen aus Ermüdungsversuchen zu gewinnen, werden statistische Verfahren angewendet, welche die Streuungen zufallsbedingt erfassen können. Ein Überblick zu statistischen Verfahren kann Mauch [Mauch, 1999] oder Radaj und Vormwald [Radaj, 2007] entnommen werden. Aus dem Streuband der Versuchsergebnisse lassen sich Ermüdungsfestigkeitskurven bestimmter Überlebenswahrscheinlichkeiten ableiten. Der Dauerschwingversuch ist nach DIN 50100 [DIN 50100, 1978] genormt, jedoch sind keine Angaben zur statistischen Auswertung enthalten. Im Gegensatz dazu gibt DIN 969 [DIN 969, 1997] Empfehlungen zur Versuchsführung und statistischen Auswertung bei Verbindungselementen mit Gewinde. Häufig findet eine getrennte Versuchsführung und statistische Auswertung für den Zeitfestigkeits- und Übergangsbereich statt.

\subsubsection{Untersuchung des Zeitfestigkeitsbereichs}

Bei der Versuchsführung im Zeitfestigkeitsbereich wird zwischen dem Horizontenund Perlenschnurverfahren unterschieden, wobei allerdings auch in gemischter Form vorgegangen werden kann. Beim Horizontenverfahren werden auf mindestens zwei Beanspruchungshorizonten mehrere Ermüdungsversuche durchgeführt, wohingegen sich beim Perlenschnurverfahren die Ermüdungsversuche auf vielen Beanspruchungshorizonten entlang der Ermüdungsfestigkeitskurve im Zeitfestigkeitsbereich erstrecken. Erwartungsgemäß nimmt die Streuung mit sinkendem Beanspruchungs- 
niveau zu. Der Vorteil des Horizontenverfahrens besteht darin, dass die Auswertung für jeden Beanspruchungshorizont getrennt erfolgen kann, sodass unterschiedliche Streuungen der Beanspruchungshorizonte berücksichtigt werden können [Mauch, 1999]. Beim Perlenschurverfahren erfolgt die Auswertung für den gesamten Zeitfestigkeitsbereich mit konstanter Streuung.

Im Allgemeinen wird im Zeitfestigkeitsbereich angenommen, dass die logarithmischen Werte der Versuchsergebnisse durch die Gauß-Normalverteilung erfasst werden können [Radaj, 2007]. Diese Annahme kann durch die Auftragung der Versuchsergebnisse im Wahrscheinlichkeitsnetz der Überschreitungshäufigkeiten überprüft werden. Können die Werte der Versuchsergebnisse einer Ausgleichsgeraden zugeordnet werden, darf als Verteilungsfunktion die Gauß-Normalverteilung der logarithmischen Werte unterstellt werden [Haibach, 2006]. Der Einfluss der Verteilungsfunktion auf die Ergebnisse im Bereich von 5 - $95 \%$ Ausfallwahrscheinlichkeit ist als gering einzustufen [Mauch, 1999]. Bei der Extrapolation der Streugeraden auf besonders niedrige bzw. hohe Ausfallwahrscheinlichkeiten zeigen sich jedoch erhebliche Unterschiede, weshalb die Ergebnisse mit Unsicherheiten behaftet sind. Für eine hinreichend abgesicherte statistische Auswertung von Ermüdungsversuchen ist ein ausreichender Stichprobenumfang notwendig.

Zur Untersuchung des Zeitfestigkeitsbereichs wird in der vorliegenden Arbeit das Horizontenverfahren angewendet. Die Angaben zur erforderlichen Anzahl der Proben (Stichprobenumfang) auf den jeweiligen Beanspruchungshorizonten für eine statistische Auswertung im Zeitfestigkeitsbereich variieren. Die Angaben bewegen sich im Allgemeinen zwischen 5 und 8 Proben je Beanspruchungshorizont. In DIN 969 [DIN 969, 1997] sind für eine zuverlässige Abschätzung der Bruchwahrscheinlichkeit mindestens zwei Beanspruchungshorizonte mit mindestens 8 Proben vorgesehen. Außerdem werden für die Beanspruchungshorizonte der 1,6-fache und 2,4-fache Wert der geschätzten Dauerfestigkeit vorgeschlagen. Nach Mauch [Mauch, 1999] sowie Gudehus und Zenner [Gudehus, 1999] sind je Beanspruchungshorizont mindestens 5 Proben erforderlich, wohingegen bei Naubereit und Weihert [Naubereit, 1999] ein Umfang von mindestens 8 Proben angegeben ist.

\subsubsection{Untersuchung des Übergangsbereichs}

Im Gegensatz zum Zeitfestigkeitsbereich ist der Zusammenhang zwischen Beanspruchungsamplitude und Schwingspielzahl im Übergangsbereich nicht eindeutig. Bei der Versuchsführung im Übergangsbereich werden mehrere Proben auf vorab oder iterativ festgelegten Beanspruchungsniveaus geprüft. Es treten auf gleichen Beanspruchungsniveaus Probenversagen (Bruch) und das Erreichen einer festgelegten Grenzschwingspielzahl (Durchläufer) auf. 
Bei der Wahl geeigneter Verfahren zur Auswertung stellt sich die Frage nach dem Verlauf der Ermüdungsfestigkeitskurve im Übergangsbereich. Zeichnet sich eine klassische Wöhlerlinie mit horizontalem Auslaufen und Anriss von der Oberfläche ausgehend ab, kann beispielsweise das Treppenstufenverfahren, das Probitverfahren, das Abgrenzungsverfahren sowie das arcsin-Verfahren angewendet werden [Mauch, 1999] [Liu, 2001] [Radaj, 2007].

Bei einem weiteren Abfall der Wöhlerlinie im Übergangsbereich und der Verlagerung des Anrisses ins Innere, ist die Ermittlung der Neigung der Ermüdungsfestigkeitskurve ab dem Abknickpunkt von Interesse. Die zuvor genannten Verfahren können dies allerdings nicht leisten und liefern als Ergebnis lediglich ein horizontales Auslaufen der Wöhlerlinie.

Bisher existiert keine einheitliche oder spezielle Methode zur Versuchsführung und Auswertung von Ermüdungsversuchen im VHCF-Bereich [Pyttel, 2010]. Schwerdt und Pyttel [Schwerdt, 2009] stellen eine Auswahl an Verfahren zusammen, mit denen eine Auswertung im Übergangsbereich bei einem weiteren Abfall der Ermüdungsfestigkeit erfolgen kann, und diskutieren deren Anwendbarkeit auf durchgeführte Ermüdungsversuche. Zu diesen Verfahren zählen die Polynomregression, das modifizierte arcsin-Verfahren, die lineare Regression im Zeitfestigkeits- und Übergangsbereich, die Maximum-Likelihood-Methode sowie die lineare Regression im Zeitfestigkeitsbereich mit den Empfehlungen von Sonsino [Sonsino, 2005a] für den Übergangsbereich.

Eine lineare Regression sowie eine Polynomregression können für den Zeitfestigkeits- und Übergangsbereich unter Vernachlässigung der Durchläufer durchgeführt werden. Die lineare Regression liefert hierbei eine bilineare Wöhlerlinie mit Neigung im Übergangsbereich sowie Abknickpunkt, wobei die Polynomregression eine kontinuierliche Ermüdungsfestigkeitskurve darstellt. Beide Verfahren führen im Übergangsbereich zu eher konservativen Ermüdungsfestigkeitskurven, wobei die Polynomregression zudem keine konventionelle Beschreibung der Wöhlerlinie darstellt. Die lineare Regression im Zeitfestigkeitsbereich verbunden mit den Empfehlungen von Sonsino [Sonsino, 2005a] für den Übergangsbereich liefert ebenfalls eine bilineare Wöhlerlinie. Allerdings basiert dieses Verfahren im Übergangsbereich auf Erfahrungswerten. Die Neigung im Übergangsbereich sowie der Abknickpunkt der Ermüdungsfestigkeitskurve sind festgelegt. Es wird keine Mindestprobenanzahl benötigt.

Das modifizierte arcsin-Verfahren sowie die Maximum-Likelihood-Methode erfordern einen Mindestprobenumfang, um realistische Ergebnisse zu erhalten. Es wird eine bilineare Wöhlerlinie ermittelt. Zunächst erfolgt die Auswertung für den Zeitfestig- 
keitsbereich entsprechend der beiden Verfahren bei festgelegter Schwingspielzahl für den Abknickpunkt, wobei alle Proben, die nach dem Abknickpunkt gebrochen sind, als Durchläufer angesehen und in der Auswertung nicht berücksichtigt werden. Im zweiten Schritt erfolgt die Auswertung der Brüche und Durchläufer nach dem Abknickpunkt entsprechend der beiden Verfahren. Hierbei wird die ermittelte Schwingfestigkeit für die Grenzschwingspielzahl angenommen. Durch das lineare Verbinden der Schwingfestigkeit am Abknickpunkt und bei der Grenzschwingspielzahl kann eine Neigung im Übergangsbereich ermittelt werden.

Der Überblick der Methoden zur Auswertung von Ermüdungsversuchen im Übergangsbereich zeigt, dass sowohl Verfahren zur Auswertung bei Vorhandensein einer Dauerfestigkeit als auch bei einem weiteren Abfall der Ermüdungsfestigkeit vorliegen. Da keines der genannten Verfahren beide Fälle umsetzen kann, empfiehlt sich im Vorfeld der Auswertung der Versuchsergebnisse eine Abschätzung des Verlaufs der Ermüdungsfestigkeitskurven [Schwerdt, 2009]. Gemäß Schwerdt und Pyttel [Schwerdt, 2009] kann das Auftreten später Brüche mit erreichten Schwingspielzahlen von größer als $10^{7}$ Lastwechseln sowie eine Änderung des Schädigungsmechanismus mit einer Verlagerung des Anrisses von der Oberfläche ins Probeninnere auf einen weiteren Abfall der Ermüdungsfestigkeitskurve hindeuten.

In den vorliegenden Untersuchungen überschreiten die ermittelten Bruch- und Anrissschwingspielzahl im Übergangsbereich einen Wert von 3.106 Lastwechsel nicht. Außerdem ergab sich keine Änderung des Schädigungsmechanismus, da der Anriss aller Probekörper einheitlich an der Oberfläche auftrat. Da sich ein horizontales Auslaufen der Ermüdungsfestigkeitskurve in den durchgeführten Ermüdungsversuchen zeigt, wurde für die Auswertung des Übergangsbereichs in der vorliegenden Arbeit das Treppenstufenverfahren gewählt.

Ursprünglich geht das Treppenstufenverfahren auf Dixon und Mood [Dixon, 1948] zurück. Das Auswerteschema ist beispielsweise in DIN 969 [DIN 969, 1997] enthalten. Das Treppenstufenverfahren kalibriert sich automatisch um den Mittelwert. Der Ablauf der Versuche entspricht einem Folgeverfahren. Eine stufenweise Veränderung der Beanspruchungsamplitude erfolgt nach jedem Versuch, wobei die Höhe der Beanspruchungsamplitude des aktuellen Versuchs anhand der Ergebnisse aus dem vorigen Versuch ermittelt wird. Tritt ein Bruch auf, so ist die Beanspruchungsamplitude bei den nächsten Proben so lange um den gewählten (in der Regel konstanten) Stufensprung zu senken, bis ein Nichtbruch auftritt. Bei einem Nichtbruch ist die Beanspruchungsamplitude so lange um den gewählten Stufensprung zu erhöhen, bis ein Bruch auftritt. Das insgesamt über die Versuchsserie weniger häufig auftretende 
Ergebnis (Bruch oder Nichtbruch) wird zur Berechnung des Mittelwertes und der Standardabweichung verwendet.

Dieses Verfahren wurde von Hück [Hück, 1983] mit dem Ergebnis überprüft, dass die Standardabweichung zu gering ermittelt wird und dass keine optimalen Vertrauensgrenzen bei der Verwendung der weniger häufig auftretenden Ergebnisse erzielt werden können. Aus diesem Grund wurde das Verfahren von Hück [Hück, 1983] weiterentwickelt. Es werden nun alle Ergebnisse in die Auswertung mit einbezogen. Für eine gute Schätzung der Standardabweichung ist allerdings ein Probenumfang mit mehr als 25 Versuchen erforderlich [Mauch, 1999].

\subsubsection{Erneute Prüfung von Durchläufern}

Aus Zeit- und Kostengründen sind die Stichprobenumfänge bei der Ermüdungsprüfung vielfach begrenzt. Um dennoch auswertbare Versuchsreihen zu erhalten und/oder die statistische Aussagekraft der Versuchsreihen zu erhöhen, aber auch Erkenntnisse über eine mögliche Vorschädigung oder einen Trainingseffekt der Proben zu erhalten, wird die erneute Prüfung von Durchläufern auf höheren Beanspruchungsniveaus durchgeführt [Kuhlmann, 2006] [Morgenstern, 2006] [Weich, 2008] [Schwerdt, 2009]. Die Höhe der gewählten Beanspruchungsniveaus bei erneuter Prüfung der Durchläufer reicht von geringfügigen Erhöhungen bis hin zur Verdoppelung der Beanspruchungsamplitude.

Die Auswirkungen bei erneuter Prüfung von Durchläufern im Treppenstufenverfahren wurde von Müller et al. [Müller, 2012] anhand von Simulationen diskutiert. Hierbei wurden drei Zustände eines Durchläufers unterschieden, die sich auf das Ergebnis der erneuten Prüfung auswirken können. Zum Einen kann eine bereits geprüfte Probe eine Vorschädigung oder einen Trainingseffekt aufweisen. Dies kann sich in einer geringeren sowie höheren Ermüdungsfestigkeit niederschlagen. Zum Anderen kann ein Durchläufer durch die Prüfung bis zur Grenzschwingspielzahl in keiner Weise beeinflusst sein. Generell stellen Durchläufer jedoch Proben mit erhöhter Festigkeit dar, die bei erneuter Prüfung zur Überschätzung des Gesamtergebnisses führen. Tritt bei der erneuten Prüfung im Übergangsbereich bei allen Durchläufern ein Bruch auf, so kann beispielweise auf eine mögliche Vorschädigung geschlossen werden. Im Gegensatz dazu stellt bei erneuter Prüfung aller Durchläufer im Übergangsbereich das einheitliche Eintreten von Durchläufern einen Hinweis auf einen möglichen Trainingseffekt dar. Im Hinblick auf die Einbeziehung von erneut geprüften Durchläufern in die Auswertung mit dem Treppenstufenverfahren wird von Müller et al. auf eine denkbare Über- bzw. Unterschätzung der Dauerfestigkeit hingewiesen und empfohlen Durchläufer lediglich ein- bis zweimal erneut zu prüfen. 
Um Informationen im Bezug auf mögliche Vorschädigungen oder Trainingseffekte aus den erzielten Durchläufern der vorliegenden Arbeit ableiten zu können, wurden diese nach erfolgter Anrissprüfung mit dem Farbeindringverfahren erneut geprüft. Die Beanspruchungsniveaus variieren dabei und liegen im Zeitfestigkeitsbereich.

\subsection{Beurteilung der Versuchsbedingungen}

Um die Ergebnisse der Ermüdungsversuche aus Versuchsreihe 2 und 3 in der hochfrequenten Prüfvorrichtung im Hinblick auf die Versuchsbedingungen beurteilen zu können, werden die Soll- und Istwerte der Kraftamplitude während des Versuchs und der Vorspannkraft zu Versuchsbeginn betrachtet. Daraufhin wird auf den in Kapitel 4.3.3 zu Versuchsbeginn beobachteten temperaturbedingten Abfall der Vorspannkraft eingegangen, aus dem auch ein Abfall des Spannungsverhältnisses resultiert.

\subsubsection{Soll- und Istwerte von Kraftamplitude und Vorspannkraft}

Zunächst werden die vorgegebenen Sollwerte und die erreichten Istwerte der Kraftamplitude $F_{a}$ über die Versuchslaufzeit und der Vorspannkraft $F_{v}$ zu Versuchsbeginn betrachtet. Außerdem wird das bei Versuchsbeginn theoretisch vorliegende Spannungsverhältnis $R_{\text {Start }}$ für die untersuchten Beanspruchungsniveaus ausgewertet. Für Versuchsreihe 2 sind die Werte auf den untersuchten Beanspruchungsniveaus in Tab. 5-3 gegenübergestellt. Die Anzahl der ausgewerteten Versuche $\mathrm{n}$ sowie die prozentuale Abweichung $p_{\%}$ vom entsprechenden Sollwert sind angegeben. Der Sollwert der Kraftamplitude $F_{a, \text { Soll }}$ wurde auf allen Beanspruchungsniveaus im Mittel mit einer Abweichung $\mathrm{p}_{\%, \mathrm{Fa}}$ von $0,17 \%$ erreicht. Der Sollwert der Vorspannkraft $\mathrm{F}_{\mathrm{v} \text {,Soll }}$ zu Versuchsbeginn wurde auf allen Beanspruchungsniveaus im Mittel mit einer Abweichung $p_{\%, F v}$ von $0,69 \%$ erzielt. Der Mittelwert des bei Versuchsbeginn theore-

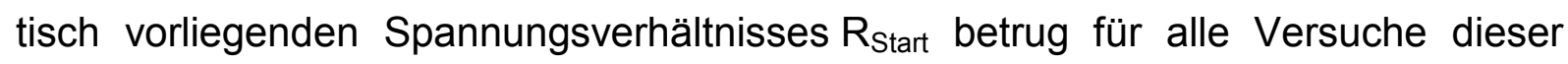
Reihe 0,102.

Tab. 5-3: Vergleich der Soll- und Istwerte von Kraftamplitude $F_{\mathrm{a}}$ und Vorspannkraft $F_{\mathrm{v}}$ für Versuchsreihe 2

\begin{tabular}{|c|c|c|c|c|c|c|c|c|}
\hline $\begin{array}{c}\Delta \boldsymbol{\sigma} \\
{\left[\mathbf{N} / \mathbf{m m}^{2}\right]}\end{array}$ & $\begin{array}{c}\mathbf{n} \\
{[-]}\end{array}$ & $\begin{array}{c}\mathbf{F}_{\mathrm{a}, \text { Soll }} \\
{[\mathbf{k N}]}\end{array}$ & $\begin{array}{c}\mathbf{F}_{\mathrm{a}, \text { st }} \\
{[\mathbf{k N}]}\end{array}$ & $\begin{array}{c}\mathbf{p}_{\%, \mathrm{Fa}} \\
{[\%]}\end{array}$ & $\begin{array}{c}\mathbf{F}_{\mathrm{v}, \text { Soll }} \\
{[\mathbf{k N}]}\end{array}$ & $\begin{array}{c}\mathbf{F}_{\mathrm{v}, \text { Ist }} \\
{[\mathbf{k N}]}\end{array}$ & $\begin{array}{c}\mathbf{p}_{\%, \mathrm{Fv}} \\
{[\%]}\end{array}$ & $\begin{array}{c}\mathbf{R}_{\text {Start }} \\
{[-]}\end{array}$ \\
\hline 277,3 & 9 & 13,87 & 13,89 & 0,15 & 16,95 & 16,97 & 0,11 & 0,100 \\
\hline 221,9 & 10 & 11,09 & 11,11 & 0,17 & 13,56 & 13,68 & 0,88 & 0,103 \\
\hline 162,5 & 2 & 8,13 & 8,14 & 0,23 & 9,93 & 10,06 & 1,26 & 0,105 \\
\hline 150,0 & 5 & 7,50 & 7,52 & 0,31 & 9,17 & 9,23 & 0,71 & 0,102 \\
\hline 137,5 & 6 & 6,88 & 6,88 & 0,11 & 8,40 & 8,40 & 0,09 & 0,099 \\
\hline 125,0 & 3 & 6,25 & 6,25 & 0,03 & 7,64 & 7,72 & 1,10 & 0,106 \\
\hline
\end{tabular}


Die Ergebnisse für die untersuchten Beanspruchungsniveaus von Versuchsreihe 3 zeigt Tab. 5-4. Im Mittel wurde der Sollwert der Kraftamplitude $F_{a, S o l l ~}$ auf allen Beanspruchungsniveaus mit einer Abweichung $p_{\%, F a}$ von $0,18 \%$ erzielt. Mit einer Abweichung $p_{\%, F v}$ von $0,49 \%$ wurde der Sollwert der Vorspannkraft $F_{v, S o l l}$ zu Versuchsbeginn auf allen Beanspruchungsniveaus im Mittel erreicht. Außerdem sind die Mittelwerte des bei Versuchsbeginn theoretisch vorliegenden Spannungsverhältnisses $R_{\text {Start }}$ für die untersuchten Beanspruchungsniveaus dargestellt. Bei Versuchsbeginn betrug der Mittelwert des theoretisch vorliegenden Spannungsverhältnisses $R_{\text {Start }}$ für alle Versuche dieser Reihe im Mittel 0,501.

Tab. 5-4: Vergleich der Soll- und Istwerte von Kraftamplitude $F_{\mathrm{a}}$ und Vorspannkraft $F_{\mathrm{v}}$ für Versuchsreihe 3

\begin{tabular}{|c|c|c|c|c|c|c|c|c|}
\hline $\begin{array}{c}\Delta \boldsymbol{\sigma} \\
{\left[\mathbf{N} / \mathbf{m m}^{2}\right]}\end{array}$ & $\begin{array}{c}\mathbf{n} \\
{[-]}\end{array}$ & $\begin{array}{c}\mathbf{F}_{\mathrm{a}, \text { Soll }} \\
{[\mathbf{k N}]}\end{array}$ & $\begin{array}{c}\mathbf{F}_{\mathrm{a}, \text { lst }} \\
{[\mathbf{k N}]}\end{array}$ & $\begin{array}{c}\mathbf{p}_{\%, \mathrm{Fa}} \\
{[\%]}\end{array}$ & $\begin{array}{c}\mathbf{F}_{\mathrm{v}, \text { Soll }} \\
{[\mathbf{k N}]}\end{array}$ & $\begin{array}{c}\mathbf{F}_{\mathrm{v}, \text { lst }} \\
{[\mathbf{k N}]}\end{array}$ & $\begin{array}{c}\mathbf{p}_{\%, \mathrm{Fv}} \\
{[\%]}\end{array}$ & $\begin{array}{c}\mathbf{R}_{\text {Start }} \\
{[-]}\end{array}$ \\
\hline 187,5 & 8 & 9,38 & 9,37 & 0,02 & 28,13 & 28,14 & 0,05 & 0,500 \\
\hline 162,5 & 9 & 8,13 & 8,15 & 0,28 & 24,38 & 24,52 & 0,59 & 0,501 \\
\hline 150,0 & 2 & 7,50 & 7,53 & 0,38 & 22,50 & 22,61 & 0,49 & 0,500 \\
\hline 143,8 & 6 & 7,19 & 7,19 & 0,04 & 21,56 & 21,80 & 1,09 & 0,504 \\
\hline 137,5 & 6 & 6,88 & 6,89 & 0,27 & 20,63 & 20,70 & 0,38 & 0,500 \\
\hline 131,3 & 2 & 6,56 & 6,57 & 0,10 & 19,69 & 19,76 & 0,37 & 0,501 \\
\hline
\end{tabular}

Der Vergleich der Soll- und Istwerte der Kraftamplitude während des Versuchs und der Vorspannkraft zu Versuchsbeginn zeigt, dass für beide Versuchsreihen eine gute Reproduzierbarkeit der vorgegebenen Versuchsbedingungen erzielt wurde.

\subsubsection{Vorspannkraftabfall zu Versuchsbeginn}

Zu Versuchsbeginn fällt die Vorspannkraft zunächst ab und erreicht ein näherungsweise konstantes Plateau bis es zu einem weiteren Abfall aufgrund des Versagens der Probe kommt (siehe Abb. 4-9). Die Ausbildung dieses Plateaus ist umso ausgeprägter, je geringer das Beanspruchungsniveau ist. Aus dem Verlauf der Vorspannkraft im Bereich des näherungsweise konstanten Plateaus werden die Vorspannkraft $F_{v, A b f a l l}$ und das zugehörige Spannungsverhältnis $R_{A b f a l l}$ als Mittelwerte ausgewertet und für die unterschiedlichen Beanspruchungsniveaus beider Versuchsreihen verglichen.

In Tab. 5-5 sind die Mittelwerte der bei Versuchsbeginn eingestellten Vorspannkraft $F_{v, \text { lst }}$ und dem zugehörigen theoretisch vorliegenden Spannungsverhältnis $R_{S t a r t}$ sowie die Vorspannkraft $F_{v, A b f a l l}$ und das zugehörige Spannungsverhältnis $R_{A b f a l l}$ für 
die untersuchten Beanspruchungsniveaus aus Versuchsreihe 2 zusammengefasst. Die Anzahl der ausgewerteten Versuche $\mathrm{n}$ sowie die mittleren Differenzen $\Delta \mathrm{F}_{\mathrm{v}}$ und $\Delta R$ sind angegeben. Es ist deutlich zu erkennen, dass der Abfall der Vorspannkraft und des Spannungsverhältnisses auf den unteren Beanspruchungsniveaus stagniert und bei steigender Spannungsschwingbreite zunimmt.

Tab. 5-5: Abfall von Vorspannkraft $F_{v}$ und Spannungsverhältnis $R$ bei Versuchsreihe 2

\begin{tabular}{|c|c|c|c|c|c|c|c|}
\hline $\begin{array}{c}\Delta \boldsymbol{\sigma} \\
{\left[\mathbf{N} / \mathbf{m m}^{2}\right]}\end{array}$ & $\begin{array}{c}\mathbf{n} \\
{[-]}\end{array}$ & $\begin{array}{c}\mathbf{F}_{\mathbf{v} \text {, st }} \\
{[\mathbf{k N}]}\end{array}$ & $\begin{array}{c}\mathbf{F}_{\mathbf{v}, \text { Abfall }} \\
{[\mathbf{k N}]}\end{array}$ & $\begin{array}{c}\mathbf{\Delta} \mathbf{F}_{\mathbf{v}} \\
{[\mathbf{k N}]}\end{array}$ & $\begin{array}{c}\mathbf{R}_{\text {Start }} \\
{[-]}\end{array}$ & $\begin{array}{c}\mathbf{R}_{\text {Abfall }} \\
{[-]}\end{array}$ & $\begin{array}{c}\mathbf{\Delta R} \\
{[-]}\end{array}$ \\
\hline 277,3 & 9 & 16,97 & 15,42 & 1,54 & 0,100 & 0,052 & 0,048 \\
\hline 221,9 & 10 & 13,68 & 12,60 & 1,08 & 0,103 & 0,063 & 0,041 \\
\hline 162,5 & 2 & 10,06 & 9,41 & 0,64 & 0,105 & 0,072 & 0,033 \\
\hline 150,0 & 5 & 9,23 & 8,89 & 0,34 & 0,102 & 0,083 & 0,019 \\
\hline 137,5 & 6 & 8,40 & 8,18 & 0,22 & 0,099 & 0,086 & 0,013 \\
\hline 125,0 & 3 & 7,72 & 7,53 & 0,19 & 0,106 & 0,093 & 0,012 \\
\hline
\end{tabular}

Die Ergebnisse für die untersuchten Beanspruchungsniveaus aus Versuchsreihe 3 sind in Tab. 5-6 dargestellt. Analog zu Versuchsreihe 2 zeigt sich deutlich, dass der Abfall der Vorspannkraft und des Spannungsverhältnisses bei steigender Spannungsschwingbreite zunimmt, während die Werte auf den unteren Beanspruchungsniveaus stagnieren.

Tab. 5-6: Abfall von Vorspannkraft $F_{v}$ und Spannungsverhältnis $R$ bei Versuchsreihe 3

\begin{tabular}{|c|c|c|c|c|c|c|c|}
\hline $\begin{array}{c}\Delta \boldsymbol{\sigma} \\
{\left[\mathbf{N} / \mathbf{m m}^{2}\right]}\end{array}$ & $\begin{array}{c}\mathbf{n} \\
{[-]}\end{array}$ & $\begin{array}{c}\mathbf{F}_{\mathbf{v}, \text { Ist }} \\
{[\mathbf{k N}]}\end{array}$ & $\begin{array}{c}\mathbf{F}_{\mathbf{v}, \text { Abfall }} \\
{[\mathbf{k N}]}\end{array}$ & $\begin{array}{c}\mathbf{\Delta} \mathbf{F}_{\mathbf{v}} \\
{[\mathbf{k N}]}\end{array}$ & $\begin{array}{c}\mathbf{R}_{\text {Start }} \\
{[-]}\end{array}$ & $\begin{array}{c}\mathbf{R}_{\text {Abfall }} \\
{[-]}\end{array}$ & $\begin{array}{c}\mathbf{\Delta R} \\
{[-]}\end{array}$ \\
\hline 187,5 & 8 & 28,14 & 27,08 & 1,06 & 0,500 & 0,486 & 0,015 \\
\hline 162,5 & 9 & 24,52 & 23,58 & 0,94 & 0,501 & 0,486 & 0,015 \\
\hline 150,0 & 2 & 22,61 & 21,82 & 0,79 & 0,500 & 0,487 & 0,014 \\
\hline 143,8 & 6 & 21,80 & 21,17 & 0,63 & 0,504 & 0,493 & 0,011 \\
\hline 137,5 & 6 & 20,70 & 20,16 & 0,54 & 0,500 & 0,490 & 0,010 \\
\hline 131,3 & 2 & 19,76 & 19,35 & 0,41 & 0,501 & 0,493 & 0,008 \\
\hline
\end{tabular}

Im Hinblick auf die Ergebnisse der Ermüdungsversuche der Versuchsreihen 2 und 3 liegen auf den oberen Beanspruchungsniveaus günstigere Versuchsbedingungen als auf den unteren Beanspruchungsniveaus vor. Diese können bei Brüchen zu erhöhten Schwingspielzahlen führen. Im Vergleich zu idealen Versuchsbedingungen mit konstantem Spannungsverhältnis sind die Versuchsbedingungen prinzipiell als günstiger 
einzustufen. Dies kann generell und besonders auf den oberen Beanspruchungsniveaus zu einer erhöhten Lebensdauer führen, was in die Diskussion in Kapitel 5.5.2 mit einbezogen wird.

\subsubsection{Erwärmung der Probekörper}

Der beobachtete Abfall der Vorspannkraft zu Versuchsbeginn wurde auf die Erwärmung des Probekörpers infolge innerer Reibung zurückgeführt (siehe Kapitel 4.3.3), wohingegen die Erwärmung beim Erreichen des Abbruchkriteriums für Versagen auf die Schädigung der Probe zurückzuführen ist. Um die Erwärmung der Probekörper beurteilen zu können, wurde der Großteil der Ermüdungsversuche mit Temperaturmessungen begleitet. Hierzu wurden zwei Thermoelemente im Abstand von 15 und $30 \mathrm{~mm}$ von der Nahtmitte aus seitlich am Probekörper in Blechmitte appliziert (siehe Abb. 4-9).

Im Anhang $\mathrm{H}$ sind die Temperaturen beider Thermoelemente zu Versuchsbeginn $T_{\text {Start }}$ und auf dem näherungsweise konstanten Vorspannkraft- bzw. Temperaturplateau $T_{\text {Anstieg }}$ der einzelnen Versuche tabellarisch zusammengefasst. Desweiteren sind die Temperaturdifferenzen $\Delta T$ von Temperaturanstieg und Starttemperatur sowie die erreichten Maximaltemperaturen $\mathrm{T}_{\max }$ beim Erreichen des Abbruchkriteriums bei Versagen der Probe angegeben. Analog zum Vorspannkraftplateau, bildet sich ein Temperaturplateau aus, auf dem die Temperaturen entsprechend ausgewertet wurden. Lediglich auf dem obersten Beanspruchungsniveau von Versuchsreihe 2 ist ein kontinuierlicher Anstieg der Temperatur zu verzeichnen. Die Auswertung der Temperaturen für dieses Beanspruchungsniveau wurde aus dem Verlauf der Vorspannkraft abgeleitet.

Eine Übersicht der Mittelwerte der Erwärmung der Probekörper ist in Tab. 5-7 für Versuchsreihe 2 gegeben. Es kann abgeleitet werden, dass die Erwärmung der Probekörper mit zunehmender Spannungsschwingbreite zunimmt.

Tab. 5-7: Mittelwerte der Erwärmung der Probekörper aus Versuchsreihe 2

\begin{tabular}{|c|c|c|c|c|c|c|c|c|}
\hline $\begin{array}{c}\Delta \boldsymbol{\sigma} \\
{\left[\mathbf{N} / \mathbf{m m}^{2}\right]}\end{array}$ & $\begin{array}{c}\mathbf{T}_{1, \text { Start }} \\
{\left[{ }^{\circ} \mathbf{C}\right]}\end{array}$ & $\begin{array}{c}\mathbf{T}_{2, \text { Start }} \\
{\left[{ }^{\circ} \mathbf{C}\right]}\end{array}$ & $\begin{array}{c}\mathbf{T}_{1, \text { Anstieg }} \\
{\left[{ }^{\circ} \mathbf{C}\right]}\end{array}$ & $\begin{array}{c}\mathbf{T}_{2, \text { Anstieg }} \\
{\left[{ }^{\circ} \mathbf{C}\right]}\end{array}$ & $\begin{array}{c}\Delta \mathbf{T}_{1} \\
{[\mathbf{K}]}\end{array}$ & $\begin{array}{c}\Delta \mathbf{T}_{2} \\
{[\mathbf{K}]}\end{array}$ & $\begin{array}{c}\mathbf{T}_{1, \max } \\
{\left[{ }^{\circ} \mathbf{C}\right]}\end{array}$ & $\begin{array}{c}\mathbf{T}_{2, \max } \\
{\left[{ }^{\circ} \mathbf{C}\right]}\end{array}$ \\
\hline 277,3 & 24,8 & 24,8 & $34,0^{*}$ & $34,5^{*}$ & $9,2^{*}$ & $9,7^{*}$ & 41,0 & 39,7 \\
\hline 221,9 & 21,7 & 21,7 & 31,1 & 31,7 & 9,4 & 10,0 & 35,3 & 33,8 \\
\hline variabel & 24,3 & 24,2 & 26,8 & 26,8 & 2,6 & 2,6 & 29,3 & 28,4 \\
\hline
\end{tabular}

* kein Temperaturplateau 
Im Übergangsbereich erwärmen sich die Probekörper im Mittel um 2,6 K. Die mittlere Erwärmung der Probekörper im Zeitfestigkeitsbereich beträgt hingegen über 9,0 K. Auch die Maximaltemperaturen $\mathrm{T}_{\max }$ beim Erreichen des Abbruchkriteriums bei Versagen der Probe nehmen mit steigender Spannungsschwingbreite zu. Im Übergangsbereich zeigen die Temperaturmessungen in Versuchen, bei denen der Probekörper nicht versagt hat, ähnliche Erwärmungen $T_{\text {Anstieg }}$ im Vergleich zu Proben mit Versagen.

In Tab. 5-8 sind die Mittelwerte der Erwärmung der Probekörper auf den unterschiedlichen Beanspruchungsniveaus von Versuchsreihe 3 angegeben. Die Erwärmung der Probekörper nimmt, wie bereits bei Versuchsreihe 2 beobachtet, mit zunehmender Spannungsschwingbreite zu. Die Probekörper erwärmen sich im Übergangsbereich im Mittel um 3,5 K. Im Zeitfestigkeitsbereich beläuft sich die mittlere Erwärmung der Probekörper hingegen auf 4,8 K sowie 7,3 K. Mit steigender Spannungsschwingbreite nehmen die Maximaltemperaturen $\mathrm{T}_{\max }$ beim Erreichen des Abbruchkriteriums bei Versagen der Probe ebenfalls zu. Analog zu Versuchsreihe 2 weisen die Probekörper, die im Übergangsbereich nicht versagt haben, eine ähnliche Erwärmung $T_{\text {Anstieg }}$ im Vergleich zu den Probekörpern, die im Übergangsbereich versagt haben, auf.

Tab. 5-8: Mittelwerte der Erwärmung der Probekörper aus Versuchsreihe 3

\begin{tabular}{|c|c|c|c|c|c|c|c|c|}
\hline $\begin{array}{c}\Delta \sigma \\
{\left[\mathrm{N} / \mathrm{mm}^{2}\right]}\end{array}$ & $\begin{array}{c}\mathrm{T}_{1, \mathrm{Start}} \\
{\left[{ }^{\circ} \mathrm{C}\right]}\end{array}$ & $\begin{array}{c}\mathbf{T}_{2, \text { Start }} \\
{\left[{ }^{\circ} \mathrm{C}\right]}\end{array}$ & $\begin{array}{c}\mathrm{T}_{1, \text { Anstieg }} \\
{\left[{ }^{\circ} \mathrm{C}\right]}\end{array}$ & $\begin{array}{c}\mathrm{T}_{2, \text { Anstieg }} \\
{\left[{ }^{\circ} \mathrm{C}\right]}\end{array}$ & $\begin{array}{l}\Delta \mathrm{T}_{1} \\
{[\mathrm{~K}]}\end{array}$ & $\begin{array}{l}\Delta \mathrm{T}_{2} \\
{[\mathrm{~K}]}\end{array}$ & $\begin{array}{l}\mathrm{T}_{1, \max } \\
{\left[{ }^{\circ} \mathrm{C}\right]}\end{array}$ & $\begin{array}{c}\mathrm{T}_{2, \max } \\
{\left[{ }^{\circ} \mathrm{C}\right]}\end{array}$ \\
\hline 187,5 & 24,1 & 24,1 & 31,3 & 31,4 & 7,2 & 7,3 & 34,1 & 33,1 \\
\hline 162,5 & 23,7 & 23,7 & 28,5 & 28,5 & 4,9 & 4,8 & 31,1 & 29,9 \\
\hline variabel & 22,0 & 22,0 & 25,4 & 25,4 & 3,4 & 3,5 & 28,3 & 27,1 \\
\hline
\end{tabular}

Die Größenordnung der gemessenen Erwärmung der Probekörper beider Versuchsreihen ist ähnlich und liegt unterhalb dem in DIN 969 [DIN 969, 1997] angegebenen Maximalwert von $50^{\circ} \mathrm{C}$. Bei Versuchen mit Ultraschallprüfmaschinen wird die Probekörpererwärmung durch Kühlung und/oder den Betrieb mit Beanspruchungspausen auf unterschiedliche Maximaltemperaturen begrenzt, wie beispielsweise $25^{\circ} \mathrm{C}$ [Holper, 2004], $35^{\circ} \mathrm{C}$ [Tsutsumi, 2009] oder $40^{\circ} \mathrm{C}$ [Schwerdt, 2009]. Die gemessenen Temperaturen sind als globale Probentemperatur zu interpretieren und zeigen die Größenordnung der Erwärmung auf. Mangels ausreichender Zugänglichkeit der Probekörper innerhalb der Prüfvorrichtung wurden keine Temperaturmessungen an den geometrischen Kerben der Schweißnaht, wo höhere Temperaturen erwarten werden können, durchgeführt. 
Beide Versuchsreihen zeigen, dass die Erwärmung der Probekörper zu Versuchsbeginn infolge innerer Reibung abhängig von der Höhe der Beanspruchung ist. Je höher das Beanspruchungsniveau ist, desto größer ist die Erwärmung des Probekörpers und desto ausgeprägter ist der Abfall von Vorspannkraft und Spannungsverhältnis. Die Ergebnisse der Temperaturmessungen weisen allerdings deutliche Streuungen auf, was auf der einen Seite auf die Genauigkeit bei der Applikation der Thermoelemente und auf der anderen Seite auf die allgemein hohe Streuung im Bezug auf Ermüdungsversuche und der lokalen Nahtgeometrie der Probekörper zurückgeführt werden kann. Die Streuungen der gemessenen Temperaturen von Versuchsreihe 3 sind im Vergleich zu Versuchsreihe 2 geringer, da auf niedrigeren Beanspruchungsniveaus im Zeitfestigkeitsbereich geprüft wurde.

Für Versuche im Übergangsbereich wird der Einfluss der Erwärmung der Probekörper und der damit verbundene Abfall von Vorspannkraft und Spannungsverhältnis auf die Ergebnisse von Ermüdungsversuchen als gering eingestuft. Im Zeitfestigkeitsbereich können der Einfluss der Erwärmung der Probekörper und die damit verbundenen Effekte nicht ohne Weiteres als gering eingestuft werden. Um zu einer Aussage zu kommen, bieten sich mehrere Möglichkeiten an. Zum einen könnten weitere Versuche bei unterschiedlichen Prüffrequenzen Aufschluss über den Einfluss der Probekörpererwärmung und die daraus resultierenden Effekte auf die Ermüdungsfestigkeit geben. Hierzu könnten die Hebelarme der Prüfmaschine verlängert werden, um eine geringerer Prüffrequenz zu realisieren. Auf der anderen Seite könnte der Erwärmung der Probekörper durch Kühlung der Probekörper und/oder einem Betrieb mit Beanspruchungspausen entgegengewirkt werden, wobei ein Betrieb mit Beanspruchungspausen die Versuchslaufzeit erhöhen würde.

\subsection{Versuchsergebnisse}

Zunächst werden die Versuchsergebnisse der einzelnen Versuchsreihen ausgewertet und dargestellt. Daraufhin wird der Einfluss von Prüffrequenz und Mittelspannung auf die Versuchsergebnisse diskutiert. Desweiteren werden das Verhalten der hochgesetzten Durchläufer sowie die Bruchflächen beurteilt. Das Verhalten der Probekörper im Bereich sehr hoher Lastwechselzahlen wird zusammengefasst betrachtet. Daran schließt der Vergleich der Versuchsergebnisse der vorliegenden Arbeit mit der normierten Auswertung von Ermüdungsversuchen aus Sonsino et al. [Sonsino, 2005b] an. Abschließend erfolgt die Auswertung der Versuchsergebnisse gemäß dem Hintergrundbericht zum Eurocode 3. Die Ergebnisse werden dargestellt und im Vergleich zur Kerbfallklasse gemäß Eurocode 3 [DIN EN 1993-1-9, 2010] für das untersuchte Konstruktionsdetail bewertet. 


\subsubsection{Allgemeine Auswertung und Darstellung der Versuchsergebnisse}

Die allgemeine Auswertung der Versuchsergebnisse im Zeitfestigkeitsbereich (A) und Übergangsbereich (B) wird im Folgenden für die jeweiligen Versuchsreihen 1 bis 3 dargestellt. Hierbei ist die Nennspannungsschwingbreite $\Delta \sigma$ für Versuchsreihe 1 über der Bruchschwingspielzahl $N_{B}$ sowie für die Versuchsreihen 2 und 3 über der Anrissschwingspielzahl $\mathrm{N}_{\mathrm{A}}$ im Wöhlerdiagramm aufgetragen. Neben den Einzelversuchen sind die ermittelten Bruch- bzw. Anrisswöhlerlinien für die Überlebenswahrscheinlichkeit $\mathrm{P}_{\mathrm{ü}}$ von $10 \%, 50 \%$ und $90 \%$ aufgetragen. Die Ergebnisse aller Einzelversuche aus den Versuchsreihen 1 bis 3 sind im Anhang I.1 bis I.3 tabellarisch aufgeführt.

Die Neigungsexponenten $k$ der Wöhlerlinien im Zeitfestigkeitsbereich (A) wurden durch lineare Regressionsrechnung ermittelt. Die Auswertung der Ergebnisse im Übergangsbereich (B) erfolgte mit dem Treppenstufenverfahren nach Hück [Hück, 1983] (siehe Anhang J.1). Aus dem Schnittpunkt der Zeitfestigkeitsgeraden und der Dauerfestigkeit ergibt sich der Abknickpunkt.

Das Horizontenverfahren ermöglicht für jeden Beanspruchungshorizont eine getrennte Auswertung der Streuung. Ein Maß für die Streuung ist die Streuspanne $T_{N}$, die sich aus den Quotienten der Bruchschwingspielzahlen $N_{B}$ bzw. der Anrissschwingspielzahlen $\mathrm{N}_{\mathrm{A}}$ für eine Überlebenswahrscheinlichkeit $\mathrm{P}_{u}$ von $10 \%$ und $90 \%$ ergibt. Im Übergangsbereich wird die Streuspanne $T_{\sigma}$, die sich aus den Quotienten der Spannungsschwingbreiten $\Delta \sigma_{D}$ der Dauerfestigkeit für eine Überlebenswahrscheinlichkeit $\mathrm{P}_{u ̈}$ von $10 \%$ und $90 \%$ ergibt, als Maß für die Streuung angegeben.

\section{Versuchsreihe 1}

Die Versuchsergebnisse aus Versuchsreihe 1-A und 1-B sind in Abb. 5-1 zusammengefasst. Der Neigungsexponent k der Wöhlerlinie im Zeitfestigkeitsbereich (1-A) wurde zu 3,8 ( $\left.P_{u}=50 \%\right)$ ermittelt. Die Auswertung der Ergebnisse im Übergangsbereich (1-B) liefert eine mittlere Dauerfestigkeit $\Delta \sigma_{D, 50 \%}$ von $160,7 \mathrm{~N} / \mathrm{mm}^{2}$. Der Abknickpunkt $\mathrm{N}_{\mathrm{k}, 50 \%}$ zur Dauerfestigkeit ergibt sich bei 913.750 Lastwechseln. 


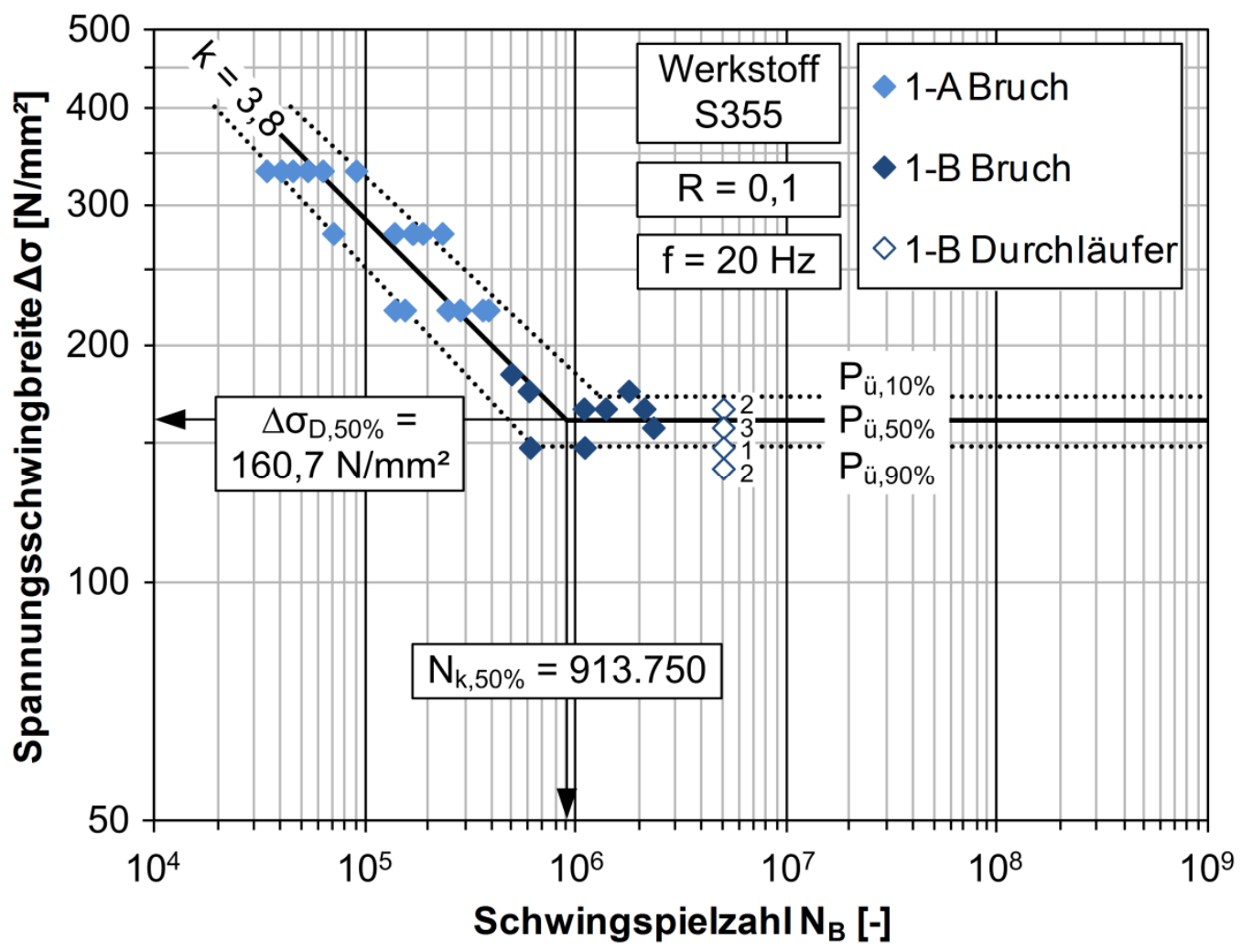

Abb. 5-1: Ergebnisse der Versuchsreihen 1-A und 1-B

Für die drei Beanspruchungshorizonte im Zeitfestigkeitsbereich (1-A) ist die Auswertung der jeweiligen Streuung in Tab. 5-9 dargestellt. Die Streuspanne $T_{N}$ auf den drei Beanspruchungshorizonten nimmt mit abnehmender Spannungsschwingbreite erwartungsgemäß zu und reicht von 2,51 bis 3,04. Im Vergleich dazu liefert die Auswertung der Ergebnisse für ein konstantes Streuband im Zeitfestigkeitsbereich (1-A) über alle Beanspruchungshorizonte eine Streuspanne $T_{N}$ von 2,76. Im Übergangsbereich (1-B) ergibt sich eine Streuspanne $T_{\sigma}$ von 1,15.

Tab. 5-9: Auswertung der Streuung auf den Beanspruchungshorizonten von Versuchsreihe 1-A

\begin{tabular}{|c|c|c|c|c|}
\hline $\begin{array}{c}\Delta \boldsymbol{\sigma} \\
{\left[\mathbf{N} / \mathbf{m m}^{2}\right]}\end{array}$ & $\begin{array}{c}\mathbf{N}_{\mathrm{B}, \mathbf{5 0} \%} \\
{[-]}\end{array}$ & $\begin{array}{c}\mathbf{N}_{\mathrm{B}, 10 \%} \\
{[-]}\end{array}$ & $\begin{array}{c}\mathbf{N}_{\mathrm{B}, 90 \%} \\
{[-]}\end{array}$ & $\begin{array}{c}\mathbf{T}_{\mathbf{N}} \\
{[-]}\end{array}$ \\
\hline 332,8 & 59.012 & 93.403 & 37.279 & 2,51 \\
\hline 277,3 & 117.177 & 193.779 & 70.855 & 2,73 \\
\hline 221,9 & 271.300 & 473.396 & 155.498 & 3,04 \\
\hline
\end{tabular}




\section{Versuchsreihe 2}

In Abb. 5-2 sind die Versuchsergebnisse aus Versuchsreihe 2-A und 2-B zusammengefasst. Im Zeitfestigkeitsbereich (2-A) ergibt sich der Neigungsexponent $k$ der Wöhlerlinie zu 4,1 ( $\mathrm{P}_{\ddot{u}}=50 \%$ ). Im Übergangsbereich (2-B) liefert die Auswertung der Ergebnisse eine mittlere Dauerfestigkeit $\Delta \sigma_{D, 50 \%}$ von $141,9 \mathrm{~N} / \mathrm{mm}^{2}$. Bei 1.901 .787 Lastwechseln ergibt sich der Abknickpunkt $\mathrm{N}_{k, 50 \%}$ zur Dauerfestigkeit.

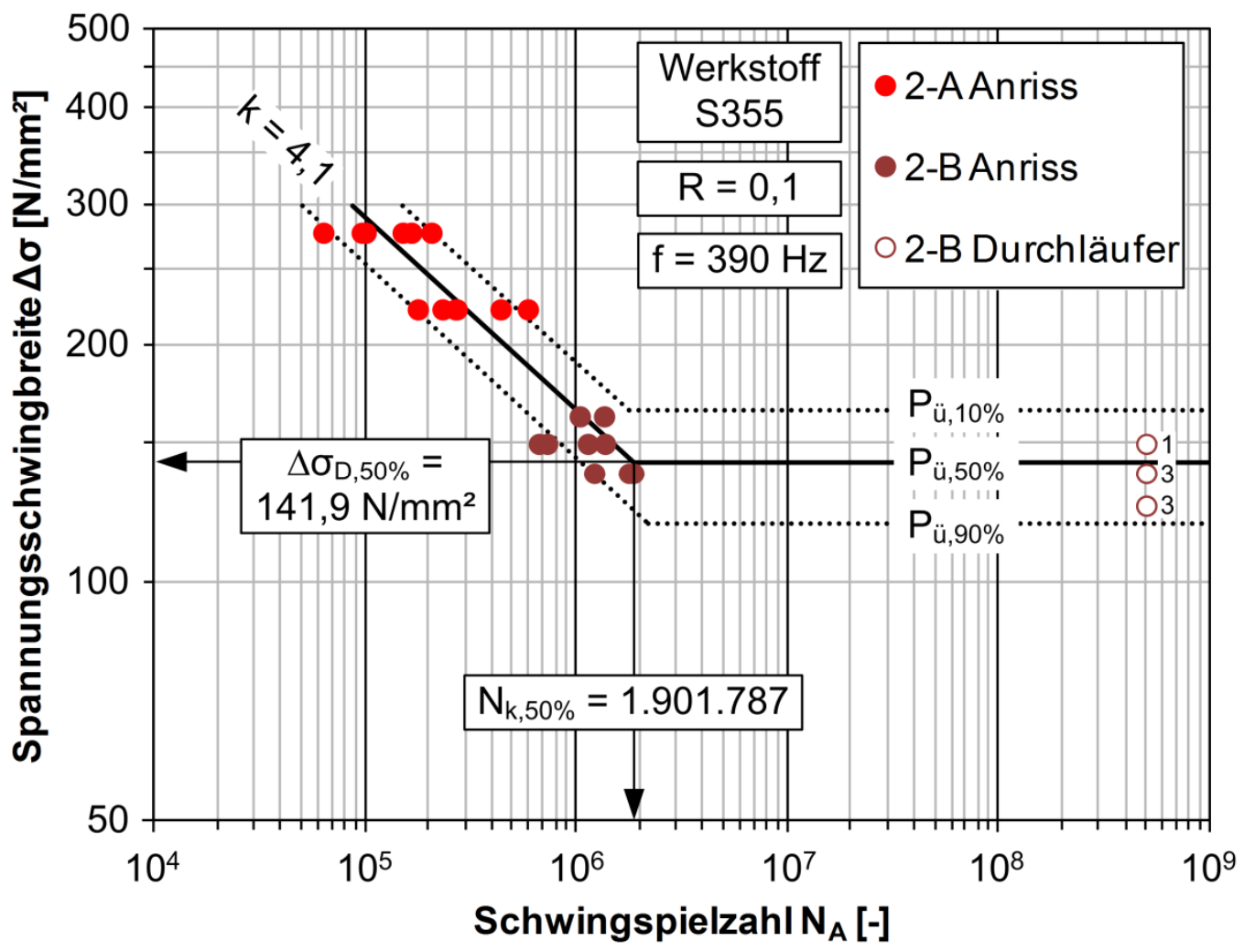

Abb. 5-2: Ergebnisse der Versuchsreihen 2-A und 2-B

In Tab. 5-10 ist die Auswertung der jeweiligen Streuung auf den beiden Beanspruchungshorizonten im Zeitfestigkeitsbereich (2-A) dargestellt. Die Streuspannen $T_{N}$ auf den beiden Beanspruchungshorizonten sind annähernd identisch. Es zeigt sich lediglich eine geringfügige Zunahme der Streuspanne $T_{N}$ bei abnehmender Spannungsschwingbreite von 3,02 auf 3,07.

Tab. 5-10: Auswertung der Streuung auf den Beanspruchungshorizonten von Versuchsreihe 2-A

\begin{tabular}{|c|c|c|c|c|}
\hline $\begin{array}{c}\Delta \boldsymbol{\sigma} \\
{\left[\mathbf{N} / \mathbf{m m}^{2}\right]}\end{array}$ & $\begin{array}{c}\mathbf{N}_{\mathrm{A}, 50 \%} \\
{[-]}\end{array}$ & $\begin{array}{c}\mathbf{N}_{\mathrm{A}, 10 \%} \\
{[-]}\end{array}$ & $\begin{array}{c}\mathbf{N}_{\mathrm{A}, 90 \%} \\
{[-]}\end{array}$ & $\begin{array}{c}\mathbf{T}_{\mathbf{N}} \\
{[-]}\end{array}$ \\
\hline 277,3 & 120.780 & 210.019 & 69.460 & 3,02 \\
\hline 221,9 & 302.512 & 530.272 & 172.579 & 3,07 \\
\hline
\end{tabular}


Die Auswertung der Ergebnisse für ein konstantes Streuband im Zeitfestigkeitsbereich (2-A) über alle Beanspruchungshorizonte liefert eine Streuspanne $T_{N}$ von 3,05. Die Streuspanne $T_{\sigma}$ im Übergangsbereich (2-B) ergibt sich zu 1,39.

\section{Versuchsreihe 3}

In Abb. 5-3 sind die Versuchsergebnisse der Reihen 3-A und 3-B dargestellt. Die Auswertung der Wöhlerlinie im Zeitfestigkeitsbereich (3-A) liefert einen Neigungsexponenten $\mathrm{k}$ von $4,7 \quad\left(P_{u}=50 \%\right)$. Eine mittlere Dauerfestigkeit $\Delta \sigma_{D, 50 \%}$ von $140,4 \mathrm{~N} / \mathrm{mm}^{2}$ wurde im Übergangsbereich (3-B) ermittelt. Der Abknickpunkt $\mathrm{N}_{k, 50 \%}$ zur Dauerfestigkeit ergibt sich bei 1.239.512 Lastwechseln.

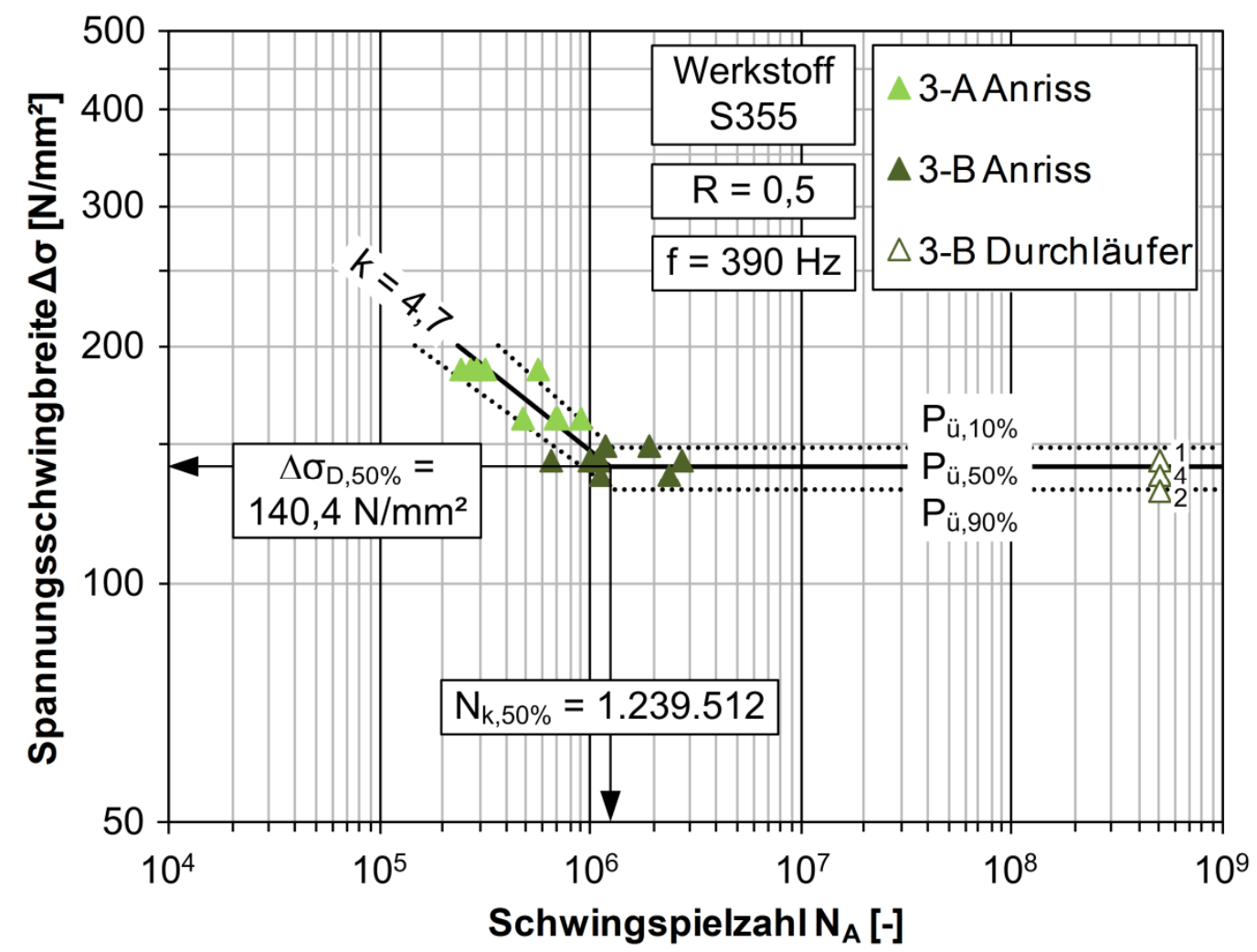

Abb. 5-3: Ergebnisse der Versuchsreihen 3-A und 3-B

Für die zwei Beanspruchungshorizonte im Zeitfestigkeitsbereich (3-A) ist die Auswertung der jeweiligen Streuung in Tab. 5-11 dargestellt. Entgegen der Erwartung nimmt die Streuspanne $T_{N}$ der beiden Beanspruchungshorizonte bei abnehmender Spannungsschwingbreite von 2,33 auf 2,01 ab. Dieses Verhalten wird auf den geringen Probenumfang bei der Prüfung in der Nähe des Übergangsbereichs, mit jeweils 5 Probekörpern pro Beanspruchungsniveau, zurückgeführt. Für diese Versuchsreihe ist demnach eine Auswertung mit einem konstanten Streuband im Zeitfestigkeitsbereich (3-A) zu bevorzugen. Hierbei ergibt sich über alle Beanspruchungshorizonte eine Streuspanne $T_{N}$ von 2,17. Im Übergangsbereich (1-B) ergibt sich eine Streuspanne $T_{\sigma}$ von 1,13 . 
Tab. 5-11: Auswertung der Streuung auf den Beanspruchungshorizonten von Versuchsreihe 3-A

\begin{tabular}{|c|c|c|c|c|}
\hline $\begin{array}{c}\Delta \boldsymbol{\sigma} \\
{\left[\mathbf{N} / \mathbf{m m}^{2}\right]}\end{array}$ & $\begin{array}{c}\mathbf{N}_{\mathrm{A}, 50 \%} \\
{[-]}\end{array}$ & $\begin{array}{c}\mathbf{N}_{\mathrm{A}, 10 \%} \\
{[-]}\end{array}$ & $\begin{array}{c}\mathbf{N}_{\mathrm{A}, 90 \%} \\
{[-]}\end{array}$ & $\begin{array}{c}\mathbf{T}_{\mathbf{N}} \\
{[-]}\end{array}$ \\
\hline 187,5 & 317.853 & 485.468 & 208.109 & 2,33 \\
\hline 162,5 & 623.572 & 884.158 & 439.789 & 2,01 \\
\hline
\end{tabular}

\subsubsection{Einfluss der Prüffrequenz}

Die Einzelergebnisse aus Versuchsreihe 1 und 2 sowie die jeweils ermittelten Wöhlerlinien mit einer Überlebenswahrscheinlichkeit $\mathrm{P}_{\ddot{u ̈}}$ von $50 \%$ sind in Abb. 5-4 dargestellt. Auf die Abbildung der Wöhlerlinien mit einer Überlebenswahrscheinlichkeit $\mathrm{P}_{\ddot{u}}$ von $10 \%$ und $90 \%$ wurde aus Gründen der Übersichtlichkeit verzichtet. Bei Versuchsreihe 1, ermittelt bei einer Prüffrequenz $f$ von $20 \mathrm{~Hz}$, handelt es sich um Bruchschwingspielzahlen und somit um eine Bruchwöhlerlinie, wohingegen es sich bei Versuchsreihe 2, ermittelt bei einer Prüffrequenz f von $390 \mathrm{~Hz}$, um Anrissschwingspielzahlen und somit um eine Anrisswöhlerlinie handelt.

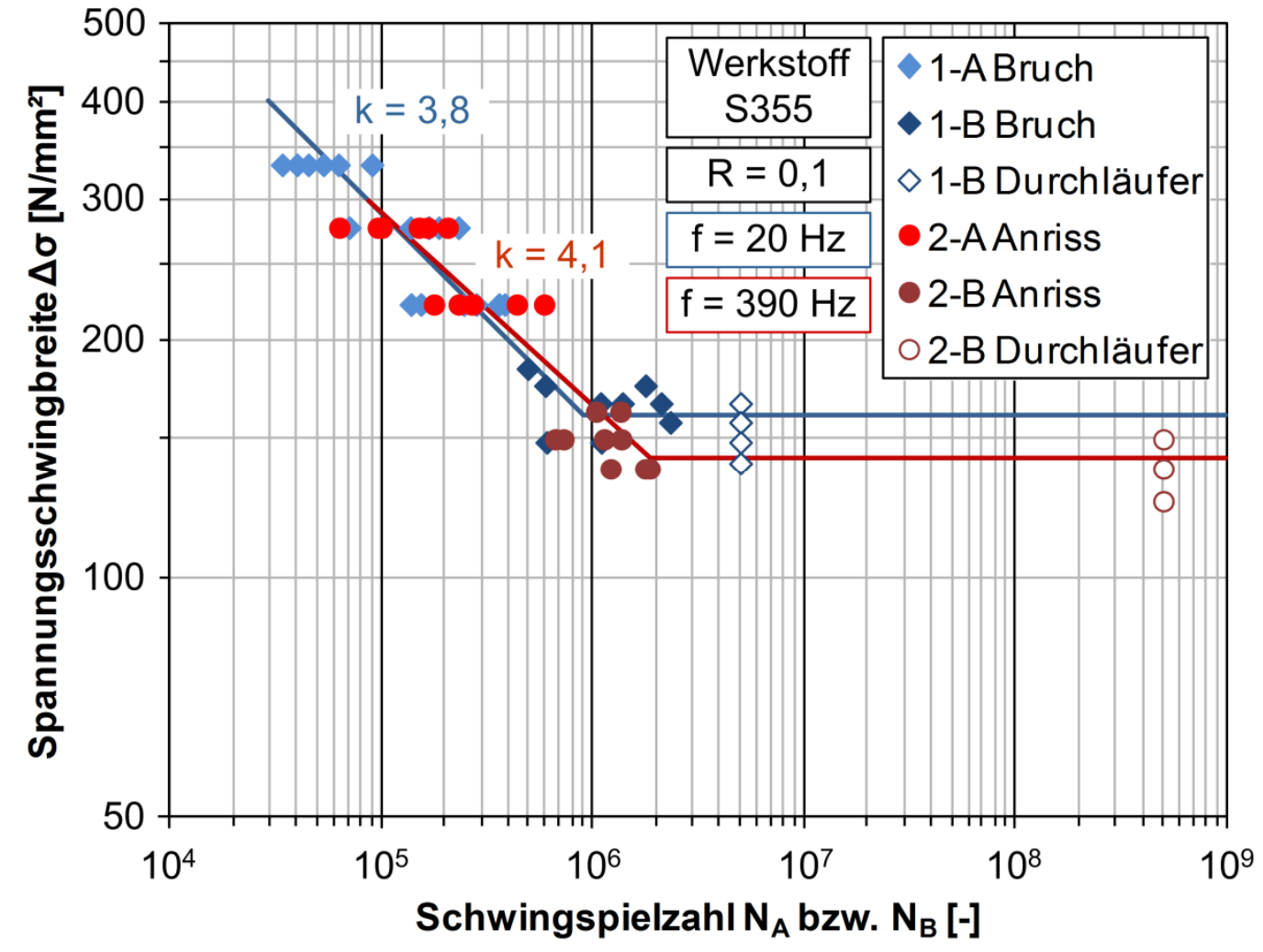

Abb. 5-4: Einfluss der Prüffrequenz 
In Tab. 5-12 sind die Grunddaten der statistischen Auswertung beider Versuchsreihen gegenübergestellt. Es sind der Neigungsexponent $k$ der Wöhlerlinie, die mittlere Dauerfestigkeit $\Delta \sigma_{D, 50 \%}$, die Schwingspielzahl $N_{k, 50 \%}$ am Abknickpunkt und die Streuung im Übergangbereich in Form der Streuspanne $T_{\sigma}$ aufgeführt.

Tab. 5-12: Grunddaten der statistischen Auswertung von Versuchsreihe 1 und 2

\begin{tabular}{|c|c|c|c|c|c|c|}
\hline $\begin{array}{c}\text { Versuchs- } \\
\text { reihe }\end{array}$ & $\begin{array}{c}\mathbf{k} \\
{[-]}\end{array}$ & $\begin{array}{c}\mathbf{\Delta} \mathbf{\sigma}_{\mathrm{D}, 50 \%} \\
{\left[\mathbf{N} / \mathbf{m m}^{2}\right]}\end{array}$ & $\begin{array}{c}\mathbf{N}_{\mathbf{k}, 50 \%} \\
{[-]}\end{array}$ & $\begin{array}{c}\mathbf{\Delta} \mathbf{\sigma}_{\mathrm{D}, 10 \%} \\
{\left[\mathbf{N} / \mathbf{m m}^{2}\right]}\end{array}$ & $\begin{array}{c}\mathbf{\Delta} \mathbf{\sigma}_{\mathrm{D}, 90 \%} \\
{\left[\mathbf{N} / \mathbf{m m}^{2}\right]}\end{array}$ & $\begin{array}{c}\mathbf{T}_{\boldsymbol{\sigma}} \\
{[-]}\end{array}$ \\
\hline 1 & 3,8 & 160,7 & 913.750 & 172,0 & 149,3 & 1,15 \\
\hline 2 & 4,1 & 141,9 & 1.901 .787 & 165,1 & 118,7 & 1,39 \\
\hline
\end{tabular}

Im Zeitfestigkeitsbereich liegen die Bruchwöhlerlinie aus Versuchsreihe 1-A und die Anrisswöhlerlinie aus Versuchsreihe 2-A dicht beieinander und unterscheiden sich nur geringfügig. Allgemein ergeben sich die ermittelten Neigungsexponenten beider Versuchsreihen oberhalb der von Haibach [Haibach, 2006] angegebenen Erfahrungswerte von 3,5 für unter einheitlichen Bedingungen und von 3,0 für unter betriebsüblichen Bedingungen angefertigte Schweißnähte aus Baustahl. Die Bruchwöhlerlinie aus Versuchsreihe 1-A mit einem Neigungsexponent $k$ von 3,8 weist im Vergleich zur Anrisswöhlerlinie aus Versuchsreihe 2-A mit einem Neigungsexponent $k$ von 4,1 einen steileren Verlauf auf. Für eine zuverlässige Abschätzung der Wöhlerlinienneigung, sollte ein ausreichender Abstand der Beanspruchungshorizonte realisiert werden. Aus versuchstechnischen Gründen konnten in Versuchsreihe 2-A keine Versuche auf dem obersten Beanspruchungsniveau von Versuchsreihe 1-A durchgeführt werden, sodass dies eine Ursache für die etwas flachere Neigung der Anrisswöhlerlinie aus Versuchsreihe 2-A sein könnte.

In Tab. 5-13 werden die einzelnen Beanspruchungsniveaus im Zeitfestigkeitsbereich von Versuchsreihe 1-A und 2-A verglichen. Die Kennwerte beider Wöhlerlinien liegen in derselben Größenordnung, wobei die mittleren Schwingspielzahlen der Anrisswöhlerlinie $\mathrm{N}_{\mathrm{A}, 50 \%}$ geringfügig oberhalb derer der Bruchwöhlerlinie $\mathrm{N}_{\mathrm{B}, 50 \%}$ liegen. Der Vergleich der Streuungen auf den Beanspruchungsniveaus ist ebenfalls in Tab. 5-13 aufgeführt. Die Streuung, ausgedrückt als Streuspanne $T_{N}$, weist auf den Beanspruchungshorizonten von Versuchsreihe 1-A und 2-A dieselbe Größenordnung auf, wobei die Streuspanne von Versuchsreihe 2-A auf dem oberen Beanspruchungsniveau geringfügig größer ist als die von Versuchsreihe 1-A. 
Tab. 5-13: Vergleich der Beanspruchungsniveaus im Zeitfestigkeitsbereich von Versuchsreihe 1-A und 2-A

\begin{tabular}{|c|c|c|c|c|c|c|c|c|}
\hline \multirow[b]{2}{*}{$\begin{array}{c}\Delta \sigma \\
{\left[\mathrm{N} / \mathrm{mm}^{2}\right]}\end{array}$} & \multicolumn{4}{|c|}{ Versuchsreihe 1-A } & \multicolumn{4}{|c|}{ Versuchsreihe 2-A } \\
\hline & $\begin{array}{c}\mathbf{N}_{\mathrm{B}, 50 \%} \\
{[-]}\end{array}$ & $\begin{array}{c}\mathbf{N}_{\mathrm{B}, 10 \%} \\
{[-]}\end{array}$ & $\begin{array}{c}\mathbf{N}_{\mathrm{B}, 90 \%} \\
{[-]}\end{array}$ & $\begin{array}{l}T_{N} \\
{[-]}\end{array}$ & $\begin{array}{c}\mathbf{N}_{\mathrm{A}, 50 \%} \\
{[-]}\end{array}$ & $\begin{array}{c}\mathbf{N}_{\mathrm{A}, 10 \%} \\
{[-]}\end{array}$ & $\begin{array}{c}\mathbf{N}_{\mathrm{A}, 90 \%} \\
{[-]}\end{array}$ & $\begin{array}{l}T_{N} \\
{[-]}\end{array}$ \\
\hline 332,8 & 59.012 & 93.403 & 37.279 & 2,51 & & & - & - \\
\hline 277,3 & 117.177 & 193.779 & 70.855 & 2,73 & 120.780 & 10.019 & 69.460 & 3,02 \\
\hline 221,9 & 271.300 & 473.396 & 155.498 & 3,04 & 302.512 & 530.272 & 172.579 & 3,07 \\
\hline
\end{tabular}

Die Auswertung der Ergebnisse für ein konstantes Streuband im Zeitfestigkeitsbereich über alle Beanspruchungshorizonte liefert für Versuchsreihe 1-A eine geringere Streuspanne $T_{N}$ von 2,76 im Vergleich zu Versuchsreihe 2-A mit einem Wert von 3,05. Diese Werte liegen im mittleren bis oberen Bereich der von Haibach [Haibach, 2006] angegebenen Erfahrungswerte von 2,5 für unter einheitlichen Bedingungen und von 3,0 für unter betriebsüblichen Bedingungen angefertigte Schweißnähte aus Baustahl.

Die im Übergangsbereich ermittelte Dauerfestigkeit $\Delta \sigma_{D, 50 \%}$ der Versuchsreihe 1-B ( $f=20 \mathrm{~Hz}$ ) von $160,7 \mathrm{~N} / \mathrm{mm}^{2}$ liegt $13,2 \%$ höher als die der Versuchsreihe 2-B $(f=390 \mathrm{~Hz})$ mit $141,9 \mathrm{~N} / \mathrm{mm}^{2}$. Die Zahlenwerte der ermittelten Anrissschwingspielzahlen heben sich jedoch nicht signifikant von den ermittelten Bruchschwingspielzahlen ab. Demnach zeigt sich bei der vorliegenden Untersuchung im Übergangsbereich keine erhöhte Lebensdauer der Versuche bei einer Prüffrequenz $f$ von $390 \mathrm{~Hz}$, was somit nicht auf eine Dehngeschwindigkeitsabhängigkeit des Materials schließen lässt. Dieses Ergebnis sollte durch weitere Untersuchungen abgesichert werden, da die Anzahl der Proben gering ist und eine Abhängigkeit von den gewählten Beanspruchungsniveaus vorliegen kann. Begründet durch den Unterschied der mittleren Dauerfestigkeiten $\Delta \sigma_{D, 50 \%}$ knickt die Bruchwöhlerlinie aus Versuchsreihe 1 $\left(N_{k, 50 \%}=913.750\right)$ früher ab als die Anrisswöhlerlinie aus Versuchsreihe 2 $\left(\mathrm{N}_{\mathrm{k}, 50 \%}=1.901 .787\right)$.

Desweiteren ist beim vorliegenden Vergleich zu beachten, dass neben unterschiedlichen Bedingungen wie Prüfmaschine und -frequenz eine Bruchwöhlerlinie mit einer Anrisswöhlerlinie verglichen wird. Unter der Voraussetzung identischer Versuchsbedingungen würde eine Anrisswöhlerlinie im Vergleich zu einer Bruchwöhlerlinie geringere Schwingspielzahlen aufweisen. Der Anteil der Anrisslebensdauer an der Gesamtlebensdauer ist nach Radaj und Vormwald [Radaj, 2007] sowie Gudehus und Zenner [Gudehus, 1999] u. a. abhängig von der definierten Anrisslänge, dem vorlie- 
genden Beanspruchungszustand sowie der Probengröße und -form. Beispielsweise kann bei einer gekerbten Probe die Anrisslebensdauer gegenüber der Restlebensdauer im Bereich höherer Beanspruchungsniveaus geringer sein [Radaj, 2007], wobei bei kleinen [Gudehus, 1999] sowie im Labor geprüften Proben [Schijve, 2009] der Anteil der Restlebensdauer an der Gesamtlebensdauer als geringer eingestuft und unter Umständen sogar vernachlässigt werden kann [Gudehus, 1999]. Die Frage nach dem zu erwartenden Anteil der Anrisslebensdauer an der Gesamtlebensdauer lässt sich somit nicht pauschal beantworten.

In Abb. 5-5 werden die Bruchschwingspielzahlen der Versuchsreihen 1 und 2 verglichen. Der Bruch bei Versuchsreihe 2 ist als das Erreichen des Abbruchkriteriums, was dem Abfall der Vorspannkraft auf das Niveau der Kraftamplitude entspricht, definiert. Der Vergleich von Anriss- und Bruchschwingspielzahlen von Versuchsreihe 2 und 3 ist in Anhang J.2 dargestellt. In Abb. 5-5 ist zu erkennen, dass die Wöhlerlinie von Versuchsreihe 2 im Zeitfestigkeitsbereich oberhalb der Wöhlerlinie von Versuchsreihe 1 liegt. Bei Auswertung der Bruchschwingspielzahlen resultiert ein steilerer Verlauf der Wöhlerlinie von Versuchsreihe 2-A mit einem Neigungsexponenten $\mathrm{k}$ von 3,9. Die Neigungsexponenten beider Versuchsreihen weichen nur geringfügig voneinander $a b$.

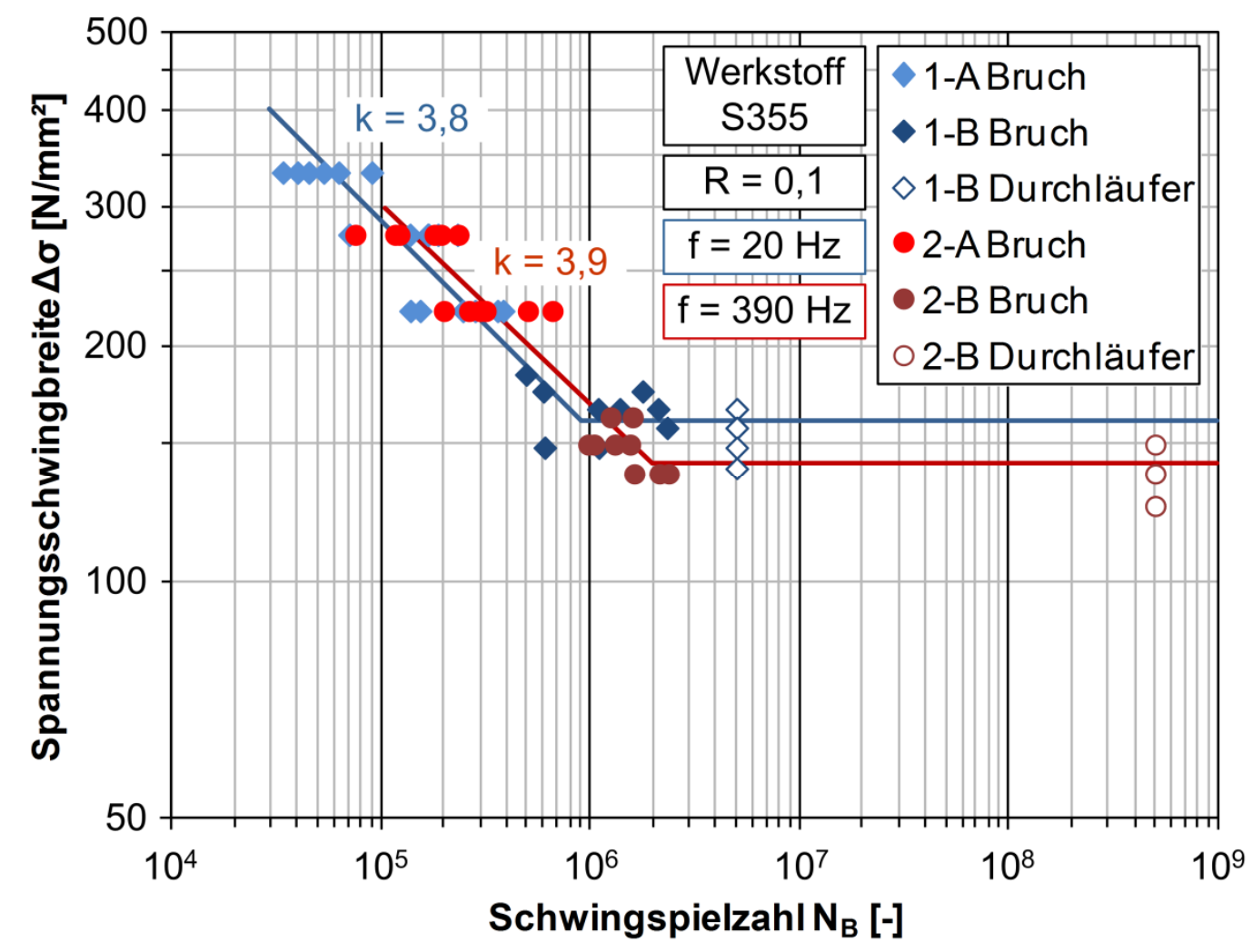

Abb. 5-5: Einfluss der Prüffrequenz bei Vergleich von Bruchschwingspielzahlen 
Die mittleren Bruchschwingspielzahl $\mathrm{N}_{\mathrm{B}, 50 \%}$ beider Versuchsreihen auf den unteren beiden Beanspruchungsniveaus im Zeitfestigkeitsbereich sind in Tab. 5-14 gegenübergestellt. Außerdem ist die Differenz $\Delta N_{B, 50 \%}$ zwischen den Ergebnissen der Versuchsreihen 1-A und 2-A sowie die prozentuale Abweichung $p_{\%, N B 50 \%}$ der mittleren Bruchschwingspielzahl $N_{B, 50 \%}$ von Versuchsreihe 2-A zu Versuchsreihe 1-A angegeben. Die Wöhlerlinie von Versuchsreihe 2-A ist im Vergleich zur Wöhlerlinie von Versuchsreihe 1-A auf den unteren beiden Beanspruchungsniveaus um $22,5 \%$ und $26,4 \%$ in Richtung höherer Bruchschwingspielzahlen verschoben. Es könnte somit ein geringer Einfluss der Prüffrequenz im Zeitfestigkeitsbereich unterstellt werden. Dies steht allerdings im Gegensatz zu den Ergebnissen im Übergangsbereich, sodass sich keine eindeutige Aussage ableiten lässt.

Tab. 5-14: Vergleich der mittleren Bruchschwingspielzahlen $\mathbf{N}_{B, 50 \%}$ auf den Beanspruchungsniveaus im Zeitfestigkeitsbereich von Versuchsreihe 1-A und 2-A

\begin{tabular}{|c|c|c|c|c|}
\hline Versuchsreihe & $1-A$ & 2-A & Differenz & Abweichung \\
\hline $\begin{array}{c}\Delta \sigma \\
{\left[\mathbf{N} / \mathrm{mm}^{2}\right]}\end{array}$ & $\begin{array}{c}\mathbf{N}_{\mathrm{B}, 50 \%} \\
{[-]}\end{array}$ & $\begin{array}{c}\mathbf{N}_{\mathrm{B}, 50 \%} \\
{[-]}\end{array}$ & $\begin{array}{c}\Delta \mathbf{N}_{\mathrm{B}, 50 \%} \\
{[-]}\end{array}$ & $\begin{array}{c}\mathbf{p}_{\%, \mathrm{NB} 50 \%} \\
{[\%]}\end{array}$ \\
\hline 277,3 & 117.177 & 143.486 & 26.309 & 22,5 \\
\hline 221,9 & 271.300 & 342.812 & 71.512 & 26,4 \\
\hline
\end{tabular}

Desweiteren ist zu beachten, dass eine Erwärmung des Probekörpers infolge innerer Reibung festgestellt wurde (vergleiche Kapitel 4.3.3 und Kapitel 5.4). Diese Erwärmung des Probekörpers hat einen Abfall der Vorspannkraft zur Folge, womit ein Abfall des Spannungsverhältnisses einhergeht. Die Versuche der Versuchsreihe 2-A sind demnach bei einem leicht abfallenden und somit geringeren Spannungsverhältnis geprüft worden als die der Versuchsreihe 1-A. Diese Versuchsbedingungen können im Vergleich zum konstanten Spannungsverhältnis aufgrund der geringeren Oberlasten ebenfalls zu erhöhten Schwingspielzahlen führen. Zudem ist die Erwärmung des Probekörpers von der Höhe des Beanspruchungsniveaus abhängig. Je höher das Beanspruchungsniveau ist, desto größer ist die Erwärmung und demnach auch der Abfall der Vorspannkraft (vergleiche Kapitel 5.4 sowie Anhang H.1 und H.2). Hieraus ließe sich schließen, dass im Bereich hoher Beanspruchungen und demnach größerer Erwärmung der beschriebene Effekt einer verlängerten Lebensdauer ausgeprägter ist, als im Bereich niedriger Beanspruchungen, was tendenziell zu einem steileren Verlauf der Wöhlerlinie führen könnte. Diese Überlegung kann anhand der vorliegenden Ergebnisse in Tab. 5-13 nicht bestätigt werden, da sich ein flacherer Verlauf sowohl der Anriss- als auch der Bruchwöhlerlinie von Versuchsreihe 2-A im Vergleich zur Bruchwöhlerlinie von Versuchsreihe 1-A einstellt. 
Zur Schwingprüfung von Schraube-Mutter-Verbindungen wurde von Alt [Alt, 2005] eine Prüfvorrichtung entwickelt und eingesetzt, die auf demselben Prüfprinzip basiert, wie die in der vorliegenden Arbeit eingesetzte Prüfvorrichtung. Der Einfluss der Prüffrequenz wurde hierbei von Alt et al. [Alt, 2007] untersucht. Für hochfeste Schrauben aus Stahl konnte für einen Bereich von $122 \mathrm{~Hz}$ bis $504 \mathrm{~Hz}$ kein Einfluss der Prüffrequenz auf die Ergebnisse der Ermüdungsversuche festgestellt werden.

In Keindorf et al. [Keindorf, 2010] wurde ein Vergleich von Versuchsergebnissen mit der in der vorliegenden Arbeit eingesetzten hochfrequenten Prüfvorrichtung für Schweißverbindungen mit einer Prüffrequenz $f$ von $380 \mathrm{~Hz}$ und einer servorhydraulischen Prüfmaschine mit einer Prüffrequenz $f$ von $15 \mathrm{~Hz}$ im Zeitfestigkeitsbereich durchgeführt. Es wurde ein höherer Neigungsexponent der ermittelten Ermüdungsfestigkeitskurven bei $380 \mathrm{~Hz}$ festgestellt, sodass ein flacherer Verlauf der Wöhlerlinie bei $380 \mathrm{~Hz}$ ermittelt wurde als bei $15 \mathrm{~Hz}$. Dieses Ergebnis konnte in den vorliegenden Untersuchungen bestätigt werden.

\section{Zusammenfassung und Erkenntnisse}

Der Vergleich der Bruchwöhlerlinie aus Versuchsreihe 1 mit der Anrisswöhlerlinie aus Versuchsreihe 2 im Bezug auf den Einfluss der Prüffrequenz lässt keine eindeutigen Aussagen zu. Es könnte ein geringfügiger Einfluss der Prüffrequenz im Zeitfestigkeitsbereich unterstellt werden, da die Wöhlerlinien sich nicht signifikant unterscheiden und eine Anrisswöhlerlinie tendenziell mit geringeren Schwingspielzahlen zu erwarten wäre. Dies könnte allerdings auch auf die vorliegenden Versuchsbedingungen zurückgeführt werden. Es ist ein einheitlicher Vergleich von Anriss- oder Bruchwöhlerlinien anzustreben, um diese Vermutung zu untermauern.

Der Vergleich von Bruchwöhlerlinien beider Versuchsreihen wurde hinzugezogen. Die Neigungsexponenten der Wöhlerlinien beider Versuchsreihen unterscheiden sich nur geringfügig. Die Wöhlerlinie, ermittelt bei einer Prüffrequenz $f$ von $390 \mathrm{~Hz}$, ist im Vergleich zur Wöhlerlinie, ermittelt bei einer Prüffrequenz $f$ von $20 \mathrm{~Hz}$, zu höheren Bruchschwingspielzahlen verschoben. Es könnte somit ein geringer Einfluss der Prüffrequenz im Zeitfestigkeitsbereich unterstellt werden. Im Übergangsbereich stellt sich eine Abweichung der ermittelten Dauerfestigkeiten $\Delta \sigma_{D, 50 \%}$ ein. Bei Versuchsreihe 2-A mit höherer Prüffrequenz wurde eine geringere Dauerfestigkeit $\Delta \sigma_{D, 50 \%}$ ermittelt, was nicht auf den Einfluss einer erhöhten Dehngeschwindigkeit schließen lässt. Dieses Ergebnis lässt ebenfalls keine einheitlichen Aussagen zu. Aufgrund der geringen Probenanzahl und zum Ausschluss einer Abhängigkeit der gewählten Beanspruchungsniveaus ist eine weitere Absicherung dieser Ergebnisse empfehlenswert. 
Die Vermutung einer verlängerten Lebensdauer auf den oberen Beanspruchungsniveaus von Versuchsreihe 2, resultierend aus der Erwärmung der Probekörper, bestätigte sich nicht durch einen steileren Verlauf der Wöhlerlinie im Vergleich zu Versuchsreihe 1. Allgemein wird der Einfluss der Prüffrequenz als nicht signifikant eingestuft, wobei die Ergebnisse keine einheitliche Aussage zulassen.

\subsubsection{Einfluss der Mittelspannung}

Die Einzelergebnisse aus Versuchsreihe 2 und 3 sowie die jeweils ermittelten Wöh-

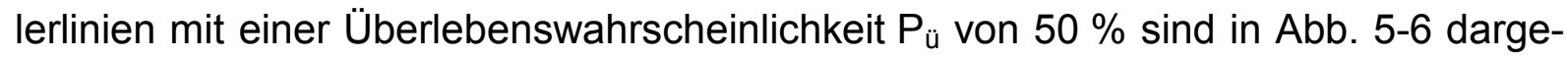
stellt. Aus Gründen der Übersichtlichkeit wurde auf die Abbildung der Wöhlerlinien mit einer Überlebenswahrscheinlichkeit $P_{u ̈}$ von $10 \%$ und $90 \%$ verzichtet. Bei beiden Versuchsreihen, ermittelt bei einer Prüffrequenz $f$ von $390 \mathrm{~Hz}$, handelt es sich um Anrissschwingspielzahlen und somit um Anrisswöhlerlinien.

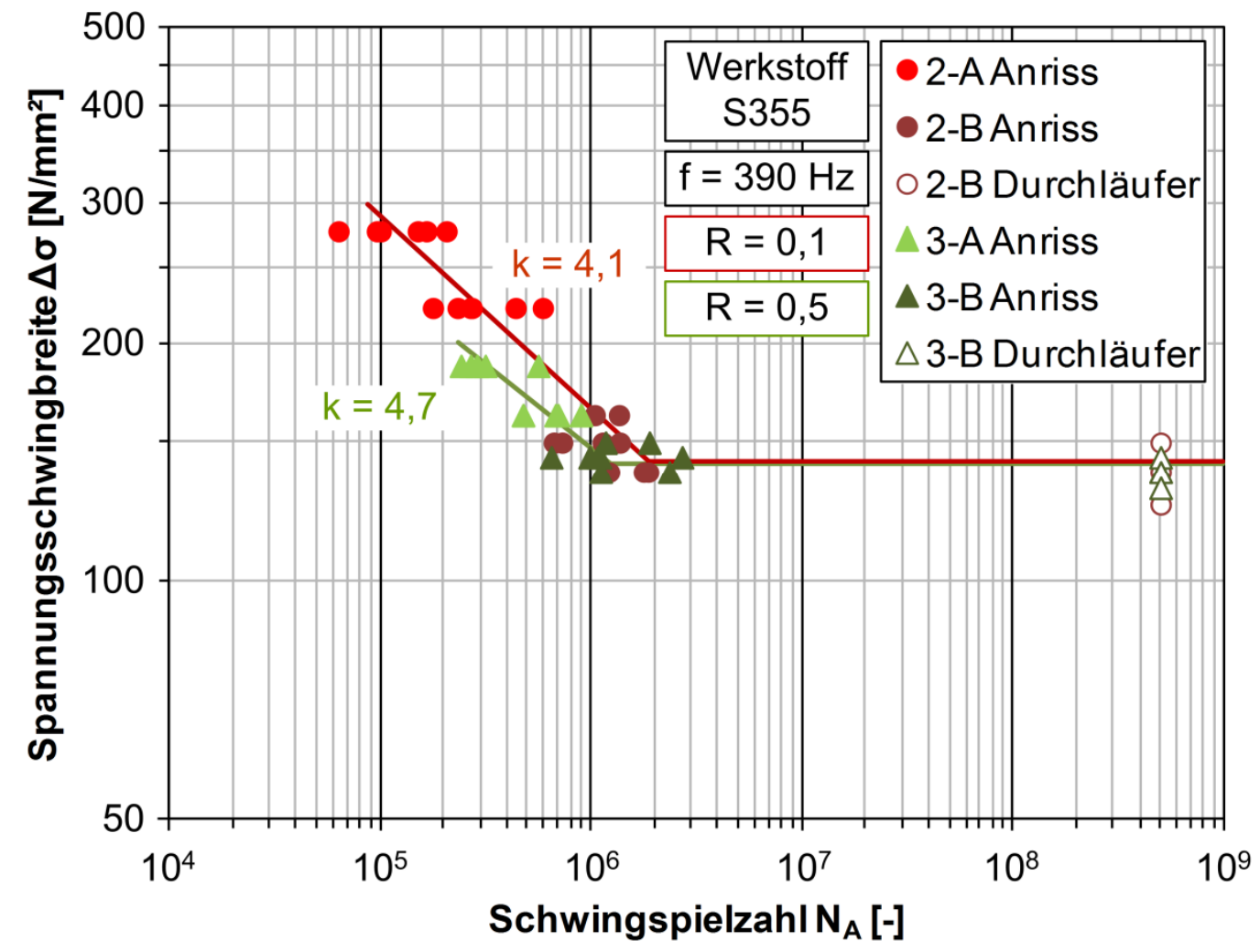

Abb. 5-6: Einfluss der Mittelspannung

In Tab. 5-15 sind die Grunddaten der statistischen Auswertung beider Versuchsreihen gegenübergestellt. Es sind die Neigungsexponenten $\mathrm{k}$ der Wöhlerlinie, die mittlere Dauerfestigkeit $\Delta \sigma_{D, 50 \%}$, die Schwingspielzahl $N_{k, 50 \%}$ am Abknickpunkt und die Streuung im Übergangsbereich in Form der Streuspanne $T_{\sigma}$ aufgeführt. Im Zeitfestigkeitsbereich weist die Anrisswöhlerlinie aus Versuchsreihe 2-A mit einem Neigungsexponenten $\mathrm{k}$ von 4,1 im Vergleich zur Anrisswöhlerlinie aus Versuchsreihe 3-A mit 
einem Neigungsexponenten k von 4,7 einen steileren Verlauf auf. Für eine zuverlässige Abschätzung der Wöhlerlinienneigung, soltte ein ausreichender Abstand der Beanspruchungshorizonte realisiert werden. Aus versuchstechnischen Gründen waren bei Versuchsreihe 3-A keine höheren Beanspruchungsniveaus realisierbar, sodass der ermittelte Neigungsexponent als Tendenz hinsichtlich eines flacheren Verlaufs der Wöhlerlinie anzusehen ist. Der flachere Verlauf der Anrisswöhlerlinie aus Versuchsreihe 3-A ist auf die Prüfung bei erhöhtem Spannungsverhältnis zurückzuführen. Bei gleicher Kraftamplitude ist die Mittellast bei höherem Spannungsverhältnis größer als bei niedrigem Spannungsverhältnis, woraus eine höhere Oberlast resultiert, die zu einer kürzeren Lebensdauer führen kann.

Tab. 5-15: Grunddaten der statistischen Auswertung von Versuchsreihe 2 und 3

\begin{tabular}{|c|c|c|c|c|c|c|}
\hline $\begin{array}{c}\text { Versuchs- } \\
\text { reihe }\end{array}$ & $\begin{array}{c}\mathbf{k} \\
{[-]}\end{array}$ & $\begin{array}{c}\mathbf{\Delta} \mathbf{\sigma}_{\mathrm{D}, 50 \%} \\
{\left[\mathbf{N} / \mathbf{m m}^{\mathbf{2}}\right]}\end{array}$ & $\begin{array}{c}\mathbf{N}_{\mathbf{k}, 50 \%} \\
{[-]}\end{array}$ & $\begin{array}{c}\mathbf{\Delta} \mathbf{\sigma}_{\mathrm{D}, 10 \%} \\
{\left[\mathbf{N} / \mathbf{m m}^{2}\right]}\end{array}$ & $\begin{array}{c}\mathbf{\Delta} \mathbf{\sigma}_{\mathrm{D}, 90 \%} \\
{\left[\mathbf{N} / \mathbf{m m}^{2}\right]}\end{array}$ & $\begin{array}{c}\mathbf{T}_{\mathbf{\sigma}} \\
{[-]}\end{array}$ \\
\hline 2 & 4,1 & 141,9 & 1.901 .787 & 165,1 & 118,7 & 1,39 \\
\hline 3 & 4,7 & 140,4 & 1.239 .512 & 149,2 & 131,6 & 1,13 \\
\hline
\end{tabular}

In Tab. 5-16 werden die Streuungen auf den Beanspruchungsniveaus der beiden Versuchsreihen verglichen, wobei zu beachten ist, dass die Beanspruchungsniveaus nicht gleich sind. Die Streuung, ausgedrückt als Streuspanne $T_{N}$ von Versuchsreihe 2, ist deutlich größer als von Versuchsreihe 3. Hierbei ist anzumerken, dass sich die Beanspruchungsniveaus von Versuchsreihe 3 zudem sehr dicht am Übergangsbereich befinden, wo in der Regel mit höheren Streuungen zu rechnen wäre.

Tab. 5-16: Vergleich der Beanspruchungsniveaus im Zeitfestigkeitsbereich von Versuchsreihe 2-A und 3-A

\begin{tabular}{|cc|c|c|c|c|}
\hline & & \multicolumn{2}{|c|}{ Versuchsreihe 2-A } & \multicolumn{2}{c|}{ Versuchsreihe 3-A } \\
\hline$\Delta \boldsymbol{\sigma}$ & {$\left[\mathbf{N} / \mathbf{m m}^{\mathbf{2}}\right]$} & 277,3 & 221,9 & 187,5 & 162,5 \\
\hline $\mathbf{N}_{\mathbf{A}, \mathbf{5 0} \%}$ & {$[-]$} & 120.780 & 302.512 & 317.853 & 623.572 \\
\hline $\mathbf{N}_{\mathbf{A}, \mathbf{1 0} \%}$ & {$[-]$} & 210.019 & 530.272 & 485.468 & 884.158 \\
\hline $\mathbf{N}_{\mathrm{A}, \mathbf{0 0} \%}$ & {$[-]$} & 69.460 & 172.579 & 208.109 & 439.789 \\
\hline $\mathbf{T}_{\mathbf{N}}$ & {$[-]$} & 3,02 & 3,07 & 2,33 & 2,01 \\
\hline
\end{tabular}

Die im Übergangsbereich ermittelten Dauerfestigkeiten $\Delta \sigma_{D, 50 \%}$ der beiden Versuchsreihen unterscheiden sich nur marginal. Die mittlere Dauerfestigkeit $\Delta \sigma_{D, 50 \%}$ der Versuchsreihe 2-B $(R=0,1)$ von $141,9 \mathrm{~N} / \mathrm{mm}^{2}$ liegt $1,1 \%$ höher als die der Versuchsreihe $3-B(R=0,5)$ mit $140,4 \mathrm{~N} / \mathrm{mm}^{2}$. Allerdings weist Versuchsreihe 3 wie im Zeitfestig- 
keitsbereich auch im Übergangsbereich deutlich geringere Streuungen auf als Versuchsreihe 2. Bedingt durch die unterschiedlichen Neigungsexponenten knickt die Anrisswöhlerlinie aus Versuchsreihe $2\left(\mathrm{~N}_{\mathrm{k}, 50 \%}=1.901 .787\right)$ später ab als die der Versuchsreihe $3\left(N_{k, 50 \%}=1.239 .512\right)$. In Versuchsreihe 3 wurden zum Teil höhere Anrissschwingspielzahlen im Übergangsbereich erzielt als in Versuchsreihe 2. Hier kann im Hinblick auf die mittlere Dauerfestigkeit $\Delta \sigma_{D, 50 \%}$ kein signifikanter Einfluss der Mittelspannung festgestellt werden. Lediglich die ermittelten Abknickpunkte unterscheiden sich.

\section{Zusammenfassung und Erkenntnisse}

Im Zeitfestigkeitsbereich konnte ein Einfluss der Mittelspannung auf die Ergebnisse der Ermüdungsversuche festgestellt werden. Die Prüfung bei einem Spannungsverhältnis $R$ von 0,5 führte zu einem flacheren Verlauf der Anrisswöhlerlinie im Vergleich zur Prüfung bei einem Spannungsverhältnis $R$ von 0,1 . Im Übergangsbereich konnte hingegen kein Einfluss der Mittelspannung auf die mittlere Dauerfestigkeit $\Delta \sigma_{D, 50 \%}$ festgestellt werden. Lediglich im Bezug auf den Abknickpunkt zeigte sich, dass die Anrisswöhlerlinie, die bei einem Spannungsverhältnis $R$ von 0,5 ermittelt wurde, früher abknickt als die Anrisswöhlerlinie, die bei einem Spannungsverhältnis $R$ von 0,1 ermittelt wurde. Allgemein ergeben sich geringere Streuungen der Versuchsergebnisse bei der Prüfung mit einem Spannungsverhältnis $R$ von 0,5. Aus versuchstechnischen Gründen war die Prüfung bei Spannungsverhältnissen $R$ größer als 0,5 nicht möglich. Um das Auftreten später Brüche bei Oberspannungen im Bereich der Streckgrenze ausschließen zu können, sollten die Untersuchungen auf entsprechend höhere Spannungsverhältnisse ausgedehnt werden.

\subsubsection{Bewertung der hochgesetzten Durchläufer}

Die erzielten Durchläufer aller Versuchsreihen im Übergangsbereich (B) wurden mit dem Farbeindringverfahren auf vorliegende Anrisse an der Oberfläche untersucht. Mit diesem Verfahren konnte bei keiner Probe ein Anriss an der Oberfläche festgestellt werden. Daraufhin wurden diese Proben erneut auf den Beanspruchungsniveaus im Zeitfestigkeitsbereich bis zum Versagen geprüft. Die hochgesetzten Durchläufer (C) der einzelnen Versuchsreihen 1 bis 3 sind in Abb. 5-7 bis Abb. 5-9 jeweils im Vergleich zu den Ergebnissen der Reihen im Zeitfestigkeitsbereich (A) und im Übergangsbereich (B) dargestellt. Außerdem sind die Streuspannen $T_{N}$ auf den einzelnen Beanspruchungsniveaus der jeweiligen Reihen im Zeitfestigkeitsbereich (A) angegeben. Die Ergebnisse der Einzelversuche der hochgesetzten Durchläufer (C) sind ebenfalls im Anhang I.1 bis I.3 für alle Versuchsreihen tabellarisch aufgeführt. 


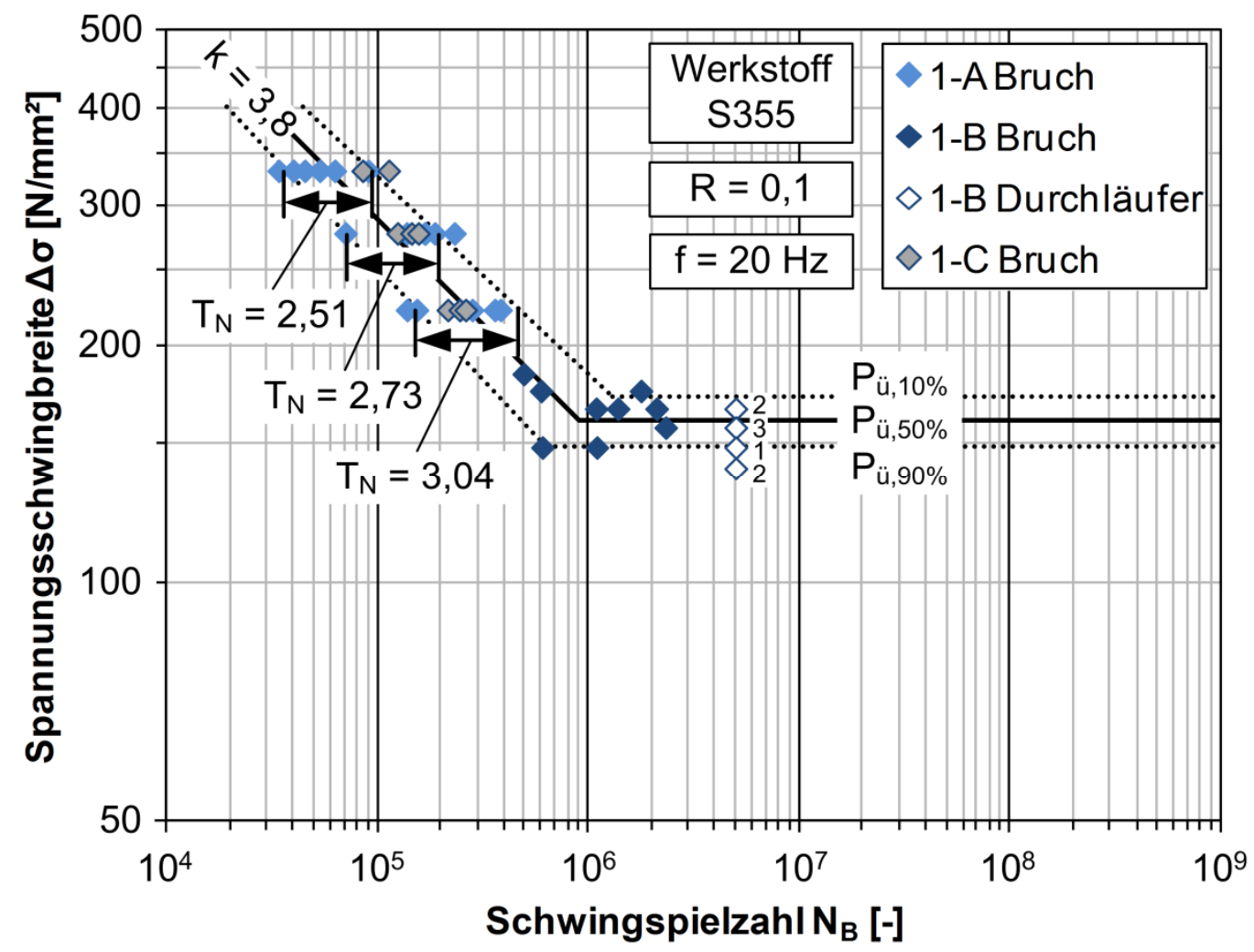

Abb. 5-7: Versuchsreihe 1-C im Vergleich zu Versuchsreihe 1-A und 1-B

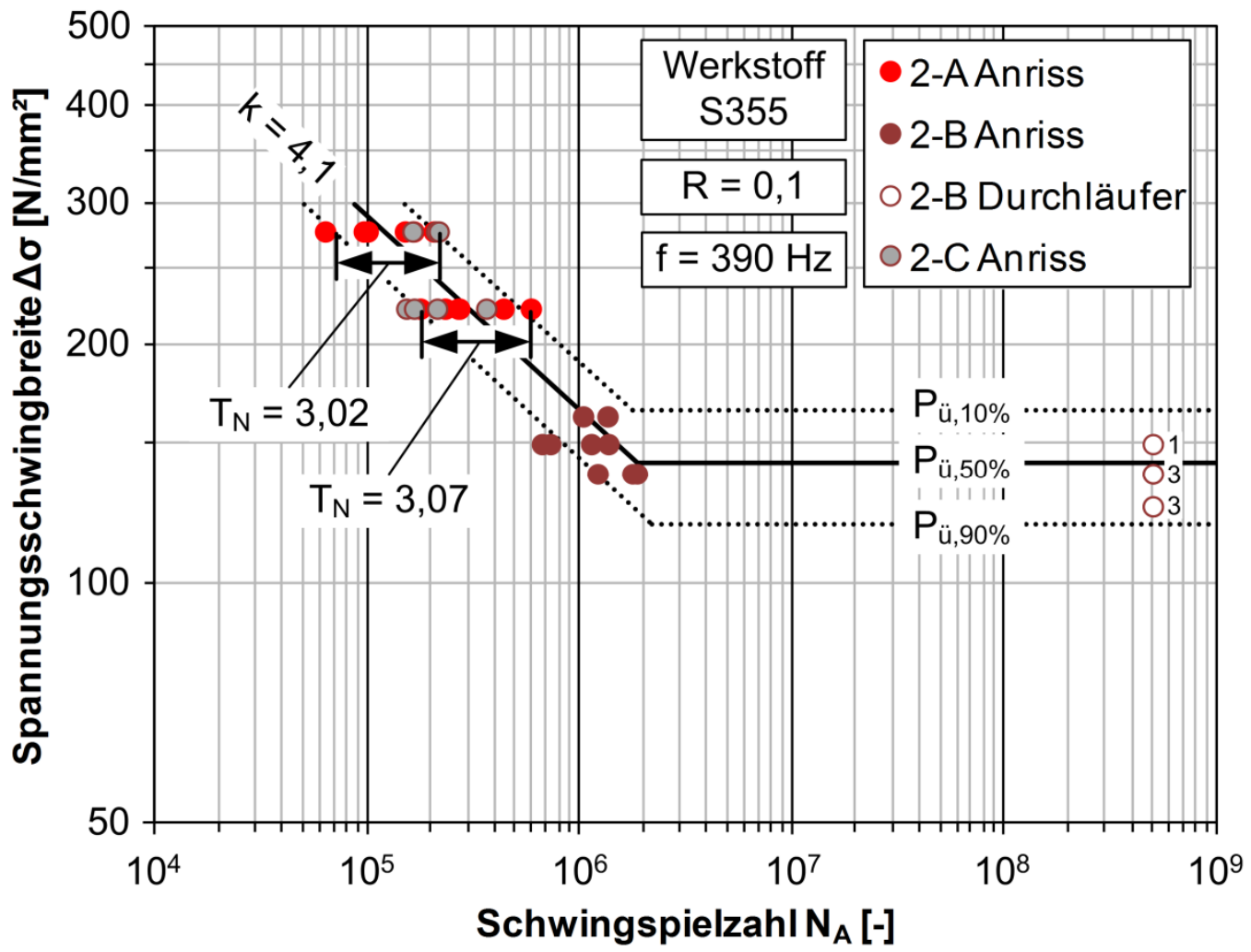

Abb. 5-8: Versuchsreihe 2-C im Vergleich Versuchsreihe 2-A und 2-B 


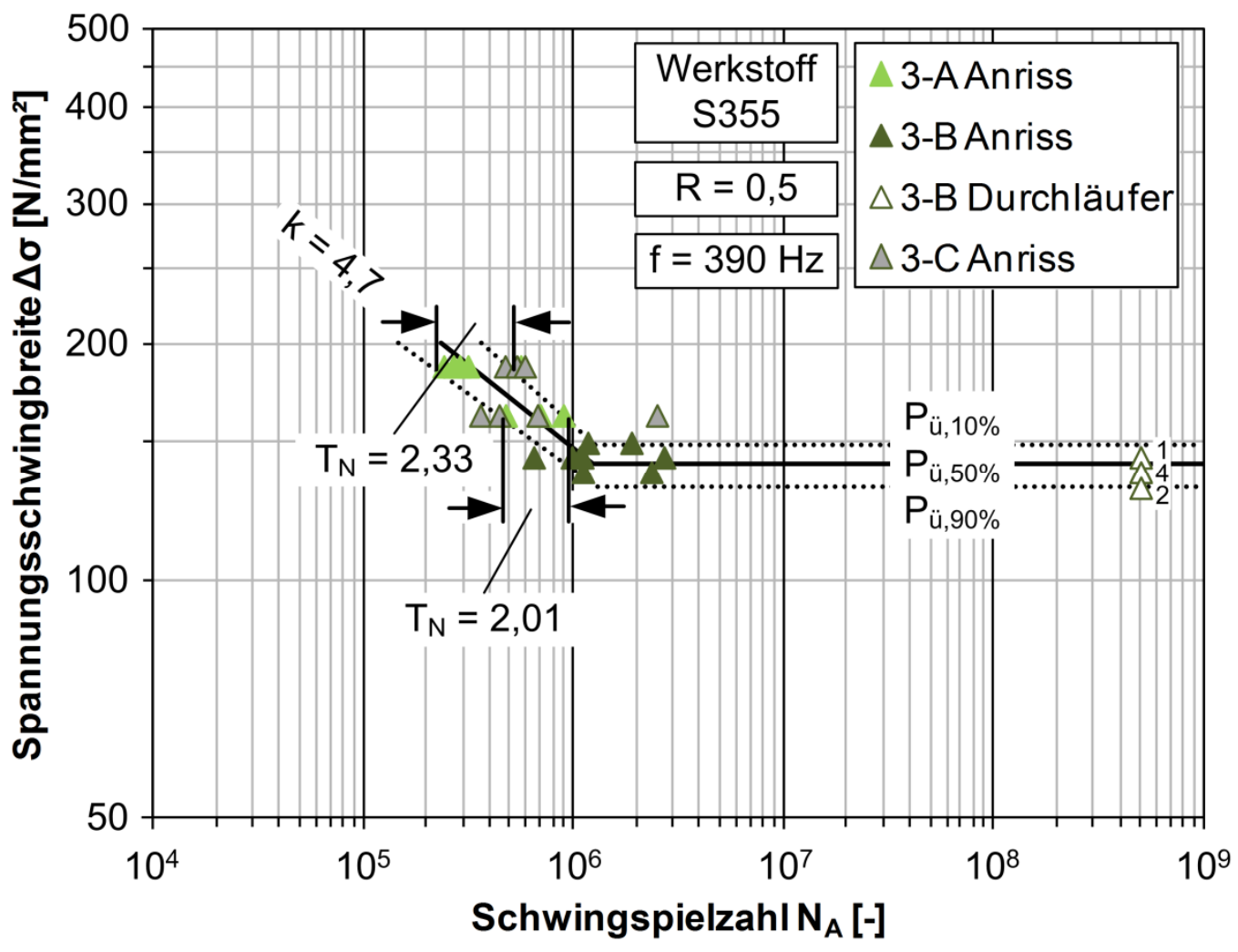

Abb. 5-9: Versuchsreihe 3-C im Vergleich Versuchsreihe 3-A und 3-B

Allgemein zeigt sich, dass die hochgesetzten Durchläufer aller Versuchsreihen mit Ausnahme des unteren Beanspruchungsniveaus von Versuchsreihe 3 dicht beieinanderliegen und sich in den Streubändern der Ergebnisse im Zeitfestigkeitsbereich befinden. Die hochgesetzten Durchläufer auf den oberen Beanspruchungsniveaus befinden sich im Bereich der ermittelten Bruch- bzw. Anrissschwingspielzahl für eine Überlebenswahrscheinlichkeit $P_{u ̈ ~}$ von $90 \%$ der Versuchsreihen $A$ und weisen eine sehr geringe Streuung auf. Mit sinkender Beanspruchungsamplitude nimmt die Streuung der hochgesetzten Durchläufer zu und die Ergebnisse verschieben sich in den Bereich der ermittelten Bruch- bzw. Anrissschwingspielzahl für eine Überlebenswahrscheinlichkeit $P_{u ̈}$ von $50 \%$ der Versuchsreihen $A$ und darunter. Auf dem unteren Beanspruchungsniveau von Versuchsreihe 3-C weist eine Probe eine besonders hohe Lebensdauer auf. Dieses Ergebnis wird auf die Höhe des Beanspruchungsniveaus in unmittelbarer Nähe zum Übergangsbereich zurückgeführt.

Die Ergebnisse der hochgesetzten Durchläufer liegen im Erwartungsbereich, da Durchläufer generell Proben mit erhöhter Festigkeit darstellen und somit von mittleren bis höheren Versagensschwingspielzahlen und einem geringeren Streuband auszugehen ist. Es ist kein deutlich frühzeitiges Versagen der hochgesetzten Durchläufer zu erkennen, das auf eine Vorschädigung der erneut geprüften Proben hindeuteten würde. Auf der anderen Seite zeigen die Ergebnisse auch keine deutlich erhöhten Bruch- bzw. Anrissschwingspielzahlen im Vergleich zu den Versuchsreihen A. Es 
lässt sich somit auch nicht auf einen ausgeprägten Trainingseffekt schließen, sondern eher auf eine neutrale Ausgangssituation der Probe trotz Vorbelastung bis zur Grenzschwingspielzahl.

Die Durchläufer wurden im vorliegenden Fall auf die Beanspruchungsniveaus im Zeitfestigkeitsbereich hochgesetzt. Für weitere Untersuchungen wäre es auch denkbar, die Proben im Übergangsbereich bis zum Eintritt eines Bruches hochsetzen, was allerdings bei erneutem Durchlaufen der Proben zu erhöhten Prüfzeiten führen würde.

\subsubsection{Beurteilung der Bruchflächen}

Die Beurteilung der Bruchflächen erfolgt visuell. Alle Bruchflächen wurden einer makroskopischen Untersuchung mit dem Lichtmikroskop (Keyence VHX-1000D) unterzogen und jeweils mit einer Übersichtsaufnahme dokumentiert. Die Übersichtsaufnahmen der Bruchflächen aller Einzelversuche aus den Versuchsreihen 1 bis 3 sind in Anhang I dokumentiert.

Die Ermüdungsversuche von Versuchsreihe 1, durchgeführt in einer konventionellen servohydraulischen Prüfmaschine, wurden bis zum vollständigen Bruch der Probe geprüft. Somit handelt es sich um Bruchflächen, die sich aus Ermüdungs- und Restbruchfläche zusammensetzen. Die Ermüdungsversuche von Versuchsreihe 2 und 3 wurden bis zu einem definierten Abfall der Vorspannkraft geprüft und im Anschluss im statischen Zugversuch getrennt. Es handelt sich bei diesen beiden Versuchsreihen folglich um Bruchflächen, die sich aus Ermüdungsbruchfläche bis zum Abbruchkriterium und statischer Restbruchfläche zusammensetzen. Bei Versuchsreihe 2 wurde als Abbruchkriterium der Abfall der Vorspannkraft auf das Beanspruchungsniveau der Prüfkraftamplitude festgelegt, sodass der Probekörper keine Druckbeanspruchung erfährt. Demnach wurden die Versuche aus Versuchsreihe 2 bei einem prozentualen Abfall von ca. 18,1\% vom Sollwert der Vorspannkraft abgebrochen. Dies entspricht je nach Höhe des Beanspruchungsniveaus einem Abfall der Vorspannkraft $F_{v}$ von ca. 1,5 bis $3,0 \mathrm{kN}$. Bei Versuchsreihe 3 wurde für den Abfall der Vorspannkraft $F_{v}$ ein konstanter Wert von $5,0 \mathrm{kN}$ festgelegt. Diese unterschiedlichen Abbruchkriterien sind im Hinblick auf den Vergleich der Bruchflächen der einzelnen Versuchsreihen zu beachten.

Aus der Betrachtung der Bruchflächen geht hervor, dass alle Bruchflächen dasselbe makroskopische Erscheinungsbild unabhängig von Beanspruchungsniveau und Prüfvorrichtung aufweisen. Der Ermüdungsbruch ist normalflächig und verläuft senkrecht zur Beanspruchungsrichtung in die Tiefe des Querschnitts. Beim Erreichen des kritischen Restquerschnitts tritt der Restbruch in Form eines Scherbruches ein (siehe Versuchsreihe 1). Bei Versuchsreihe 2 und 3 wurden die Proben nach Erreichen der 
jeweiligen Abbruchkriterien im statischen Zugversuch getrennt. Bei Betrachtung des Verhältnisses von Ermüdungs- und Restbruchfläche auf den Bruchflächen der Proben ist eine Abhängigkeit von der Beanspruchungshöhe zu erkennen. Je geringer das Beanspruchungsniveau ist, desto größer ist die Ermüdungsbruchfläche im Vergleich zur Restbruchfläche. Da die Proben bei konstanter Beanspruchungsamplitude geprüft wurden, sind in der Regel keine Rastlinien auf den Bruchflächen zu erkennen. Es ist somit nur möglich den Endzustand der Rissausbreitung zu beurteilen und indirekt auf die Rissausgangsorte sowie den Rissverlauf und die Rissform zu schließen.

Der Ermüdungsbruch weist bei allen Proben unabhängig von Beanspruchungsniveau und Prüfvorrichtung einen Bruchausgang an den Kerben im Bereich der Schweißnaht von der Oberfläche aus auf. Dies ist auf die makroskopische Kerbwirkung der unbehandelten Schweißnaht zurückzuführen. Es konnte keine Verlagerung des Anrissortes von der Oberfläche ins Innere der Proben festgestellt werden. Der Anriss trat vorwiegend am Wurzelübergang auf. Vereinzelt kam es zu einer Verlagerung des Anrissortes in den Bereich des Nahtübergangs. Dies bestätigt die aus der Untersuchung der lokalen Nahtgeometrie hervorgegangene Vermutung aus Kapitel 3.5.

In Abb. 5-10 sind aus Versuchsreihe 1 exemplarisch Bruchflächen mit Ausgang des Anrisses vom Wurzelübergang (links) und vom Nahtübergang (rechts) dargestellt. Beim Anriss am Wurzelübergang verläuft der Riss durch die Wärmeeinflusszone sowie das Schmelzbad der Schweißverbindung, wohingegen der Riss bei Anriss am Nahtübergang durch das Grundmaterial verläuft. Bedingt durch die Korngröße zeigt sich bei Anriss am Nahtübergang im Grundmaterial eine deutlich feinere Ermüdungsbruchfläche im Vergleich zur Wärmeeinflusszone bzw. zum Schmelzbad bei Anriss am Wurzelübergang.
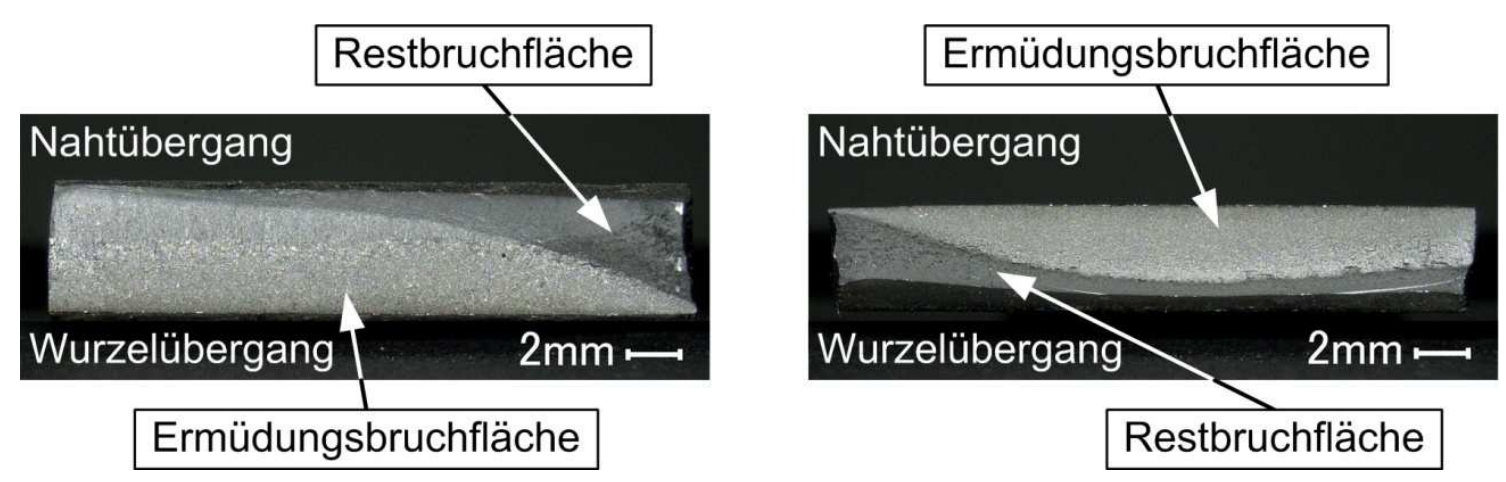

Abb. 5-10: Anriss am Wurzelübergang (links) und am Nahtübergang (rechts)

Von insgesamt 89 Probekörpern verlagerte sich bei 8 Probekörpern der Anrissort vom Wurzel- zum Nahtübergang, was einem Anteil von etwa $9 \%$ entspricht. Da die Ergebnisse der Proben mit Anriss am Nahtübergang im Streuband der Proben mit 
Anriss am Wurzelübergang liegen, wurde in der Auswertung keine Unterscheidung nach dem Anrissort vorgenommen. Eine Prognose des Anrissortes kann anhand der in Kapitel 3.5 als Maß für die Kerbwirkung ermittelten Kerbformzahlen der einzelnen Übergänge aus den Profilschrieben von 20 Probekörpern vorgenommen werden. Bei Betrachtung der Ergebnisse kann für jede Probe anhand der maximalen Kerbformzahl eine Vorhersage für den Anrissort gegeben werden. Der Anriss kann an den Naht- oder Wurzelübergängen sowie auf der rechten oder linken Seite auftreten. Der Vergleich dieser Prognose, abgeleitet aus den numerisch ermittelten Kerbformzahlen, mit dem tatsächlich eingetreten Ereignis zeigt, dass bei ungefähr $63 \%$ der Proben eine Übereinstimmung vorliegt. Es konnte hierbei keine Abhängigkeit von der Beanspruchungshöhe festgestellt werden. Um eine zuverlässigere Prognose des Anrissortes einzelner Probekörper zu erzielen, ist eine flächige Aufnahme der lokalen Nahtgeometrie zu empfehlen. Im vorliegenden Fall wurden lediglich 3 Profilschriebe auf einer Probe ausgewertet. Die hohe Streuung der ermittelten lokalen Nahtgeometrieparameter bedingt ein solches Ergebnis.

Die Ermüdungsbruchflächen wurden unterschiedlichen Erscheinungsformen zugeordnet, wobei diese Zuordnung visuell anhand des Erscheinungsbildes der Bruchfläche im Endzustand der Rissausbreitung erfolgte. Einen Überblick der auftretenden Rissformen aller Versuchsreihen gibt Abb. 5-11. Es zeigen sich durchgehende Risse (Rissform A), halbelliptische Oberflächenanrisse (Rissform B) und viertelliptische Eckanrisse (Rissform C) sowie Mischformen, wobei es sich um einzelne und mehrere Anrisse handeln kann, die sich fortpflanzen und zusammenschließen.

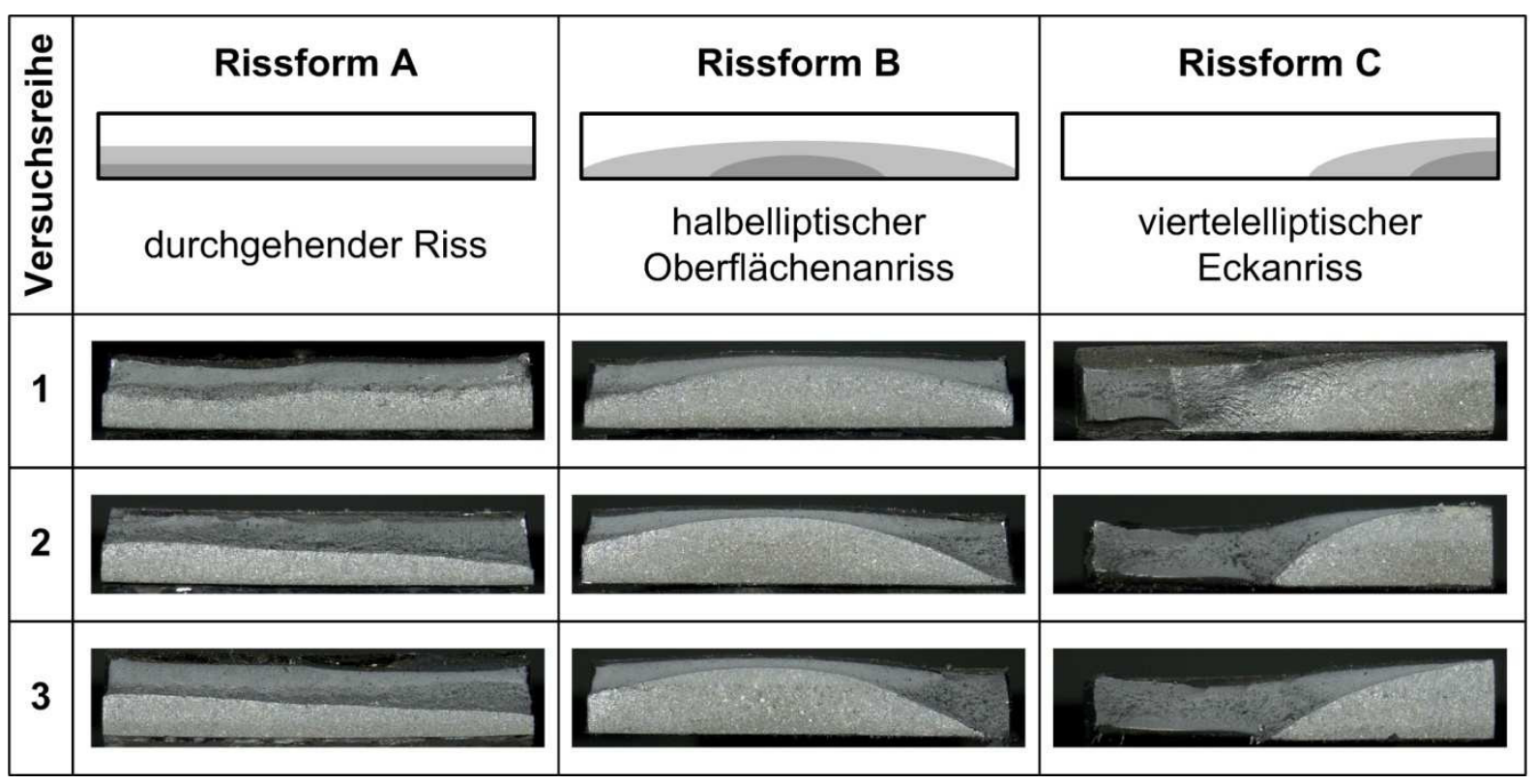

Abb. 5-11: Überblick der aufgetretenen Rissformen aller Versuchsreihen 
Bei Rissform A werden entlang dem Verlauf des Wurzelübergangs viele einzelne Risse initiiert, die sich zu einer durchgehenden, annähernd parallel über die gesamte Probenbreite wachsenden Rissfront zusammenschließen. Zum Teil ist die Ausbreitung des Risses auf einer Seite etwas ausgeprägter, wo es dann auch zum Versagen gekommen ist. Bei Rissform B handelt es sich um halbelliptische Oberflächenanrisse, die sich flächig im Probenquerschnitt bis zum Versagen fortsetzen. Zum Teil schließen sich mehrere einzelne halbelliptische Oberflächenanrisse zu einer Rissfront zusammen. Wenn die Risse nicht in der Mitte der Probe auftreten, breiten sie sich halbelliptisch bis zum Erreichen einer Seite aus. Dann folgt eine deutlichere Ausbreitung auf dieser Seite bis zum Versagen. Zum Teil sind die Ausprägungen mehrerer einzelner halbelliptischer Oberflächenanrisse auf der Bruchfläche erkennbar. Bei Rissform $C$ handelt es sich um viertelelliptische Eckanrisse, die sich flächig im Probenquerschnitt bis zum Versagen fortsetzen. Sie treten an den Rändern der Proben im Bereich der Nahtwurzel auf. Von dieser Ecke aus wächst der Riss bis zum Restbruch, der sich auf der anderen Probenseite ereignet. Teilweise zeigt sich eine sehr geradlinige Rissfront.

Bei allen Versuchsreihen kann beobachtet werden, dass im Übergangsbereich (B) ausschließlich die Rissformen $B$ und $C$ auftreten, wohingegen sich im Zeitfestigkeitsbereich (A) alle Rissformen sowie Mischformen daraus vertreten sind. Besonders auf den höheren Beanspruchungsniveaus im Zeitfestigkeitsbereich (A) der Versuchsreihen 1 und 2 kann vermehrt Rissform A festgestellt werden. Auf den unteren Beanspruchungsniveaus im Zeitfestigkeitsbereich (A) von Versuchsreihe 3 zeigt sich vielfach Rissform B, wohingegen bei Versuchsreihe 2 in diesem Bereich vorwiegend Rissform C auftritt. Die Bruchflächen der Versuchsreihen 2 und 3 weisen im Zeitfestigkeitsbereich weitere Anrisse auf den Bruchflächen auf. Dies kann darauf zurückgeführt werden, dass die Probekörper dieser Reihen bis zum unterschiedlichen Abbruchkriterium geprüft und anschließend im statischen Zugversuch getrennt wurden. Die Ausbreitung der Rissfront ist zum Teil nicht so weit fortgeschritten wie im Vergleich zu Versuchsreihe 1, wo bis zum vollständigen Bruch der Probekörper geprüft wurde. Dies lässt den Vergleich der Bruchflächen nur bedingt zu. Vereinzelt zeigen sich auch weitere kleine Anrisse am gegenüberliegenden Wurzelübergang oder im Bereich der Nahtübergänge.

Die erneut geprüften Durchläufer (C) der Versuchsreihen 2 und 3 weisen überwiegend die Rissformen $B$ und $C$ auf. Einzelne Proben dieser Reihen weisen darüber hinaus weitere Anrisse am gegenüberliegenden Wurzelübergang oder im Bereich der Nahtübergänge mit sehr deutlicher Ausprägung auf. In Abb. 5-12 sind exemplarisch die Wurzelüberhöhung in der Draufsicht (links) sowie die Bruchflächen der Probe aus Versuch V2-C-2 dargestellt. Allerdings liegen die Ergebnisse der hochgesetzten 
Durchläufer im Streuband der übrigen Versuche im Zeitfestigkeitsbereich, wie in Kapitel 5.5.4 gezeigt.
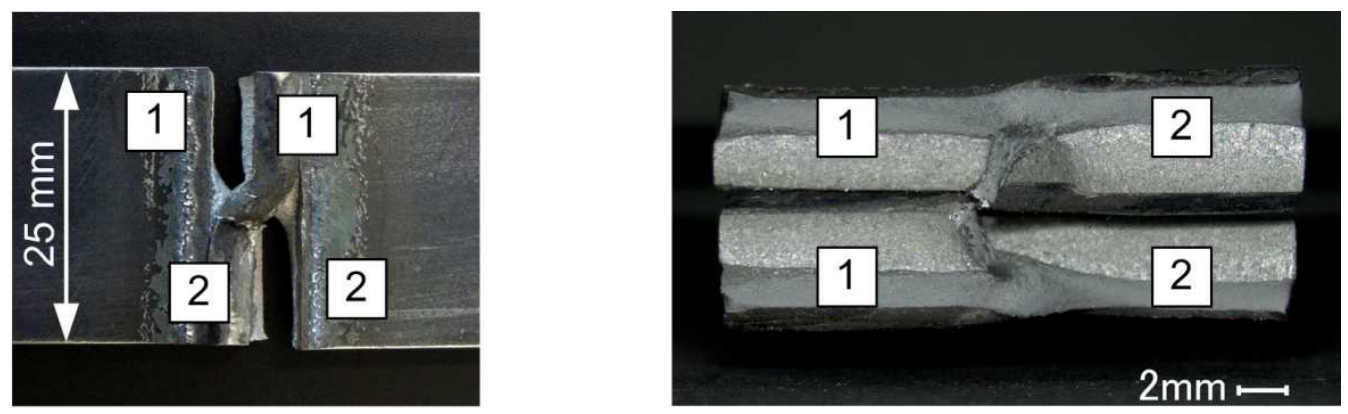

Abb. 5-12: Draufsicht der Wurzelüberhöhung (links) und Bruchflächen (rechts) von Versuch V2-C-2

Alle Probekörper, bei denen der Anriss am Nahtübergang initiiert wurde, weisen Rissform B auf. Der Anriss am Nahtübergang wurde fast ausschließlich im Zeitfestigkeitsbereich (A) beobachtet. Allgemein werden die Anrissorte und Erscheinungsformen der Bruchflächen aller Versuchsreihen als gleichartig eingestuft.

\subsubsection{Verhalten im Bereich sehr hoher Lastwechselzahlen}

Das Verhalten der Probekörper im Bereich sehr hoher Lastwechselzahlen wird über die maximal erreichten Bruch- bzw. Anrissschwingspielzahlen der einzelnen Versuchsreihen sowie die Ergebnisse der Bruchflächenuntersuchung und der Prüfung der hochgesetzten Durchläufer beurteilt. Bei Versuchsreihe 1 ergibt sich die maximal erreichte Bruchschwingspielzahl $N_{B}$ im Übergangsbereich (B) zu 2.327.013 Lastwechseln. In den Versuchsreihen 2 und 3 liegen die maximal erreichten Anrissschwingspielzahlen $\mathrm{N}_{\mathrm{A}}$ bei 1.858.886 Lastwechseln und 2.342.732 Lastwechseln. Außerdem trat bei Prüfung der hochgesetzten Durchläufer (C) von Versuchsreihe 3 das Versagen einer Probe auf dem untersten Beanspruchungsniveau nahe dem Übergangsbereich bei 2.491.698 Lastwechseln auf. Der Anriss ereignete sich in diesem Fall am Nahtübergang.

Die maximal erreichten Bruch- bzw. Anrissschwingspielzahlen im Übergangsbereich aller Versuchsreihen übersteigen den Wert von $3 \cdot 10^{6}$ Lastwechseln nicht und liegen deutlich unterhalb von $10^{7}$ Lastwechseln. Es treten demnach keine späten Brüche auf. Dieser Sachverhalt sowie die Erkenntnis, dass die Anrisse aller Proben von der Oberfläche ausgehen, deuten somit nicht auf einen weiteren Abfall der Ermüdungsfestigkeit im Bereich sehr hoher Lastwechselzahlen hin. Außerdem konnten mit dem Farbeindringverfahren keine Oberflächenanrisse bei den Durchläufern der einzelnen Versuchsreihen festgestellt werden. Auch die erneute Prüfung der Durchläufer auf den Beanspruchungsniveaus im Zeitfestigkeitsbereich (siehe Kapitel 5.5.4) zeigt im 
Vergleich zu den übrigen Versuchen im Zeitfestigkeitsbereich keine besonderen Auffälligkeiten. Im vorliegenden Fall kann von einem horizontalen Auslaufen der Wöhlerlinie und demnach von einer vorliegenden Dauerfestigkeit ausgegangen werden.

\subsubsection{Vergleich der Versuchsergebnisse mit Sonsino et al.}

Eine normierte Auswertung von Ermüdungsversuchen (siehe Abb. 1-1), die bis in den Bereich sehr hoher Lastwechselzahlen reichen, erfolgte in Sonsino et al. [Sonsino, 2005b]. Insgesamt wurden 240 Versuchsergebnisse ([Sonsino, 1994], [Nakamura, 1988], [Maddox, 1998], [Buitrago, 2003]) unterschiedlicher Schweißverbindungen, ermittelt bei Prüffrequenzen $\mathrm{f}$ bis $125 \mathrm{~Hz}$ und bis zu Grenzschwingspielzahlen $N_{G}$ von $10^{8}$ Lastwechseln, normiert ausgewertet. Hierbei wurde für jede Versuchsreihe die Spannungsschwingbreite $\Delta \sigma$ bei $2 \cdot 10^{6}$ Lastwechseln ermittelt und auf 1,0 normiert.

Zunächst zeigt die Auswertung, dass bisher nur wenige experimentelle Ergebnisse für Schweißverbindungen aus Stahl im Bereich sehr hoher Lastwechselzahlen vorliegen. Die zusammengestellten Versuchsergebnisse aus Sonsino et al. [Sonsino, 2005b] reichen lediglich bis zu einer Grenzschwingspielzahl $N_{G}$ von $10^{8}$ Lastwechseln, wohingegen die Versuchsreihen der vorliegenden Arbeit bis zu einer Grenzschwingspielzahl $N_{G}$ von $5 \cdot 10^{8}$ Lastwechseln geprüft wurden. Bis zu einer Schwingspielzahl von $10^{7}$ Lastwechseln werden die Versuchsergebnisse durch einen Neigungsexponenten $\mathrm{k}$ von 3,0 angenähert. Nach einer Schwingspielzahl von $10^{7}$ Lastwechseln zeigt sich ein weiterer Abfall der Ermüdungsfestigkeit bis zu einer Schwingspielzahl von bis zu $10^{8}$ Lastwechseln, der mit einem Neigungsexponenten $\mathrm{k}^{*}$ von 22 angenähert wird. Es wird angemerkt, dass das Auftreten von Durchläufern in diesem Bereich auf eine Veränderung des Schädigungsmechanismus hindeutet.

Die Versuchsergebnisse der vorliegenden Arbeit aus den Versuchsreihen 1 bis 3 wurden für den Vergleich ebenfalls normiert. Zur Ermittlung der Spannungsschwingbreite $\Delta \sigma$ bei $2 \cdot 10^{6}$ Lastwechseln wurden die Ergebnisse aus den jeweiligen Versuchsreihen 1-A, 2-A und 3-A herangezogen. Für die Auswertung wurden die Bruchschwingspielzahlen der Versuchsreihen (entsprechend der jeweiligen Definition von Bruch) zugrundegelegt. Die normierten Versuchsergebnisse wurden ebenfalls in das Diagramm aus Abb. 1-1 eingetragen. In Abb. 5-13 ist der Vergleich der Versuche aus der vorliegenden Arbeit mit der normierten Auswertung von Ermüdungsversuchen aus Sonsino et al. [Sonsino, 2005b] dargestellt. Es ist zu erkennen, dass der Großteil der Versuchsergebnisse der vorliegenden Arbeit innerhalb des Streubandes der anderen Versuchsergebnisse liegt. Allerdings zeigt sich bei den Versuchsergebnissen 
der vorliegenden Arbeit ein deutlich früherer Abknickpunkt im Bereich von $10^{6}$ bis $2 \cdot 10^{6}$ Lastwechseln.

Bei Sonsino et al. wird angegeben, dass der Vorschlag für den dargestellten Verlauf der Ermüdungsfestigkeitskurven bei sehr hohen Schwingspielzahlen mit konstanter Spannungsschwingbreite für Schweißverbindungen mit hohen Zugeigenspannungen sowie metallurgischen Diskontinuitäten wie Einschlüsse oder Poren in ermüdungskritischen Bereichen der Schweißnaht gültig ist. Liegen günstigere Bedingungen der Schweißnaht vor, so können andere Ansätze erforderlich werden [Sonsino, 2005b]. Für den Fall, dass Zugeigenspannungen verringert oder vermieden werden können, wird darauf hingewiesen, dass der Abknickpunkt auf ein höheres Level in Abhängigkeit der Höhe der Spannungskonzentration sogar in Richtung $10^{6}$ Lastwechsel verschoben werden kann.

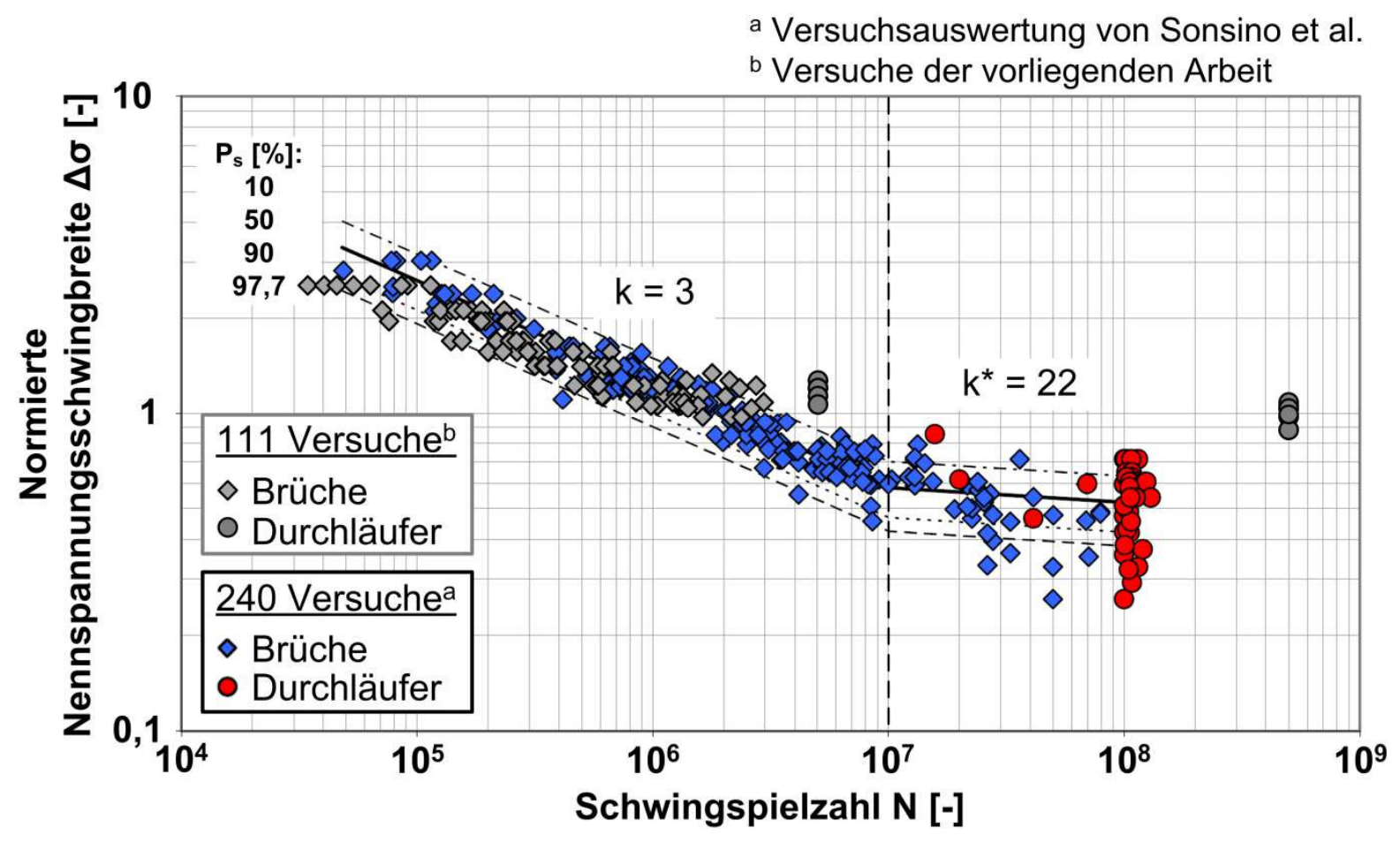

Abb. 5-13: Vergleich der Versuchsergebnisse der vorliegenden Arbeit mit Sonsino et al. [Sonsino, 2005b]

Die Verschiebung des Abknickpunktes in Richtung $10^{6}$ Lastwechsel kann durch die Versuchsergebnisse der vorliegenden Arbeit bestätigt werden. Versuchsreihe 3 wurde zur näherungsweisen Berücksichtigung von Zugeigenspannungen bei einem Spannungsverhältnis $\mathrm{R}$ von 0,5 ermittelt. Im Hinblick auf den Abknickpunkt unterscheidet sich Versuchsreihe 3 allerdings nicht signifikant von den Versuchsreihen 1 und 2. Das Auftreten später Brüche bzw. eines weiteren Abfalls der Ermüdungsfestigkeit nach $10^{7}$ Lastwechseln bzw. nach dem Abknickpunkt kann durch die Ver- 
suchsreihen der vorliegenden Arbeit nicht bestätigt werden. Im vorliegenden Fall wird von einer Dauerfestigkeit ausgegangen.

\subsubsection{Vergleich der Versuchsergebnisse mit Eurocode 3}

Die Einstufung der Konstruktionsdetails für die Berechnung mit dem Nennspannungskonzept erfolgt nach Eurocode 3 [DIN EN 1993-1-9, 2010] unabhängig von Streckgrenze und Spannungsverhältnis, wobei der Neigungsexponent k der Ermüdungsfestigkeitskurven für Längsspannungsschwingbreiten zu 3,0 und der Abknickpunkt $N_{D}$ zur Dauerfestigkeit bei $5 \cdot 10^{6}$ für konstante Spannungsschwingbreiten festgelegt sind. Einseitig geschweißte Stumpfnähte ohne Schweißbadsicherung werden gemäß Tabelle 8.3 in Eurocode 3 [DIN EN 1993-1-9, 2010] in Kerbfallklasse 36 bzw. bei Inspektion der Wurzellage durch ZFP (Zerstörungsfreie Prüfverfahren) in Kerbfallklasse 71 eingestuft (siehe Abb. 5-14). Bei Blechdicken von $t>25 \mathrm{~mm}$ ist eine Abminderung der Ermüdungsfestigkeit über den Faktor $k_{s}$ vorgesehen.

\begin{tabular}{|c|l|l|l|l|}
\hline Kerbfall & Konstruktionsdetail & Beschreibung & Anforderungen \\
\hline 36 & \multicolumn{2}{|l|}{$\begin{array}{l}\text { Blechdi- } \\
\text { ckenabh. f. } \\
t>25 \mathrm{~mm}: \\
k_{\mathrm{s}}=(25 / t)^{0,2}\end{array}$}
\end{tabular}

Abb. 5-14: Auszug aus Tabelle 8.3 in Eurocode 3 [DIN EN 1993-1-9, 2010] zur Kerbfalleinstufung quer laufender Stumpfnähte

Um die Einstufung der Kerbfallklasse mit den Versuchsergebnissen der vorliegenden Arbeit vergleichen zu können, erfolgte die statistische Auswertung der Ermüdungsversuche im Zeitfestigkeitsbereich der Versuchsreihen 1-A, 2-A und 3-A nach dem Hintergrundbericht zum Eurocode 3, wie bei Dürr [Dürr, 2007] erläutert. Die Auswertung wurde auf Basis der Bruchschwingspielzahlen der Versuchsreihen (entsprechend der jeweiligen Definition von Bruch) durchgeführt. Es wurden ein fester Neigungsexponent $k$ der Ermüdungsfestigkeitskurven von 3,0 sowie der Abknickpunkt $N_{D}$ zur Dauerfestigkeit bei $5 \cdot 10^{6}$ zugrundegelegt. In Abb. 5-15 sind neben den Einzelversuchen der jeweiligen Versuchsreihen die ermittelten Bruchwöhlerlinien gemäß dem Hintergrundbericht zum Eurocode 3 im Vergleich zu den Wöhlerlinien der Kerbfallklassen 36 und 71 dargestellt. Die Ergebnisse der einzelnen Versuchsreihen sind mit konstantem sowie variablem Neigungsexponenten im Anhang J.3.1 bis J.3.3 dargestellt. 


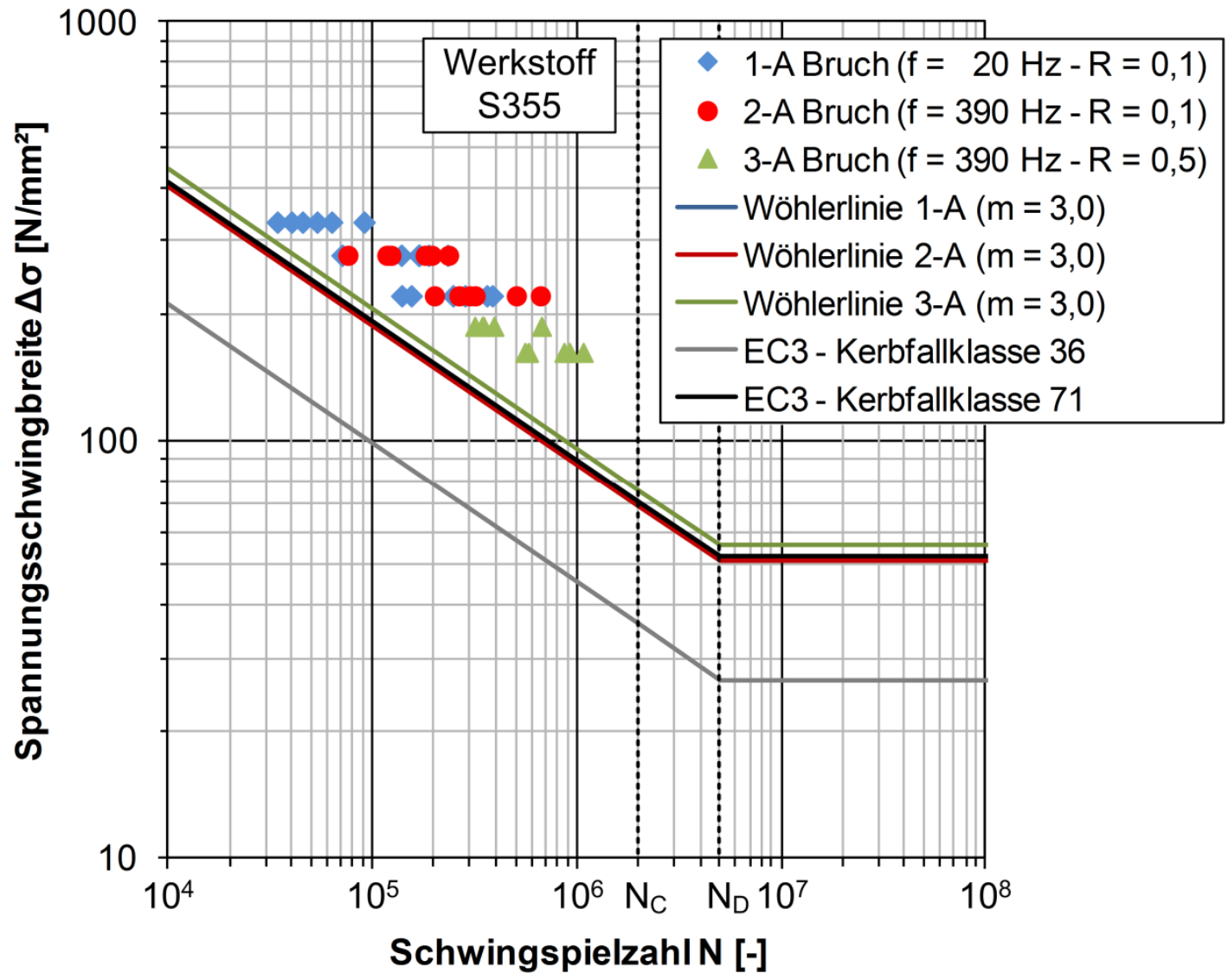

Abb. 5-15: Vergleich der Versuchsergebnisse der vorliegenden Arbeit mit Eurocode 3

Die ermittelten Wöhlerlinien aller Versuchsreihen liegen deutlich oberhalb der Wöhlerlinie von Kerbfallklasse 36. Die Wöhlerlinien von Versuchsreihe 1-A und 2-A liegen geringfügig unterhalb der Wöhlerlinie von Kerbfallklasse 71 , wohingegen die Wöhlerlinie von Versuchsreihe 3-A geringfügig oberhalb der Wöhlerlinie von Kerbfallklasse 71 liegt. In Tab. 5-17 sind die charakteristischen Ermüdungsfestigkeiten $\Delta \sigma_{\mathrm{C}}$ bei $2 \cdot 10^{6}$ Lastwechseln der einzelnen Versuchsreihen bei festem Neigungsexponenten $\mathrm{k}$ von 3,0 und variabel ermitteltem Neigungsexponenten gegenübergestellt. AuBerdem ist die prozentuale Abweichung $\mathrm{p}_{\%, \mathrm{KF} 71}$ der ermittelten charakteristischen Ermüdungsfestigkeiten von Kerbfallklasse 71 angegeben.

Tab. 5-17: Gegenüberstellung der ermittelten charakteristischen Ermüdungsfestigkeiten $\Delta \sigma_{C}$ der Versuchsreihen 1-A, 2-A und 3-A

\begin{tabular}{|c|c|c|c|c|c|c|c|c|}
\hline \multicolumn{3}{|c|}{ Versuchsreihe 1-A } & \multicolumn{3}{|c|}{ Versuchsreihe 2-A } & \multicolumn{3}{|c|}{ Versuchsreihe 3-A } \\
\hline $\begin{array}{c}\mathbf{k} \\
{[-]}\end{array}$ & $\begin{array}{c}\Delta \sigma_{\mathrm{C}} \\
{\left[\mathrm{N} / \mathrm{mm}^{2}\right]}\end{array}$ & $\begin{array}{c}\mathbf{p}_{\%, \mathrm{KF} 11} \\
{[\%]}\end{array}$ & $\begin{array}{c}\mathbf{k} \\
{[-]}\end{array}$ & $\begin{array}{c}\Delta \sigma_{\mathrm{C}} \\
{\left[\mathrm{N} / \mathrm{mm}^{2}\right]}\end{array}$ & $\begin{array}{c}\mathbf{p}_{\%, K F 71} \\
{[\%]}\end{array}$ & $\begin{array}{c}\mathbf{k} \\
{[-]}\end{array}$ & $\begin{array}{c}\Delta \sigma_{\mathrm{c}} \\
{\left[\mathrm{N} / \mathrm{mm}^{2}\right]}\end{array}$ & $\begin{array}{c}\mathbf{p}_{\%, \mathrm{KF71}} \\
{[\%]}\end{array}$ \\
\hline 3,0 & 70,8 & $-0,3$ & 3,0 & 69,1 & $-2,7$ & 3,0 & 75,7 & $+6,6$ \\
\hline 3,8 & 97,4 & $+37,2$ & 3,9 & 98,9 & $+39,3$ & 4,6 & 109,0 & $+53,5$ \\
\hline
\end{tabular}


Bei der Auswertung mit einem festen Neigungsexponenten $k$ von 3,0 betragen die prozentualen Abweichungen $\mathrm{p}_{\%, \mathrm{KF} 71}$ der ermittelten charakteristischen Ermüdungsfestigkeiten $-0,3 \%$ bei Versuchsreihe 1-A sowie $-2,7 \%$ bei Versuchsreihe 2-A im Verhältnis zu Kerbfallklasse 71. Versuchsreihe 3-A weist hingegen eine prozentuale Abweichung $\mathrm{p}_{\%, \mathrm{KF} 71}$ von $+6,6 \%$ auf. Bei der Berechnung mit variablem Neigungsexponenten zeigt sich eine Erhöhung der charakteristischen Ermüdungsfestigkeit aller Versuchsreihen in Abhängigkeit von den ermittelten Neigungsexponenten. Da die Neigungsexponenten $\mathrm{k}$ Werte größer als 3,0 annehmen, weisen die Wöhlerlinien einen flacheren Verlauf auf, was mit einer höheren charakteristischen Ermüdungsfestigkeit einhergeht. Für die vorliegenden Versuchsreihen kann die Einstufung des Konstruktionsdetails nach Eurocode 3 [DIN EN 1993-1-9, 2010] in Kerbfallklasse 71 annähernd bestätigt werden.

Die ermittelten Neigungsexponenten der einzelnen Versuchsreihen liegen oberhalb des in Eurocode 3 [DIN EN 1993-1-9, 2010] angegebenen Neigungsexponenten $k$ von 3,0. Ein Überblick von Sonsino et al. [Sonsino, 2010] zeigt, dass die Ermüdungsfestigkeitskurven bei dünnen Blechen $(t<5 \mathrm{~mm}$ ) üblicherweise eine hohe Streuung der Neigungsexponenten $\mathrm{k}$ zwischen 3,0 und 6,0 aufweisen und demnach flacher verlaufen als in den Regelwerken einheitlich angegeben. Dies kann zu einer Überschätzung der Ermüdungsfestigkeit im Bereich hoher Spannungsschwingbreiten sowie zu einer Unterschätzung der Ermüdungsfestigkeit im Bereich niedriger Spannungsschwingbreiten führen. Die von Haibach [Haibach, 2006] angegebenen Erfahrungswerte liegen bei 3,5 für unter einheitlichen Bedingungen und bei 3,0 für unter betriebsüblichen Bedingungen angefertigten Schweißnähten aus Baustahl. Die vorliegenden Schweißverbindungen wurden unter einheitlichen Bedingungen geschweißt. Die ermittelten Neigungsexponenten $\mathrm{k}$ von Versuchsreihe 1-A mit 3,8 und von Versuchsreihe 2-A mit 3,9 weichen nur geringfügig von diesem Erfahrungswert $a b$, wohingegen der Neigungsexponent $k$ von Versuchsreihe 3-A mit 4,6 deutlich flacher ermittelt wurde. Dies kann auf die Prüfung bei einem Spannungsverhältnis $R$ von 0,5 und dem Sachverhalt, dass die Beanspruchungsniveaus im Zeitfestigkeitsbereich sehr dicht beieinander und zudem sehr nah am Übergangsbereich liegen, zurückgeführt werden.

\subsection{Zusammenfassung und Erkenntnisse}

In drei Versuchsreihen wurden insgesamt 111 Ermüdungsversuche an axial beanspruchten bauteilähnlichen Schweißverbindungen im Zeitfestigkeits- und Übergangsbereich bis zu sehr hohen Lastwechselzahlen bei konstanter Beanspruchungsamplitude durchgeführt. Die Versuche in der hochfrequenten Prüfvorrichtung 
reichen hierbei bis zu einer Grenzschwingspielzahl $N_{G}$ von $5 \cdot 10^{8}$ Lastwechseln. Die einzelnen Versuchsreihen wurden ausgewertet und dokumentiert.

Die Versuchsbedingungen der Versuchsreihen 2 und 3, durchgeführt in der hochfrequenten Prüfvorrichtung, wurden im Hinblick die Ergebnisse der Ermüdungsversuche bewertet. Die vorgegebenen Versuchsbedingungen der Kraftamplitude während des Versuchs und der Vorspannkraft zu Versuchsbeginn wurden mit einer guten Reproduzierbarkeit erzielt. Die Betrachtung der Erwärmung des Probekörpers durch innere Reibung sowie des damit einhergehenden Abfalls der Vorspannkraft zeigten, dass auf den oberen Beanspruchungsniveaus günstigere Versuchsbedingungen als auf den unteren Beanspruchungsniveaus und generell als im Vergleich zu idealen Versuchsbedingungen mit konstantem Spannungsverhältnis vorliegen. Dies kann generell und besonders auf den oberen Beanspruchungsniveaus zu einer erhöhten Lebensdauer führen.

Zur Untersuchung des Einflusses der Prüffrequenz wurden zwei Versuchsreihen, die bei unterschiedlichen Prüffrequenzen $(20 \mathrm{~Hz}$ und $390 \mathrm{~Hz})$ getestet wurden, verglichen. Im Zeitfestigkeitsbereich ist die Wöhlerlinie mit höherer Prüffrequenz im Vergleich zur Wöhlerlinie mit niedriger Prüffrequenz zu höheren Bruchschwingspielzahlen verschoben. Im Übergangsbereich liegt die Wöhlerlinie mit höherer Prüffrequenz unterhalb der Wöhlerlinien mit niedriger Prüffrequenz. Der Vergleich der beiden Versuchsreihen ließ demnach keine eindeutigen Aussagen zu. Ein signifikanter Einfluss der Prüffrequenz auf die Versuchsergebnisse konnte nicht festgestellt werden.

Um Aussagen über den Einfluss von Zugeigenspannungen auf die Ermüdungsfestigkeit anhand kleiner, als nahezu eigenspannungsfrei anzusehender Probekörper treffen zu können, wurden die Zugeigenspannungen näherungsweise als Mittelspannungen berücksichtigt. Der Einfluss der Mittelspannung wurde in zwei Versuchsreihen, die bei unterschiedlichen Spannungsverhältnissen $(R=0,1$ und $R=0,5)$ getestet wurden, untersucht. Im Zeitfestigkeitsbereich weist die Wöhlerlinie mit höherem Spannungsverhältnis einen flacheren Verlauf auf, als die Wöhlerlinie mit geringerem Spannungsverhältnis. Im Übergangsbereich liegen die Ergebnisse beider Versuchsreihen sehr dicht beieinander. Der Vergleich der beiden Versuchsreihen zeigte den Einfluss der Mittelspannung auf die Versuchsergebnisse im Zeitfestigkeitsbereich. Bei höherem Spannungsverhältnis verläuft die Wöhlerlinie flacher als bei geringerem Spannungsverhältnis.

Die Auswertung der hochgesetzten Durchläufer zeigte, dass die Ergebnisse der Versuchsreihen in den jeweiligen Streubändern der Ergebnisse im Zeitfestigkeitsbereich liegen. Auf den oberen Beanspruchungsniveaus sind die Ergebnisse der hochgesetzten Durchläufer im Bereich der ermittelten Bruch- bzw. Anrissschwingspielzahl für 
eine Überlebenswahrscheinlichkeit $\mathrm{P}_{u ̈}$ von $90 \%$ im Zeitfestigkeitsbereich zu finden. Zudem liegt in diesem Bereich eine geringe Streuung der erneut geprüften Durchläufer vor, die mit sinkendem Beanspruchungsniveau zunimmt. Hierbei kam es zu einer Verschiebung der hochgesetzten Durchläufer in den Bereich der ermittelten Bruchbzw. Anrissschwingspielzahl für eine Überlebenswahrscheinlichkeit $P_{u ̈}$ von $50 \%$ und darunter. Da Durchläufer generell Proben mit erhöhter Festigkeit darstellen, lag dieses Ergebnis mittlerer bis höherer Versagensschwingspielzahlen und einer geringen Streuung im Erwartungsbereich. Es konnte weder eine deutlich geringere noch eine deutlich höhere Lebensdauer der hochgesetzten Durchläufer im Vergleich zu den anderen Ergebnissen im Zeitfestigkeitsbereich festgestellt werden. Aus diesem Grund kann trotz Vorbelastung von einer neutralen Ausgangssituation der Probekörper ausgegangen werden.

Unabhängig von Beanspruchungsniveau und Prüfvorrichtung zeigten die Bruchflächen aller Versuchsreihen ein einheitliches makroskopisches Erscheinungsbild. Der Ermüdungsbruch ist normalflächig und verläuft senkrecht zur Beanspruchungsrichtung. Aufgrund der makroskopischen Kerbwirkung der unbehandelten Schweißnaht traten die Ermüdungsbrüche der Proben an den Kerben der Übergänge von Nahtund Wurzelüberhöhung im Bereich der Schweißnaht auf. Die Anrisse gingen von der Oberfläche aus. Es konnte keine Verlagerung des Anrissortes ins Innere der Proben festgestellt werden. Der Anriss trat vorwiegend am Wurzelübergang auf, wobei es vereinzelt zu einer Verlagerung des Anrissortes in den Bereich des Nahtübergangs kam. Im Übergangsbereich traten ausschließlich halbelliptische Oberflächenanrisse sowie viertelelliptische Eckanrisse auf, wohingegen sich im Zeitfestigkeitsbereich besonders auf den höheren Beanspruchungsniveaus auch durchgehende Risse zeigten. Bei Initiierung des Anrisses am Nahtübergang wiesen die Proben halbelliptische Oberflächenanrisse auf. Die Anrissorte und Erscheinungsformen der Bruchflächen sind für alle Versuchsreihen als gleichartig einzustufen.

Im Übergangsbereich traten in keiner Versuchsreihe Brüche bzw. Anrisse bei Schwingspielzahlen von größer als $3 \cdot 10^{6}$ Lastwechseln auf. Die maximal erreichten Bruch- bzw. Anrissschwingspielzahlen liegen deutlich unterhalb von $10^{7}$ Lastwechseln. Zudem gab es keinen Hinweis auf eine Änderung des Schädigungsmechanismus in Form einer Verlagerung des Anrissortes von der Oberfläche ins Innere der Probe. Bei den Durchläufern der Versuchsreihen konnten keine Oberflächenanrisse mit dem Farbeindringverfahren festgestellt werden. Die erneut geprüften Durchläufer auf den Beanspruchungsniveaus im Zeitfestigkeitsbereich liegen im Streuband der übrigen Versuche im Zeitfestigkeitsbereich und zeigten keine wesentlichen Auffälligkeiten. Diese Erkenntnisse deuten daher nicht auf einen weiteren Abfall der Ermüdungsfestigkeit, sondern auf ein horizontales Auslaufen der Wöhlerlinien im Bereich 
sehr hoher Lastwechselzahlen hin. Im vorliegenden Fall kann demnach von einer Dauerfestigkeit ausgegangen werden.

Der Vergleich der Versuche der vorliegenden Arbeit mit der normierten Auswertung von Ermüdungsversuchen aus Sonsino et al. [Sonsino, 2005b] zeigte, dass ein Großteil der Versuchsergebnisse der vorliegenden Arbeit innerhalb des Streubandes der anderen Versuchsergebnisse liegt. Allerdings sind der frühere Abknickpunkt der Versuchsergebnisse der vorliegenden Arbeit im Bereich von $10^{6}$ bis $2 \cdot 10^{6}$ Lastwechseln sowie das horizontale Auslaufen deutlich zu erkennen. Die Versuche der vorliegenden Arbeit reichen bis zu einer Grenzschwingspielzahl von $5 \cdot 10^{8}$ Lastwechseln, wohingegen die anderen Versuche lediglich bis zu $10^{8}$ Lastwechseln reichen.

Der Vergleich der Versuchsergebnisse mit Eurocode 3 [DIN EN 1993-1-9, 2010] zeigte, dass die ermittelten Wöhlerlinien der Versuchsreihen 1 und 2 geringfügig unterhalb sowie von Versuchsreihe 3 geringfügig oberhalb der Wöhlerlinie von Kerbfallklasse 71 für einseitig geschweißte Stumpfnähte ohne Schweißbadsicherung mit ZFP liegen. Für die vorliegenden Versuchsreihen konnte somit annähernd die Einstufung des Konstruktionsdetails nach Eurocode 3 in Kerbfallklasse 71 bestätigt werden. Allerdings liegen die ermittelten Neigungsexponenten der einzelnen Versuchsreihen oberhalb des im Eurocode 3 angegebenen Neigungsexponenten. 


\section{Zusammenfassung und Ausblick}

\subsection{Zusammenfassung}

Die vorliegende Arbeit widmet sich der Untersuchung des Ermüdungsverhaltens einer stählernen Stumpfnahtverbindung bis in den Bereich sehr hoher Lastwechselzahlen. Auf der einen Seite bestand die Zielsetzung der Arbeit in der Weiterentwicklung der Prüftechnik zur Realisierung von Ermüdungsversuchen an Schweißverbindungen im Bereich sehr hoher Lastwechselzahlen in einem akzeptablen Zeitraum. Auf der anderen Seite war das Ziel der Arbeit die Durchführung und Ausweitung der experimentellen Untersuchungen an Schweißverbindungen bei konstanter Spannungsschwingbreite bis in den Bereich sehr hoher Schwingspielzahlen zur Klärung der Fragestellung des Auftretens später Brüche.

Als Ausgangspunkt wurde der im Kontext dieser Arbeit stehende aktuelle Stand der Forschung in Kapitel 2 dargestellt.

Die Herstellung der Probekörper für die experimentellen Untersuchungen wurde beschrieben. Die Bewertung der Reproduzierbarkeit des Schweißprozesses sowie die Überprüfung der Qualität der Probekörper erfolgten durch die Charakterisierung des Ausgangszustandes in Kapitel 3.

Der Schweißprozess stellte sich bei der Beurteilung der herstellungsbegleitend durchgeführten Temperaturmessungen während der Schweiß- und Abkühlphase als reproduzierbar dar. Zur Beurteilung des Einflusses des Temperatur-Zeit-Verlaufs der Schweiß- und Abkühlphase auf die mechanischen Eigenschaften der Schweißverbindung wurden die Abkühlzeiten $t_{8 / 5}$ ausgewertet. Die ermittelten Werte der Abkühlzeiten $t_{8 / 5}$ lagen im oberen Bereich der Richtwerte.

Trotz mechanisierter Fertigung der Probekörper wurden große Streuungen der lokalen Nahtgeometrieparameter ermittelt. An den Wurzelübergängen zeigten sich im Mittel kleinere Kerbradien sowie größere Anstiegswinkel als an den Nahtübergängen, wobei sich die Extremalwerte an den Naht- und Wurzelübergängen nur geringfügig unterscheiden.

Die Kerbformzahlen der Übergänge an der Naht- und Wurzelüberhöhung wurden als Maß zur Beurteilung der Kerbwirkung analytisch anhand von Näherungsformeln sowie numerisch mit Hilfe der Methode der Finiten Elemente ermittelt. Der Vergleich ergab, dass die Näherungsformeln für doppeltsymmetrische Stumpfstöße allgemein zur Abschätzung des im vorliegenden Fall nicht doppeltsymmetrischen Stumpfstoßes herangezogen werden können. Am Nahtübergang ergab sich eine gute Übereinstimmung der Ergebnisse, wohingegen am Wurzelübergang in der numerischen Be- 
rechnung höhere Kerbformzahlen als mit den Näherungsformeln ermittelt wurden. Die maximalen Kerbformzahlen traten bei der numerischen Berechnung ausschließlich am Wurzelübergang auf, was im Hinblick auf die Ermüdungsversuche den Übergang an der Wurzelüberhöhung als vorwiegenden Anrissort erwarten ließ.

Die Festigkeitseigenschaften der Schweißverbindung zeigten, dass die Anforderung an die Schweißverbindung einer mindestens gleichwertigen Festigkeit im Vergleich zum Grundwerkstoff eingehalten wurde. Die metallographische Untersuchung zeigte keine Auffälligkeiten. Die maximalen Härtewerte wurden im Bereich der Grobkornzone der Wärmeeinflusszone in der Nähe der Schmelzlinie ermittelt und lagen deutlich unterhalb der zulässigen Höchstwerte. Es lag eine sehr gute Übereinstimmung der gemessenen maximalen Härtewerte mit den nach Näherungsformeln analytisch berechneten Härtewerten vor. Nach ausführlicher Charakterisierung des Ausgangszustandes der Schweißproben konnte festgehalten werden, dass die Probekörper im Hinblick auf die Ermüdungsversuche als repräsentativ zu bewerten sind.

Die im Rahmen dieser Arbeit verwendete Prüfvorrichtung wurde in Kapitel 4 erläutert. Mit einer Prüffrequenz von bis zu $400 \mathrm{~Hz}$ konnten Ermüdungsversuche an Schweißverbindungen bis in den Bereich sehr hoher Lastwechselzahlen in einem akzeptablen Zeitraum realisiert werden. Neben der Untersuchung der zeitlich veränderlichen Messgrößen, die Aufschluss über das Verhalten der Prüfvorrichtung und die Aussagekraft der erzielten Versuchsergebnisse geben, wurden die Weiterentwicklungen vorgestellt.

Der beobachtete temperaturbedingte Abfall der Vorspannkraft zu Versuchsbeginn führt zu einem Abfall des Spannungsverhältnisses während des Versuchs, da die Vorspannkraft der Prüfvorrichtung im Laufe des Versuchs prinzipiell nicht nachgeregelt werden kann. Dies kann zu höheren Schwingspielzahlen führen. Im Langzeitbetrieb wurde teilweise ein geringfügiger Anstieg der Vorspannkraft festgestellt und auf Abnutzungserscheinungen, verursacht durch Reibkorrosion im Bereich der Spannklötze, zurückgeführt.

Durch die Weiterentwicklung der Vorspannvorrichtung entfiel die separate Vorspannvorrichtung mittels Spannklötzen und Exzenterwellen. Die Vorspannkraft kann nun direkt eingestellt werden und der durch die Umlagerung des Kraftflusses hervorgerufene Verlust an Vorspannkraft entfällt.

Außerdem wurde über den Prüfzeitraum bis zum Erreichen der Grenzschwingspielzahl eine Änderung die Steifigkeit von Probekörper und Einspannung festgestellt, die mit einer Änderung der Proportionalität der Beanspruchungen von Prüfrahmen und Probekörper einhergeht und einer Frequenzabhängigkeit des dynamischen Kalibrierfaktors entspricht. Um den Einfluss des Steifigkeitsverlustes auf den dynamischen 
Kalibrierfaktor zu kompensieren, wurde die Regelung um eine frequenzabhängige Sollwertvorgabe erweitert. Im relevanten Frequenzbereich konnte eine näherungsweise konstante Beanspruchung im Probekörper erzielen werden.

Bei Eintritt eines Anrisses verliert die aufgestellte Proportionalitätsbeziehung zwischen Prüfkraft und zugehöriger Probendehnung ihre Gültigkeit. Die Prüfkraftamplitude kann zu Versuchsende nicht mehr exakt aufgebracht werden. Um eine einheitliche Auswertung der Versuchsergebnisse zu gewährleistet, war die Definition eines Kriteriums zur Ermittlung der Anrissschwingspielzahl erforderlich. Die prozentuale Abweichung von $0,01 \%$ des aktuellen Wertes der Prüffrequenz von einer im leicht linear abfallenden Bereich eingeführten Regressionsgeraden wurde als Kriterium festgelegt.

Um Aufschluss über die Größe des vorliegenden Anrisses beim Erreichen des definierten Anrisskriteriums zu erlangen, wurden Versuche zur Markierung der Rissfront auf der Bruchfläche durchgeführt. Hier wurde festgestellt, dass die Anrisstiefe von der Rissform abhängig ist und die Anzahl der sichtbaren Rastlinien begrenzt war. Da die Breite der Rastlinien auf den Bruchflächen nur in wenigen Fällen auswertbar war, konnten keine Aussagen über die Anrissbreite getroffen werden. Bei viertelelliptischen Eckanrissen konnte eine Anrisstiefe im Bereich von 1,5 mm festgestellt werden, wohingegen in den übrigen Fällen Anrisstiefen von kleiner als $1,2 \mathrm{~mm}$ anzunehmen sind.

Die durchgeführten experimentellen Untersuchungen an bauteilähnlichen Schweißverbindungen wurden in Kapitel 5 dokumentiert, ausgewertet und diskutiert. In drei Versuchsreihen mit insgesamt 111 Ermüdungsversuchen im Zeitfestigkeits- und Übergangsbereich wurde der Einfluss von Prüffrequenz und Zugeigenspannungen auf die Ermüdungsfestigkeit untersucht. Da die Probengröße keine direkte Prüfung von eigenspannungsbehafteten Probekörpern zuließ, wurden aus dem Schweißprozess resultierende Zugeigenspannungen näherungsweise als Mittelspannungen in Form eines erhöhten Spannungsverhältnisses berücksichtigt. Die Versuche wurden an axial beanspruchten Stumpfnahtverbindungen bei konstanter Beanspruchungsamplitude bis zu Grenzschwingspielzahlen von $5 \cdot 10^{8}$ Lastwechseln geprüft.

Die Bewertung der Versuchsbedingungen in der vorgestellten Prüfvorrichtung von Versuchsreihe 2 und 3 im Hinblick auf die Ergebnisse der Ermüdungsversuche ergab, dass die vorgegebenen Parameter mit einer guten Reproduzierbarkeit erzielt werden konnten. Aufgrund des temperaturbedingten Abfalls der Vorspannkraft lagen auf den oberen Beanspruchungsniveaus günstigere Versuchsbedingungen als auf den unteren Beanspruchungsniveaus vor, was im Allgemeinen und besonders auf den oberen Beanspruchungsniveaus zu einer erhöhten Lebensdauer führen kann. 
In zwei Versuchsreihen wurden Schweißverbindungen bei unterschiedlichen Prüffrequenzen $(20 \mathrm{~Hz}$ und $390 \mathrm{~Hz})$ geprüft und verglichen. Die Ergebnisse zeigten keinen signifikanten Einfluss der Prüffrequenz. Die Wöhlerlinie mit höherer Prüffrequenz verläuft im Zeitfestigkeitsbereich oberhalb der Wöhlerlinie mit niedriger Prüffrequenz, wohingegen im Übergangsbereich die Wöhlerlinie mit höherer Prüffrequenz unterhalb der Wöhlerlinie mit niedriger Prüffrequenz liegt. Eine eindeutige Aussage ließ sich aus diesem Vergleich nicht ableiten.

Da kleine Probekörper als nahezu eigenspannungsfrei anzusehen sind, wurden die Zugeigenspannungen bei der Untersuchung des Einflusses der Zugeigenspannungen auf die Ermüdungsfestigkeit näherungsweise als Mittelspannungen betrachtet. In zwei Versuchsreihen wurden somit Schweißverbindungen bei unterschiedlichen Spannungsverhältnissen $(R=0,1$ und $R=0,5)$ geprüft und verglichen. Die Wöhlerlinie mit höherem Spannungsverhältnis zeigte im Zeitfestigkeitsbereich einen flacheren Verlauf auf als die Wöhlerlinie mit geringerem Spannungsverhältnis. Im Übergangsbereich lagen beide Wöhlerlinien hingegen sehr dicht beieinander.

Die Ergebnisse der hochgesetzten Durchläufer befanden sich im Bereich mittlerer bis höherer Versagensschwingspielzahlen innerhalb der Streubänder im Zeitfestigkeitsbereich. Da Durchläufer im Allgemeinen Proben mit erhöhter Festigkeit darstellen, lag dieses Ergebnis im Erwartungsbereich. Generell wiesen die Ergebnisse eine geringe Streuung auf, was sich besonders im Bereich höherer Beanspruchungsniveaus zeigte. Da weder eine deutlich geringere noch eine deutlich höhere Lebensdauer der hochgesetzten Durchläufer im Vergleich zu den übrigen Ergebnisse im Zeitfestigkeitsbereich festzustellen war, kann trotz Vorbelastung bis zur Grenzschwingspielzahl von einer neutralen Ausgangssituation der Probekörper ausgegangen werden.

Die Bruchflächen aller Versuchsreihen zeigten unabhängig von Beanspruchungsniveau und Prüfvorrichtung ein einheitliches makroskopisches Erscheinungsbild. Der Ermüdungsbruch ist normalflächig und trat aufgrund der makroskopischen Kerbwirkung an den Übergängen der Naht- und Wurzelüberhöhung auf. Die Anrisse gingen von der Oberfläche aus und es kam zu keiner Verlagerung des Anrissortes ins Innere der Proben. Der Anrissort war vorwiegend am Wurzelübergang, wobei es vereinzelt zu einer Verlagerung zum Nahtübergang kam. Im Übergangsbereich traten ausschließlich halbelliptische Oberflächenanrisse sowie viertelelliptische Eckanrisse auf, wohingegen sich im Zeitfestigkeitsbereich besonders auf den höheren Beanspruchungsniveaus auch durchgehende Risse zeigten. Die Anrissorte und Erscheinungsformen der Bruchflächen sind für alle Versuchsreihen als gleichartig einzustufen.

Aus den Versuchsergebnissen, die bis in den Bereich von $5 \cdot 10^{8}$ Lastwechseln reichen, ließ sich zusammenfassend ableiten, dass es zu keinem weiteren Abfall der 
Ermüdungsfestigkeit kommt. Die Ermüdungsversuche deuten auf ein horizontales Auslaufen der Wöhlerlinien im Bereich sehr hoher Lastwechselzahlen hin, sodass von dem Vorliegen einer Dauerfestigkeit auszugehen ist. Zum Einen lagen keine Brüche bzw. Anrisse bei Schwingspielzahlen von größer als $3 \cdot 10^{6}$ Lastwechseln vor und zum Anderen zeigte sich keine Verlagerung des Anrissortes von der Oberfläche ins Innere der Probe. Bei den erzielten Durchläufern konnten mit dem Farbeindringverfahren keine Oberflächenanrisse festgestellt werden. Die erneute Prüfung der Durchläufer der Versuchsreihen auf den Beanspruchungsniveaus im Zeitfestigkeitsbereich zeigte ebenfalls keine Hinweise auf eine Vorschädigung.

Der Vergleich der Versuchsergebnisse der vorliegenden Arbeit mit der normierten Auswertung von Ermüdungsversuchen aus Sonsino et al. [Sonsino, 2005b] zeigte, dass ein Großteil der in dieser Arbeit erzielten Versuchsergebnisse zwar innerhalb des Streubandes der anderen Versuchsergebnisse liegt, aber ein früheres Abknicken im Bereich von $10^{6}$ bis $2 \cdot 10^{6}$ Lastwechseln zu erkennen ist. Zudem wurde das horizontale Auslaufen der Versuchsergebnisse der vorliegenden Arbeit im Bereich sehr hoher Lastwechselzahlen deutlich. Außerdem wurde veranschaulicht, dass nun Versuchsergebnisse an Schweißverbindungen bis zu einer Grenzschwingspielzahl von $5 \cdot 10^{8}$ Lastwechseln vorliegen.

Die Einstufung des vorliegenden Konstruktionsdetails, einseitig geschweißte Stumpfnaht ohne Schweißbadsicherung mit ZFP, in die Kerbfallklasse 71 nach Eurocode 3 konnte annähernd bestätigt werden. Allerdings liegen die ermittelten Neigungsexponenten der einzelnen Versuchsreihen oberhalb des im Eurocode 3 angegebenen Neigungsexponenten.

\subsection{Ausblick}

Die bisher vorliegenden Ermüdungsversuche an bauteilähnlichen Probekörpern, die bis in den Bereich sehr hoher Lastwechselzahlen reichen, sind als äußerst begrenzt anzusehen. In der vorliegenden Arbeit wurde ein Betrag zur Ausweitung der Ermüdungsversuche an Schweißverbindungen bis in den Bereich von $5 \cdot 10^{8}$ Lastwechseln realisiert. Allerdings sollten die Untersuchungen auf weitere Nahtformen und Beanspruchungsarten sowie dickere Bleche und Stähle höherer Festigkeit ausgeweitet werden, um eine sichere und wirtschaftliche Auslegung von Schweißverbindungen bis in den Bereich sehr hoher Lastwechselzahlen gewährleisten zu können.

Auch zukünftig werden experimentelle Untersuchungen durch die verfügbaren Prüftechniken begrenzt sein. Die vorliegende Arbeit zeigt auf, dass weiterhin Potential besteht, die bestehende Prüftechnik weiterzuentwickeln, um belastbare Ermüdungs- 
versuche an bauteilähnlichen Probekörpern bis in den Bereich sehr hoher Lastwechselzahlen in einem akzeptablen Zeitraum erzielen zu können.

Die durchgeführte Untersuchung zum Einfluss der Prüffrequenz auf die Ergebnisse der Ermüdungsversuche ergab kein einheitliches Ergebnis für den Zeitfestigkeitsund Übergangsbereich. Aufgrund der geringen Probenanzahl und zum Ausschluss einer Abhängigkeit der gewählten Beanspruchungsniveaus ist eine weitere Absicherung dieser Ergebnisse durch weiterführende Untersuchungen empfehlenswert.

Um Aufschluss über den Einfluss der Probekörpererwärmung und den daraus resultierenden Effekten auf die Ermüdungsfestigkeit zu erlangen, sollten weitere Untersuchungen in der hochfrequenten Prüfvorrichtung bei unterschiedlichen Prüffrequenzen erfolgen. Hierzu könnten die Hebelarme der Prüfmaschine verlängert werden, um eine geringerer Prüffrequenz zu realisieren. Allerdings könnte der Erwärmung der Probekörper ebenso durch Kühlung der Probekörper und/oder einem Betrieb mit Beanspruchungspausen entgegengewirkt werden, wobei ein Betrieb mit Beanspruchungspausen die Versuchslaufzeit erhöhen würde.

Bei der Untersuchung zum Einfluss der Zugeigenspannungen auf die Ermüdungsfestigkeit in der hochfrequenten Prüfvorrichtung war die Höhe der Mittelspannung begrenzt. Weiterführende Untersuchungen mit höheren Spannungsverhältnissen und mit der vorgeschlagenen Methode von Otha et al. [Otha, 1986] sollten durchgeführt werden, um das Auftreten später Brüche bei Oberspannungen im Bereich der Streckgrenze ausschließen zu können. Um dies zu realisieren, könnte beispielweise der Prüfquerschnitt der Probekörper verringert oder die Exzenterwellen angepasst werden. 


\section{Literatur}

\subsection{Normen und Richtlinien}

[DIN 969, 1997] DIN 969: Schwingfestigkeitsversuch bei Axialbelastung, Prüfverfahren und Auswertung der Ergebnisse, Verbindungselemente mit Gewinde. DIN Deutsches Institut für Normung e.V., Berlin, Dezember 1997.

[DIN 50100, 1978] DIN 50100: Dauerschwingversuch, Begriffe, Zeichen, Durchführung, Auswertung, Werkstoffprüfung. DIN Deutsches Institut für Normung e.V., Berlin, Februar 1978.

[DIN 50125, 2009] DIN 50125: Prüfung metallischer Werkstoffe - Zugproben. DIN Deutsches Institut für Normung e.V., Berlin, Juli 2009.

[DIN EN 1993-1-9, 2010] DIN EN 1993-1-9: Eurocode 3: Bemessung und Konstruktion von Stahlbauten - Teil 1-9: Ermüdung. Deutsche Fassung EN 19931-9:2005 + AC:2009, DIN Deutsches Institut für Normung e.V., Berlin, Dezember 2010.

[DIN EN 10025-1, 2005] DIN EN 10025-1: Warmgewalzte Erzeugnisse aus Baustählen - Teil 1: Allgemeine technische Lieferbedingungen. Deutsche Fassung EN 10025-1:2004, DIN Deutsches Institut für Normung e.V., Berlin, Februar 2005.

[DIN EN 14610, 2005] DIN EN 14610: Schweißen und verwandte Prozesse Begriffe für Metallschweißprozesse. Dreisprachige Fassung EN 14610, DIN Deutsches Institut für Normung e.V., Berlin, Februar 2005.

[DIN EN ISO 5817, 2006] DIN EN ISO 5817: Schweißen - Schmelzschweißverbindungen an Stahl, Nickel, Titan und deren Legierungen (ohne Strahlschweißen) - Bewertungsgruppen von Unregelmäßigkeiten (ISO 5817:2003 + Cor. 1:2006). Deutsche Fassung EN ISO 5817:2003 + AC:2006), DIN Deutsches Institut für Normung e.V., Berlin, Oktober 2006.

[DIN EN ISO 6892-1, 2009] DIN EN ISO 6892-1: Metallische Werkstoffe - Zugversuch - Teil 1: Prüfverfahren bei Raumtemperatur (ISO 6892-1:2009). Deutsche Fassung EN ISO 6892-1:2009, DIN Deutsches Institut für Normung e.V., Berlin, Dezember 2009. 
[DIN EN ISO 9015-1, 2011] DIN EN ISO 9015-1: Zerstörende Prüfung von Schweißverbindungen an metallischen Werkstoffen - Härteprüfung - Teil 1: Härteprüfung für Lichtbogenschweißverbindungen (ISO 9015-1:2001). Deutsche Fassung EN ISO 9015-1:2011, DIN Deutsches Institut für Normung e.V., Berlin, Mai 2011.

[DIN EN ISO 9015-2, 2011] DIN EN ISO 9015-2: Zerstörende Prüfung von Schweißverbindungen an metallischen Werkstoffen - Härteprüfung - Teil 2: Mikrohärteprüfung für Lichtbogenschweißverbindungen (ISO 9015-2:2003). Deutsche Fassung EN ISO 9015-2:2011, DIN Deutsches Institut für Normung e.V., Berlin, Mai 2011.

[DIN EN ISO 9692-1, 2004] DIN EN ISO 9692-1: Schweißen und verwandte Prozesse - Empfehlungen zur Schweißnahtvorbereitung - Teil 1: Lichtbogenhandschweißen, Schutzgasschweißen, Gasschweißen, WIG-Schweißen und Strahlschweißen von Stählen (ISO 9692-1:2003). Deutsche Fassung EN ISO 9692-1:2003, DIN Deutsches Institut für Normung e.V., Berlin, Mai 2004.

[DIN EN ISO 14341, 2011] DIN EN ISO 14341: Schweißzusätze - Drahtelektroden und Schweißgut zum Metall-Schutzgasschweißen von unlegierten Stählen und Feinkornstählen - Einteilung (ISO 14341:2010). Deutsche Fassung EN ISO 14341:2011, DIN Deutsches Institut für Normung e.V., Berlin, April 2011.

[DIN EN ISO 15614-1, 2008] DIN EN ISO 15614-1: Anforderung und Qualifizierung von Schweißverfahren für metallische Werkstoffe - Schweißverfahrensprüfung - Teil 1: Lichtbogen- und Gasschweißen von Stählen und Lichtbogenschweißen von Nickel und Nickellegierungen (ISO 15614-1:2004 + Amd 1:2008). Deutsche Fassung EN ISO 15614-1:2004 + A1:2008, DIN Deutsches Institut für Normung e.V., Berlin, September 2008.

[IIW, 2009] Hobbacher, A. F. (Ed): IIW Recommendations for Fatigue Design of Welded Joints and Components. IIW-1829-07 ex XIII-2151r4-07/XV1254r-07, Welding Research Council, New York, 2009.

[SEW 088, 1993] SEW 088: Schweißgeeignete Feinkornbaustähle, Richtlinie für die Verarbeitung, besonders für das Schmelzschweißen. Stahl-EisenWerkstoffblätter (SEW) des Vereins Deutscher Eisenhüttenleute, 4. Ausgabe, Oktober 1993. 


\subsection{Fachbücher, Monographien, Aufsätze und Berichte}

[Akiniwa, 2006] Akiniwa, Y.; Miyamoto, N.; Tsuru, H.; Tanaka, K.: Notch effect on fatigue strength reduction of bearing steel in the very high cycle regime. International Journal of Fatigue 28, S. 1555-1565, 2006.

[Allison, 2007] Allison, J. E.; Jones, J. W.; Larsen, J. M.; Ritchie, R. O. (Ed.): Proceedings of the Fourth International Conference on Very High Cycle Fatigue. VHCF-4, August 19-22, 2007, University of Michigan, Ann Arbor, Michigan, 2007.

[Alt, 2005] Alt, A.: Dauerfestigkeitsprüfung und Dauerfestigkeit von SchraubeMutter-Verbindungen unter kombinierter Zug- und Biegebelastung. Dissertation, Fortschritt-Berichte VDI, Reihe 1, Nr. 381, VDI Verlag GmbH, Düsseldorf, 2005.

[Alt, 2007] Alt, A.; Mertens, H.; Arz, U.; Blessing, L.; Berger, C.: Schwingfestigkeit von Schraube-Mutter-Verbindungen. Materialwissenschaften und Werkstofftechnik 38, Heft 5, S. 402-409, 2007.

[Anthes, 1993] Anthes, R. J.; Köttgen, V. B.; Seeger, T.: Kerbformzahlen von Stumpfstößen und Doppel-T-Stößen. Schweißen und Schneiden 45, Heft 12, S. 685-688, 1993.

[Bacher-Höchst, 2008] Bacher-Höchst, M.; Berger, C.; Sonsino, C. M.; Vormwald, M.: Current developments and trends on structural durability. Materialwissenschaften und Werkstofftechnik 39, Heft 10, S. 680-687, 2008.

[Bannantine, 1990] Bannantine, J. A.; Comer, J. J.; Handrock, J. L.: Fundamentals of metal fatigue analysis. Prentice Hall, Englewood Cliffs, New Jersey, 1990.

[Bathias, 1999] Bathias, C.: Gigacycle Fatigue. Selected Papers, Euromech Colloquium 382, Juni 1998, Paris, In: Fatigue and Fracture Fatigue and Fracture of Engineering Materials and Structures 22, Heft 7 und 8 , S. 545-641 und S. 647-728, 1999.

[Bathias, 2001] Bathias, C.; Drouillac, L.; Le Francois, P.: How and why the fatigue S-N curve does not approach a horizontal asymptote. International Journal of Fatigue 23, S. 143-151, 2001.

[Berger, 2011] Berger, C.; Christ, H.-J. (Ed.): Proceedings of the Fifth International Conference on Very High Cycle Fatigue. VHCF-5, June 28-30, 2011, Deutscher Verband für Materialforschung und -prüfung, Berlin, 2011. 
[Buitrago, 2003] Buitrago, J.; Weir, M. S.; Kan, W. C.: Fatigue design and performance verification of deepwater risers. In: Proceedings of OMAE03, $22^{\text {nd }}$ International Conference on Offshore Mechanics and Arctic Engineering, June 8-13, 2013, Cancun, Paper No. 37492, S. 351-366, 2003.

[Cremer, 2010] Cremer, M.; Zimmermann, M.; Christ, H.-J.: Ultraschallermüdungsprüfung zur Charakterisierung des Verhaltens von Aluminiumschweißverbindungen bei sehr hohen Lastspielzahlen (VHCF). Tagung Werkstoffprüfung 2010 - Konstruktion, Werkstoffentwicklung und Schadensanalyse, Hrsg.: Pohl, M., S. 125-130, Neu-Ulm, 2010.

[Cremer, 2011] Cremer, M.; Zimmermann, M.; Christ, H.-J.: Fatigue life evaluation of the different zones of a welded aluminum joint in the high cycle to the very high cycle regime. In: Proceedings of the Fifth International Conference on Very High Cycle Fatigue, VHCF-5, June 28-30, 2011, S. 581-586, Berlin, 2011.

[Cremer, 2013] Cremer, M.; Zimmermann, M.; Christ, H.-J.: High-frequency cyclic testing of welded aluminium alloy joints in the region of very high cycle fatigue (VHCF). International Journal of Fatigue 57, S. 120-130, 2013.

[Degenkolbe, 1985] Degenkolbe, J.; Uwer, D.; Wegmann, H. G.: Kennzeichnung von Schweißtemperaturzyklen hinsichtlich ihrer Auswirkung auf die mechanischen Eigenschaften von Schweißverbindungen durch die Abkühlzeit $t_{8 / 5}$ und deren Ermittlung. Thyssen Technische Berichte, Heft 1, S. 57-73, 1985.

[Deutsches Patent 10204258.6, 2005] Deutsches Patent 10204258.6: Prüfvorrichtung zur Dauerschwingprüfung von Prüflingen. Alt, A., 2005.

[DFG-Paketantrag] Deutsche Forschungsgesellschaft (DFG), Paketantrag: Ermüdungsschädigung im Übergangsbereich zwischen High Cycle Fatigue (HCF) und Very High Cycle Fatigue (VHCF). www.vhcf-paketantrag.uni-siegen.de (09.03.2014).

[DFG-SPP1466] Deutsche Forschungsgesellschaft (DFG), Schwerpunktprogramm (SPP) 1466: Life $\infty$. www.spp1466.uni-siegen.de (09.03.2014).

[Dilthey, 2005] Dilthey, U.: Schweißtechnische Fertigungsverfahren 2, Verhalten der Werkstoffe beim Schweißen. 3. Auflage, Springer-Verlag Berlin Heidelberg, 2005.

[Dixon, 1948] Dixon, W. J.; Mood, A. M.: A Method for Obtaining and Analyzing Sensitivity Data. Journal of the American Statistical Association 43, S. 108-126, 1948. 
[Dürr, 2007] Dürr, A.: Zur Ermüdungsfestigkeit von Schweißkonstruktionen aus höherfesten Baustählen bei Anwendung von UIT-Nachbehandlung. Dissertation, Mitteilungen des Instituts für Konstruktion und Entwurf, Nr. 2006-3, Stuttgart, 2007.

[Fischer, 1974] Fischer, J. W.; Albrecht, P. A.; Yen, B. T.; Klingermann, D. J.; McNamee, B. M.: Fatigue strength of steel beams with welded stiffeners and attachments. National Cooperative Highway Research Program, Report 147, National Research Council, Washington D. C., 1974.

[Fischer, 1997] Fischer, J. W.: Improved performance through large scale dynamic testing of structures. In: Proceedings of the international conference of performance of dynamically loaded welded structures, S. 332-353, San Francisco, 1997.

[Fricke, 2010] Fricke, W.: Guideline for the Fatigue Assessment by Notch Stress Analysis for Welded Structures. IIW-Doc. XIII-2240r2-08/XV-1289r2-08, July 2010.

[Gudehus, 1999] Gudehus, H.; Zenner, H.: Leitfaden für eine Betriebsfestigkeitsrechnung, Empfehlung zur Lebensdauerabschätzung von Maschinenbauteilen. 4. Auflage, Verlag Stahleisen $\mathrm{GmbH}$, Düsseldorf, 1999.

[Gurney, 1972] Gurney, T. R.; Maddox, S. J.: A re-analysis of fatigue data for welded joints. Research Report E/44/72, The Welding Institute, Abington Publishing, Cambridge, 1972.

[Gurney, 1977] Gurney, T. R.: Some recent work relating to the influence of residual stresses on fatigue strength. In: Proceedings of the Conference on Residual Stresses in Welded Construction and their Effects, Paper 23, S. 151-163, The Welding Institute, 1977.

[Gurney, 1979] Gurney, T. R.: Fatigue of welded structures. $2^{\text {nd }}$ Edition, Cambridge University Press, 1979.

[Haibach, 1971] Haibach, E.: Die Dauerfestigkeit von Schweißverbindungen bei Grenzschwingspielzahlen größer als $2 \cdot 10^{6}$. Archiv für das Eisenhüttenwesen, 42. Jahrgang, Heft 12, S. 901-908, 1971.

[Haibach, 2006] Haibach, E.: Betriebsfestigkeit, Verfahren und Daten zur Bauteilberechnung. 3. Auflage, Springer-Verlag Berlin Heidelberg, 2006. 
[Hanus, 1996] Hanus, F.; Schütz, R.; Grünewald, J.: Härte in der WEZ - Wirkung der chemischen Zusammensetzung des Stahls, der Schweißbedingungen und des Spannungsarmglühens. Schweißen \& Schneiden '96, DVS-Berichte, Band 176, S. 150-153, DVS-Verlag, Düsseldorf, 1996.

[Harrison, 1981] Harrison, J. D.: The effect of residual stresses on fatigue behaviour. In: Residual stresses and their effect, The Welding Institute, Cambridge, 1981.

[Haydrych, 2001] Haydrych, I.: Wachstum von Ermüdungsrissen an Niet- und Schraubenlöchern unter Berücksichtigung von Vorspannkräften der Verbindungsmittel. Fortschritt-Berichte VDI, Reihe 4, Nr. 169, VDI Verlag GmbH, Düsseldorf, 2001.

[Heeschen, 1986] Heeschen, J.: Untersuchungen zum Dauerschwingverhalten von Schweißverbindungen aus hochfesten Baustählen unter besonderer Berücksichtigung des Eigenspannungszustandes und der Nahtgeometrie. Dissertation, Universität - Gesamthochschule Kassel, Institut für Werkstofftechnik, Kassel, 1986.

[Heeschen, 1990] Heeschen, J.; Nitschke, T.; Wohlfahrt, H.: Der Einfluss der Nahtgeometrie auf die Dauerschwingfestigkeit von Schweißverbindungen aus einem hochfesten Feinkornbaustahl StE690. Abschlussbericht, DFG-Wo 344/1, Kassel, 1990.

[Hobbacher, 2009] Hobbacher, A. F.: The new IIW recommendations for fatigue assessment of welded joints and components - A comprehensive code recently updated. International Journal of Fatigue 31, S. 50-58, 2009.

[Holper, 2004] Holper, B.; Mayer, H.; Vasudevan, A. K.; Stanzl-Tschegg, S. E.: Near threshold fatigue crack growth at positive load ratio in aluminium alloys at low and ultrasonic frequency: influences of strain rate, slip behaviour and air humidity. International Journal of Fatigue 26, S. 27-38, 2004.

[Hück, 1983] Hück, M.: Ein verbessertes Verfahren für die Auswertung von Treppenstufenversuchen. Zeitschrift für Werkstofftechnik 14, S. 406-417, 1983.

[Kaffenberger, 2012] Kaffenberger, M.: Schwingfestigkeit von Schweißnahtenden und Übertragbarkeit von Schweißverbindungswöhlerlinien. Dissertation, Veröffentlichungen des Instituts für Stahlbau und Werkstoffmechanik der Technischen Universität Darmstadt, Heft 97, Darmstadt, 2012. 
[Keindorf, 2009] Keindorf, C.: Zur Tragfähigkeit und Ermüdungsfähigkeit von Sandwichtürmen für Windenergieanlagen. Dissertation, Schriftenreihe des Instituts für Stahlbau der Leibniz Universität Hannover, Heft 25, Hannover, 2009.

[Keindorf, 2010] Keindorf, C.; Schaumann, P.; Ahmed, H.; Goes, P.; Vermeulen, M.: High Frequency Fatigue Testing of Butt Welds with a New Magnet Resonance Machine. $63^{\text {rd }}$ Annual Assembly \& International Conference of the International Institute of Welding, 11-17 July 2010, Istanbul, S. 149-156, 2010.

[Koller, 1997] Koller, R. E.: Einflüsse auf die Schwingfestigkeit. In: Grundlagen der Betriebsfestigkeit, Seminar der EMPA Dübendorf, Sankt Gallen, S. 1-25, 1997.

[Krebs, 2012] Krebs, J.; Hübner, P.; Kaßner, M.: Eigenspannungseinfluss auf Schwingfestigkeit und Bewertung in geschweißten Bauteilen. 2. überarbeitete und erweiterte Auflage, DVS-Berichte, Band 234, DVS-Verlag, Düsseldorf, 2012.

[Kuhlmann, 2006] Kuhlmann, U.; Dürr, A.; Bergmann, J.; Thumser, R.: Effizienter Stahlbau aus höherfesten Stählen unter Ermüdungsbeanspruchung. Forschungsbericht P 620, Forschungsvereinigung Stahlanwendung, Düsseldorf, 2006.

[Kulka, 1987] Kulka, C.; Sonsino, C. M.; Buxbaum, O.: Verlauf der Wöhlerlinie von Schweißverbindungen bei hohen Schwingspielzahlen. Abschlussbericht, LBF-Bericht Nr. 4772, Darmstadt, 1987.

[Lachmann, 2003] Lachmann, C.: Einfluss von Eigenspannungen und Mikrostruktur auf die Lebensdauervorhersage von Schweißverbindungen. Dissertation, Technische Universität Braunschweig, Forschungsberichte des Instituts für Schweißtechnik, Band 8, Braunschweig, 2003.

[Laird, 1982] Laird, C.; Charsley, P.: Strain rate sensitivity effects in cyclic deformation and fatigue fracture. In: Ultrasonic Fatigue Proceedings of the First International Conference on Fatigue and Corrosion Fatigue up to Ultrasonic Frequencies, The Metallugical Society of AIME, Philadelphia, PA, S. 187-205, 1982.

[Liu, 2001] Liu, J.: Dauerfestigkeitsberechnung metallischer Bauteile. Habilitation, Technische Universität Clausthal, Clausthal-Zellerfeld, 2001. 
[Lohrmann, 1995] Lohrmann, G. R.; Lueb, H.: Kleine Werkstoffkunde für das Schweißen von Stahl und Eisen. 8. Auflage, Fachbuchreihe Schweißtechnik, Band 8, Deutscher Verlag für Schweißtechnik DVS-Verlag GmbH, Düsseldorf, 1995.

[Macherauch, 1973] Macherauch, E.; Wohlfahrt, H.; Wolfstieg, E.: Zur zweckmäßigen Definition von Eigenspannungen. Härterei-Technische Mitteilungen 28, Heft 3, S. 201-211, 1973.

[Macherauch, 1976] Macherauch, E.; Mayr, P.: Strukturmechanische Grundlagen der Ermüdung metallischer Werkstoffe. In: Werkstoff- und Bauteilverhalten unter Schwingbeanspruchung, VDI-Berichte, Nr. 268, S. 5-20, VDI-Verlag $\mathrm{GmbH}$, Düsseldorf, 1976.

[Maddox, 1982] Maddox, S. J.: Influence of Tensile Residual Stresses on the Fatigue Behavior of Welded Joints in Steel. Symposium on Residual Stress Effects in Fatigue, ASTM STP 776, American Society of Testing and Materials, S. 63-96, Philadelphia, 1982.

[Maddox, 1991] Maddox, S. J.: Fatigue Strength of Welded Structures. $2^{\text {nd }}$ Edition, Abington Publishing, Cambridge, 1991.

[Maddox, 1998] Maddox, S. J.; Razmjoo, G. R.: Fatigue performance of large girth welded steel tubes. In: Proceedings of OMAE98, $17^{\text {nd }}$ International Conference on Offshore Mechanics and Arctic Engineering, July 5-9, 1998, Lisbon, Paper No. 2355, 1998.

[Mauch, 1999] Mauch, H.: Statistische Methoden zur Beurteilung von Bauteillebensdauer und Zuverlässigkeit und ihre beispielhafte Anwendung auf Zahnräder. Abschlussbericht, Forschungsvorhaben Nr. 304 "Lebensdauerstatistik", FVA-Heft 580, FVA, Frankfurt a. M., 1999.

[Mayer, 1999] Mayer, H.: Fatigue crack growth and threshold measurements at very high frequencies. International Materials Reviews 44, Heft 1, S. 1-35, 1999.

[Mayer, 2007] Mayer, H.: Fatigue damage of low amplitude cycles under variable amplitude loading condition. In: Proceedings of the Fourth International Conference on Very High Cycle Fatigue, VHCF-4, August 19-22, 2007, S. 333-340, University of Michigan, Ann Arbor, Michigan, 2007. 
[Morgenstern, 2006] Morgenstern, C.: Kerbgrundkonzepte für die schwingfeste Auslegung von Aluminiumschweißverbindungen am Beispiel der naturharten Legierung AIMg4,5Mn (AW-5083) und der warmausgehärteten Legierung AlMgSi1 T6 (AW-6082 T6). Dissertation, Technische Universität Darmstadt, Darmstadt, 2006.

[Müller, 2012] Müller, C.; Hinkelmann, K.; Wächter, M.; Masendorf, R.; Esderts, A.: Zur Wiederverwendung von Durchläufern im Treppenstufenversuch. Materials Testing 54, Heft 11-12/2012, S. 786-792, 2012.

[Müller-Bollenhagen, 2011] Müller-Bollenhagen, C.: Verformungsinduzierte Martensitbildung bei mehrstufiger Umformung und deren Nutzung zur Optimierung der HCF und VHCF Eigenschaften von austenitischem Edelstahlbleche. Dissertation, Universität Siegen, Siegener Werkstoffkundliche Berichte, Siegen, 2011.

[Murakami, 2002] Murakami, Y.; Yokoyama, N. N.; Nagata, J.: Mechanism of fatigue failure in ultralong life regime. Fatigue \& Fracture of Engineering Materials \& Structures 25, S. 735-746, 2002.

[Nakamura, 1988] Nakamura, H.; Nishijimia, S.; Ohta, A.; Maeda, Y., Uchino, K.; Kohno, T.; Toyomasu, K.; Soya, I.: A Method for Obtaining Conservative S-N Data for Welded Structures. Journal of Testing and Evaluation 16, Heft 3 , S. 280-285, 1988.

[Naubereit, 1999] Naubereit, H.; Weihert, J.: Einführung in die Ermüdungsfestigkeit. Carl Hanser Verlag München Wien, 1999.

[Nishijima, 1999] Nishijima, S.; Kanazawa, K.: Stepwise S-N curve and fish-eye failure in gigacycle fatigue. Fatigue \& Fracture of Engineering Materials \& Structures 22, S. 601-607, 1999.

[Nitschke-Pagel, 1995] Nitschke-Pagel, T.: Eigenspannungen und Schwingfestigkeitsverhalten geschweißter Feinkornbaustähle. Dissertation, Technische Universität Braunschweig, Braunschweig, 1995.

[Nitschke-Pagel, 2006] Nitschke-Pagel, T. ;Wohlfahrt, H.: Anwendung des lokalen Dauerfestigkeitskonzepts zur Bewertung der Wirksamkeit von Schweißnahtnachbehandlungsmaßnahmen. International Journal of Materials Research 97, Heft 12, S. 1697-1705, 2006.

[Olivier, 1979-1985] Olivier, R.; Ritter, W.: Wöhlerlinienkatalog für Schweißverbindungen aus Baustählen. DVS Berichte, Nr. 56, Deutscher Verlag für Schweißtechnik, Düsseldorf, 1979-1985. 
[Olivier, 1989] Olivier, R.; Köttgen, V. B.; Seeger, T.: Schwingfestigkeitsnachweise für Schweißverbindungen auf der Grundlage örtlicher Beanspruchungen. Forschungshefte, FKM-Heft 143, Forschungskuratorium Maschinenbau, Frankfurt am Main, 1989.

[Olivier, 1994] Olivier, R.; Köttgen, V. B.; Seeger, T.: Untersuchungen zur Einbindung eines neuartigen Zeit- und Dauerfestigkeitsnachweises für Schweißverbindungen aus Stahl in Regelwerke. Forschungshefte, FKM-Heft 180, Forschungskuratorium Maschinenbau, Frankfurt am Main, 1994.

[Olivier, 2000] Olivier, R.: Experimentelle und numerische Untersuchungen zur Bemessung schwingbeanspruchter Schweißverbindungen auf der Grundlage örtlicher Beanspruchungen. Dissertation, Veröffentlichungen des Instituts für Stahlbau und Werkstoffmechanik der Technischen Hochschule Darmstadt, Heft 62, Darmstadt, 2000.

[Otha, 1986] Otha, A.; Meada, Y.; Mawari, T.; Nishijima, S.; Nakamura, H.: Fatigue strength evaluation of welded joints containing high tensile residual stresses. International Journal of Fatigue 8, Heft 3, S. 147-150.

[Otha, 1997] Otha, A.; Suzuki, N.; Maeda, Y.: Effect of Residual Stresses on Fatigue of Weldment. In: Proceedings of the International Conference on Performance of Dynamically Loaded Welded Structures, Welding Research Council, New York, S. 108-122, 1997.

[Pöting, 2002] Pöting, S.; Zenner, H.: Lebensdauer von Schweißverbindungen im Bereich hoher Lastspielzahlen (HCF). Schlussbericht, Förderkennzeichen AIF-Nr. 11.661N, DVS-Nr. 9.018, Clausthal, 2002.

[Pyttel, 2007] Pyttel, B.; Schwerdt, D.; Berger, C.: Very high cycle fatigue of two different aluminium wrought alloys. In: Proceedings of the Fourth International Conference on Very High Cycle Fatigue, VHCF-4, August 19-22, 2007, S. 313-318, University of Michigan, Ann Arbor, Michigan, 2007.

[Pyttel, 2010] Pyttel, B.; Schwerdt, D.; Berger, C.: Fatigue strength and failure mechanisms in the VHCF-region for quenched and tempered steel $42 \mathrm{CrMoS} 4$ and consequences to fatigue design. Fatigue 2010, Procedia Engineering 2, S. 1327-1336, 2010.

[Pyttel, 2011] Pyttel, B.; Schwerdt, D.; Berger, C.: Very high cycle fatigue - Is there a fatigue limit?. International Journal of Fatigue 33, S. 49-58, 2011.

[Radaj, 1970] Radaj, D.: Untersuchungen zur geometrischen Form von Stumpf- und Kehlnähten. Schweißen und Schneiden 22, Heft 5, S. 206-209, 1970. 
[Radaj, 2000] Radaj, D.; Sonsino, C. M.: Ermüdungsfestigkeit von Schweißverbindungen nach lokalen Konzepten. Fachbuchreihe Schweißtechnik, Band 142, Verlag für Schweißen und verwandte Verfahren, DVS-Verlag GmbH, Düsseldorf, 2000.

[Radaj, 2006] Radaj, D.; Sonsino, C. M.; Fricke, W.: Fatigue assessment of welded joints by local approaches. 2. Edition, Woodhead Publishing, Cambridge, 2006.

[Radaj, 2007] Radaj, D.; Vormwald, M.: Ermüdungsfestigkeit, Grundlagen für Ingenieure. 3. Auflage, Springer-Verlag Berlin Heidelberg, 2007.

[Rainer, 1979] Rainer, G.: Errechnen von Spannungen in Schweißverbindungen mit der Methode der Finiten Elemente. Dissertation, Forschungshefte, Forschungskuratorium Maschinenbau e.V., Heft 74, 1979.

[Rappe, 1972] Rappe, H.-A.: Beitrag zur Frage der Schweißeigenspannungen. Dissertation, Technische Universität Hannover, Hannover, 1972.

[Ritter, 1984] Ritter, W.; Olivier, R.: Einfluss fertigungsbedingter Eigenspannungen auf die Schwingfestigkeit von Schweißverbindungen. In: Buxbaum, O.: Vorträge des vierten LBF-Kolloquium am 31. Januar und 2. Februar 1984 in Darmstadt, Bericht Nr. TB-171, Fraunhofer Institut für Betriebsfestigkeit, Darmstadt, 1984.

[Ritter, 1994] Ritter, W.: Kenngrößen der Wöhlerlinien für Schweißverbindungen aus Stählen. Dissertation, Veröffentlichungen des Instituts für Stahlbau und Werkstoffmechanik der Technischen Hochschule Darmstadt, Heft 53, Darmstadt, 1994.

[Ruge, 1980] Ruge, J.; Wösle, H.: Einfluss der Schweißparameter und Herstellbedingungen auf das Schwingfestigkeitsverhalten metall-aktivgasgeschweißter Proben. Schweißen und Schneiden 32, Heft 9, S. 374-379, 1980.

[Sakai, 2004] Sakai, T.; Ochi, Y. (Ed.): Proceedings of the Third International Conference on Fatigue in the Very High Cycle Fatigue Regime, VHCF-3. Juli 2-4, 2004, Ritsumeikan University, Kyoto/Kusatzu, 2004.

[Sakai, 2007] Sakai, T.: Review and prospects for current studies on very high cycle fatigue of metallic materials for machine structural use. In: Proceedings of the Fourth International Conference on Very High Cycle Fatigue, VHCF-4, August 19-22, 2007, S. 3-12, University of Michigan, Ann Arbor, Michigan, 2007. 
[Sander, 2008] Sander, M.: Sicherheit und Betriebsfestigkeit von Maschinen und Anlagen, Konzepte und Methoden zur Lebensdauervorhersage. SpringerVerlag Berlin Heidelberg, 2008.

[Schaumann, 2008] Schaumann, P.; Keindorf, C.; Alt, A.: Hochfrequente Ermüdungstests an Schweißverbindungen mit einem neu entwickelten Magnetresonanzprüfrahmen. Große Schweißtechnische Tagung 2008 Dresden, 17. bis 19. September 2008, Dresden, 2008.

[Schaumann, 2009] Schaumann, P.; Steppeler, S.: Fatigue Strength of Welded Components of Support Structures of Wind Energy Converters in the VHCF Regime, EAWE PhD Seminar 2009, Durham, 2009.

[Schaumann, 2011a] Schaumann, P.; Steppeler, S.: Ermüdungsverhalten von Schweißverbindungen von Tragstrukturen für Windenergieanlagen bei sehr hohen Lastwechselzahlen. Sondertagung Schweißen im Schiffbau und Ingenieurbau, Hamburg, 27. September 2011, DVS Berichte, Heft 277, S. 53-58, 2011.

[Schaumann, 2011b] Schaumann, P.; Lochte-Holtgreven, S.; Steppeler, S.: Special Fatigue Aspects in Support Structures of Offshore Wind Turbines. Materialwissenschaft und Werkstofftechnik 42, Special Issue: Structural Durability, Heft 12, S. 1075-1081, 2011.

[Schaumann, 2013a] Schaumann, P.; Collmann, M.; Bach, F.-W.; Hassel, T.; Konya, R.; Deißer, A.; Priebe, S.: Ökologische und ökonomische Hochleistungsfügetechniken für Stahlrohrtürme von Windenergieanlagen OPTIWELD. Sachbericht, Förderkennzeichen BMBF 0325063 A-C, 2013.

[Schaumann, 2013b] Schaumann, P.; Steppeler, S.: Fatigue tests of axially loaded butt welds up to very high cycles. $5^{\text {th }}$ Fatigue Design Conference, Fatigue Design 2013, Procedia Engineering 66, S. 88-97, 2013.

[Schijve, 2009] Schijve, J.: Fatigue of Structures and Materials. 2. Edition, Springer, 2009.

[Schimmöller, 1992] Schimmöller, H.: Entstehung und Bestimmung von Schweißeigenspannungen. Institut für Schiffbau der Universität Hamburg, Bericht Nr. 523, Hamburg, 1992. 
[Schmid, 2011] Schmid, S.; Hahn, M.; Issler, S.; Bacher-Hoechst, M.; Bomas, H.; Zoch, H.-W.: Effect of frequency and environment on very high cycle fatigue behaviour of a high strength steel. In: Proceedings of the Fifth International Conference on Very High Cycle Fatigue, VHCF-5, June 28-30, 2011, S. 287-294, Berlin, 2011.

[Schulze, 2010] Schulze, G.: Die Metallurgie des Schweißens, Eisenwerkstoffe Nichteisenmetallische Werkstoffe. 4. Auflage, Springer-Verlag Berlin Heidelberg, 2010.

[Schwerdt, 2009] Schwerdt, D.; Pyttel, B.: VHC- und UHC-Ermüdungsfestigkeit: Vorhaben Nr. 277 Ermüdungsfestigkeit von Konstruktionswerkstoffen bei sehr hohen Schwingspielzahlen (im VHCF- und UHCF-Bereich). Abschlussbericht, Forschen im Maschinenbau, Heft 304, Frankfurt am Main, 2009.

[Seeger, 1987] Seeger, T.; Olivier, R.: Ertragbare und zulässige Schubspannungen schwingbeanspruchter Schweißverbindungen. Stahlbau 8, Heft 8, S. 231-238, 1987.

[Seeger, 1992] Seeger, T.; Olivier, R.: Neigung und Abknickpunkt der Wöhlerlinie von schubbeanspruchten Kehlnähten. Stahlbau 61, Heft 5, S. 137-142, 1992.

[Seeger, 1996] Seeger, T.: Grundlagen für Betriebsfestigkeitsnachweise. In: Stahlbau Handbuch, Band 1, Teil B, S. 5-123, Stahlbau-Verlagsgesellschaft mbH, Köln, 1996.

[Shiozawa, 2001] Shiozawa, K.; Lu, L.; Ishihara, S.: S-N curve characteristics and subsurface crack initiation behaviour in ultra-long life fatigue of a high carbon-chromium bearing steel. Fatigue \& Fracture of Engineering Materials \& Structures 24, S. 781-790, 2001.

[Signes, 1967] Signes, E. G.; Baker, R. G.; Harrison, J. D.; Burdekin, F. M.: Factors affecting the fatigue strength of welded high strength steels. British Welding Journal, Heft 3, S. 108-116, 1967.

[Sonsino, 1987] Sonsino, C. M.; Böhme, D.; Kulka, C.; Helwig, R.: Slope of the $\mathrm{S}-\mathrm{N}$-curve and high-cylce fatigue behaviour of longitudinal stiffeners in aswelded and stress-relieved states. IIW-Doc. No XIII-1242-87, 1987.

[Sonsino, 1994] Sonsino, C. M.: Über den Einfluss von Eigenspannungen, Nahtgeometrie und mehrachsigen Spannungszuständen auf die Betriebsfestigkeit geschweißter Konstruktionen aus Baustählen. Materialwissenschaften und Werkstofftechnik 25, Heft 3, S. 97-109, 1994. 
[Sonsino, 2005a] Sonsino, C. M.: „Dauerfestigkeit” - Eine Fiktion. Konstruktion 4, April, S. 87-92, 2005.

[Sonsino, 2005b] Sonsino, C. M.; Maddox, S. J.; Haagensen, P.: A Short Study on the Form of the SN-Curves for Weld Details in the High-Cycle-Fatigue Regime. IIW-Doc. No. XIII-2045-05, 2005.

[Sonsino, 2007] Sonsino, C. M.: Course of SN-curves especially in the high-cycle fatigue regime with regard to component design and safety. International Journal of Fatigue 29, S. 2247-2258, 2007.

[Sonsino, 2010] Sonsino, C. M.; Bruder, T.; Baumgartner, J.: S-N lines for welded thin joints - Suggested slopes and FAT values for applying the notch stress concept with various reference radii. Welding in the World 54, Heft 11/12, 2010.

[Stanzl-Tschegg, 2001] Stanzl-Tschegg, S.; Mayer, H. (Ed.): Proceedings of the International Conference on Fatigue in the Very High Cycle Fatigue Regime. Juli 2-4, 2001, Universität für Bodenkultur Wien, Wien, 2001.

[Stanzl-Tschegg, 2014] Stanzl-Tschegg, S.: Very high cycle fatigue measuring techniques. International Journal of Fatigue 60, S. 2-17, 2014.

[Stephenson, 1958] Stephenson, N.: A review of the literature on the effect of frequency on the fatigue properties of metals an alloys. National Gas Turbine Establishment Memorandum No. M.320, Pyestock, Hants, 1958.

[Tostmann, 2001] Tostmann, K.-H.: Korrosion, Ursachen und Vermeidung. Whiley$\mathrm{VCH}$, Weinheim, 2001.

[Tsutsumi, 2009] Tsutsumi, N.; Murakami, Y.; Doquet, V.: Effect of test frequency on fatigue strength of low carbon steel. Fatigue \& Fracture of Engineering Materials \& Structures 32, S. 473-483, 2009.

[Weich, 2008] Weich, I.: Ermüdungsverhalten mechanisch nachbehandelter Schweißverbindungen in Abhängigkeit des Randschichtzustandes. Dissertation, Technische Universität Braunschweig, Braunschweig 2008.

[Wohlfahrt, 1983] Wohlfahrt, H.: Schweißeigenspannungen. Entstehung - Berechnung - Bewertung. In: Eigenspannungen. Entstehung - Messung - Bewertung. Band 1, Hrsg. Macherauch, E. und Hauk, V., Deutsche Gesellschaft für Materialkunde, Oberursel, 1983. 
[Zeine El Dine, 2007] Zein El Dine, S.: Ermüdungssicherheit der Schweißnähte an Ringflanschverbindungen in turmartigen Stahlbauten. Dissertation, Universität Duisburg-Essen, Duisburg-Essen, 2007.

[Zhao, 2012] Zhao, X.; Wang, D.; Deng, C.; Liu, Y.; Song, Z.: The fatigue behaviors of butt welds ground flush in the super-long life regime. International Journal of Fatigue 36, S. 1-8, 2012.

\subsection{Programmsysteme}

[ANSYS, 2010] ANSYS Release 13.0, ANSYS, Inc., 2010.

[LabVIEW, 2007] LabView 8.5, National Instruments, 2007.

\subsection{Studentische Arbeiten}

Ahmad, F.: Statistische Auswertung von Ermüdungsversuchen im Zeitfestigkeits- und Übergangsbereich. Projektarbeit am Institut für Stahlbau, Leibniz Universität Hannover, 2012.

Fricke, S.: Theoretische und numerische Untersuchungen zum Kerbspannungskonzept für Schweißverbindungen. Studienarbeit am Institut für Stahlbau, Leibniz Universität Hannover, 2010.

Fricke, S.: Experimentelle und numerische Untersuchungen zum transienten Temperaturfeld beim Schweißen einer Stumpfnahtverbindung. Diplomarbeit am Institut für Stahlbau, Leibniz Universität Hannover, 2010.

Fuchs, J.: Theoretische und numerische Untersuchungen zum Einfluss der Kerbwirkung auf die Ermüdungsfestigkeit von Schweißverbindungen. Bachelorarbeit am Institut für Stahlbau, Leibniz Universität Hannover, 2013.

Gates, R. L.: Einfluss der Prüffrequenz auf die Ergebnisse von Ermüdungsversuchen am Beispiel einer Stumpfnahtverbindung. Projektarbeit am Institut für Stahlbau, Leibniz Universität Hannover, 2011.

Greve, M.: Theoretical and numerical investigations on the notch stress approach for welded joints. Studienarbeit am Institut für Stahlbau, Leibniz Universität Hannover, 2010.

Wagner, L.: Theoretische Untersuchungen zum Rissfortschritt bei Schweißverbindungen. Bachelorarbeit am Institut für Stahlbau, Leibniz Universität Hannover, 2013. 


\section{Verzeichnisse}

\subsection{Abbildungsverzeichnis}

Abb. 1-1: Normierte Auswertung von Ermüdungsversuchen an Schweißverbindungen aus Stahl [Sonsino, 2005b] .............................................. 2

Abb. 2-1: Phasen des Ermüdungsprozesses [Gudehus, 1999] [Radaj, 2007] ...... 5

Abb. 2-2: Schematischer Verlauf der Wöhlerlinie [Haibach, 2006]....................... 7

Abb. 2-3: Schematische Verläufe von Wöhlerlinien bis in den VHCF-Bereich in doppeltlogarithmischer Darstellung [Pyttel, 2011].............................. 8

Abb. 2-4: Risseinleitungsarten glatter Proben im LCF-, HCF- und VHCFBereich [Bathias, 2001]

Abb. 2-5: Einflussgrößen auf die Ermüdungsfestigkeit [Radaj, 2000] ................ 10

Abb. 2-6: Eigenspannungen einer Stumpfnahtverbindung in Längsrichtung (links) und in Querrichtung (rechts) zur Naht [Rappe, 1972] .............. 14

Abb. 2-7: Überlagerung von Last- und Eigenspannungen [Gurney, 1979].......... 15

Abb. 2-8: Einfluss der Höhe der schweißbedingten Zugeigenspannungen auf die Mittelspannungsabhängigkeit [Haibach, 2006] 16

Abb. 2-9: Ermüdungsversuche an einer Stumpfnahtverbindung aus Otha et al. [Otha, 1986].

Abb. 2-10: Ermüdungsversuche an abgeschliffenen Schweißproben und Grundwerkstoffproben aus Zhao et al. [Zhao, 2012]

Abb. 2-11: Ermüdungsversuche an Flachstäben mit Quersteifen aus Haibach [Haibach, 1971]

Abb. 2-12: Ermüdungsversuche an Längssteifen aus Kulka et al. [Kulka, 1987] .. 24

Abb. 2-13: Ermüdungsfestigkeitskurve für Längsspannungsschwingbreiten aus Eurocode 3 [DIN EN 1993-1-9, 2010].

Abb. 2-14: Einfluss schweißbedingter Zugeigenspannungen auf die Mittelspannungsabhängigkeit nach Eurocode 3 (links) und nach den Empfehlungen des IIW (rechts) [Haibach, 2006]

Abb. 2-15: Ermüdungsfestigkeitskurven für konstante Längsspannungsschwingbreiten bei Anwendungen im VHCF-Bereich aus den Empfehlungen des IIW [IIW, 2009] 
Abb. 3-1: $\quad$ Schweißnahtvorbereitung in Anlehnung an DIN EN ISO 9692-1

[DIN EN ISO 9692-1, 2004] 34

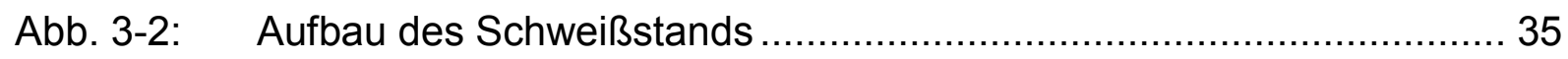

Abb. 3-3: Geometrie der Probekörper ............................................... 36

Abb. 3-4: $\quad$ Position der Thermoelemente auf der Schweißprobe im Querschnitt.. 36

Abb. 3-5: Position der Thermoelemente auf der Schweißprobe in der Draufsicht.

Abb. 3-6: Temperatur-Zeit-Verläufe der einzelnen Thermoelemente während der Schweiß- und Abkühlphase in Messung 5

Abb. 3-7: $\quad$ Aufbau der Lasermessung (links) und Beispiel eines Messschriebs (rechts)

Abb. 3-8: Definition der lokalen Nahtgeometrieparameter an den Übergängen.. 42

Abb. 3-9: Darstellung der Auswertung der lokalen Nahtgeometrie an einem exemplarischen Übergang.

Abb. 3-10: Grenzwerte für Oberflächenunregelmäßigkeiten für Bewertungsgruppe B nach DIN EN ISO 5817 [DIN EN ISO 5817, 2006]. 46

Abb. 3-11: Darstellung des numerischen Modells (oben) und der Verteilung der 1. Hauptspannung $\sigma_{1}$ (unten)

Abb. 3-12: Metallographische Untersuchung

Abb. 3-13: Lage der Härtereihen im Makroschliff (oben) und Härtemessung HV1 der einzelnen Härtereihen (unten)

Abb. 4-1: Aufbau der Prüfvorrichtung. 60

Abb. 4-2: Funktionsprinzip der Prüfvorrichtung.... 60

Abb. 4-3: Vorspannvorrichtung

Abb. 4-4: Verlauf der zeitlich veränderlichen Messgrößen über die Schwingspielzahl $\mathrm{N}$ bei Probenversagen .

Abb. 4-5: Verlauf der zeitlich veränderlichen Messgrößen über die Schwingspielzahl $\mathrm{N}$ beim Erreichen der Grenzschwingspielzahl $\mathrm{N}_{\mathrm{G}}$.

Abb. 4-6: Verlauf der Prüffrequenz f über die Schwingspielzahl N bei Probenversagen

Abb. 4-7: Verlauf der Prüffrequenz $f$ über die Schwingspielzahl $N$ bis zum Erreichen der Grenzschwingspielzahl $\mathrm{N}_{\mathrm{G}}$ 
Abb. 4-8: Hochfahrverhalten der Prüfkraftamplitude $F_{a}$ auf den Beanspruchungsniveaus $\mathrm{H} 1$ und $\mathrm{H} 2$ 68

Abb. 4-9: Verlauf von Vorspannkraft $F_{v}$ und Temperaturdifferenz $\Delta T$ gegenüber der Temperatur bei Versuchsbeginn während der Versuchslaufzeit ... 70

Abb. 4-10: Abhängigkeit des Verlaufes der Vorspannkraft $F_{v}$ von der erreichten Schwingspielzahl N für die Versuche von Beanspruchungsniveau H2 71

Abb. 4-11: Temperaturdifferenz $\Delta T$ der Versuche auf den Beanspruchungsniveaus $\mathrm{H} 1$ und $\mathrm{H} 2$

Abb. 4-12: Verlauf der Vorspannkraft $F_{\mathrm{v}}$ über die Schwingspielzahl $\mathrm{N}$ bis zum Erreichen der Grenzschwingspielzahl

Abb. 4-13: Verlauf von Vorspannkraft $F_{v}$ und Temperaturdifferenz $\Delta T$ über die Versuchslaufzeit bei Versuchsbeginn (links) und bei Versuchsende (rechts)

Abb. 4-14: Abnutzungserscheinungen auf den Kontaktflächen der Spannklötze zum Prüfrahmen nach Langzeitversuch .

Abb. 4-15: Ermittlung der Anrissschwingspielzahl $\mathrm{N}_{\mathrm{A}}$ 76

Abb. 4-16: Schematische Versuchsführung beim Verfahren der Blocklastfolgen . 77

Abb. 4-17: Bruchfläche aus Versuchs- und Markierungsblock mit Rastlinien . 77

Abb. 4-18: Zeitlich veränderliche Messgrößen während des Versuchs R-1-1 zur Markierung der Rissfront auf der Bruchfläche 78

Abb. 4-19: Aufbereitung der zeitlich veränderlichen Messgrößen von Versuch R-1-1 zur Auswertung des Kriteriums für den Anriss. 79

Abb. 4-20: Ermittlung der Anrissschwingspielzahl $\mathrm{N}_{\mathrm{A}}$ für Versuch R-1-1 ........... 80

Abb. 4-21: Definition der Tiefe und Breite bei der Vermessung der Rastlinien ..... 81

Abb. 4-22: Kalibrierprobe 83

Abb. 4-23: Ermittlung des statischen Kalibrierfaktor kfs 84

Abb. 4-24: Frequenzabhängigkeit des dynamischen Kalibrierfaktors kfd 86

Abb. 4-25: Regelung Rahmendehnung: konstant (links) und frequenzabhängig (rechts)

Abb. 4-26: Datengrundlage zur Ermittlung der Korrekturfaktoren $p_{k}$ und kfd $_{\text {Bezug }}$ der frequenzabhängigen Sollwertvorgabe 
Abb. 4-27: Verlauf der Prüfkraftamplitude $F_{a}$ von Rahmen und Probe über die Prüffrequenz $\mathrm{f}$ beim Erreichen der Grenzschwingspielzahl $\mathrm{N}_{\mathrm{G}}$...... 89

Abb. 4-28: Vergleich zeitlich veränderlicher Messgrößen von Probe und Rahmen über die Schwingspielzahl $\mathrm{N}$ bei Probenversagen 90

Abb. 5-1: Ergebnisse der Versuchsreihen 1-A und 1-B …............................ 108

Abb. 5-2: Ergebnisse der Versuchsreihen 2-A und 2-B ............................... 109

Abb. 5-3: Ergebnisse der Versuchsreihen 3-A und 3-B .............................. 110

Abb. 5-4: Einfluss der Prüffrequenz ………………................................. 111

Abb. 5-5: Einfluss der Prüffrequenz bei Vergleich von Bruchschwingspiel-

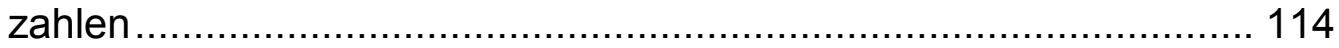

Abb. 5-6: Einfluss der Mittelspannung ………......................................... 117

Abb. 5-7: Versuchsreihe 1-C im Vergleich zu Versuchsreihe 1-A und 1-B ...... 120

Abb. 5-8: Versuchsreihe 2-C im Vergleich Versuchsreihe 2-A und 2-B ........... 120

Abb. 5-9: Versuchsreine 3-C im Vergleich Versuchsreihe 3-A und 3-B ........... 121

Abb. 5-10: Anriss am Wurzelübergang (links) und am Nahtübergang (rechts) ... 123

Abb. 5-11: Überblick der aufgetretenen Rissformen aller Versuchsreihen.......... 124

Abb. 5-12: Draufsicht der Wurzelüberhöhung (links) und Bruchflächen (rechts) von Versuch V2-C-2 ……................................................ 126

Abb. 5-13: Vergleich der Versuchsergebnisse der vorliegenden Arbeit mit Sonsino et al. [Sonsino, 2005b]

Abb. 5-14: Auszug aus Tabelle 8.3 in Eurocode 3 [DIN EN 1993-1-9, 2010] zur Kerbfalleinstufung quer laufender Stumpfnähte 129

Abb. 5-15: Vergleich der Versuchsergebnisse der vorliegenden Arbeit mit Eurocode 3

\subsection{Tabellenverzeichnis}

Tab. 2-1: Empfohlene Abknickpunkte, Neigungen nach dem Abknickpunkt und Streumaße, Auszug aus [Sonsino, 2005a] [Sonsino, 2007] 29

Tab. 2-2: Prüfmaschinen mit maximaler Prüffrequenz und entsprechender Versuchsdauer bis zu $10^{9}$ Lastwechseln [Pyttel, 2011] [Stanzl-Tschegg, 2014]. 
Tab. 3-1: $\quad$ Chemische Zusammensetzung des Werkstoffes S355J2 +N $(\mathrm{t}=4 \mathrm{~mm})$ 33

Tab. 3-2: $\quad$ Überblick der Schweißparameter ........................................... 34

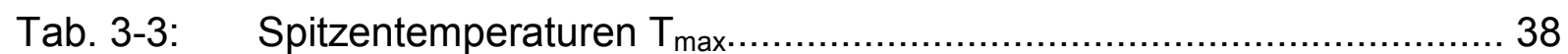

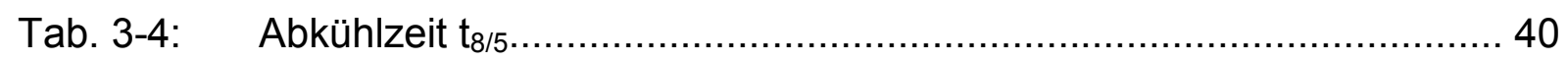

Tab. 3-5: Ergebnisübersicht der Auswertung der lokalen Nahtgeometrieparameter

Tab. 3-6: Zusammenfassung der ermittelten Kerbformzahlen $\mathrm{K}_{\mathrm{t}}$ nach

Anthes et al. [Anthes, 1993] und nach Rainer [Rainer, 1979]

Tab. 3-7: $\quad$ Vergleich der Kerbformzahlen $\mathrm{K}_{\mathrm{t}}$ der einzelnen Profilschriebe von

Probe M.

Tab. 3-8: Zusammenfassung der numerisch ermittelten Kerbform-

zahlen $\mathrm{K}_{\mathrm{t} \_\mathrm{FEM}}$

Tab. 3-9: Vergleich der Ergebnisse der analytisch und numerisch ermittelten

Kerbformzahlen $\mathrm{K}_{\mathrm{t} \_\mathrm{N}}$ am Nahtübergang .

Tab. 3-10: Vergleich der Ergebnisse der analytisch und numerisch ermittelten Kerbformzahlen $\mathrm{K}_{\mathrm{t} \_\mathrm{w}}$ am Wurzelübergang

Tab. 3-11: $\quad$ Vergleich der mittleren prozentualen Abweichungen $\mathrm{p}_{\%}$

Tab. 4-1: $\quad$ Bezeichnungen der Versuche

Tab. 4-2: Übersicht der Versuche zur Markierung der Rissfront auf der

Bruchfläche

Tab. 4-3: Überblick der Ergebnisse der Versuche zur Markierung der

Rissfront

Tab. 4-4: $\quad$ Ergebnisse der Vermessung der Rastlinien

Tab. 5-1: $\quad$ Übersicht der Prüfmaschinen

Tab. 5-2: $\quad$ Übersicht über das Versuchsprogramm ....

Tab. 5-3: Vergleich der Soll- und Istwerte von Kraftamplitude $F_{a}$ und

Vorspannkraft $F_{v}$ für Versuchsreihe 2.

Tab. 5-4: Vergleich der Soll- und Istwerte von Kraftamplitude $F_{a}$ und

Vorspannkraft $F_{v}$ für Versuchsreihe 3.

Tab. 5-5: $\quad$ Abfall von Vorspannkraft $F_{v}$ und Spannungsverhältnis $R$ bei

Versuchsreihe 2 . 
Tab. 5-6: $\quad$ Abfall von Vorspannkraft $F_{v}$ und Spannungsverhältnis $R$ bei Versuchsreihe 3 103

Tab. 5-7: $\quad$ Mittelwerte der Erwärmung der Probekörper aus Versuchsreihe 2 ... 104

Tab. 5-8: $\quad$ Mittelwerte der Erwärmung der Probekörper aus Versuchsreihe 3 ... 105

Tab. 5-9: $\quad$ Auswertung der Streuung auf den Beanspruchungshorizonten von

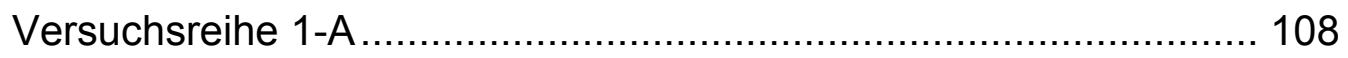

Tab. 5-10: Auswertung der Streuung auf den Beanspruchungshorizonten von Versuchsreihe 2-A. 109

Tab. 5-11: Auswertung der Streuung auf den Beanspruchungshorizonten von Versuchsreihe 3-A

Tab. 5-12: Grunddaten der statistischen Auswertung von Versuchsreihe 1 und 2

Tab. 5-13: Vergleich der Beanspruchungsniveaus im Zeitfestigkeitsbereich von Versuchsreihe 1-A und 2-A

Tab. 5-14: $\quad$ Vergleich der mittleren Bruchschwingspielzahlen $\mathrm{N}_{\mathrm{B}, 50 \%}$ auf den Beanspruchungsniveaus im Zeitfestigkeitsbereich von Versuchsreihe $1-A$ und $2-A$

Tab. 5-15: Grunddaten der statistischen Auswertung von Versuchsreihe 2 und 3

Tab. 5-16: Vergleich der Beanspruchungsniveaus im Zeitfestigkeitsbereich von Versuchsreihe 2-A und 3-A

Tab. 5-17: Gegenüberstellung der ermittelten charakteristischen Ermüdungsfestigkeiten $\Delta \sigma_{C}$ der Versuchsreihen 1-A, 2-A und 3-A ... 130 


\section{Anhang}

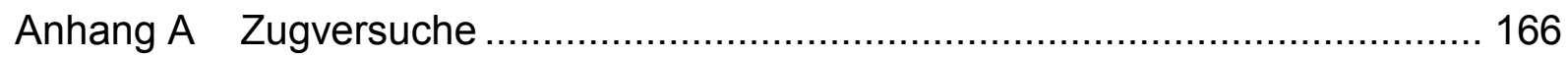

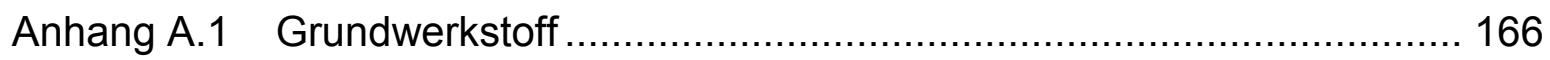

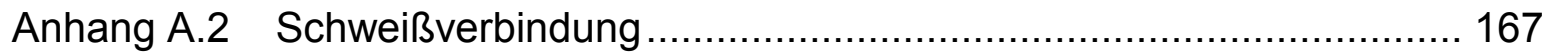

Anhang B Temperaturmessungen während des Schweißens ........................... 168

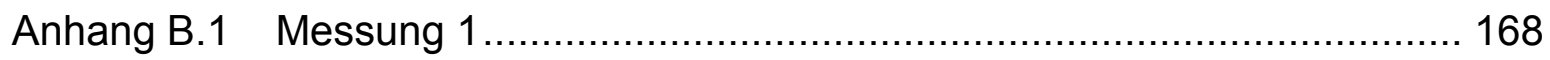

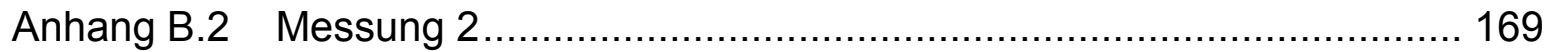

Anhang B.3 Messung 3 …................................................................. 170

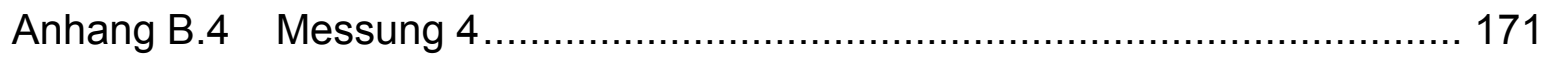

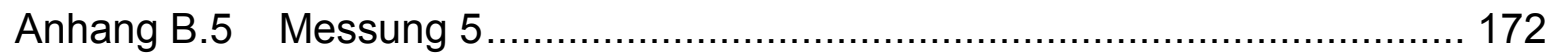

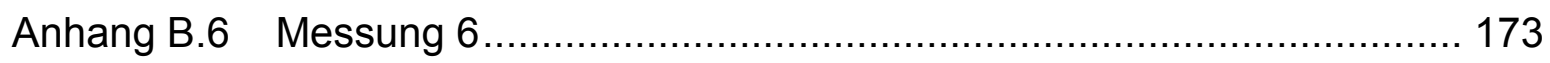

Anhang C Rauheitsmessung nach dem Tastschnittverfahren .......................... 174

Anhang D Algorithmus zur Auswertung der lokalen Nahtgeometrieparameter .... 175

Anhang E Lokalen Schweißnahtgeometrie .................................................. 176

Anhang E.1 Einzelergebnisse der lokalen Nahtgeometrieparameter ............... 176

Anhang E.2 Einzelergebnisse der ermittelten Kerbformzahlen $\mathrm{K}_{\mathrm{t}} \ldots \ldots \ldots \ldots \ldots \ldots \ldots \ldots$

Anhang F Ergebnisse der Versuche zur Markierung der Rissfront..................... 182

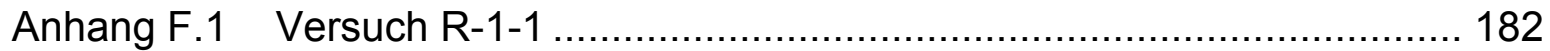

Anhang F.1.1 Auswertung der zeitlich veränderlichen Messgrößen ................ 182

Anhang F.1.2 Vermessung der Rastlinien auf der Bruchfläche ...................... 183

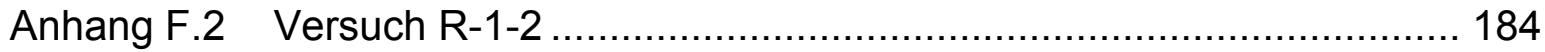

Anhang F.2.1 Auswertung der zeitlich veränderlichen Messgrößen ................ 184

Anhang F.2.2 Vermessung der Rastlinien auf der Bruchfläche ..................... 185

Anhang F.3 Versuch R-1-3 ….......................................................... 186

Anhang F.3.1 Auswertung der zeitlich veränderlichen Messgrößen ................ 186

Anhang F.3.2 Vermessung der Rastlinien auf der Bruchfläche ..................... 187

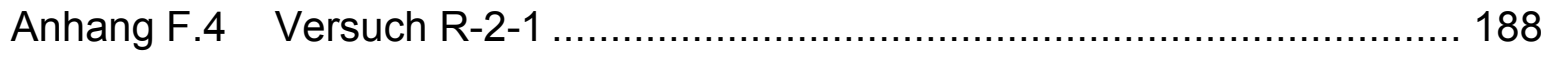

Anhang F.4.1 Auswertung der zeitlich veränderlichen Messgrößen ................ 188 
Anhang F.4.2 Vermessung der Rastlinien auf der Bruchfläche .................... 189

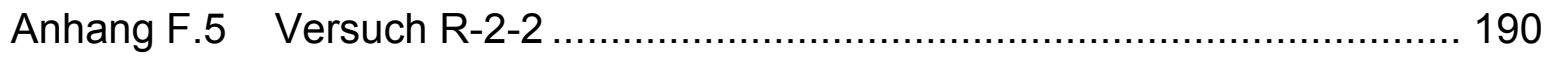

Anhang F.5.1 Auswertung der zeitlich veränderlichen Messgrößen ............... 190

Anhang F.5.2 Vermessung der Rastlinien auf der Bruchfläche .................... 191

Anhang F.6 Versuch R-2-3 ...................................................... 192

Anhang F.6.1 Auswertung der zeitlich veränderlichen Messgrößen ............... 192

Anhang F.6.2 Vermessung der Rastlinien auf der Bruchfläche .................... 193

Anhang G Übertragungsfaktoren kf der Kalibrierproben ............................... 194

Anhang H Temperaturmessungen während der Ermüdungsversuche ............... 195

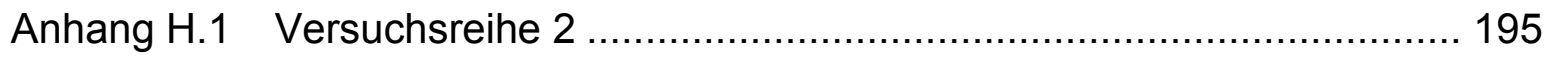

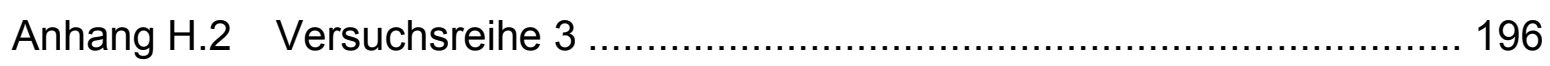

Anhang I Einzelversuche und Bruchflächen der Versuchsreihen ...................... 197

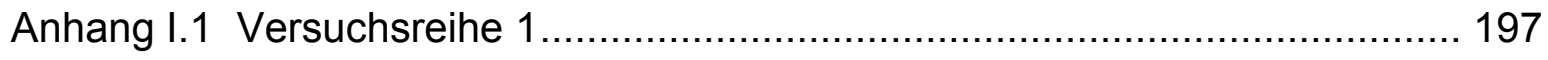

Anhang I.1.1 A - Zeitfestigkeitsbereich........................................... 197

Anhang I.1.2 B - Übergangsbereich ................................................. 200

Anhang I.1.3 C - Hochgesetzte Durchläufer ........................................ 202

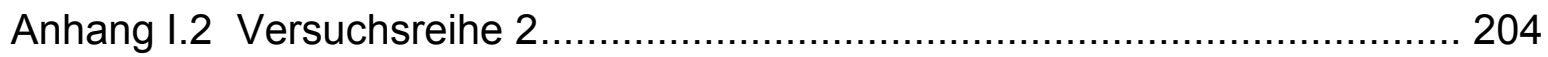

Anhang I.2.1 A - Zeitfestigkeitsbereich ............................................... 204

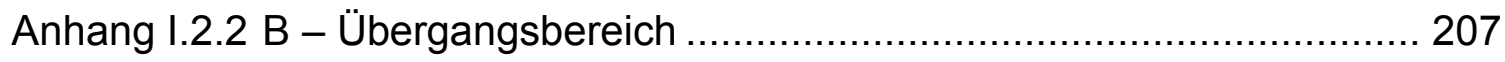

Anhang I.2.3 C - Hochgesetzte Durchläufer ........................................ 209

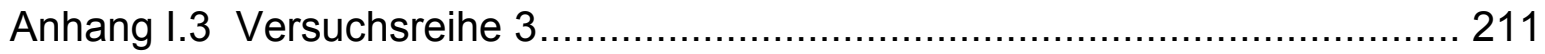

Anhang I.3.1 A - Zeitfestigkeitsbereich ......................................... 211

Anhang I.3.2 B - Übergangsbereich ...................................................... 213

Anhang I.3.3 C - Hochgesetzte Durchläufer ..................................... 215

Anhang J Auswertung der Ermüdungsversuche .................................... 217

Anhang J.1 Übergangsbereich - Treppenstufenverfahren ......................... 217

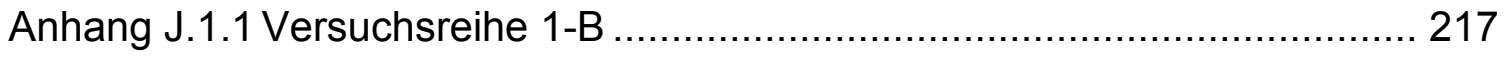

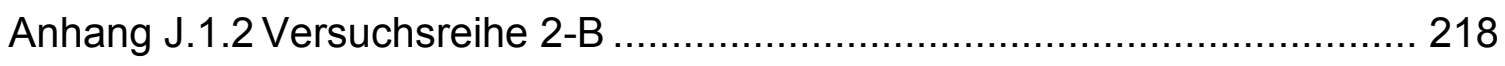

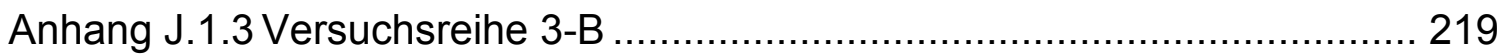


Anhang J.2 Vergleich von Anriss- und Bruchwöhlerlinie............................... 220

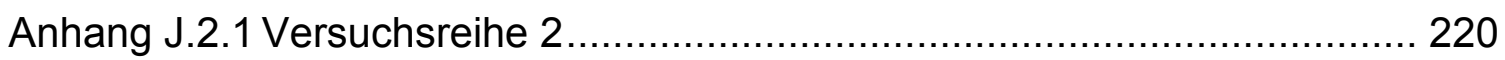

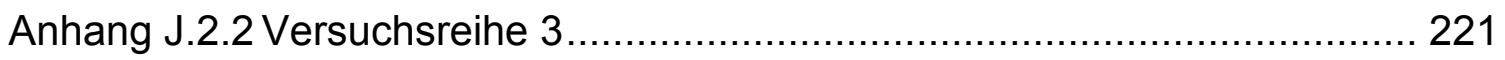

Anhang J.3 Statistische Auswertung nach dem Hintergrundbericht zu EC3...... 222

Anhang J.3.1 Versuchsreihe 1-A ................................................. 222

Anhang J.3.2 Versuchsreihe 2-A ....................................................... 223

Anhang J.3.3 Versuchsreihe 3-A ....................................................... 224 
Anhang A Zugversuche

Anhang A.1 Grundwerkstoff

Einzelergebnisse und statistische Kenngrößen

\begin{tabular}{|c|c|c|c|}
\hline $\begin{array}{c}\text { Probe } \\
{[-]}\end{array}$ & $\begin{array}{c}\mathbf{R}_{\text {eH }} \\
{\left[\mathbf{N} / \mathbf{m m}^{2}\right]}\end{array}$ & $\begin{array}{c}\mathbf{R}_{\mathbf{m}} \\
{\left[\mathbf{N} / \mathbf{m m}^{2}\right]}\end{array}$ & $\begin{array}{c}\mathbf{A} \\
{[\%]}\end{array}$ \\
\hline G-Z1-1 & 502,57 & 588,13 & 27,16 \\
\hline G-Z1-2 & 504,52 & 591,41 & 28,06 \\
\hline G-Z1-3 & 503,12 & 590,58 & 26,98 \\
\hline Mittelwert & 503,41 & 590,04 & 27,40 \\
\hline $\begin{array}{c}\text { Standard- } \\
\text { abweichung }\end{array}$ & 1,38 & 1,71 & 0,58 \\
\hline Varianz & 1,01 & 2,92 & 0,33 \\
\hline
\end{tabular}

Spannungs-Dehnungs-Diagramm

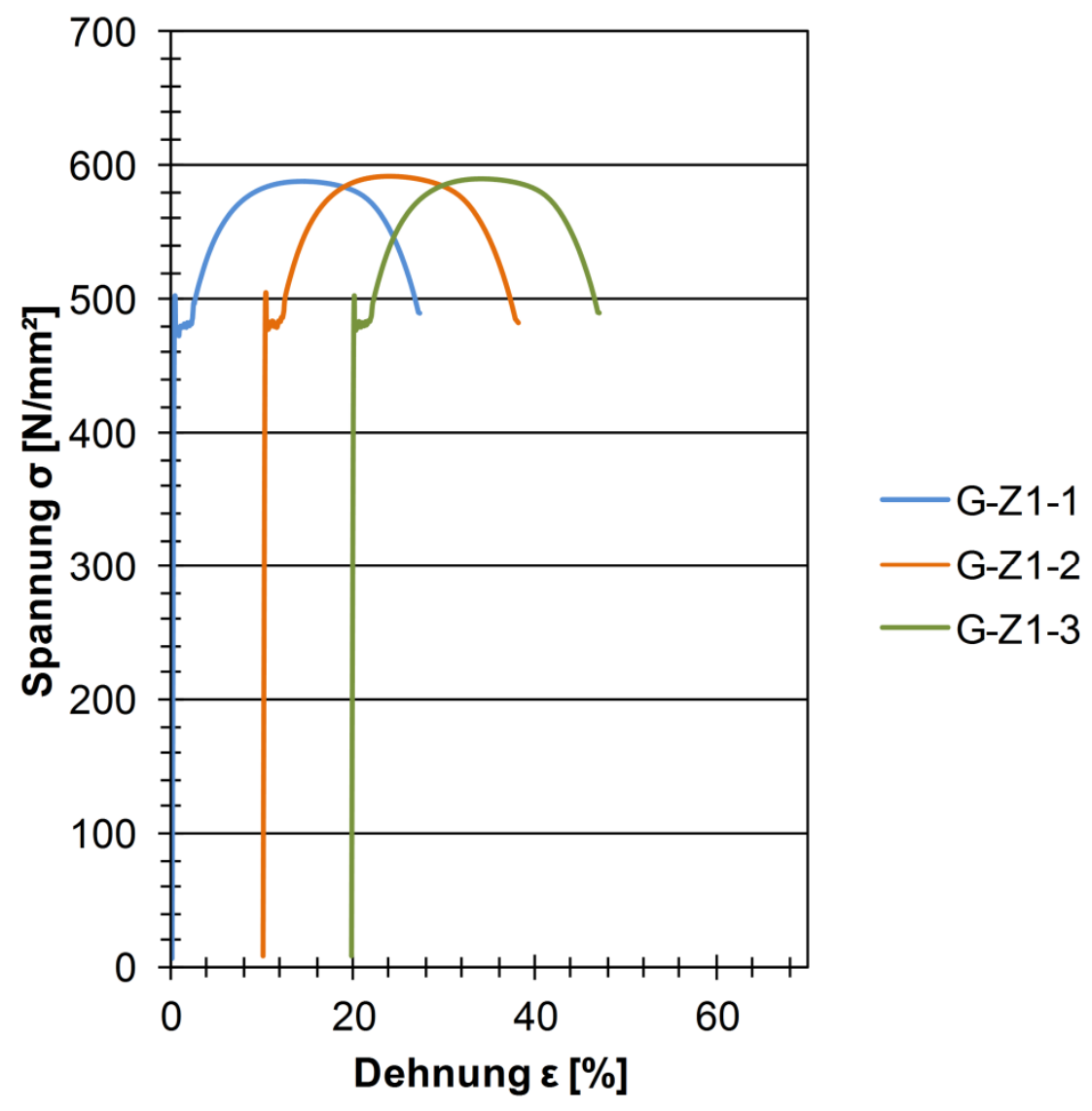


Anhang A.2 Schweißverbindung

Einzelergebnisse und statistische Kenngrößen

\begin{tabular}{|c|c|c|c|c|}
\hline $\begin{array}{c}\text { Probe } \\
{[-]}\end{array}$ & $\begin{array}{c}\mathbf{R}_{\mathbf{e H}} \\
{\left[\mathbf{N} / \mathbf{m m}^{2}\right]}\end{array}$ & $\begin{array}{c}\mathbf{R}_{\mathbf{m}} \\
{\left[\mathbf{N} / \mathbf{m m}^{2}\right]}\end{array}$ & $\begin{array}{c}\mathbf{A} \\
{[\%]}\end{array}$ \\
\hline S-Z1-1 & 507,94 & 598,21 & 25,15 \\
\hline S-Z1-2 & 506,56 & 599,38 & 24,04 \\
\hline S-Z2-1 & 491,85 & 581,33 & 19,69 \\
\hline S-Z2-2 & 490,87 & 586,57 & 18,64 \\
\hline S-Z3-1 & 495,25 & 561,88 & 17,59 \\
\hline S-Z3-2 & 505,73 & 571,96 & 16,19 \\
\hline Mittelwert & 499,70 & 583,22 & 20,22 \\
\hline $\begin{array}{c}\text { Standard- } \\
\text { abweichung }\end{array}$ & 7,88 & 14,72 & 3,60 \\
\hline Varianz & 62,14 & 216,54 & 12,97 \\
\hline
\end{tabular}

\section{Spannungs-Dehnungs-Diagramm}

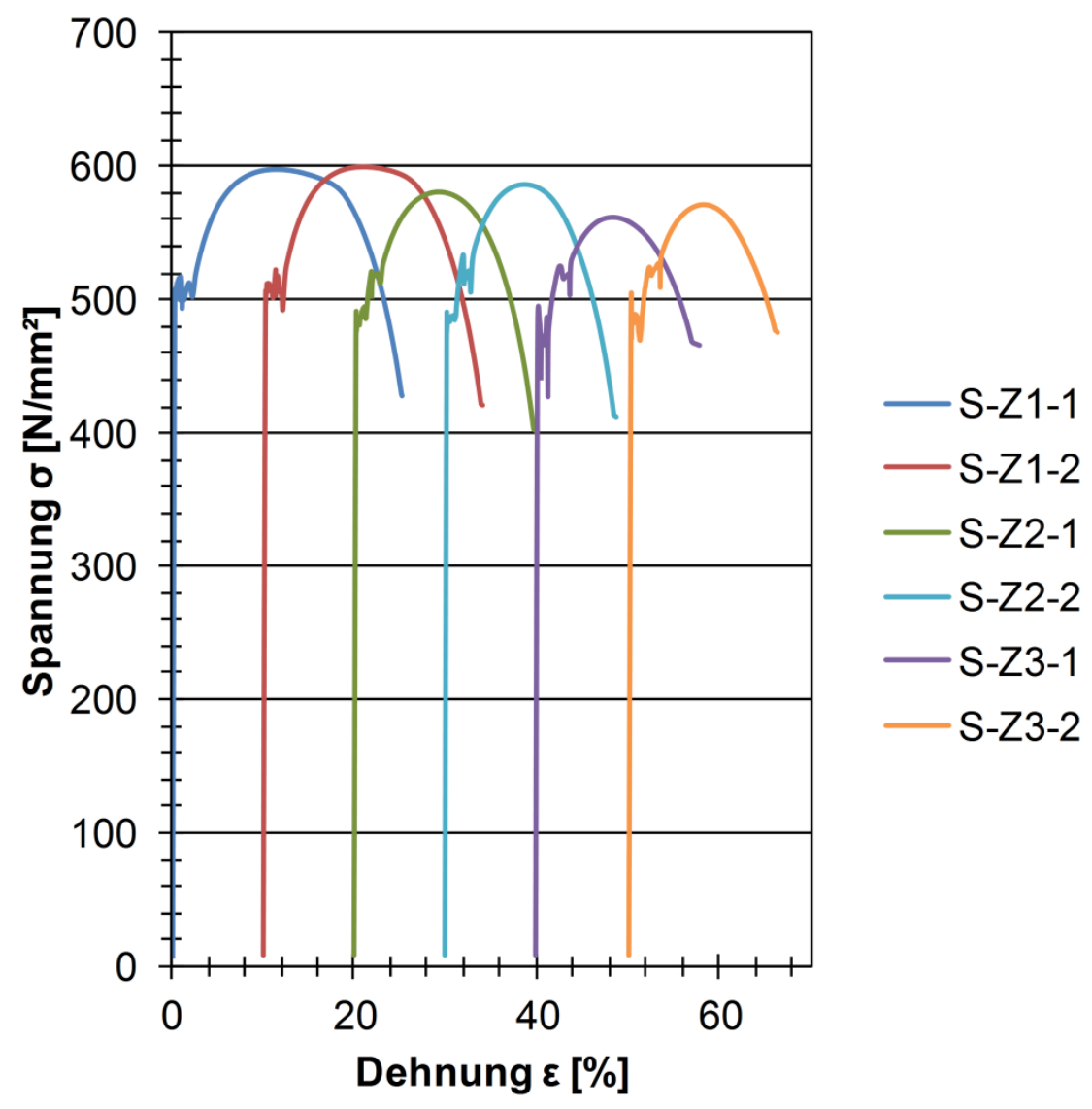




\section{Anhang B Temperaturmessungen während des Schweißens}

\section{Anhang B.1 Messung 1}

Temperatur-Zeit-Verlauf

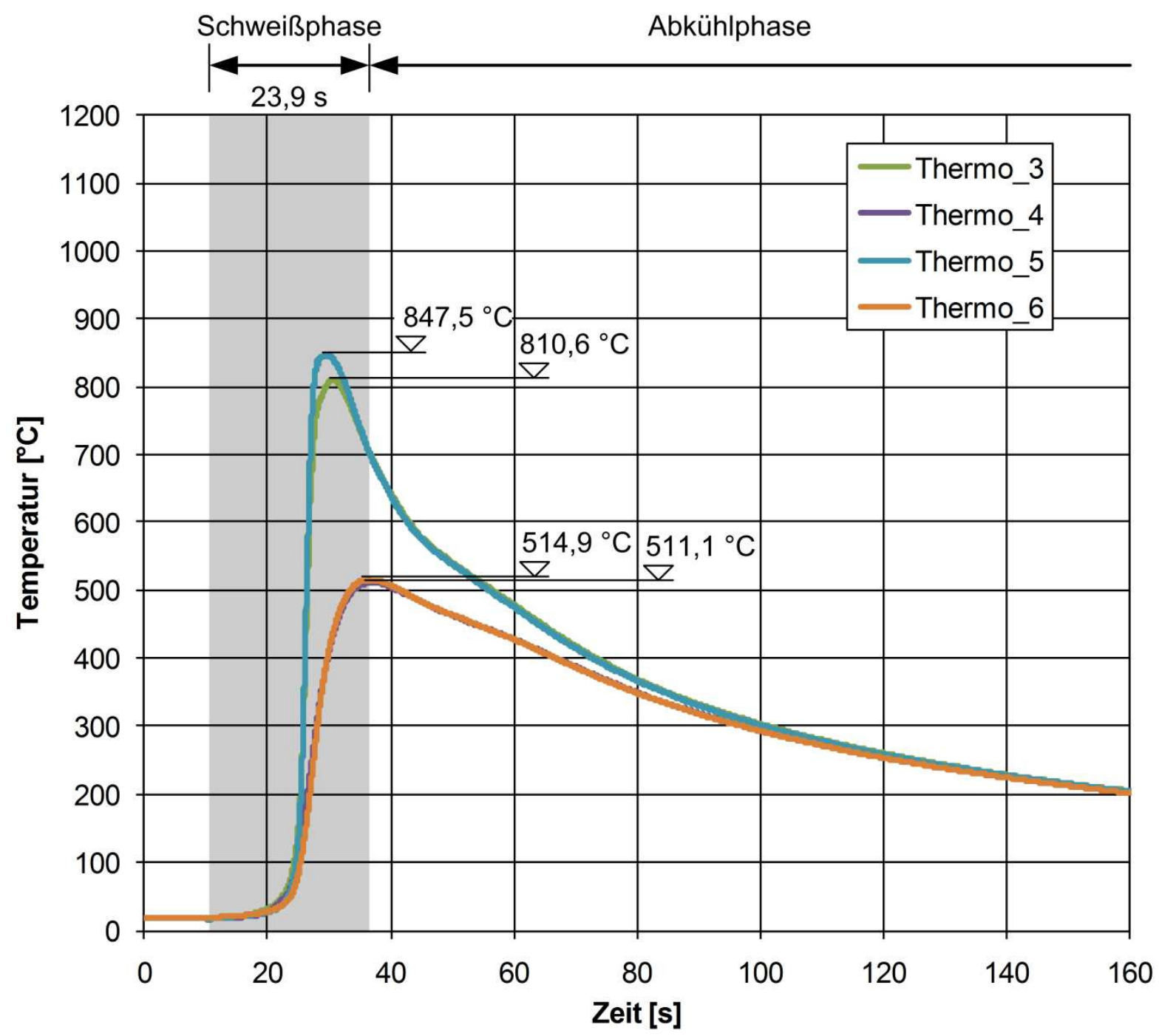

Spitzentemperaturen $\mathrm{T}_{\max }$ der Thermoelemente

\begin{tabular}{|c|c|c|c|c|c|}
\hline Thermo_1 & Thermo_2 & Thermo_3 & Thermo_4 & Thermo_5 & Thermo_6 \\
\hline- & - & $810,6{ }^{\circ} \mathrm{C}$ & $511,1^{\circ} \mathrm{C}$ & $847,5^{\circ} \mathrm{C}$ & $514,9^{\circ} \mathrm{C}$ \\
\hline
\end{tabular}

- Ausfall Thermoelement 


\section{Anhang B.2 Messung 2}

\section{Temperatur-Zeit-Verlauf}

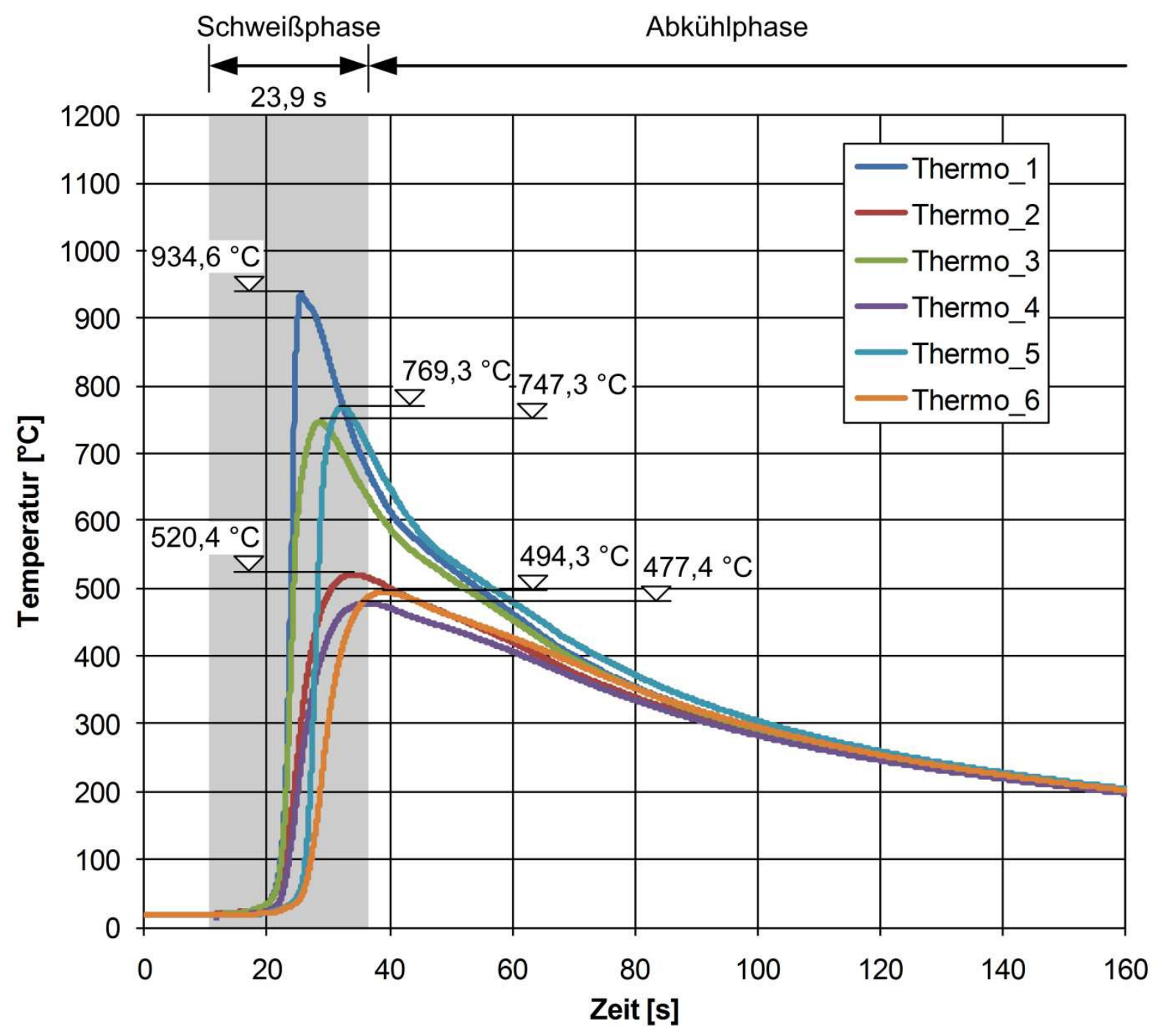

Spitzentemperaturen $\mathrm{T}_{\max }$ der Thermoelemente

\begin{tabular}{|c|c|c|c|c|c|}
\hline Thermo_1 & Thermo_2 & Thermo_3 & Thermo_4 & Thermo_5 & Thermo_6 \\
\hline $934,6{ }^{\circ} \mathrm{C}$ & $520,4^{\circ} \mathrm{C}$ & $747,3^{\circ} \mathrm{C}$ & $477,4{ }^{\circ} \mathrm{C}$ & $769,3^{\circ} \mathrm{C}$ & $494,3^{\circ} \mathrm{C}$ \\
\hline
\end{tabular}




\section{Anhang B.3 Messung 3}

\section{Temperatur-Zeit-Verlauf}

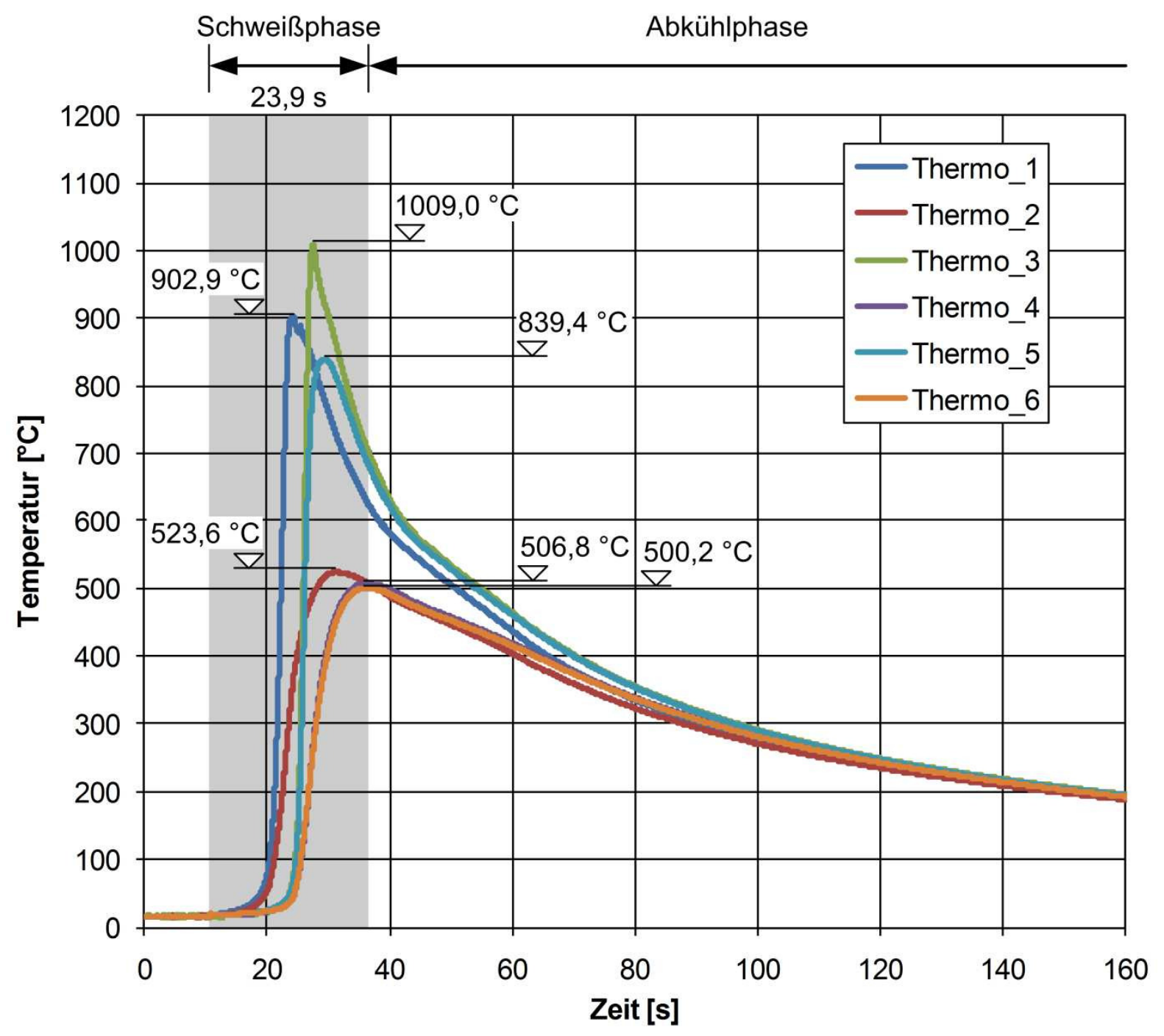

Spitzentemperaturen $T_{\max }$ der Thermoelemente

\begin{tabular}{|c|c|c|c|c|c|}
\hline Thermo_1 & Thermo_2 & Thermo_3 & Thermo_4 & Thermo_5 & Thermo_6 \\
\hline $902,9^{\circ} \mathrm{C}$ & $523,6{ }^{\circ} \mathrm{C}$ & $1009,0^{\circ} \mathrm{C}$ & $506,8^{\circ} \mathrm{C}$ & $839,4^{\circ} \mathrm{C}$ & $500,2^{\circ} \mathrm{C}$ \\
\hline
\end{tabular}




\section{Anhang B.4 Messung 4}

\section{Temperatur-Zeit-Verlauf}

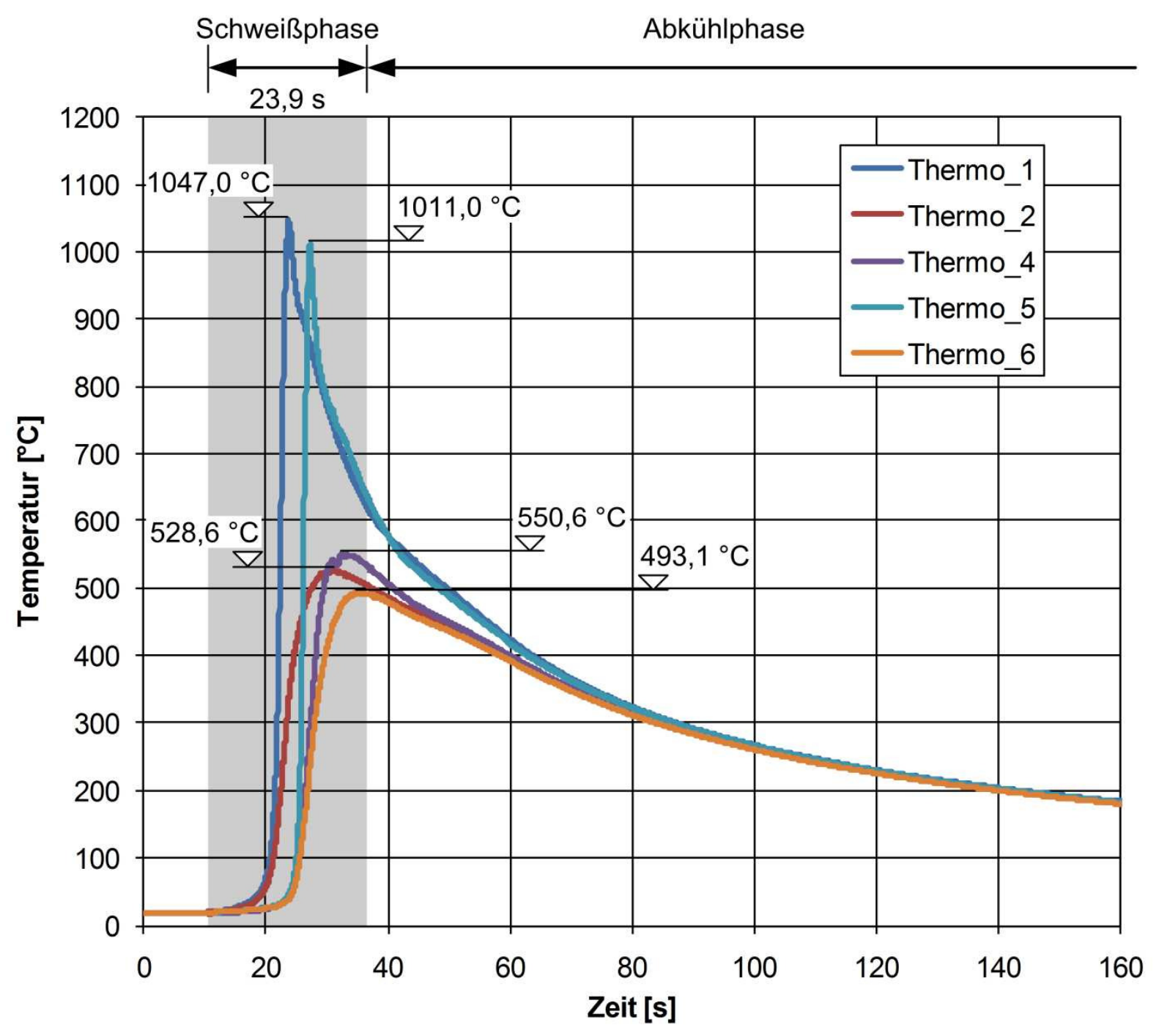

Spitzentemperaturen $\mathrm{T}_{\max }$ der Thermoelemente

\begin{tabular}{|c|c|c|c|c|c|}
\hline Thermo_1 & Thermo_2 & Thermo_3 & Thermo_4 & Thermo_5 & Thermo_6 \\
\hline $1047,0^{\circ} \mathrm{C}$ & $528,6^{\circ} \mathrm{C}$ & - & $550,6{ }^{\circ} \mathrm{C}$ & $1011,0^{\circ} \mathrm{C}$ & $493,1^{\circ} \mathrm{C}$ \\
\hline
\end{tabular}

- Ausfall Thermoelement 


\section{Anhang B.5 Messung 5}

\section{Temperatur-Zeit-Verlauf}

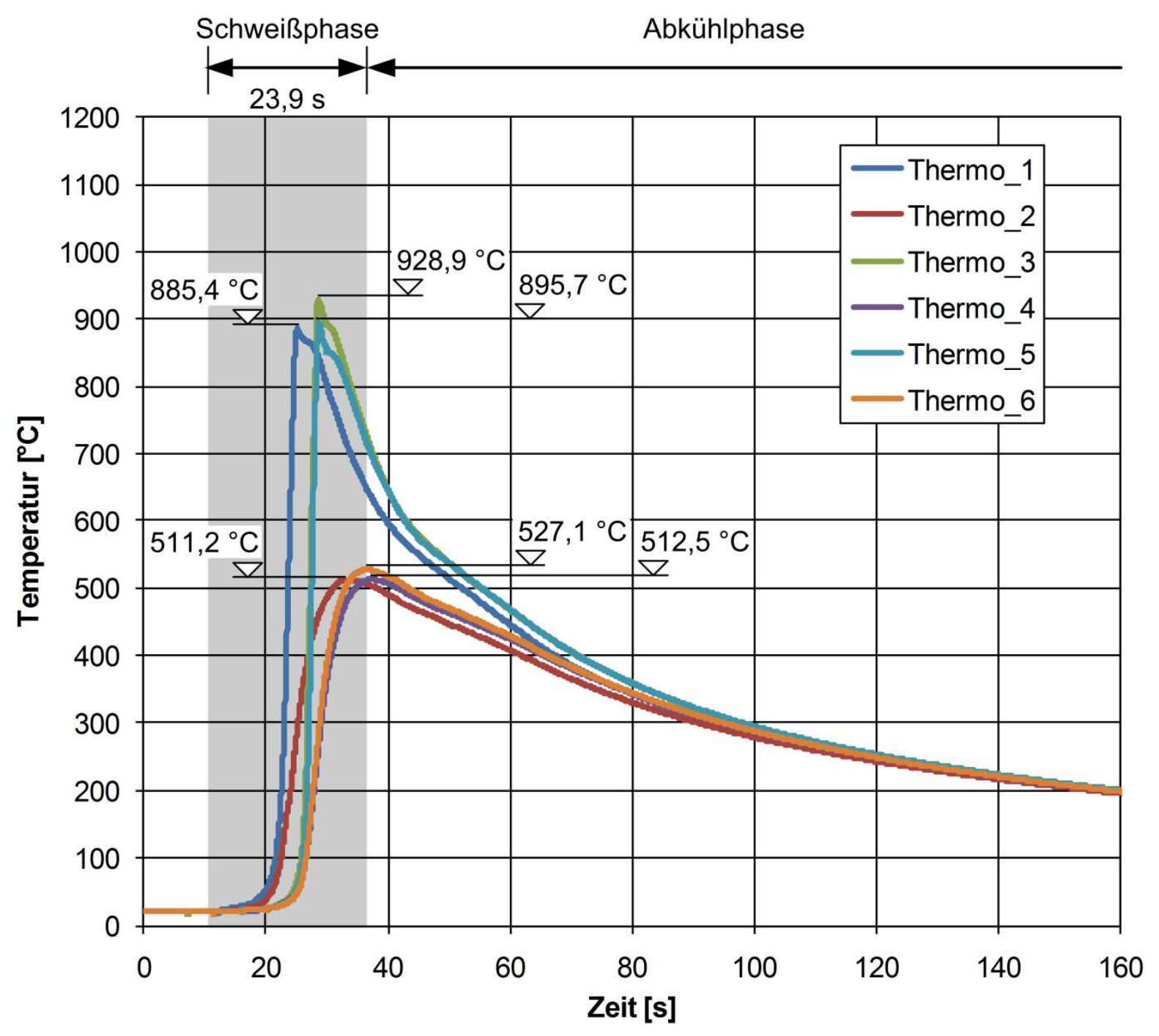

Spitzentemperaturen $\mathrm{T}_{\max }$ der Thermoelemente

\begin{tabular}{|c|c|c|c|c|c|}
\hline Thermo_1 & Thermo_2 & Thermo_3 & Thermo_4 & Thermo_5 & Thermo_6 \\
\hline $885,4^{\circ} \mathrm{C}$ & $511,2^{\circ} \mathrm{C}$ & $928,9^{\circ} \mathrm{C}$ & $512,5^{\circ} \mathrm{C}$ & $895,7^{\circ} \mathrm{C}$ & $527,1^{\circ} \mathrm{C}$ \\
\hline
\end{tabular}




\section{Anhang B.6 Messung 6}

\section{Temperatur-Zeit-Verlauf}

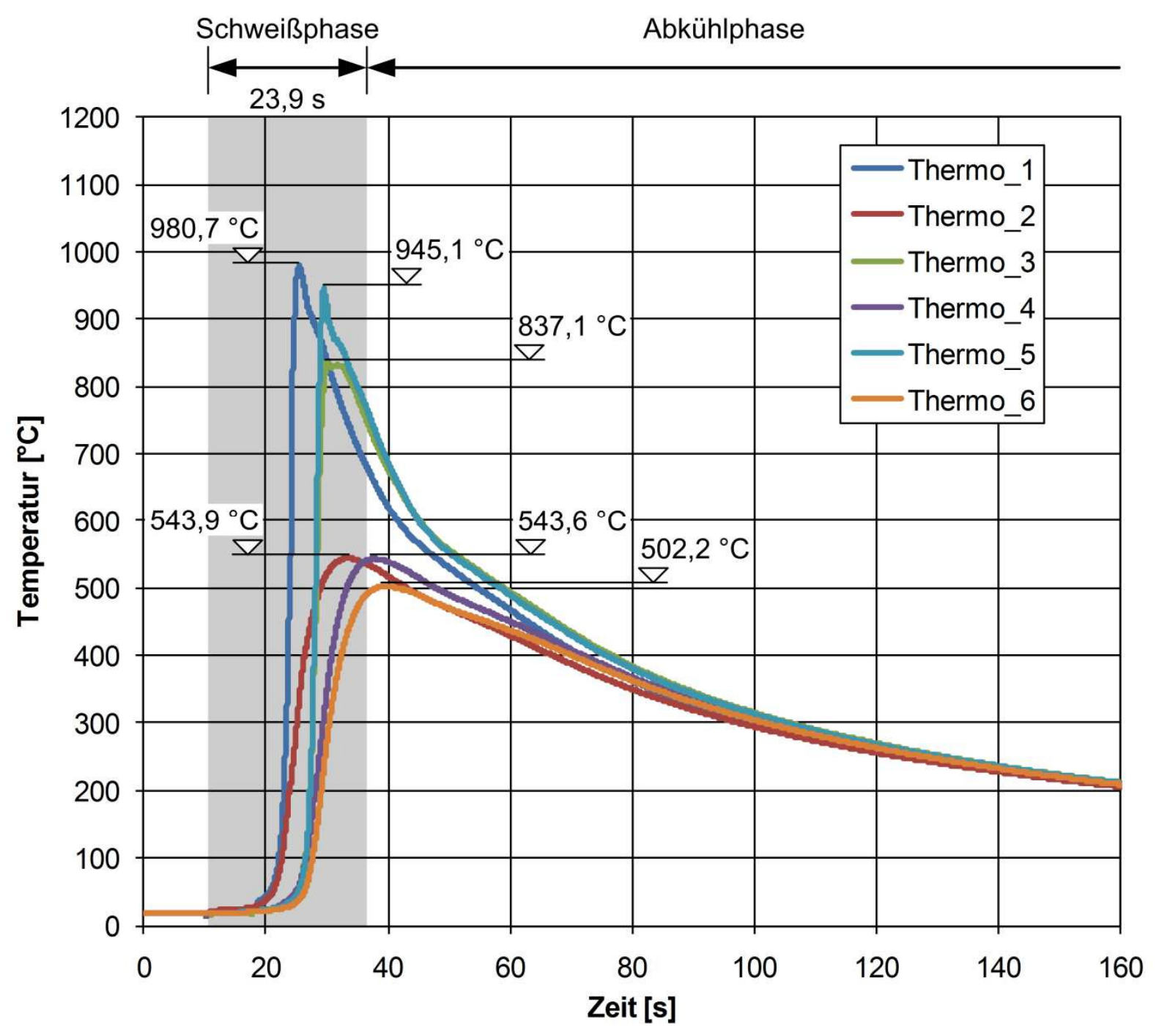

Spitzentemperaturen $\mathrm{T}_{\max }$ der Thermoelemente

\begin{tabular}{|c|c|c|c|c|c|}
\hline Thermo_1 & Thermo_2 & Thermo_3 & Thermo_4 & Thermo_5 & Thermo_6 \\
\hline $980,7^{\circ} \mathrm{C}$ & $543,9^{\circ} \mathrm{C}$ & $837,1^{\circ} \mathrm{C}$ & $543,6^{\circ} \mathrm{C}$ & $945,1^{\circ} \mathrm{C}$ & $502,2^{\circ} \mathrm{C}$ \\
\hline
\end{tabular}




\section{Anhang C Rauheitsmessung nach dem Tastschnittverfahren}

Die Oberflächenrauheit der Probekörper wurde mit dem Tastschnittverfahren ermittelt, wobei die Oberfläche mit einer Diamantspitze mechanisch abgetastet wird. Die Auswertung der Taststrecke erfolgt durch Filterung des ungefilterten Primärprofils $(P-$ Profil) nach DIN EN ISO 11562. Durch das Trennen der kurzwelligen und langwelligen Profilelemente werden das Rauheitsprofil (R-Profil) und das Welligkeitsprofil (WProfil) ermittelt.

Für die unterschiedlichen Profile (gekennzeichnet mit $\mathrm{P}, \mathrm{W}$ und $\mathrm{R}$ ) wurden die folgenden Kenngrößen (definiert nach DIN 4287) ausgewertet:

- Gesamthöhe des Profils $\left(P_{t} / W_{t} / R_{t}\right)$

- Maximale Profilhöhe $\left(P_{z} / W_{z} / R_{z}\right)$

- $\quad$ Arithmetischer Mittenwert $\left(\mathrm{P}_{\mathrm{a}} / \mathrm{W}_{\mathrm{a}} / \mathrm{R}_{\mathrm{a}}\right)$

- Quadratischer Mittenwert $\left(\mathrm{P}_{\mathrm{q}} / \mathrm{W}_{\mathrm{q}} / \mathrm{R}_{\mathrm{q}}\right)$

\begin{tabular}{|c|c|c|c|c|c|c|c|c|c|c|c|c|}
\hline $\begin{array}{c}\text { Mes- } \\
\text { sung } \\
\mathbf{N r} .\end{array}$ & $\begin{array}{c}\mathbf{P}_{\mathbf{t}} \\
{[\boldsymbol{\mu m}]}\end{array}$ & $\begin{array}{c}\mathbf{P}_{\mathbf{z}} \\
{[\boldsymbol{\mu m}]}\end{array}$ & $\begin{array}{c}\mathbf{P}_{\mathbf{a}} \\
{[\mu \mathrm{m}]}\end{array}$ & $\begin{array}{c}\mathbf{P}_{\mathbf{q}} \\
{[\boldsymbol{\mu m}]}\end{array}$ & $\begin{array}{c}\mathbf{W}_{\mathbf{t}} \\
{[\boldsymbol{\mu m}]}\end{array}$ & $\begin{array}{c}\mathbf{W}_{\mathbf{z}} \\
{[\boldsymbol{\mu m}]}\end{array}$ & $\begin{array}{c}\mathbf{W}_{\mathbf{a}} \\
{[\boldsymbol{\mu m}]}\end{array}$ & $\begin{array}{c}\mathbf{W}_{\mathbf{q}} \\
{[\boldsymbol{\mu m}]}\end{array}$ & $\begin{array}{c}\mathbf{R}_{\mathbf{t}} \\
{[\boldsymbol{\mu m}]}\end{array}$ & $\begin{array}{c}\mathbf{R}_{\mathbf{z}} \\
{[\boldsymbol{\mu m}]}\end{array}$ & $\begin{array}{c}\mathbf{R}_{\mathbf{a}} \\
{[\boldsymbol{\mu m}]}\end{array}$ & $\begin{array}{c}\mathbf{R}_{\mathbf{q}} \\
{[\boldsymbol{\mu m}]}\end{array}$ \\
\hline 1 & 16,00 & 8,77 & 2,55 & 2,92 & 5,88 & 1,78 & 1,52 & 1,76 & 10,82 & 7,97 & 1,17 & 1,51 \\
\hline 2 & 11,42 & 7,93 & 1,97 & 2,19 & 2,92 & 0,98 & 0,93 & 0,98 & 9,29 & 7,72 & 1,29 & 1,60 \\
\hline 3 & 9,40 & 7,07 & 1,63 & 1,77 & 2,51 & 1,02 & 0,51 & 0,63 & 8,32 & 6,72 & 1,13 & 1,36 \\
\hline 4 & 17,29 & 11,16 & 1,62 & 1,93 & 2,16 & 0,85 & 0,43 & 0,56 & 16,40 & 10,75 & 1,27 & 1,65 \\
\hline 5 & 24,46 & 9,68 & 5,84 & 2,92 & 13,94 & 4,52 & 1,52 & 4,46 & 9,34 & 7,03 & 1,13 & 1,39 \\
\hline 6 & 21,07 & 10,87 & 4,89 & 2,19 & 11,34 & 3,62 & 0,93 & 3,57 & 14,14 & 9,82 & 1,14 & 1,57 \\
\hline 7 & 13,36 & 9,10 & 1,67 & 1,77 & 3,19 & 1,22 & 0,51 & 0,91 & 10,16 & 8,84 & 0,96 & 1,38 \\
\hline 8 & 15,83 & 11,18 & 3,01 & 1,93 & 7,01 & 2,65 & 0,43 & 2,06 & 10,17 & 9,63 & 1,59 & 2,03 \\
\hline 9 & 22,42 & 15,63 & 3,98 & 4,36 & 5,40 & 2,15 & 1,43 & 1,63 & 20,19 & 14,95 & 2,72 & 3,32 \\
\hline 10 & 10,83 & 6,80 & 1,46 & 1,64 & 3,19 & 1,08 & 0,80 & 0,91 & 8,84 & 6,33 & 0,85 & 1,10 \\
\hline 11 & 9,01 & 6,93 & 1,62 & 1,83 & 2,86 & 0,85 & 0,63 & 0,71 & 8,58 & 6,97 & 1,05 & 1,30 \\
\hline 12 & 27,60 & 14,33 & 2,52 & 3,13 & 3,71 & 2,01 & 0,82 & 1,00 & 21,98 & 13,31 & 1,80 & 2,40 \\
\hline 13 & 10,78 & 6,18 & 1,75 & 2,00 & 3,49 & 1,21 & 0,77 & 0,94 & 6,98 & 5,67 & 0,98 & 1,20 \\
\hline 14 & 18,96 & 10,87 & 1,66 & 2,01 & 2,99 & 1,02 & 0,68 & 0,81 & 16,91 & 10,66 & 1,23 & 1,65 \\
\hline 15 & 9,94 & 6,10 & 2,18 & 2,35 & 4,53 & 1,39 & 1,46 & 1,59 & 6,68 & 5,63 & 0,98 & 1,20 \\
\hline 16 & 24,15 & 18,28 & 3,52 & 4,14 & 6,27 & 3,31 & 1,31 & 1,59 & 19,79 & 16,06 & 2,39 & 3,21 \\
\hline \hline $\begin{array}{c}\text { Mittel- } \\
\text { wert }\end{array}$ & $\mathbf{1 6 , 4 1}$ & $\mathbf{1 0 , 0 6}$ & $\mathbf{2 , 6 2}$ & $\mathbf{2 , 4 4}$ & $\mathbf{5 , 0 9}$ & $\mathbf{1 , 8 5}$ & $\mathbf{0 , 9 2}$ & $\mathbf{1 , 5 1}$ & $\mathbf{1 2 , 4 1}$ & $\mathbf{9 , 2 5}$ & $\mathbf{1 , 3 5}$ & $\mathbf{1 , 7 4}$ \\
\hline \hline
\end{tabular}




\section{Anhang D Algorithmus zur Auswertung der lokalen Nahtgeomet- rieparameter}

Der angegebene Algorithmus wurde aus Zeine El Dine [Zeine El Dine, 2007] entnommen, wobei Parameter wie beispielsweise die Anzahl der Messpunkte des Nachlaufs an die vorliegende Situation angepasst wurden.

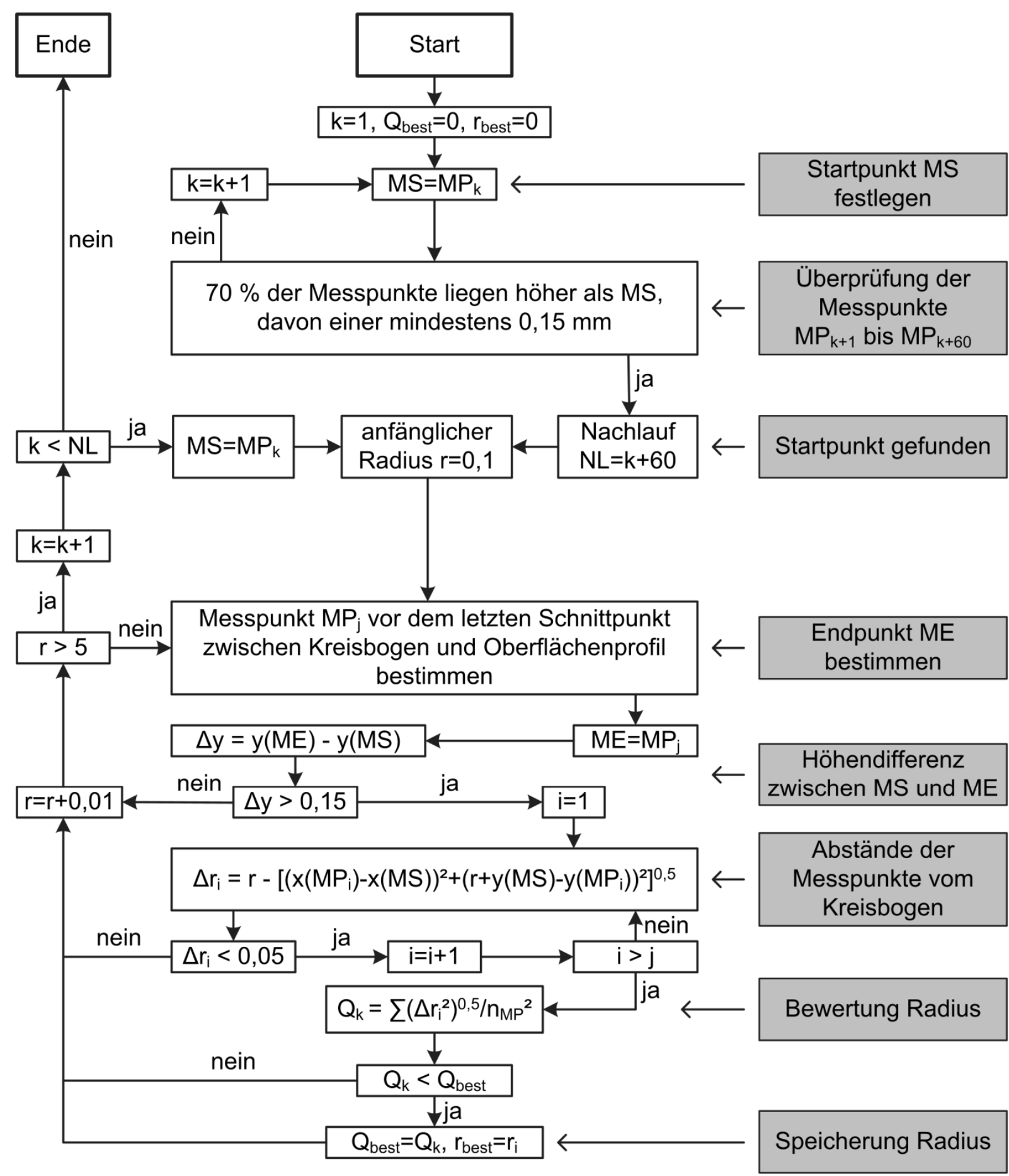




\section{Anhang E Lokalen Schweißnahtgeometrie}

\section{Anhang E.1 Einzelergebnisse der lokalen Nahtgeometrieparameter}

\begin{tabular}{|c|c|c|c|c|c|c|c|c|c|c|c|}
\hline \multicolumn{2}{|c|}{$\begin{array}{c}\text { Probe } \\
\text { Nr. }\end{array}$} & $\begin{array}{c}\mathbf{b}_{\mathrm{N}} \\
{[\mathrm{mm}]}\end{array}$ & $\begin{array}{c}\mathbf{b}_{\mathrm{w}} \\
{[\mathrm{mm}]}\end{array}$ & $\begin{array}{c}\mathrm{h}_{\mathrm{N}} \\
{[\mathrm{mm}]}\end{array}$ & $\begin{array}{c}\mathbf{h}_{\mathrm{w}} \\
{[\mathrm{mm}]}\end{array}$ & $\begin{array}{c}\mathbf{t}_{\mathrm{v}} \\
{[\mathrm{mm}]}\end{array}$ & $\begin{array}{l}\rho_{v} \\
{\left[{ }^{\circ}\right]}\end{array}$ & $\begin{array}{c}\mathrm{r}_{\mathrm{N}} \\
{[\mathrm{mm}]}\end{array}$ & $\begin{array}{c}r_{w} \\
{[\mathrm{~mm}]}\end{array}$ & $\begin{array}{l}\Theta_{\mathrm{N}} \\
{\left[^{\circ}\right]}\end{array}$ & $\begin{array}{l}\Theta_{w} \\
{\left[^{\circ}\right]}\end{array}$ \\
\hline \multirow{2}{*}{$A-1$} & links & \multirow{2}{*}{10,00} & \multirow{2}{*}{4,01} & \multirow{2}{*}{1,13} & \multirow{2}{*}{0,92} & \multirow{2}{*}{0,04} & \multirow{2}{*}{0,76} & 3,77 & 1,04 & 19,98 & 48,84 \\
\hline & rechts & & & & & & & 2,20 & 0,43 & 27,81 & 49,00 \\
\hline \multirow{2}{*}{ A-2 } & links & \multirow{2}{*}{10,75} & \multirow{2}{*}{4,37} & \multirow{2}{*}{1,07} & \multirow{2}{*}{0,89} & \multirow{2}{*}{0,13} & \multirow{2}{*}{0,78} & 3,96 & 0,95 & 19,21 & 26,07 \\
\hline & rechts & & & & & & & 1,64 & 1,71 & 25,37 & 23,25 \\
\hline \multirow{2}{*}{ A-3 } & links & \multirow{2}{*}{10,42} & \multirow{2}{*}{4,17} & \multirow{2}{*}{0,99} & \multirow{2}{*}{0,88} & \multirow{2}{*}{0,06} & \multirow{2}{*}{0,77} & 3,61 & 1,16 & 25,96 & 35,36 \\
\hline & rechts & & & & & & & 3,46 & 1,30 & 25,70 & 39,63 \\
\hline \multirow{2}{*}{ B-1 } & links & \multirow{2}{*}{9,45} & \multirow{2}{*}{3,97} & & & & & 3,05 & 0,62 & 22,85 & 33,38 \\
\hline & rechts & & & 1,14 & 0,85 & 0,22 & 0,61 & 3,71 & 1,40 & 24,45 & 25,38 \\
\hline & links & & & & & & & 3,11 & 1,52 & 20,57 & 27,30 \\
\hline B-2 & rechts & $y, 51$ & 4,50 & 0,89 & $0,8 /$ & $0, Z 1$ & 0,62 & 3,25 & 1,81 & 23,10 & 31,48 \\
\hline $\mathrm{R}-3$ & links & & 381 & & & & 066 & 2,03 & 1,93 & 28,81 & 30,84 \\
\hline B-3 & rechts & 9,02 & 3,81 & $1,0 /$ & 0,90 & 0,19 & 0,06 & 3,12 & 1,02 & 23,05 & 34,64 \\
\hline & links & & & & & & & 2,47 & 0,46 & 27,01 & 48,95 \\
\hline$C-1$ & rechts & 8,80 & 3,73 & 1,07 & 1,01 & 0,01 & 0,14 & 2,57 & 0,91 & 32,31 & 55,15 \\
\hline & links & & & & & & & 3,63 & 0,91 & 22,89 & 41,21 \\
\hline$C-2$ & rechts & 9,16 & 4,44 & 1,07 & 1,05 & 0,02 & 0,81 & 3,66 & 1,19 & 22,99 & 39,07 \\
\hline$C-3$ & links & 962 & 461 & & & & 087 & 3,48 & 1,09 & 21,62 & 40,58 \\
\hline$C-3$ & rechts & 9,62 & 4,61 & 1,00 & 1,20 & 0,00 & 0,81 & 1,33 & 1,18 & 38,95 & 22,92 \\
\hline $\mathrm{n}_{-1}$ & links & 823 & 17 & 075 & & 008 & 067 & 2,18 & 0,50 & 28,49 & 50,00 \\
\hline D-1 & rechts & 8,23 & 4,17 & 0,15 & 1,21 & 0,08 & $0,6 /$ & 3,62 & 0,75 & 24,64 & 52,96 \\
\hline ?-1 & links & 827 & 132 & 088 & 112 & 0.05 & 077 & 1,16 & 1,06 & 39,24 & 29,94 \\
\hline U-2 & rechts & 8,21 & 4,32 & 0,ठర & 1,13 & 0,05 & 0,17 & 2,97 & 1,09 & 23,18 & 36,50 \\
\hline & links & & & & & & & 1,99 & 0,78 & 20,50 & 50,42 \\
\hline$D-3$ & rechts & 8,29 & 4,32 & $0,7 /$ & 1,07 & 0,05 & 0,60 & 0,87 & 1,10 & 32,39 & 24,15 \\
\hline & links & & & & & & & 1,47 & 0,71 & 44,97 & 32,84 \\
\hline$E-1$ & rechts & 9,55 & 3,46 & 1,21 & 0,61 & 0,15 & 0,25 & 2,14 & 2,74 & 19,12 & 15,57 \\
\hline E-2 & links & & & 131 & & & & 2,04 & 1,03 & 42,02 & 26,41 \\
\hline$E-2$ & rechts & 9,58 & 3,32 & 1,34 & 0,62 & 0,14 & 0,27 & 2,59 & 2,29 & 30,74 & 29,42 \\
\hline$F_{-} 3$ & links & 942 & 310 & 146 & & & 032 & 3,51 & 1,24 & 28,05 & 24,39 \\
\hline$E-3$ & rechts & 9,42 & 3,10 & 1,46 & 0,45 & 0,15 & 0,32 & 1,27 & 0,56 & 38,43 & 35,87 \\
\hline$F_{-1}$ & links & $100 ?$ & 437 & 121 & 077 & רחת ח & 070 & 0,86 & 2,49 & 28,21 & 19,31 \\
\hline$r-1$ & rechts & 10,02 & 4,31 & 1,21 & $0, \pi 7$ & 0,02 & 0,10 & 3,45 & 1,02 & 27,96 & 42,23 \\
\hline$F_{-} 2$ & links & 1057 & 468 & 128 & 079 & 005 & (9 0 & 2,59 & 1,31 & 29,11 & 39,45 \\
\hline $1-2$ & rechts & 10,51 & 4,68 & 1,28 & 0,19 & 0,05 & 0,90 & 1,63 & 2,24 & 33,95 & 17,11 \\
\hline$F_{-}-3$ & links & 1042 & 368 & 109 & 073 & 0.07 & 077 & 4,19 & 0,64 & 21,41 & 32,47 \\
\hline$r-3$ & rechts & 10,42 & 0,00 & 1,09 & 0,10 & 0,0 & 0,17 & 2,85 & 0,80 & 30,76 & 42,70 \\
\hline
\end{tabular}




\begin{tabular}{|c|c|c|c|c|c|c|c|c|c|c|c|}
\hline \multicolumn{2}{|c|}{$\begin{array}{c}\text { Probe } \\
\mathrm{Nr} .\end{array}$} & $\begin{array}{c}\mathbf{b}_{\mathrm{N}} \\
{[\mathrm{mm}]}\end{array}$ & $\begin{array}{c}\mathbf{b}_{\mathrm{w}} \\
{[\mathrm{mm}]}\end{array}$ & $\begin{array}{c}\mathrm{h}_{\mathrm{N}} \\
{[\mathrm{mm}]}\end{array}$ & $\begin{array}{c}h_{w} \\
{[\mathrm{~mm}]}\end{array}$ & $\begin{array}{c}\mathbf{t}_{\mathrm{v}} \\
{[\mathrm{mm}]}\end{array}$ & $\begin{array}{l}\rho_{v} \\
{\left[{ }^{\circ}\right]}\end{array}$ & $\begin{array}{c}\mathbf{r}_{\mathrm{N}} \\
{[\mathrm{mm}]}\end{array}$ & $\begin{array}{c}r_{\mathrm{w}} \\
{[\mathrm{mm}]}\end{array}$ & $\begin{array}{l}\Theta_{N} \\
{\left[{ }^{\circ}\right]}\end{array}$ & $\begin{array}{l}\Theta_{w} \\
{\left[{ }^{\circ}\right]}\end{array}$ \\
\hline \multirow{2}{*}{ G-1 } & links & \multirow{2}{*}{10,57} & \multirow{2}{*}{5,46} & \multirow{2}{*}{1,01} & \multirow{2}{*}{1,27} & \multirow{2}{*}{0,11} & \multirow{2}{*}{0,58} & 3,67 & 2,28 & 20,95 & 29,57 \\
\hline & rechts & & & & & & & 4,03 & 1,04 & 18,91 & 27,91 \\
\hline \multirow{2}{*}{ G-2 } & links & \multirow{2}{*}{10,88} & \multirow{2}{*}{4,63} & \multirow{2}{*}{0,86} & \multirow{2}{*}{1,24} & \multirow{2}{*}{0,13} & \multirow{2}{*}{0,52} & 4,47 & 0,94 & 17,18 & 39,64 \\
\hline & rechts & & & & & & & 4,31 & 0,45 & 16,66 & 58,50 \\
\hline \multirow{2}{*}{ G-3 } & links & \multirow{2}{*}{10,78} & \multirow{2}{*}{4,79} & \multirow{2}{*}{0,89} & \multirow{2}{*}{1,36} & \multirow{2}{*}{0,11} & \multirow{2}{*}{0,61} & 4,44 & 0,58 & 17,81 & 38,76 \\
\hline & rechts & & & & & & & 4,53 & 1,18 & 18,89 & 23,57 \\
\hline $\mathrm{H}_{1}$ & links & 1125 & 360 & 120 & 72 & 001 & 057 & 2,48 & 1,53 & 28,32 & 33,01 \\
\hline$\pi-1$ & rechts & 11,30 & 3,00 & $1, \angle 0$ & 0,13 & 0,04 & 0,01 & 4,16 & 0,90 & 29,79 & 35,66 \\
\hline H? & links & 1100 & 172 & 121 & 71 & כחת ח & 011 & 1,67 & 1,33 & 38,03 & 39,04 \\
\hline$\pi-2$ & rechts & 11,90 & 4,12 & 1,31 & 0,14 & 0,02 & 0,41 & 4,30 & 2,80 & 18,78 & 27,39 \\
\hline $\mathrm{H}_{3}$ & links & 1100 & 381 & 121 & 55 & 10 & 071 & 3,64 & 1,33 & 26,08 & 29,31 \\
\hline ח-3 & rechts & (1,09 & 0,01 & 1,01 & 0,55 & 0,10 & 0,71 & 1,67 & 1,16 & 35,05 & 29,62 \\
\hline 1 & links & 967 & 399 & 141 & 092 & 08 & 077 & 2,32 & 0,49 & 31,90 & 38,85 \\
\hline $1-1$ & rechts & $9,6 /$ & 3,99 & 1,41 & 0,92 & 0,08 & $0, \pi 7$ & 0,79 & 0,84 & 48,09 & 28,22 \\
\hline $1-2$ & links & 1016 & 414 & 132 & 0.93 & $0 \cap 7$ & 064 & 1,72 & 0,82 & 31,79 & 34,85 \\
\hline $1-2$ & rechts & 10,10 & 4,14 & & & $0,0 /$ & 0,64 & 1,74 & 1,15 & 37,00 & 24,10 \\
\hline $1-3$ & links & 1075 & 372 & 152 & 0.87 & 006 & 074 & 3,45 & 0,62 & 26,28 & 35,68 \\
\hline & rechts & & & & & & 0,14 & 1,37 & 0,76 & 43,35 & 52,31 \\
\hline & links & & & & & & & 0,91 & 1,48 & 33,15 & 33,58 \\
\hline$J-1$ & rechts & 10,49 & 4,81 & 1,03 & 0,80 & 0,25 & 0,31 & 4,25 & 2,06 & 23,95 & 18,26 \\
\hline & links & & & & & & & 1,54 & 0,47 & 25,52 & 69,35 \\
\hline $\mathrm{J}-2$ & rechts & 10,51 & 4,10 & 1,09 & 1,03 & 0,22 & 0,21 & 3,15 & 0,91 & 24,53 & 32,62 \\
\hline & links & & & & & & & 1,04 & 1,04 & 35,64 & 35,19 \\
\hline$J-3$ & rechts & 9,93 & 4,17 & 0,96 & 0,19 & 0,25 & 0,30 & 0,95 & 0,63 & 45,22 & 51,82 \\
\hline & links & & & & & & & 2,20 & 1,31 & 20,13 & 40,57 \\
\hline K-1 & rechts & 9,22 & $4, / 7$ & 1,26 & 1,01 & 0,31 & 0,42 & 1,73 & 2,30 & 41,43 & 27,23 \\
\hline$K=2$ & links & & & & & & & 1,51 & 0,58 & 32,26 & 41,00 \\
\hline$K-2$ & rechts & 9,60 & 4,10 & 1,06 & $1,1 /$ & 0,36 & 0,32 & 3,88 & 2,05 & 29,37 & 18,29 \\
\hline$K 3$ & links & & 141 & 103 & 111 & 035 & 012 & 3,46 & 0,41 & 23,48 & 41,61 \\
\hline K-3 & rechts & 9,86 & 4,44 & 1,03 & 1,11 & 0,35 & 0,42 & 2,60 & 2,19 & 33,96 & 18,24 \\
\hline $1-1$ & links & 949 & 404 & 125 & 075 & 009 & 042 & 2,92 & 1,55 & 21,32 & 32,45 \\
\hline$L-1$ & rechts & 9,49 & 4,04 & 1,25 & 0,15 & 0,09 & 0,42 & 0,95 & 0,72 & 42,35 & 47,30 \\
\hline $1-2$ & links & 962 & 205 & 122 & 040 & $\cap \cap 9$ & 050 & 1,59 & 1,57 & 34,97 & 29,72 \\
\hline L-2 & rechts & 9,62 & 2,95 & 1,22 & 0,40 & 0,09 & 0,50 & 3,72 & 0,76 & 31,31 & 30,15 \\
\hline $1-3$ & links & 100 & 333 & 149 & 063 & 0.7 & 050 & 3,00 & 0,94 & 34,22 & 36,73 \\
\hline L-3 & rechts & 10,09 & 3,33 & 1,49 & 0,03 & 0,07 & 0,50 & 2,29 & 1,00 & 17,72 & 31,87 \\
\hline 1 & links & 1001 & ? 10 & 100 & & & & 3,63 & 0,90 & 22,24 & 33,05 \\
\hline$|M|-1$ & rechts & 10,84 & 4,23 & 1,08 & 0,99 & 0,08 & 0,40 & 4,66 & 0,84 & 14,04 & 49,15 \\
\hline$M-2$ & links & 1067 & 406 & 106 & 078 & 006 & 050 & 3,03 & 1,19 & 18,50 & 35,46 \\
\hline $\mathrm{IV}-\mathrm{L}$ & rechts & 10,61 & 4,Ub & 1,Ub & 0,18 & 0,06 & U,50 & 0,88 & 1,35 & 27,65 & 28,84 \\
\hline & links & 1027 & 115 & 000 & 001 & ת06 & 051 & 2,75 & 1,55 & 24,91 & 27,03 \\
\hline $\mathrm{IV}-3$ & rechts & 10,31 & 4,15 & 0,99 & 0,81 & 0,06 & 0,54 & 4,18 & 1,13 & 22,51 & 28,73 \\
\hline
\end{tabular}




\begin{tabular}{|c|c|c|c|c|c|c|c|c|c|c|c|}
\hline \multicolumn{2}{|c|}{$\begin{array}{c}\text { Probe } \\
\text { Nr. }\end{array}$} & $\begin{array}{c}b_{\mathrm{N}} \\
{[\mathrm{mm}]}\end{array}$ & $\begin{array}{c}\mathbf{b}_{\mathrm{w}} \\
{[\mathrm{mm}]}\end{array}$ & $\begin{array}{c}\mathbf{h}_{\mathrm{N}} \\
{[\mathrm{mm}]}\end{array}$ & $\begin{array}{c}\mathrm{h}_{\mathrm{W}} \\
{[\mathrm{mm}]}\end{array}$ & $\begin{array}{c}\mathbf{t}_{\mathrm{V}} \\
{[\mathrm{mm}]}\end{array}$ & $\begin{array}{l}\rho_{v} \\
{\left[{ }^{\circ}\right]}\end{array}$ & $\begin{array}{c}\mathbf{r}_{\mathrm{N}} \\
{[\mathrm{mm}]}\end{array}$ & $\begin{array}{c}r_{w} \\
{[\mathrm{~mm}]}\end{array}$ & $\begin{array}{l}\Theta_{\mathrm{N}} \\
{\left[{ }^{\circ}\right]}\end{array}$ & $\begin{array}{l}\Theta_{w} \\
{\left[{ }^{\circ}\right]}\end{array}$ \\
\hline \multirow{2}{*}{$\mathrm{N}-1$} & links & \multirow{2}{*}{9,95} & \multirow{2}{*}{4,08} & \multirow{2}{*}{1,12} & \multirow{2}{*}{0,84} & \multirow{2}{*}{0,15} & \multirow{2}{*}{0,76} & 1,35 & 0,75 & 29,64 & 50,68 \\
\hline & rechts & & & & & & & 3,39 & 1,26 & 30,47 & 43,54 \\
\hline \multirow{2}{*}{$\mathrm{N}-2$} & links & \multirow{2}{*}{10,20} & \multirow{2}{*}{4,68} & \multirow{2}{*}{1,24} & \multirow{2}{*}{1,01} & \multirow{2}{*}{0,11} & \multirow{2}{*}{0,78} & 4,96 & 1,66 & 17,23 & 29,20 \\
\hline & rechts & & & & & & & 1,68 & 1,15 & 35,47 & 46,57 \\
\hline \multirow{2}{*}{$\mathrm{N}-3$} & links & \multirow{2}{*}{9,73} & \multirow{2}{*}{4,59} & \multirow{2}{*}{0,91} & \multirow{2}{*}{1,19} & \multirow{2}{*}{0,09} & \multirow{2}{*}{0,75} & 3,99 & 1,04 & 17,00 & 45,58 \\
\hline & rechts & & & & & & & 2,14 & 0,53 & 22,28 & 48,55 \\
\hline & links & & & & & & & 1,47 & 0,72 & 21,66 & 51,25 \\
\hline $0-1$ & rechts & 10,60 & 4,92 & 0,81 & 1,40 & 0,08 & U,88 & 4,16 & 1,21 & 15,45 & 24,55 \\
\hline & links & & & & & & & 2,33 & 1,35 & 18,97 & 37,24 \\
\hline $0-2$ & rechts & 9,02 & 5,51 & 0,11 & 1,35 & 0,0ठ & 0,80 & 3,60 & 1,55 & 15,81 & 22,60 \\
\hline & links & & & & & & & 4,54 & 0,43 & 18,40 & 49,13 \\
\hline $0-3$ & rechts & 11,29 & 5,06 & 0,10 & 1,44 & 0,10 & 0,84 & 3,11 & 1,01 & 16,79 & 52,37 \\
\hline & links & & & & & & & 1,42 & 0,71 & 33,77 & 39,83 \\
\hline$P-1$ & rechts & 11,55 & 4,57 & 0,98 & 0,95 & 0,00 & 0,39 & 3,06 & 1,43 & 26,64 & 25,16 \\
\hline P_2 & links & & & & & & & 3,42 & 0,71 & 27,16 & 34,01 \\
\hline$P-2$ & rechts & 10,40 & $4,5 /$ & 1,01 & 0,91 & 0,01 & 0,63 & 3,11 & 1,41 & 28,11 & 38,62 \\
\hline$P_{3} 3$ & links & & & & & & & 3,85 & 1,13 & 16,74 & 29,86 \\
\hline$P-3$ & rechts & 11,48 & 4,85 & 1,10 & 0,99 & 0,04 & 0,48 & 2,19 & 1,04 & 27,01 & 31,95 \\
\hline $0_{1}$ & links & & & & & & 083 & 1,88 & 0,29 & 35,51 & 54,92 \\
\hline Q-1 & rechts & 10,91 & 2,13 & 1,45 & 0,55 & 0,11 & 0,83 & 3,49 & 1,07 & 29,28 & 25,93 \\
\hline & links & & & & & & & 1,46 & 1,06 & 41,03 & 43,02 \\
\hline$Q-2$ & rechts & 10,46 & 3,68 & 1,59 & 0,63 & 0,14 & 0,81 & 1,27 & 1,08 & 28,79 & 35,73 \\
\hline & links & & & & & & & 1,87 & 1,01 & 29,71 & 26,40 \\
\hline$Q-3$ & rechts & 11,24 & 3,22 & $1, / 2$ & 0,40 & 0,17 & 0,95 & 4,15 & 1,15 & 28,40 & 32,07 \\
\hline & links & & & & & & & 3,82 & 2,56 & 20,95 & 22,34 \\
\hline R-T & rechts & $11, / 1$ & 3,88 & 1,36 & 0,51 & 0,08 & 0,83 & 3,02 & 2,01 & 27,05 & 26,29 \\
\hline R-2 & links & 1055 & & & & & & 1,52 & 1,51 & 39,79 & 30,95 \\
\hline R-2 & rechts & 10,55 & 3,64 & 1,44 & 0,47 & 0,03 & 0,81 & 1,87 & 1,66 & 35,68 & 27,62 \\
\hline R_3 & links & & & & & & & 1,13 & 0,98 & 31,31 & 38,86 \\
\hline R-3 & rechts & 11,11 & 4,12 & 1,41 & & 0,00 & 0,65 & 1,25 & 3,10 & 34,11 & 15,95 \\
\hline$S_{-1}$ & links & 1111 & & 124 & & & & 0,53 & 1,26 & 50,08 & 38,45 \\
\hline$s-1$ & rechts & 11,11 & 4,92 & 1,24 & 0,90 & 0,02 & 1,30 & 3,44 & 2,50 & 25,26 & 17,49 \\
\hline S-2 & links & 1117 & 483 & 124 & 100 & 0,04 & 126 & 0,53 & 1,35 & 69,97 & 44,67 \\
\hline $3-2$ & rechts & 71,17 & 4,83 & $1, \angle 4$ & 1,09 & 0,04 & $1, \angle 0$ & 3,81 & 0,72 & 26,60 & 36,55 \\
\hline$S-3$ & links & 1108 & 430 & 122 & 100 & 0 : & 132 & 2,69 & 0,79 & 30,65 & 42,72 \\
\hline $3-3$ & rechts & 11,08 & 4,30 & $1, \angle 2$ & 1,00 & 0,01 & 1,32 & 0,99 & 1,26 & 46,69 & 28,48 \\
\hline T-1 & links & 1204 & 423 & 130 & 095 & 028 & 122 & 2,22 & 1,02 & 29,99 & 40,84 \\
\hline $1-1$ & rechts & 12,04 & $4, \angle 3$ & 1,30 & 0,90 & $0, \angle 8$ & $1, \angle 2$ & 4,09 & 1,36 & 23,72 & 26,29 \\
\hline T-2 & links & 1133 & 383 & 126 & 074 & 029 & 109 & 3,13 & 0,68 & 28,11 & 35,67 \\
\hline $1-2$ & rechts & 11,00 & 3,00 & $1, \angle 0$ & 0,14 & $0, \angle Y$ & 1,09 & 3,59 & 0,91 & 21,27 & 36,06 \\
\hline T-3 & links & 1175 & 414 & 129 & 083 & 025 & & 1,88 & 0,73 & 21,39 & 48,33 \\
\hline $1-3$ & rechts & 11,10 & 4,14 & $1, \angle Y$ & 0,83 & 0,25 & 1,04 & 1,86 & 1,14 & 33,43 & 43,89 \\
\hline
\end{tabular}


Anhang E.2 Einzelergebnisse der ermittelten Kerbformzahlen $K_{t}$

\begin{tabular}{|c|c|c|c|c|c|c|c|}
\hline \multicolumn{2}{|c|}{$\begin{array}{c}\text { Probe } \\
\mathrm{Nr} .\end{array}$} & \multirow{2}{*}{ 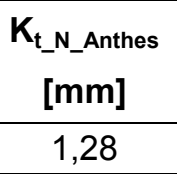 } & \multirow{2}{*}{ 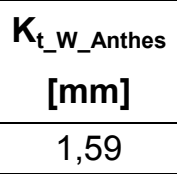 } & \multirow{2}{*}{ 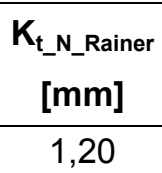 } & \multirow{2}{*}{ 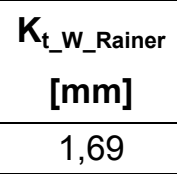 } & \multirow{2}{*}{$\begin{array}{c}\mathbf{K}_{\mathbf{t} \_ \text {N_FEM }} \\
\left.\text { [ }^{\circ}\right]\end{array}$} & \multirow{2}{*}{ 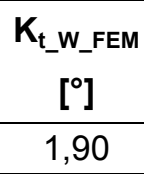 } \\
\hline & links & & & & & & \\
\hline$A-1$ & rechts & 1,39 & 1,93 & 1,37 & 2,23 & 1,30 & 2,41 \\
\hline \multirow{2}{*}{$A-2$} & links & 1,27 & 1,52 & 1,19 & 1,73 & 1,19 & 1,92 \\
\hline & rechts & 1,42 & 1,40 & 1,48 & 1,45 & 1,36 & 1,74 \\
\hline \multirow{2}{*}{ A-3 } & links & 1,31 & 1,54 & 1,21 & 1,63 & 1,20 & 1,85 \\
\hline & rechts & 1,32 & 1,53 & 1,22 & 1,57 & 1,21 & 1,81 \\
\hline \multirow{2}{*}{ B-1 } & links & 1,32 & 1,69 & 1,26 & 1,97 & 1,24 & 2,10 \\
\hline & rechts & 1,31 & 1,45 & 1,20 & 1,54 & 1,20 & 1,76 \\
\hline \multirow{2}{*}{ B-2 } & links & 1,31 & 1,45 & 1,25 & 1,50 & 1,23 & 1,70 \\
\hline & rechts & 1,32 & 1,44 & 1,24 & 1,43 & 1,22 & 1,65 \\
\hline \multirow{2}{*}{ B-3 } & links & 1,41 & 1,42 & 1,39 & 1,41 & 1,32 & 1,65 \\
\hline & rechts & 1,32 & 1,57 & 1,25 & 1,69 & 1,23 & 1,84 \\
\hline \multirow{2}{*}{$C-1$} & links & 1,37 & 1,90 & 1,32 & 2,21 & 1,28 & 2,29 \\
\hline & rechts & 1,38 & 1,64 & 1,31 & 1,77 & 1,28 & 1,93 \\
\hline \multirow{2}{*}{$C-2$} & links & 1,30 & 1,62 & 1,21 & 1,78 & 1,21 & 1,95 \\
\hline & rechts & 1,30 & 1,55 & 1,21 & 1,63 & 1,20 & 1,84 \\
\hline \multirow{2}{*}{$C-3$} & links & 1,30 & 1,57 & 1,22 & 1,69 & 1,21 & 1,84 \\
\hline & rechts & 1,52 & 1,45 & 1,57 & 1,65 & 1,45 & 1,86 \\
\hline \multirow{2}{*}{ D-1 } & links & 1,39 & 1,86 & 1,35 & 2,19 & 1,32 & 2,20 \\
\hline & rechts & 1,31 & 1,71 & 1,21 & 1,91 & 1,22 & 1,95 \\
\hline \multirow{2}{*}{ D-2 } & links & 1,55 & 1,53 & 1,63 & 1,70 & 1,50 & 1,85 \\
\hline & rechts & 1,33 & 1,56 & 1,26 & 1,69 & 1,25 & 1,79 \\
\hline \multirow{2}{*}{ D-3 } & links & 1,36 & 1,69 & 1,39 & 1,87 & 1,32 & 1,94 \\
\hline & rechts & 1,60 & 1,48 & 1,75 & 1,68 & 1,57 & 1,81 \\
\hline \multirow{2}{*}{$E-1$} & links & 1,50 & 1,65 & 1,54 & 1,82 & 1,42 & 2,03 \\
\hline & rechts & 1,34 & 1,28 & 1,38 & 1,27 & 1,29 & 1,57 \\
\hline \multirow{2}{*}{ E-2 } & links & 1,42 & 1,51 & 1,40 & 1,64 & 1,33 & 1,86 \\
\hline & rechts & 1,37 & 1,39 & 1,31 & 1,33 & 1,27 & 1,61 \\
\hline \multirow{2}{*}{ E-3 } & links & 1,32 & 1,46 & 1,22 & 1,52 & 1,21 & 1,77 \\
\hline & rechts & 1,53 & 1,74 & 1,63 & 1,87 & 1,46 & 2,12 \\
\hline \multirow{2}{*}{$F-1$} & links & 1,57 & 1,32 & 1,83 & 1,31 & 1,56 & 1,64 \\
\hline & rechts & 1,32 & 1,59 & 1,22 & 1,67 & 1,21 & 1,90 \\
\hline \multirow{2}{*}{$\mathrm{F}-2$} & links & 1,37 & 1,52 & 1,31 & 1,56 & 1,27 & 1,85 \\
\hline & rechts & 1,46 & 1,31 & 1,49 & 1,35 & 1,38 & 1,68 \\
\hline \multirow{2}{*}{$F-3$} & links & 1,28 & 1,68 & 1,18 & 1,91 & 1,18 & 2,09 \\
\hline & rechts & 1,36 & 1,67 & 1,28 & 1,79 & 1,25 & 2,03 \\
\hline
\end{tabular}




\begin{tabular}{|c|c|c|c|c|c|c|c|}
\hline \multicolumn{2}{|c|}{$\begin{array}{c}\text { Probe } \\
\text { Nr. }\end{array}$} & \multirow{2}{*}{$\begin{array}{c}\mathbf{K}_{\mathrm{t} \_\mathrm{N} \_ \text {Anthes }} \\
\text { [mm] }\end{array}$} & \multirow{2}{*}{$\begin{array}{c}\mathbf{K}_{\mathbf{t} \_\mathbf{W} \_ \text {Anthes }} \\
\text { [mm] }\end{array}$} & \multirow{2}{*}{ 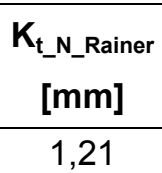 } & \multirow{2}{*}{ 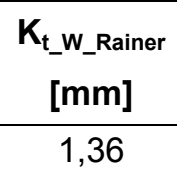 } & \multirow{2}{*}{$\begin{array}{c}\mathbf{K}_{\mathbf{t} \_ \text {N_FEM }} \\
\left.\text { [ }^{\circ}\right]\end{array}$} & \multirow{2}{*}{ 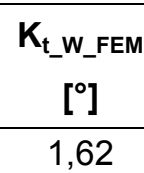 } \\
\hline$C_{1}$ & links & & & & & & \\
\hline G-1 & rechts & 1,27 & 1,52 & 1,18 & 1,72 & 1,19 & 1,88 \\
\hline \multirow{2}{*}{ G-2 } & links & 1,25 & 1,61 & 1,16 & 1,78 & 1,17 & 1,94 \\
\hline & rechts & 1,25 & 1,94 & 1,17 & 2,28 & 1,17 & 2,37 \\
\hline \multirow{2}{*}{ G-3 } & links & 1,25 & 1,75 & 1,16 & 2,11 & 1,17 & 2,19 \\
\hline & rechts & 1,26 & 1,46 & 1,16 & 1,66 & 1,17 & 1,84 \\
\hline \multirow{2}{*}{$\mathrm{H}-1$} & links & 1,37 & 1,47 & 1,33 & 1,49 & 1,27 & 1,79 \\
\hline & rechts & 1,30 & 1,61 & 1,18 & 1,73 & 1,18 & 1,98 \\
\hline \multirow{2}{*}{$\mathrm{H}-2$} & links & 1,47 & 1,52 & 1,48 & 1,55 & 1,37 & 1,90 \\
\hline & rechts & 1,26 & 1,35 & 1,17 & 1,27 & 1,17 & 1,61 \\
\hline \multirow{2}{*}{$\mathrm{H}-3$} & links & 1,31 & 1,48 & 1,21 & 1,51 & 1,20 & 1,81 \\
\hline & rechts & 1,46 & 1,51 & 1,48 & 1,57 & 1,37 & 1,89 \\
\hline \multirow{2}{*}{$\mathrm{I}-1$} & links & 1,39 & 1,81 & 1,35 & 2,14 & 1,30 & 2,34 \\
\hline & rechts & 1,68 & 1,57 & 1,90 & 1,80 & 1,64 & 2,07 \\
\hline \multirow{2}{*}{$\mathrm{I}-2$} & links & 1,45 & 1,63 & 1,47 & 1,82 & 1,37 & 2,06 \\
\hline & rechts & 1,46 & 1,47 & 1,47 & 1,64 & 1,37 & 1,91 \\
\hline \multirow{2}{*}{$\mathrm{I}-3$} & links & 1,32 & 1,71 & 1,23 & 1,97 & 1,21 & 2,20 \\
\hline & rechts & 1,52 & 1,70 & 1,59 & 1,85 & 1,44 & 2,15 \\
\hline \multirow{2}{*}{$\mathrm{J}-1$} & links & 1,59 & 1,48 & 1,77 & 1,51 & 1,55 & 1,80 \\
\hline & rechts & 1,29 & 1,33 & 1,17 & 1,38 & 1,18 & 1,64 \\
\hline \multirow{2}{*}{$\mathrm{J}-2$} & links & 1,43 & 1,94 & 1,51 & 2,20 & 1,38 & 2,39 \\
\hline & rechts & 1,33 & 1,59 & 1,25 & 1,77 & 1,23 & 1,99 \\
\hline \multirow{2}{*}{$\mathrm{J}-3$} & links & 1,57 & 1,57 & 1,69 & 1,67 & 1,51 & 1,92 \\
\hline & rechts & 1,62 & 1,77 & 1,74 & 1,94 & 1,56 & 2,19 \\
\hline \multirow{2}{*}{$\mathrm{K}-1$} & links & 1,34 & 1,52 & 1,37 & 1,58 & 1,30 & 1,79 \\
\hline & rechts & 1,46 & 1,38 & 1,47 & 1,35 & 1,38 & 1,64 \\
\hline \multirow{2}{*}{$\mathrm{K}-2$} & links & 1,47 & 1,77 & 1,52 & 2,08 & 1,41 & 2,23 \\
\hline & rechts & 1,31 & 1,33 & 1,19 & 1,39 & 1,20 & 1,65 \\
\hline \multirow{2}{*}{$\mathrm{K}-3$} & links & 1,31 & 1,90 & 1,22 & 2,32 & 1,21 & 2,39 \\
\hline & rechts & 1,38 & 1,32 & 1,30 & 1,37 & 1,27 & 1,66 \\
\hline \multirow{2}{*}{$\mathrm{L}-1$} & links & 1,30 & 1,47 & 1,25 & 1,48 & 1,24 & 1,74 \\
\hline & rechts & 1,63 & 1,71 & 1,80 & 1,85 & 1,57 & 2,11 \\
\hline \multirow{2}{*}{ L-2 } & links & 1,47 & 1,46 & 1,50 & 1,42 & 1,39 & 1,70 \\
\hline & rechts & 1,32 & 1,61 & 1,20 & 1,69 & 1,20 & 1,91 \\
\hline \multirow{2}{*}{ L-3 } & links & 1,35 & 1,60 & 1,27 & 1,68 & 1,24 & 1,93 \\
\hline & rechts & 1,32 & 1,56 & 1,36 & 1,65 & 1,28 & 1,90 \\
\hline & links & 1,30 & 1,59 & 1,21 & 1,78 & 1,20 & 1,99 \\
\hline M-1 & rechts & 1,22 & 1,66 & 1,15 & 1,82 & 1,16 & 2,00 \\
\hline & links & 1,29 & 1,54 & 1,26 & 1,60 & 1,23 & 1,84 \\
\hline M-2 & rechts & 1,55 & 1,48 & 1,80 & 1,54 & 1,54 & 1,82 \\
\hline & links & 1,34 & 1,44 & 1,29 & 1,49 & 1,25 & 1,74 \\
\hline M-3 & rechts & 1,28 & 1,51 & 1,18 & 1,63 & 1,18 & 1,83 \\
\hline
\end{tabular}




\begin{tabular}{|c|c|c|c|c|c|c|c|}
\hline \multicolumn{2}{|c|}{$\begin{array}{c}\text { Probe } \\
\mathrm{Nr} .\end{array}$} & \multirow{2}{*}{$\begin{array}{c}\mathbf{K}_{\mathbf{t} \_\mathbf{N} \_A n t h e s} \\
\text { [mm] }\end{array}$} & \multirow{2}{*}{$\begin{array}{c}\mathbf{K}_{\mathbf{t} \_\mathbf{W} \_ \text {Anthes }} \\
\text { [mm] }\end{array}$} & \multirow{2}{*}{$\begin{array}{c}\begin{array}{c}\mathbf{K}_{\mathbf{t} \_N} \mathbf{N} \text { Rainer } \\
\text { [mm] }\end{array} \\
1,58\end{array}$} & \multirow{2}{*}{$\begin{array}{c}\begin{array}{c}\mathbf{K}_{\mathbf{t} \_\mathbf{W} \_ \text {Rainer }} \\
\text { [mm] }\end{array} \\
1,85\end{array}$} & \multirow{2}{*}{ 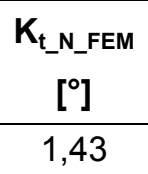 } & \multirow{2}{*}{$\begin{array}{c}\begin{array}{c}\mathrm{K}_{\mathrm{t} \_\mathrm{W}} \text { _FEM } \\
{\left[{ }^{\circ}\right]}\end{array} \\
2,09\end{array}$} \\
\hline & links & & & & & & \\
\hline$N-1$ & rechts & 1,33 & 1,54 & 1,23 & 1,58 & 1,22 & 1,83 \\
\hline \multirow{2}{*}{$\mathrm{N}-2$} & links & 1,24 & 1,44 & 1,14 & 1,47 & 1,15 & 1,71 \\
\hline & rechts & 1,46 & 1,56 & 1,48 & 1,65 & 1,38 & 1,92 \\
\hline \multirow{2}{*}{$\mathrm{N}-3$} & links & 1,26 & 1,59 & 1,19 & 1,72 & 1,19 & 1,87 \\
\hline & rechts & 1,36 & 1,83 & 1,37 & 2,15 & 1,30 & 2,26 \\
\hline \multirow{2}{*}{$0-1$} & links & 1,41 & 1,72 & 1,51 & 1,97 & 1,37 & 2,10 \\
\hline & rechts & 1,24 & 1,47 & 1,18 & 1,65 & 1,17 & 1,80 \\
\hline \multirow{2}{*}{$0-2$} & links & 1,33 & 1,51 & 1,33 & 1,59 & 1,28 & 1,73 \\
\hline & rechts & 1,26 & 1,41 & 1,21 & 1,52 & 1,20 & 1,65 \\
\hline \multirow{2}{*}{$0-3$} & links & 1,26 & 1,93 & 1,16 & 2,36 & 1,16 & 2,39 \\
\hline & rechts & 1,28 & 1,60 & 1,24 & 1,75 & 1,21 & 1,90 \\
\hline \multirow{2}{*}{ P-1 } & links & 1,49 & 1,69 & 1,54 & 1,91 & 1,41 & 2,16 \\
\hline & rechts & 1,34 & 1,44 & 1,25 & 1,54 & 1,23 & 1,81 \\
\hline \multirow{2}{*}{ P-2 } & links & 1,32 & 1,66 & 1,22 & 1,91 & 1,21 & 2,09 \\
\hline & rechts & 1,34 & 1,50 & 1,25 & 1,54 & 1,23 & 1,80 \\
\hline \multirow{2}{*}{ P-3 } & links & 1,26 & 1,52 & 1,20 & 1,65 & 1,19 & 1,89 \\
\hline & rechts & 1,39 & 1,55 & 1,37 & 1,70 & 1,30 & 1,97 \\
\hline \multirow{2}{*}{ Q-1 } & links & 1,44 & 2,16 & 1,44 & 2,32 & 1,34 & 2,57 \\
\hline & rechts & 1,33 & 1,50 & 1,22 & 1,61 & 1,21 & 1,87 \\
\hline \multirow{2}{*}{ Q-2 } & links & 1,50 & 1,58 & 1,56 & 1,63 & 1,42 & 1,95 \\
\hline & rechts & 1,49 & 1,56 & 1,63 & 1,62 & 1,44 & 1,93 \\
\hline \multirow{2}{*}{ Q-3 } & links & 1,42 & 1,52 & 1,44 & 1,58 & 1,34 & 1,90 \\
\hline & rechts & 1,30 & 1,53 & 1,18 & 1,53 & 1,18 & 1,85 \\
\hline \multirow{2}{*}{ R-1 } & links & 1,29 & 1,34 & 1,20 & 1,29 & 1,19 & 1,62 \\
\hline & rechts & 1,34 & 1,40 & 1,26 & 1,36 & 1,23 & 1,69 \\
\hline \multirow{2}{*}{ R-2 } & links & 1,49 & 1,47 & 1,53 & 1,45 & 1,40 & 1,78 \\
\hline & rechts & 1,44 & 1,43 & 1,44 & 1,42 & 1,34 & 1,75 \\
\hline \multirow{2}{*}{ R-3 } & links & 1,53 & 1,60 & 1,69 & 1,67 & 1,48 & 1,98 \\
\hline & rechts & 1,52 & 1,27 & 1,63 & 1,24 & 1,45 & 1,62 \\
\hline \multirow{2}{*}{ S-1 } & links & 1,84 & 1,53 & 2,16 & 1,59 & 1,82 & 1,93 \\
\hline & rechts & 1,32 & 1,30 & 1,22 & 1,31 & 1,21 & 1,64 \\
\hline \multirow{2}{*}{ S-2 } & links & 1,87 & 1,52 & 2,16 & 1,57 & 1,83 & 1,94 \\
\hline & rechts & 1,31 & 1,67 & 1,20 & 1,92 & 1,19 & 2,15 \\
\hline \multirow{2}{*}{ S-3 } & links & 1,37 & 1,67 & 1,30 & 1,85 & 1,26 & 2,10 \\
\hline & rechts & 1,61 & 1,49 & 1,75 & 1,60 & 1,55 & 1,92 \\
\hline$T-1$ & links & 1,40 & 1,59 & 1,37 & 1,70 & 1,30 & 2,01 \\
\hline $1-1$ & rechts & 1,29 & 1,46 & 1,18 & 1,56 & 1,18 & 1,86 \\
\hline T2 & links & 1,34 & 1,68 & 1,25 & 1,88 & 1,23 & 2,14 \\
\hline $1-2$ & rechts & 1,30 & 1,61 & 1,21 & 1,73 & 1,20 & 1,99 \\
\hline$T 3$ & links & 1,37 & 1,71 & 1,43 & 1,87 & 1,32 & 2,15 \\
\hline $1-3$ & rechts & 1,44 & 1,56 & 1,44 & 1,63 & 1,34 & 1,96 \\
\hline
\end{tabular}




\section{Anhang F Ergebnisse der Versuche zur Markierung der Rissfront}

\section{Anhang F.1 Versuch R-1-1}

Anhang F.1.1 Auswertung der zeitlich veränderlichen Messgrößen
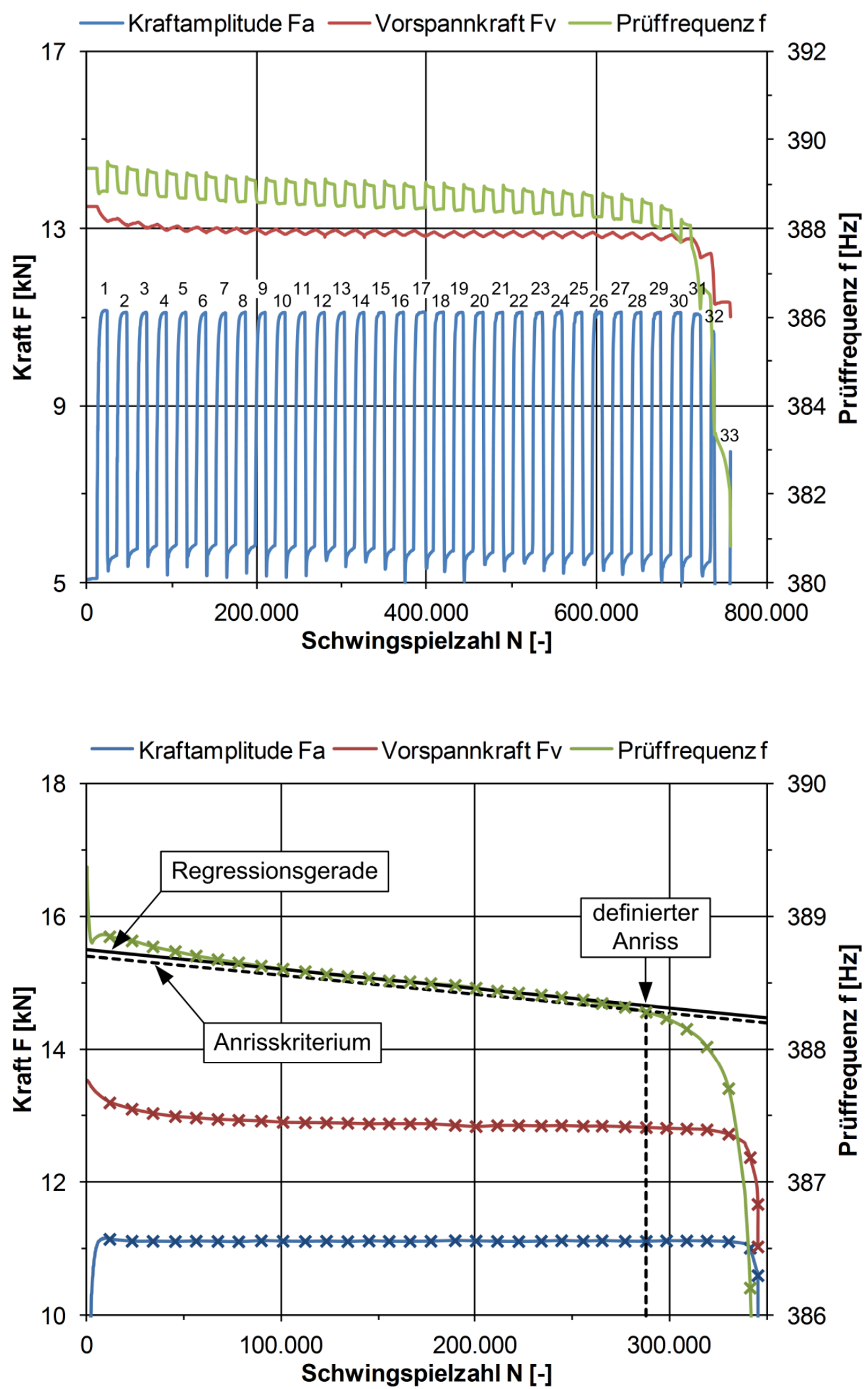
Anhang F.1.2 Vermessung der Rastlinien auf der Bruchfläche

\section{Übersichtsaufnahmen}
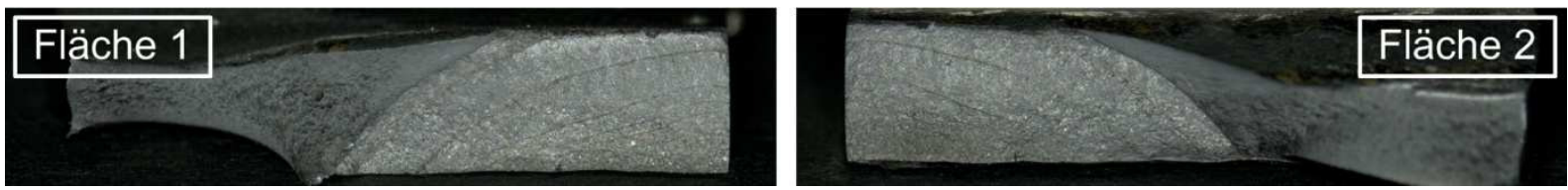

\section{Ausschnitte (20-fache Vergrößerung)}
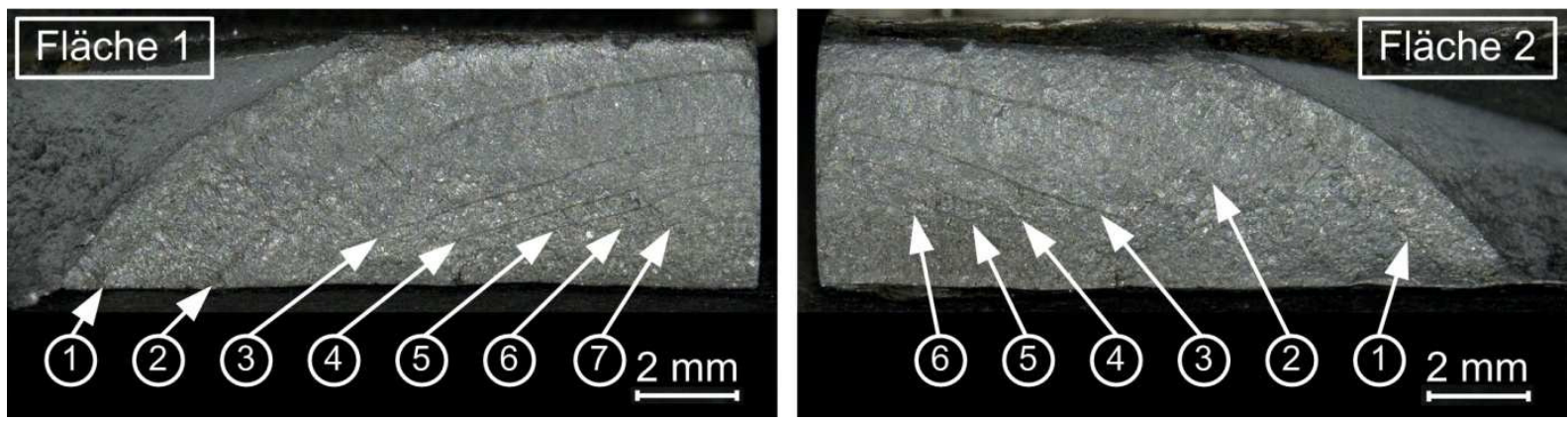

\section{Ergebnisse}

\begin{tabular}{|c|c|c|c|c|}
\hline $\begin{array}{c}\text { Rast- } \\
\text { linie }\end{array}$ & $\begin{array}{c}\text { Tiefe 1 } \\
\mathbf{t}_{\mathbf{1}} \text { [mm] }\end{array}$ & $\begin{array}{c}\text { Breite 1 } \\
\mathbf{b}_{\mathbf{1}} \text { [mm] }\end{array}$ & $\begin{array}{c}\text { Tiefe 2 } \\
\mathbf{t}_{\mathbf{2}} \text { [mm] }\end{array}$ & $\begin{array}{c}\text { Breite 2 } \\
\mathbf{b}_{\mathbf{2}} \text { [mm] }\end{array}$ \\
\hline 1 & 5,0 & 13,2 & 4,8 & 12,8 \\
\hline 2 & 4,3 & 11,0 & 4,3 & 10,9 \\
\hline 3 & 3,1 & 9,8 & 3,0 & 9,7 \\
\hline 4 & 2,5 & 8,7 & 2,5 & - \\
\hline 5 & 2,1 & - & 2,1 & - \\
\hline 6 & 1,8 & - & 1,8 & - \\
\hline 7 & 1,5 & - & - & \\
\hline
\end{tabular}

Tiefe $=$ maximale Ausdehnung der Rastlinie über die Probendicke

Breite $=$ maximale Ausdehnung der Rastlinie über die Probenbreite 
Anhang F.2 Versuch R-1-2

Anhang F.2.1 Auswertung der zeitlich veränderlichen Messgrößen
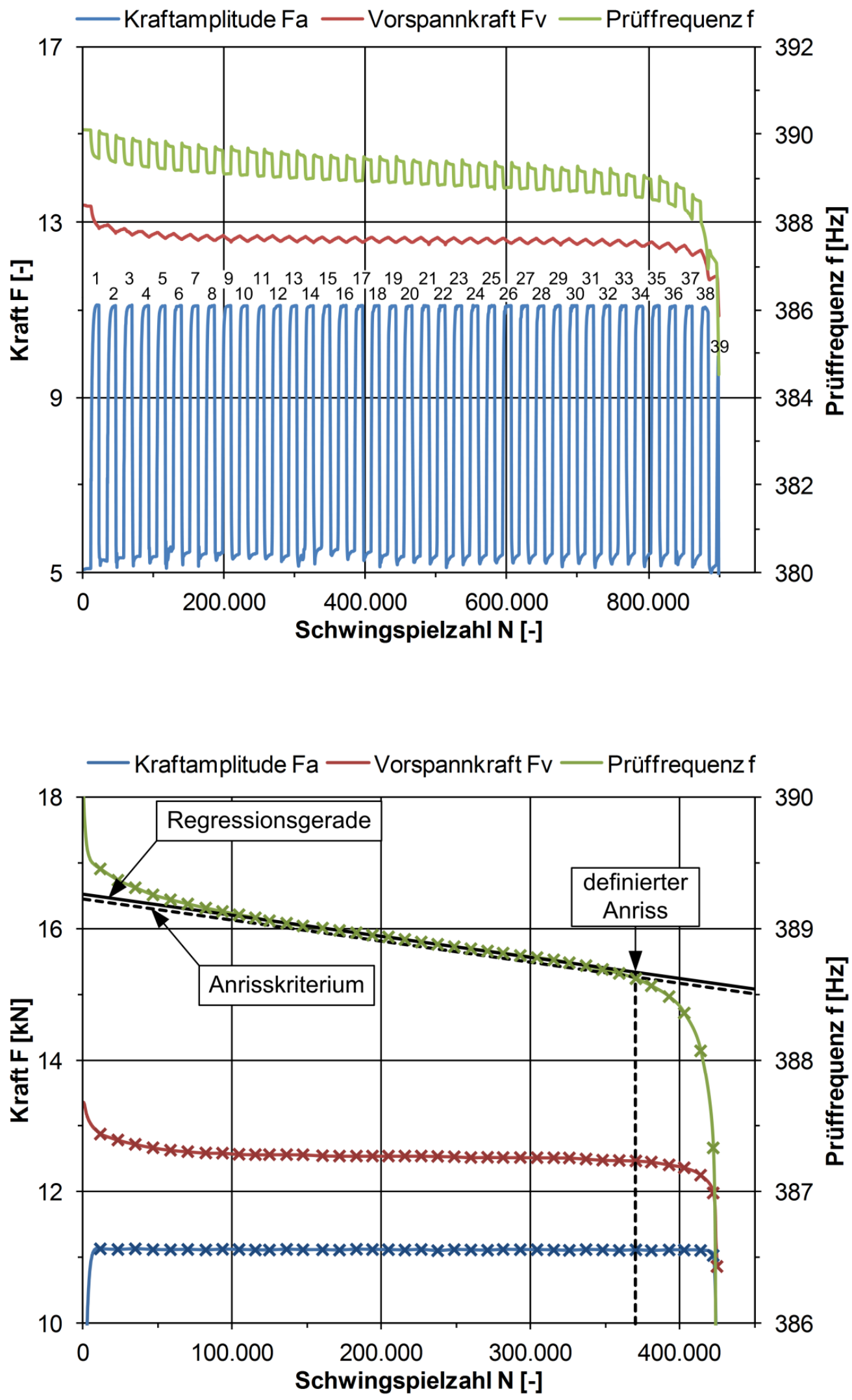
Anhang F.2.2 Vermessung der Rastlinien auf der Bruchfläche

\section{Übersichtsaufnahmen}
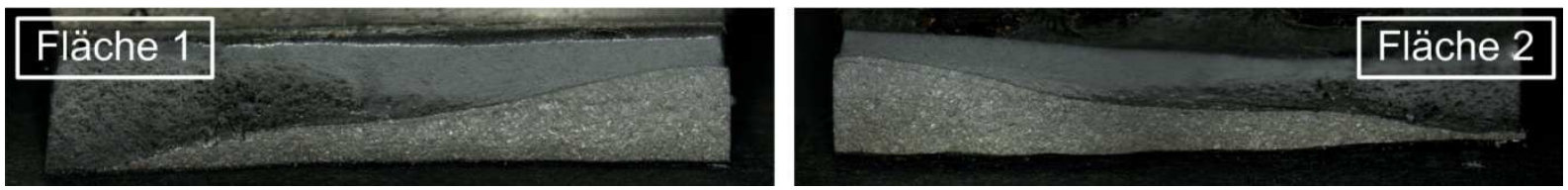

\section{Ausschnitte (20-fache Vergrößerung)}
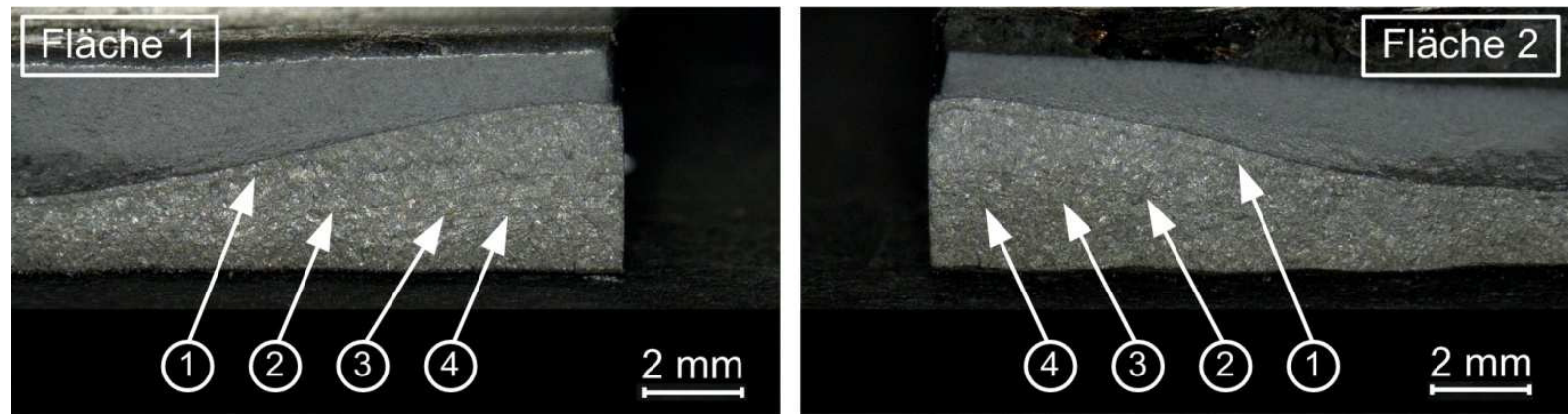

\section{Ergebnisse}

\begin{tabular}{|c|c|c|c|c|}
\hline $\begin{array}{c}\text { Rast- } \\
\text { linie }\end{array}$ & $\begin{array}{c}\text { Tiefe 1 } \\
\mathbf{t}_{\mathbf{1}} \text { [mm] }\end{array}$ & $\begin{array}{c}\text { Breite 1 } \\
\mathbf{b}_{\mathbf{1}}[\mathbf{m m}]\end{array}$ & $\begin{array}{c}\text { Tiefe 2 } \\
\mathbf{t}_{\mathbf{2}} \text { [mm] }\end{array}$ & $\begin{array}{c}\text { Breite 2 } \\
\mathbf{b}_{\mathbf{2}} \text { [mm] }\end{array}$ \\
\hline 1 & 3,1 & - & 3,2 & - \\
\hline 2 & 2,0 & - & 1,9 & - \\
\hline 3 & 1,5 & - & 1,5 & - \\
\hline 4 & 1,2 & - & 1,3 & - \\
\hline
\end{tabular}

Tiefe $=$ maximale Ausdehnung der Rastlinie über die Probendicke

Breite $=$ maximale Ausdehnung der Rastlinie über die Probenbreite 
Anhang F.3 Versuch R-1-3

Anhang F.3.1 Auswertung der zeitlich veränderlichen Messgrößen
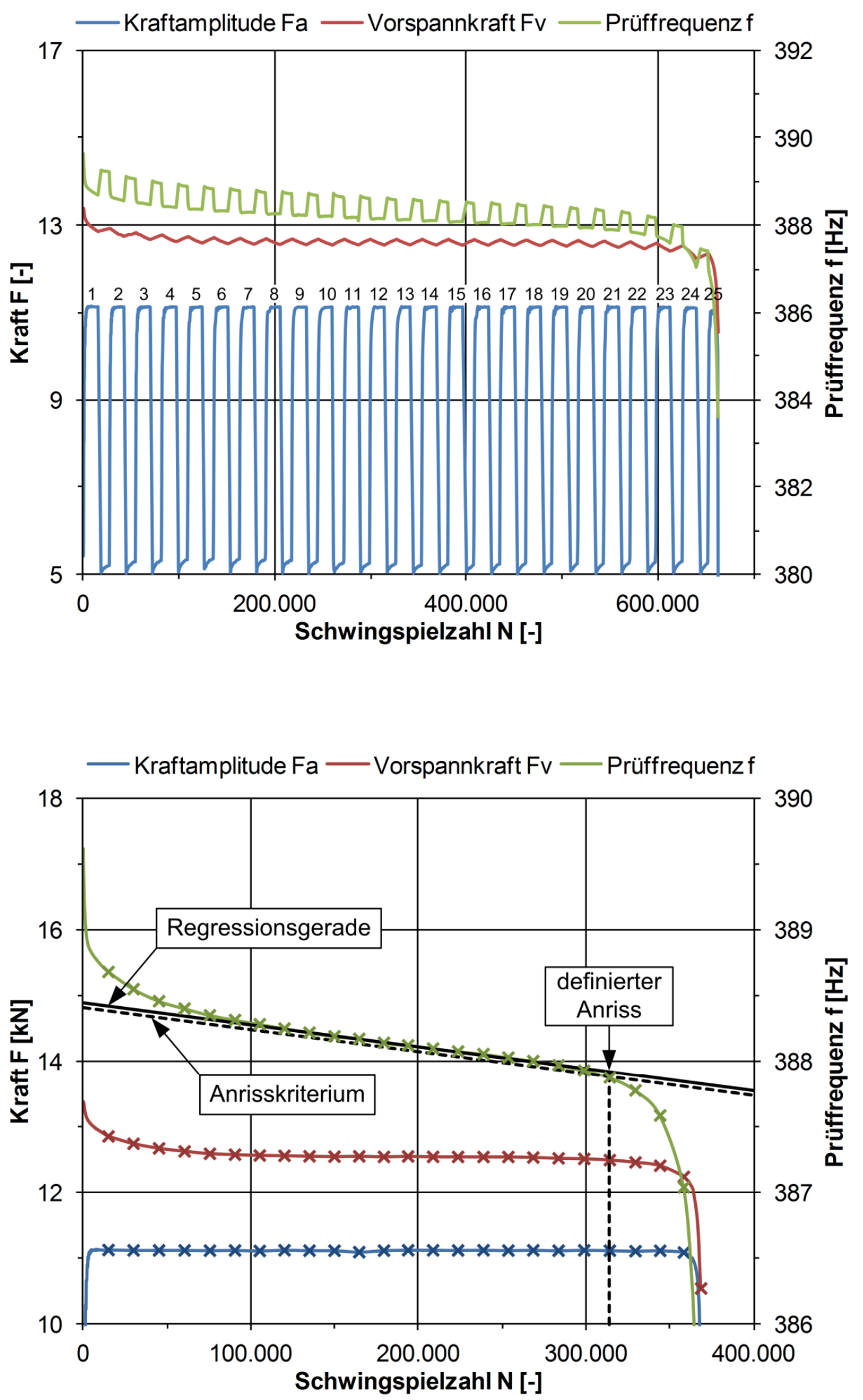
Anhang F.3.2 Vermessung der Rastlinien auf der Bruchfläche

\section{Übersichtsaufnahmen}
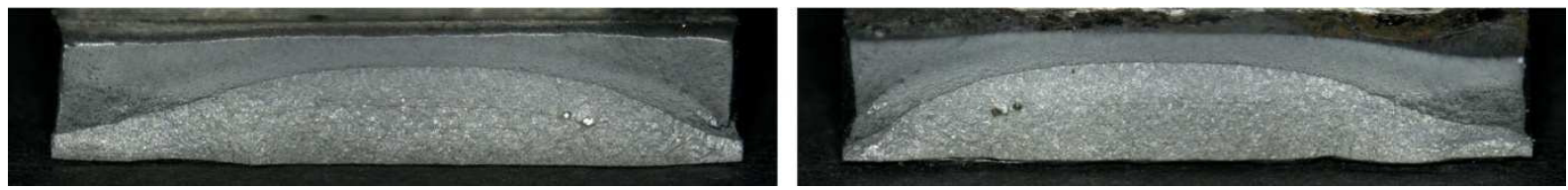

\section{Ausschnitte (20-fache Vergrößerung)}
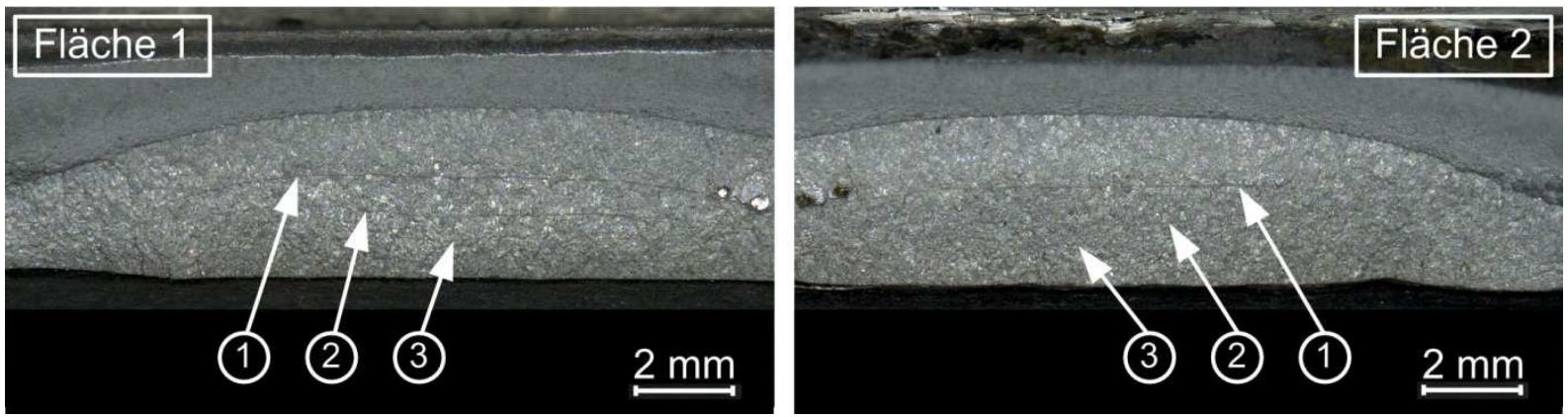

\section{Ergebnisse der Vermessung}

\begin{tabular}{|c|c|c|c|c|}
\hline $\begin{array}{c}\text { Rast- } \\
\text { linie }\end{array}$ & $\begin{array}{c}\text { Tiefe 1 } \\
\mathbf{t}_{\mathbf{1}}[\mathrm{mm}]\end{array}$ & $\begin{array}{c}\text { Breite 1 } \\
\mathbf{b}_{\mathbf{1}}[\mathrm{mm}]\end{array}$ & $\begin{array}{c}\text { Tiefe 2 } \\
\mathbf{t}_{\mathbf{2}}[\mathrm{mm}]\end{array}$ & $\begin{array}{c}\text { Breite 2 } \\
\mathbf{b}_{\mathbf{2}} \text { [mm] }\end{array}$ \\
\hline 1 & 2,1 & - & 2,1 & - \\
\hline 2 & 1,5 & - & 1,5 & - \\
\hline 3 & 1,2 & - & 1,2 & - \\
\hline
\end{tabular}

Tiefe = maximale Ausdehnung der Rastlinie über die Probendicke

Breite $=$ maximale Ausdehnung der Rastlinie über die Probenbreite 


\section{Anhang F.4 Versuch R-2-1}

\section{Anhang F.4.1 Auswertung der zeitlich veränderlichen Messgrößen}
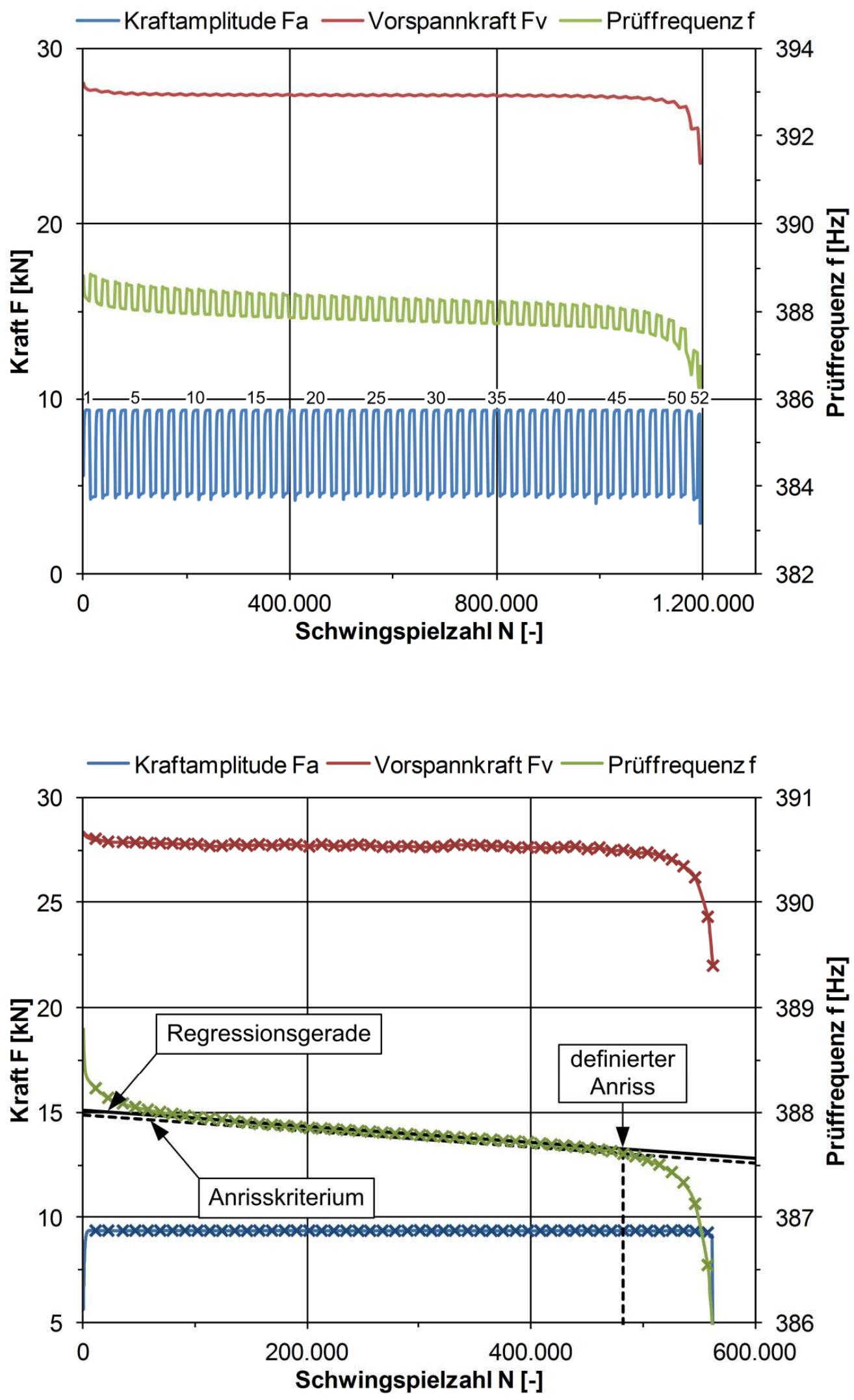
Anhang F.4.2 Vermessung der Rastlinien auf der Bruchfläche

\section{Übersichtsaufnahmen}
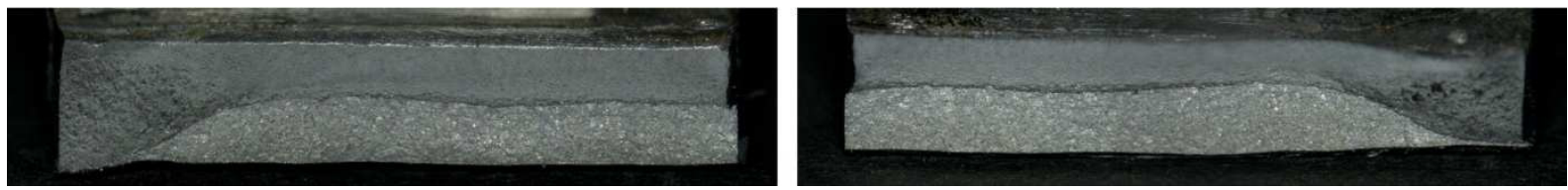

\section{Ausschnitte (20-fache Vergrößerung)}
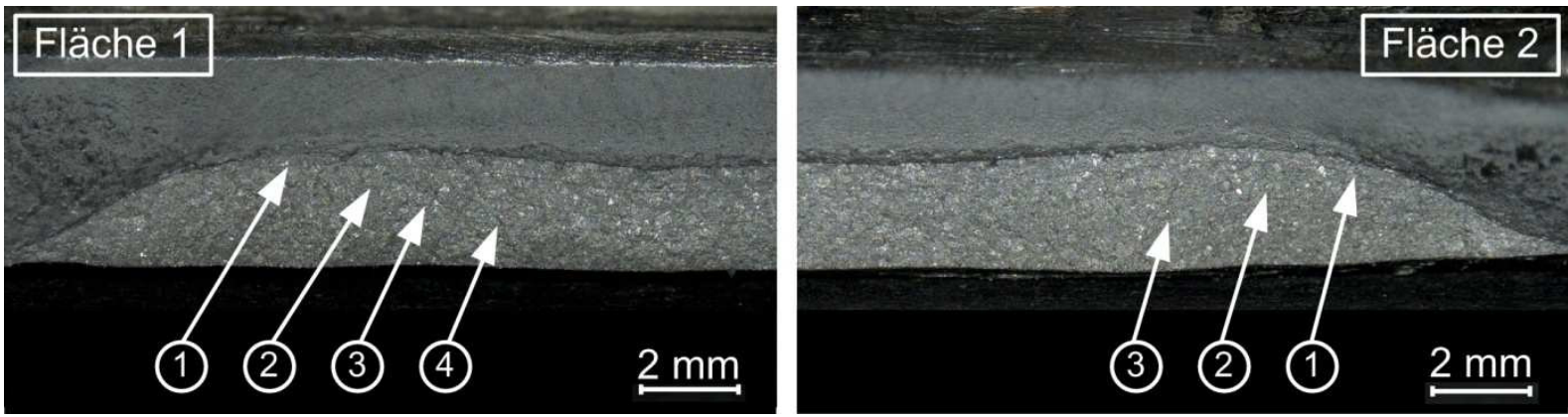

\section{Ergebnisse der Vermessung}

\begin{tabular}{|c|c|c|c|c|}
\hline $\begin{array}{c}\text { Rast- } \\
\text { linie }\end{array}$ & $\begin{array}{c}\text { Tiefe 1 } \\
\mathbf{t}_{\mathbf{1}} \text { [mm] }\end{array}$ & $\begin{array}{c}\text { Breite 1 } \\
\mathbf{b}_{\mathbf{1}}[\mathbf{m m}]\end{array}$ & $\begin{array}{c}\text { Tiefe 2 } \\
\mathbf{t}_{\mathbf{2}} \text { [mm] }\end{array}$ & $\begin{array}{c}\text { Breite 2 } \\
\mathbf{b}_{\mathbf{2}} \text { [mm] }\end{array}$ \\
\hline 1 & 2,1 & - & 2,2 & - \\
\hline 2 & 1,7 & - & 1,8 & - \\
\hline 3 & 1,4 & - & 1,4 & - \\
\hline 4 & 1,2 & - & - & - \\
\hline
\end{tabular}

Tiefe = maximale Ausdehnung der Rastlinie über die Probendicke

Breite $=$ maximale Ausdehnung der Rastlinie über die Probenbreite 


\section{Anhang F.5 Versuch R-2-2}

\section{Anhang F.5.1 Auswertung der zeitlich veränderlichen Messgrößen}
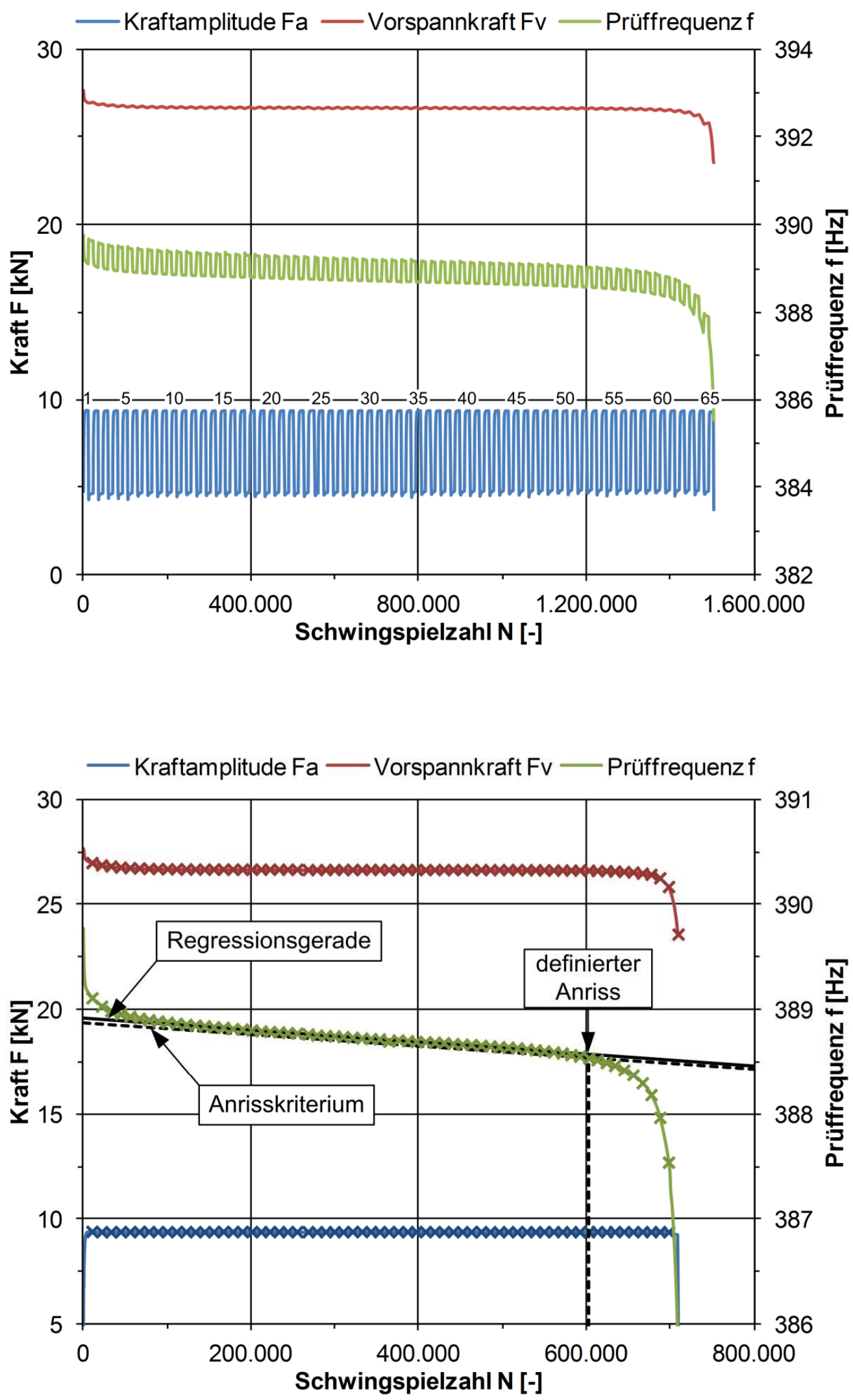
Anhang F.5.2 Vermessung der Rastlinien auf der Bruchfläche

Übersichtsaufnahmen
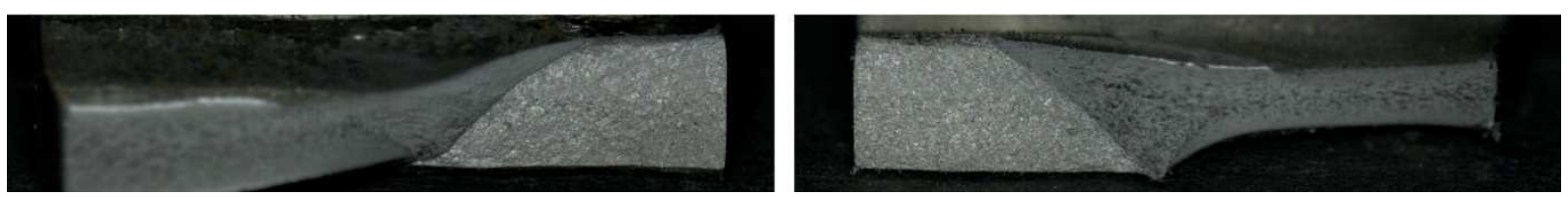

Ausschnitte (20-fache Vergrößerung)
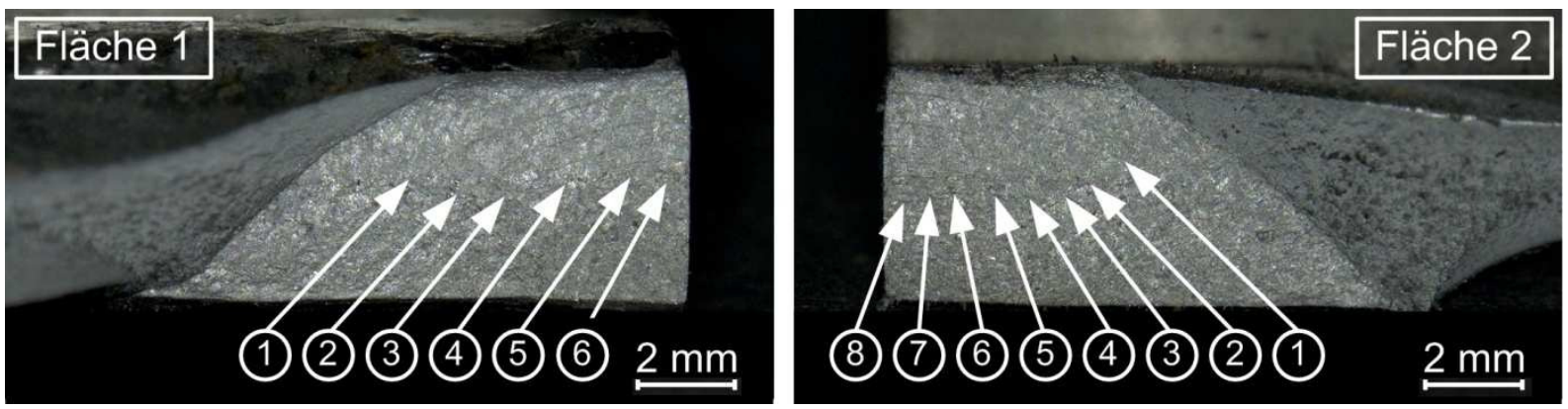

Ergebnisse der Vermessung

\begin{tabular}{|c|c|c|c|c|}
\hline $\begin{array}{c}\text { Rast- } \\
\text { linie }\end{array}$ & $\begin{array}{c}\text { Tiefe 1 } \\
\mathbf{t}_{\mathbf{1}} \text { [mm] }\end{array}$ & $\begin{array}{c}\text { Breite 1 } \\
\mathbf{b}_{\mathbf{1}}[\mathbf{m m}]\end{array}$ & $\begin{array}{c}\text { Tiefe 2 } \\
\mathbf{t}_{\mathbf{2}} \text { [mm] }\end{array}$ & $\begin{array}{c}\text { Breite 2 } \\
\mathbf{b}_{\mathbf{2}} \text { [mm] }\end{array}$ \\
\hline 1 & 4,4 & 8,2 & 4,4 & 8,3 \\
\hline 2 & 3,8 & 7,1 & 3,8 & 7,2 \\
\hline 3 & 3,3 & 6,6 & 3,3 & 6,2 \\
\hline 4 & 3,0 & 6,0 & 2,9 & 5,9 \\
\hline 5 & 2,7 & 5,5 & 2,7 & 5,4 \\
\hline 6 & 2,5 & - & 2,5 & - \\
\hline 7 & - & - & 2,3 & - \\
\hline 8 & - & - & 2,1 & - \\
\hline
\end{tabular}

Tiefe = maximale Ausdehnung der Rastlinie über die Probendicke

Breite $=$ maximale Ausdehnung der Rastlinie über die Probenbreite 
Anhang F.6 Versuch R-2-3

Anhang F.6.1 Auswertung der zeitlich veränderlichen Messgrößen
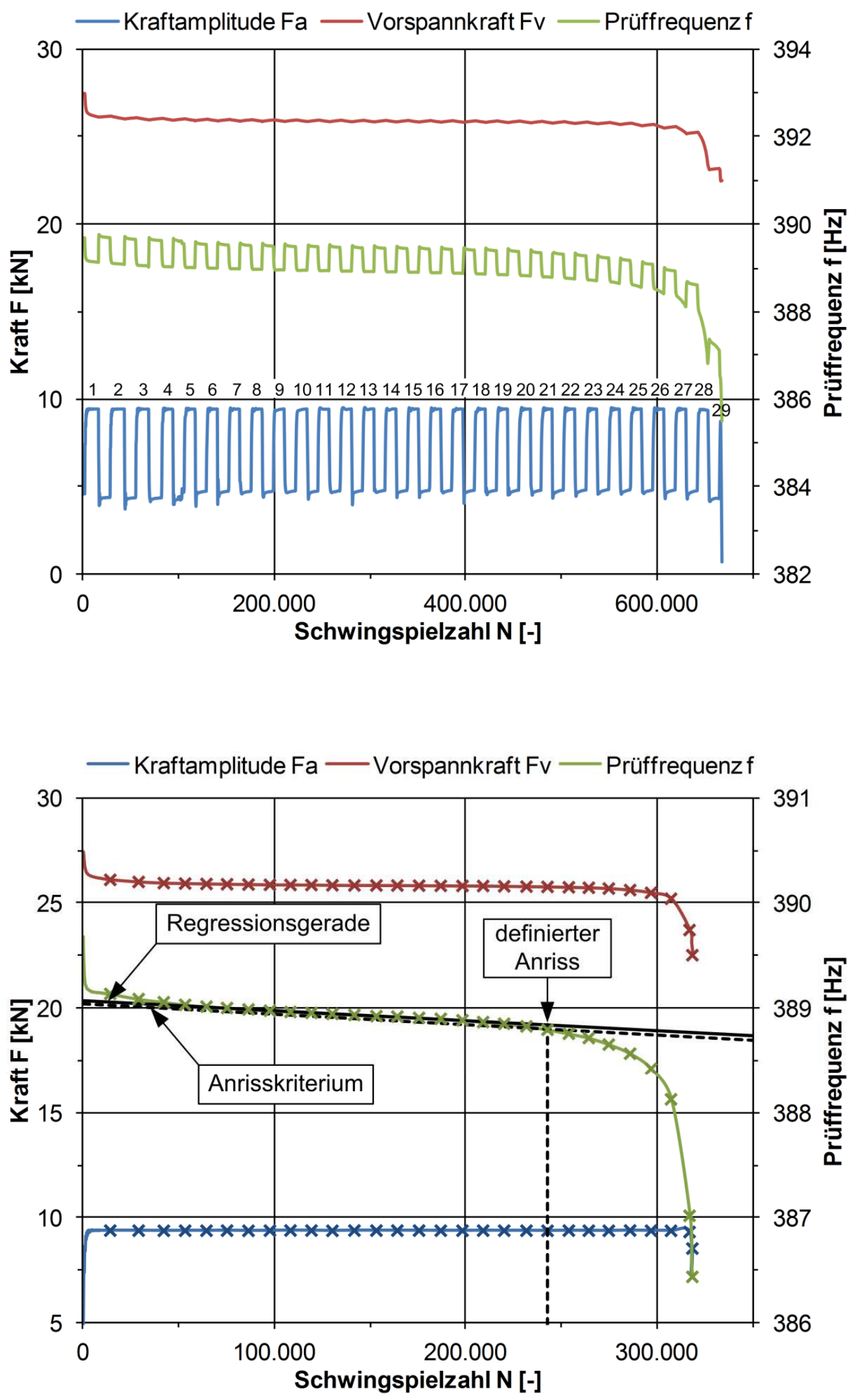
Anhang F.6.2 Vermessung der Rastlinien auf der Bruchfläche

Übersichtsaufnahmen

$$
\text { Fläche } 1
$$

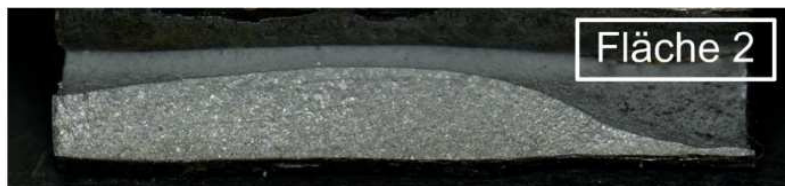

Ausschnitte (20-fache Vergrößerung)
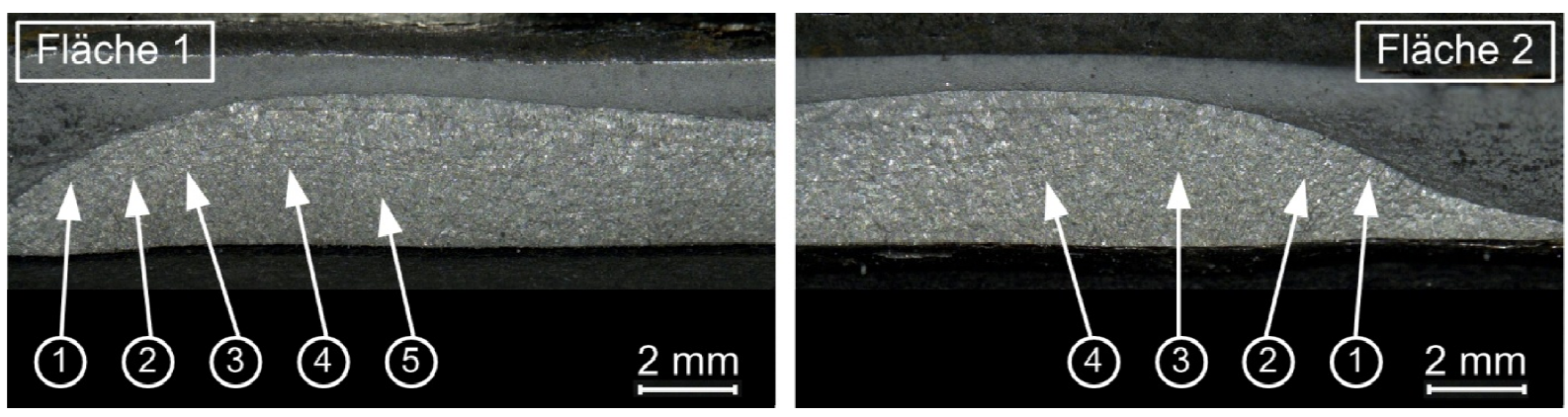

Ergebnisse der Vermessung

\begin{tabular}{|c|c|c|c|c|}
\hline $\begin{array}{c}\text { Rast- } \\
\text { linie }\end{array}$ & $\begin{array}{c}\text { Tiefe 1 } \\
\mathbf{t}_{\mathbf{1}} \text { [mm] }\end{array}$ & $\begin{array}{c}\text { Breite 1 } \\
\mathbf{b}_{\mathbf{1}} \text { [mm] }\end{array}$ & $\begin{array}{c}\text { Tiefe 2 } \\
\mathbf{t}_{\mathbf{2}} \text { [mm] }\end{array}$ & $\begin{array}{c}\text { Breite 2 } \\
\mathbf{b}_{\mathbf{2}} \text { [mm] }\end{array}$ \\
\hline 1 & 2,8 & - & 2,9 & - \\
\hline 2 & 2,1 & - & 2,3 & - \\
\hline 3 & 1,8 & - & 1,8 & - \\
\hline 4 & 1,6 & - & 1,6 & - \\
\hline 5 & 1,3 & - & - & - \\
\hline
\end{tabular}

Tiefe = maximale Ausdehnung der Rastlinie über die Probendicke Breite $=$ maximale Ausdehnung der Rastlinie über die Probenbreite 


\section{Anhang G Übertragungsfaktoren kf der Kalibrierproben}

Kalibrierprobe 1: $\quad \mathrm{kf}=\mathbf{4 , 0 0 0}$

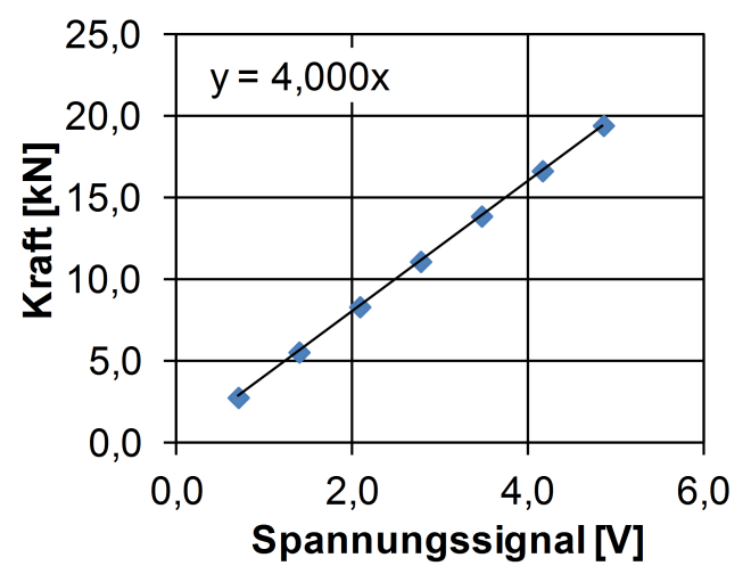

Kalibrierprobe 3: $\mathrm{kf}=4,125$

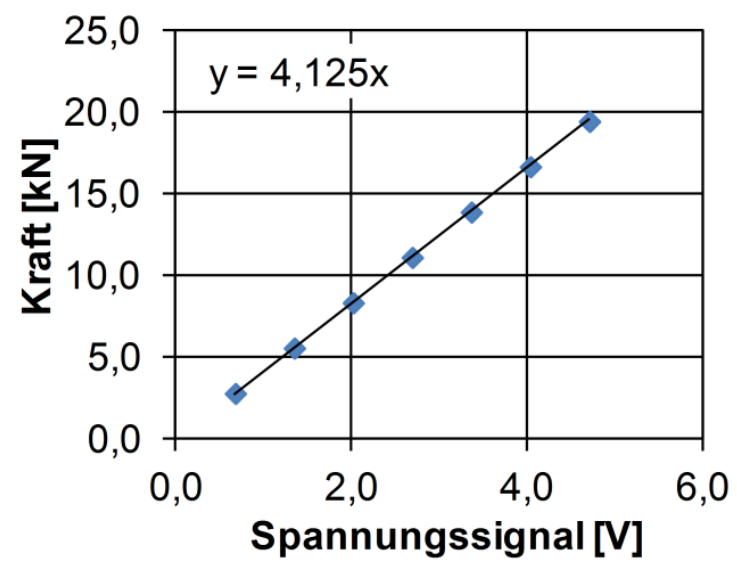

Kalibrierprobe 5: $\quad$ kf $=3,882$

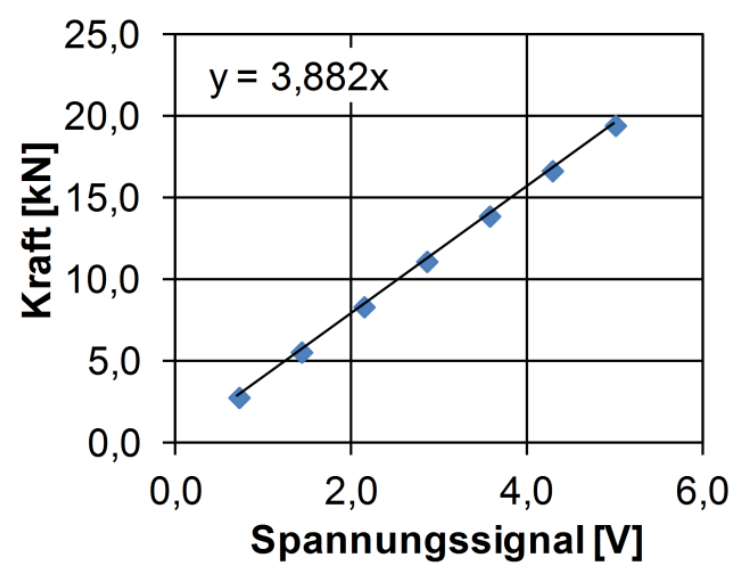

Kalibrierprobe 2: $\mathbf{k f}=3,982$

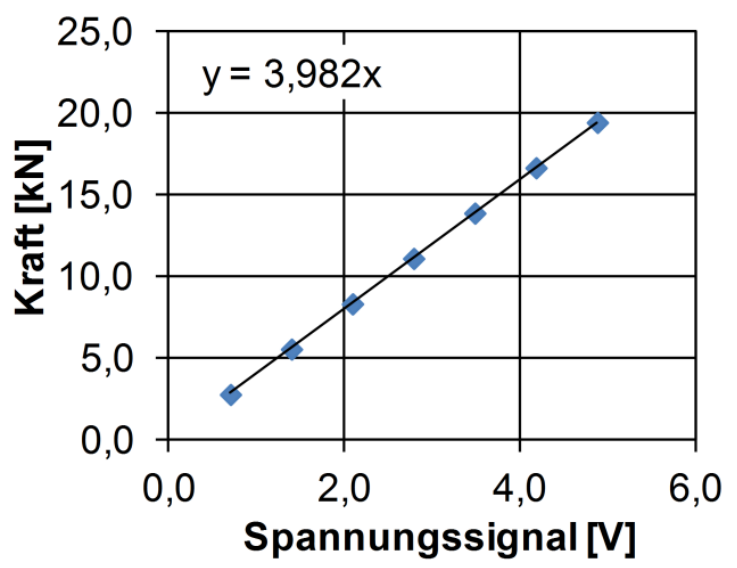

Kalibrierprobe 4: $\mathrm{kf}=4,008$

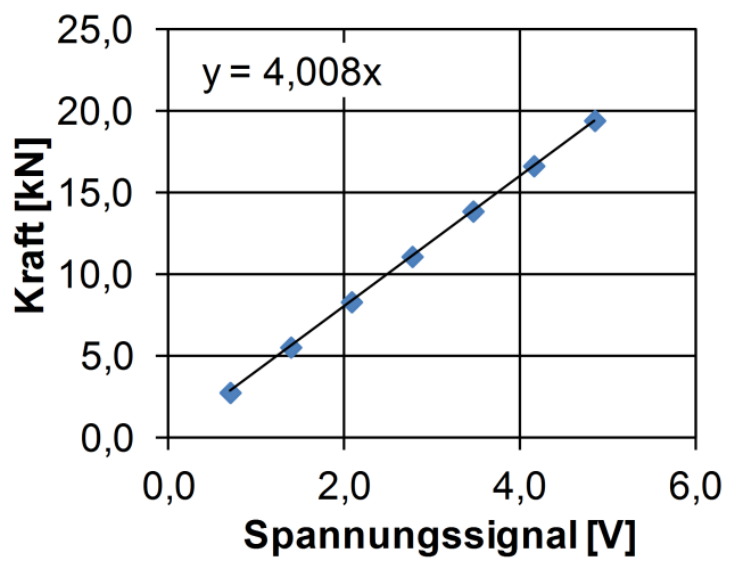




\section{Anhang H Temperaturmessungen während der Ermüdungsver- suche}

\section{Anhang H.1 Versuchsreihe 2}

\begin{tabular}{|c|c|c|c|c|c|c|c|c|c|}
\hline $\begin{array}{c}\Delta \sigma \\
{\left[\mathrm{N} / \mathrm{mm}^{2}\right]}\end{array}$ & $\begin{array}{l}\text { Ver- } \\
\text { such }\end{array}$ & $\begin{array}{c}\mathrm{T}_{1, \text { Start }} \\
{\left[{ }^{\circ} \mathrm{C}\right]}\end{array}$ & $\begin{array}{c}\mathrm{T}_{2, \text { Start }} \\
{\left[{ }^{\circ} \mathrm{C}\right]}\end{array}$ & $\begin{array}{c}\mathrm{T}_{1, \text { Anstieg }} \\
{\left[{ }^{\circ} \mathrm{C}\right]}\end{array}$ & $\begin{array}{c}\mathrm{T}_{2, \text { Anstieg }} \\
{\left[{ }^{\circ} \mathrm{C}\right]}\end{array}$ & $\begin{array}{l}\Delta \mathrm{T}_{1} \\
{[\mathrm{~K}]}\end{array}$ & $\begin{array}{l}\Delta \mathrm{T}_{2} \\
{[\mathrm{~K}]}\end{array}$ & $\begin{array}{l}\mathrm{T}_{1, \max } \\
{\left[{ }^{\circ} \mathrm{C}\right]}\end{array}$ & $\begin{array}{c}\mathrm{T}_{2, \max } \\
{\left[{ }^{\circ} \mathrm{C}\right]}\end{array}$ \\
\hline 277,33 & V2-A-1 & 24,5 & 24,5 & $30,5^{*}$ & $31,7^{*}$ & 6,0 & $7,2^{*}$ & $39,3^{*}$ & 37,9 \\
\hline 277,33 & V2-A-2 & 25,3 & 25,7 & $34,8^{*}$ & $36,5^{\star}$ & 9,5 & $10,8^{*}$ & $42,5^{*}$ & 41,9 \\
\hline 277,33 & V2-A-3 & 26,4 & 26,2 & $36,5^{*}$ & $35,8^{*}$ & 10,1 & $9,6^{*}$ & $41,7^{*}$ & 43,9 \\
\hline 277,33 & V2-A-4 & 24,2 & 24,2 & $31,8^{*}$ & $32,3^{*}$ & 7,6 & $8,1^{*}$ & $39,9^{*}$ & 37,0 \\
\hline 277,33 & V2-A-5 & 24,1 & 24,1 & $33,3^{*}$ & $32,7^{*}$ & $9,2^{*}$ & $8,6^{*}$ & $39,8^{*}$ & 36,5 \\
\hline 277,33 & V2-A-6 & 24,4 & 24,4 & $37,2^{*}$ & $38,2^{*}$ & $12,8^{*}$ & $13,8^{*}$ & 43,0 & 41,1 \\
\hline \multicolumn{2}{|c|}{ Mittelwerte } & 24,8 & 24,8 & $34,0^{*}$ & $34,5^{*}$ & $9,2^{*}$ & $9,7^{*}$ & 41,0 & 39,7 \\
\hline 221,86 & V2-A-7 & 22,4 & 22,4 & 31,8 & 32,3 & 9,4 & 9,9 & 36,4 & 34,8 \\
\hline 221,86 & V2-A-8 & 21,7 & 21,6 & 31,4 & 31,7 & 9,7 & 10,1 & 36,4 & 34,1 \\
\hline 221,86 & V2-A-9 & 21,3 & 21,9 & 29,6 & 30,2 & 8,3 & 8,9 & 34,9 & 32,5 \\
\hline 221,86 & V2-A-10 & 21,5 & 21,5 & 30,3 & 31,8 & 8,8 & 10,3 & 35,0 & 34,4 \\
\hline 221,86 & V2-A-11 & 22,1 & 22,1 & 32,7 & 33,1 & 10,6 & 10,9 & 35,2 & 34,4 \\
\hline 221,86 & V2-A-12 & 21,3 & 21,3 & 30,7 & 31,0 & 9,4 & 9,7 & 33,8 & 32,7 \\
\hline \multicolumn{2}{|c|}{ Mittelwerte } & 21,7 & 21,7 & 31,1 & 31,7 & 9,4 & 10,0 & 35,3 & 33,8 \\
\hline 162,50 & V2-B-2 & 21,2 & 21,1 & 25,6 & 25,2 & 4,4 & 4,1 & 27,1 & 27,8 \\
\hline 150,00 & V2-B-3 & 25,3 & 25,1 & 27,5 & 27,3 & 2,1 & 2,2 & 29,6 & 28,5 \\
\hline 150,00 & V2-B-4 & 24,0 & 24,0 & 25,9 & 25,9 & 1,9 & 1,9 & 28,4 & 27,1 \\
\hline 150,00 & V2-B-5 & 23,8 & 23,7 & 28,5 & 28,4 & 4,8 & 4,7 & 32,0 & 30,2 \\
\hline 150,00 & V2-B-6 & 23,7 & 23,8 & 27,0 & 27,2 & 3,3 & 3,5 & 29,6 & 29,0 \\
\hline 150,00 & V2-B-7 & 25,1 & 25,1 & 27,0 & 26,9 & 1,9 & 1,8 & - & - \\
\hline 137,50 & V2-B-8 & 25,6 & 25,7 & 27,0 & 27,1 & 1,3 & 1,5 & 29,0 & 28,0 \\
\hline 137,50 & V2-B-11 & 25,5 & 25,6 & 27,8 & 27,3 & 1,7 & 1,7 & - & - \\
\hline 137,50 & V2-B-12 & 23,9 & 23,9 & 26,1 & 26,2 & 2,2 & 2,3 & - & - \\
\hline 125,00 & V2-B-14 & 24,2 & 24,2 & 26,4 & 26,5 & 2,2 & 2,3 & - & - \\
\hline \multicolumn{2}{|c|}{ Mittelwerte } & 24,3 & 24,2 & 26,8 & 26,8 & 2,6 & 2,6 & 29,3 & 28,4 \\
\hline
\end{tabular}

* kein Temperaturplateau - Durchläufer 


\section{Anhang H.2 Versuchsreihe 3}

\begin{tabular}{|c|c|c|c|c|c|c|c|c|c|}
\hline $\begin{array}{c}\Delta \sigma \\
{\left[\mathrm{N} / \mathrm{mm}^{2}\right]}\end{array}$ & $\begin{array}{l}\text { Ver- } \\
\text { such }\end{array}$ & $\begin{array}{c}\mathrm{T}_{1, \text { Start }} \\
{\left[{ }^{\circ} \mathrm{C}\right]}\end{array}$ & $\begin{array}{c}\mathrm{T}_{2, \text { Start }} \\
{\left[{ }^{\circ} \mathrm{C}\right]}\end{array}$ & $\begin{array}{c}\mathrm{T}_{1, \text { Anstieg }} \\
{\left[{ }^{\circ} \mathrm{C}\right]}\end{array}$ & $\begin{array}{c}T_{2, \text { Anstieg }} \\
{\left[{ }^{\circ} \mathrm{C}\right]}\end{array}$ & $\begin{array}{l}\Delta \mathrm{T}_{1} \\
{[\mathrm{~K}]}\end{array}$ & $\begin{array}{l}\Delta \mathrm{T}_{2} \\
{[\mathrm{~K}]}\end{array}$ & $\begin{array}{l}\mathrm{T}_{1, \max } \\
{\left[{ }^{\circ} \mathrm{C}\right]}\end{array}$ & $\begin{array}{c}\mathrm{T}_{2, \max } \\
{\left[{ }^{\circ} \mathrm{C}\right]}\end{array}$ \\
\hline 187,50 & V3-A-1 & 23,7 & 23,7 & 31,1 & 30,4 & 7,4 & 6,7 & 33,8 & 32,1 \\
\hline 187,50 & V3-A-2 & 23,2 & 23,2 & 30,5 & 30,9 & 7,3 & 7,7 & 33,6 & 32,9 \\
\hline 187,50 & V3-A-3 & 24,7 & 24,7 & 32,4 & 32,7 & 7,7 & 8,0 & 35,5 & 34,7 \\
\hline 187,50 & V3-A-4 & 24,0 & 24,0 & 31,1 & 31,5 & 7,2 & 7,4 & 33,7 & 33,1 \\
\hline 187,50 & V3-A-5 & 25,0 & 25,0 & 31,5 & 31,5 & 6,5 & 6,5 & 33,9 & 32,8 \\
\hline 187,50 & V3-A-1 & 23,7 & 23,7 & 31,1 & 30,4 & 7,4 & 6,7 & 33,8 & 32,1 \\
\hline \multicolumn{2}{|c|}{ Mittelwerte } & 24,1 & 24,1 & 31,3 & 31,4 & 7,2 & 7,3 & 34,1 & 33,1 \\
\hline 162,50 & V3-A-6 & 22,8 & 22,8 & 28,9 & 29,0 & 6,1 & 6,2 & 33,4 & 31,4 \\
\hline 162,50 & V3-A-7 & 23,0 & 23,0 & 26,6 & 26,3 & 3,6 & 3,3 & 28,0 & 27,2 \\
\hline 162,50 & V3-A-8 & 23,6 & 23,6 & 28,8 & 28,6 & 5,2 & 5,1 & 31,7 & 30,3 \\
\hline 162,50 & V3-A-9 & 24,0 & 24,0 & 28,6 & 28,5 & 4,6 & 4,5 & 30,5 & 29,6 \\
\hline 162,50 & V3-A-10 & 24,9 & 25,0 & 29,8 & 29,9 & 4,9 & 4,9 & 31,7 & 30,8 \\
\hline 162,50 & V3-A-6 & 22,8 & 22,8 & 28,9 & 29,0 & 6,0 & 6,2 & 33,4 & 31,4 \\
\hline \multicolumn{2}{|c|}{ Mittelwerte } & 23,7 & 23,7 & 28,5 & 28,5 & 4,9 & 4,8 & 31,1 & 29,9 \\
\hline 150,00 & V3-B-1 & 21,4 & 21,4 & 25,9 & 26,0 & 4,4 & 4,6 & 30,0 & 28,3 \\
\hline 150,00 & V3-B-2 & 22,8 & 22,8 & 26,2 & 26,3 & 3,4 & 3,5 & 32,4 & 30,1 \\
\hline 143,75 & V3-B-3 & 22,5 & 22,5 & 25,6 & 25,7 & 3,1 & 3,1 & 27,7 & 26,8 \\
\hline 143,75 & V3-B-4 & 23,5 & 23,5 & 26,9 & 27,1 & 3,4 & 3,6 & 28,9 & 28,2 \\
\hline 143,75 & V3-B-5 & 21,0 & 21,0 & 24,3 & 24,5 & 3,3 & 3,5 & 27,3 & 26,1 \\
\hline 143,75 & V3-B-6 & \# & 21,2 & \# & 24,4 & \# & 3,2 & \# & 25,8 \\
\hline 143,75 & V3-B-7 & 21,1 & 21,1 & 24,9 & 25,2 & 3,8 & 4,0 & 28,8 & 27,4 \\
\hline 143,75 & V3-B-8 & 20,3 & 20,3 & 23,6 & 23,8 & 3,3 & 3,5 & - & - \\
\hline 137,50 & V3-B-9 & 20,9 & 20,9 & 23,6 & 23,5 & 2,7 & 2,6 & 24,8 & 25,9 \\
\hline 137,50 & V3-B-10 & 21,7 & 21,6 & 23,9 & 23,9 & 2,2 & 2,3 & 26,5 & 25,3 \\
\hline 137,50 & V3-B-11 & 23,0 & 23,0 & 27,1 & 27,2 & 4,1 & 4,2 & - & - \\
\hline 137,50 & V3-B-12 & 23,2 & 23,2 & 27,2 & 27,3 & 4,0 & 4,1 & - & - \\
\hline 137,50 & V3-B-13 & 23,2 & 23,2 & 27,6 & 27,8 & 4,4 & 4,6 & - & - \\
\hline 137,50 & V3-B-14 & 23,5 & 23,5 & 27,1 & 27,3 & 3,6 & 3,7 & - & - \\
\hline 131,25 & V3-B-15 & 20,8 & 20,8 & 23,4 & 23,4 & 2,5 & 2,6 & - & - \\
\hline 131,25 & V3-B-16 & 21,3 & 21,3 & 23,6 & 23,7 & 2,3 & 2,4 & - & - \\
\hline \multicolumn{2}{|c|}{ Mittelwerte } & 22,0 & 22,0 & 25,4 & 25,4 & 3,4 & 3,5 & 28,3 & 27,1 \\
\hline
\end{tabular}

- Durchläufer \# Ausfall des Thermoelementes 


\section{Anhang I Einzelversuche und Bruchflächen der Versuchsreihen}

\section{Anhang I.1 Versuchsreihe 1}

Prüfmaschine: Servohydraulische Prüfmaschine

Prüffrequenz $\quad f=20 \mathrm{~Hz}$

Spannungsverhältnis $\quad R=0,1$

\section{Anhang l.1.1 A - Zeitfestigkeitsbereich}

\section{Datenübersicht}

\begin{tabular}{|c|c|c|c|c|c|}
\hline Versuch & Probe & $\begin{array}{c}\Delta \boldsymbol{\sigma} \\
{\left[\mathbf{N} / \mathbf{m m}^{2}\right]}\end{array}$ & $\begin{array}{c}\mathbf{N}_{\mathbf{B}} \\
{[-]}\end{array}$ & Ergebnis & Rissausgang \\
\hline V1-A-1 & 1 & 332,80 & 33.963 & Bruch & W \\
\hline V1-A-2 & 2 & 332,80 & 39.867 & Bruch & W \\
\hline V1-A-3 & 3 & 332,80 & 45.129 & Bruch & W \\
\hline V1-A-4 & 4 & 332,80 & 53.160 & Bruch & W \\
\hline V1-A-5 & 5 & 332,80 & 62.658 & Bruch & W \\
\hline V1-A-6 & 6 & 332,80 & 90.292 & Bruch & W \\
\hline V1-A-7 & 7 & 277,33 & 70.457 & Bruch & W \\
\hline V1-A-8 & 8 & 277,33 & 137.306 & Bruch & N \\
\hline V1-A-9 & 9 & 277,33 & 166.873 & Bruch & N \\
\hline V1-A-10 & 10 & 277,33 & 168.155 & Bruch & W \\
\hline V1-A-11 & 11 & 277,33 & 186.802 & Bruch & W \\
\hline V1-A-12 & 12 & 277,33 & 231.498 & Bruch & W \\
\hline V1-A-13 & 13 & 221,86 & 137.998 & Bruch & W \\
\hline V1-A-14 & 14 & 221,86 & 153.650 & Bruch & W \\
\hline V1-A-15 & 15 & 221,86 & 245.813 & Bruch & W \\
\hline V1-A-16 & 16 & 221,86 & 281.151 & Bruch & $\mathrm{N}$ \\
\hline V1-A-17 & 17 & 221,86 & 359.971 & Bruch & W \\
\hline V1-A-18 & 18 & 221,86 & 383.037 & Bruch & W \\
\hline
\end{tabular}

W - Übergang an Wurzelüberhöhung

$\mathrm{N}$ - Übergang an Nahtüberhöhung 


\section{Übersichtsaufnahmen der Bruchflächen}

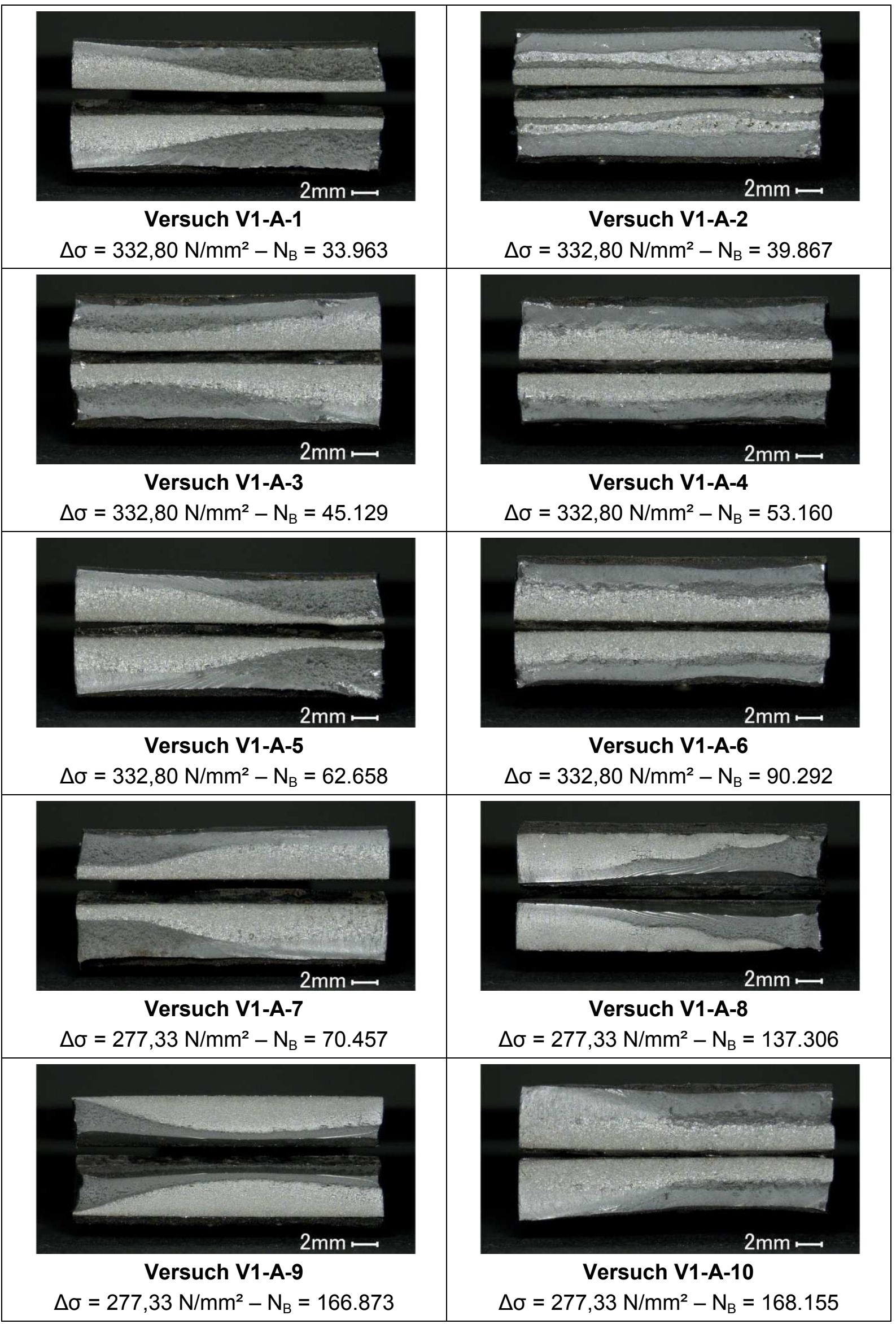




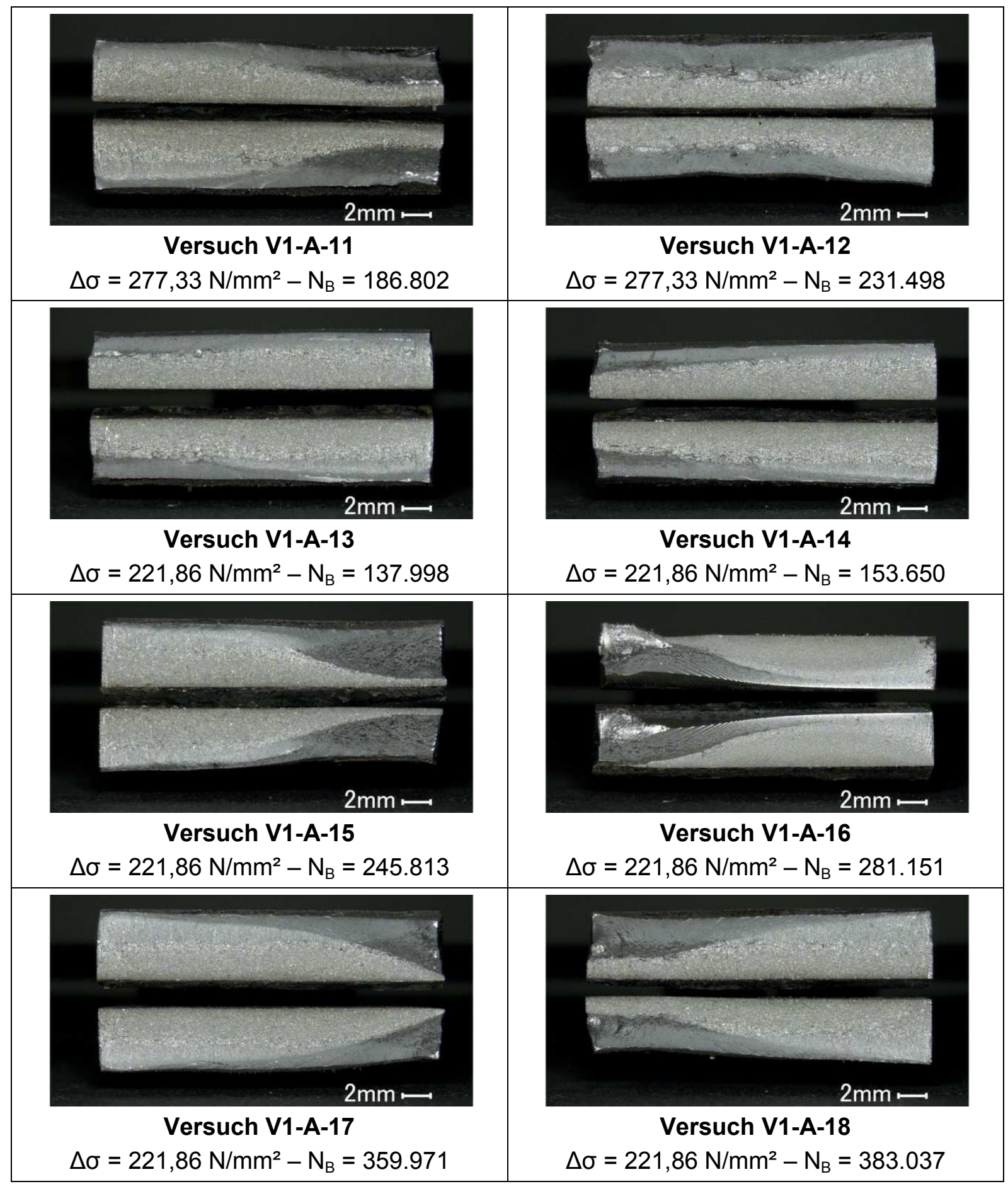




\section{Anhang I.1.2 B - Übergangsbereich}

\section{Datenübersicht}

\begin{tabular}{|c|c|c|c|c|c|}
\hline Versuch & Probe & $\begin{array}{c}\boldsymbol{\Delta} \boldsymbol{\sigma} \\
{\left[\mathbf{N} / \mathbf{m m}^{2}\right]}\end{array}$ & $\begin{array}{c}\mathbf{N}_{\mathbf{B}} \\
{[-]}\end{array}$ & Ergebnis & Rissausgang \\
\hline V1-B-1 & 19 & 184,14 & 493.444 & Bruch & W \\
\hline V1-B-2 & 20 & 175,27 & 596.600 & Bruch & W \\
\hline V1-B-3 & 21 & 175,27 & 1.776 .271 & Bruch & W \\
\hline V1-B-4 & 22 & 166,40 & 1.088 .945 & Bruch & W \\
\hline V1-B-5 & 23 & 166,40 & 1.381 .754 & Bruch & W \\
\hline V1-B-6 & 24 & 166,40 & 2.106 .449 & Bruch & W \\
\hline V1-B-7 & 25 & 166,40 & 5.000 .000 & Durchläufer & - \\
\hline V1-B-8 & 26 & 166,40 & 5.000 .000 & Durchläufer & - \\
\hline V1-B-9 & 27 & 157,52 & 2.327 .013 & Bruch & W \\
\hline V1-B-10 & 28 & 157,52 & 5.000 .000 & Durchläufer & - \\
\hline V1-B-11 & 29 & 157,52 & 5.000 .000 & Durchläufer & - \\
\hline V1-B-12 & 30 & 157,52 & 5.000 .000 & Durchläufer & - \\
\hline V1-B-13 & 31 & 148,65 & 605.408 & Bruch & W \\
\hline V1-B-14 & 32 & 148,65 & 1.097 .209 & Bruch & W \\
\hline V1-B-15 & 33 & 148,65 & 5.000 .000 & Durchläufer & - \\
\hline V1-B-16 & 34 & 139,77 & 5.000 .000 & Durchläufer & - \\
\hline V1-B-17 & 35 & 139,77 & 5.000 .000 & Durchläufer & - \\
\hline
\end{tabular}

W - Übergang an Wurzelüberhöhung

$\mathrm{N}$ - Übergang an Nahtüberhöhung 


\section{Übersichtsaufnahmen der Bruchflächen}

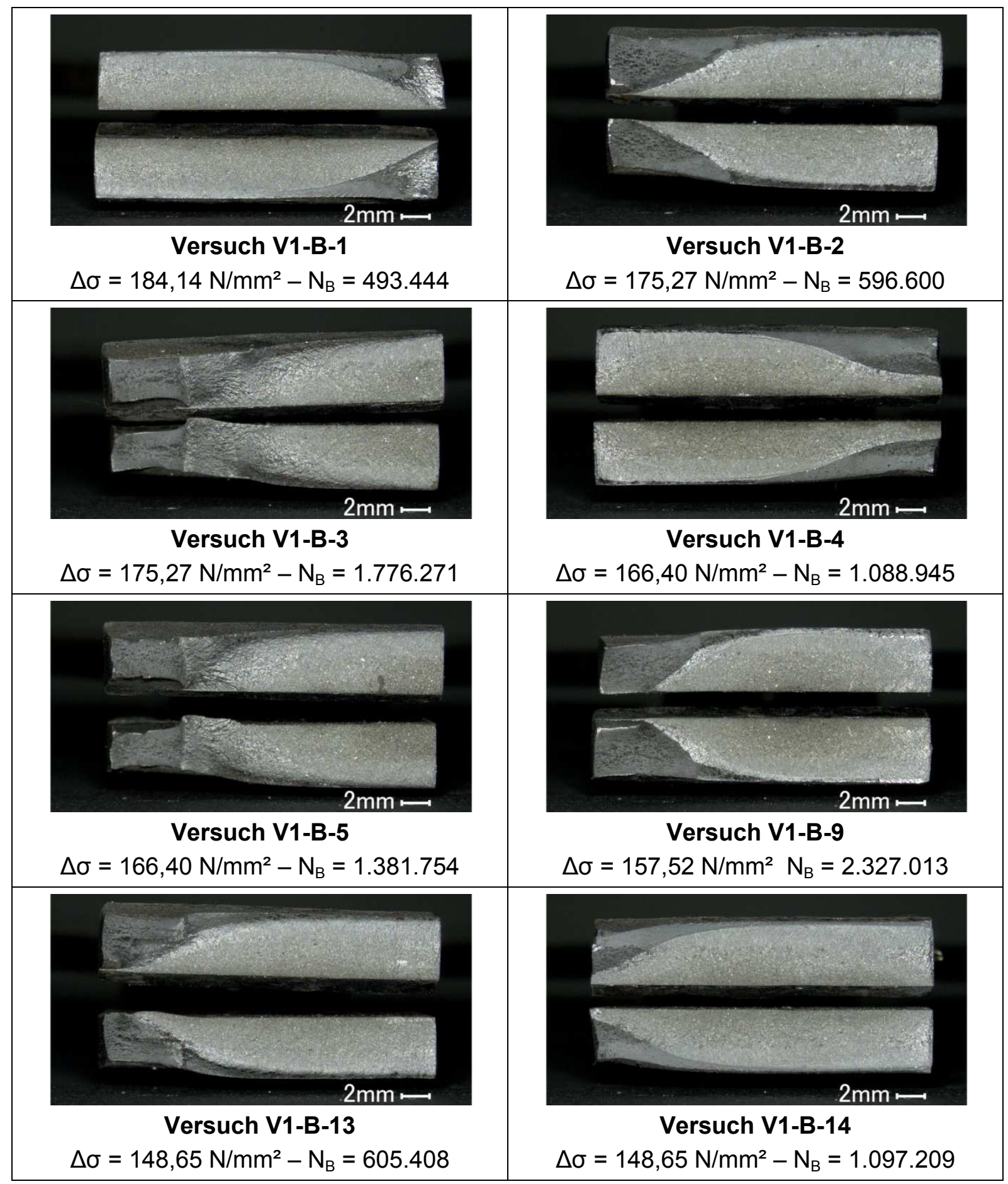


Anhang l.1.3 C - Hochgesetzte Durchläufer

Datenübersicht

\begin{tabular}{|c|c|c|c|c|c|}
\hline Versuch & Probe & $\begin{array}{c}\boldsymbol{\Delta} \boldsymbol{\sigma} \\
{\left[\mathbf{N} / \mathbf{m m}^{2}\right]}\end{array}$ & $\begin{array}{c}\mathbf{N}_{\mathbf{B}} \\
{[-]}\end{array}$ & Ergebnis & Rissausgang \\
\hline V1-C-1 & 30 & 332,80 & 84.981 & Bruch & W \\
\hline V1-C-2 & 26 & 332,80 & 113.017 & Bruch & W \\
\hline V1-C-3 & 29 & 277,33 & 124.365 & Bruch & W \\
\hline V1-C-4 & 25 & 277,33 & 144.789 & Bruch & N \\
\hline V1-C-5 & 35 & 277,33 & 156.145 & Bruch & W \\
\hline V1-C-6 & 34 & 221,86 & 215.360 & Bruch & W \\
\hline V1-C-7 & 28 & 221,86 & 245.091 & Bruch & N \\
\hline V1-C-8 & 33 & 221,86 & 261.589 & Bruch & W \\
\hline
\end{tabular}

W - Übergang an Wurzelüberhöhung

$\mathrm{N}$ - Übergang an Nahtüberhöhung 


\section{Übersichtsaufnahmen der Bruchflächen}

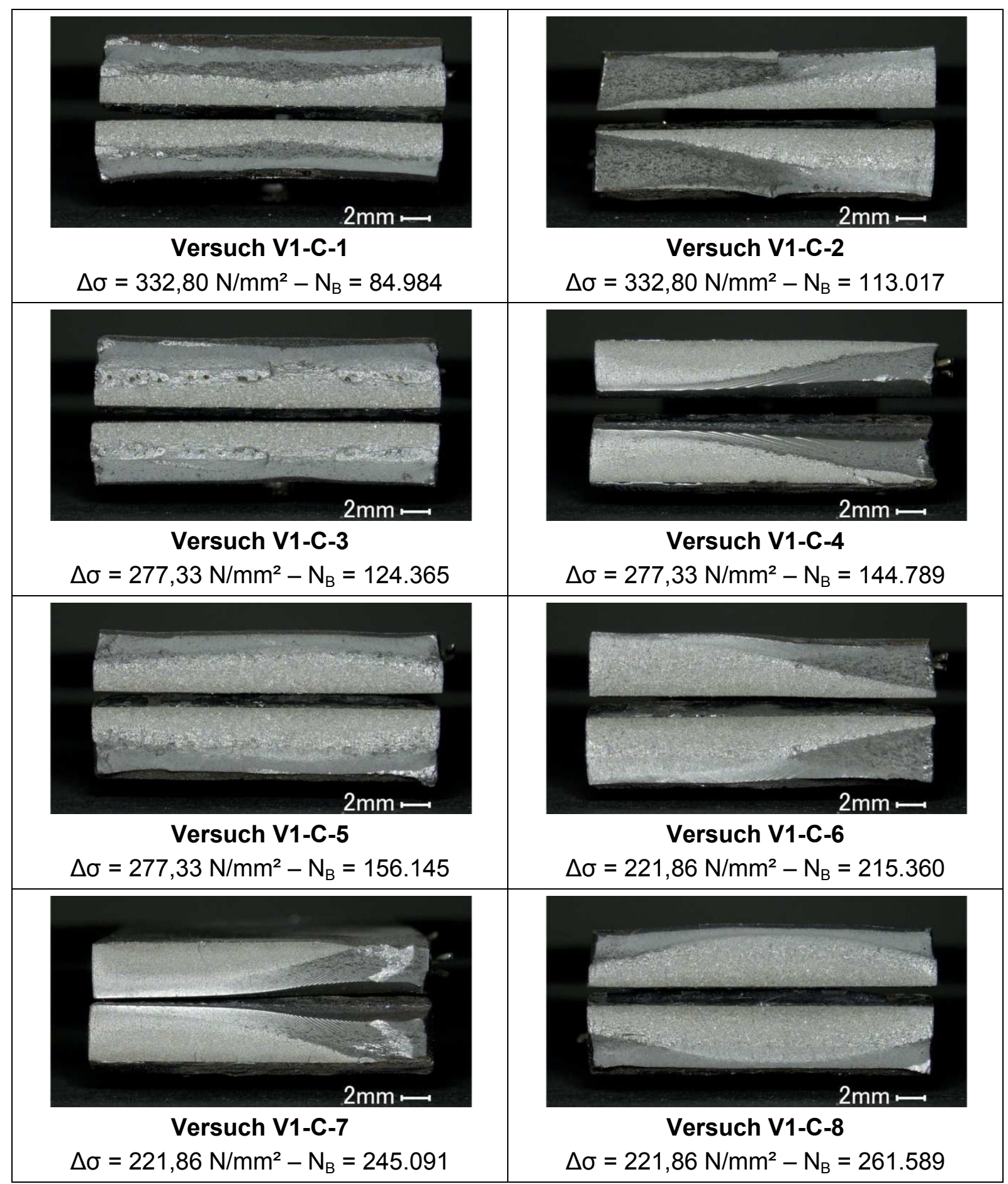




\section{Anhang 1.2 Versuchsreihe 2}

Prüfmaschine: hochfrequente Prüfvorrichtung

Prüffrequenz $\quad f=390 \mathrm{~Hz}$

Spannungsverhältnis $\quad R=0,1$

\section{Anhang l.2.1 A - Zeitfestigkeitsbereich}

\section{Datenübersicht}

\begin{tabular}{|c|c|c|c|c|c|c|}
\hline Versuch & Probe & $\begin{array}{c}\Delta \boldsymbol{\sigma} \\
{\left[\mathbf{N} / \mathbf{m m}^{2}\right]}\end{array}$ & $\begin{array}{c}\mathbf{N}_{\mathbf{A}} \\
{[-]}\end{array}$ & $\begin{array}{c}\mathbf{N}_{\mathrm{B}}{ }^{*} \\
{[-]}\end{array}$ & Ergebnis & $\begin{array}{c}\text { Riss- } \\
\text { ausgang }\end{array}$ \\
\hline V2-A-1 & 36 & 277,33 & 63.211 & 75.266 & Bruch $^{*}$ & $\mathrm{~W}$ \\
\hline V2-A-2 & 37 & 277,33 & 96.123 & 116.704 & Bruch $^{*}$ & $\mathrm{~W}$ \\
\hline V2-A-3 & 38 & 277,33 & 100.233 & 122.143 & Bruch $^{*}$ & $\mathrm{~W}$ \\
\hline V2-A-4 & 39 & 277,33 & 150.284 & 179.912 & Bruch $^{*}$ & $\mathrm{~W}$ \\
\hline V2-A-5 & 40 & 277,33 & 165.011 & 193.302 & Bruch $^{*}$ & $\mathrm{~W}$ \\
\hline V2-A-6 & 41 & 277,33 & 205.550 & 233.891 & Bruch $^{*}$ & $\mathrm{~W}$ \\
\hline V2-A-7 & 42 & 221,86 & 177.286 & 199.433 & Bruch $^{*}$ & $\mathrm{~W}$ \\
\hline V2-A-8 & 43 & 221,86 & 232.006 & 262.942 & Bruch $^{*}$ & $\mathrm{~W}$ \\
\hline V2-A-9 & 44 & 221,86 & 267.585 & 296.771 & Bruch $^{*}$ & $\mathrm{~W}$ \\
\hline V2-A-10 & 45 & 221,86 & 270.756 & 315.401 & Bruch $^{*}$ & $\mathrm{~W}$ \\
\hline V2-A-11 & 46 & 221,86 & 437.079 & 502.197 & Bruch $^{*}$ & $\mathrm{~W}$ \\
\hline V2-A-12 & 47 & 221,86 & 588.418 & 658.443 & Bruch $^{*}$ & $\mathrm{~W}$ \\
\hline
\end{tabular}

* Bruch ist definiert bei Erreichen des Abbruchkriteriums $F_{v}=F_{a}$

W - Übergang an Wurzelüberhöhung $\quad$ N - Übergang an Nahtüberhöhung 
Übersichtsaufnahmen der Bruchflächen

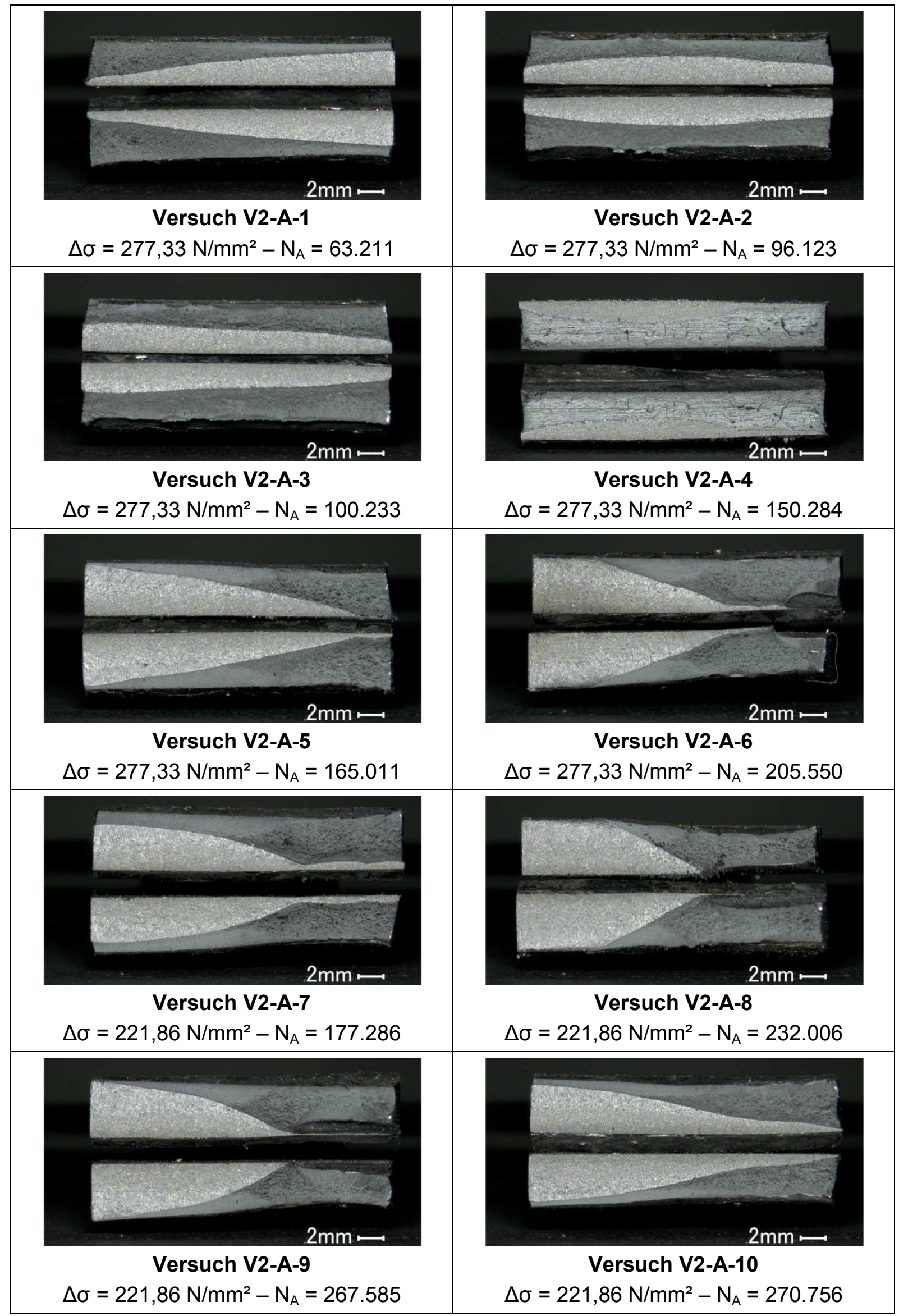




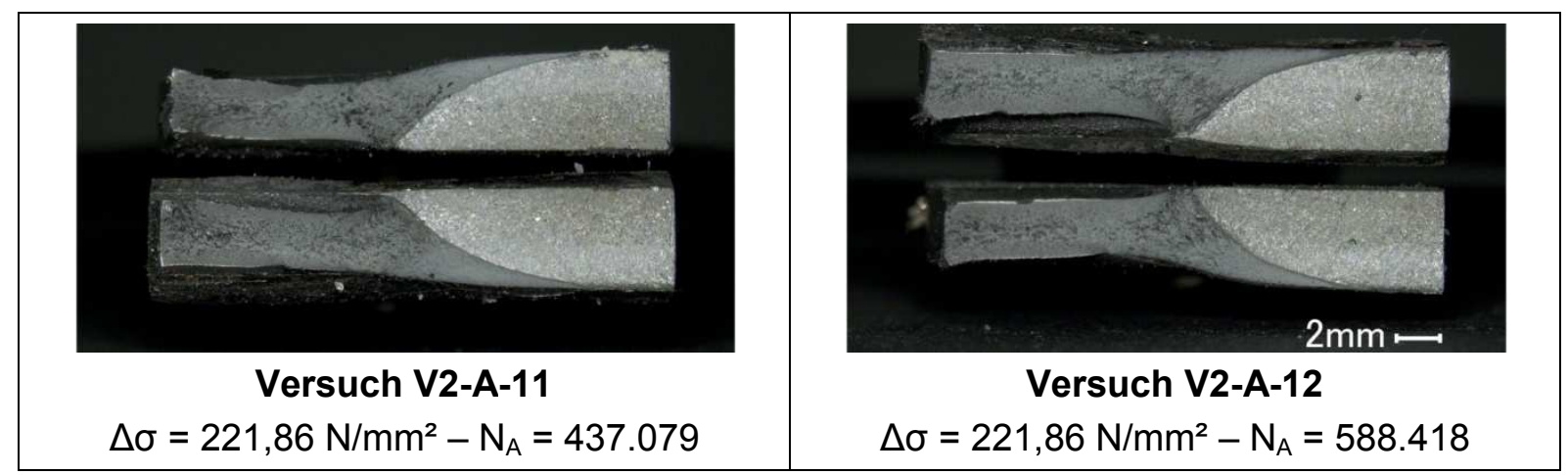




\section{Anhang I.2.2 B - Übergangsbereich}

\section{Datenübersicht}

\begin{tabular}{|c|c|c|c|c|c|c|}
\hline Versuch & Probe & $\begin{array}{c}\mathbf{\Delta} \boldsymbol{\sigma} \\
{\left[\mathbf{N} / \mathbf{m m}^{2}\right]}\end{array}$ & $\begin{array}{c}\mathbf{N}_{\mathbf{A}} \\
{[-]}\end{array}$ & $\begin{array}{c}\mathbf{N}_{\mathbf{B}}{ }^{*} \\
{[-]}\end{array}$ & Ergebnis & $\begin{array}{c}\text { Riss- } \\
\text { ausgang }\end{array}$ \\
\hline V2-B-1 & 48 & 162,50 & 1.036 .609 & 1.248 .461 & Bruch $^{*}$ & W \\
\hline V2-B-2 & 49 & 162,50 & 1.351 .004 & 1.591 .389 & Bruch* $^{*}$ & W \\
\hline V2-B-3 & 50 & 150,00 & 663.686 & 1.039 .646 & Bruch* $^{*}$ & W \\
\hline V2-B-4 & 51 & 150,00 & 726.273 & 975.917 & Bruch* $^{*}$ & W \\
\hline V2-B-5 & 52 & 150,00 & 1.131 .763 & 1.307 .895 & Bruch* $^{*}$ & W \\
\hline V2-B-6 & 53 & 150,00 & 1.364 .258 & 1.546 .649 & Bruch $^{*}$ & W \\
\hline V2-B-7 & 54 & 150,00 & 500.000 .000 & 500.000 .000 & Durchläufer $^{*}$ & - \\
\hline V2-B-8 & 55 & 137,50 & 1.213 .444 & 1.620 .230 & Bruch* $^{*}$ & W \\
\hline V2-B-9 & 56 & 137,50 & 1.769 .963 & 2.135 .217 & Bruch* $^{*}$ & W \\
\hline V2-B-10 & 57 & 137,50 & 1.858 .886 & 2.365 .094 & Bruch* $^{*}$ & W \\
\hline V2-B-11 & 58 & 137,50 & 500.000 .000 & 500.000 .000 & Durchläufer & - \\
\hline V2-B-12 & 59 & 137,50 & 500.000 .000 & 500.000 .000 & Durchläufer & - \\
\hline V2-B-13 & 60 & 137,50 & 500.000 .000 & 500.000 .000 & Durchläufer & - \\
\hline V2-B-14 & 61 & 125,00 & 500.000 .000 & 500.000 .000 & Durchläufer & - \\
\hline V2-B-15 & 62 & 125,00 & 500.000 .000 & 500.000 .000 & Durchläufer & - \\
\hline V2-B-16 & 63 & 125,00 & 500.000 .000 & 500.000 .000 & Durchläufer & - \\
\hline
\end{tabular}

* Bruch ist definiert bei Erreichen des Abbruchkriteriums $F_{v}=F_{a}$

W - Übergang an Wurzelüberhöhung

$\mathrm{N}$ - Übergang an Nahtüberhöhung 


\section{Übersichtsaufnahmen der Bruchflächen}

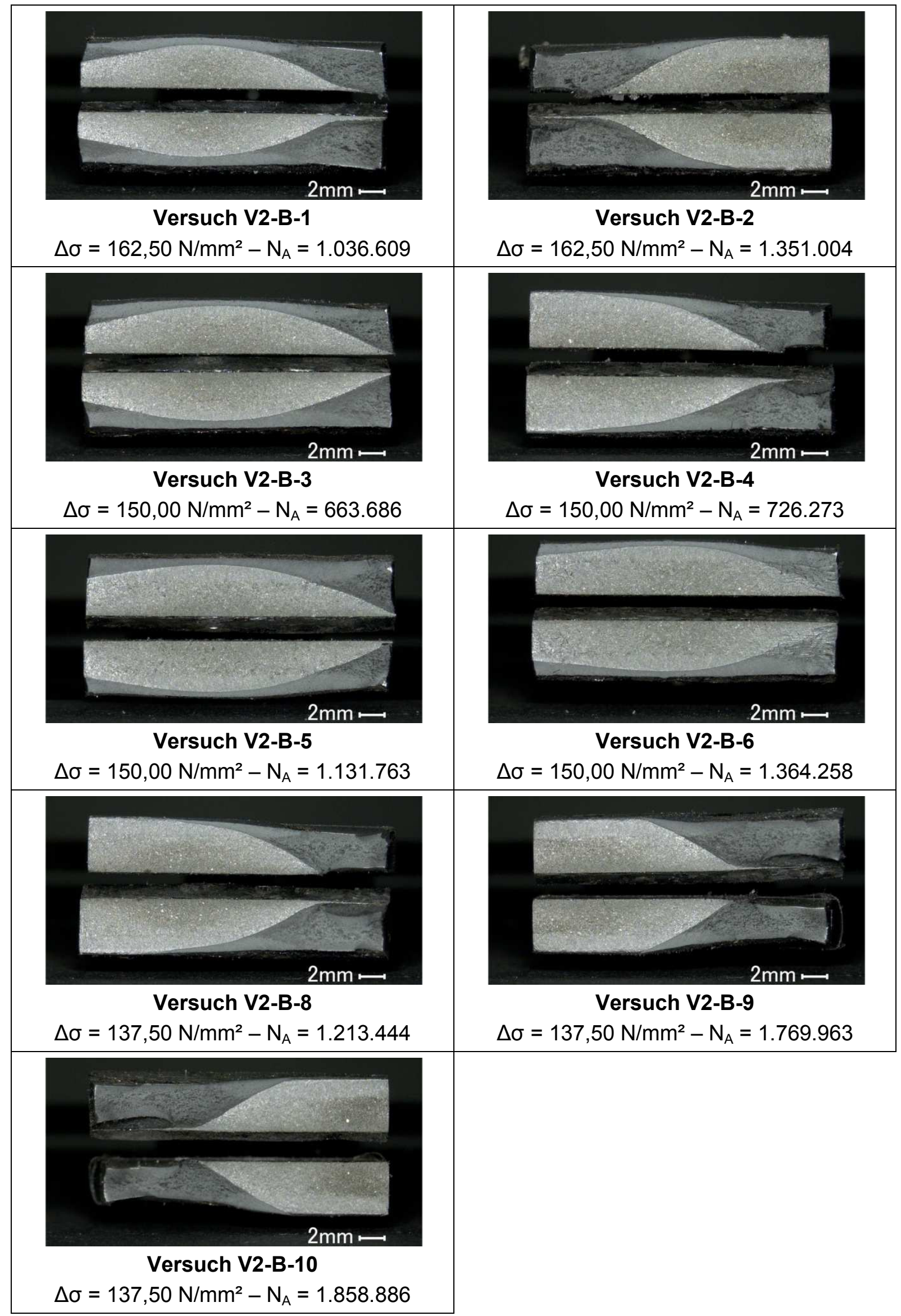




\section{Anhang l.2.3 C - Hochgesetzte Durchläufer}

Datenübersicht

\begin{tabular}{|c|c|c|c|c|c|c|}
\hline Versuch & Probe & $\begin{array}{c}\mathbf{\Delta} \boldsymbol{\sigma} \\
{\left[\mathbf{N} / \mathbf{m m}^{2}\right]}\end{array}$ & $\begin{array}{c}\mathbf{N}_{\mathbf{A}} \\
{[-]}\end{array}$ & $\begin{array}{c}\mathbf{N}_{\mathbf{B}}{ }^{*} \\
{[-]}\end{array}$ & Ergebnis & $\begin{array}{c}\text { Riss- } \\
\text { ausgang }\end{array}$ \\
\hline V2-C-1 & 62 & 277,33 & 163.048 & 185.386 & Bruch $^{*}$ & $\mathrm{~W}$ \\
\hline V2-C-2 & 59 & 277,33 & 210.486 & 253.657 & Bruch $^{*}$ & $\mathrm{~W}$ \\
\hline V2-C-3 & 58 & 277,33 & 216.604 & 238.924 & Bruch* $^{*}$ & $\mathrm{~N}$ \\
\hline V2-C-4 & 54 & 221,86 & 152.457 & 198.068 & Bruch $^{*}$ & $\mathrm{~W}$ \\
\hline V2-C-5 & 63 & 221,86 & 165.800 & 229.275 & Bruch $^{*}$ & $\mathrm{~W}$ \\
\hline V2-C-6 & 61 & 221,86 & 212.924 & 292.508 & Bruch $^{*}$ & $\mathrm{~W}$ \\
\hline V2-C-7 & 60 & 221,86 & 362.663 & 456.208 & Bruch $^{*}$ & $\mathrm{~W}$ \\
\hline
\end{tabular}

* Bruch ist definiert bei Erreichen des Abbruchkriteriums $F_{v}=F_{a}$

W - Übergang an Wurzelüberhöhung N N N Übergang an Nahtüberhöhung 


\section{Übersichtsaufnahmen der Bruchflächen}

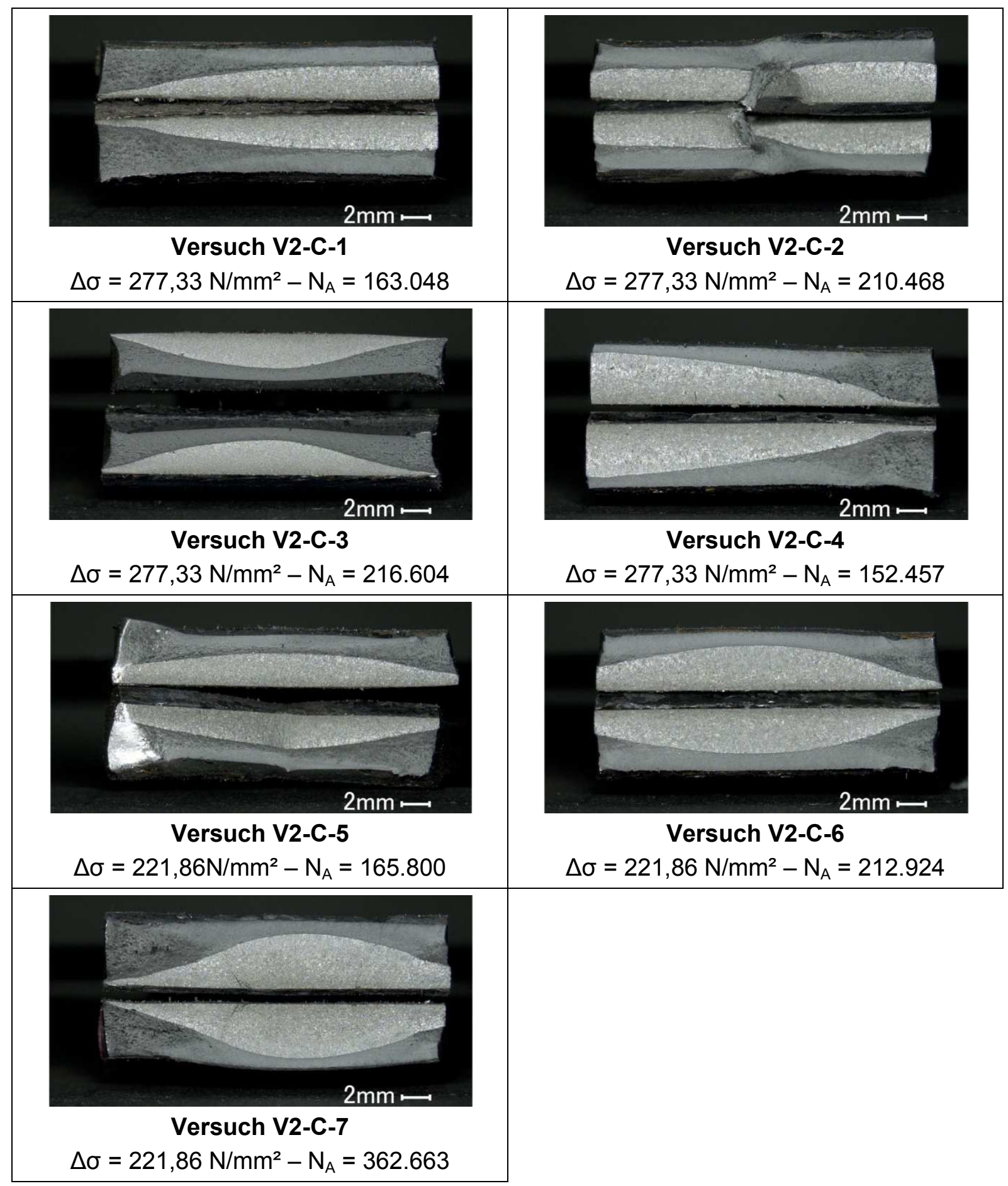




\section{Anhang I.3 Versuchsreihe 3}

Prüfmaschine: hochfrequente Prüfvorrichtung

Prüffrequenz $\quad f=390 \mathrm{~Hz}$

Spannungsverhältnis $\quad R=0,5$

\section{Anhang l.3.1 A-Zeitfestigkeitsbereich}

\section{Datenübersicht}

\begin{tabular}{|c|c|c|c|c|c|c|}
\hline Versuch & Probe & $\begin{array}{c}\Delta \boldsymbol{\sigma} \\
{\left[\mathbf{N} / \mathbf{m m}^{2}\right]}\end{array}$ & $\begin{array}{c}\mathbf{N}_{\mathbf{A}} \\
{[-]}\end{array}$ & $\begin{array}{c}\mathbf{N}_{\mathbf{B}}{ }^{* *} \\
{[-]}\end{array}$ & Ergebnis & $\begin{array}{c}\text { Riss- } \\
\text { ausgang }\end{array}$ \\
\hline V3-A-1 & 64 & 187,50 & 240.836 & 314.192 & Bruch $^{* *}$ & W \\
\hline V3-A-2 & 65 & 187,50 & 267.436 & 341.951 & Bruch $^{* *}$ & W \\
\hline V3-A-3 & 66 & 187,50 & 286.576 & 346.629 & Bruch $^{* *}$ & W \\
\hline V3-A-4 & 67 & 187,50 & 314.022 & 389.783 & Bruch $^{* *}$ & W \\
\hline V3-A-5 & 68 & 187,50 & 559.743 & 666.441 & Bruch $^{* *}$ & W \\
\hline V3-A-6 & 69 & 162,50 & 473.664 & 552.907 & Bruch $^{* *}$ & W \\
\hline V3-A-7 & 70 & 162,50 & 474.521 & 575.575 & Bruch $^{* *}$ & W \\
\hline V3-A-8 & 71 & 162,50 & 676.241 & 858.928 & Bruch $^{* *}$ & W \\
\hline V3-A-9 & 72 & 162,50 & 694.297 & 912.729 & Bruch $^{* *}$ & W \\
\hline V3-A-10 & 73 & 162,50 & 893.432 & 1.066 .427 & Bruch $^{* *}$ & W \\
\hline
\end{tabular}

** Bruch ist definiert bei Erreichen des Abbruchkriteriums eines Vorspankraftabfalls von $5 \mathrm{kN}$ W - Übergang an Wurzelüberhöhung $\mathrm{N}$ - Übergang an Nahtüberhöhung 


\section{Übersichtsaufnahmen der Bruchflächen}

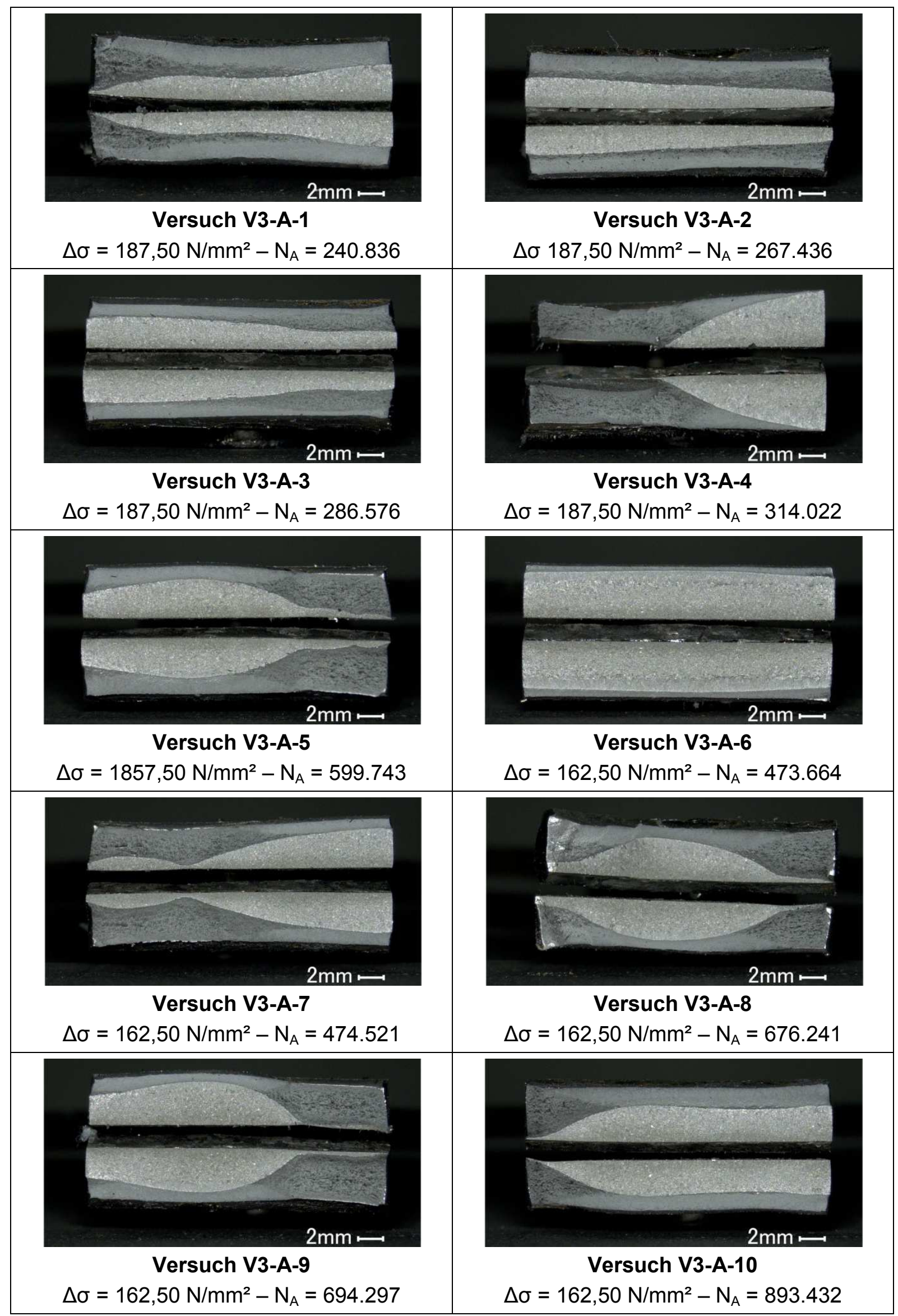




\section{Anhang I.3.2 B - Übergangsbereich}

Datenübersicht

\begin{tabular}{|c|c|c|c|c|c|c|}
\hline Versuch & Probe & $\begin{array}{c}\mathbf{\Delta} \boldsymbol{\sigma} \\
{\left[\mathbf{N} / \mathbf{m m}^{2}\right]}\end{array}$ & $\begin{array}{c}\mathbf{N}_{\mathbf{A}} \\
{[-]}\end{array}$ & $\begin{array}{c}\mathbf{N}_{\mathbf{B}}{ }^{* *} \\
{[-]}\end{array}$ & Ergebnis & $\begin{array}{c}\text { Riss- } \\
\text { ausgang }\end{array}$ \\
\hline V3-B-1 & 74 & 150,00 & 1.167 .484 & 1.335 .299 & Bruch $^{* *}$ & W \\
\hline V3-B-2 & 75 & 150,00 & 1.883 .461 & 2.023 .369 & Bruch $^{* *}$ & W \\
\hline V3-B-3 & 76 & 143,75 & 645.254 & 843.097 & Bruch $^{* *}$ & W \\
\hline V3-B-4 & 77 & 143,75 & 980.478 & 1.208 .097 & Bruch $^{* *}$ & W \\
\hline V3-B-5 & 78 & 143,75 & 1.068 .806 & 1.282 .093 & Bruch $^{* *}$ & W \\
\hline V3-B-6 & 79 & 143,75 & 1.099 .166 & 1.354 .891 & Bruch $^{* *}$ & W \\
\hline V3-B-7 & 80 & 143,75 & 2.692 .584 & 2.941 .678 & Bruch $^{* *}$ & N \\
\hline V3-B-8 & 81 & 143,75 & 500.000 .000 & 500.000 .000 & Durchläufer $^{*}$ & - \\
\hline V3-B-9 & 82 & 137,50 & 1.106 .733 & 1.401 .889 & Bruch $^{* *}$ & W \\
\hline V3-B-10 & 83 & 137,50 & 2.342 .732 & 2.610 .084 & Bruch $^{* *}$ & W \\
\hline V3-B-11 & 84 & 137,50 & 500.000 .000 & 500.000 .000 & Durchläufer & - \\
\hline V3-B-12 & 85 & 137,50 & 500.000 .000 & 500.000 .000 & Durchläufer & - \\
\hline V3-B-13 & 86 & 137,50 & 500.000 .000 & 500.000 .000 & Durchläufer & - \\
\hline V3-B-14 & 87 & 137,50 & 500.000 .000 & 500.000 .000 & Durchläufer & - \\
\hline V3-B-15 & 88 & 131,25 & 500.000 .000 & 500.000 .000 & Durchläufer & - \\
\hline V3-B-16 & 89 & 131,25 & 500.000 .000 & 500.000 .000 & Durchläufer & - \\
\hline
\end{tabular}

** Bruch ist definiert bei Erreichen des Abbruchkriteriums eines Vorspankraftabfalls von $5 \mathrm{kN}$ W - Übergang an Wurzelüberhöhung $\mathrm{N}$ - Übergang an Nahtüberhöhung 


\section{Übersichtsaufnahmen der Bruchflächen}

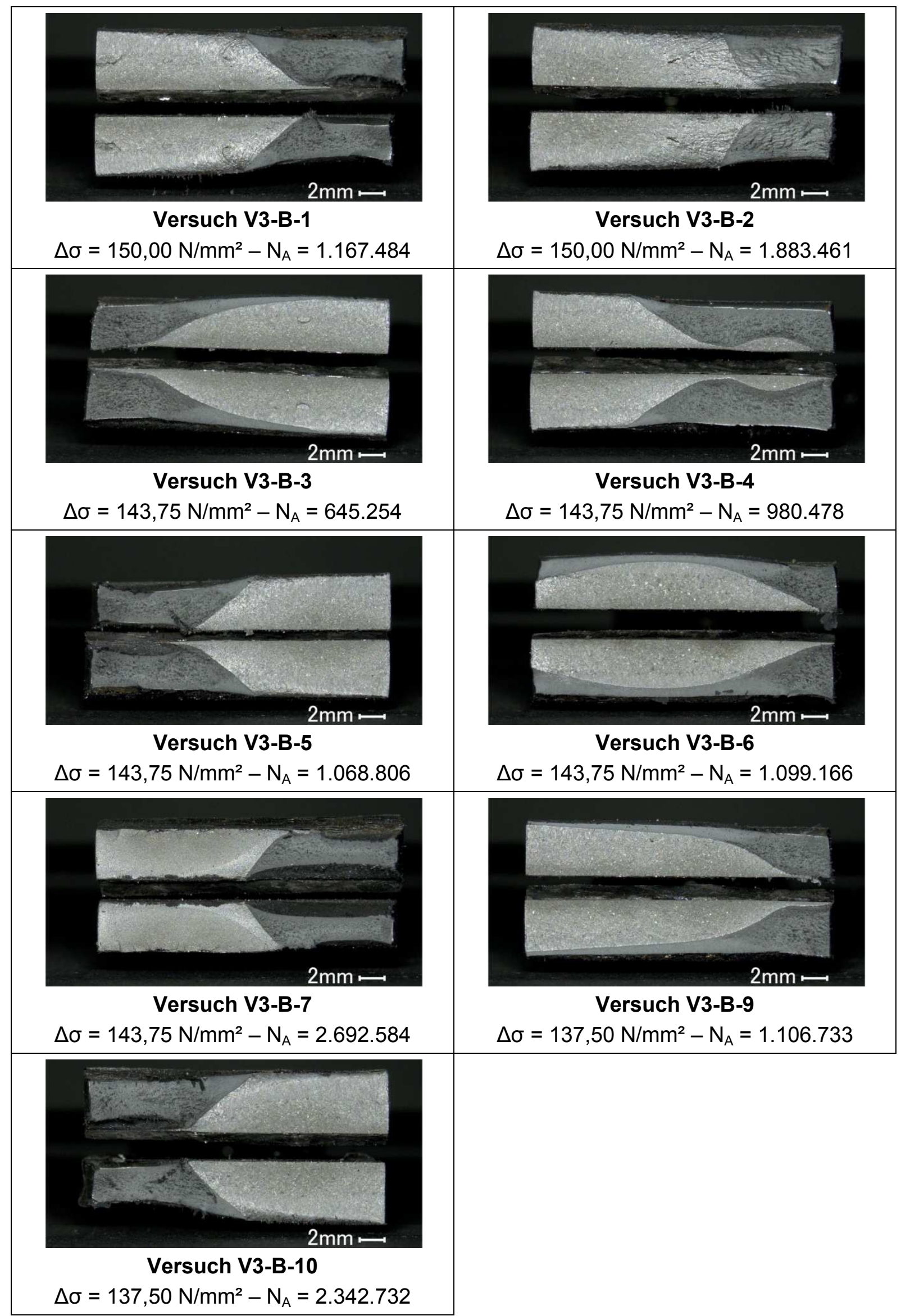




\section{Anhang l.3.3 C - Hochgesetzte Durchläufer}

Datenübersicht

\begin{tabular}{|c|c|c|c|c|c|c|}
\hline Versuch & Probe & $\begin{array}{c}\boldsymbol{\Delta} \boldsymbol{\sigma} \\
{\left[\mathbf{N} / \mathbf{m m}^{2}\right]}\end{array}$ & $\begin{array}{c}\mathbf{N}_{\mathbf{A}} \\
{[-]}\end{array}$ & $\begin{array}{c}\mathbf{N}_{\mathbf{B}}{ }^{* *} \\
{[-]}\end{array}$ & Ergebnis & $\begin{array}{c}\text { Riss- } \\
\text { ausgang }\end{array}$ \\
\hline V3-C-1 & 89 & 187,50 & 471.518 & 579.301 & Bruch $^{* *}$ & W \\
\hline V3-C-2 & 81 & 187,50 & 536.176 & 619.695 & Bruch $^{* *}$ & W \\
\hline V3-C-3 & 84 & 187,50 & 585.273 & 674.546 & Bruch $^{* *}$ & W \\
\hline V3-C-4 & 85 & 162,50 & 359.283 & 462.232 & Bruch $^{* *}$ & W \\
\hline V3-C-5 & 86 & 162,50 & 441.711 & 583.667 & Bruch $^{* *}$ & W \\
\hline V3-C-6 & 87 & 162,50 & 672.639 & 824.104 & Bruch $^{* *}$ & W \\
\hline V3-C-7 & 88 & 162,50 & 2.491 .698 & 2.731 .805 & Bruch $^{* *}$ & N \\
\hline
\end{tabular}

${ }^{* *}$ Bruch ist definiert bei Erreichen des Abbruchkriteriums eines Vorspankraftabfalls von $5 \mathrm{kN}$

W - Übergang an Wurzelüberhöhung

$\mathrm{N}$ - Übergang an Nahtüberhöhung 


\section{Übersichtsaufnahmen der Bruchflächen}

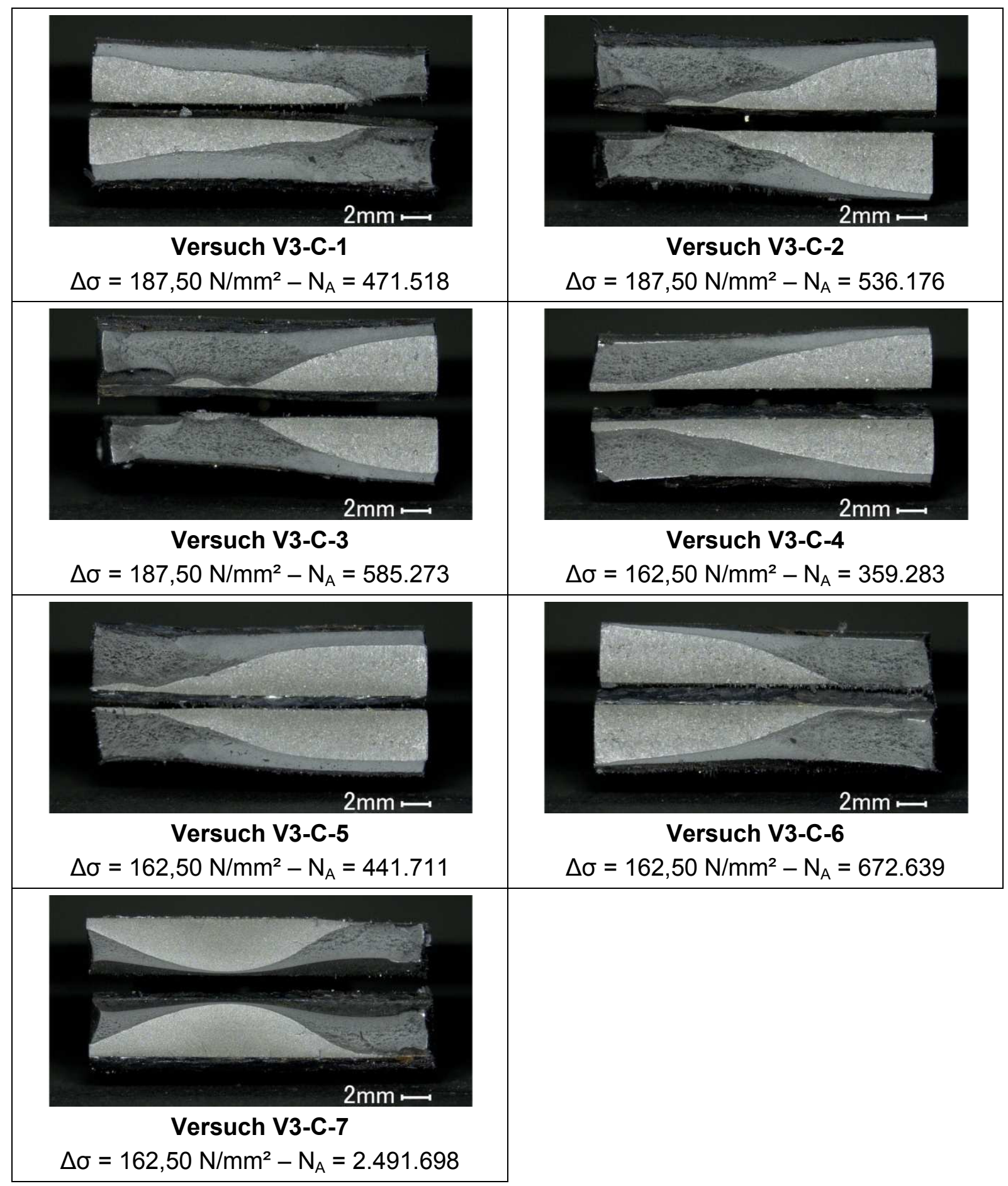




\section{Anhang J Auswertung der Ermüdungsversuche}

\section{Anhang J.1 Übergangsbereich - Treppenstufenverfahren}

Die einzelnen Versuchsreihen im Übergangsbereich wurden mit dem verbesserten Treppenstufenverfahren nach Hück [Hück, 1983] angewendet. Diagramme und Tabellen aus denen Hilfswerte abgelesen wurden, sind dort zu entnehmen.

\section{Anhang J.1.1 Versuchsreihe 1-B}

\section{Treppenstufenfolge}

\begin{tabular}{|c|c|c|c|c|c|c|c|c|c|c|c|c|c|c|c|c|c|c|c|c|c|}
\hline$\left[\mathrm{N} / \mathrm{mm}^{2}\right]$ & $\begin{array}{c}N \\
\dot{\omega} \\
1 \\
\dot{>} \\
\end{array}$ & 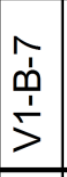 & $\begin{array}{l}m \\
1 \\
1 \\
\dot{>} \\
>\end{array}$ & $\left|\begin{array}{l}\infty \\
1 \\
1 \\
1 \\
1 \\
>\end{array}\right|$ & 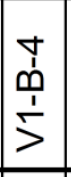 & 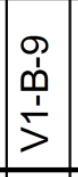 & 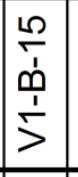 & $\begin{array}{c}m \\
\stackrel{m}{\prime} \\
\dot{1} \\
\stackrel{1}{>}\end{array}$ & $\begin{array}{l}\stackrel{0}{1} \\
\stackrel{1}{1} \\
\stackrel{1}{>}\end{array}$ & $\begin{array}{l}+ \\
\stackrel{1}{1} \\
\stackrel{1}{1} \\
\dot{>}\end{array}$ & 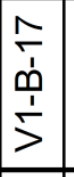 & $\begin{array}{l}\stackrel{0}{1} \\
\text { m} \\
\stackrel{1}{>} \\
>\end{array}$ & \begin{tabular}{|l|} 
\\
0 \\
1 \\
1 \\
1 \\
$>$ \\
\end{tabular} & 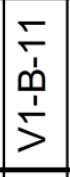 & \begin{tabular}{l}
0 \\
0 \\
1 \\
1 \\
\multicolumn{1}{|c|}{} \\
\end{tabular} & $\begin{array}{c}\stackrel{N}{\prime} \\
\text { m} \\
\stackrel{1}{>} \\
>\end{array}$ & 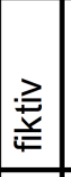 & $\mathbf{i}$ & fi & $i^{*} \mathrm{fi}$ & $\left|\mathbf{i}{ }^{*} \mathbf{f i}\right|$ \\
\hline 175,27 & $\mathbf{X}$ & & $\mathbf{X}$ & & $X$ & & & & & & & & $\mathbf{X}$ & & $\bar{x}$ & & + & 2 & 6 & 12 & 24 \\
\hline 157,52 & & 0 & & 0 & & X & & $X$ & & X & & 0 & & 0 & & 0 & & 1 & 8 & 8 & 8 \\
\hline 139,77 & & & & & & & 0 & & 0 & & 0 & & & & & & & 0 & 3 & 0 & 0 \\
\hline Probe & 20 & 25 & 21 & 26 & 22 & 27 & 33 & 31 & 34 & 32 & 35 & 28 & 23 & 29 & 24 & 30 & - & $\Sigma$ & 17 & 20 & 32 \\
\hline & & & & & & & & & & & & & & & & & & & $\mathbf{F}$ & A & B \\
\hline
\end{tabular}

\section{Auswertung}

Stufensprung

Dauerfestigkeit $\left(P_{u}=50 \%\right)$

Varianz

Hilfswert (Ablesung)

Standardabweichung

Hilfswert (Ablesung)

Dauerfestigkeit $\left(P_{\ddot{u}}=10 \%\right)$

Dauerfestigkeit $\left(P_{u}=90 \%\right)$ $d=17,75 \mathrm{~N} / \mathrm{mm}^{2}$ (verdoppelt, da sonst nicht auswertbar)

$\Delta \sigma_{D, 50 \%}=\overline{\mathrm{x}}=\mathrm{x}_{0}+\mathrm{d} \cdot \frac{\mathrm{A}}{\mathrm{F}}=139,77+17,75 \cdot \frac{20}{17}=160,66 \mathrm{~N} / \mathrm{mm}^{2}$

$k=\frac{F \cdot B-A^{2}}{F^{2}}=\frac{17 \cdot 32-20^{2}}{17^{2}}=0,5$

$\frac{s}{d}=0,5$

$s=0,5 \cdot d=0,5 \cdot 17,75=8,88 \mathrm{~N} / \mathrm{mm}^{2}$

$K_{2}=1,28$

$\Delta \sigma_{D, 10 \%}=\overline{\mathrm{x}}+K_{2} \cdot \mathrm{s}=160,66+1,28 \cdot 8,88=172,02 \mathrm{~N} / \mathrm{mm}^{2}$

$\Delta \sigma_{D, 90 \%}=\overline{\mathrm{x}}-K_{2} \cdot \mathrm{s}=160,66-1,28 \cdot 8,88=149,30 \mathrm{~N} / \mathrm{mm}^{2}$ 
Anhang J.1.2 Versuchsreihe 2-B

\section{Treppenstufenfolge}

\begin{tabular}{|c|c|c|c|c|c|c|c|c|c|c|c|c|c|c|c|c|c|c|c|c|}
\hline$\left[\mathrm{N} / \mathrm{mm}^{2}\right]$ & 㐫 & $\begin{array}{l}m \\
\dot{1} \\
\dot{1} \\
\text { Ny }\end{array}$ & $\begin{array}{l}\infty \\
\infty \\
\stackrel{1}{1} \\
\text { ஸे }\end{array}$ & 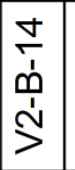 & 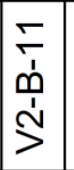 & 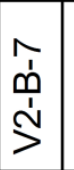 & 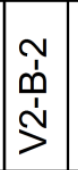 & 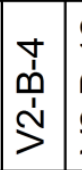 & 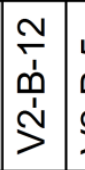 & $\begin{array}{ll}L & 0 \\
\dot{1} & \\
\dot{1} & \\
\text { N } & \end{array}$ & 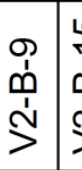 & 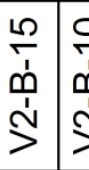 & 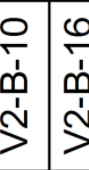 & 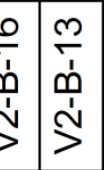 & $\begin{array}{l}0 \\
\dot{1} \\
\dot{1} \\
\stackrel{3}{>}\end{array}$ & 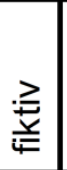 & i & fi & $i^{*} \mathrm{fi}$ & $i^{2 *} \mathbf{f i}$ \\
\hline 162,50 & $x$ & & & & & & $\mathbf{x}$ & & & & & & & & & & 3 & 2 & 6 & 18 \\
\hline 150,00 & & $\mathbf{X}$ & & & & 0 & & $\mathbf{X}$ & & $X$ & & & & & $X$ & & 2 & 5 & 10 & 20 \\
\hline 137,50 & & & $X$ & & 0 & & & & 0 & & $\mathbf{X}$ & & 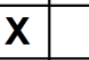 & 0 & & + & 1 & 7 & 7 & 7 \\
\hline 125,00 & & & & 0 & & & & & & & & 0 & 0 & 0 & & & 0 & 3 & 0 & 0 \\
\hline Probe & 48 & 50 & 55 & 61 & 58 & 54 & 49 & \begin{tabular}{|l|}
51 \\
\end{tabular} & \begin{tabular}{l|l}
58 & 5
\end{tabular} & \begin{tabular}{|l|l}
52 & 5 \\
\end{tabular} & \begin{tabular}{l|l}
56 & 6
\end{tabular} & \begin{tabular}{l|l}
62 & 5 \\
\end{tabular} & \begin{tabular}{l|l}
57 & 6 \\
\end{tabular} & \begin{tabular}{|l|l|}
33 & 60 \\
\end{tabular} & 53 & - & $\Sigma$ & 17 & 23 & 45 \\
\hline & & & & & & & & & & & & & & & & & & $\mathbf{F}$ & A & B \\
\hline
\end{tabular}

\section{Auswertung}

Stufensprung

$d=12,50 \mathrm{~N} / \mathrm{mm}^{2}$

Dauerfestigkeit $\left(P_{u ̈}=50 \%\right)$

$\Delta \sigma_{D, 50 \%}=\overline{\mathrm{x}}=\mathrm{x}_{0}+\mathrm{d} \cdot \frac{\mathrm{A}}{\mathrm{F}}=125,00+12,50 \cdot \frac{23}{17}=141,91 \mathrm{~N} / \mathrm{mm}^{2}$

Varianz

$k=\frac{F \cdot B-A^{2}}{F^{2}}=\frac{17 \cdot 45-23^{2}}{17^{2}}=0,8$

Hilfswert (Ablesung)

$\frac{s}{d}=1,45$

Standardabweichung

$s=1,45 \cdot d=1,45 \cdot 12,50=18,13 \mathrm{~N} / \mathrm{mm}^{2}$

Hilfswert (Ablesung)

$K_{2}=1,28$

Dauerfestigkeit $\left(\mathrm{P}_{\mathrm{u}}=10 \%\right)$

$\Delta \sigma_{D, 10 \%}=\overline{\mathrm{x}}+K_{2} \cdot \mathrm{s}=141,91+1,28 \cdot 18,13=165,11 \mathrm{~N} / \mathrm{mm}^{2}$

Dauerfestigkeit $\left(P_{u}=90 \%\right)$

$\Delta \sigma_{D, 90 \%}=\overline{\mathrm{x}}-K_{2} \cdot \mathrm{s}=141,91-1,28 \cdot 18,13=118,71 \mathrm{~N} / \mathrm{mm}^{2}$ 
Anhang J.1.3 Versuchsreihe 3-B

\section{Treppenstufenfolge}

\begin{tabular}{|c|c|c|c|c|c|c|c|c|c|c|c|c|c|c|c|c|c|c|c|c|}
\hline$\left[\mathrm{N} / \mathrm{mm}^{2}\right]$ & $\begin{array}{l}\overline{\dot{m}} \\
\dot{\rho} \\
\end{array}$ & $\begin{array}{l}m \\
\text { m } \\
\text { m่ }\end{array}$ & $\begin{array}{l}\check{c} \\
\dot{m} \\
\dot{m}\end{array}$ & $\begin{array}{l}\infty \\
1 \\
m \\
m \\
m\end{array}$ & $\begin{array}{l}\text { N } \\
\text { m} \\
\text { m } \\
\end{array}$ & 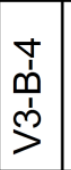 & $\begin{array}{l}\stackrel{N}{5} \\
\text { के } \\
\stackrel{3}{>}\end{array}$ & 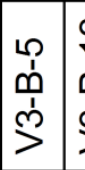 & $\begin{array}{l}m \\
\\
\dot{m} \\
m \\
\stackrel{s}{s}\end{array}$ & 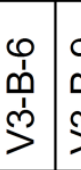 & 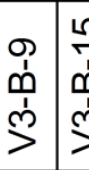 & 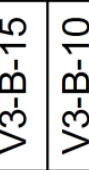 & 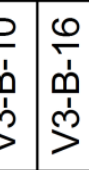 & 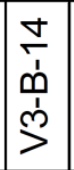 & $\begin{array}{l}\hat{n} \\
\dot{m} \\
\dot{s} \\
\end{array}$ & 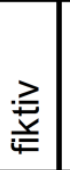 & $\mathbf{i}$ & fi & $i^{*} \mathbf{f i}$ & $i^{2 *} f i$ \\
\hline 150,00 & $\bar{x}$ & & & & $\bar{x}$ & & & & & & & & & & & & 3 & 2 & 6 & 18 \\
\hline 143,75 & & $\mathbf{X}$ & & 0 & & $\mathbf{X}$ & & $\mathbf{X}$ & & $\bar{X}$ & & & & & $\mathbf{X}$ & & 2 & 6 & 12 & 24 \\
\hline 137,50 & & & 0 & & & & 0 & & 0 & & $X$ & & $x$ & 0 & & + & 1 & 7 & 7 & 7 \\
\hline 131,25 & & & & & & & & & & & & 0 & 0 & & & & 0 & 2 & 0 & 0 \\
\hline Probe & 74 & 76 & 84 & 81 & 75 & 77 & \begin{tabular}{l|l}
85 & 7
\end{tabular} & \begin{tabular}{l|l}
78 & $\varepsilon$ \\
\end{tabular} & \begin{tabular}{l|l}
87 & 7 \\
\end{tabular} & \begin{tabular}{l|l}
79 & 8 \\
\end{tabular} & \begin{tabular}{l|l}
82 & 8 \\
\end{tabular} & \begin{tabular}{l|l}
88 & 83 \\
\end{tabular} & \begin{tabular}{l|l}
33 & 89 \\
\end{tabular} & 87 & 80 & - & $\Sigma$ & 17 & 25 & 49 \\
\hline & & & & & & & & & & & & & & & & & & $\mathbf{F}$ & $\mathbf{A}$ & B \\
\hline
\end{tabular}

\section{Auswertung}

Stufensprung

$$
d=6,25 \mathrm{~N} / \mathrm{mm}^{2}
$$

Dauerfestigkeit $\left(\mathrm{P}_{\mathrm{u}}=50 \%\right) \quad \Delta \sigma_{D, 50 \%}=\overline{\mathrm{x}}=\mathrm{x}_{0}+\mathrm{d} \cdot \frac{\mathrm{A}}{\mathrm{F}}=131,25+6,25 \cdot \frac{25}{17}=140,44 \mathrm{~N} / \mathrm{mm}^{2}$

Varianz

$$
k=\frac{F \cdot B-A^{2}}{F^{2}}=\frac{17 \cdot 49-25^{2}}{17^{2}}=0,7
$$

Hilfswert (Ablesung)

$$
\frac{s}{d}=1,10
$$

Standardabweichung

$s=1,10 \cdot d=1,45 \cdot 6,25=6,88 \mathrm{~N} / \mathrm{mm}^{2}$

Hilfswert (Ablesung)

$K_{2}=1,28$

Dauerfestigkeit $\left(\mathrm{P}_{\mathrm{u}}=10 \%\right) \quad \Delta \sigma_{D, 10 \%}=\overline{\mathrm{x}}+K_{2} \cdot \mathrm{s}=140,44+1,28 \cdot 6,88=149,24 \mathrm{~N} / \mathrm{mm}^{2}$

Dauerfestigkeit $\left(\mathrm{P}_{\ddot{u}}=90 \%\right) \quad \Delta \sigma_{D, 90 \%}=\overline{\mathrm{x}}-K_{2} \cdot \mathrm{s}=140,44-1,28 \cdot 6,88=131,64 \mathrm{~N} / \mathrm{mm}^{2}$ 


\section{Anhang J.2 Vergleich von Anriss- und Bruchwöhlerlinie}

\section{Anhang J.2.1 Versuchsreihe 2}

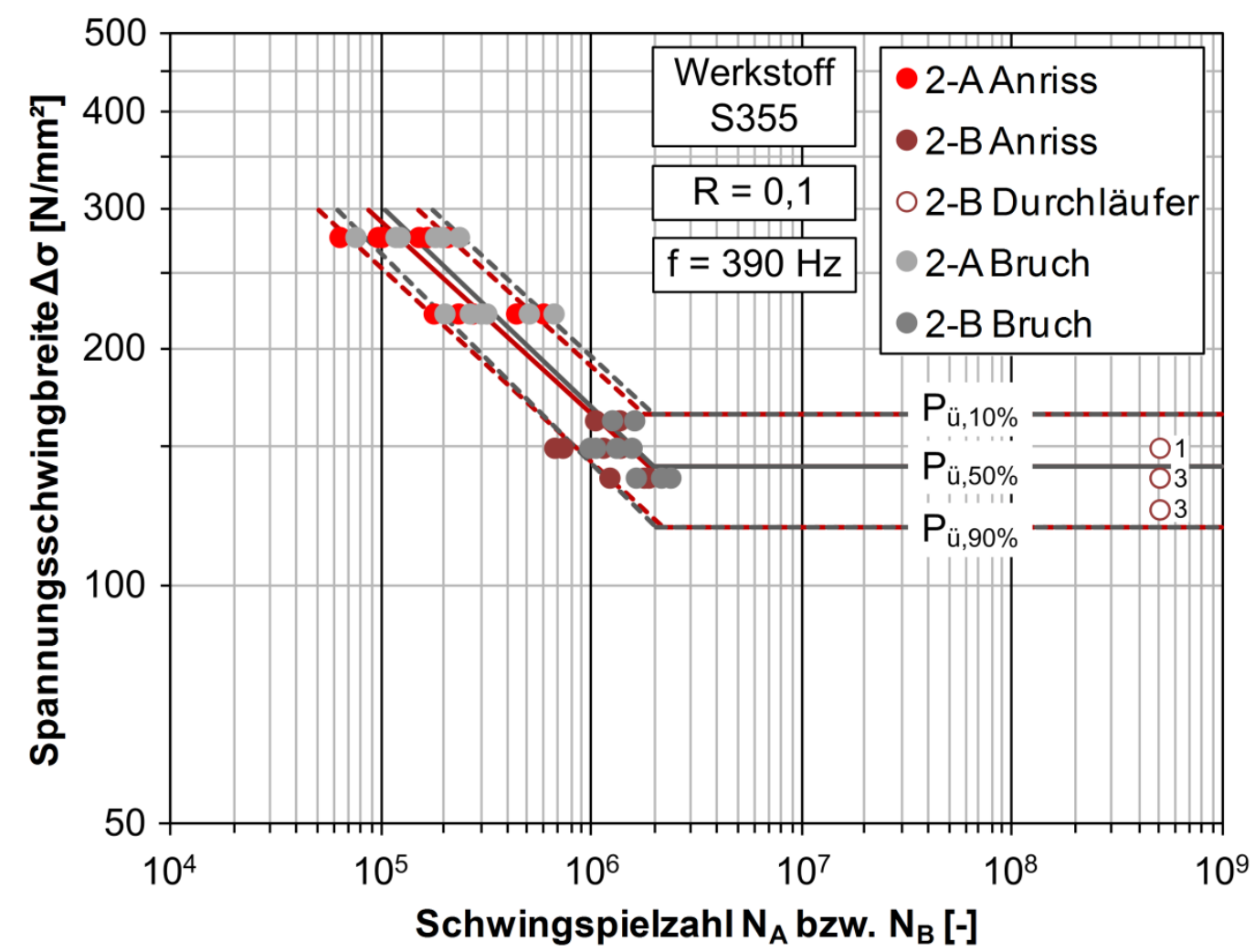

\begin{tabular}{|l|c|c|}
\hline \multicolumn{2}{|c|}{$\begin{array}{c}\text { Neigungsexponent der } \\
\text { Ermüdungsfestigkeitskurve } \\
\mathrm{k}[-]\end{array}$} & $\begin{array}{c}\text { mittlere } \\
\text { Dauerfestigkeit } \\
\Delta \sigma_{\mathrm{D}, 50 \%}\left[\mathrm{~N} / \mathrm{mm}^{2}\right]\end{array}$ \\
\hline Anriss & 4,1 & 1.901 .787 \\
\hline Bruch $^{*}$ & 3,9 & 1.960 .753 \\
\hline
\end{tabular}

* Bruch ist definiert bei Erreichen des Abbruchkriteriums $F_{v}=F_{a}$ 


\section{Anhang J.2.2 Versuchsreihe 3}

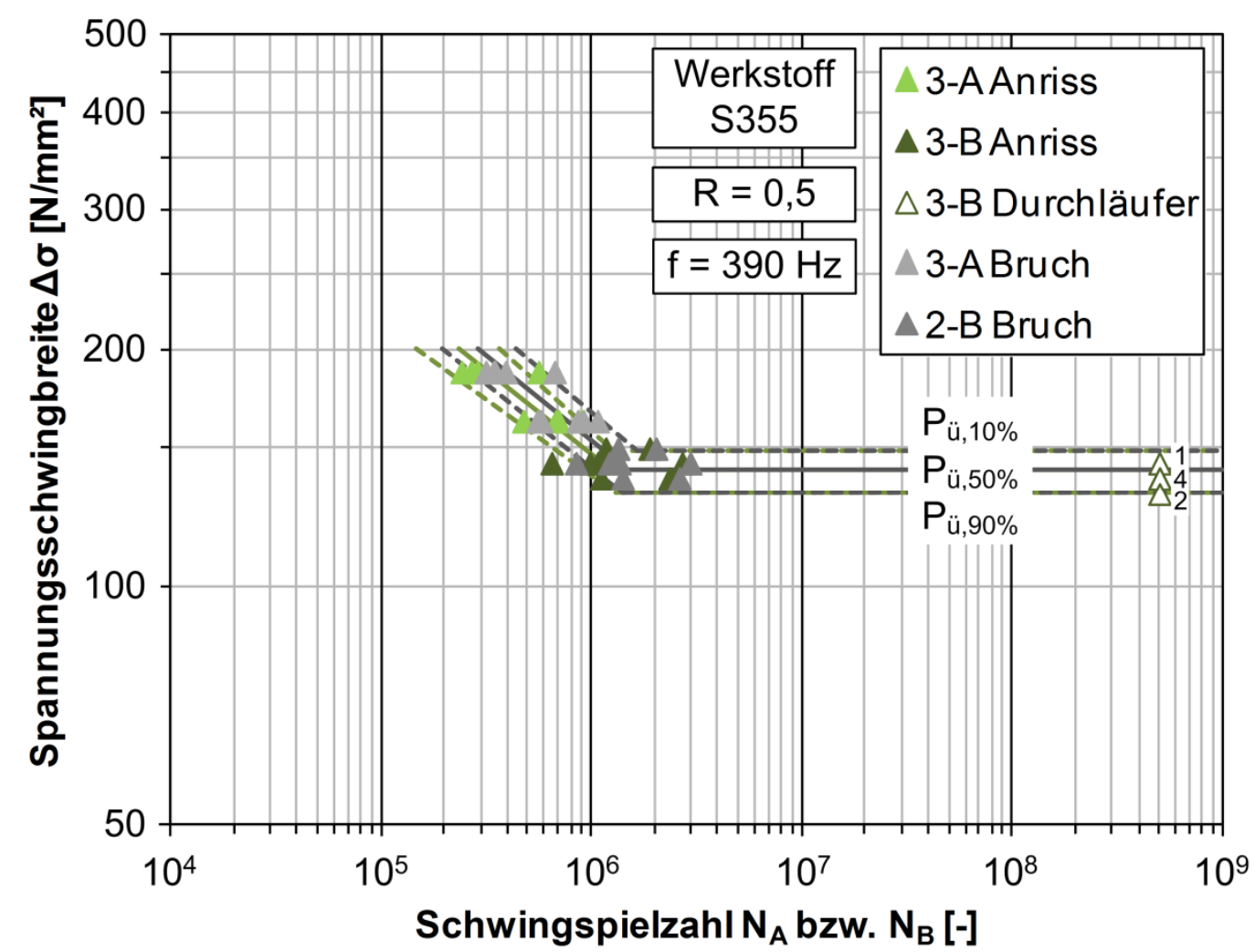

\begin{tabular}{|l|c|c|}
\hline \multicolumn{2}{|c|}{$\begin{array}{c}\text { Neigungsexponent der } \\
\text { Ermüdungsfestigkeitskurve } \\
\mathrm{k}[-]\end{array}$} & $\begin{array}{c}\text { mittlere } \\
\text { Dauerfestigkeit } \\
\Delta \sigma_{\mathrm{D}, 50 \%}\left[\mathrm{~N} / \mathrm{mm}^{2}\right]\end{array}$ \\
\hline Anriss & 4,7 & 1.239 .512 \\
\hline Bruch $^{* *}$ & 4,6 & 1.508 .278 \\
\hline
\end{tabular}

** Bruch ist definiert bei Erreichen des Abbruchkriteriums eines Vorspankraftabfalls von $5 \mathrm{kN}$ 


\section{Anhang J.3 Statistische Auswertung nach dem Hintergrundbericht zu EC3}

Die statistische Auswertung nach dem Hintergrundbericht zum Eurocode 3 erfolgte, wie bei Dürr [Dürr, 2007] erläutert, mit variablem und festem Neigungsexponenten k. Im Folgenden sind die ermittelten Ermüdungsfestigkeitskurven der statistischen Auswertung nach dem Hintergrundbericht zum Eurocode 3 für alle Versuchsreihen einzeln dargestellt.

\section{Anhang J.3.1 Versuchsreihe 1-A}

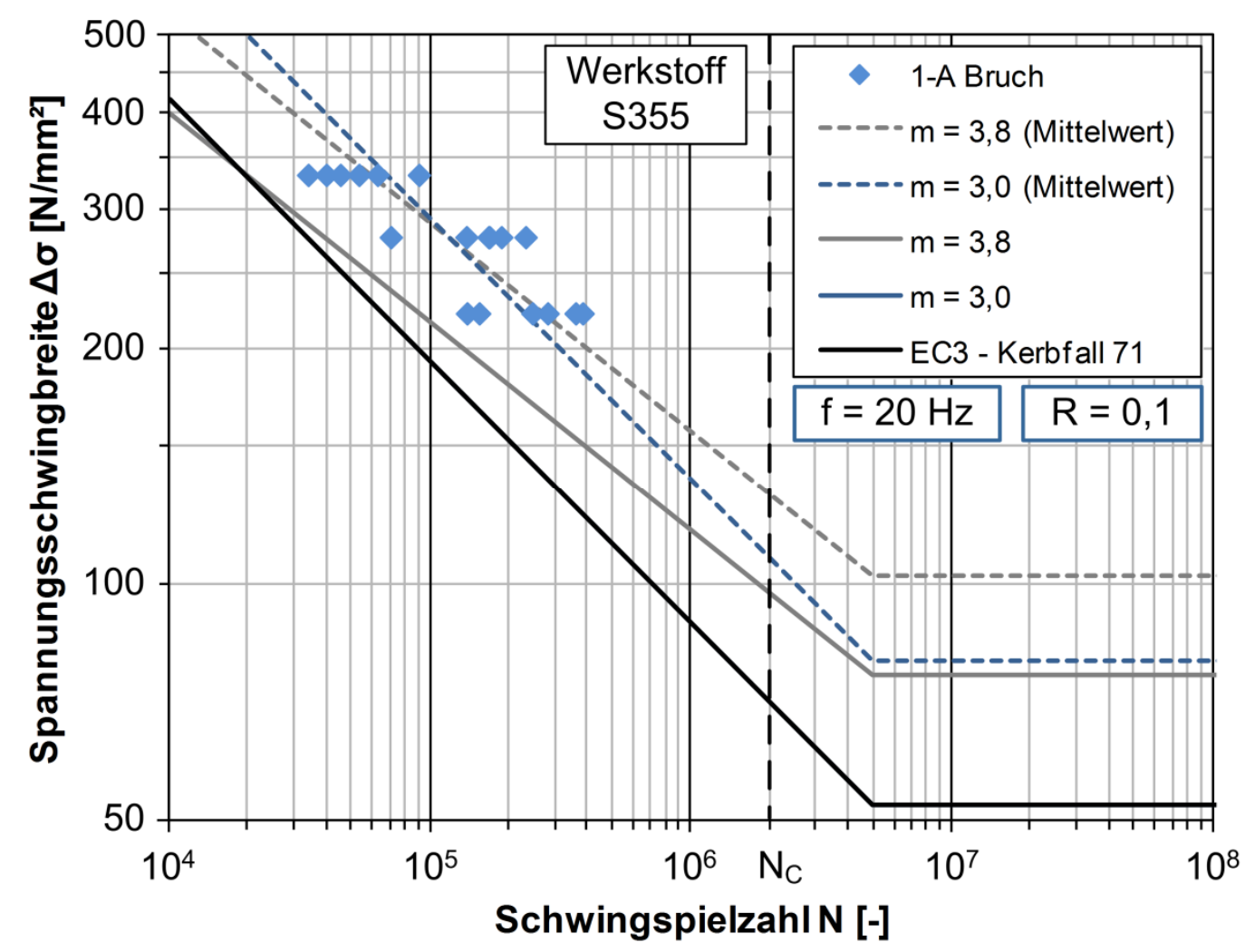

\begin{tabular}{|c|c|c|}
\hline \multicolumn{2}{|c|}{$\begin{array}{c}\text { Neigungsexponent der } \\
\text { Ermüdungsfestigkeitskurve } \\
\mathrm{k}[-]\end{array}$} & $\begin{array}{c}\text { charakteristische Ermüdungsfestigkeit } \\
\text { gemäß Hintergrundbericht zu EC3 } \\
\Delta \sigma_{\mathrm{C}}\left[\mathrm{N} / \mathrm{mm}^{2}\right]\end{array}$ \\
\hline variabel & 3,8 & 97,4 \\
\hline fest & 3,0 & 70,8 \\
\hline
\end{tabular}




\section{Anhang J.3.2 Versuchsreihe 2-A}

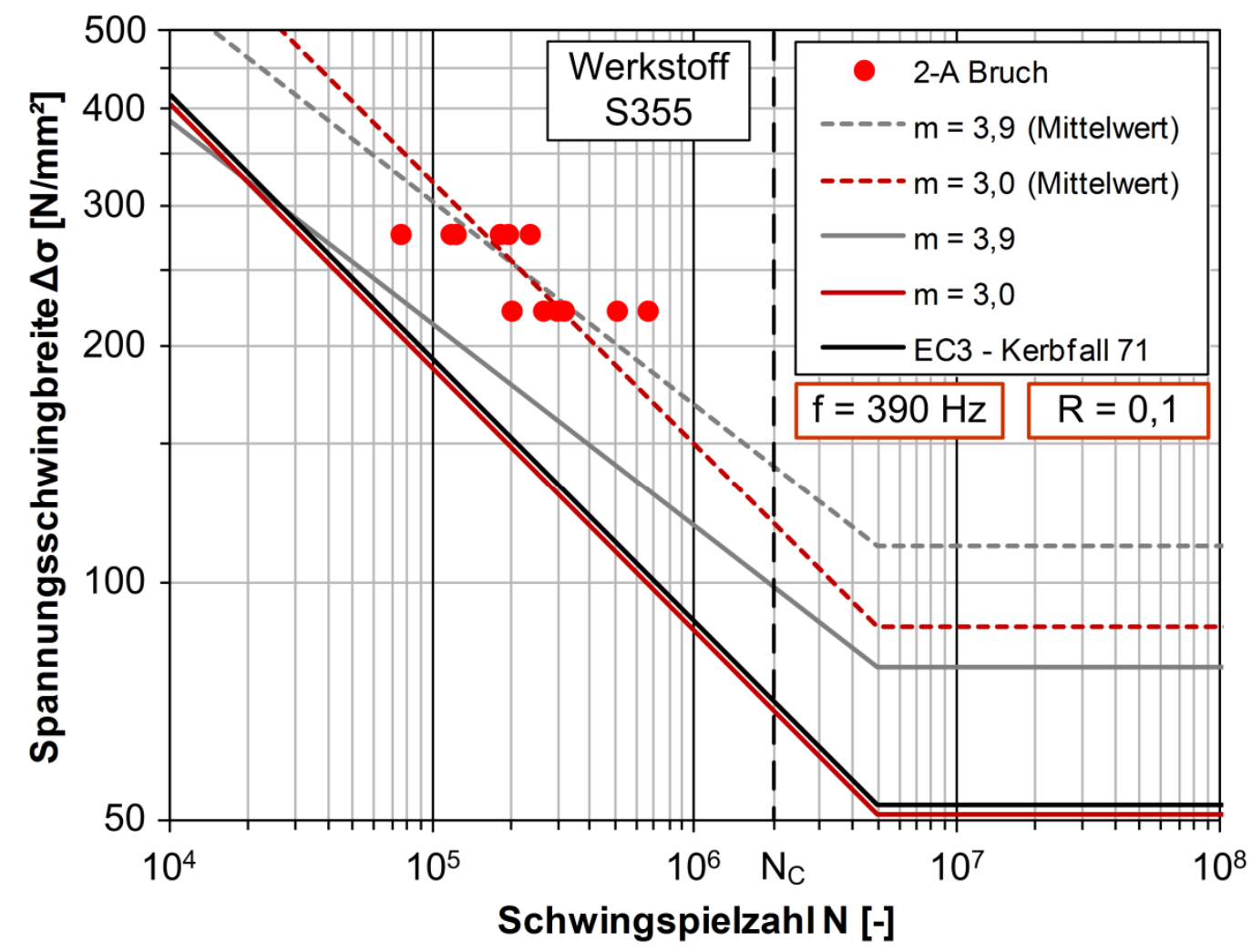

\begin{tabular}{|c|c|c|}
\hline \multicolumn{2}{|c|}{$\begin{array}{c}\text { Neigungsexponent der } \\
\text { Ermüdungsfestigkeitskurve } \\
\mathrm{k}[-]\end{array}$} & $\begin{array}{c}\text { charakteristische Ermüdungsfestigkeit } \\
\text { gemäß Hintergrundbericht zu EC3 } \\
\Delta \sigma_{\mathrm{C}}\left[\mathrm{N} / \mathrm{mm}^{2}\right]\end{array}$ \\
\hline variabel & 3,9 & 98,9 \\
\hline fest & 3,0 & 69,1 \\
\hline
\end{tabular}




\section{Anhang J.3.3 Versuchsreihe 3-A}

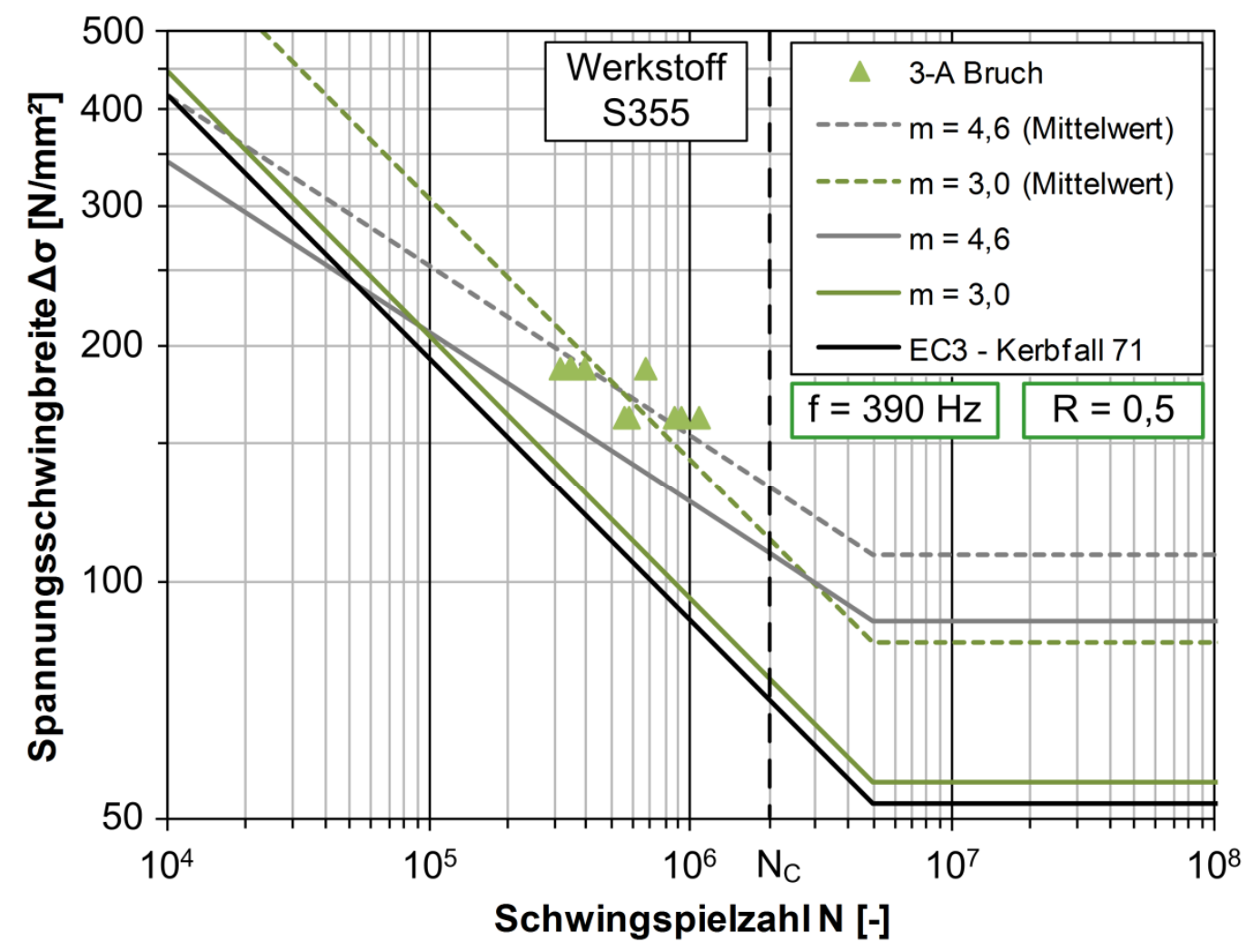

\begin{tabular}{|c|c|c|}
\hline \multicolumn{2}{|c|}{$\begin{array}{c}\text { Neigungsexponent der } \\
\text { Ermüdungsfestigkeitskurve } \\
\mathrm{k}[-]\end{array}$} & $\begin{array}{c}\text { charakteristische Ermüdungsfestigkeit } \\
\text { gemäß Hintergrundbericht zu EC3 } \\
\Delta \sigma_{\mathrm{C}}\left[\mathrm{N} / \mathrm{mm}^{2}\right]\end{array}$ \\
\hline variabel & 4,6 & 109,0 \\
\hline fest & 3,0 & 75,7 \\
\hline
\end{tabular}




\section{Lebenslauf}

Stefanie Steppeler

geboren am 19. März 1983 in Gütersloh

\section{Schulische Ausbildung und Studium}

1993 - 2002 Städtisches Gymnasium in Gütersloh

Juni $2002 \quad$ Abitur

$2002-2007 \quad$ Studium des Bauingenieurwesens an der Leibniz Universität Hannover mit der Vertiefungsrichtung Konstruktiver Ingenieurbau

September 2007 Diplom-Ingenieurin

\section{Praktische Erfahrungen und Auslandsaufenthalte}

$2003-2007 \quad$ Wissenschaftliche Hilfskraft am Institut für Stahlbau der Leibniz Universität Hannover

04/07 - 07/07 Forschungsaufenthalt an der University of British Columbia in Vancouver (Kanada)

\section{Berufliche Tätigkeiten}

$2007-2013 \quad$ Wissenschaftliche Mitarbeiterin am Institut für Stahlbau der Leibniz Universität Hannover

seit 2014

Mitarbeiterin der TÜV NORD EnSys Hannover GmbH \& Co. KG in Hannover 